
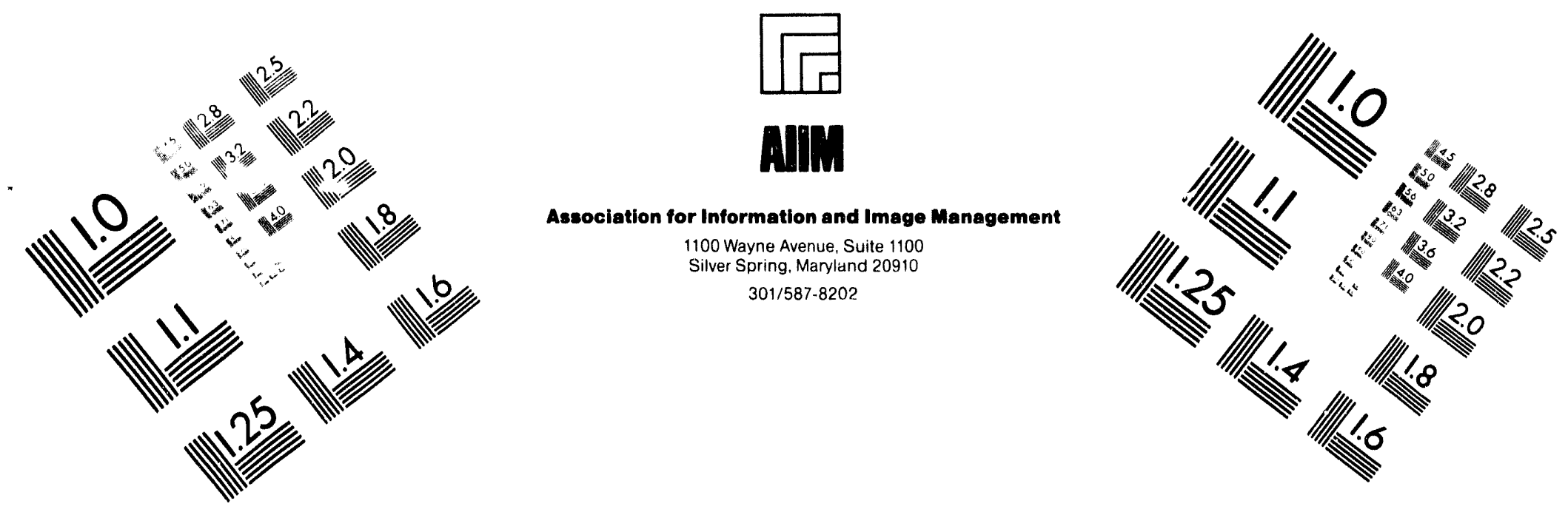

\title{
Centimeter
}

$\begin{array}{llllllllllllllll}1 & 2 & 3 & 4 & 5 & 6 & 7 & 8 & 9 & 10 & 11 & 12 & 13 & 14 & 15 & m m\end{array}$

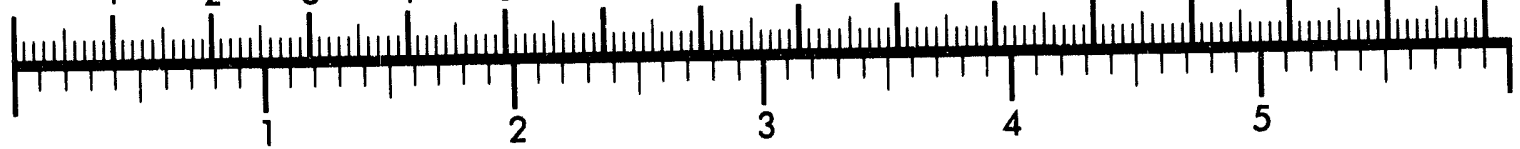
Inches
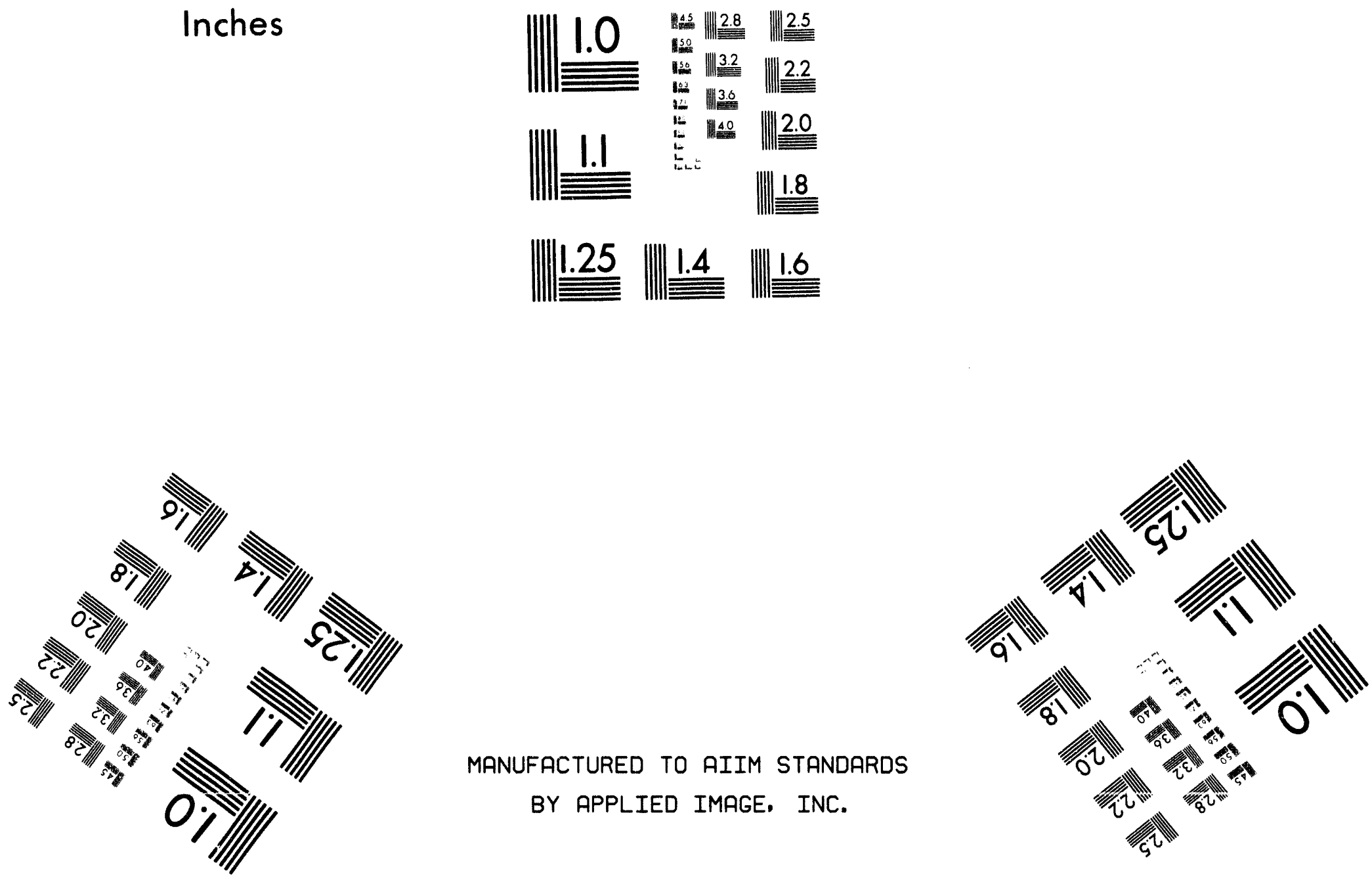

RI,:S-EIS DOCUMEN T: LOE/EIS-0185D-074-vol.2

REMEDIAL INVESTIGATION: DOE/OR/21548-074, VOL. ॥

\section{Remedial Investigation for the Chemical Plant Area of the Weldon Spring Site}

\section{Volume II}

November 1992

prepared by

MK-Ferguson Company and Jacobs Engineering Group

prepared for

U.S. Department of Energy, Oak Ridge Field Office, Weldon Spring Site Remedial Action Project, under Contract DE-AC05-860R21548

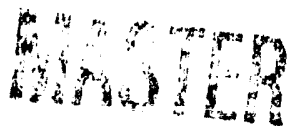




\section{VOLUME II \\ LIST OF FIGURES}

FIGURE NUMBER

ES-1

ES-2

ES-3

ES-4

ES-5

ES-6

ES-7

$1.2-1$

1.3-1

$3.1-1$

$3.1-2$

$3.1-3$

\section{TITLE}

Area and Vicinity Map of the Weldon Spring Site, Weldon Spring Missouri

Weldon Spring Site Vicinity Map

Isopach Map of Total Overburden

Regional Structural Features

Potentiometric Surface, Shallow Wells - December 1988

Surface Water Drainages Near the WSS

Conceptual Model for Contaminant Source, Transport \& Exposure

Area and Vicinity Map of the Weldon Spring Site, Weldon Spring Missouri

Weldon Spring Site Vicinity Map

Weldon Spring Chemical Plant and Raffinate Pits

Conceptual Model for Contaminant Source, Transport \& Exposure

Contaminant Source Areas

Sample Locations of Exterior Bulk Sampling for ACM

Potential Chemical Contamination Source Areas Associated with the Former Weldon Spring Ordnance Works

Phase I Soil Sampling Locations

Radiologically Contaminated Property Locations

Background Sample Locations 


\section{LIST OF FIGURES (Continued)}

\section{FIGURE NUMBER}

$3.4-2$

3.4-3

3.4-4

4.1-1

4.1-2

4.1-3

$4.2-1$

$4.2-2$

4.2-3

4.3-1

4.3-2

4.3-3

4.3-4

4.3-5

4.3-6

Extended Monitoring Well Network

Deep USGS Monitoring Well Cluster Locations

TITLE

Pumping and Observation Well Locations

Water Tracing Sample Locations

Preliminary Map of Private Water Wells in the Vicinity of the WSS

Surface Hydrological Features in the Vicinity of the Raffinate Pits and Chemical Plant Area

Topographic Map of the Weldon Spring Area (Elevations in Feet Above MSL)

Map of the WSCP/WSRP

Annual Wind Rose for the WSS-1985

Monthly Precipitation 1983-1988

Location of USGS Rain Gauges

Generalized Stratigraphic Column

Borehole and Trench Location Map with Cross Sections

Typical Borehole Lithology

Geologic Cross Section A-A'

Geologic Cross Section B-B'

Geologic Cross Section C-C' 


\section{LIST OF FIGURES (Continued)}

FIGURE NUMBER

4.3-8

4.3-9

4.3-10

4.3-11

$4.3-12$

4.3-13

4.3-14

$4.3-15$

4.3-16

4.3-17

4.3-22

4.3-23

4.3 .24

\section{TITLE}

Geologic Cross Section D-D'

Geologic Cross Section E-E'

Isopach Map of Total Overburden

Isometric View of Total Overburden Thickness

Isopach Map of Topsoil/Fill

Isopach Map of Ferrelview Formation

Isopach Map of Clay Till

Isopach Map of Basal Till

Isopach Map of Residuum

Isopach Map of Weathered Limestone

Contour Map of Top of Weathered Limestone

Isometric View of Top of Weathered Limestone

Contour Map of Top of Competent Limestone

Isometric View of Top of Competent Limestone

Structure on Top of Fern Glen Formation

Structure on Top of Bushberg Sandstone

Structure on Top of St. Peter Sandstone

Regional Structural Features 


\section{LIST OF FIGURES (Continued)}

\section{FIGURE NUMBER}

4.3-25

4.4-1

4.4-3

4.4-5

$4.4-6$

$4.5-4$

$4.5-5$

\section{TITLE}

Block Diagram Depicting Genesis of Weathered Bedrock Surface

Surface Water Drainages Near the WSS

Surface Water Drainage at the Weldon Spring Site

NPDES Surface Water Sampling Locations

Springs and Seeps in the Vicinity of the WSS

Hydrographs of Burgermeister Spring and a Nearby Wet-Weather Spring, March 1985 to April 1986

Seepage Run Results - Schote Creek and Vicinity, December 11, 1984

Seepage Run Results - Tributary to Dardenne Creek Upstream of Lake 34, April 2-3, 1985

Seepage Run Results - Schote Creek Upstream of Lake 35, April 2-3, 1985

Losing Stream Reaches Identified From Seepage Runs

Dye Traces Near the WSS

Water Tracing Study Southeast Drainage, October 28-November 2, 1987

Vadose Zone Thickness, July 1988

Storm Sewer Locations

Lysimeter Locations

Overburden Monitoring Wells and Piezometers

Cross-Section Through Raffinate Pits 3 and 4 


\section{LIST OF FIGURES (Continued)}

FIGURE NUMBER

4.5-6

4.6-1

4.6-2

4.6-3

4.6-4

4.6-5

4.6-6

4.6-7

4.6-8

4.6-9

4.6-10

4.6-11

4.6-12

4.6-13

4.6-14

4.6-15

4.6-16

4.6-17
Perched/Mounded Groundwater at the WSS

Potentiometric Surface of the Shallow Bedrock Aquifer, Summer 1984

Potentiometric Surface of the Deep Bedrock Aquifer, Summer 1984

Potentiometric Surface, Shallow Bedrock Aquifer - WSS Vicinity, July 1987

Variation in Hydraulic Conductivity with Depth

Potentiometric Surface, Shallow Wells - July 1988

Potentiometric Surface, Deep Wells - July 1988

Potentiometric Surface, Shallow Wells - December 1988

Potentiometric Surface, Deep Wells - December 1988

Hydrogeologic Cross-Section Locations

Hydrogeologic Cross-Section A-A'

Hydrogeologic Cross-Section B-B'

Hydrogeologic Cross-Section C-C'

MW 2001 Hydrograph

MW 2004 Hydrograph

MW 2010 Hydrograph

MW 2012 Hydrograph

Water Level Fluctuation Map 


\section{LIST OF FIGURES (Continued)}

FIGURE NUMBER

4.6-18

$5.1-1$

$5.1-2$

$5.1-3$

$5.1-4$

$5.1-5$

$5.1-6$

$5.2-1$

$5.2-2$

$5.2-3$

$5.2-4$

$5.2-5$

$5.2-6$

$5.2-7$

$5.2-8$

$5.2-9$

$5.2-10$
Depth to Groundwater in Upper 10 Feet of Bedrock, December 1988

Potential Nitroaromatic Source Areas at the WSCP/WSRP

Potential Chemical Contamination Source Areas

Potential Chemical Contamination Source Areas Associated with the WSCP/WSRP

Buildings and Electrical Equipment Containing PCBs at the Weldon Spring Site

Sample Locations of Exterior Bulk Sampling for ACM

Southeast Site Drainage

Location of Elevated Nitroaromatics Concentration

Location of Elevated Nitrate Concentration in Soils

Location of Elevated Nitrite Concentration in Soils

Location of Elevated Sulfate Concentration in Soils

Location of Elevated Fluoride Concentration in Soils

Location of Elevated Chloride Concentration in Soils

Location of Elevated Silver Concentration in Soils

Location of Elevated Aluminum Concentration in Soils

Location of Elevated Arsenic Concentration in Soils

Location of Elevated Barium Concentration in Soils 


\section{LIST OF FIGURES (Continued)}

FIGURE NUMBER

\section{TITLE}

Location of Elevated Calcium Concentration in Soils

Location of Elevated Cadmium Concentration in Soils

Location of Elevated Cobalt Concentration in Soils

Location of Elevated Chromium Concentration in Soils

Location of Elevated Copper Concentration in Soils

Location of Elevated Iron Concentration in Soils

Location of Elevated Mercury Concentration in Soils

Location of Elevated Potassium Concentration in Soils

Location of Elevated Lithium Concentration in Soils

Location of Elevated Magnesium Concentration in Soils

Location of Elevated Manganese Concentration in Soils

Location of Elevated Molybdenum Concentration in Soils

Location of Elevated Sodium Concentration in Soils

Location of Elevated Nickel Concentration in Soils

Location of Elevated Lead Concentration in Soils

Location of Elevated Selenium Concentration in Soils

Location of Elevated Thallium Concentration in Soils 


\section{LIST OF FIGURES (Continued)}

FIGURE NUMBER

$5.2-30$

$5.2-31$

$5.2-32$

$5.2-33$

$5.2-44$
Location of Elevated Vanadium Concentration in Soils

Location of Elevated Zinc Concentration in Soils

Locations of Detected Semi-Volatile Compound Concentrations in Soils

Location of Contaminated Areas on Army Reserve Property

Contamination Limits for U.S. Army Reserve Property \# 1 (DA 1)

Contamination Limits for U.S. Army Reserve Property \# 2 (DA 2)

Contamination Limits for U.S. Army Vicinity Property \# 3 (DA 3)

Contamination Limits for U.S. Army Vicinity Property \# 5 (DA 5)

Contamination Limits for U.S. Army Vicinity Property \# 6 (DA 6)

Contamination Limits for U.S. Army Vicinity Property \# 7 (DA 7)

Locations of Contamination Identified on the Weldon Spring and August Busch Wildlife Areas

Contamination Limits for Department of Conservation Vicinity Property \# 3 (DOC 3)

Contamination Limits for Department of Conservation Vicinity Property \# 4 (DOC 4)

Contamination Limits for Department of Conservation Vicnity Property \# 5 (DOC 5)

Area of Contamination (DOC 6) Near the Quarry Perimeter Fence

Contamination Limits for Department of Conservation Vicinity Property \# 8 (DOC 8) 


\section{LIST OF FIGURES (Continued)}

FIGURE NUMBER

$5.2-45$

$5.3-1$

$5.3-2$

$5.3-4$

$5.3-6$

\section{TITLE}

Contamination Limits for the Southeast Drainage Easement (SDE)

Surface Water and NPDES Sampling Locations

Total Uranium Concentrations (pCi/l) - Surface Water

Total Uranium Concentrations ( $\mathrm{pCi} / \mathrm{l})$ - Springs

Detectable Total Uranium Concentrations (pCi/l) - Sediment

Nitrate Concentrations (mg/l) - Surface Water

Nitrate Concentrations $(\mathrm{mg} / \mathrm{l})$ - Springs

The Concentration of Nitrate as a Function of the Discharge at Burgermeister Spring

Detectable Nitroaromatic Compound Concentrations $(\mu \mathrm{g} / \mathrm{l})$ - Surface Water

Detectable Nitroaromatic Compound Concentrations $(\mu \mathrm{g} / \mathrm{l})$ - Springs

Weathered Limestone Uranium Concentrations (pCi/l)

Competent Limestone Uranium Concentrations (pCi/l)

Weathered Limestone Nitrate Concentrations (mg/l)

Competent Limestone Nitrate Concentrations ( $\mathrm{mg} / \mathrm{l}$ )

Weathered Limestone Sulfate Concentrations (mg/l)

Competent Limestone Sulfate Concentrations (mg/l)

Weathered Limestone Chromium Concentrations $(\mu \mathrm{g} / \mathrm{l})$ 


\section{LIST OF FIGURES (Continued)}

FIGURE NUMBER

Weathered Limestone Nickel Concentrations $(\mu \mathrm{g} / \mathrm{l})$

$5.4-10$

$5.5-3$ $5.5-4$

\section{TITLE}

Weathered Limestone Aluminum Concentrations $(\mu \mathrm{g} / \mathrm{l})$

Weathered Limestone 2,4,6-Trinitrotoluene Concentrations $(\mu \mathrm{g} / \mathrm{l})$

Competent Limestone 2,4,6-Trinitrotoluene Concentrations ( $\mu \mathrm{g} / \mathrm{l})$

Weathered Limestone 1,3,5-Trinitrobenzene Concentrations ( $\mu \mathrm{g} / \mathrm{l})$

Competent Limestone 1,3,5-Trinitrobenzene Concentrations $(\mu \mathrm{g} / \mathrm{l})$

Weathered Limestone 2,4-Dinitrotoluene Concentrations ( $\mu \mathrm{g} / \mathrm{l})$

Competent Limestone 2,4-Dinitrotoluene Concentrations ( $\mu \mathrm{g} / \mathrm{l})$

Weathered Limestone 2,6-Dinitrotoluene Concentrations ( $\mu \mathrm{g} / \mathrm{l})$

Competent Limestone 2,6-Dinitrotoluene Concentrations ( $\mu \mathrm{g} / \mathrm{l})$

Weathered Limestone 1,3-Dinitrobenzene Concentrations $(\mu \mathrm{g} / \mathrm{l})$

Competent Limestone 1,3-Dinitrobenzene Concentrations $(\mu \mathrm{g} / \mathrm{l})$

Weathered Limestone Nitrobenzene Concentrations $(\mu \mathrm{g} / \mathrm{l})$

Competent Limestone Nitrobenzene Concentrations $(\mu \mathrm{g} / \mathrm{l})$

August A. Busch Wildlife Complex

Terrestrial and Aquatic Habitats at the Weldon Spring Site

Location of Lakes on the Busch Wildlife Area

Small Mammal Sampling Areas at WSS 


\section{LIST OF FIGURES (Continued)}

FIGURE NUMBER

Location of Air Monitoring Stations

$6.1-1$

Surface Water Flow Paths from the WSS

$6.1-2$

Possible Flow Paths in the Vadose Zone

$6.1-3$

Permeability Zones in Fractured Carbonate Rock

6.1-4

Surface Water-Groundwater Relationship Along S.E. Drainage Easement

6.1-5

Subsurface Flow Paths

$6.3-1$

Nitrate Flux as a Function of Discharge at Burgermeister Spring

6.3-2 


$$
\ldots
$$




\section{VOLUME II \\ LIST OF TABLES}

TABLE NUMBER

\section{TITLE}

1.2-1 Previous Investigations

1.2-2 Interim Response Actions (IRAs)

1.3-1 Chemicals Used in Former Explosives Production Facility and Uranium Feed Materials Processing Plant

1.3-2 Chemical Substances Analyzed in Water and Soil Investigations

1.3-3 Primary Contaminants for the Weldon Spring Site

1.4-1 Laws and Orders Potentially Applicable or Relevant and Appropriate to the Weldon Spring Site Remedial Action Project

2.1-1 Quality Assurance Program Plan Elements

2.3-1 Analytical Methods

2.5-1 Data Quality Assessments

3.1-1 Summary of Contaminant Sources

3.4-1 Groundwater Sample Analysis and Preservation

3.7-1 Summary of Boreholes and Trenches Used in Database

3.7-2 Summary of Geotechnical Investigations

4.1-1 Major Buildings, Structures and Facilities at the Chemical Plant

4.2-1 Precipitation

4.2-2 Frequency of Extreme Precipitation

4.2-3 Temperatures

4.2-4 Frequency of Temperature Extremes 


\section{LIST OF TABLES (Continued)}

TABLE NUMBER

\section{TITLE}

4.2-5 Relative Humidity and Heat Stress Factors

4.2-6 Wind Speed and Direction

4.2-7 Precipitation Recorded at the Weldon Spring Site

4.2-8 Temperatures Recorded at the Weldon Spring Site

4.3-1 Borehole and Trench Numbering System

4.3-2 Boreholes and Trenches Used in the Geologic Database

4.3-3 Summary of Laboratory Testing of Overburden Soil Samples (October 1988 to May 1989)

4.3-4 Summary of Laboratory Testing of Overburden Soil Samples (from BNI 1987)

4.3-5 Summary of Rock Quality Designation (RQD)

4.4-1 Physical Properties of the Four Raffinate Pits

4.4-2 Drainage Areas of Surface Streams in the Vicinity of the Weldon Spring Site

4.4-3 Average Measured Flow Rate from NPDES Discharge Points in 1987 and Estimated Average Annual Runoff

4.4-4 Mean Daily Discharge for Burgermeister Spring, March 20, 1985 through April 30, 1986

4.4-5 Mean Daily Discharge for Wet-Weather Spring, March 20, 1985 through April 30, 1986

4.5-1 Lysimeter Installations

4.5-2 Overburden Monitoring Well and Piezometer Construction Data 


\section{LIST OF TABLES (Continued)}

TABLE NUMBER

4.5-3 Water Elevations Recorded in Overburden Monitoring Wells at the Weldon Spring Site

4.6-1 Monitoring Well Construction Data

4.6-2 Hydraulic Conductivity in Bedrock as Determined by Packer Tests

4.6-3 Hydraulic Conductivity Values in Shallow Wells from In Situ Tests

4.6-4 Hydraulic Conductivity Values in Paired Deep and Shallow Monitoring Wells from In Situ Tests

4.6-5 Comparison of Hydraulic Conductivity Values from Packer Tests and In Situ Tests

4.6-6 Angled Borehole Packer Test Results

4.6-7 Aquifer Parameters Determined from Pumping Tests

4.6-8 Water Level Elevations in Shallow and Deep Monitoring Wells

4.6-9 Monitoring Well Dye Injection Results

4.6-10 Hydraulic Gradients and Hydraulic Conductivities and Resulting Range of Linear Velocities of Groundwater

4.7-1 Population in the St. Louis Standard Metropolitan Statistical Area

4.7-2 Population of the Region Surrounding the Weldon Spring Site, 1960-1990

5.1-1 Potential Sources of Contaminants at the Weldon Spring Site

5.1-2 Preliminary Listing of On-Site Tanks

5.1-3 Summary of PCB Measurements

5.1-4 Asbestos Content of Pipe Insulation on Overhead Utilities 


\section{LIST OF TABLES (Continued)}

\section{TABLE NUMBER}

\section{TITLE}

5.1-5 Summary of Asbestos Measurements

5.1-6 Compilation of Asbestos Data, Samples Suspected of Radiological Contamination

5.1-7 Compilation of Asbestos Data, Samples Not Suspected of Radiological Contamination

5.1-8 Weldon Spring Raffinate Pits Sludige Sample Radionuclide Activities

5.1-9 Inorganic Anion and Water Quality Data for the Raffinate Pits

5.1-10 Historical Concentrations of Inorganic Anions in Raffinate Pit Water and Sludge

5.1-11 Metals Concentrations in Raffinate Pit Water

5.1-12 Radiological Test Results - Raffinate Pit Water

5.1-13 Summary of the Characteristics of the Raffinate Pits

5.1-14 Raffinate Pit Sludge Anions Summary

5.1-15 Raffinate Pit Sludge Metals Summary

5.1-16 Raffinate Pit Radionuclide Contents Determined from the WSSRAP Project Management Contractor Study 1988

5.1-17 Total Activity Inventory of Ra-226 per Pit at the Time of Sampling and at 200 Years and 1000 Years after Sampling

5.1-18 Average Concentration of Ra-226 per Pit at the Time of Sampling and at 200 Years and 1000 Years after Sampling

5.1-19 Radiological Analyses Results of Surface Water from the Weldon Spring Raffinate Pits Determined from WSSRAP Project Management Contractor Study 1989

5.1.20 Raffinate Pit Water Data - Metals 


\section{LIST OF TABLES (Continued)}

TABLE NUMBER

5.2-1 Statistical Summary of Background Soil Analysis

5.2-2 Soil Contaminant Concentrations Above Background

5.2-3 Borehole and Surface Soil Sample Locations Not Shown On Plate 2

5.2-4 Summary of Estimated Areas, Depths, and Volumes in Zones

5.2-5 Summary of Contamination on Vicinity Property DA 1

5.2-6 Summary of Contamination on Vicinity Property DA 2

5.2-7 Summary of Vicinity Propery Estimated Areas, Depths, Volumes, and Average Primary Contaminant Concentrations

5.3-1 Summary of Chemical Analyses of Surface Water and Springs

5.3-2 Analyses of Uranium as Presented in Figures 5.3-2 and 5.3-3

5.3-3 Analyses of Nitrate as Presented in Figures 5.3-5 and 5.3-6

5.3-4 Analyses of 2,4,6-Trinitrotoluene as Presented in Figures 5.3-8 and 5.3-9

5.3-5 Analyses of 1,3,5-Trinitrobenzene as Presented in Figures 5.3-8 and 5.3-9

5.3-6 Analyses of 2,4-Dinitrotoluene as Presented in Figures 5.3-8 and 5.3-9

5.3-7 Analyses of 2,6-Dinitrotoluene as Presented in Figures 5.3-8 and 5.3-9

5.3-8 Analyses of 1,3-Dinitrobenzene as Presented in Figures 5.3-8 and 5.3-9

5.3-9 Analyses of Nitrobenzene as Presented in Figures 5.3-8 and 5.3-9

5.4-1 Analysis of Total Constituents (Dissolved and Suspended) in Water from Raffinate Pits, Lysimeters, and Overburden Monitoring Wells

m:lusersireneldocument|ri-revfltext|tb|-list.vii

xvii

102992 


\section{LIST OF TABLES (Continued)}

TABLE NUMBER

5.4-2 Summary of Chemical Analyses of Groundwater

5.4-3 Analyses of Uranium as Presented in Figures 5.4-1 and 5.4-2

5.4-4 Analyses of Nitrate as Presented in Figures 5.4-3 and 5.4-4

5.4-5 Analyses of Sulfate as Presented in Figures 5.4-5 and 5.4-6

5.4-6 Analyses of Chromium as Presented in Figure 5.4-7

5.4-7 Analyses of Nickel as Presented in Figure 5.4-8

5.4-8 Analyses of Aluminum as Presented in Figure 5.4-9

5.4-9 Analyses of 2,4,6-Trinitrotoluene as Presented in Figures 5.4-10 and 5.4-11

5.4-10 Analyses of 1,3,5-Trinitrobenzene as Presented in Figures 5.4-12 and 5.4-13

5.4-11 Analyses of 2,4-Dinitrotoluene as Presented in Figures 5.4-14 and 5.4-15

5.4-12 Analyses of 2,6-Dinitrotoluene as Presented in Figures 5.4-16 and 5.4-17

5.4-13 Analyses of 1,3-Dinitrobenzene as Presented in Figures 5.4-18 and 5.4-19

5.4-14 Analyses of Nitrobenzene as Presented in Figures 5.4-20 and 5.4-21

5.5-1 Vegetation Surveyed at the Weldon Spring Chemical Plant Area

5.5-2 Bird and Waterfowl Species Observed at the Weldon Spring Chemical Plant Area

5.5-3 List of Birds Observed During the Haul Road Ecological Survey June 24-28, 1991

5.5-4 List of Mammals Found in the Weldon Spring Wildlife Area

5.5-5 List of Reptiles and Amphibians Found in the Weldon Spring Wildlife Area 


\section{LIST OF TABLES (Continued)}

TABLE NUMBER

TITLE

5.5-6 Threateneci, Endangered, or Special Concern Species Reported from St. Charles County, Missouri, and Potentially Occurring on the Weldon Spring Site

5.5-7 Vertebrate Species Sampled at the Weldon Spring Site

5.5-8 Distribution of Uranium, Thorium and Radium-226 in the Frog Pond System

5.5-9 Natural Uranium Concentrations in Fish and Water Bodies of the Busch Wildlife Area

5.5-10 Radiological Concentrations in Fish Biota

5.5-11 Biouptake PCB Results for Fish

5.5-12 Biouptake CLP Metals Results for Fish Mixed Species Composite Samples

5.5-13 Comparison of Bioaccumulation Faciors for Uranium

5.5-14 Small Mammal Radiological Results

5.5-15 Raffinate Pit 4 Biota Radionuclide Concentrations

5.5-16 Bioaccumulation Factors for Raffinate Pit 4 Biota

5.6-1 Radon Measurements at the Weldon Spring Site in 1989

5.6-2 Gamma Radiation Exposure Rate Monitoring Results

5.6-3 Radiological Air Particulate Results 1989

5.6-4 Asbestos Monitoring Results for 1988-1989

6.2-1 Chemical Properties of Nitroaromatics 


\section{VOLUME II \\ LIST OF PLATES}

\section{PLATE NUMBER}

Plate 1

Plate 2

\section{TITLE}

1943 Weldon Spring Ordnance Works

Site Soil Zones with Greater than $15 \mathrm{pCi} / \mathrm{g} \mathrm{U}-238,5 \mathrm{pCi} / \mathrm{g} \mathrm{Ra}-226$, and 5 pCi/g Th-230 
REMEDIAL INVESTIGATION REPORT

FIGURES 


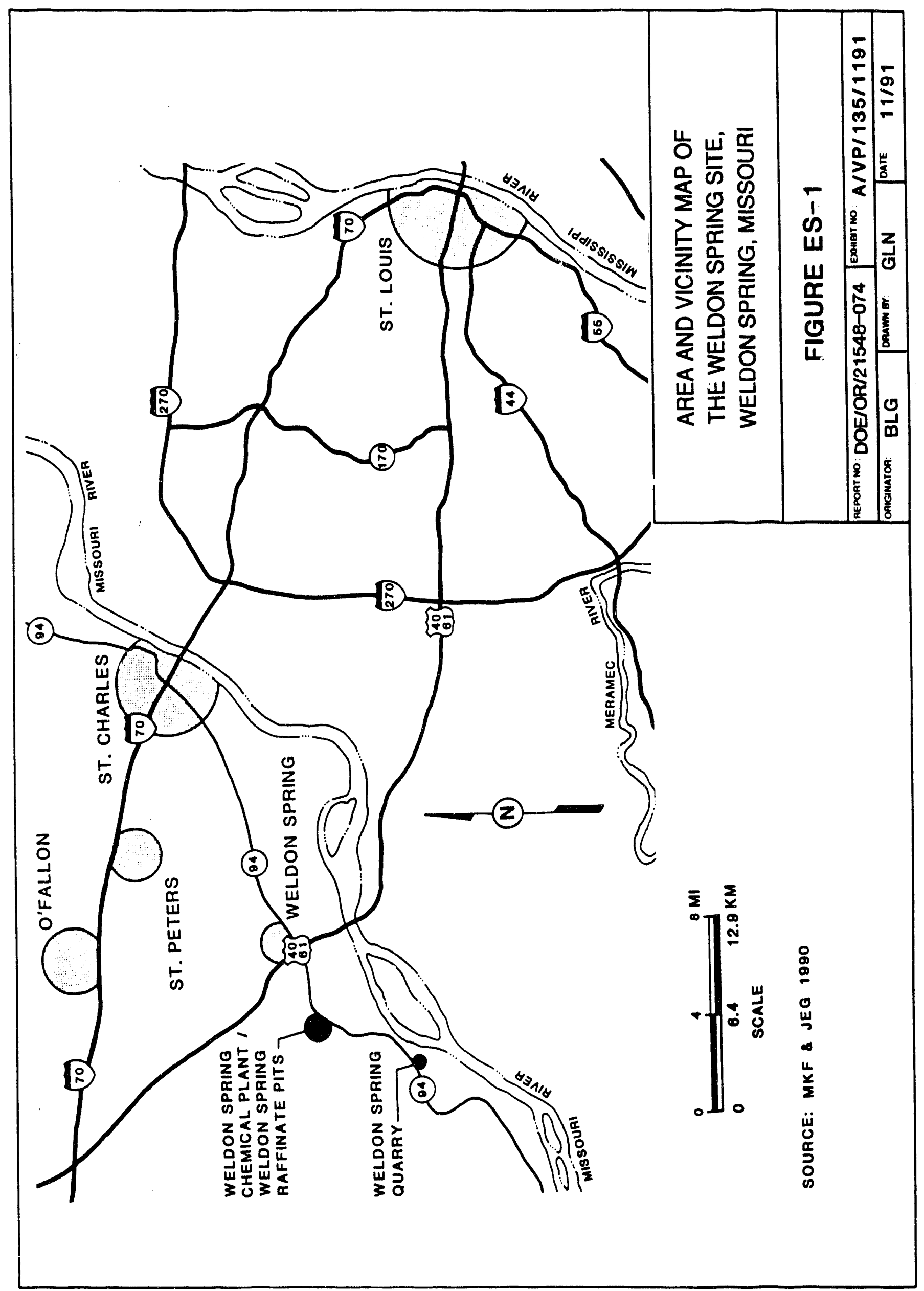




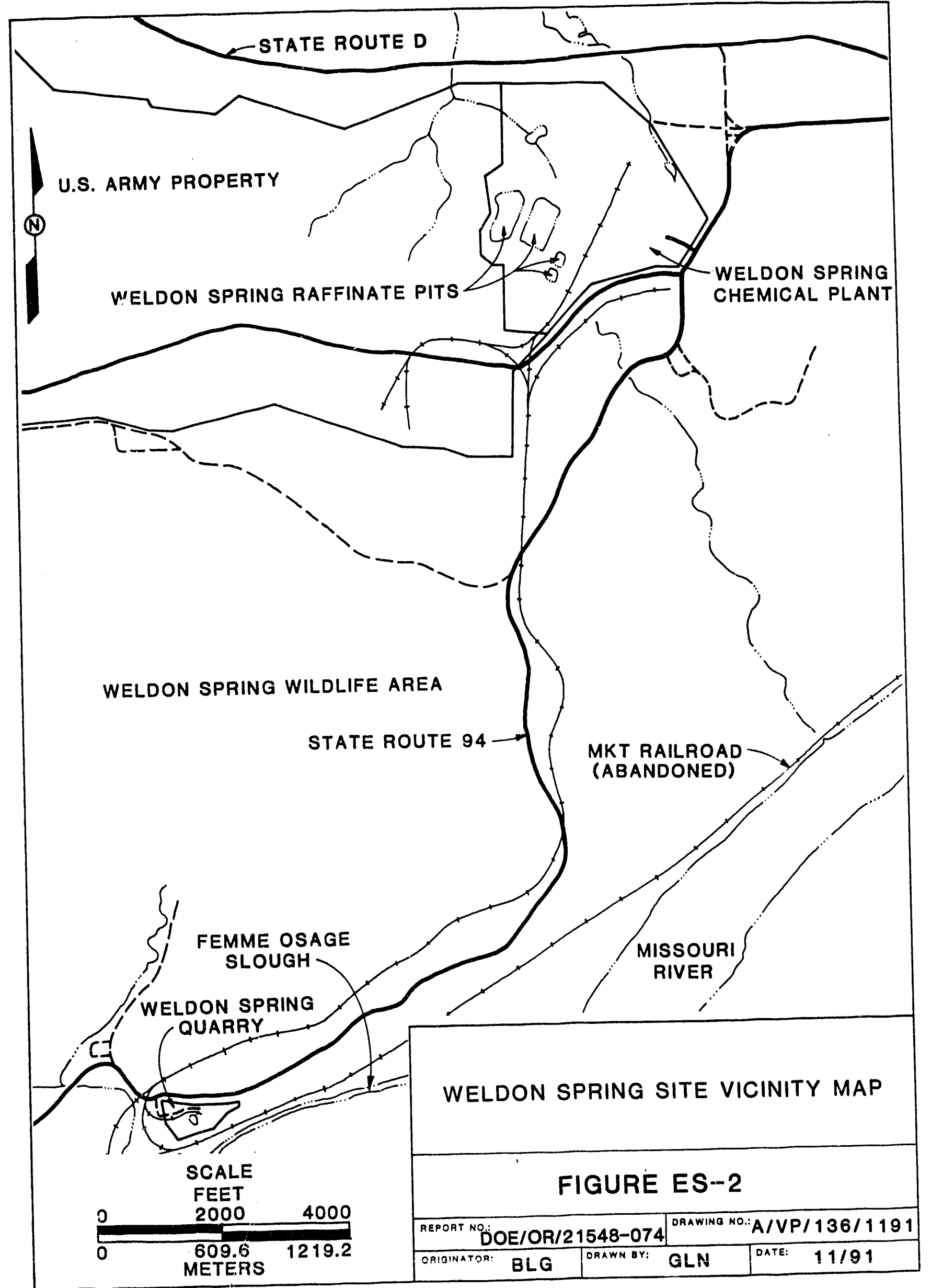




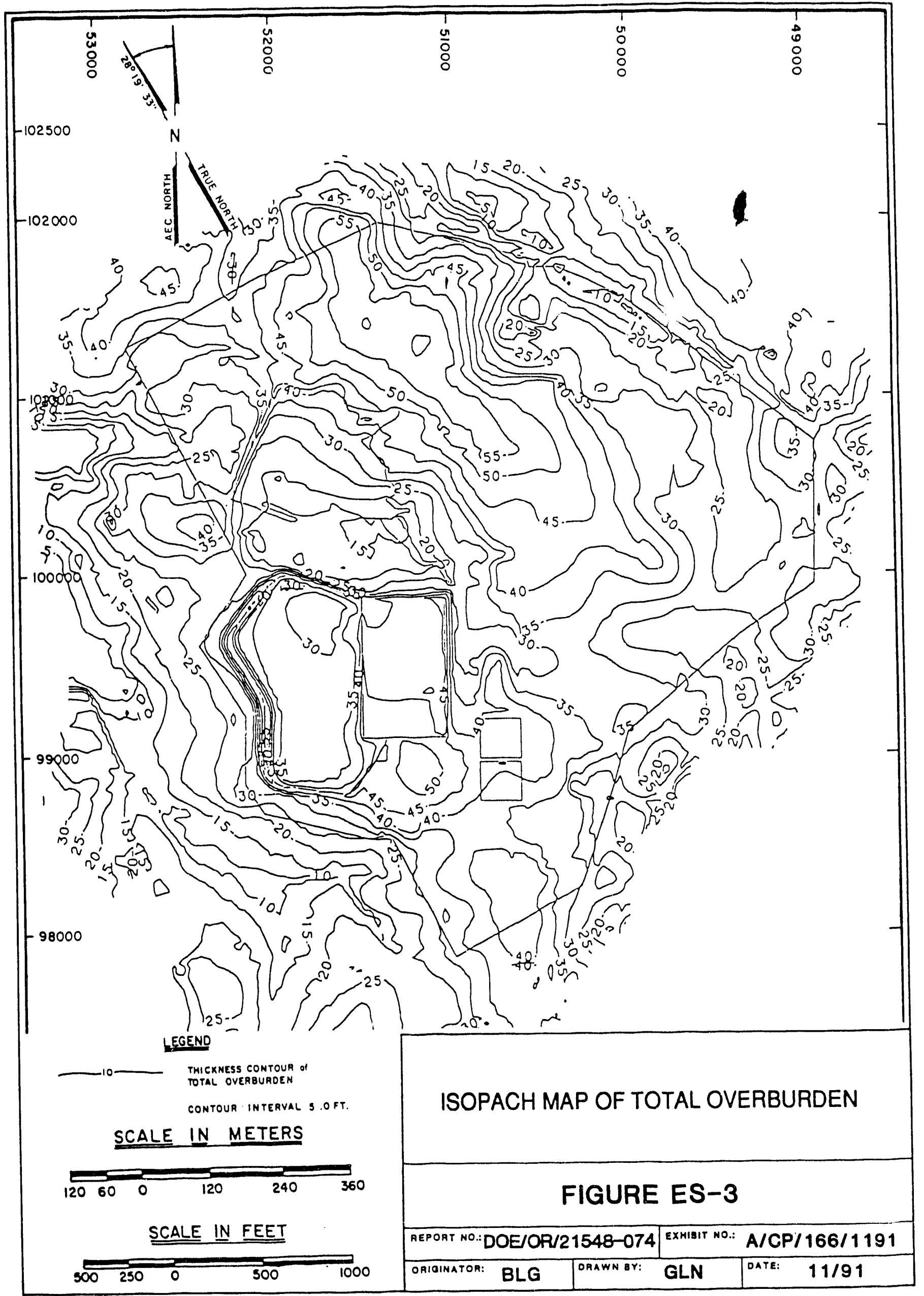




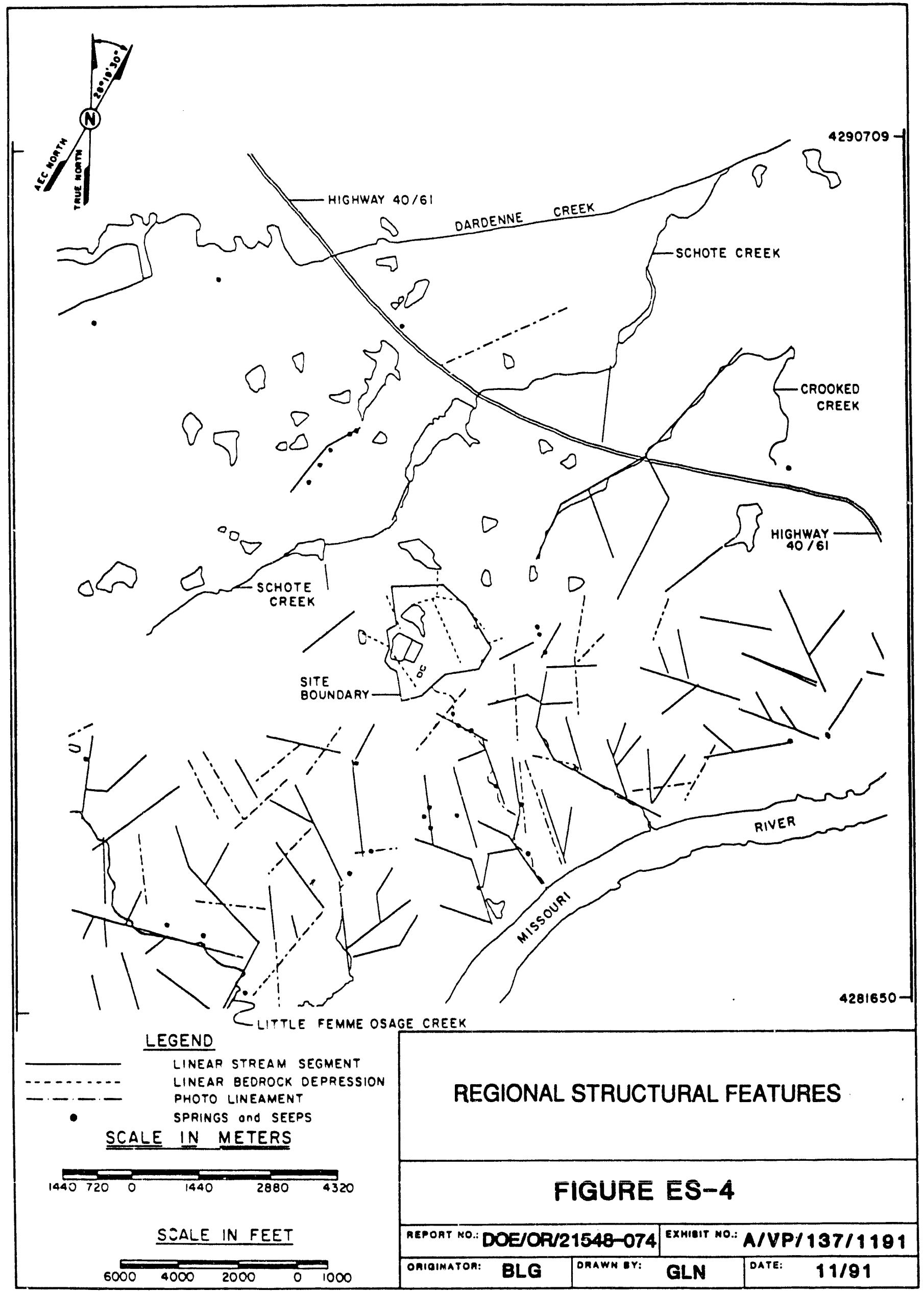




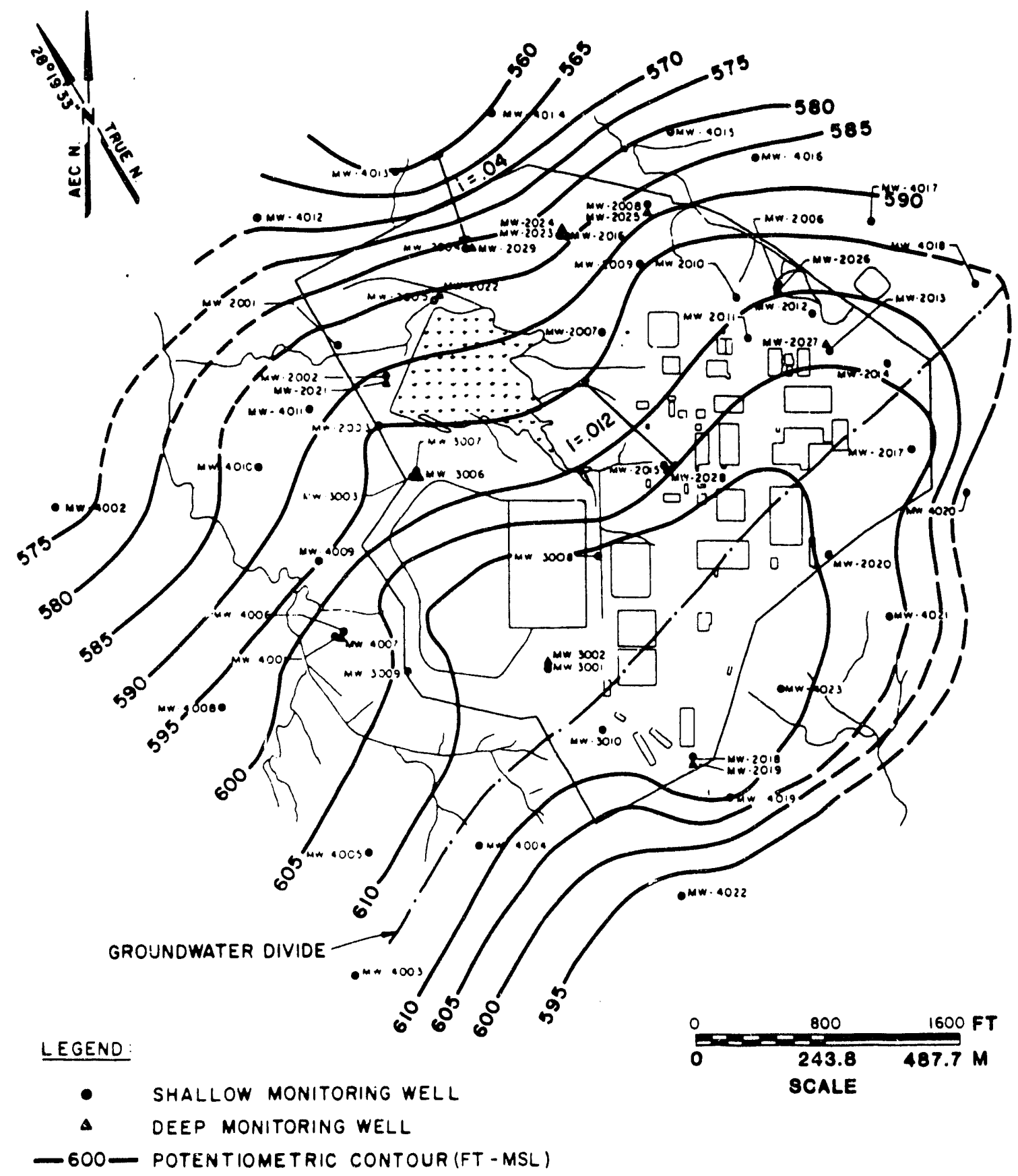

SOURCE: TABLE $4.6-8$

POTENTIOMETRIC SURFACE, SHALLOW WELLS - DECEMBER 1988

FIGURE ES-5

\begin{tabular}{|c|c|c|c|c|c|}
\hline REPOAT NO & DOE/OF & $1548-074$ & EXHIBT NO. & A/CP & $7 / 1191$ \\
\hline OAKGNATOR & BLG & Dramen or & GLN & DATE & $11 / 91$ \\
\hline
\end{tabular}




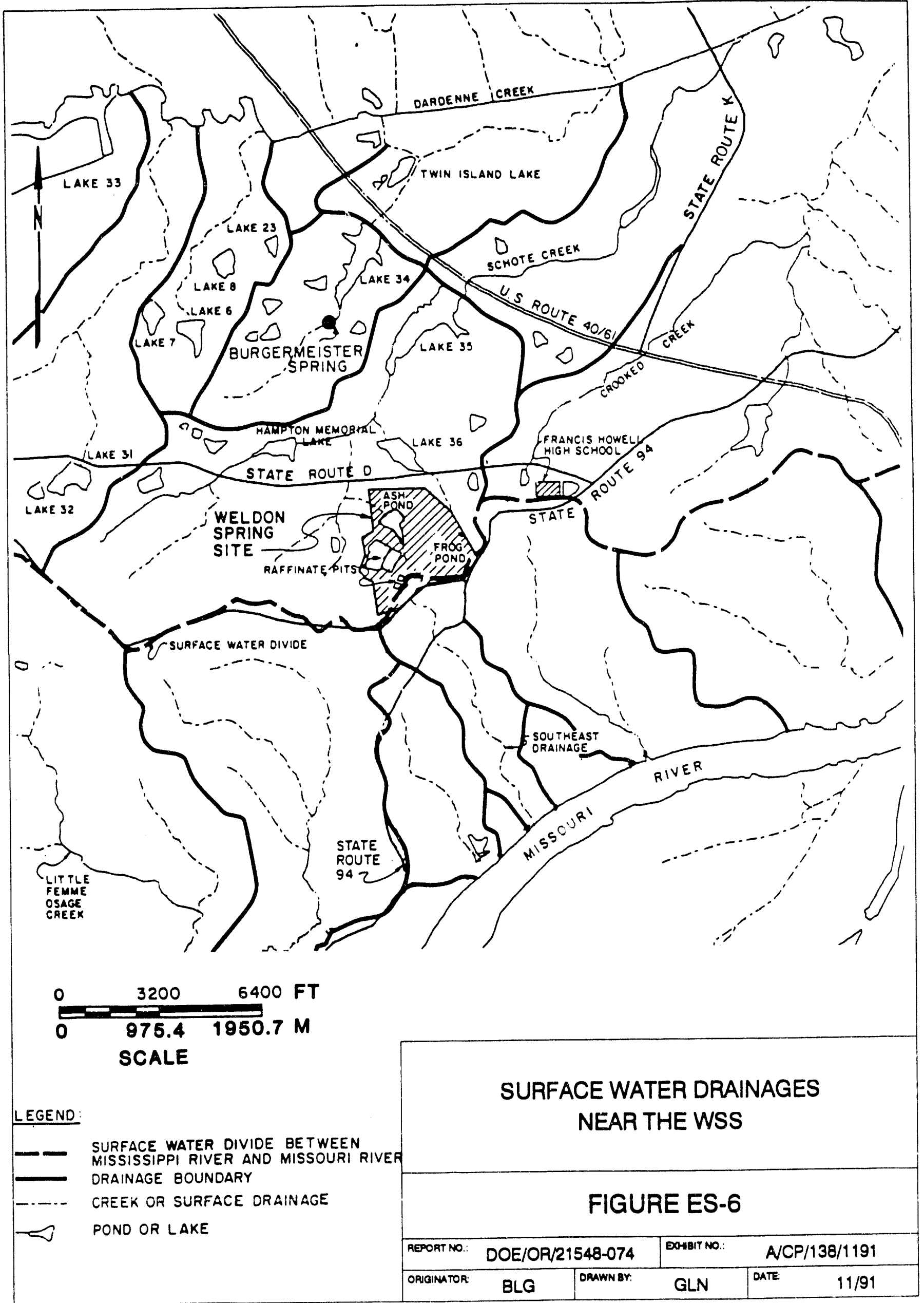




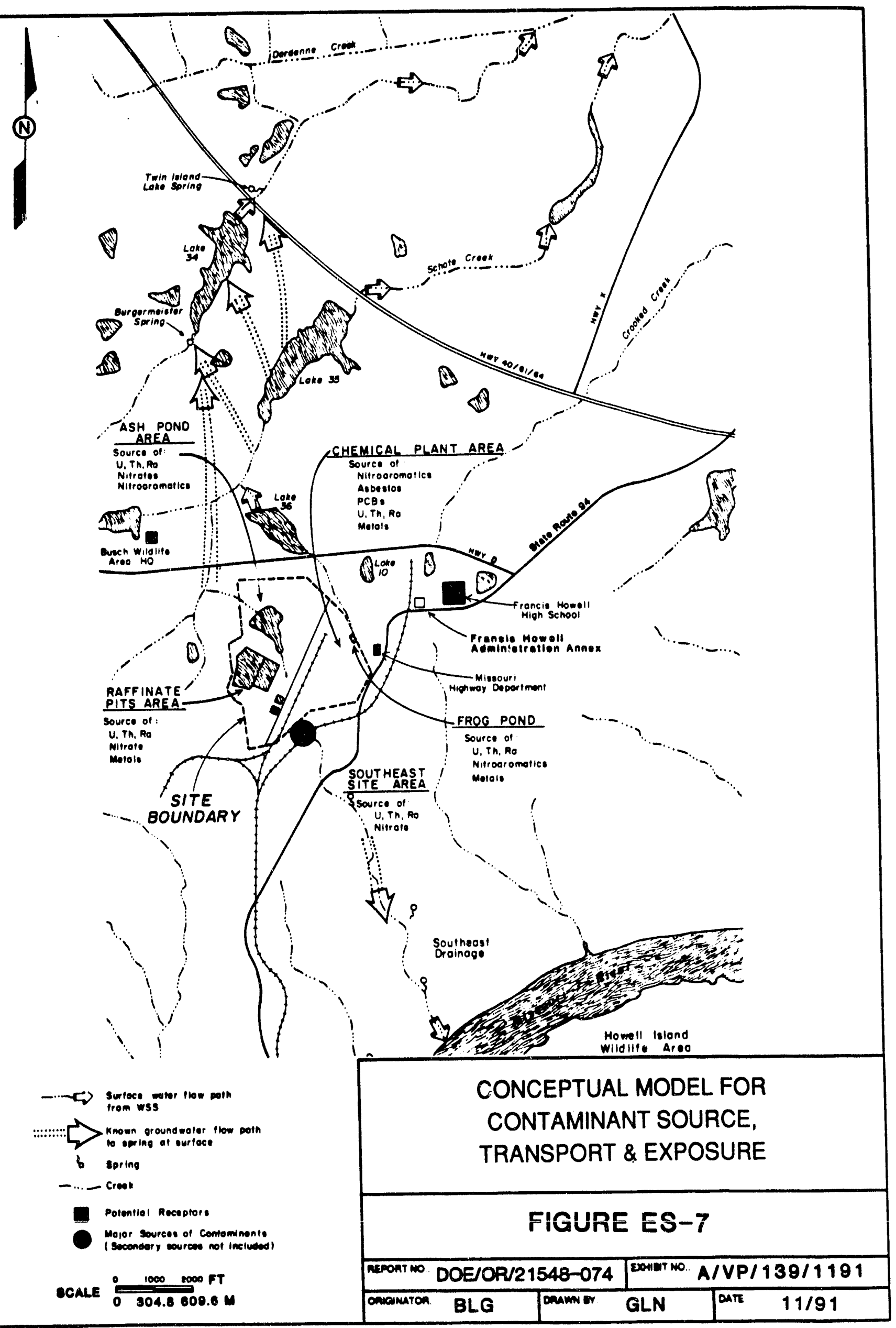




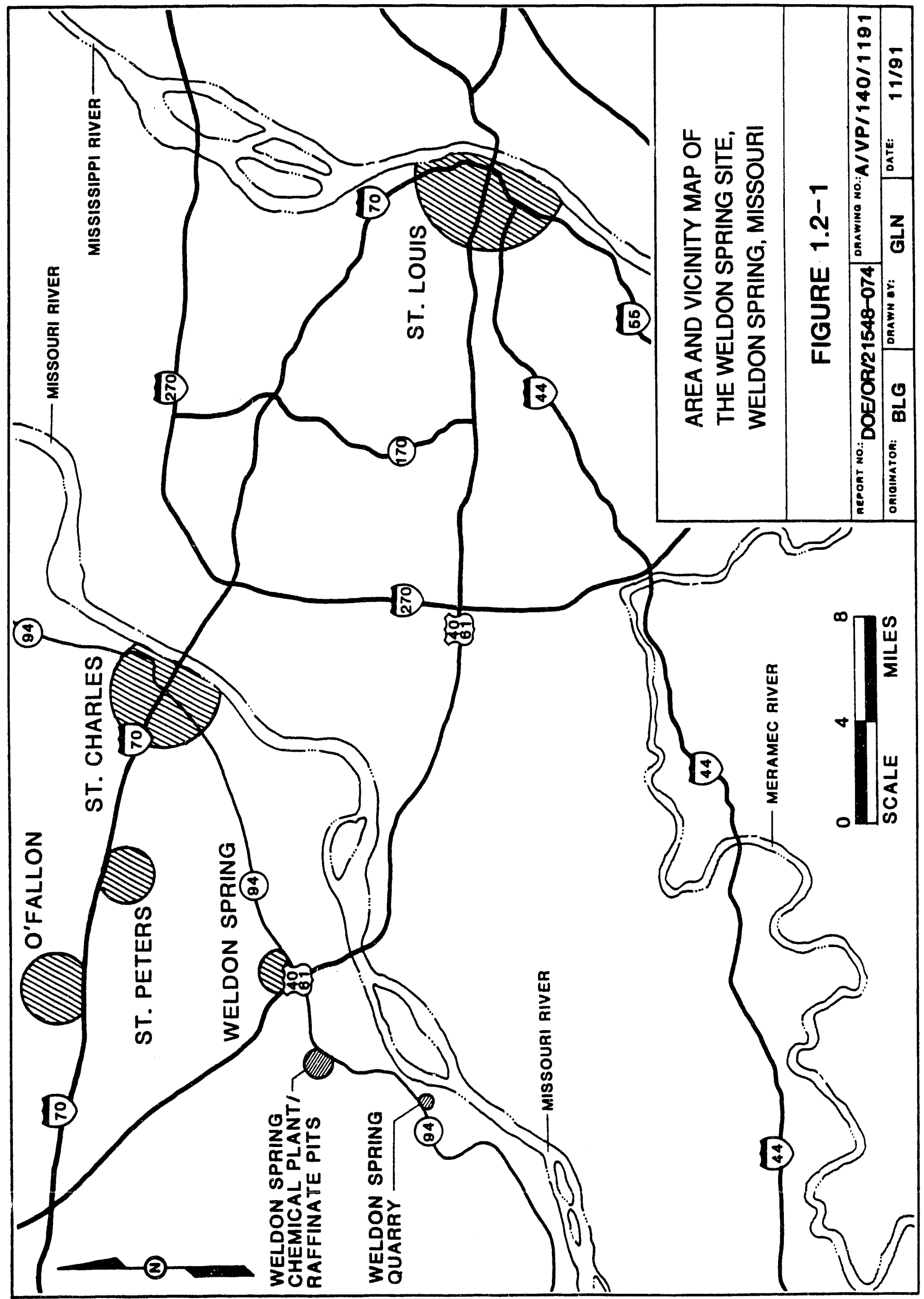




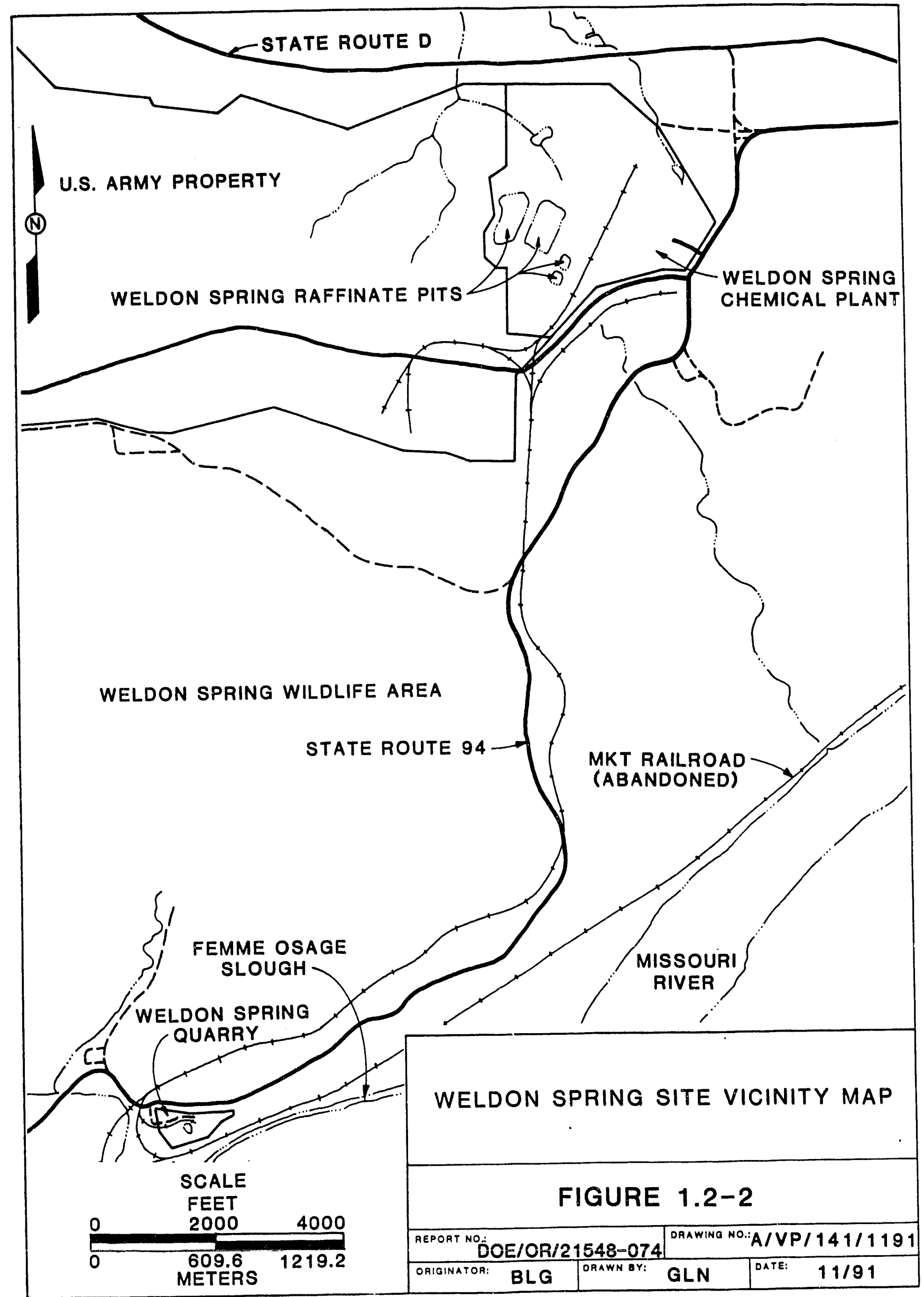




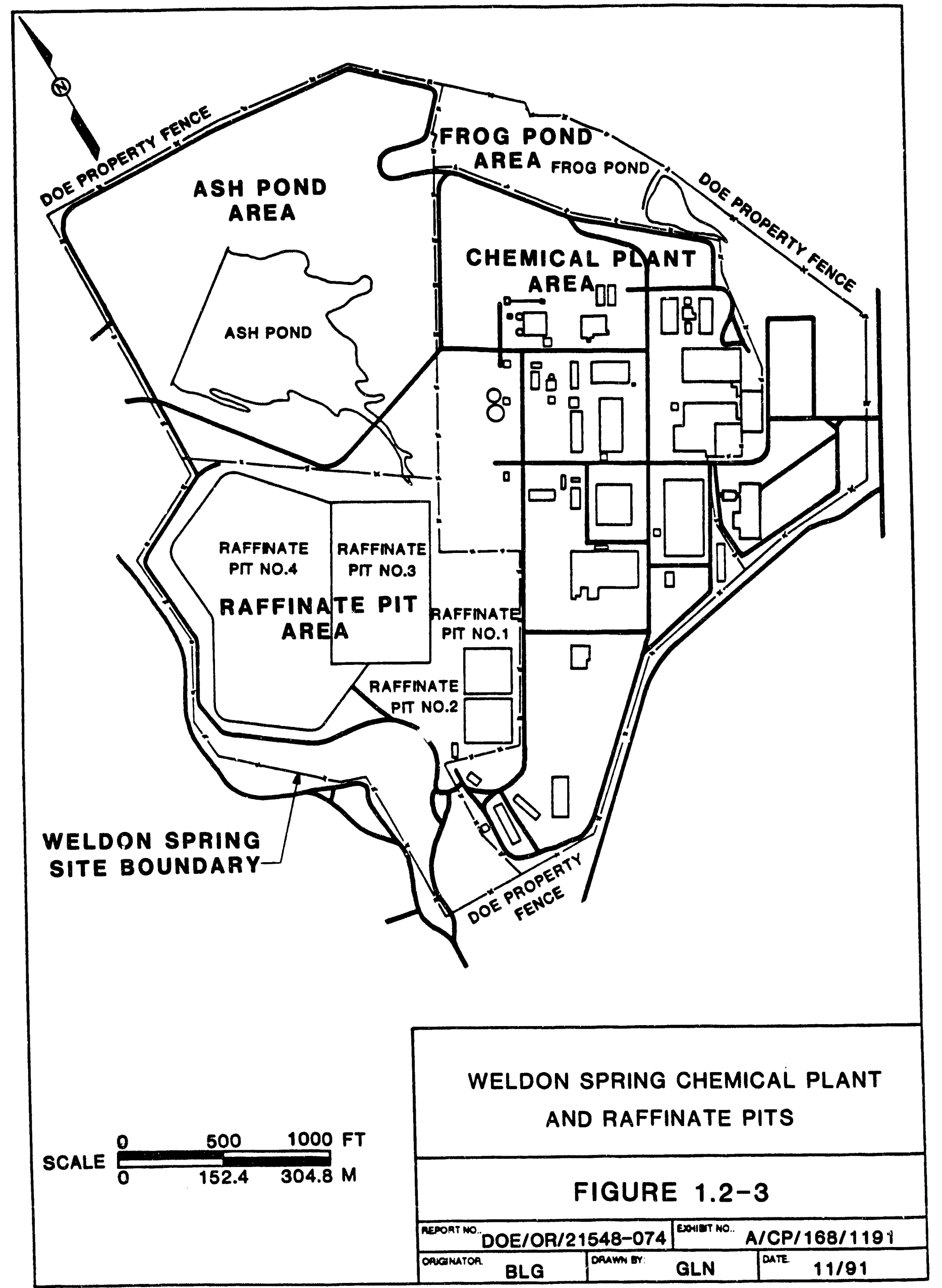




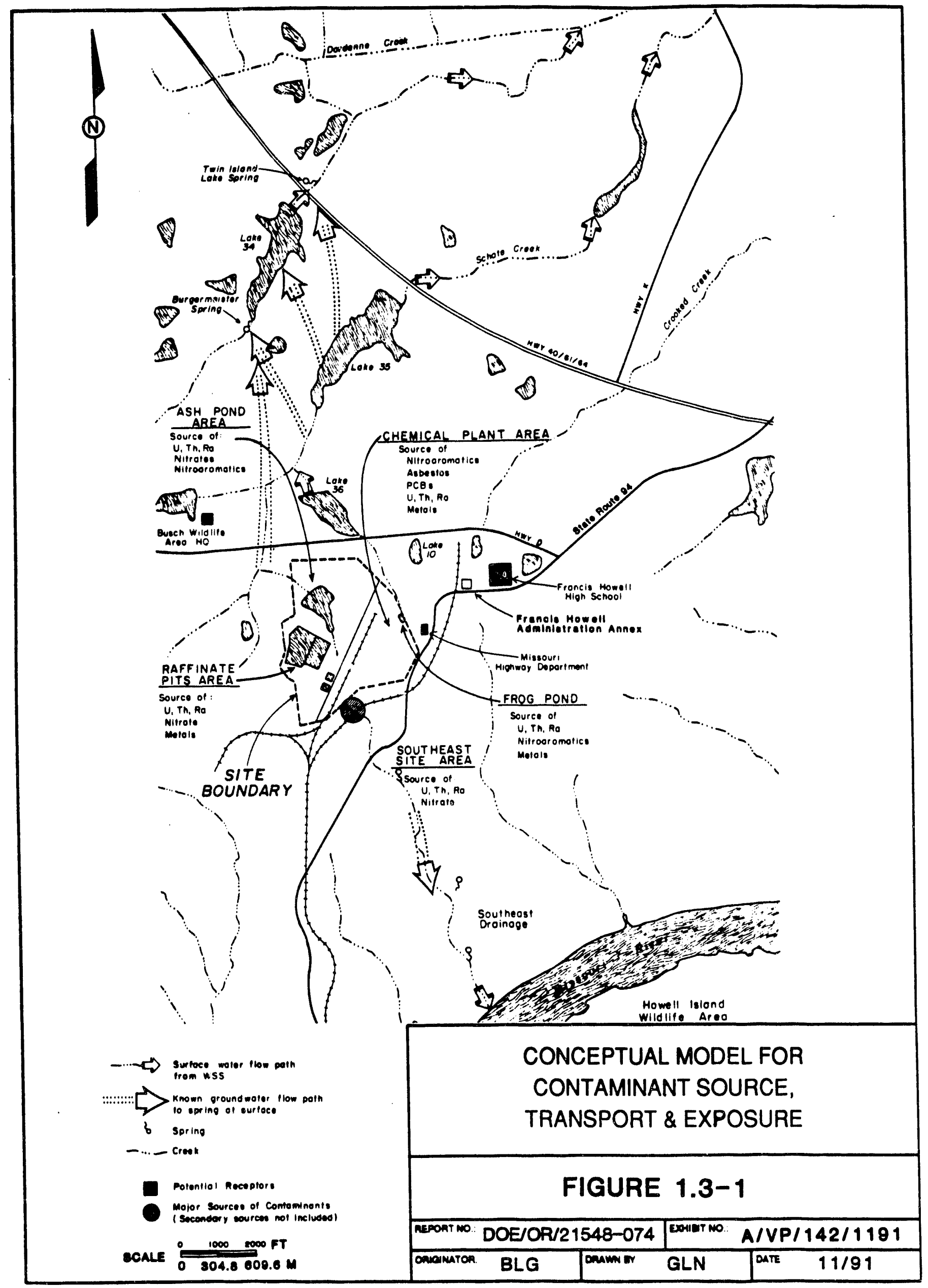




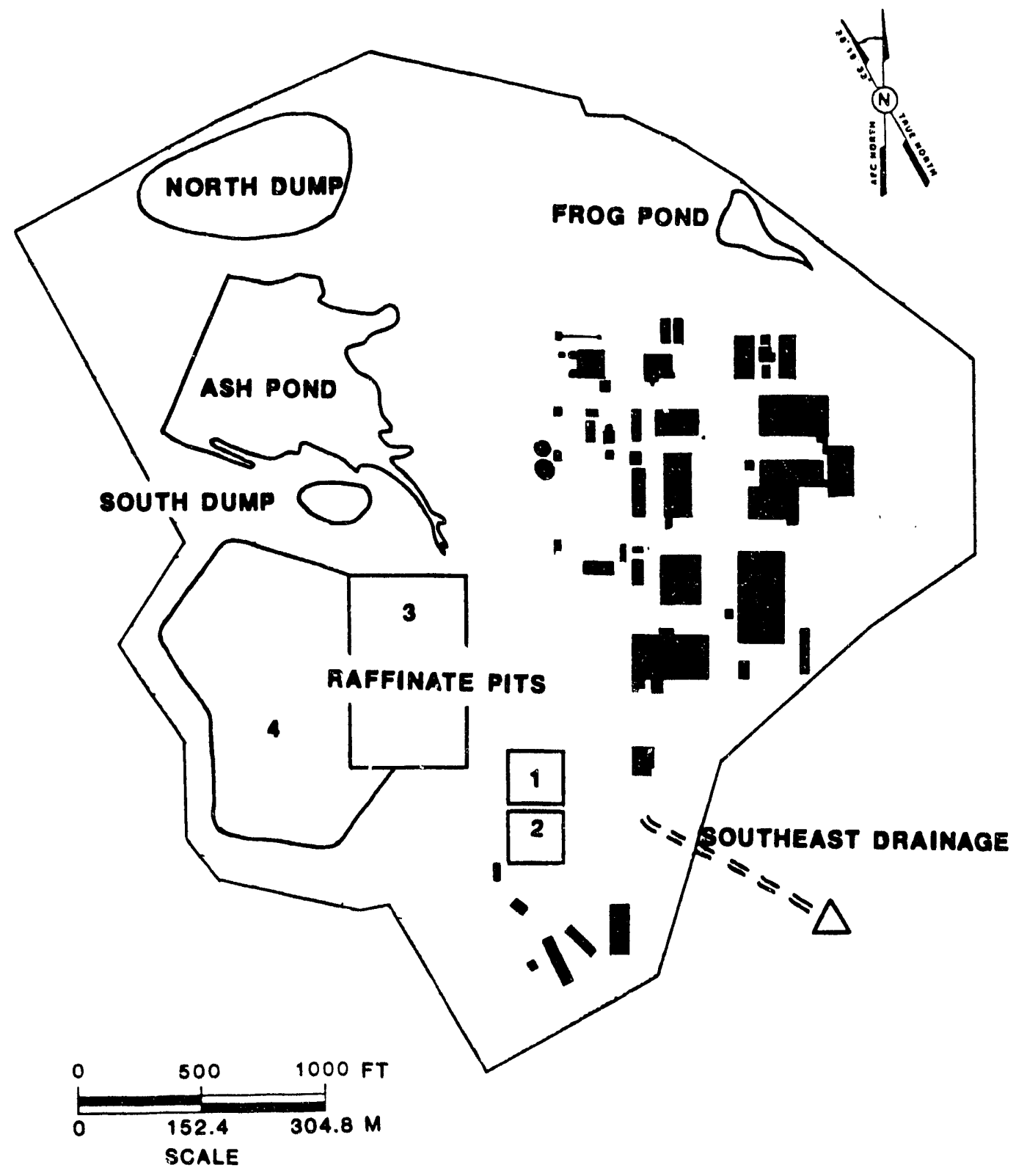

NOTE: WSCP Bulldinge chaded In black.

CONTAMINANT SOURCE AREAS

FIGURE 3.1-1

\begin{tabular}{|c|c|c|c|c|}
\hline REPOAT NO & DOEJOF & $1548-074$ & EXMEUTT NO & $A / C P / 169 / 1181$ \\
\hline ORIGINATOR & BLG & Dramin Br & GLN & TDATE $11 / 91$ \\
\hline
\end{tabular}




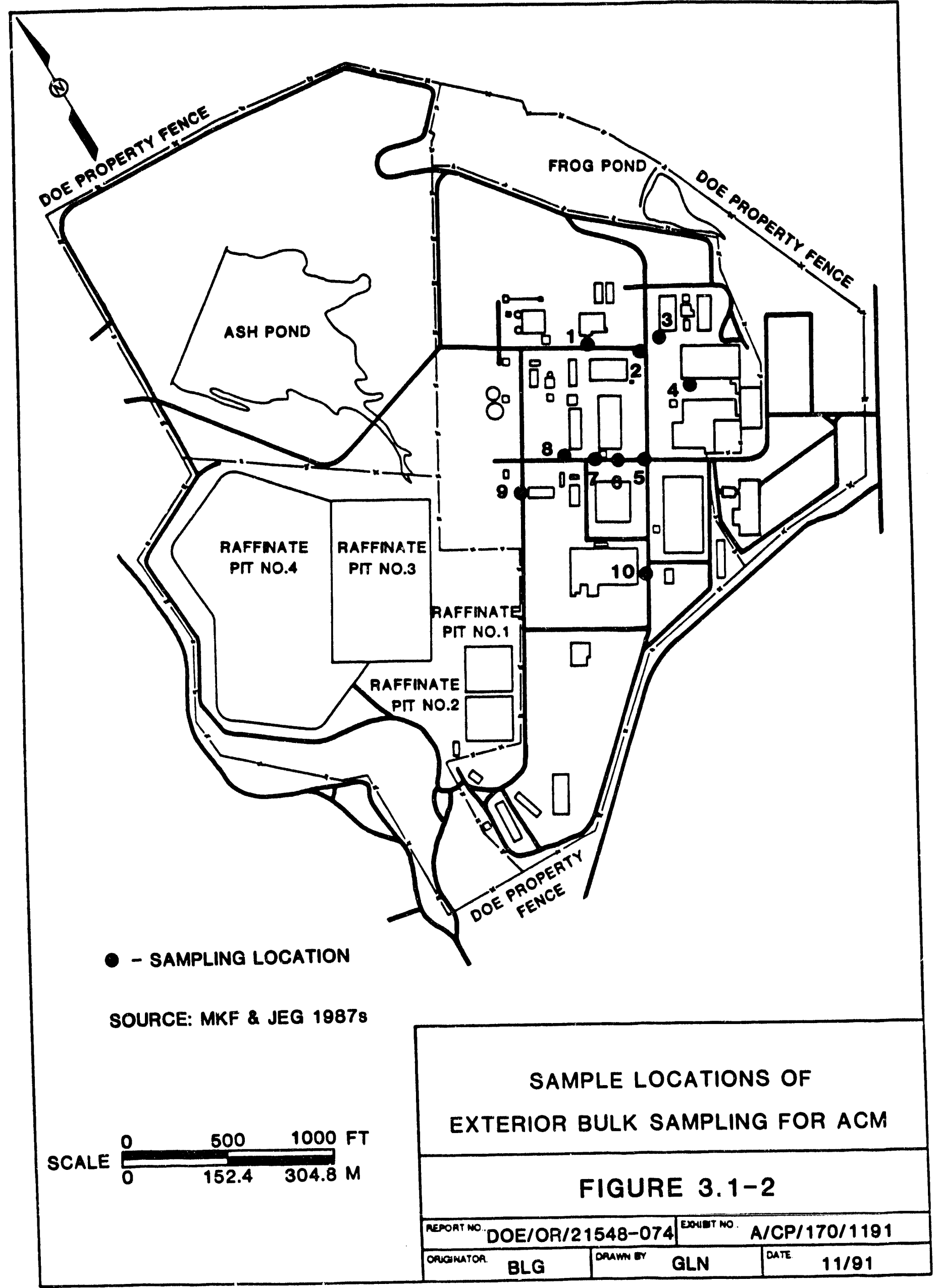




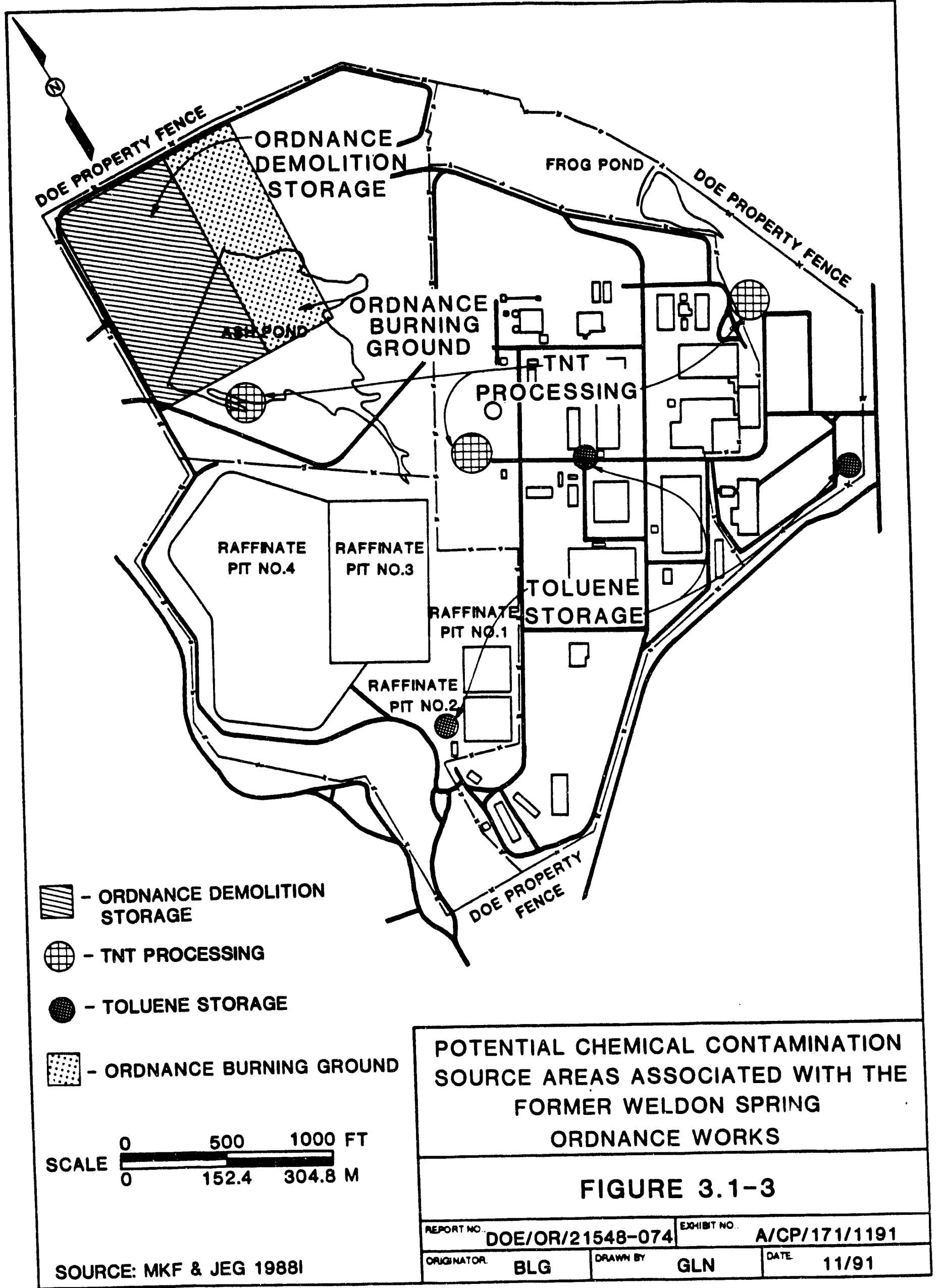




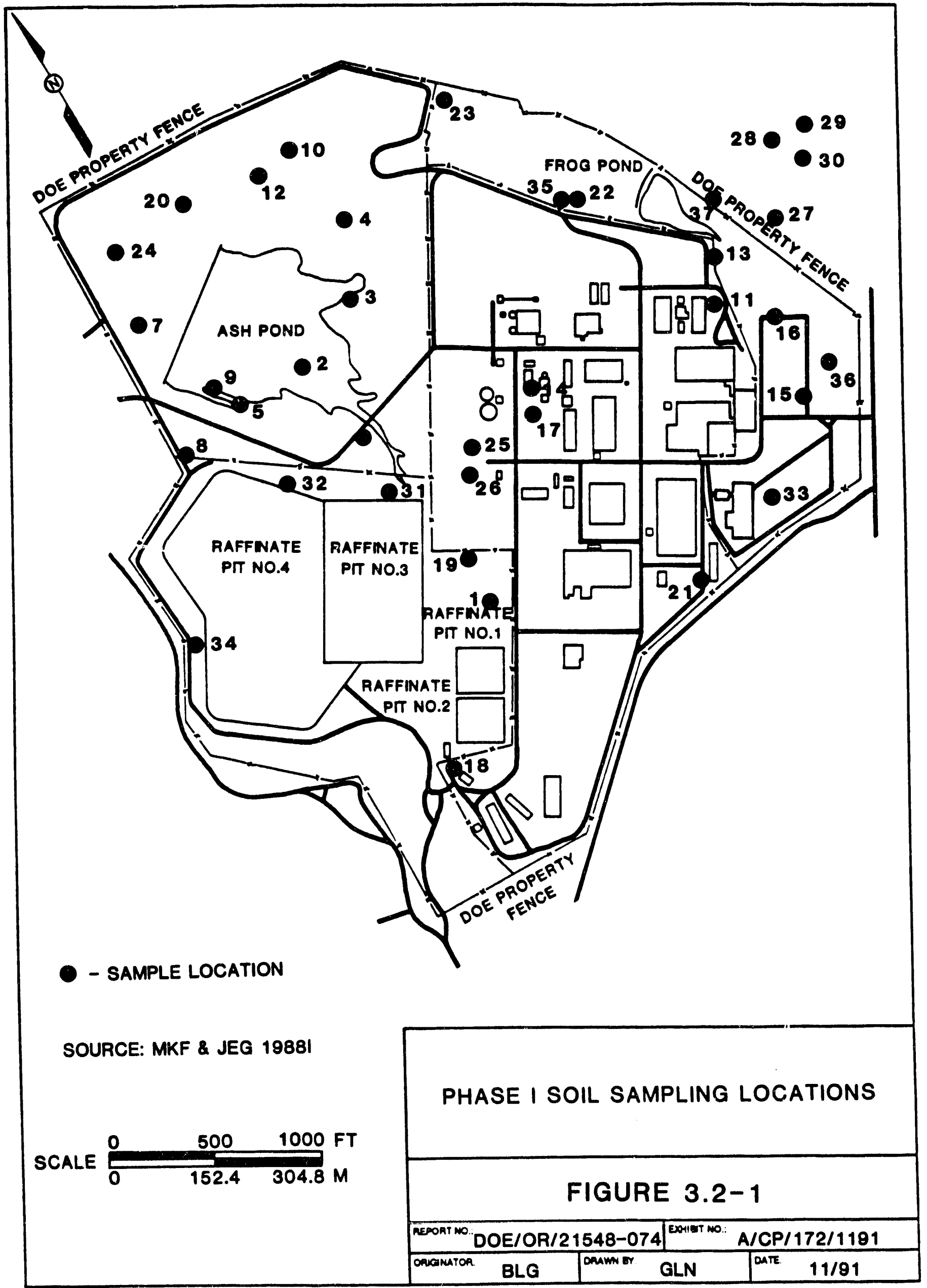




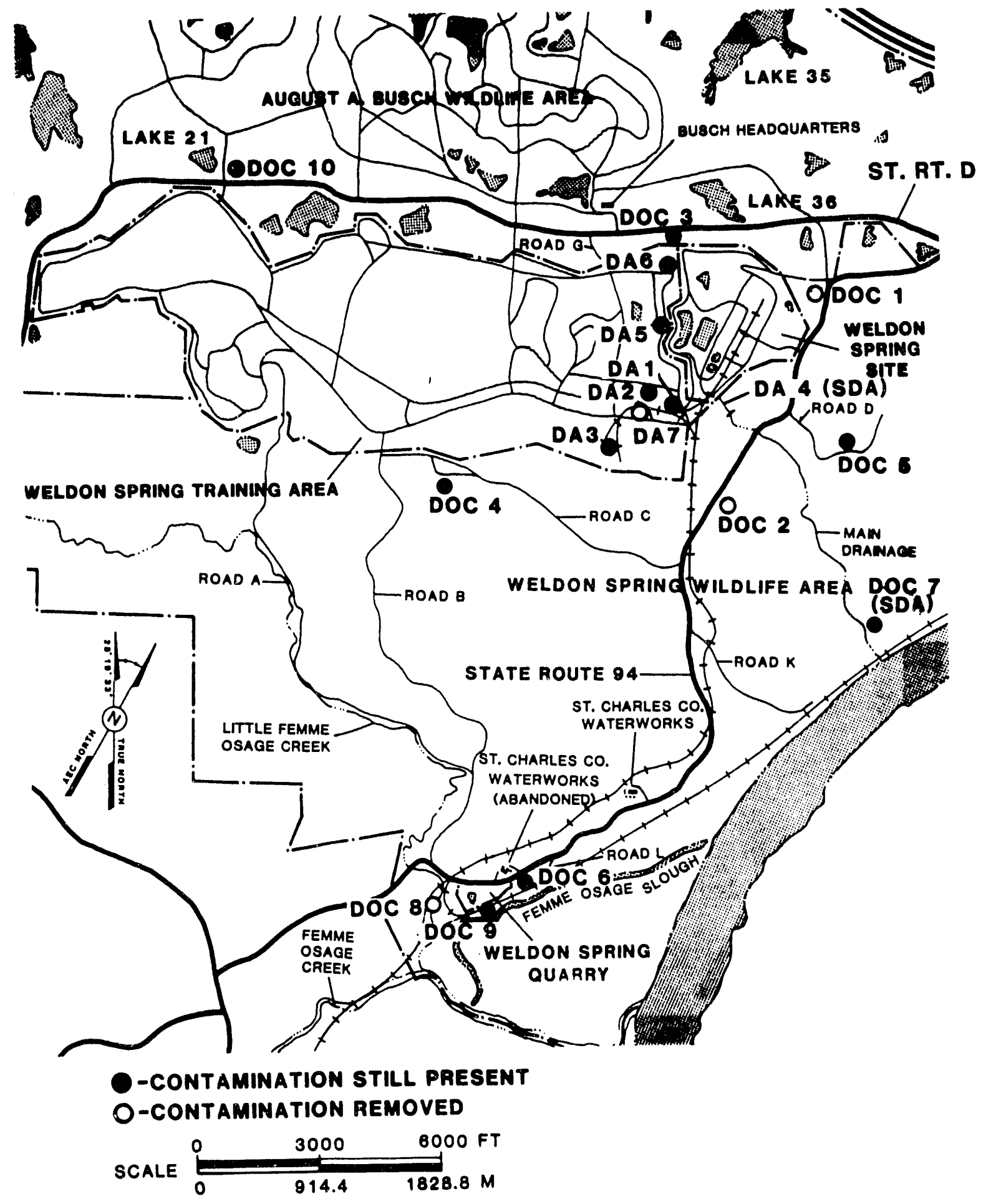

RADIOLOGICALLY CONTAMINATED PROPERTY LOCATIONS

FIGURE 3.2-2

\begin{tabular}{|c|c|c|c|c|}
\hline REPOAT NO & DOEIOF & $548-074$ & EXXHBAT NO & A/VP/143/1191 \\
\hline ORIGINATOR & BLG & DRAWN GY & GLN & $11 / 91$ \\
\hline
\end{tabular}




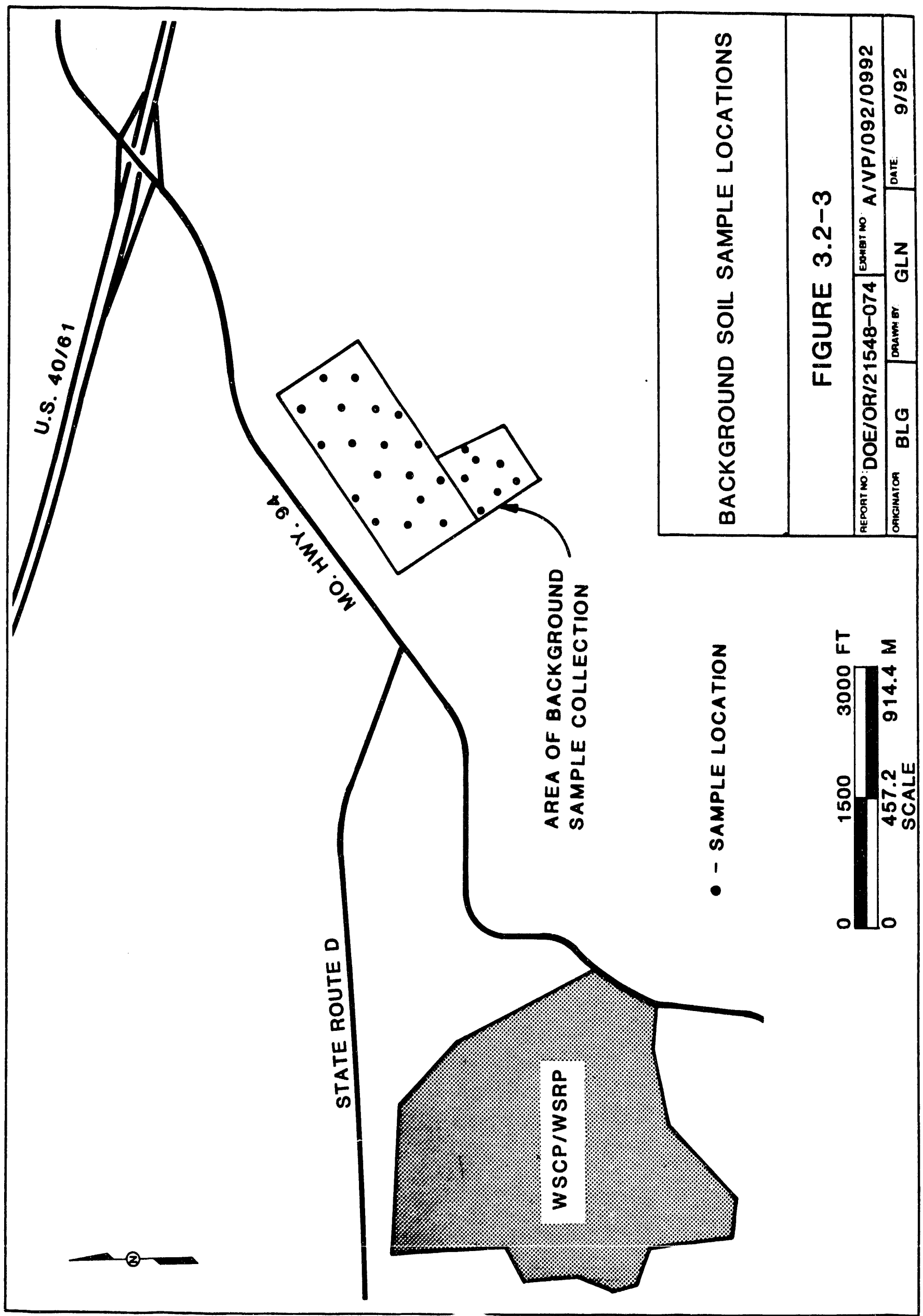




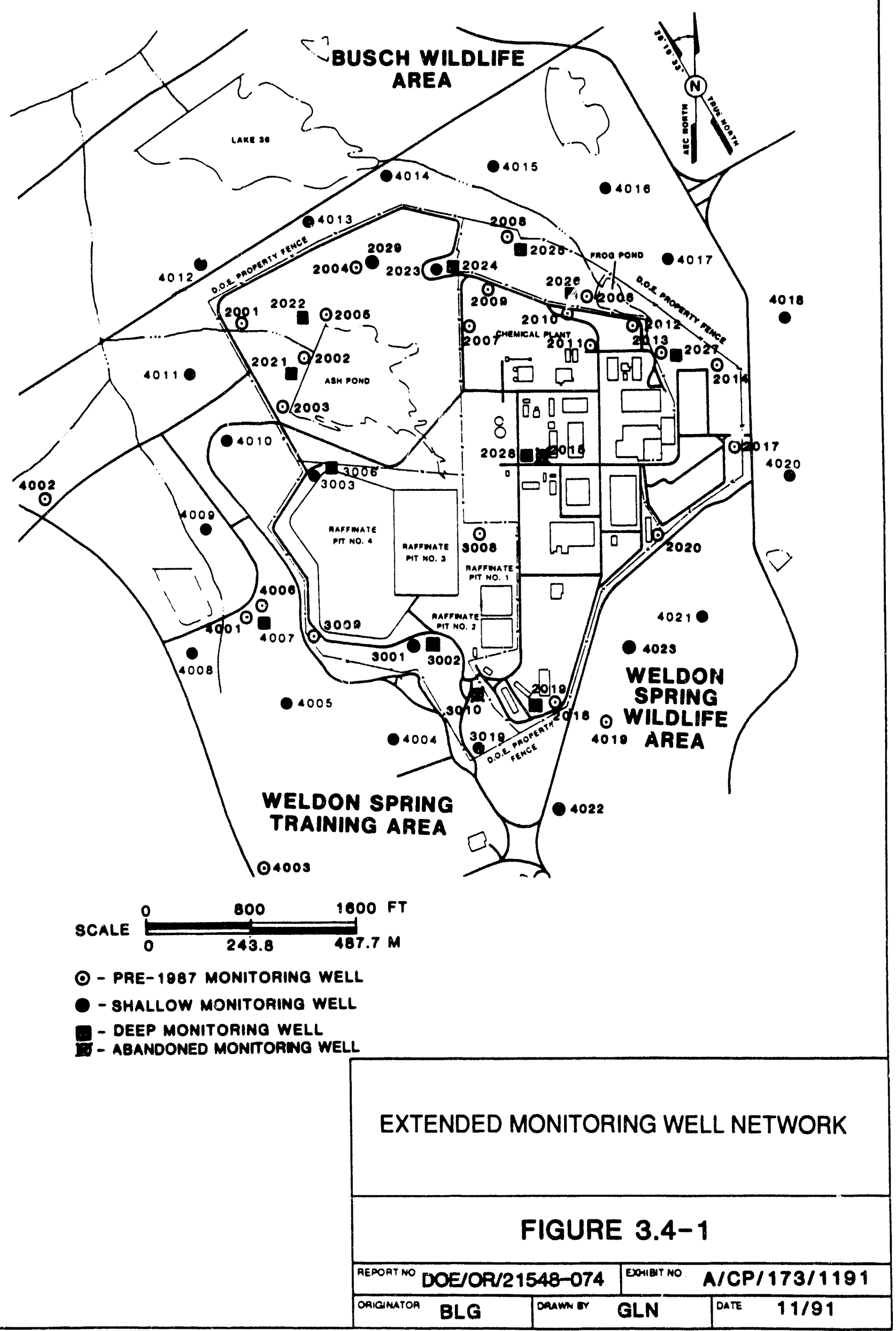




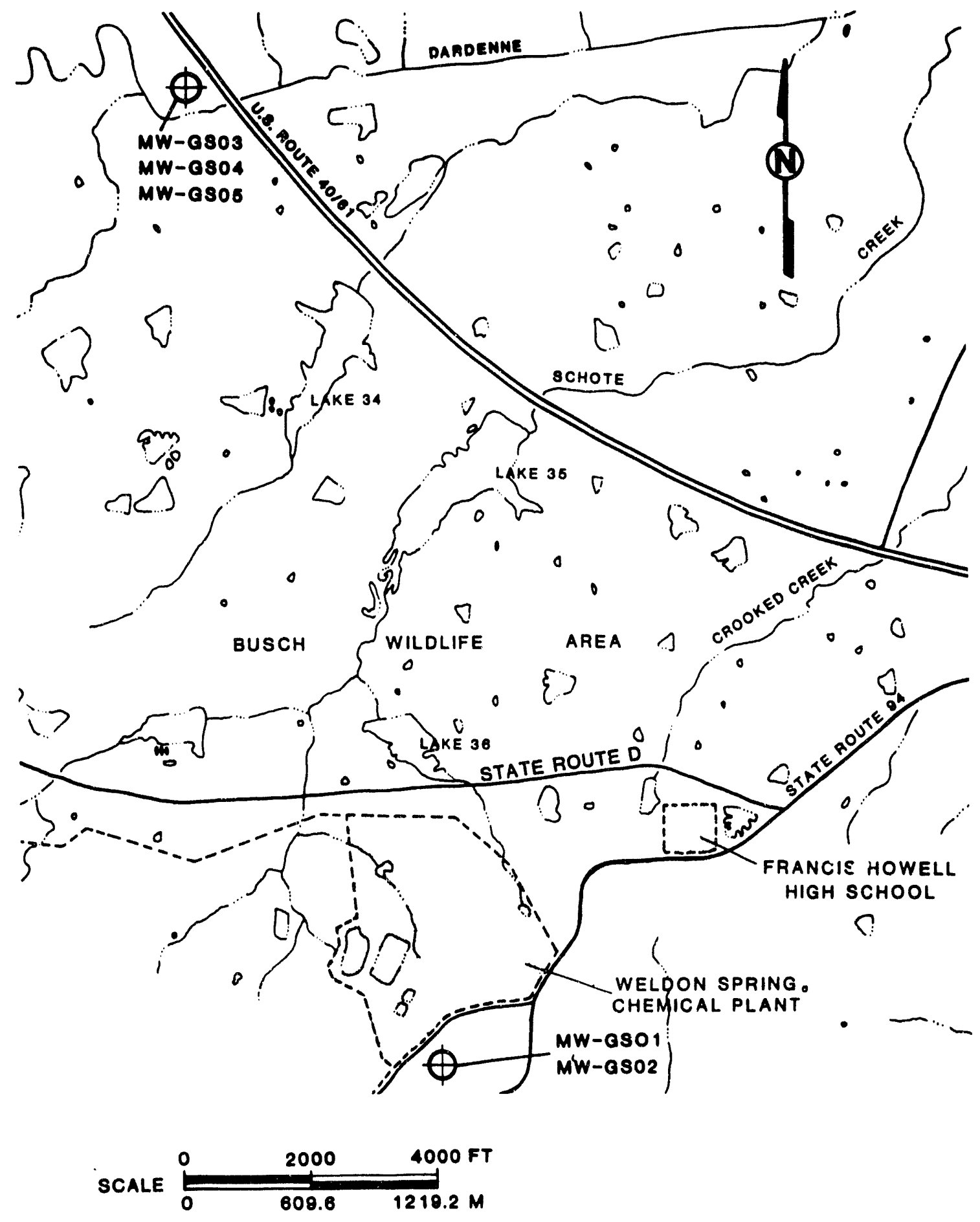

SOURCE MKF JEG 1990

DEEP USGS MONITORING WELL CLUSTER LOCATION

FIGURE 3.4-2

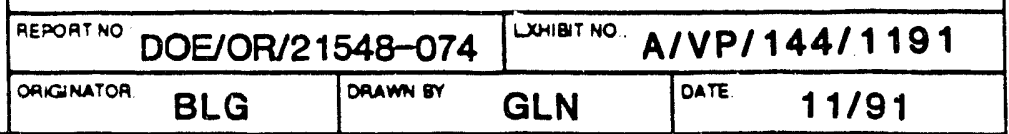




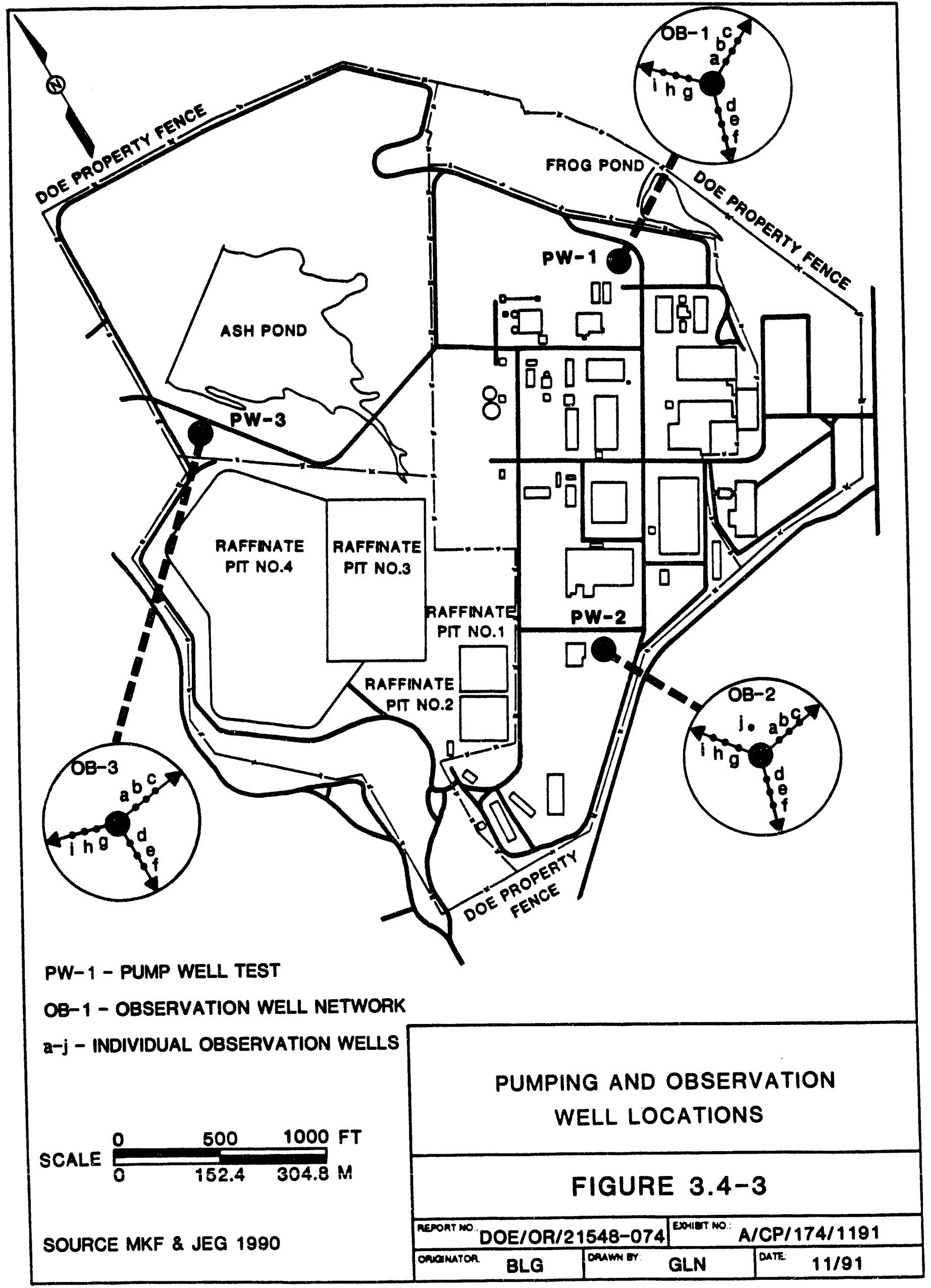




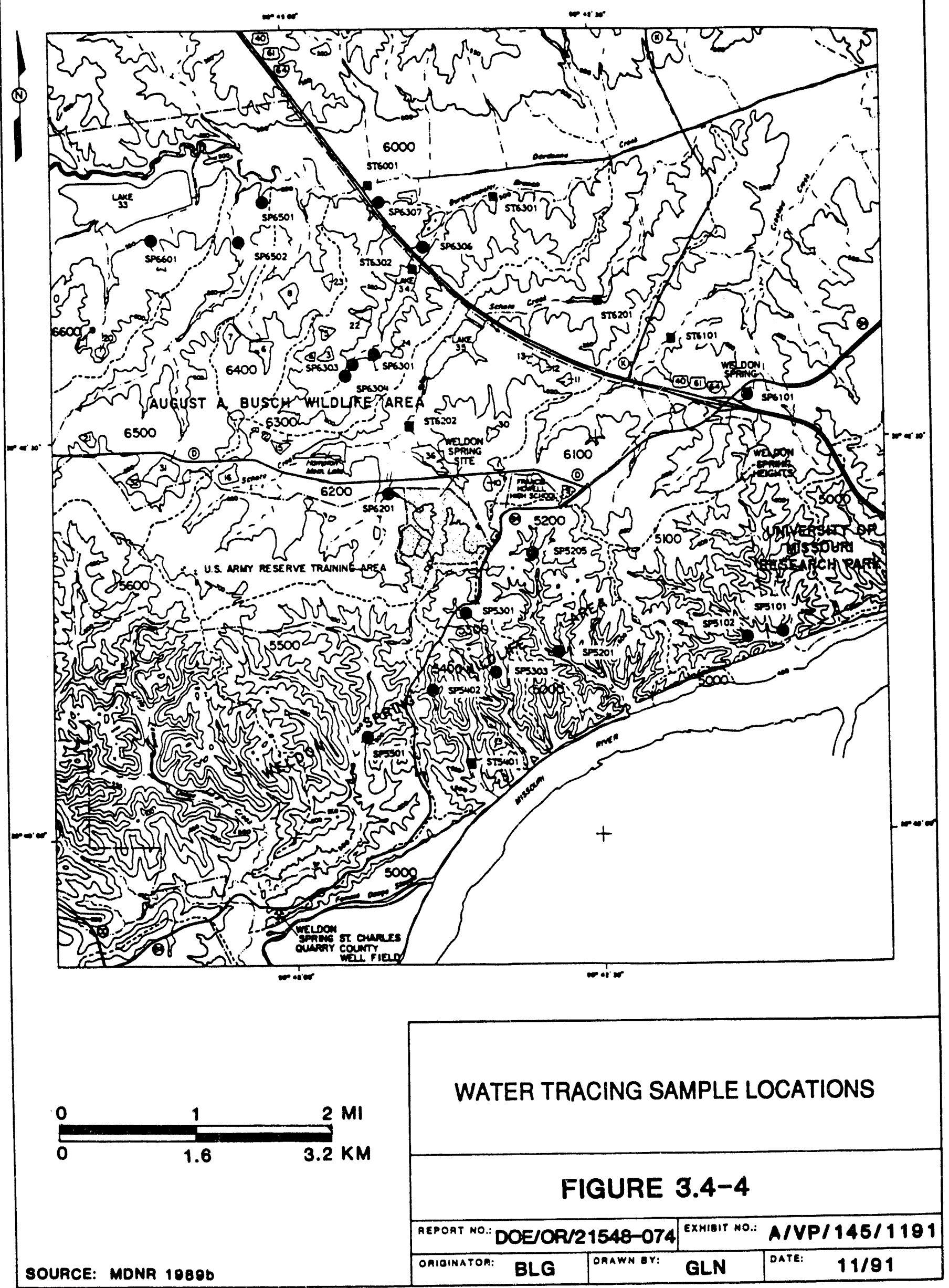




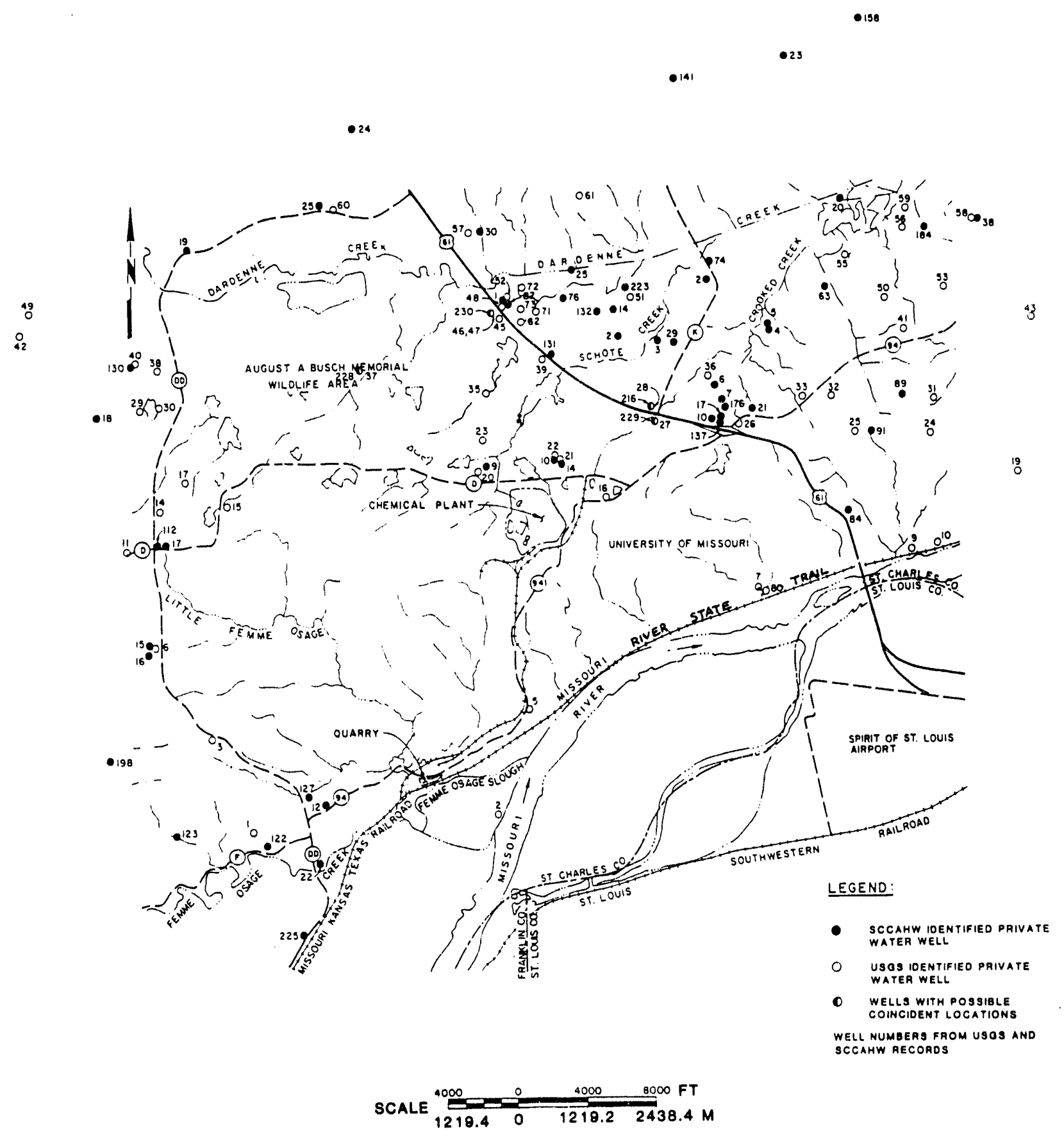

SOURCE MKF A JEG 1990

\section{PRELIMINARY MAP OF PRIVATE WATER WELLS IN THE VICINITY OF THE WSS}

FIGURE 3.4-5

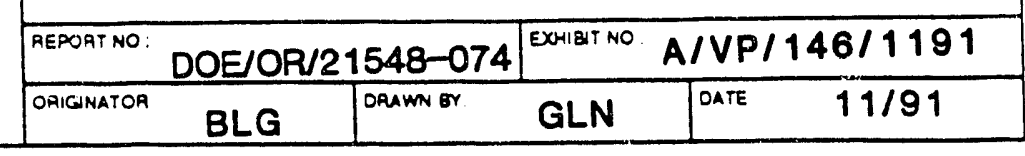




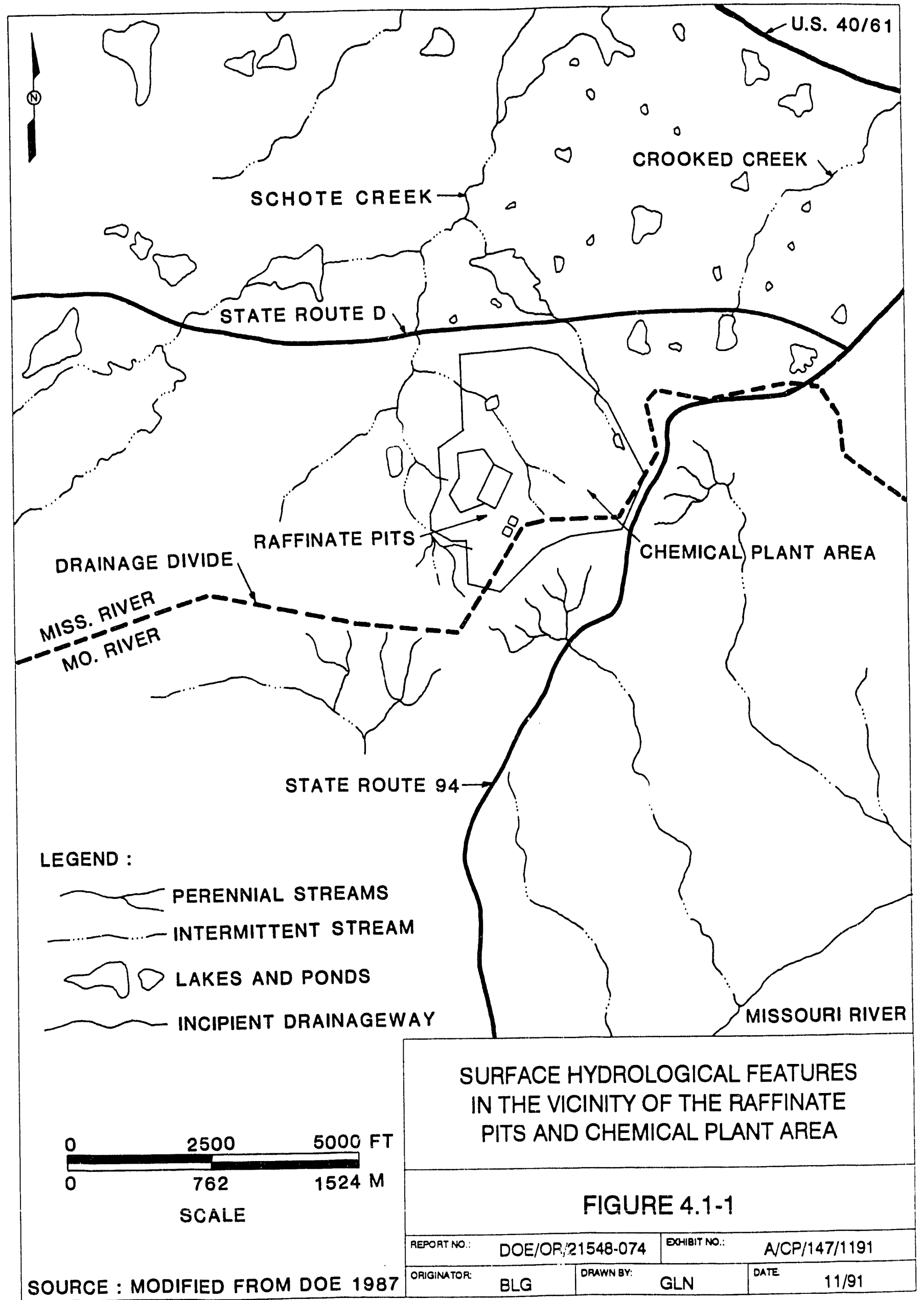



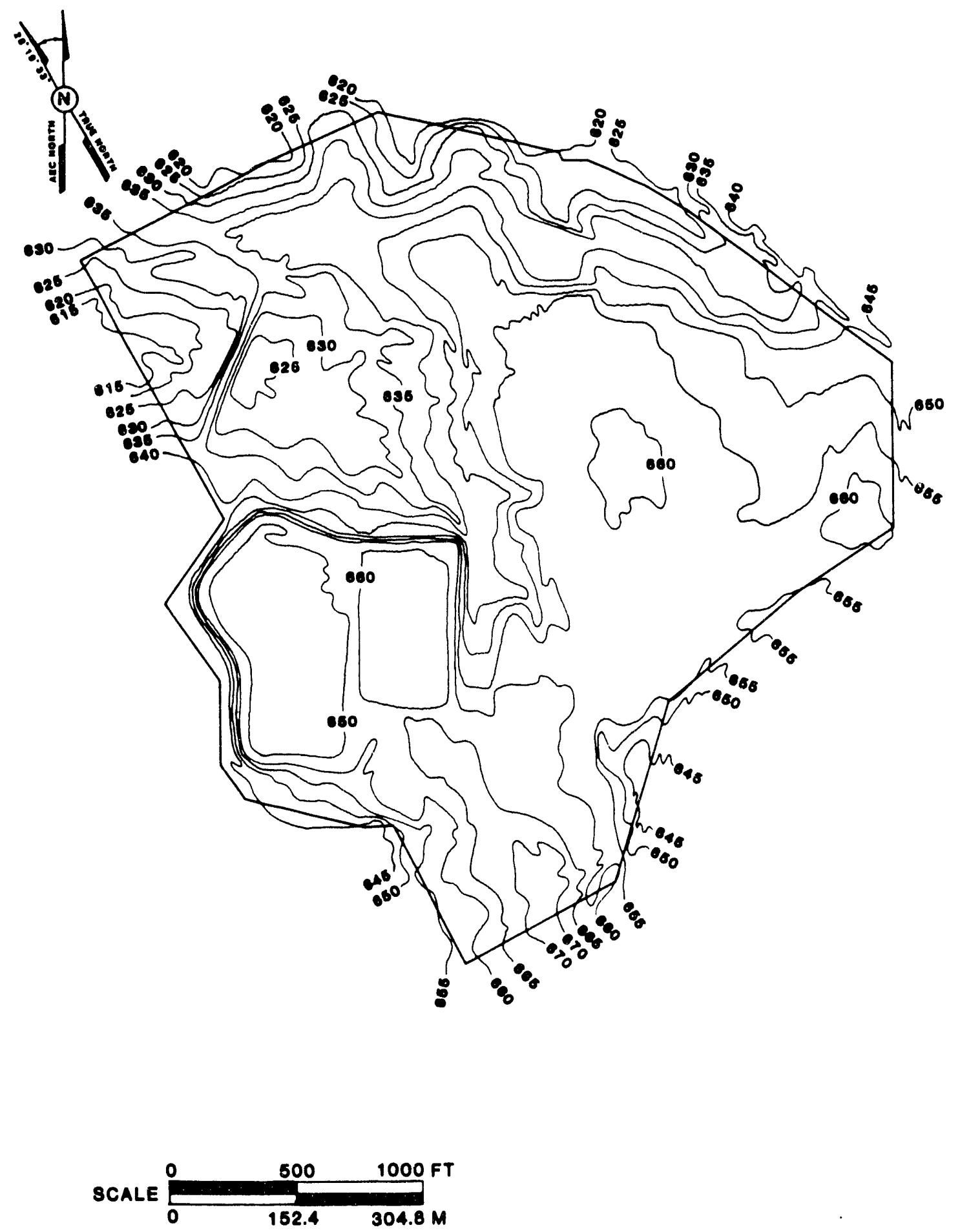

TOPOGRAPHIC MAP OF THE WELDON SPRING AREA (ELEVATIONS IN FEET ABOVE MSL)

FIGURE 4.1-2

\begin{tabular}{|c|c|c|c|c|}
\hline AEPOAT NO & DOEJOF & $1548-074$ & EOHIIT NO. & $A / C P / 175 / 1191$ \\
\hline ORIGINATOR & BLG & oreum or & GLN & $11 / 91$ \\
\hline
\end{tabular}




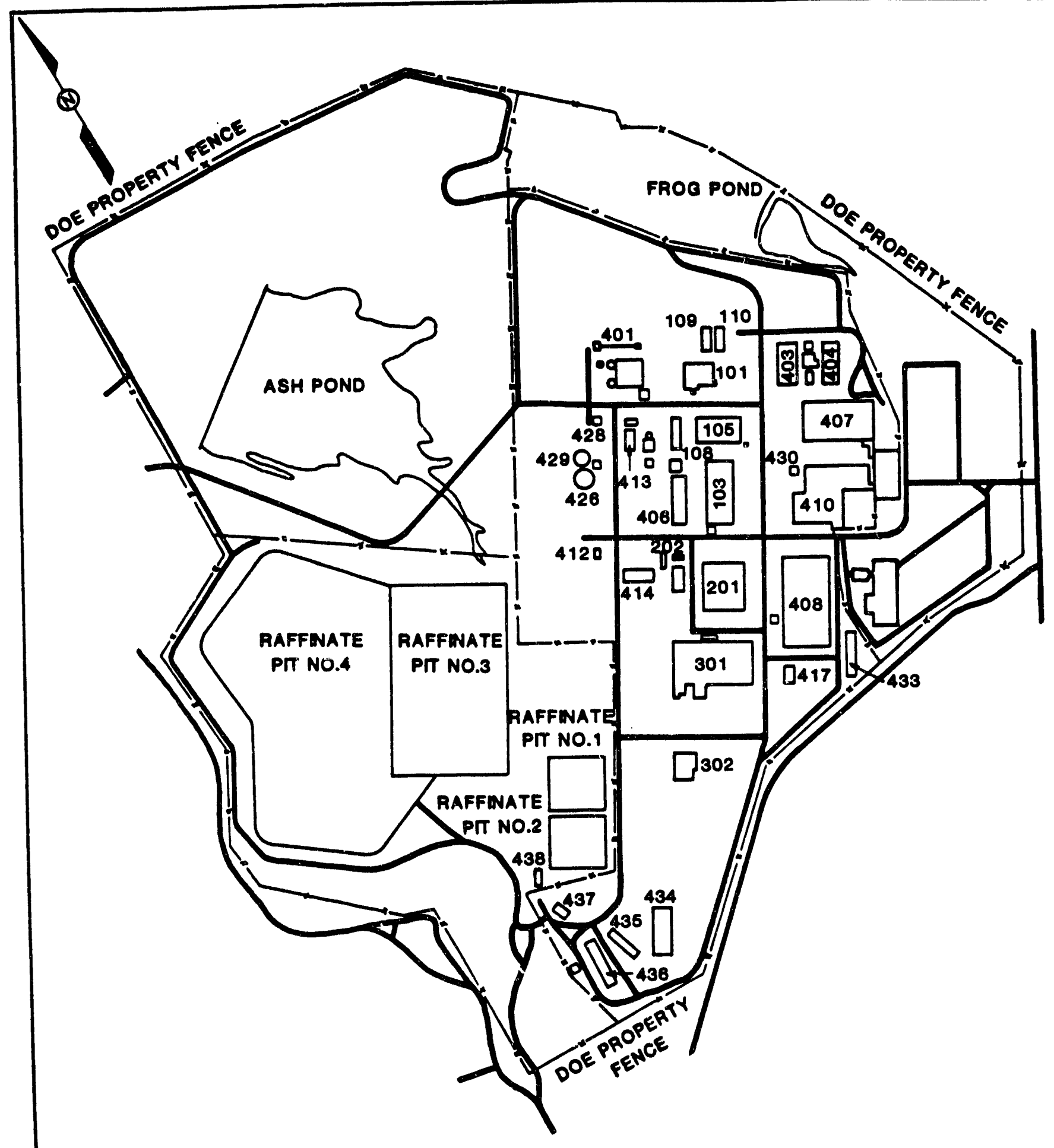

SOURCE MKF \& JEG 1987

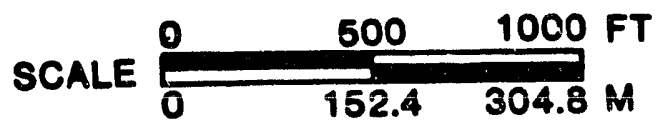

MAP OF THE WSCP/WSRP

FIGURE 4.1-3

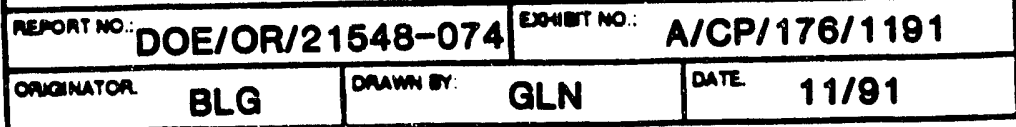



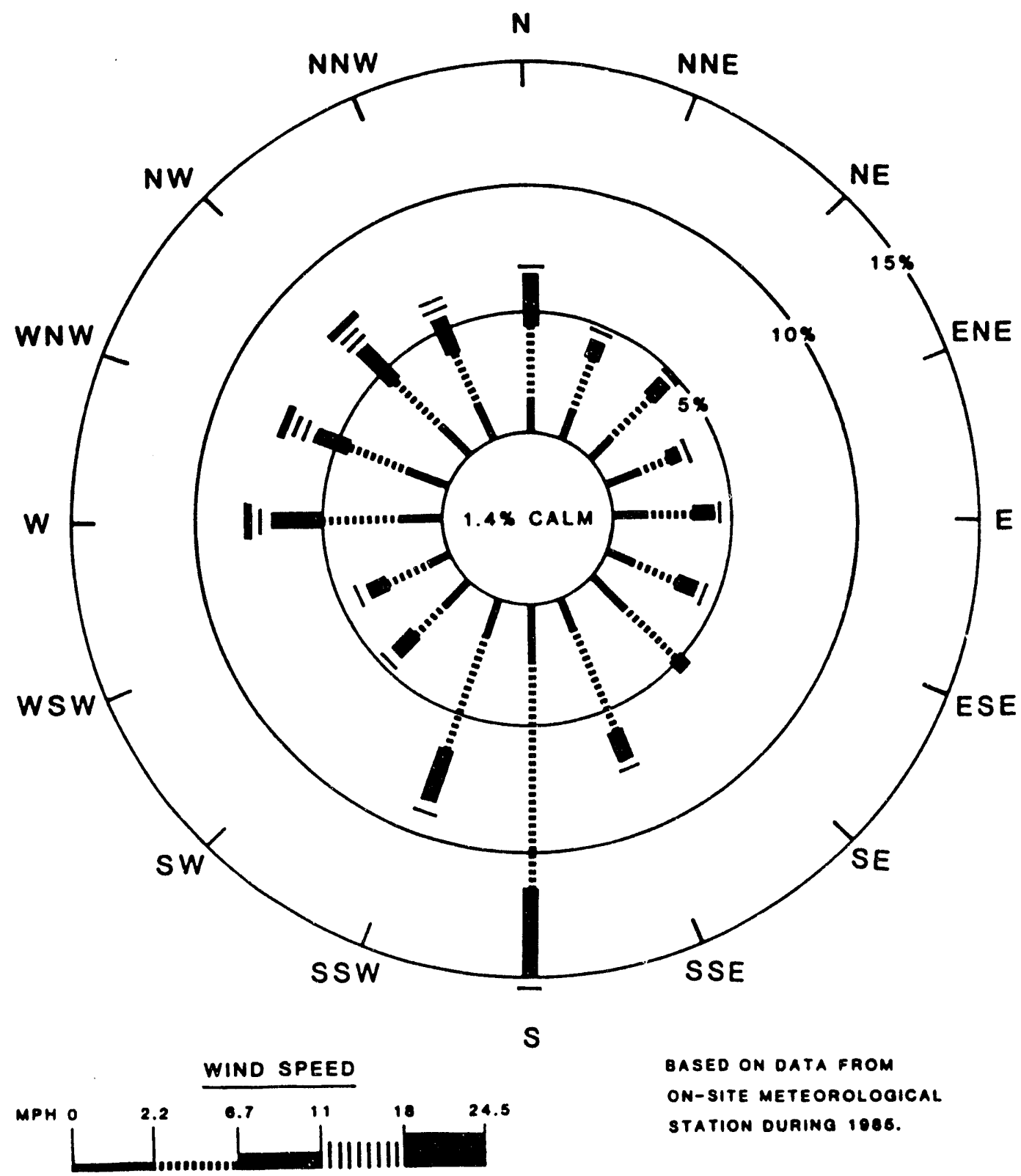

STATION DURING 1986.

SOURCE : BNI $1986 \mathrm{C}$

ANNUAL WIND ROSE FOR

THE WSS - 1985

FIGURE 4.2-1

REPOAT NO DOE/OR/21548-074 DOHLTT NO. A/PI/227/1191

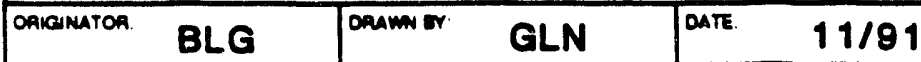




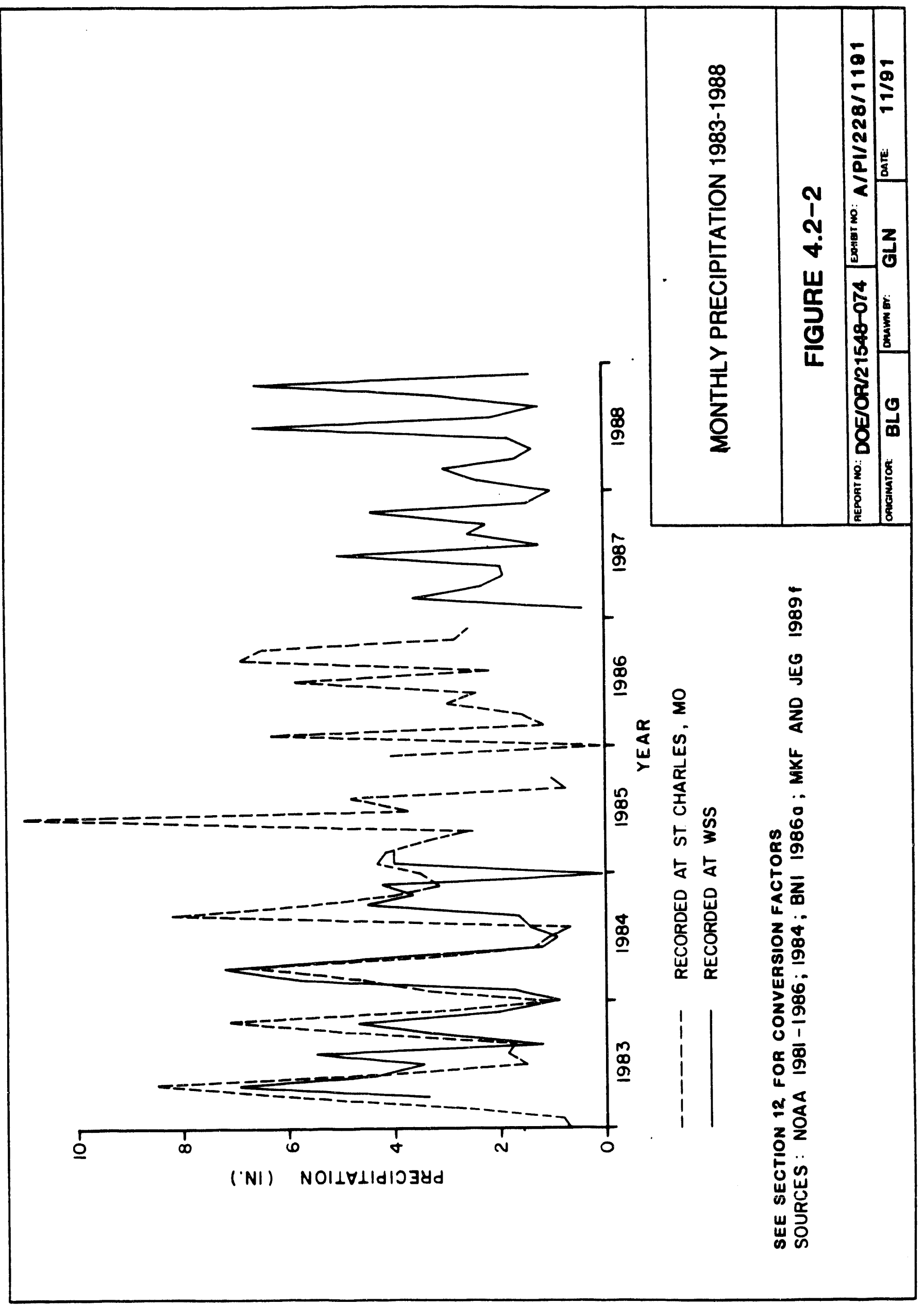




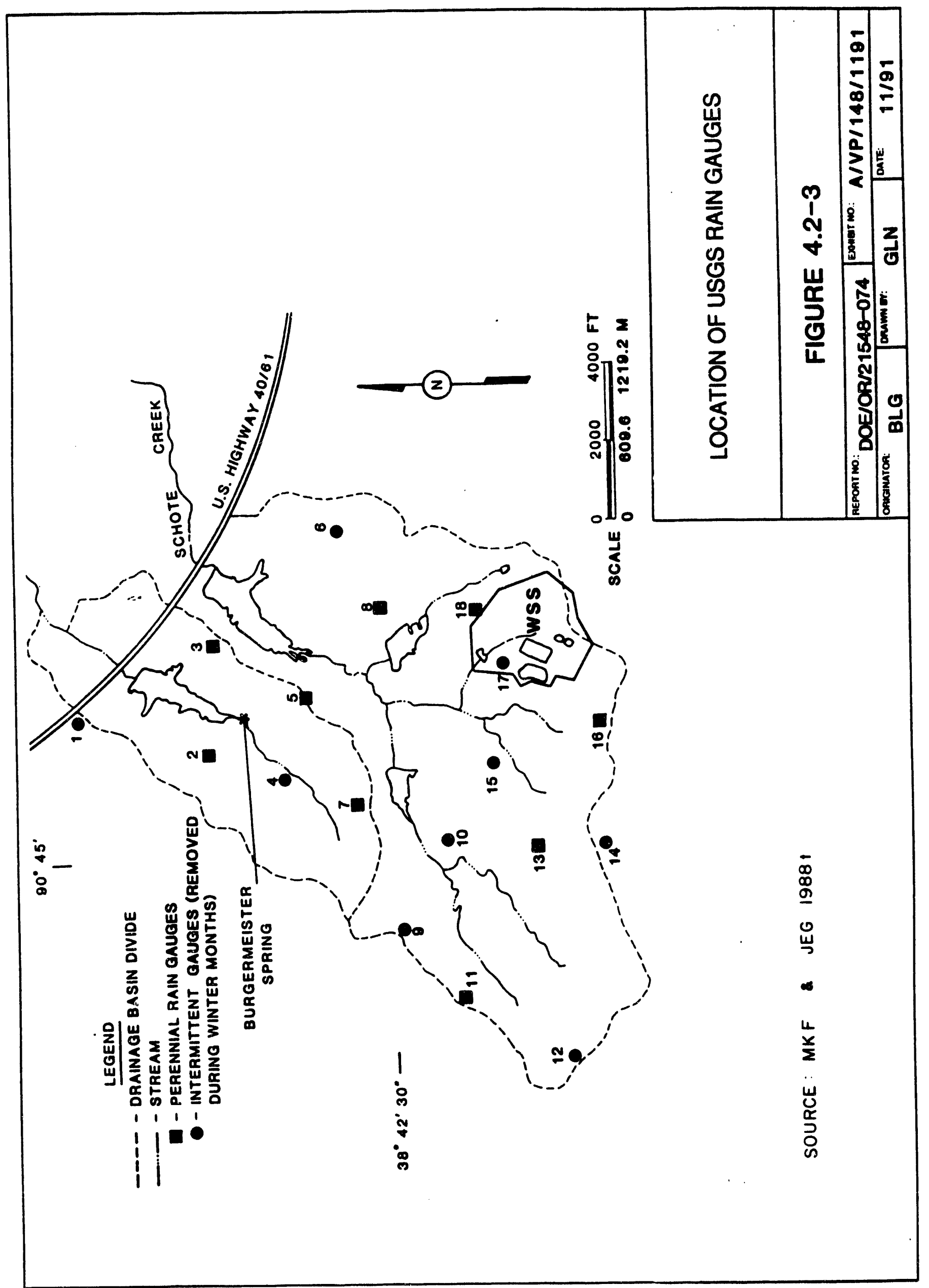




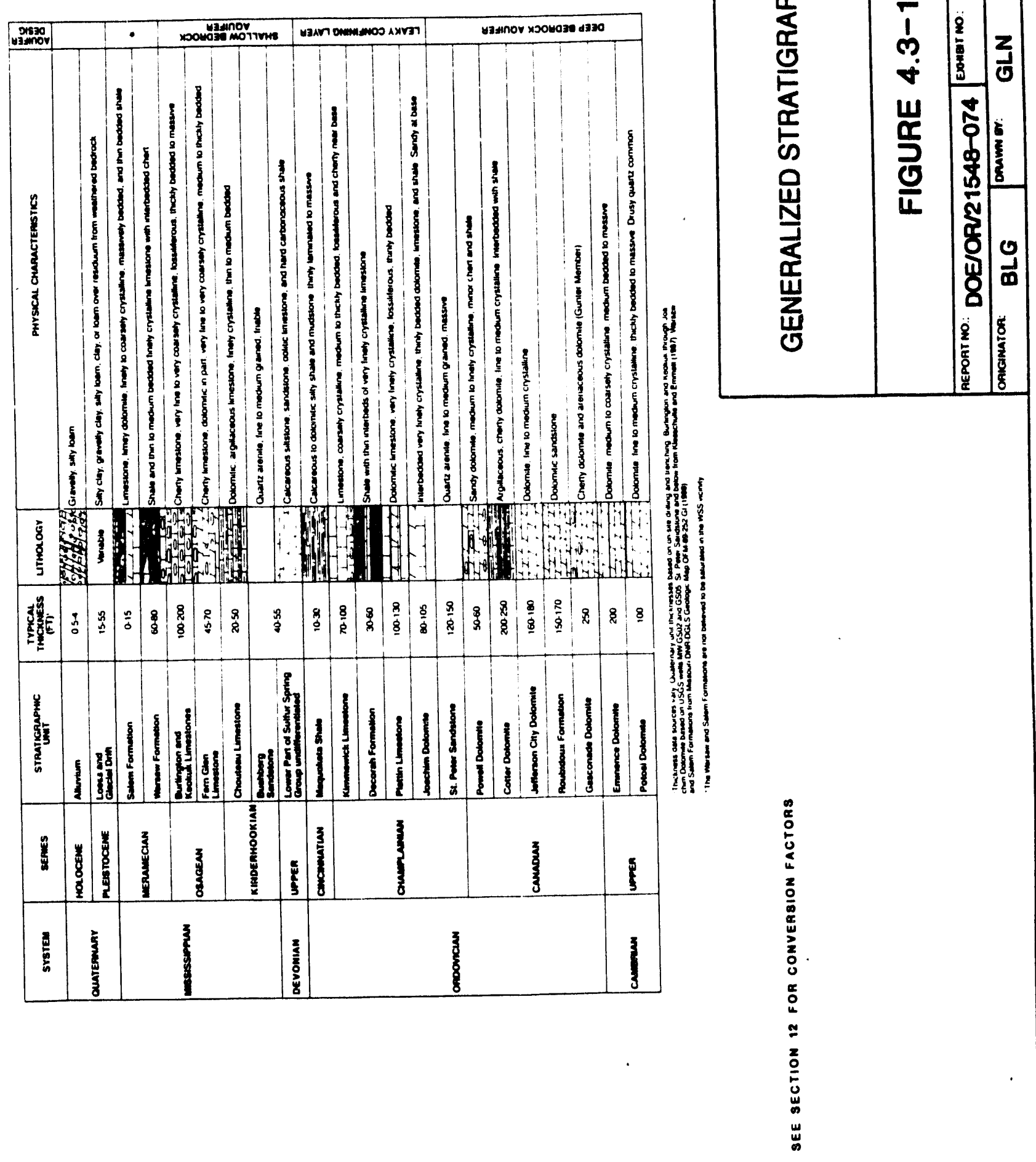




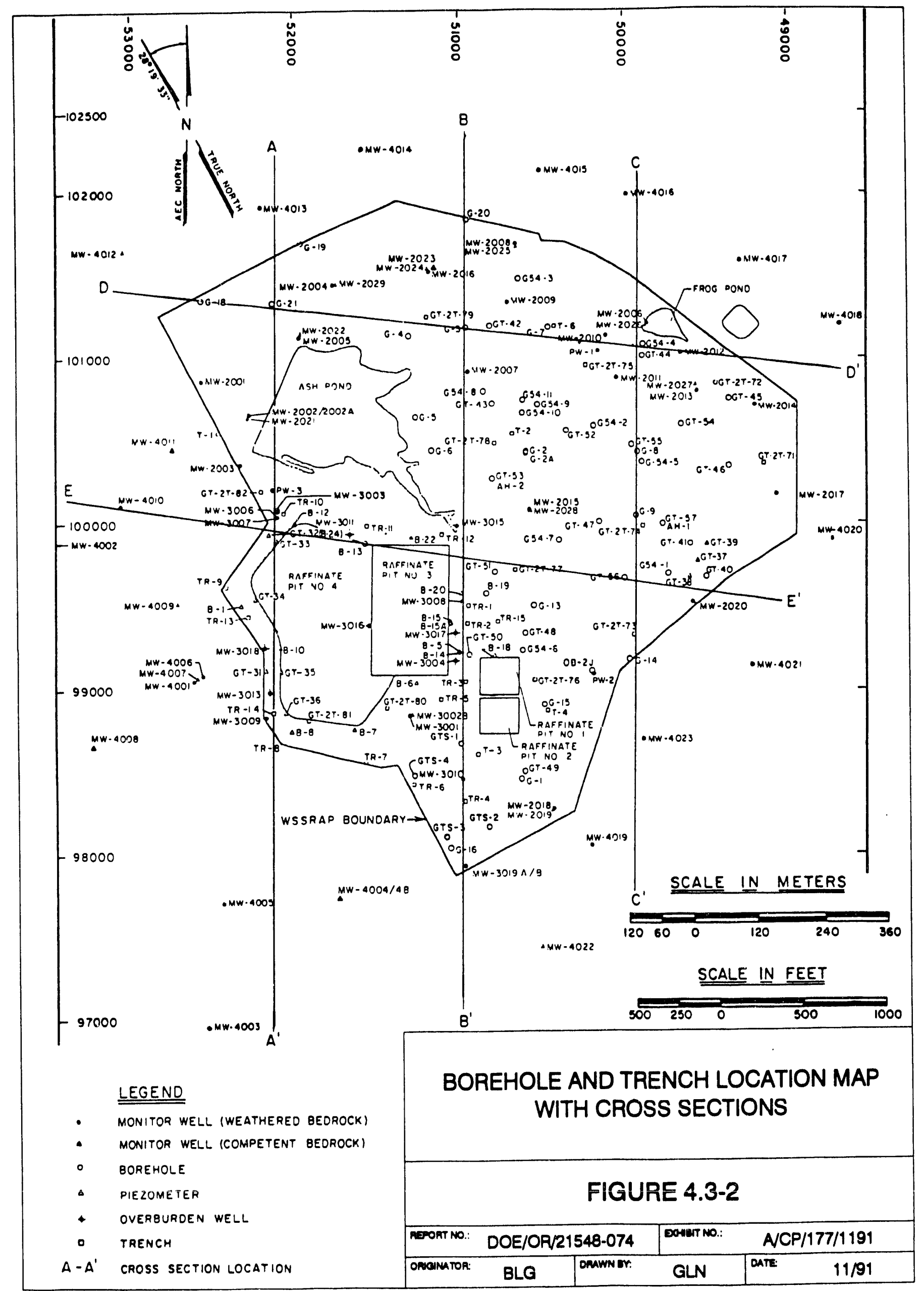




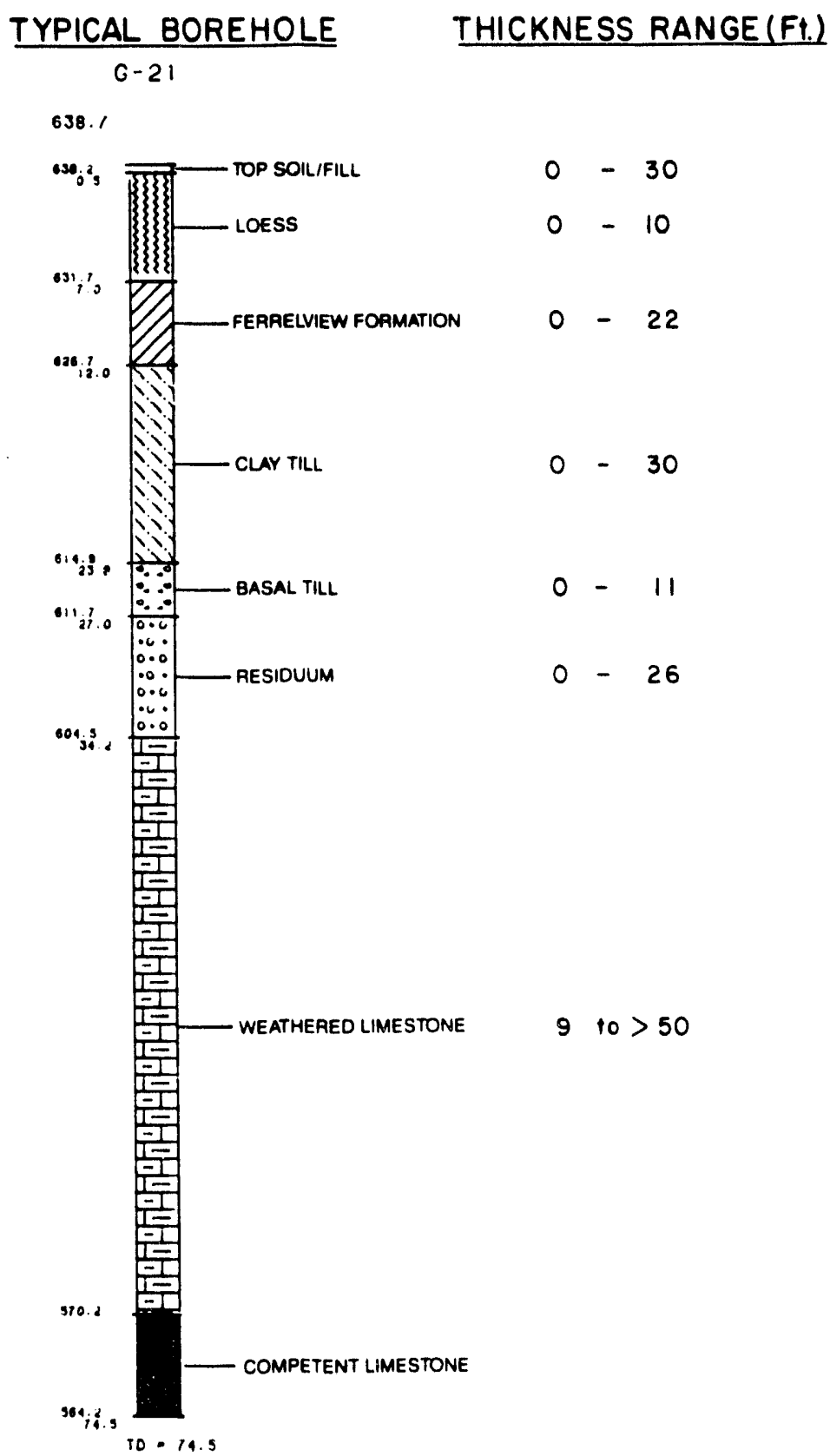

TYPICAL BOREHOLE LITHOLOGY

FIGURE 4.3-3

\begin{tabular}{|c|c|c|}
\hline AEPOAT NO & DOE/OR/21548-074 & ExHET No $A / P \mid / 230 / 1191$ \\
\hline
\end{tabular}




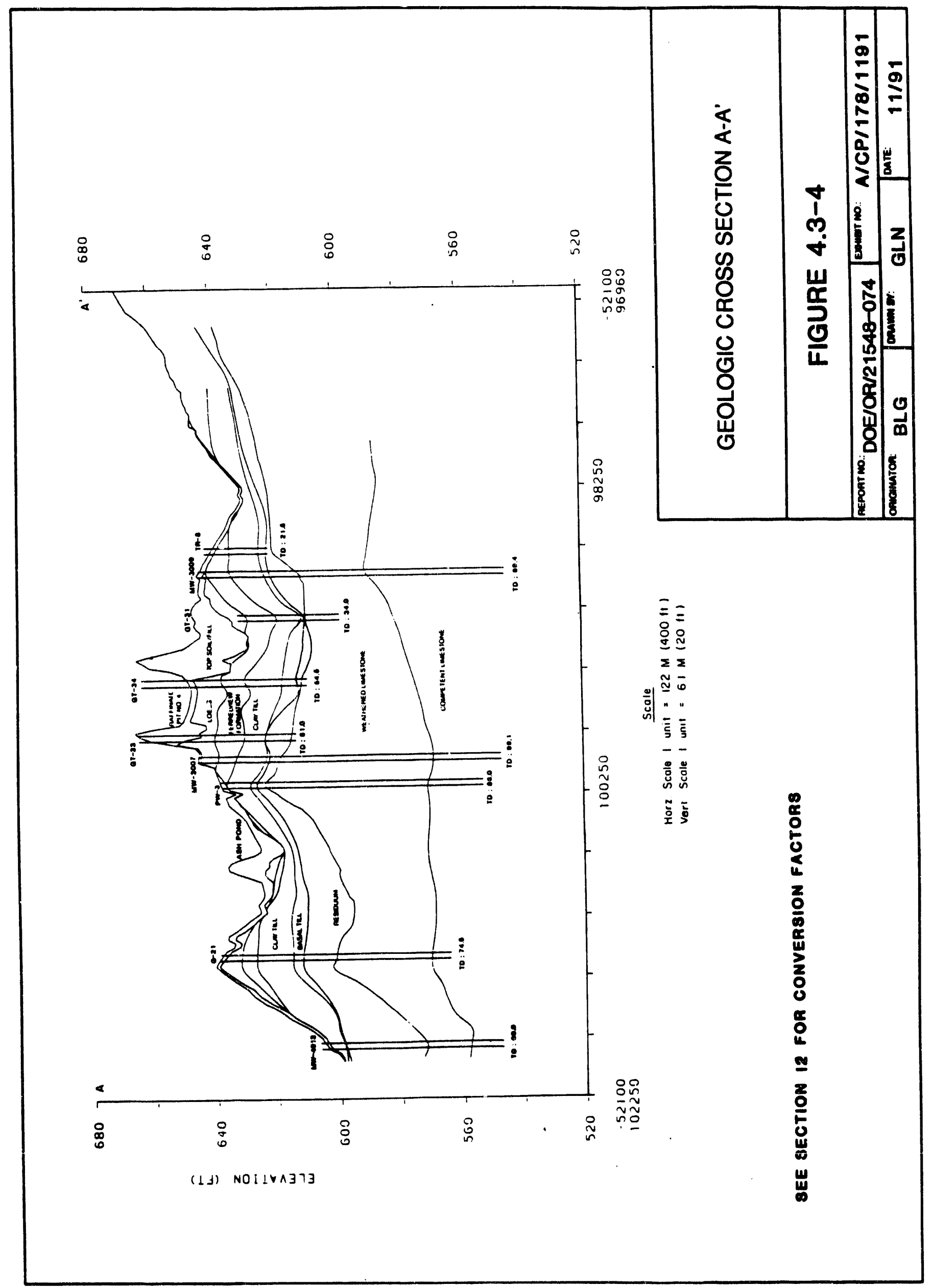




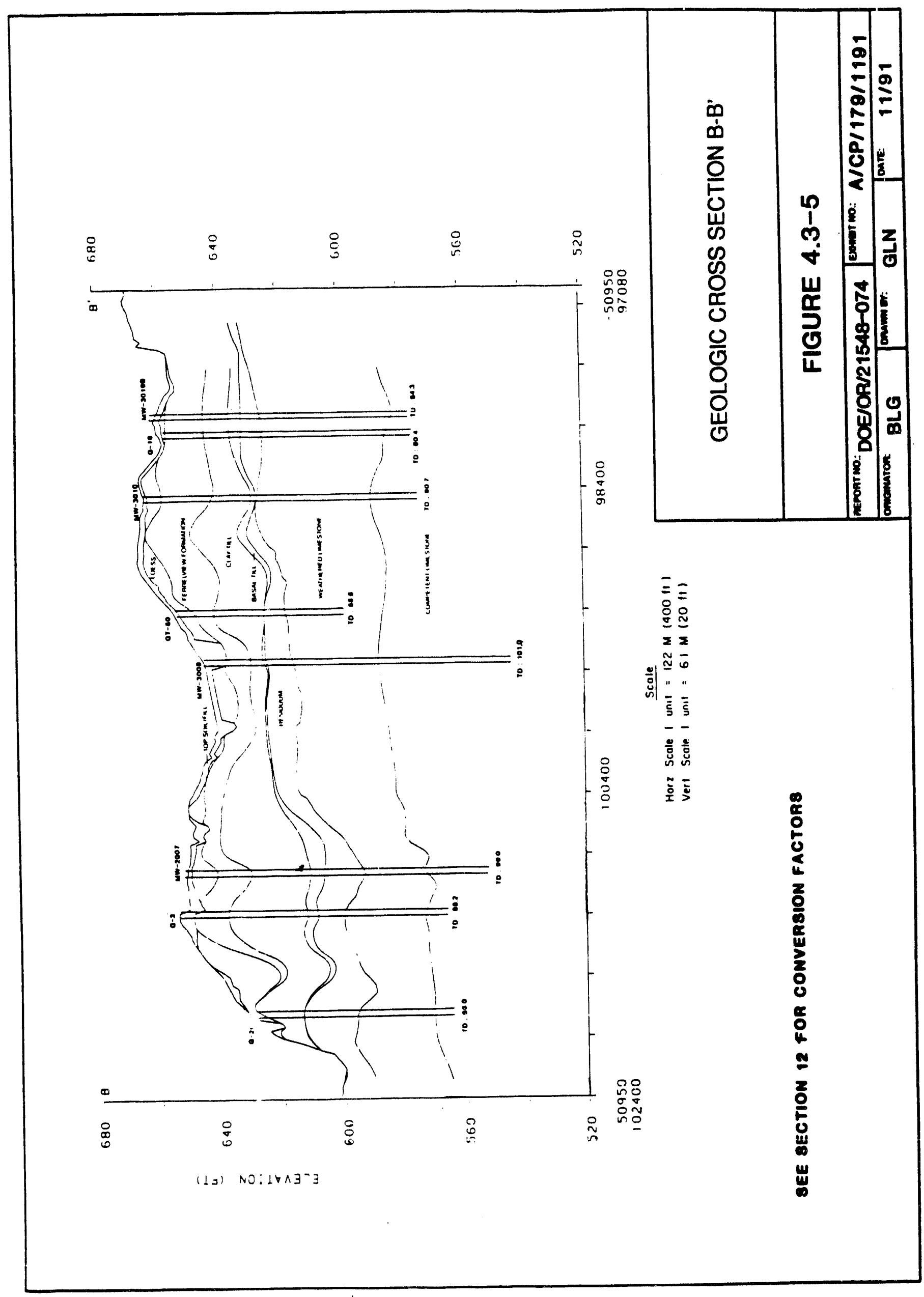




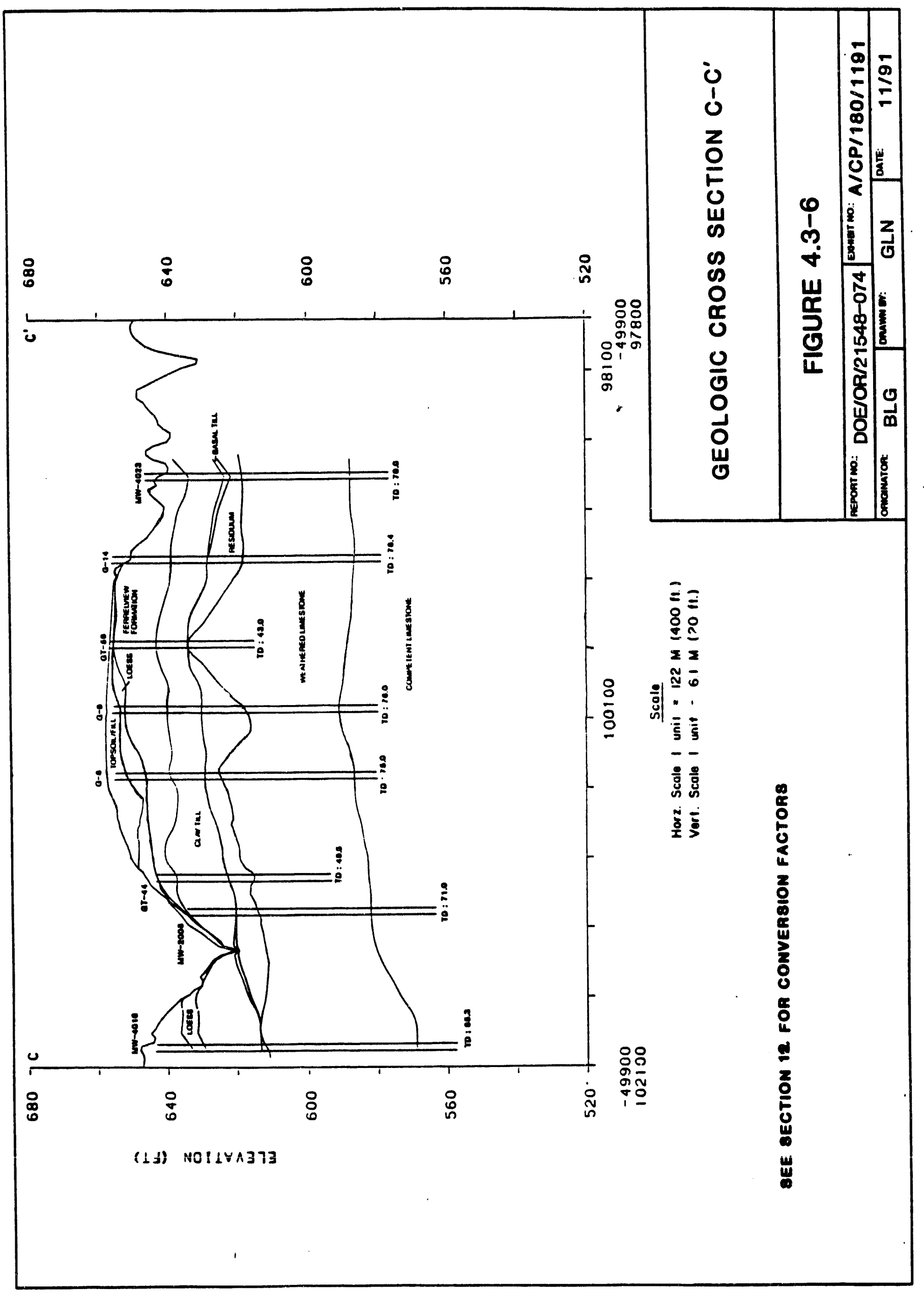




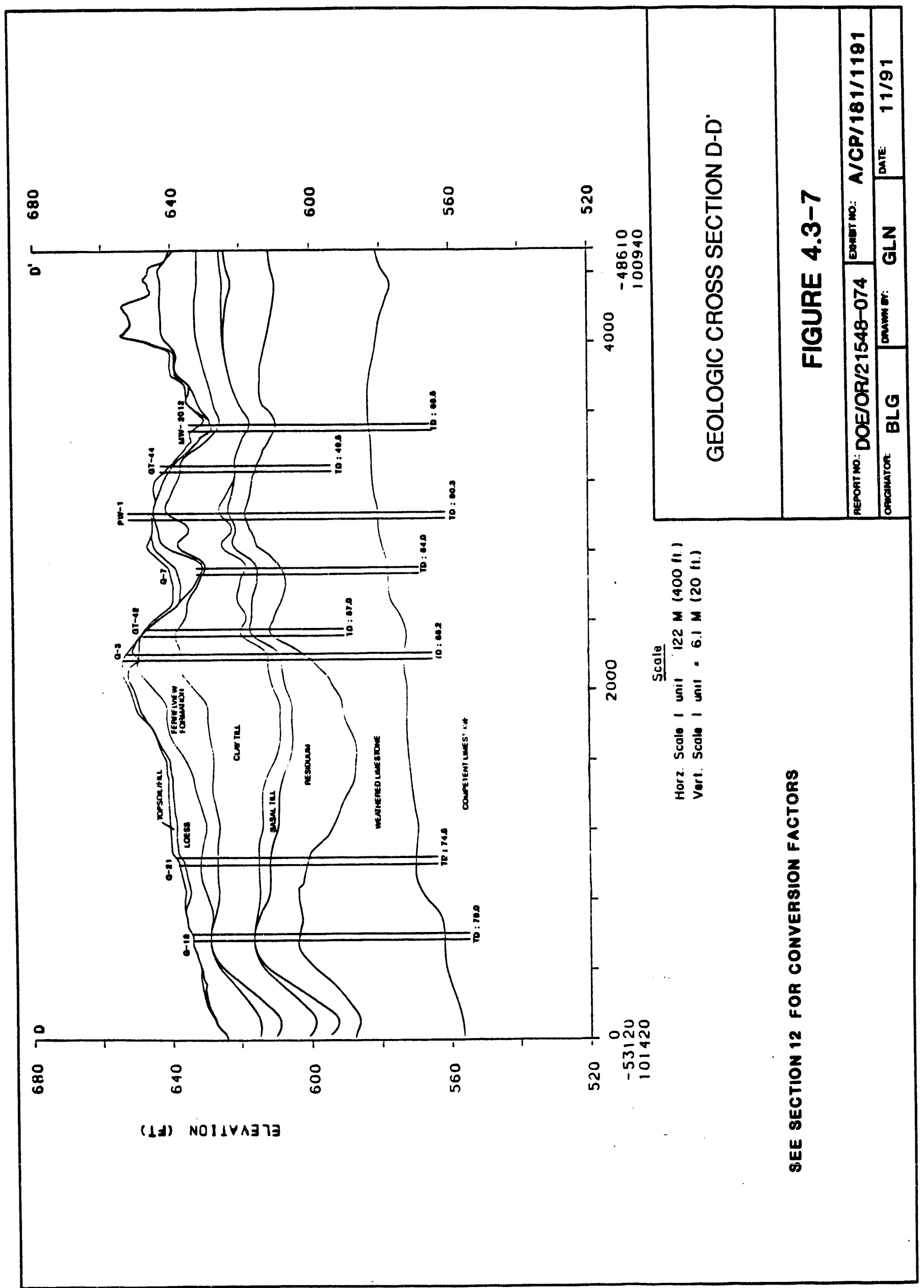




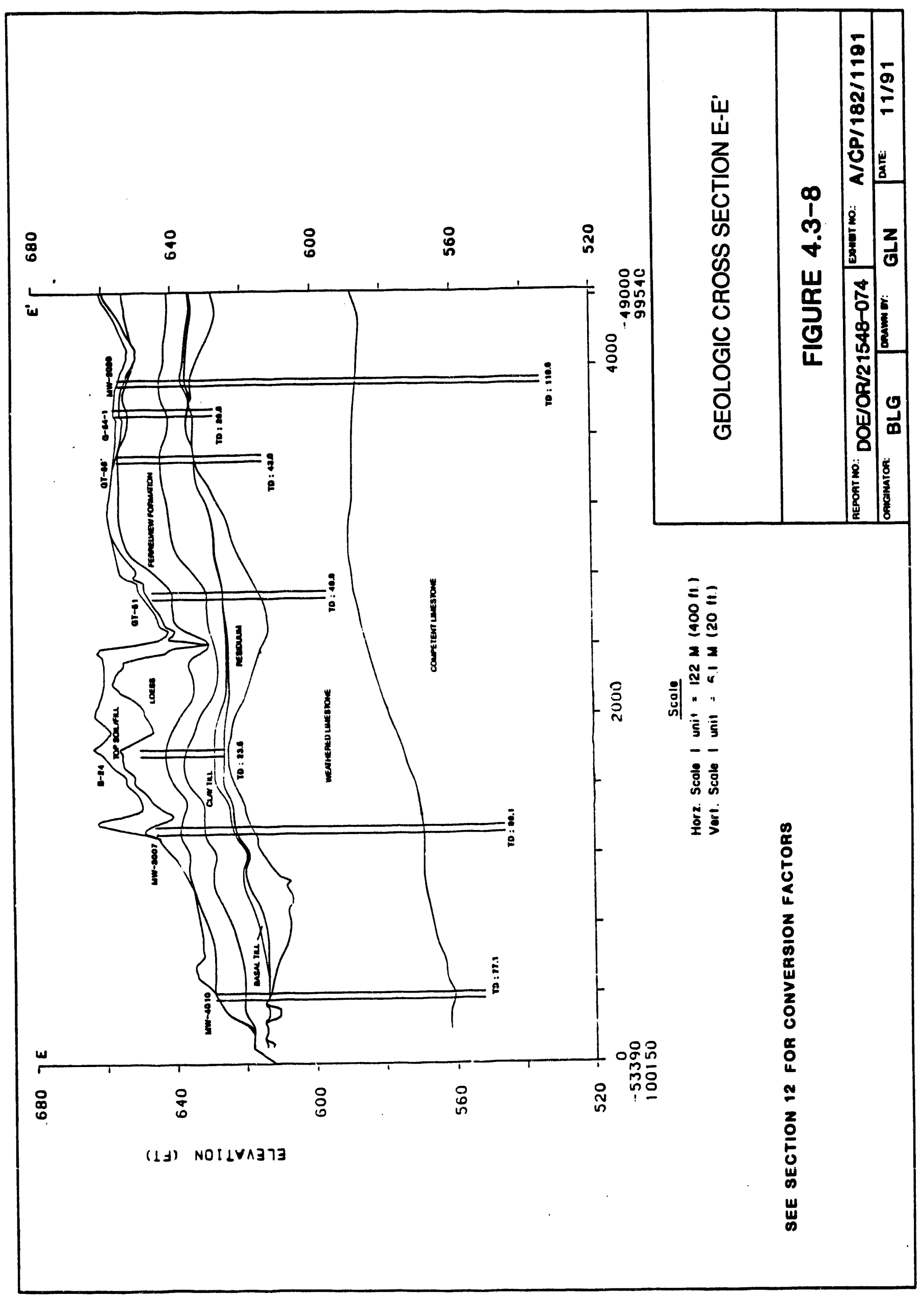




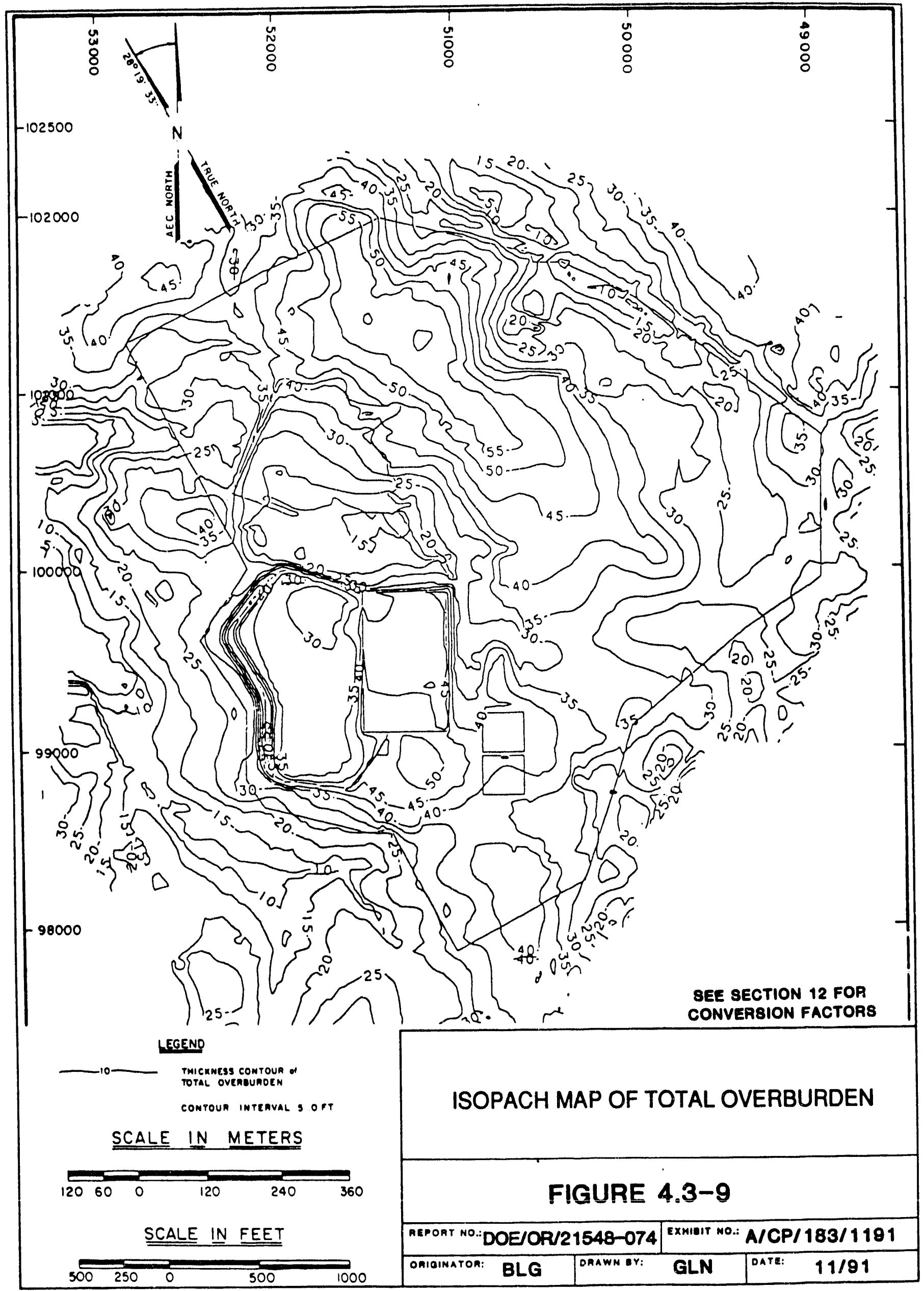




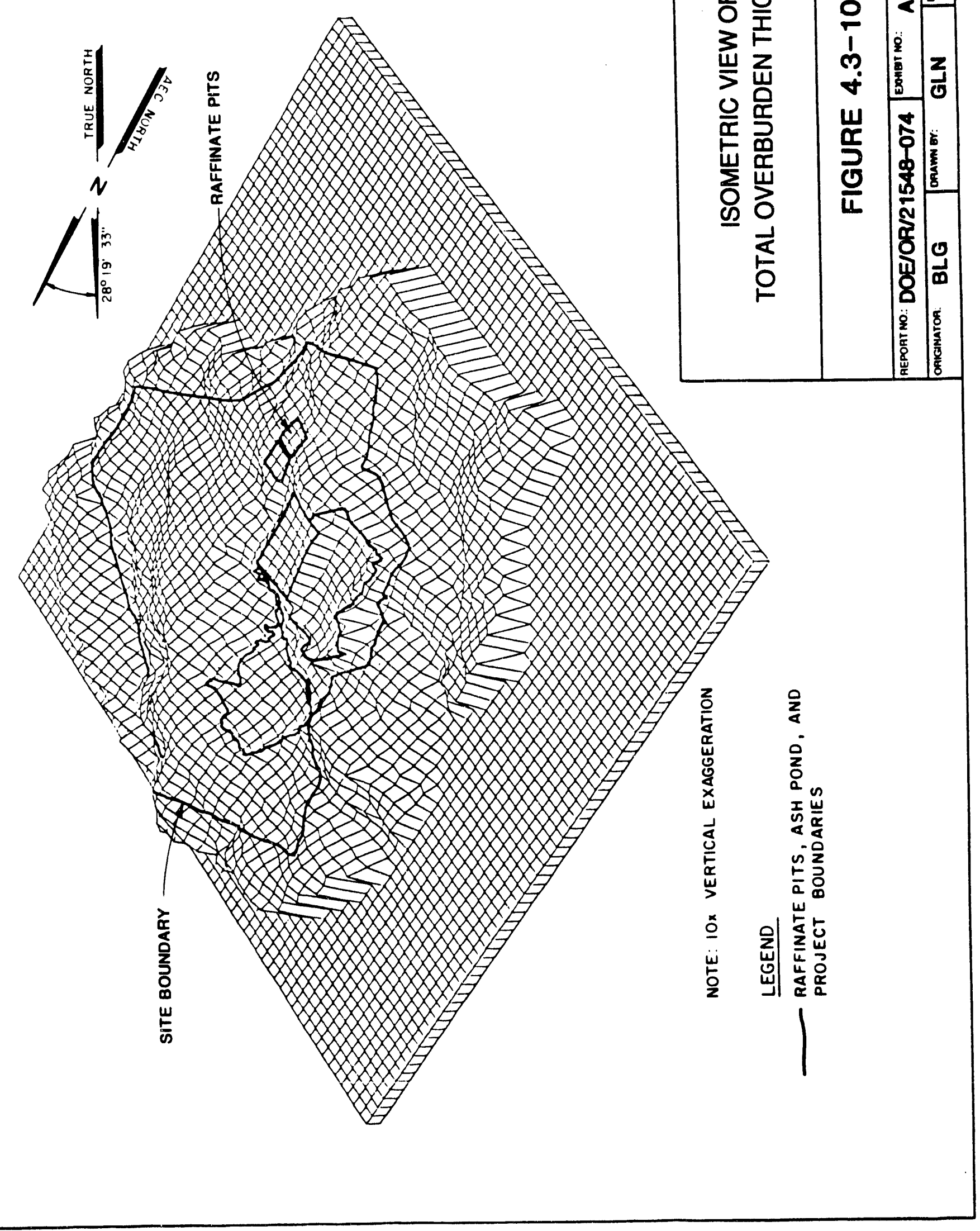




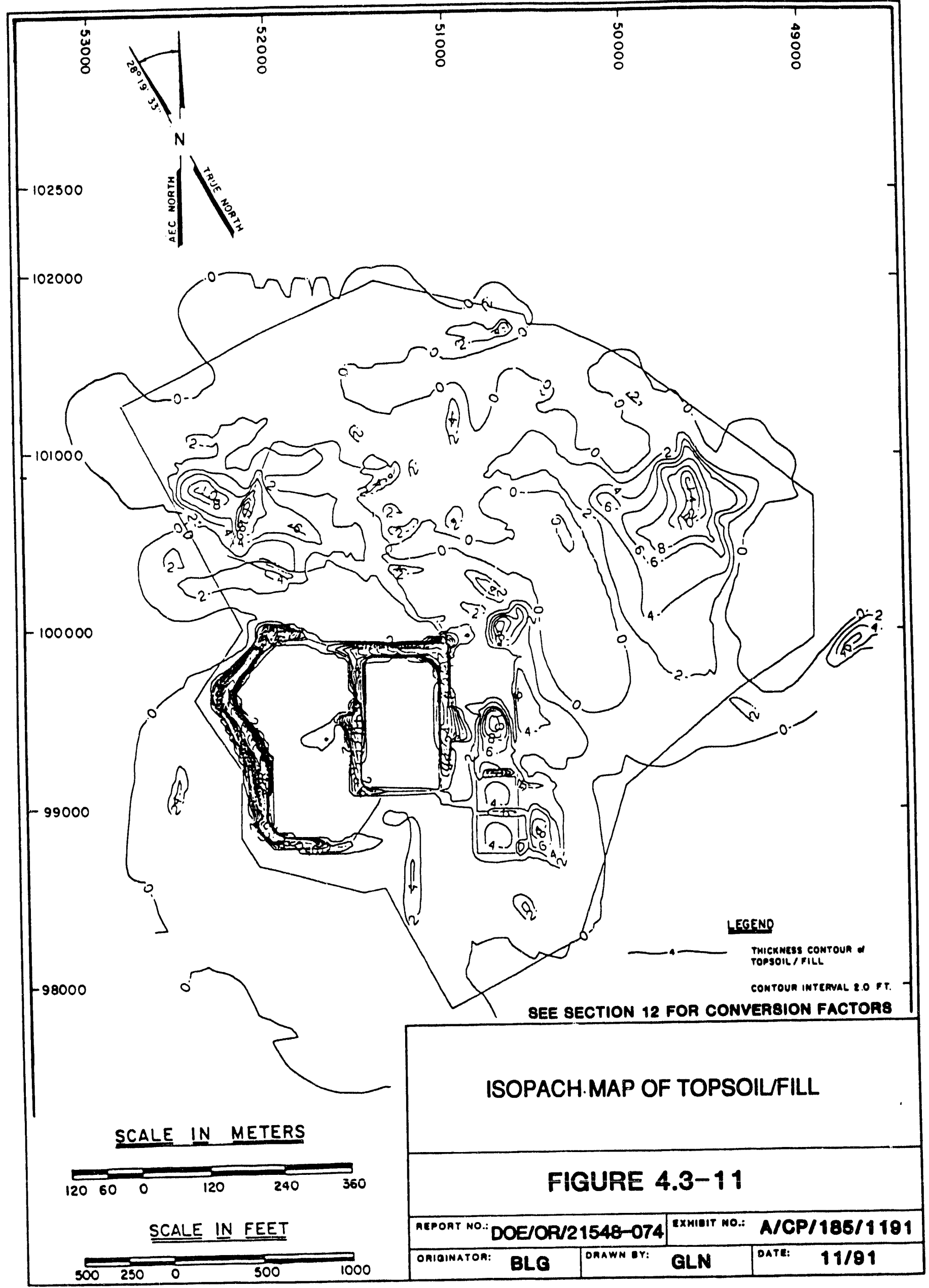




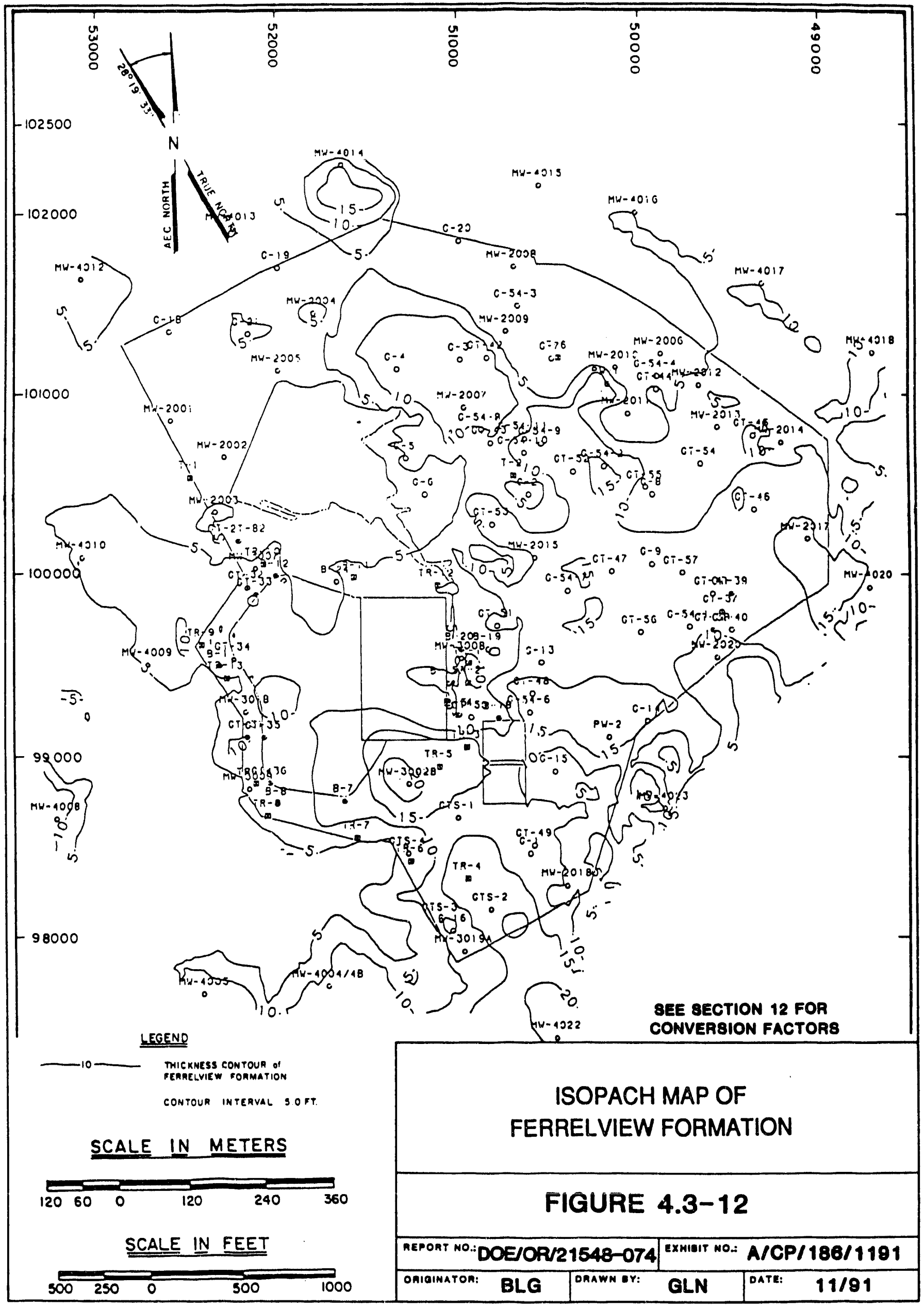




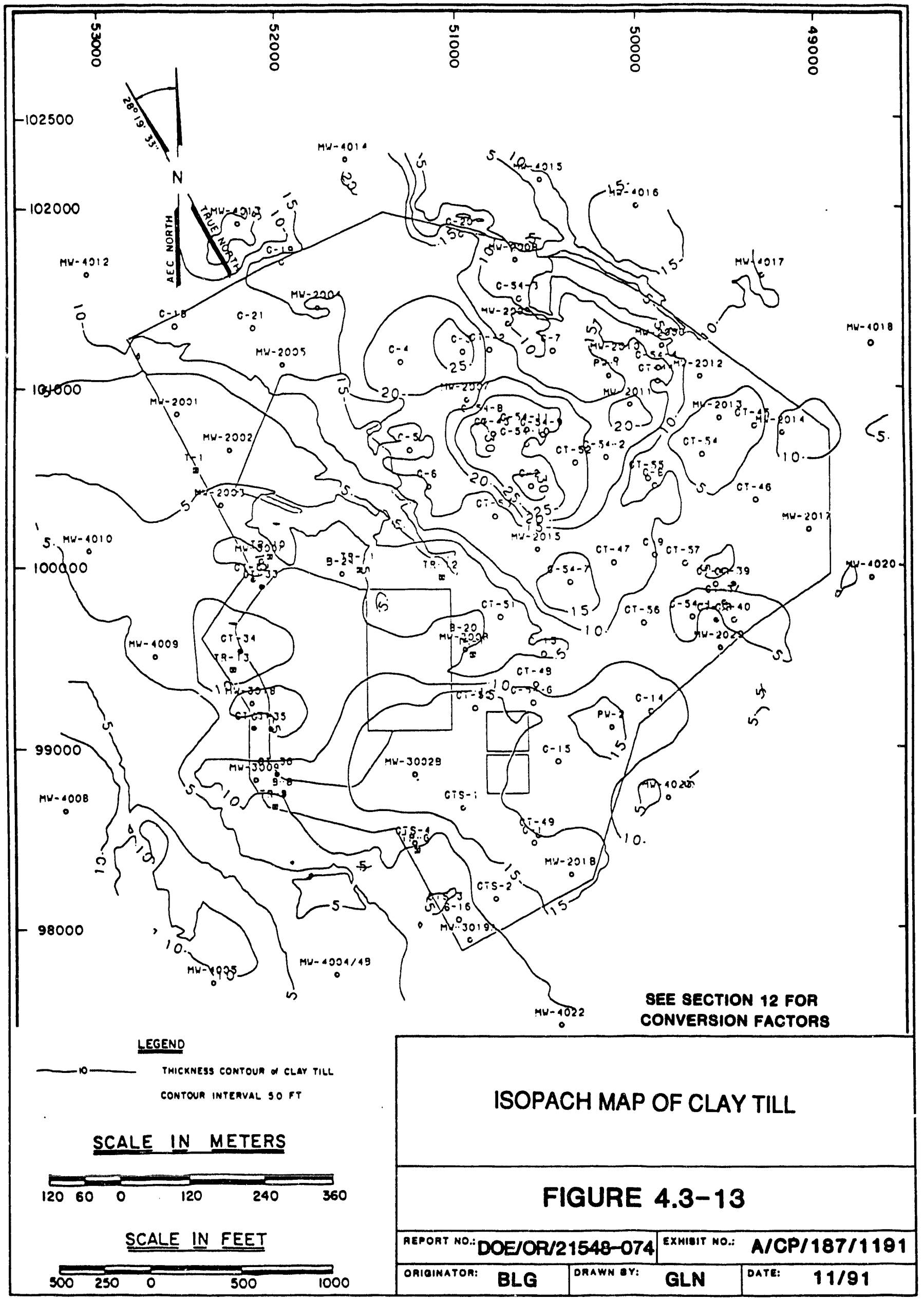




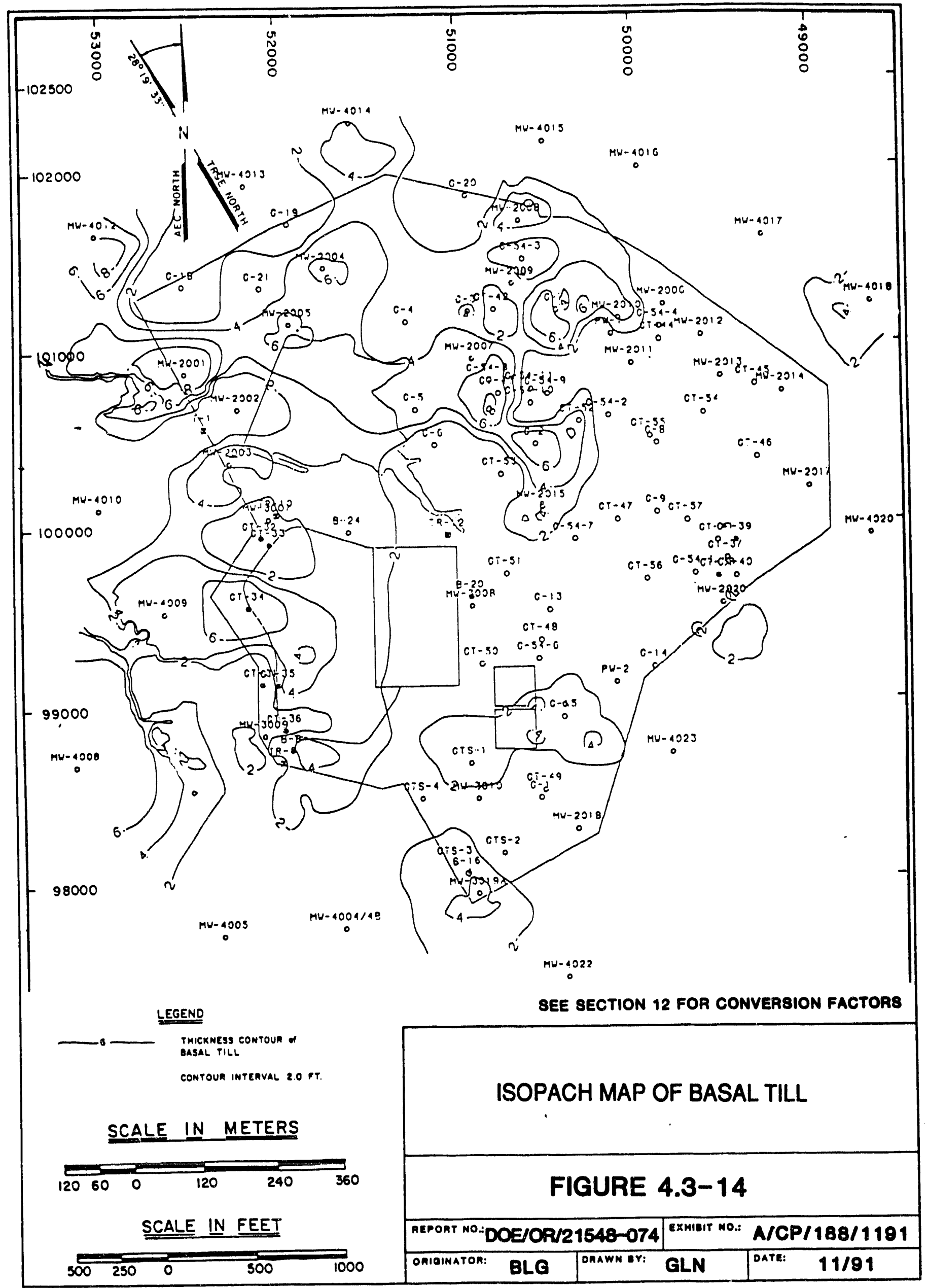




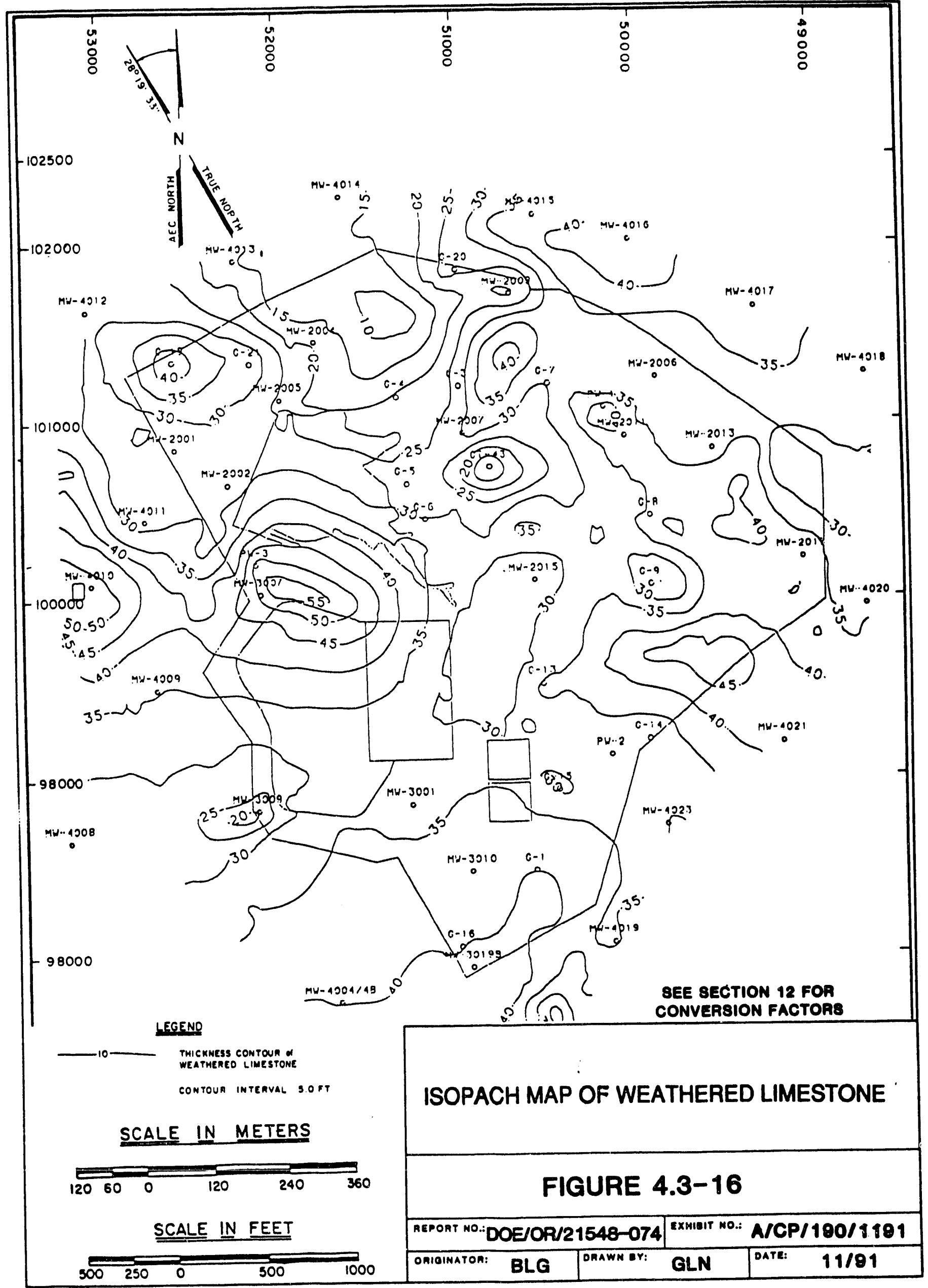




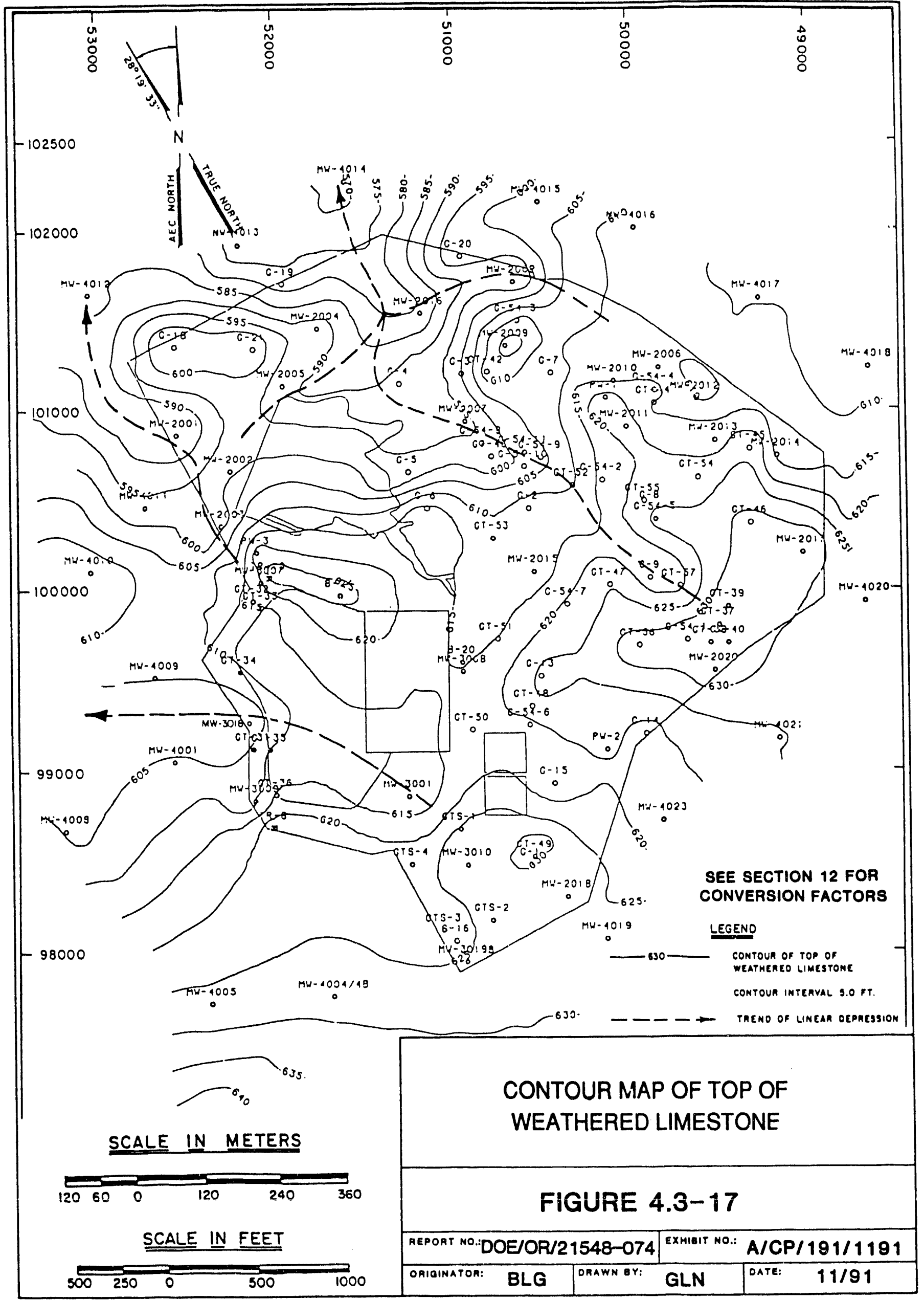




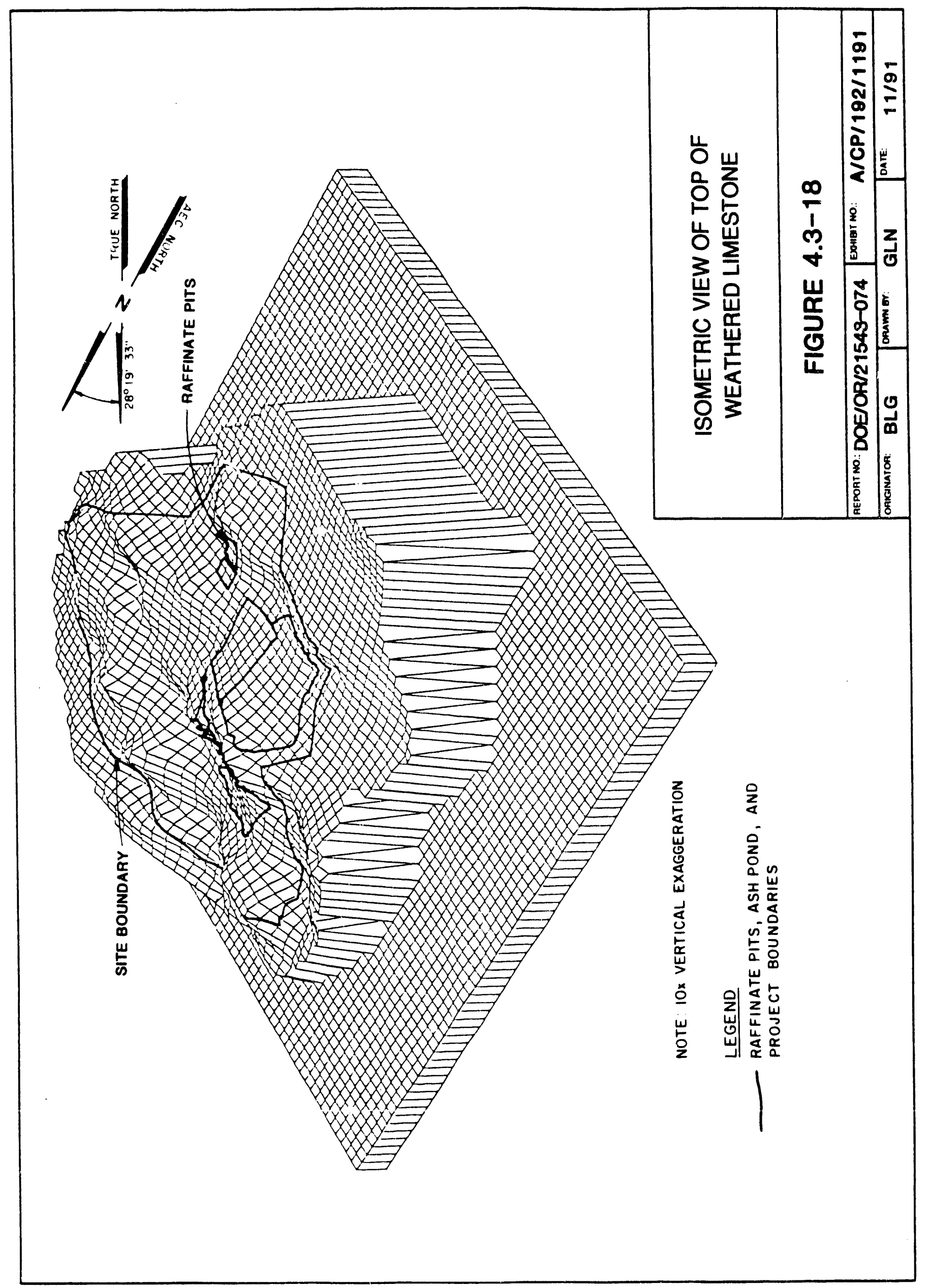




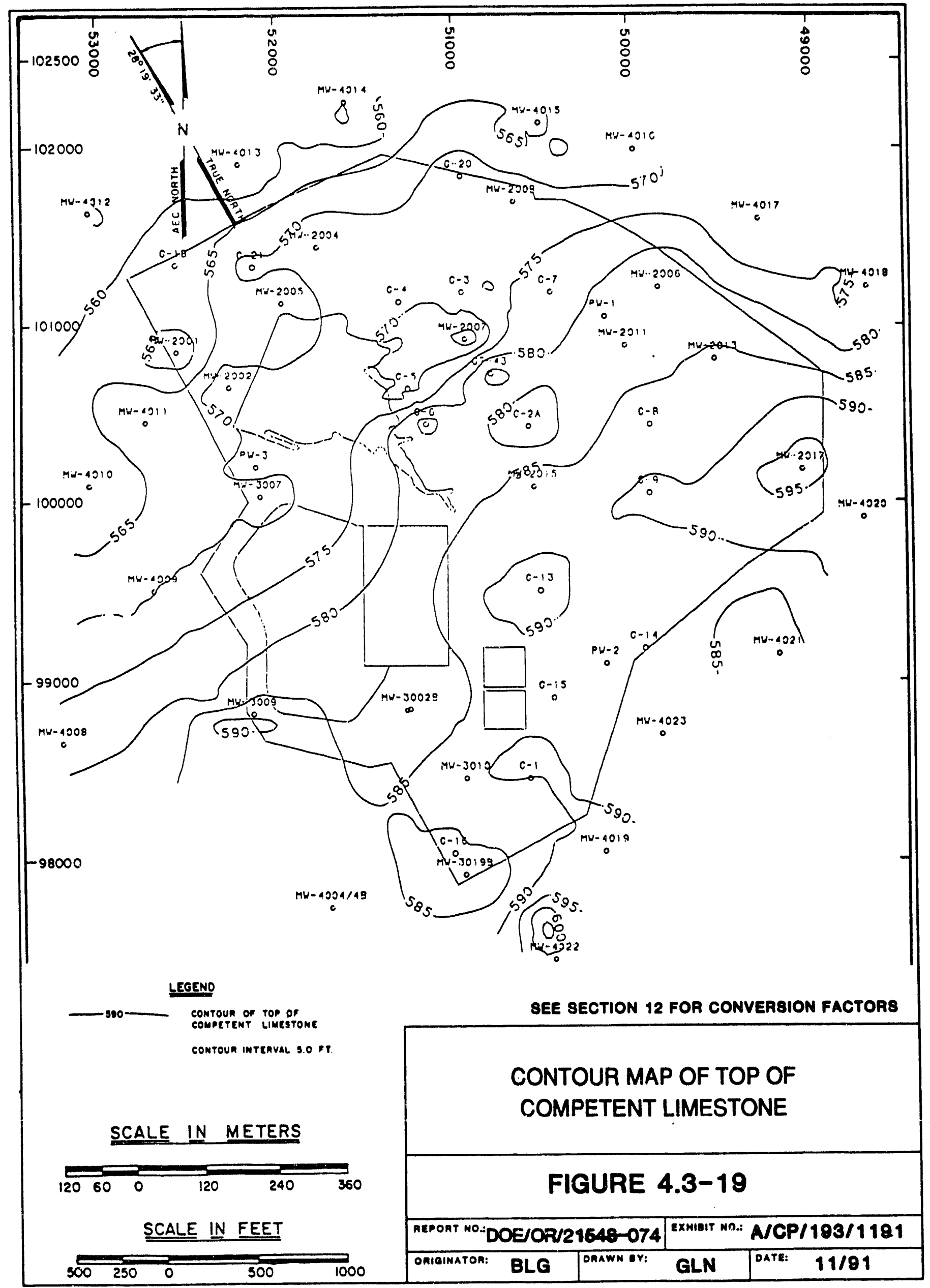




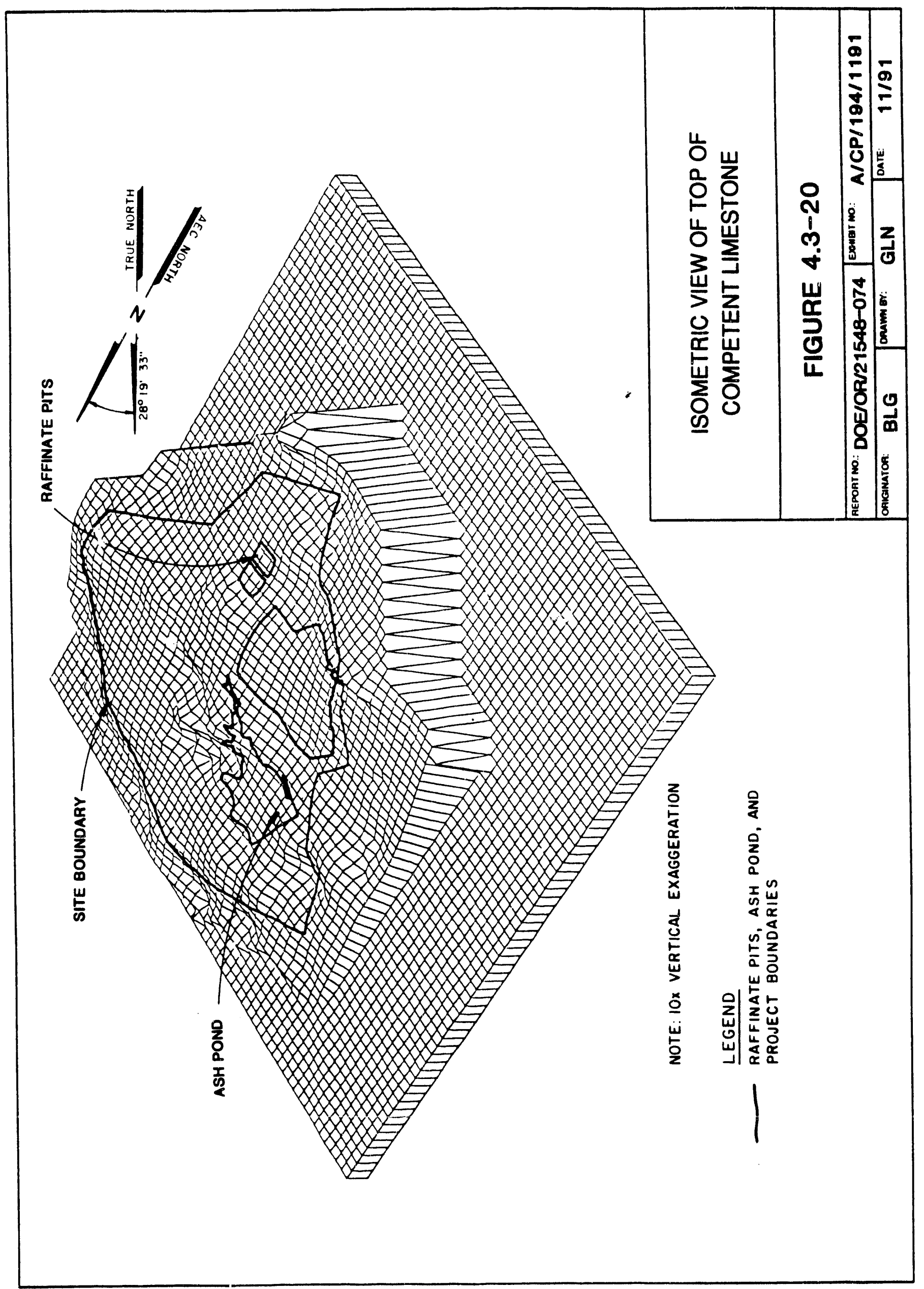




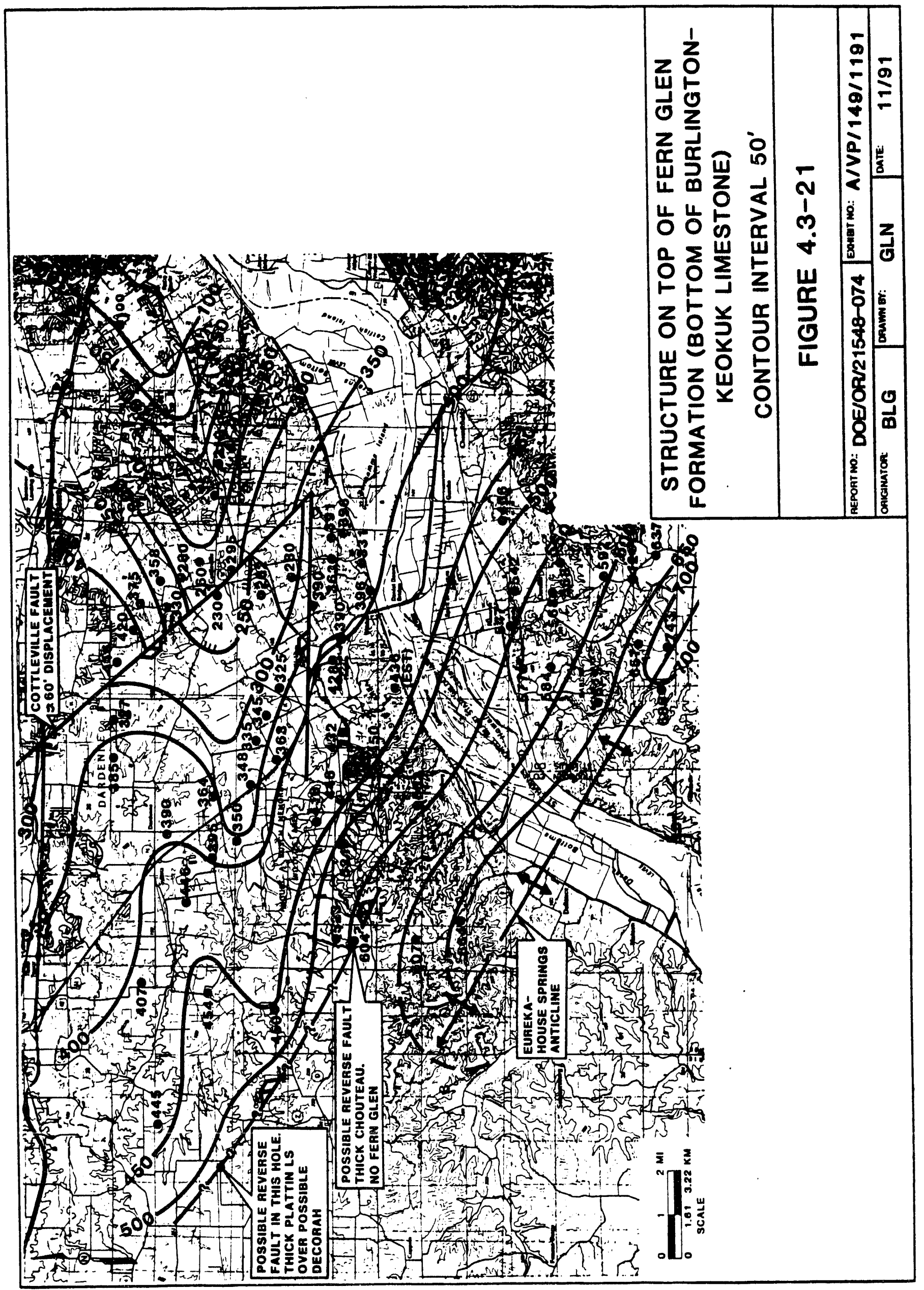




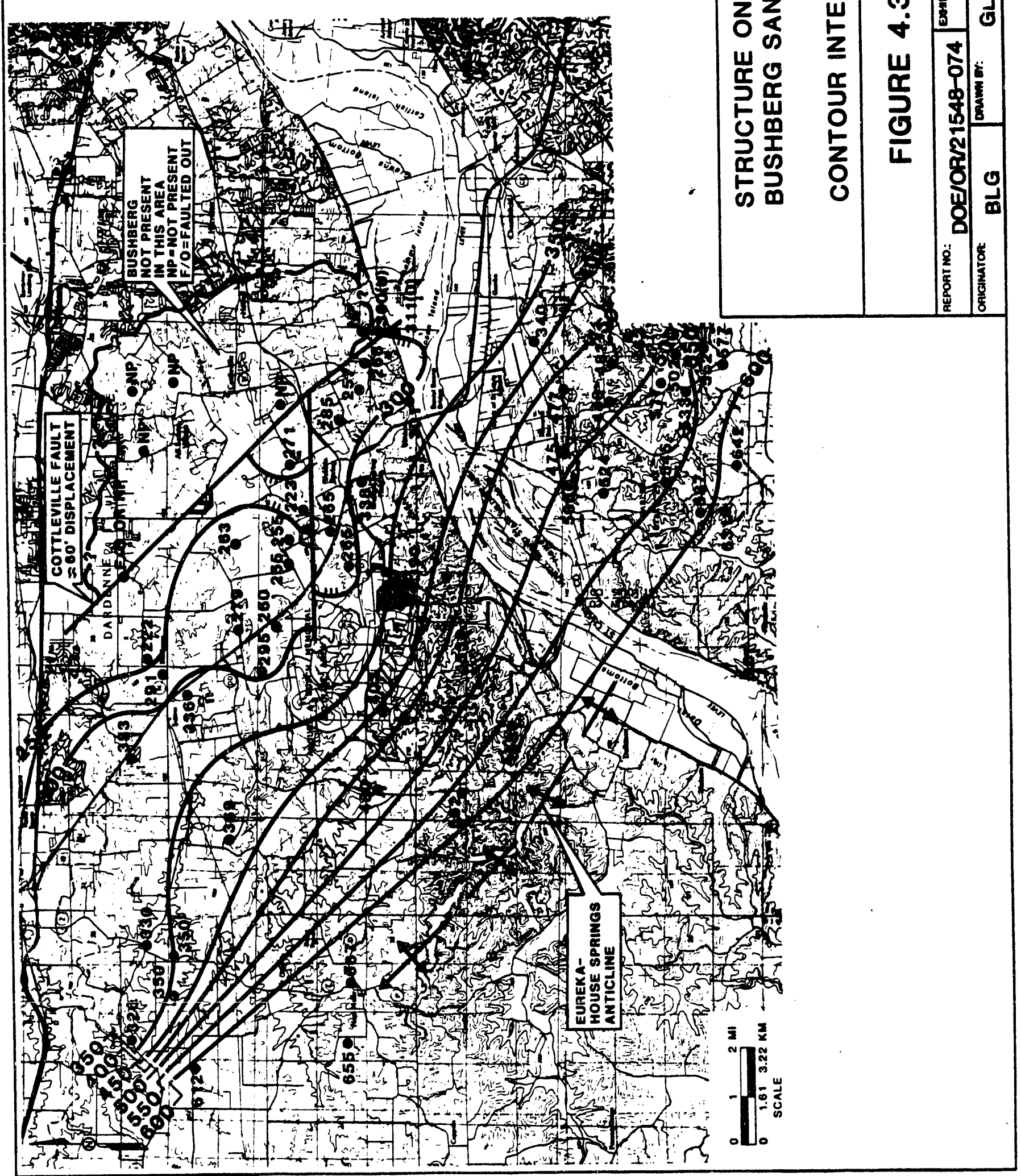




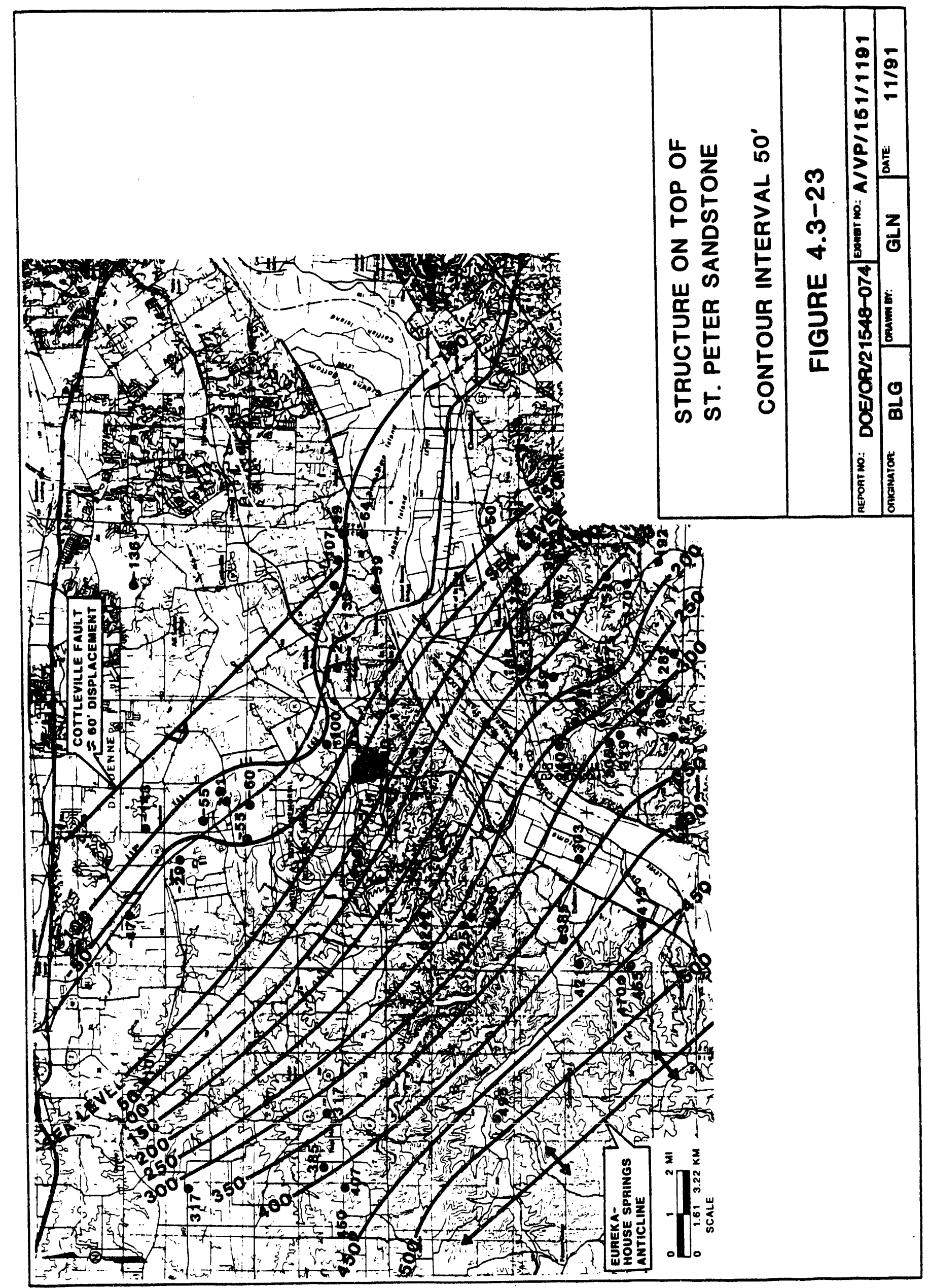




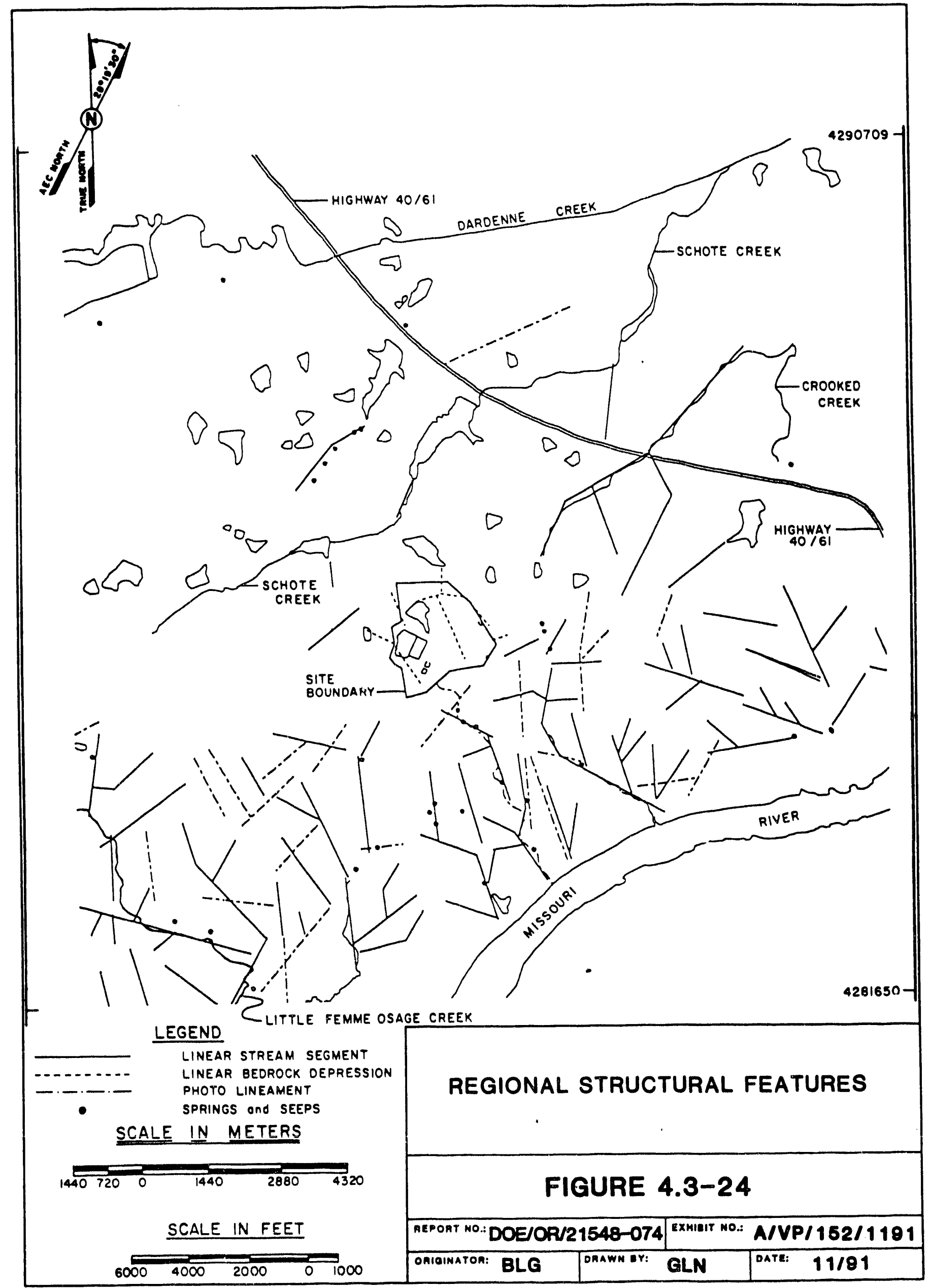



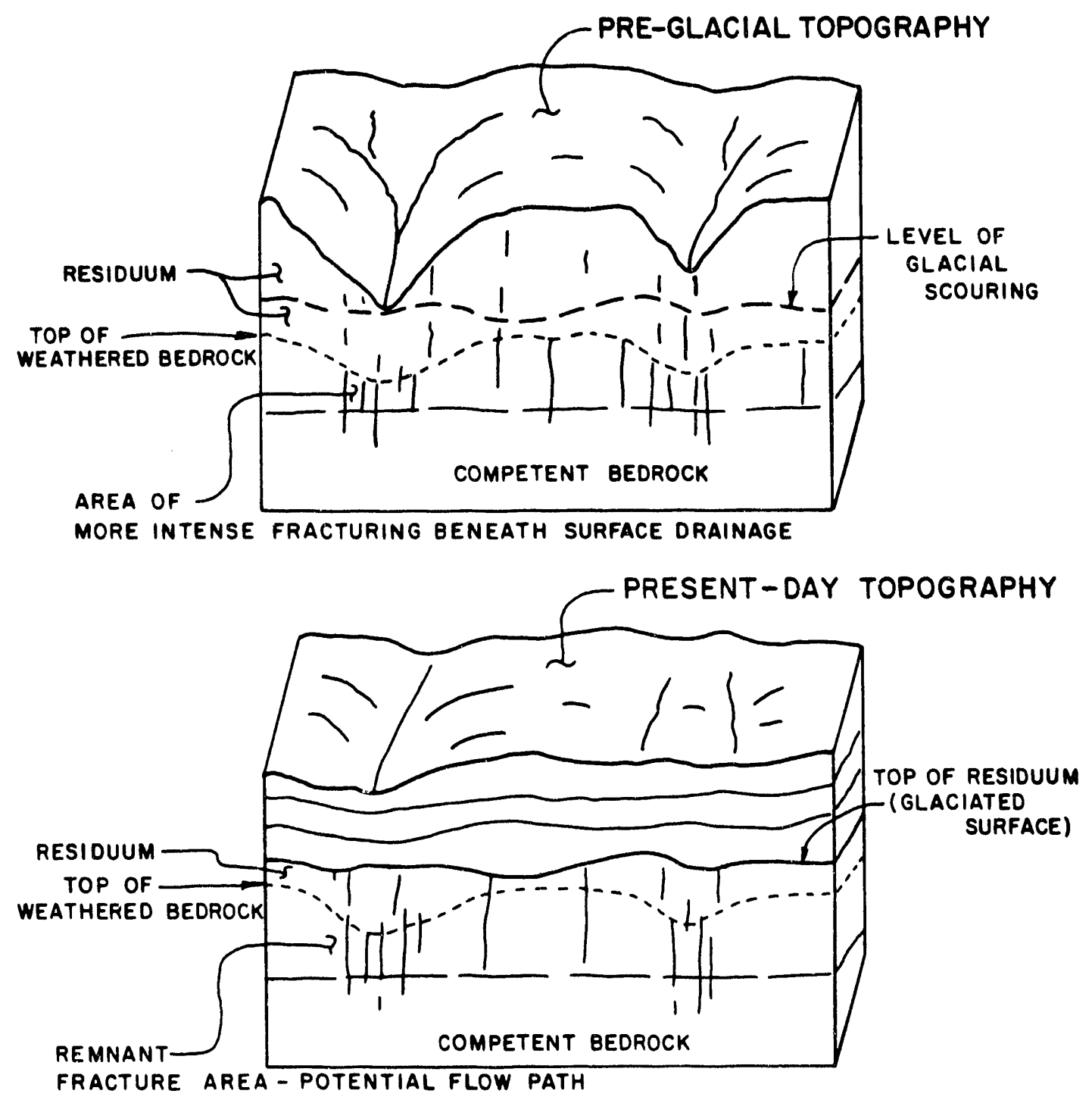

( NOT TO SCALE)

BLOCK DIAGRAM DEPICTING GENESIS OF WEATHERED BEDROCK SURFACE

FIGURE 4.3-25

\begin{tabular}{|c|c|c|c|c|c|}
\hline AERORT NO: & DOEJOF & $1548-074$ & EDHAT No & $\mid \mathbf{P I}$ & $1 / 1181$ \\
\hline OARGIMATOA & BLG & Deanim or & GLN & DATE & $11 / 91$ \\
\hline
\end{tabular}




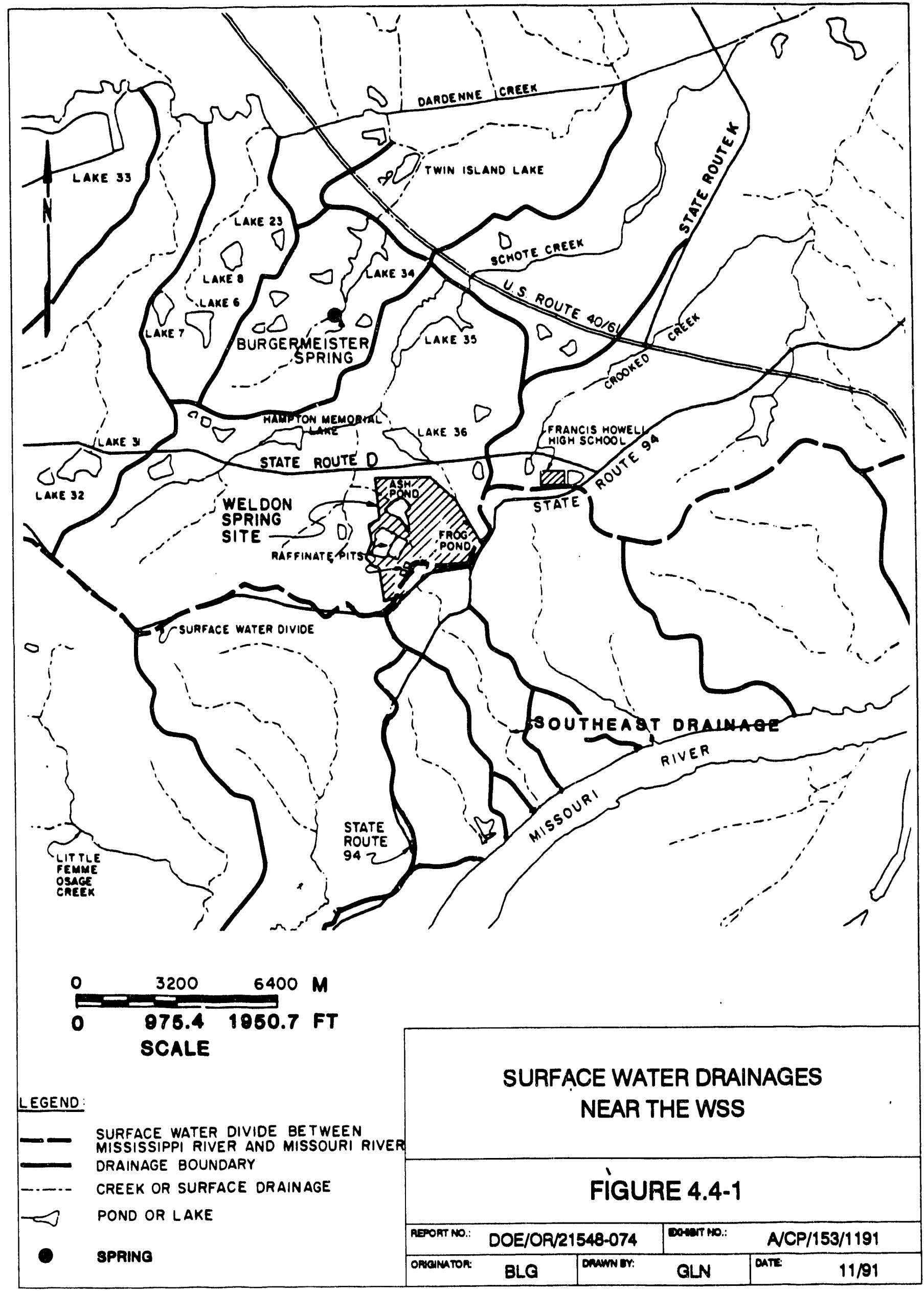




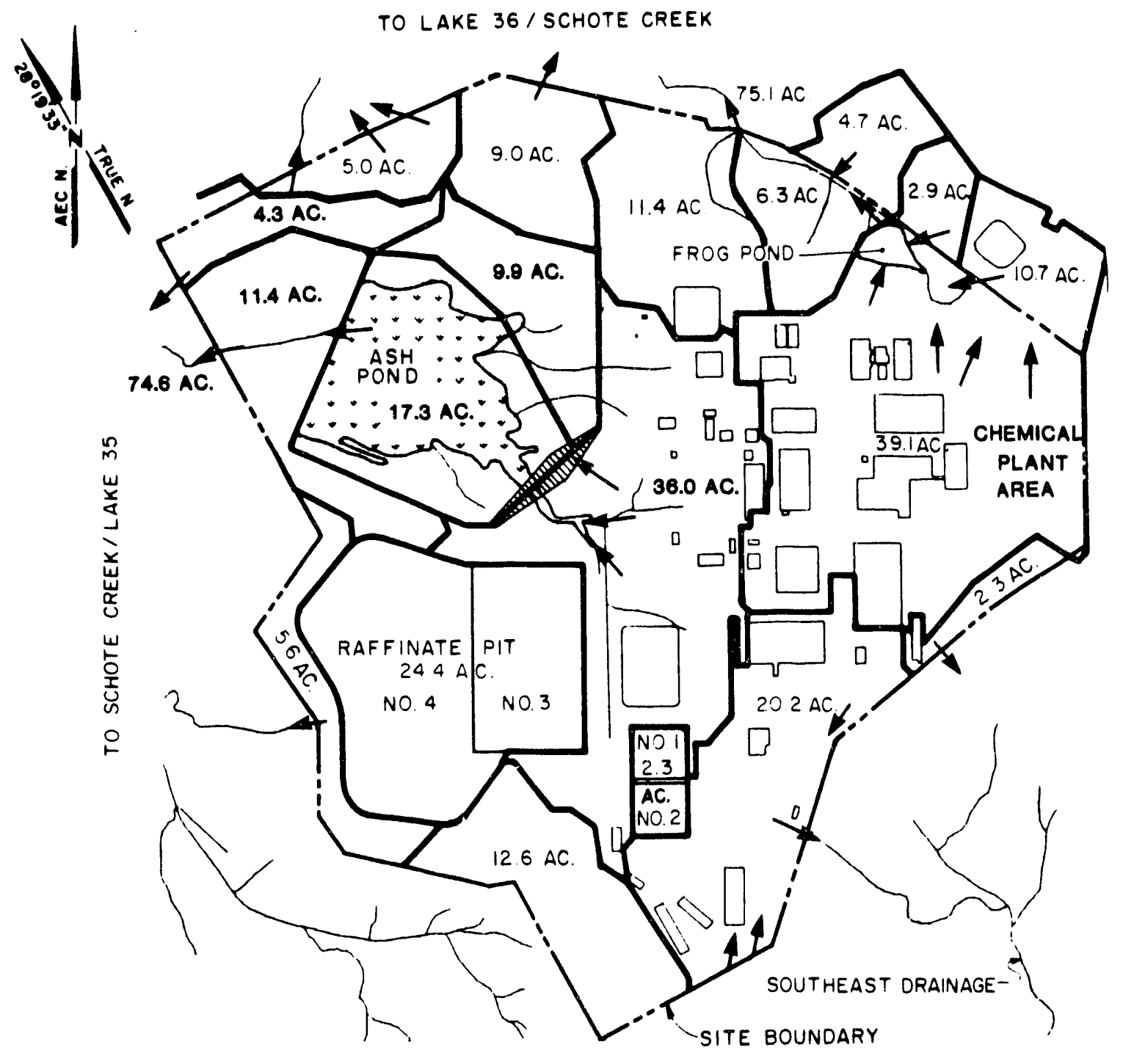

LEGEND:

- CREEK OR DRAINAGE DITCH

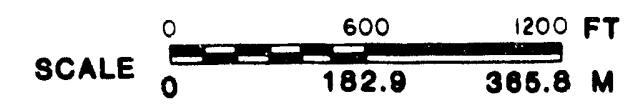

\section{DRAINAGE BASIN BOUNDARY}

$\longleftarrow$ FLOW DIRECTION

\section{SURFACE WATER DRAINAGE AT THE WELDON SPRING SITE}

FIGURE 4.4-2

\begin{tabular}{|c|c|c|c|c|}
\hline PEPOAT NO. & DOEIOF & $548-074$ & EOHETT HO & A/CP/186/1191 \\
\hline ORIGIMATOR. & BLG & Dram or & GLN & $11 / 91$ \\
\hline
\end{tabular}




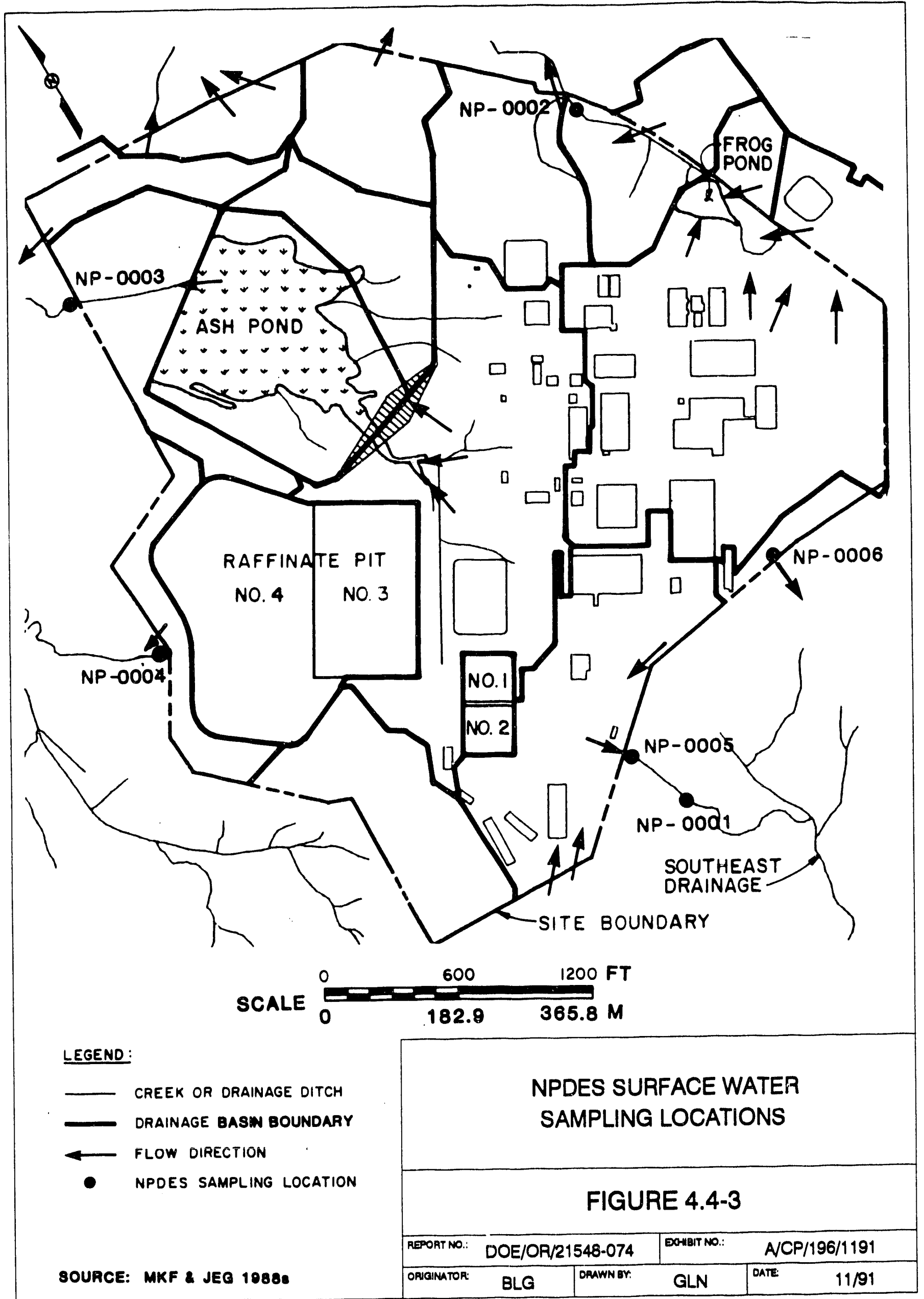




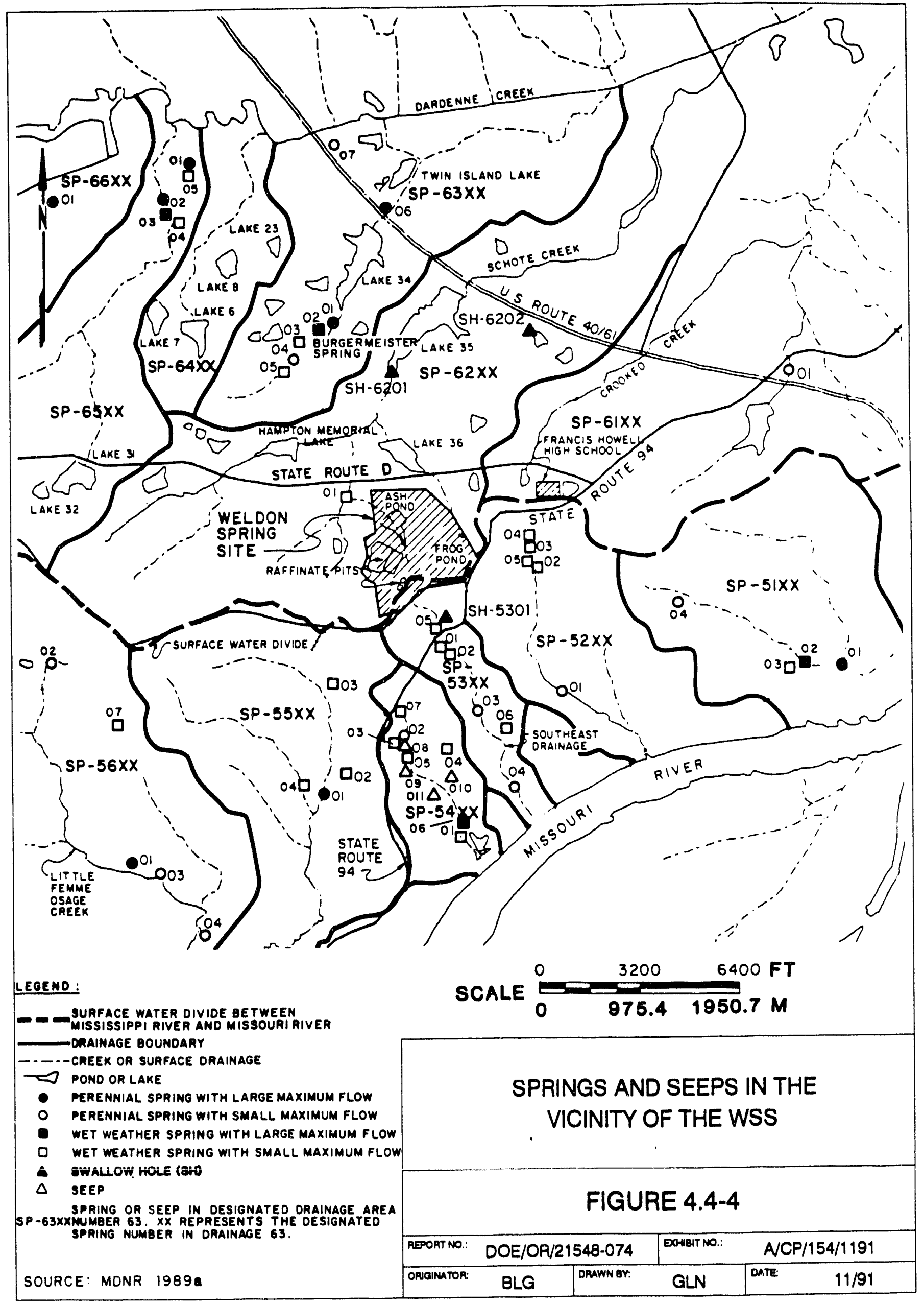



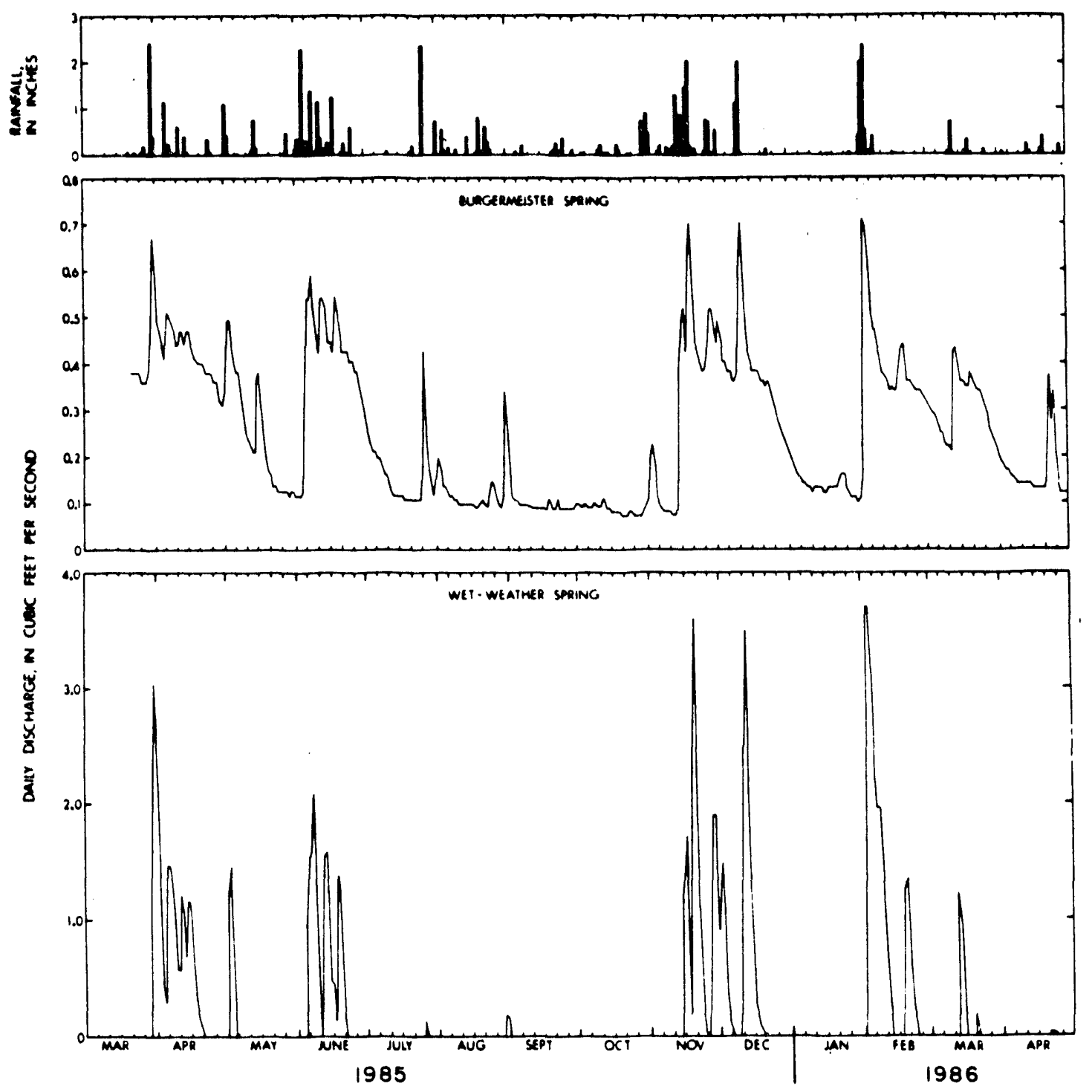

SEE SECTION 12 FOR CONVERSION FACTORS

SOURCE : KLEESCHULTE AND EMMETT 1987

\begin{tabular}{|c|c|}
\hline $\begin{array}{r}\text { HYDROGRAPHS OF BU } \\
\text { AND NEARBY WE- } \\
\text { MARCH } 1985\end{array}$ & $\begin{array}{l}\text { JRGERMEISTER SPRING } \\
\text {-WEATHER SPRING, } \\
\text { TO APRIL } 1986\end{array}$ \\
\hline \multicolumn{2}{|c|}{ FIGURE 4.4-5 } \\
\hline AEPOAT NO DOE/OR/21548-074 & एontwit wo: $A / P 1 / 232 / 1101$ \\
\hline onlemation: BLG Domum or: & |pare $11 / 91$ \\
\hline
\end{tabular}




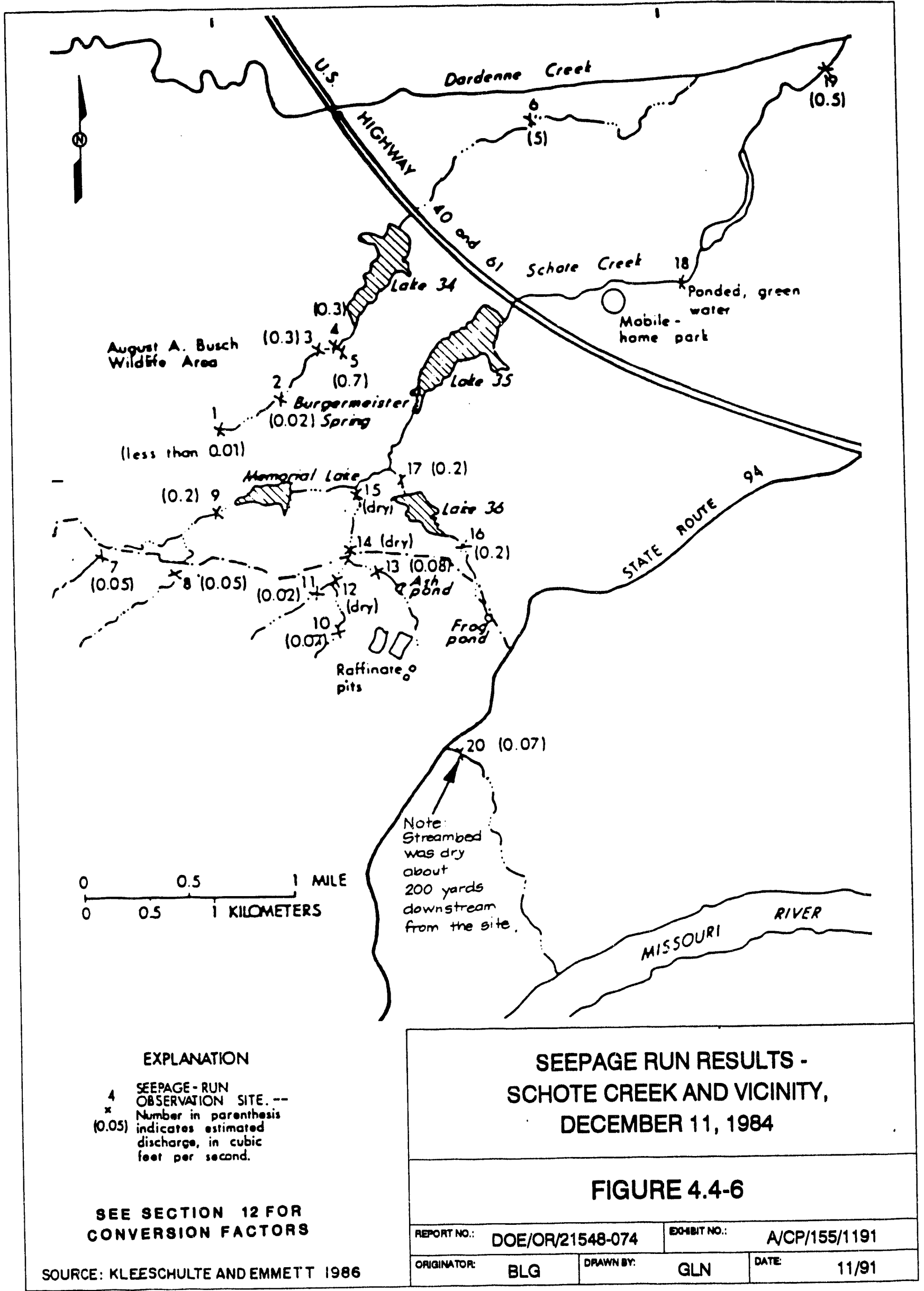




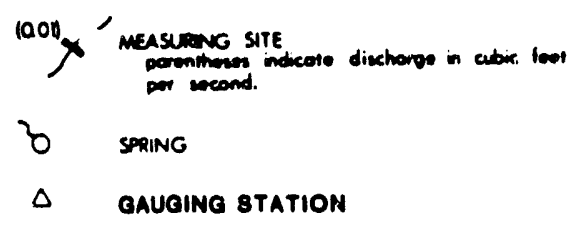

D gavaing 8Tation

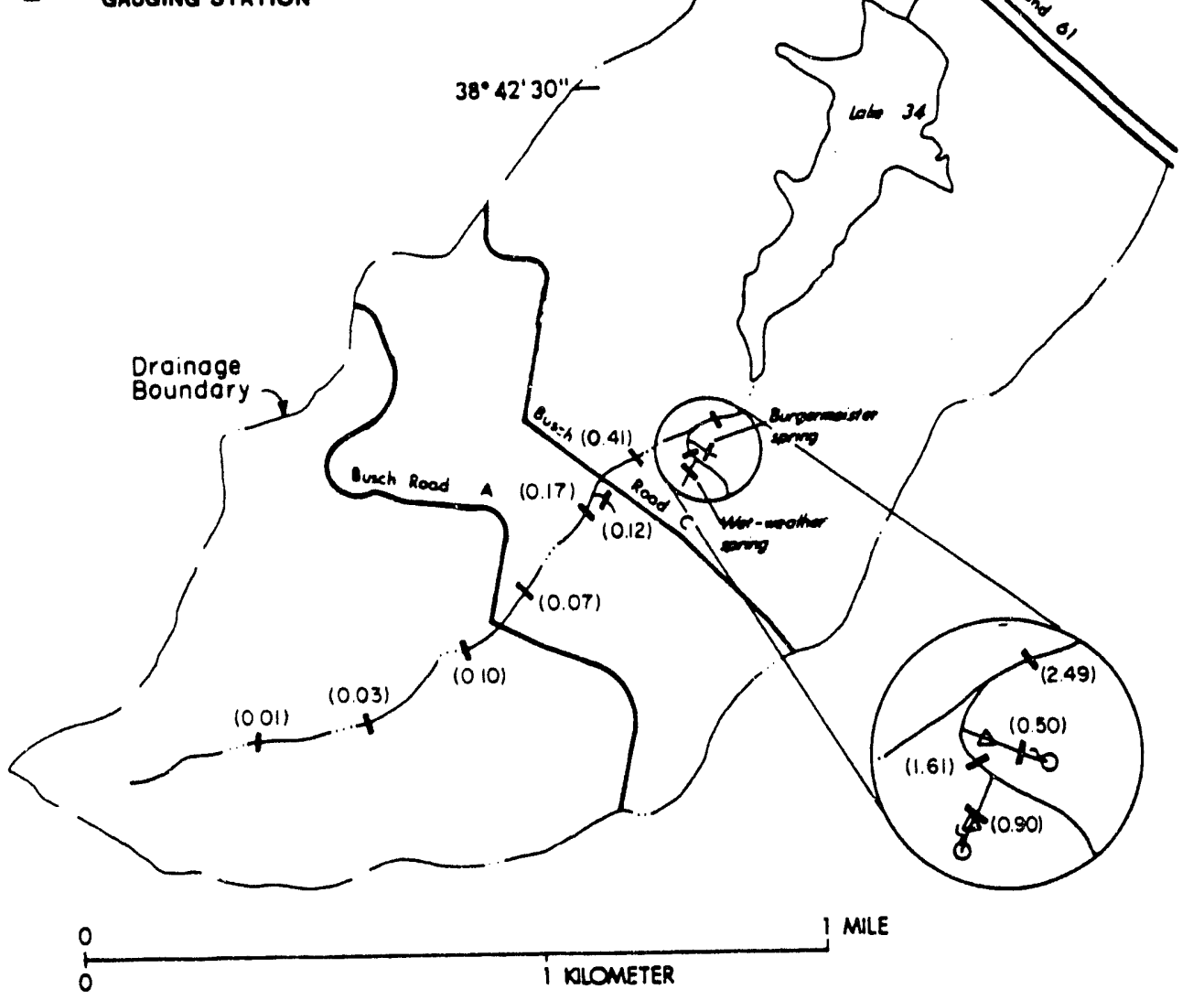

SOURCE: KLEESCHULTE, 1088

\begin{tabular}{|c|c|c|c|c|}
\hline \multicolumn{5}{|c|}{$\begin{array}{c}\text { SEEPAGE RUN RESULTS - } \\
\text { TRIBUTARY TO DARDEENE CREEK } \\
\text { UPSTF,EAM OF LAKE } 34, \\
\text { APRIL } 2-3,1985\end{array}$} \\
\hline \multicolumn{5}{|c|}{ FIGURE 4.4-7 } \\
\hline \multicolumn{2}{|c|}{ AEPDAT NO: DOE/OR/21548-074 } & \multicolumn{3}{|c|}{ Eomlat io. A/VP/166/1191 } \\
\hline Oalematon BLG & pooum or: & $\overline{G L N}$ & TaAte & $11 / 81$ \\
\hline
\end{tabular}




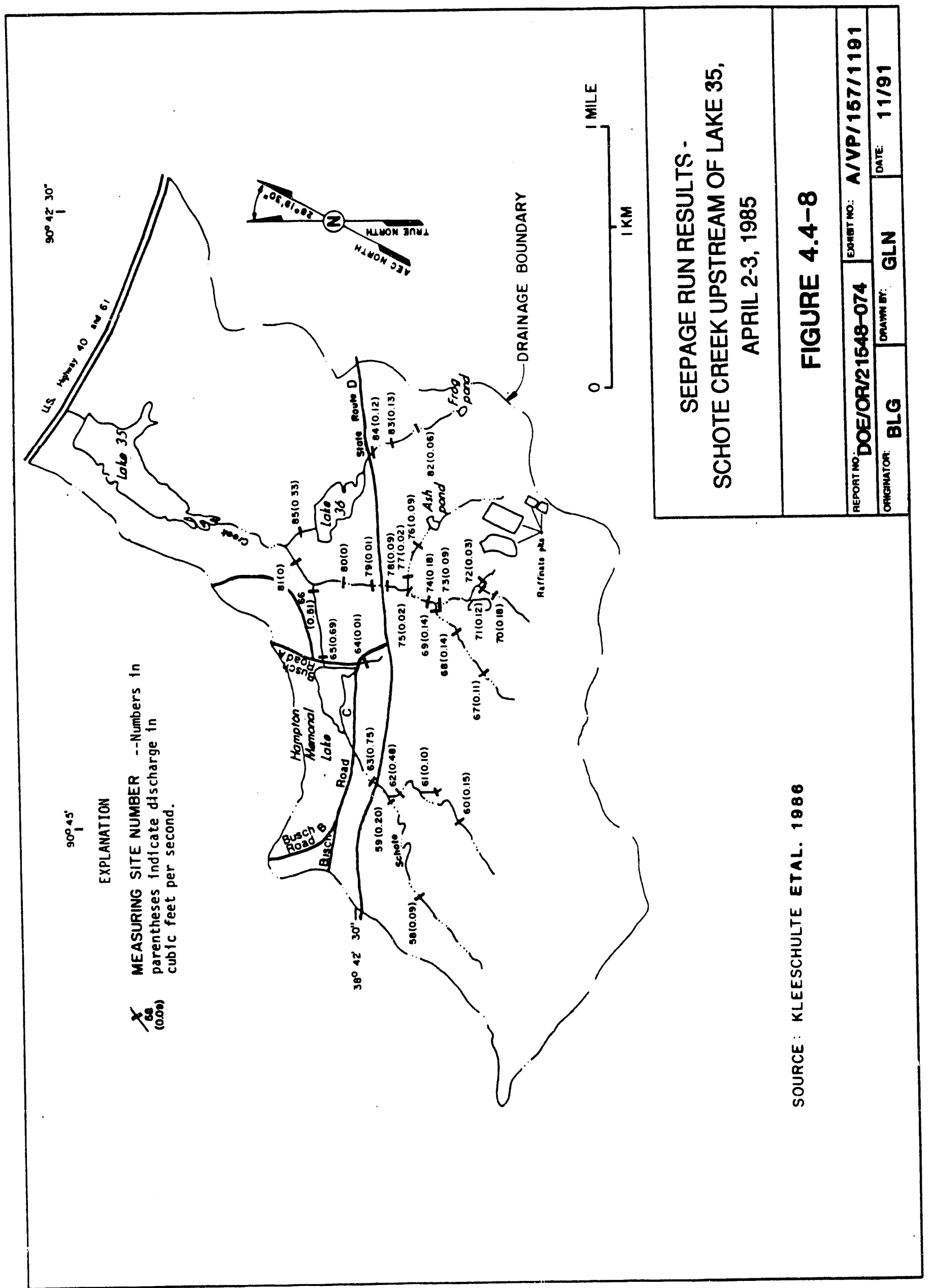




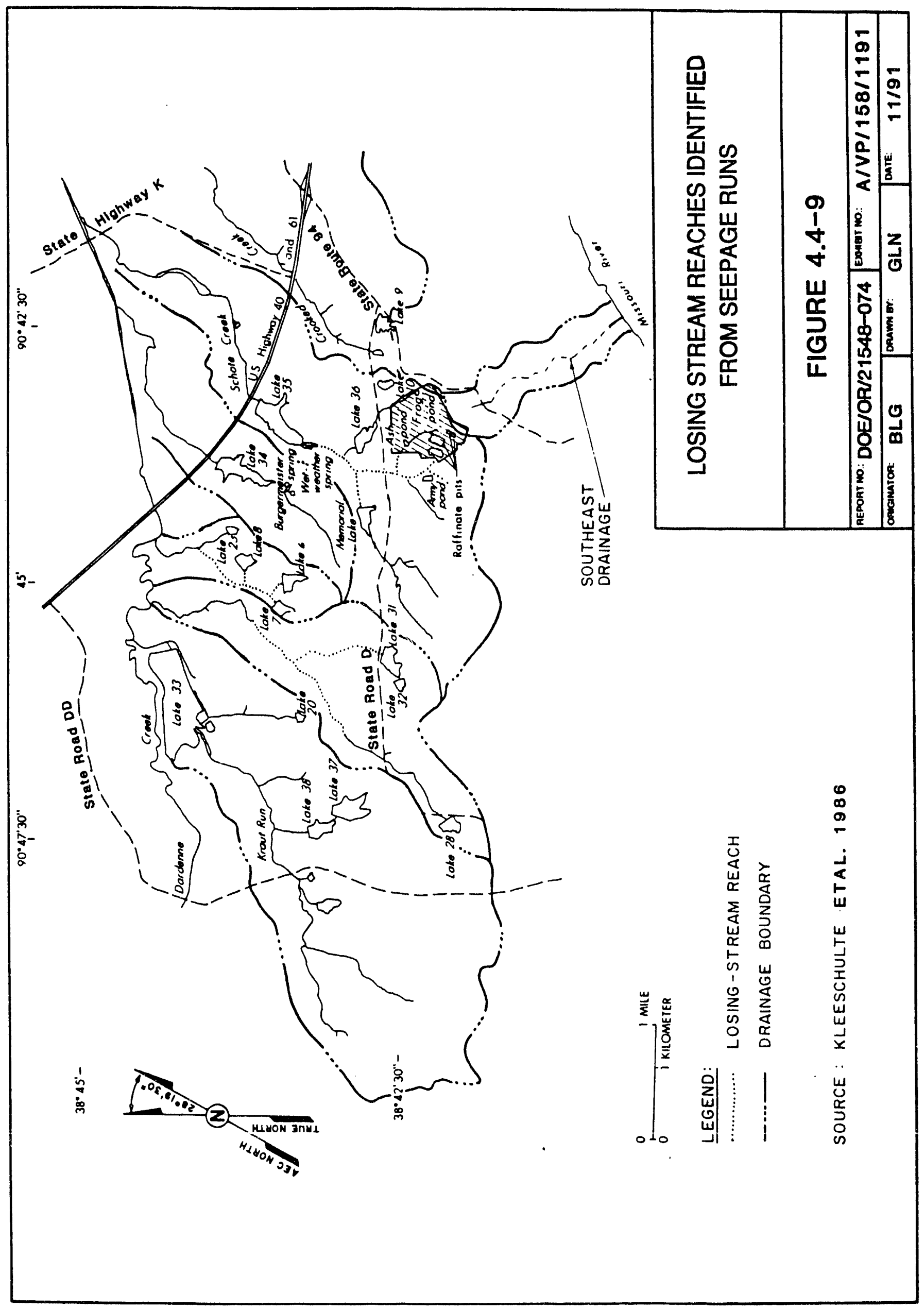




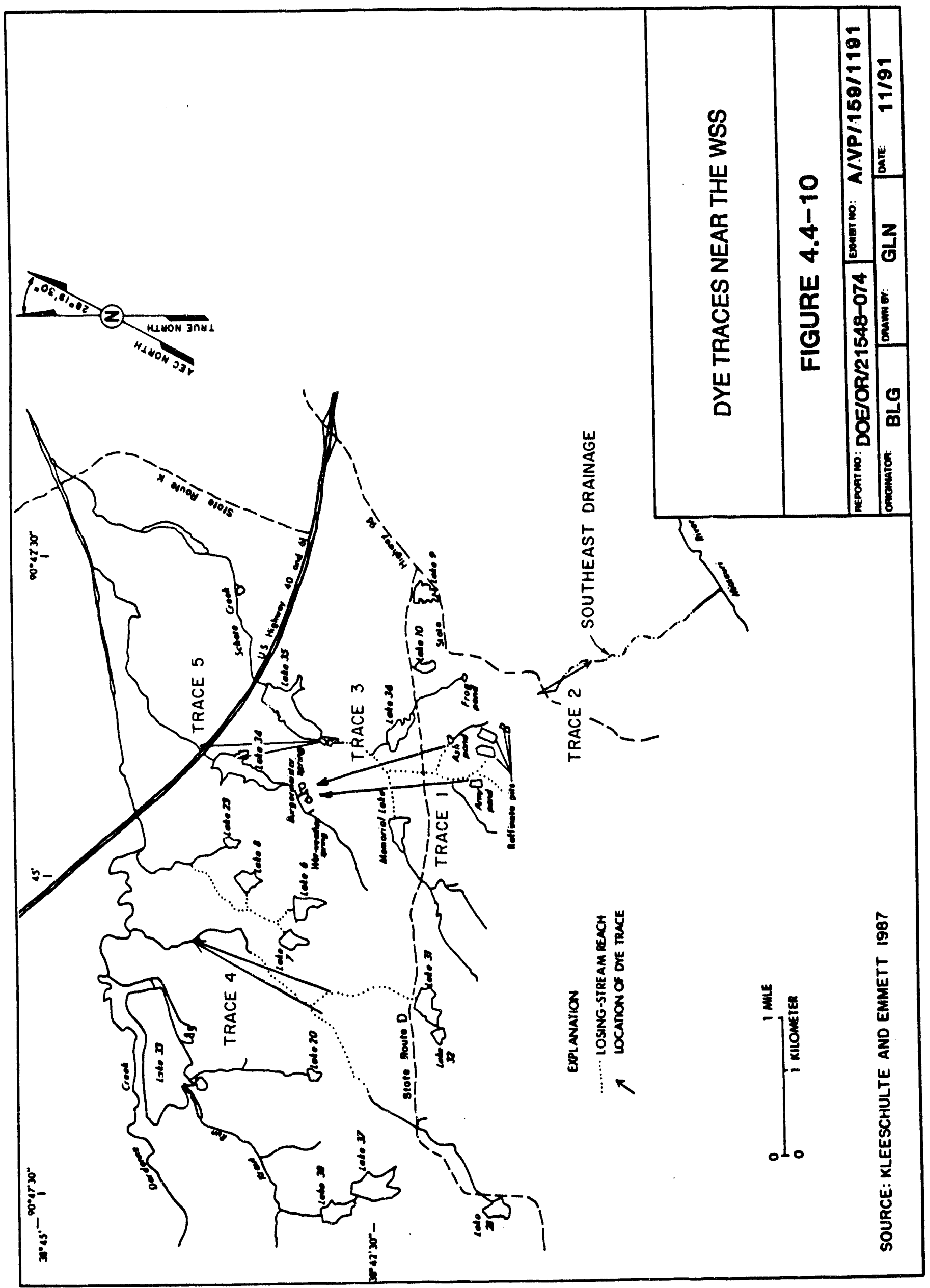




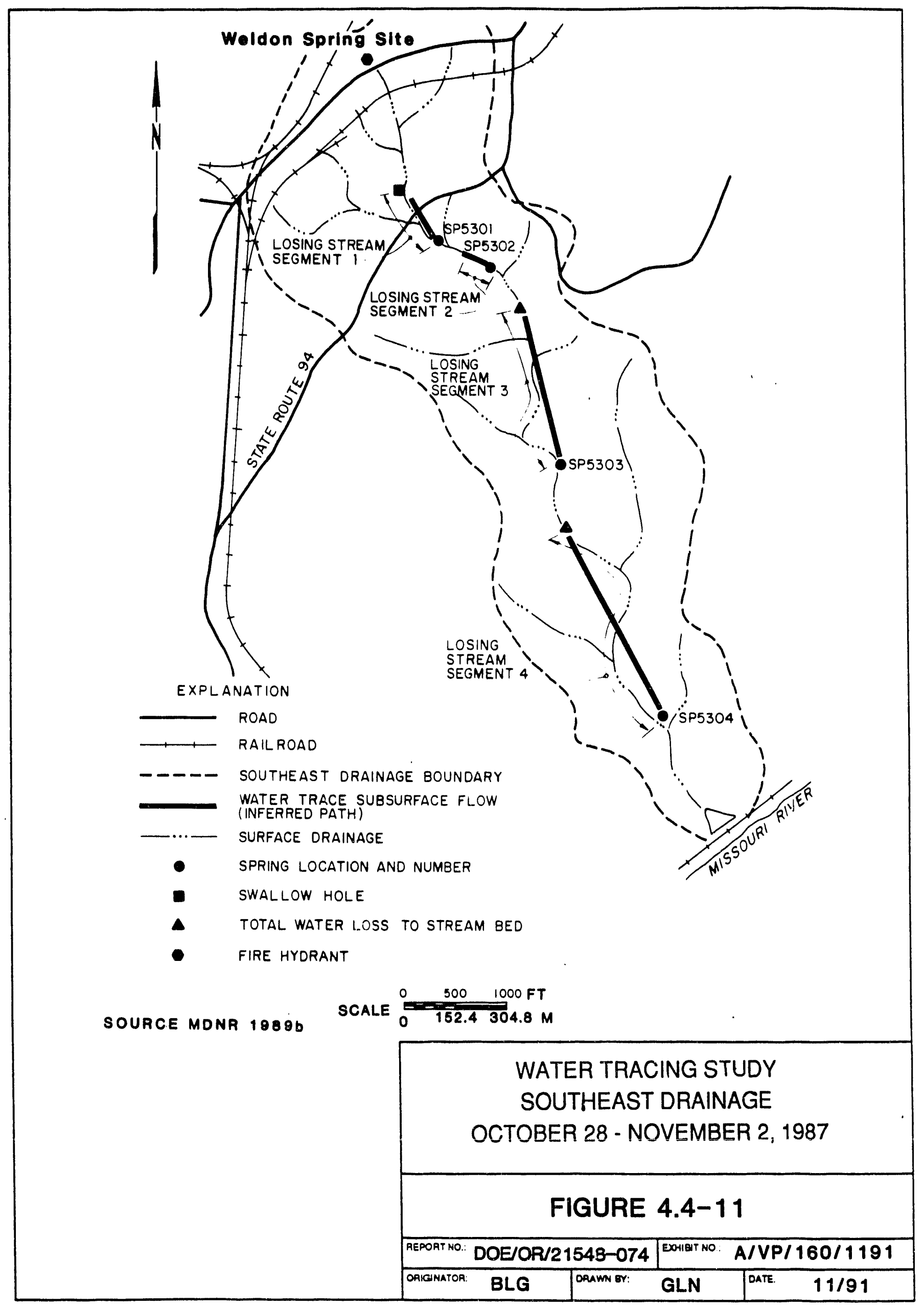



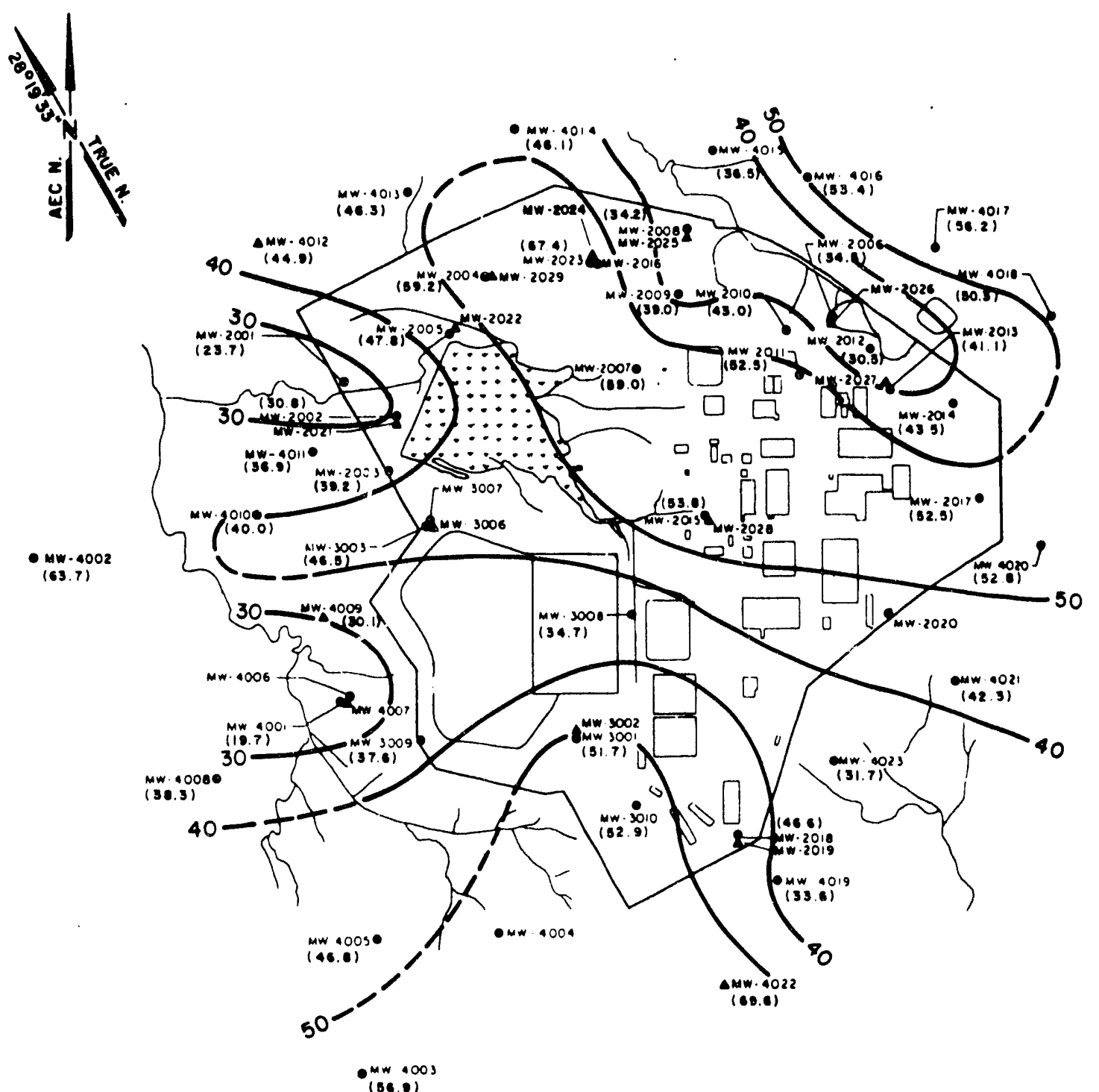

LEGEND:

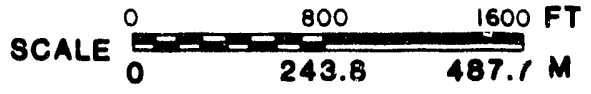

$\begin{array}{ll}\text {-50 } & \text { VADOSE ZONE THICKNESS CONTOUR } \\ & \text { (IO FEET INTERVALI(FT-MSL) } \\ & \text { DATA POINT WITH GROUNOWATER } \\ \text { MW-4005 } & \text { MONITORING WELL NUMBER AND } \\ \text { (46.1) } & \text { VADOSE ZONE THICKNESS IN FEET. }\end{array}$

NCTE:

VADOSE ZONE THICKNFSS BASED ON GROUND ELEVATIONS GIVEN IN TABLE 4.6-I AND JULY 1988 WATER LEVEL

ELEVATIONS GIVEN IN TABLE 4.6-8.

VADOSE ZONE THICKNESS, JULY 1988

FIGURE 4.5-1

\begin{tabular}{|c|c|c|c|c|c|}
\hline AEPOAT NO: & DOE/OF & $1548-074$ & EOHET No. & A/CP & $97 / 1181$ \\
\hline OAlGIMATOA & BLG & Doum or: & GLN & DATE. & $11 / 91$ \\
\hline
\end{tabular}



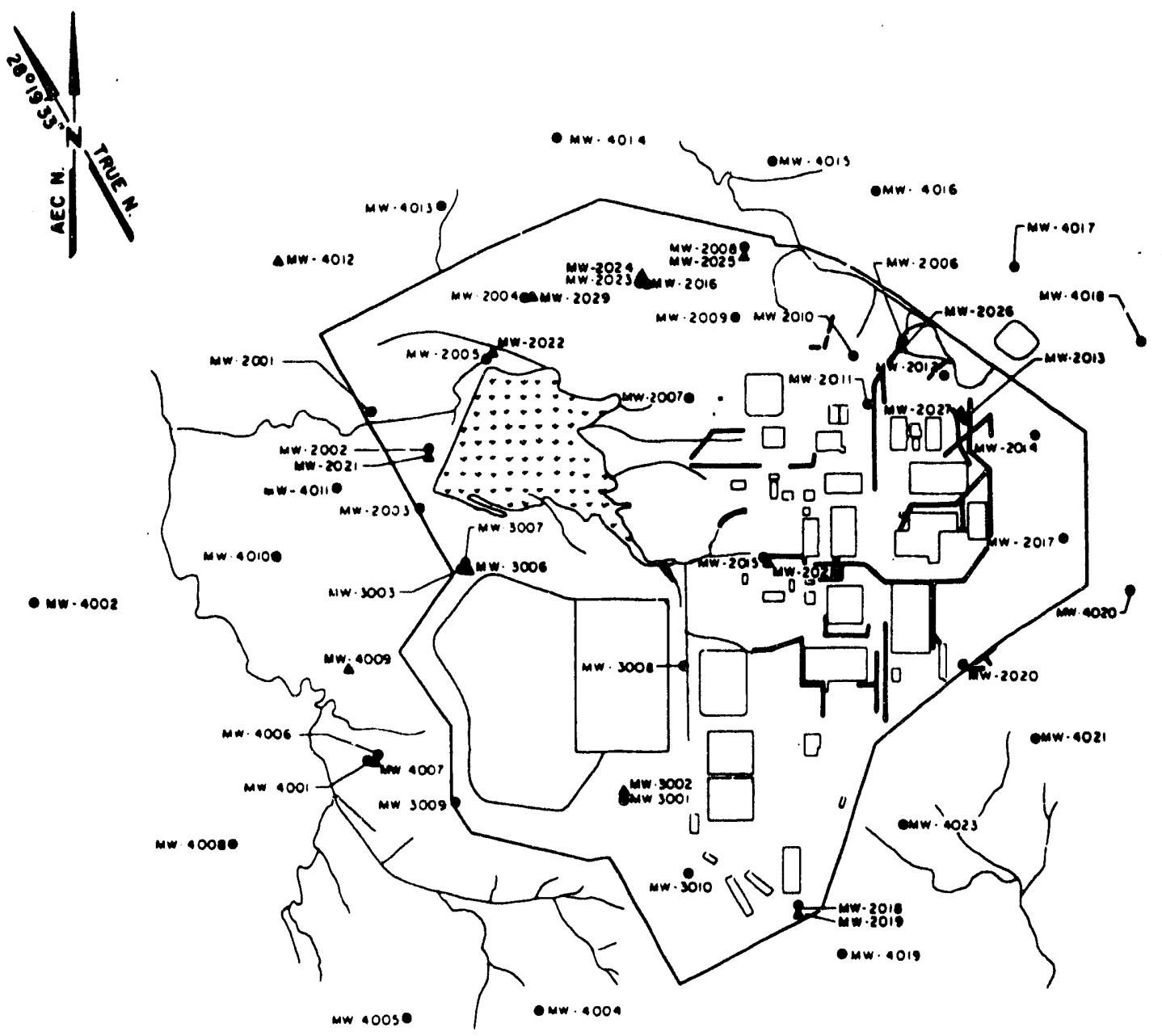

$\Delta$ Aw 4022

- $m w \cdot 1003$

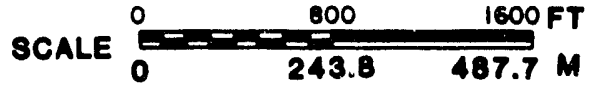

\section{STORM SEWER LOCATIONS}

FIGURE 4.5-2

\begin{tabular}{|c|c|c|c|c|}
\hline REPOAT NO: & DOE/O & $1548-074$ & DOHIOT MO: & A/CP/188/1191 \\
\hline OARGIMATOA: & BLG & onum ar: & GLN & $11 / 91$ \\
\hline
\end{tabular}




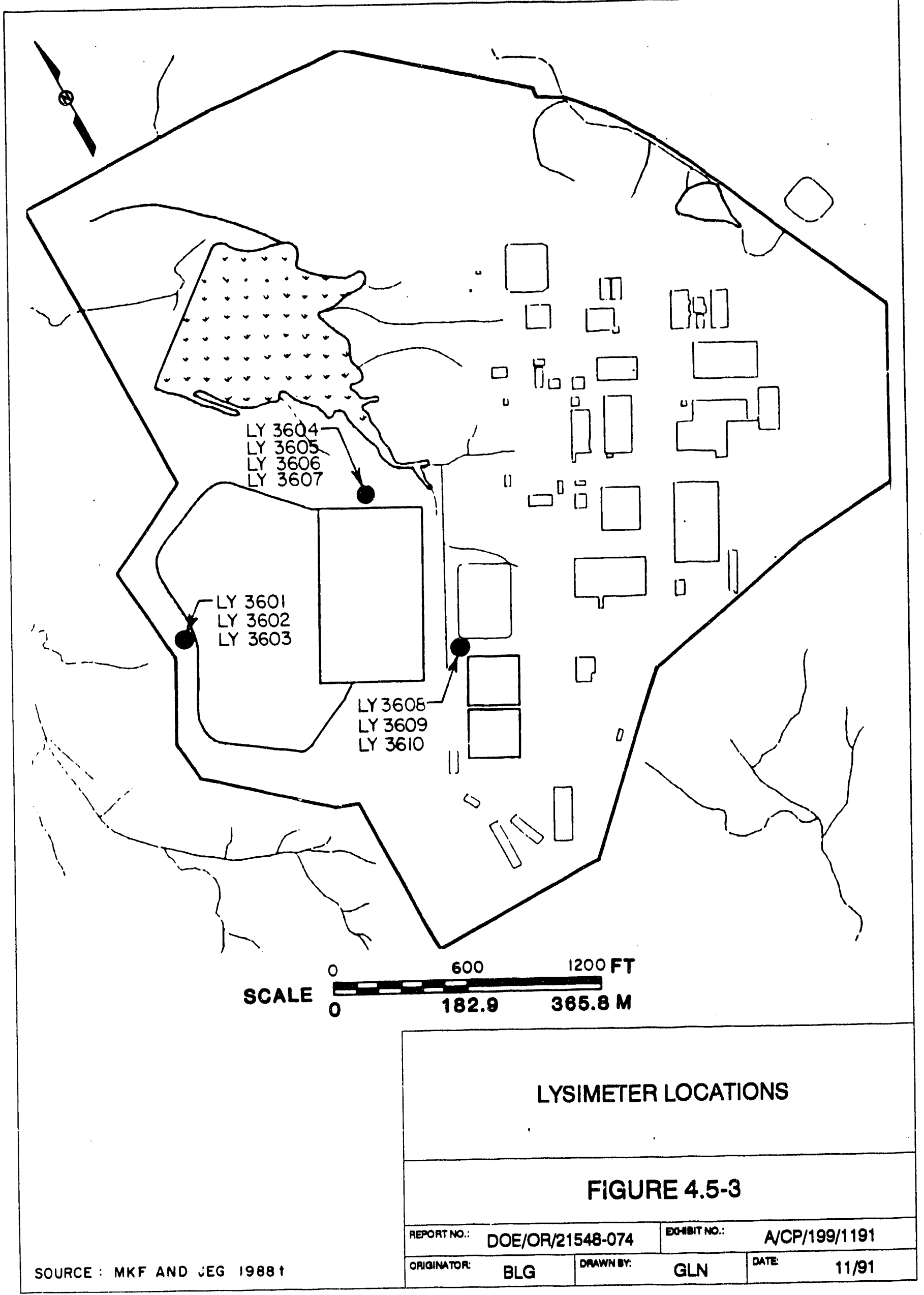




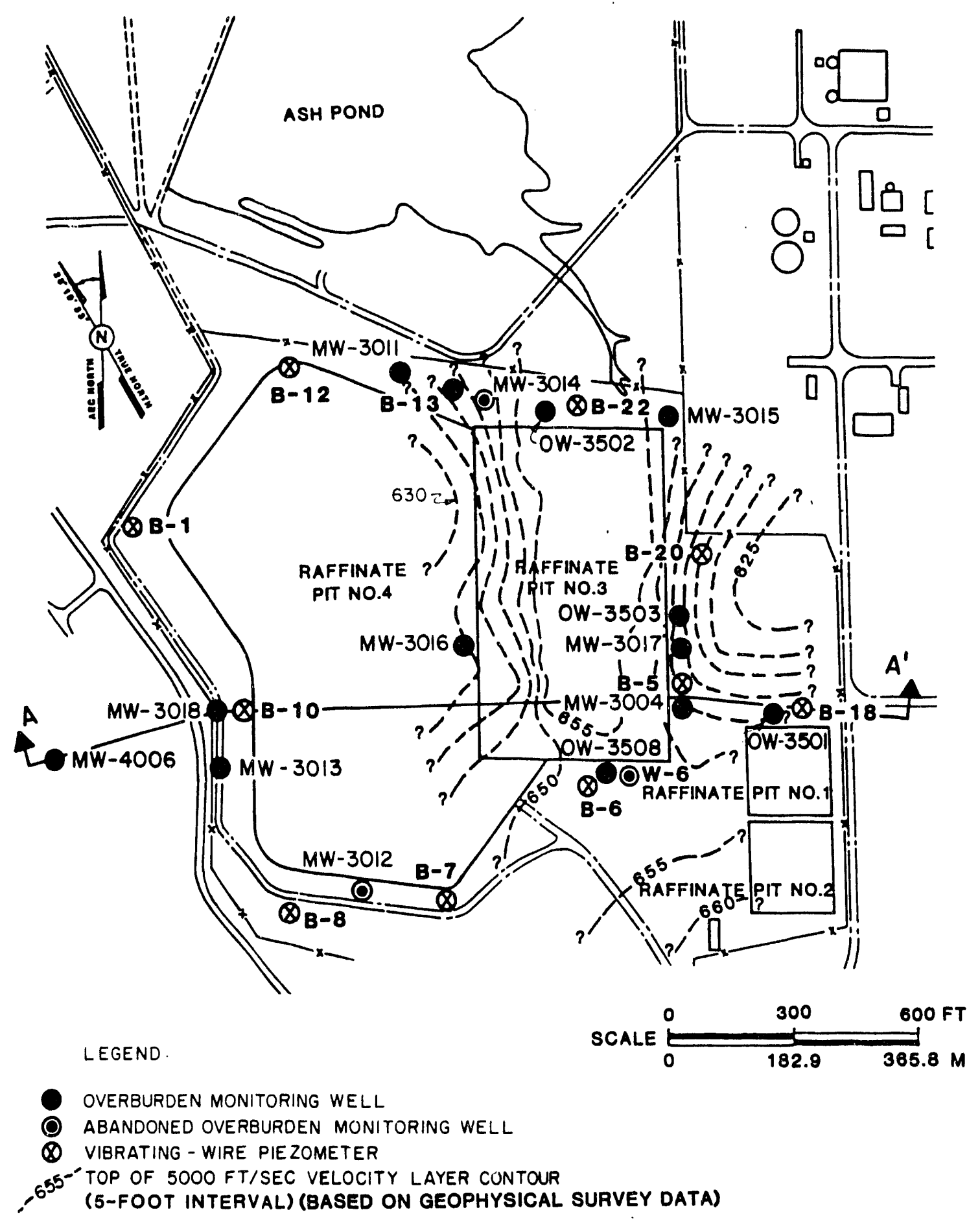

SOURCES: BNI 1984C; MKF AND JEG:\1988†

OVERBURDEN MONITORING WELLS AND PIEZOMETERS

FIGURE 4.5-4

\begin{tabular}{|c|c|c|c|c|}
\hline REPOAT NO & E/OR & $548-074$ & EXHBT NO: & A/RP/003/1191 \\
\hline ORGGINATOA: & BLG & DRAWN EY: & GLN & $11 / 91$ \\
\hline
\end{tabular}



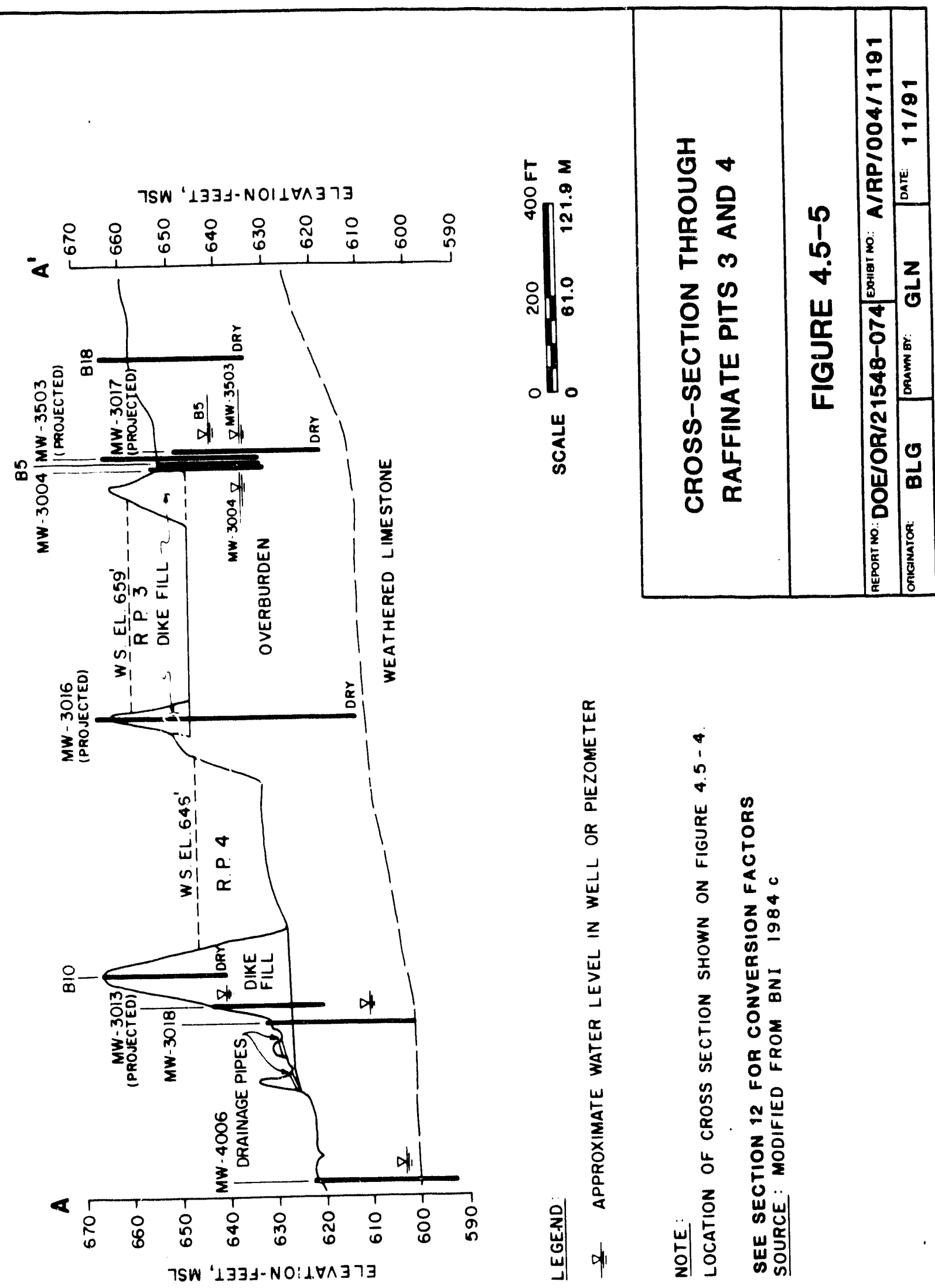


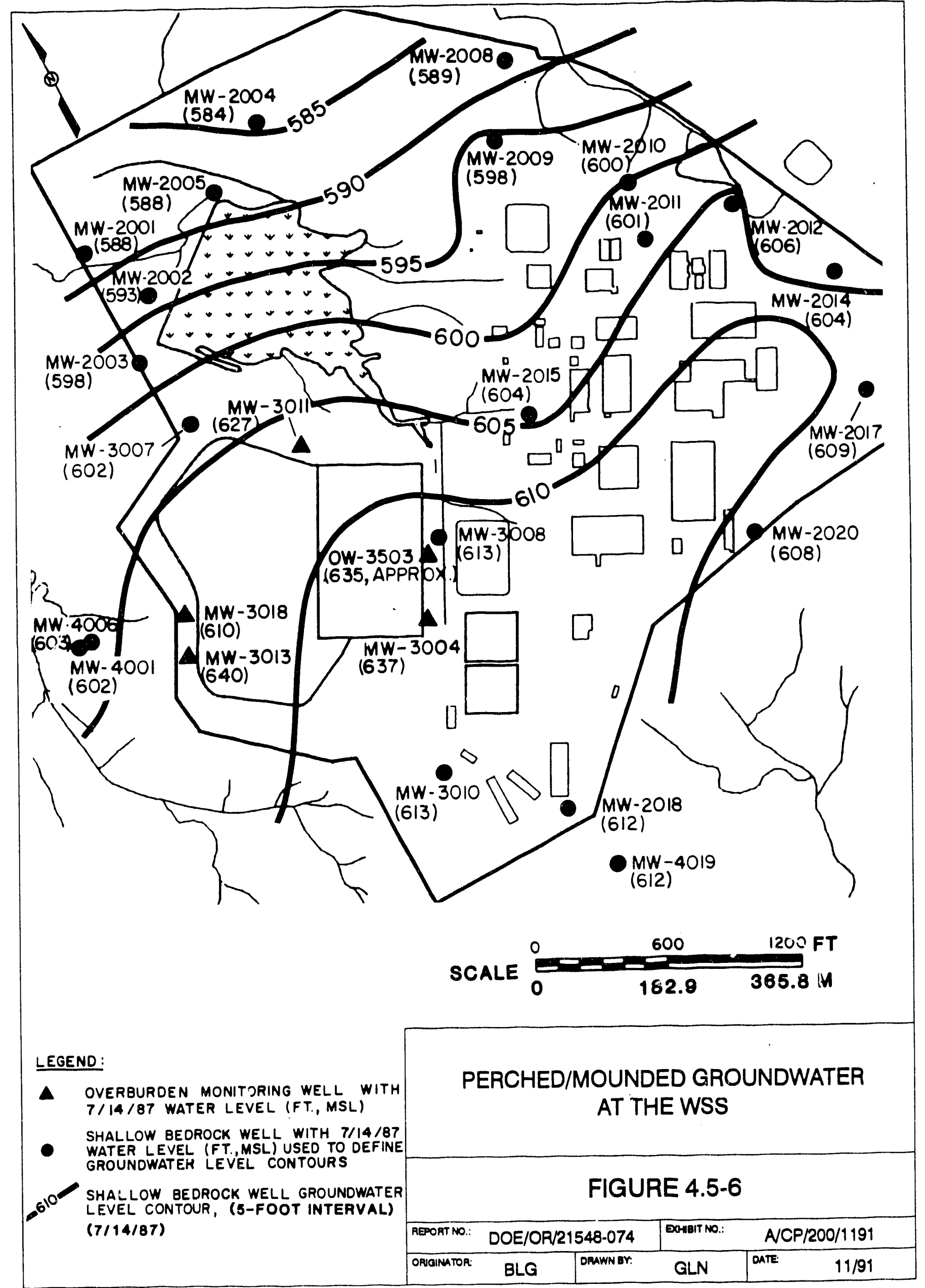




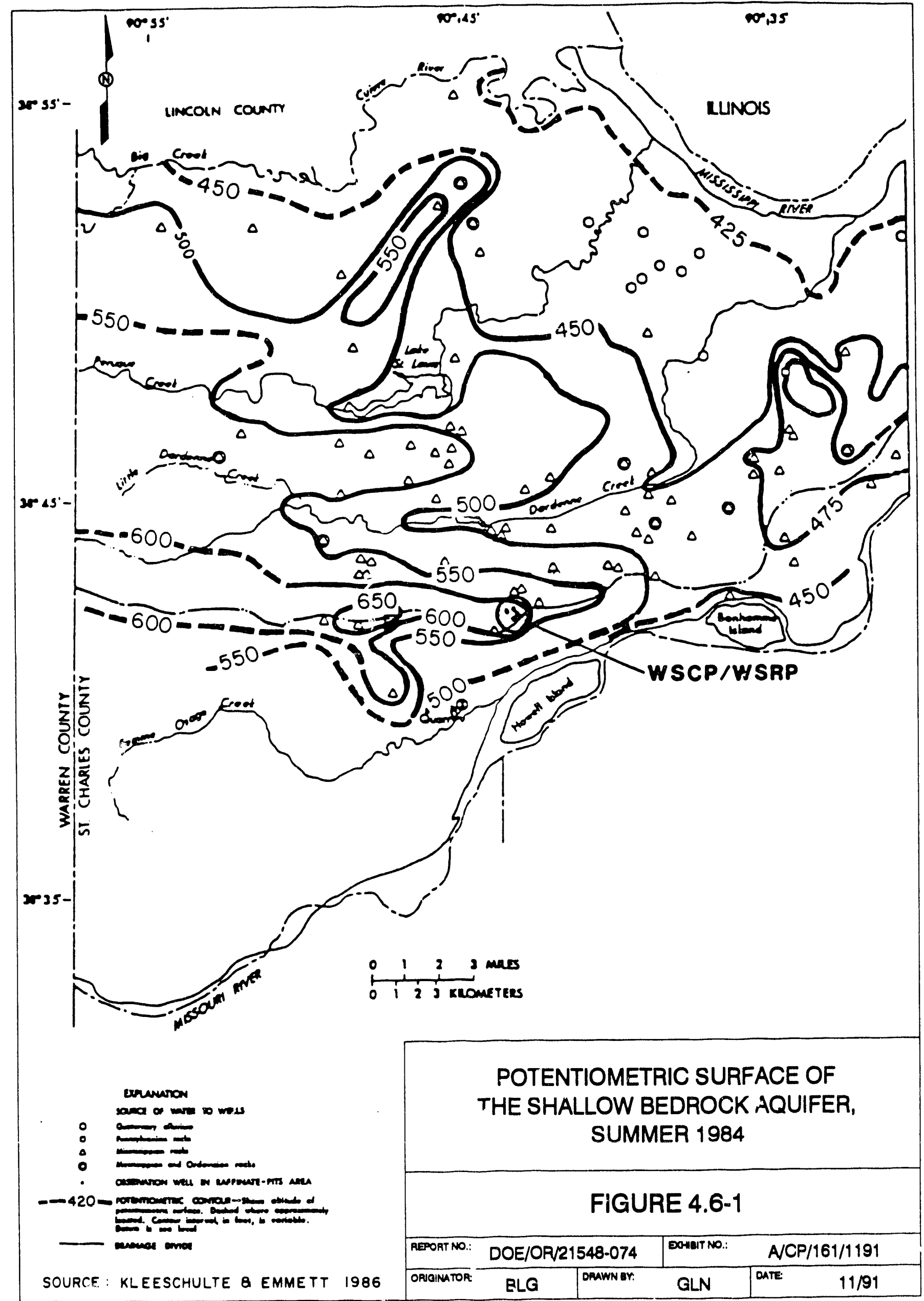




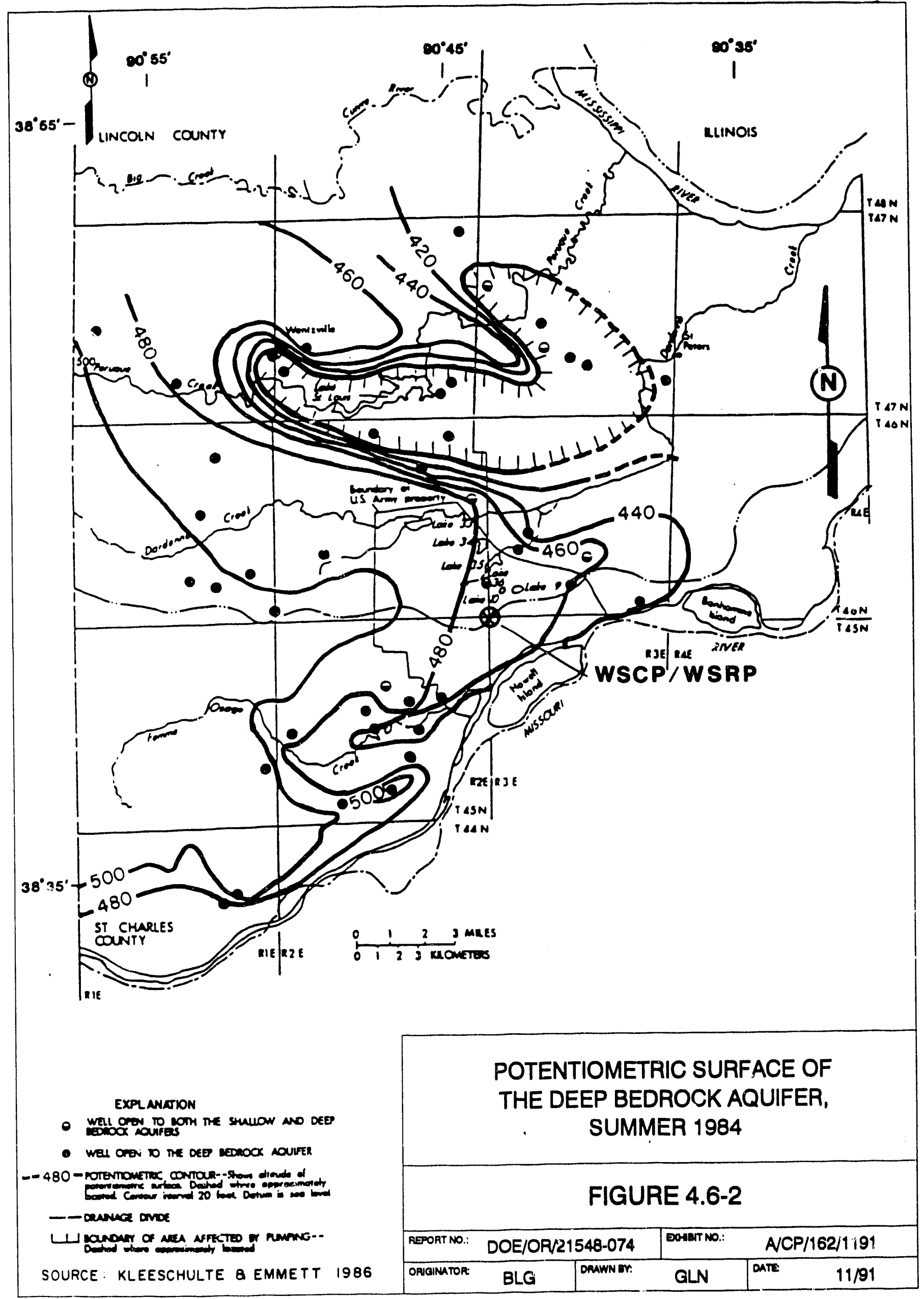




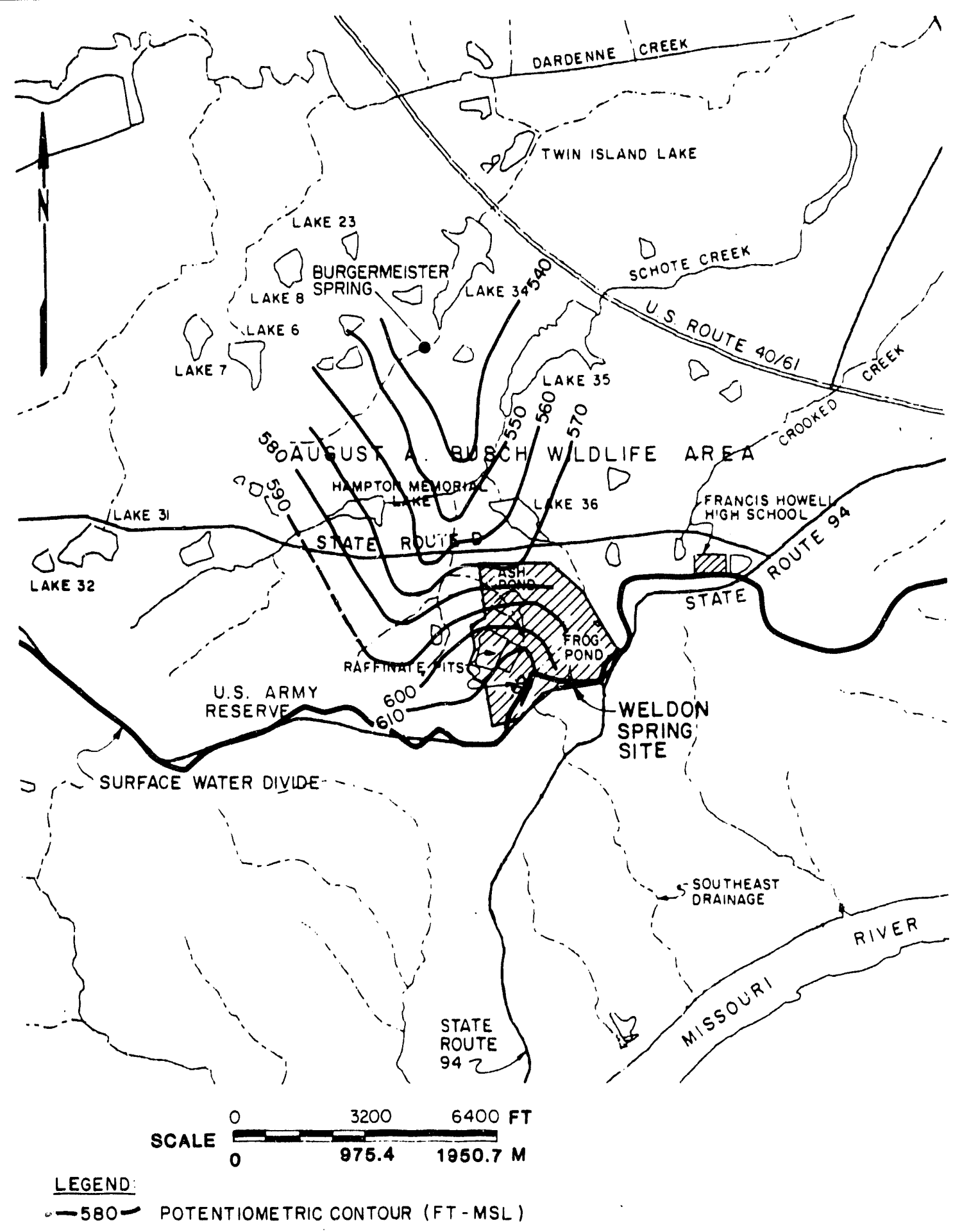

SOURCE: KLEESCHULTE \& EMMETT 1987

FOTENTIOMETRIC SURFACE, SHALLOW BEDROCK AQUIFER WSS VICINITY, JULY 1987

FIGURE 4.6-3

\begin{tabular}{|c|c|c|c|c|}
\hline REPOAT NO: & OE/OF & $548-074$ & EXH1BT NO: & A/VP/163/1191 \\
\hline OAGGINATOA: & BLG & DeaWN EY. & GLN & $11 / 91$ \\
\hline
\end{tabular}



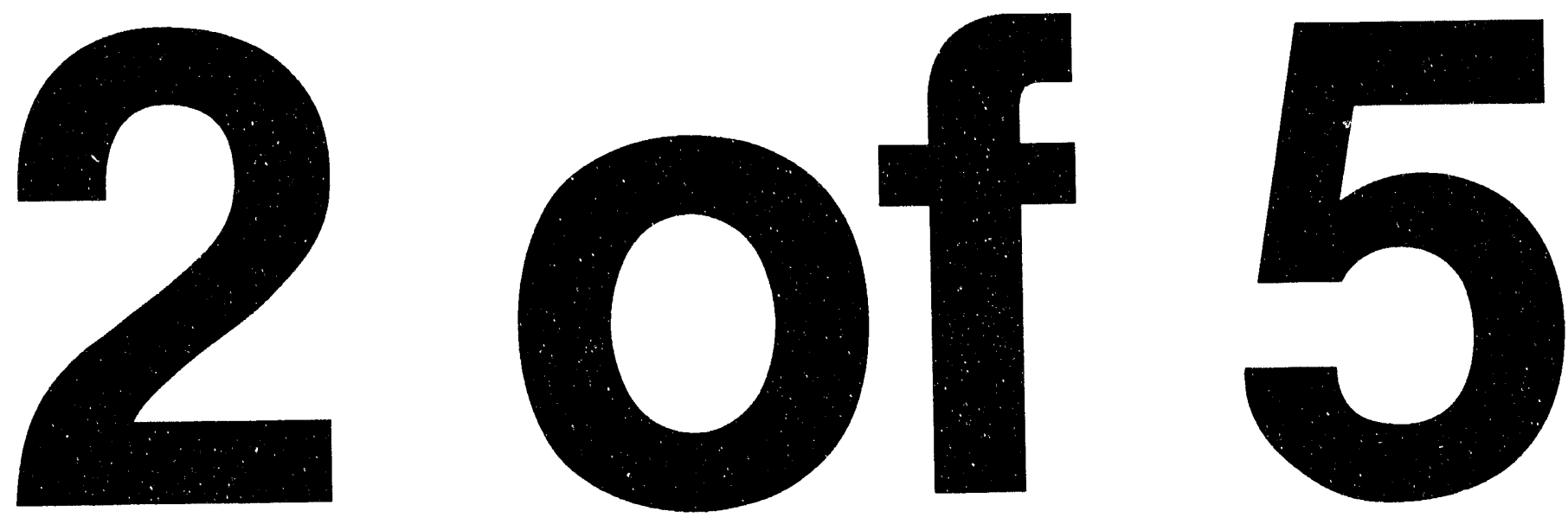


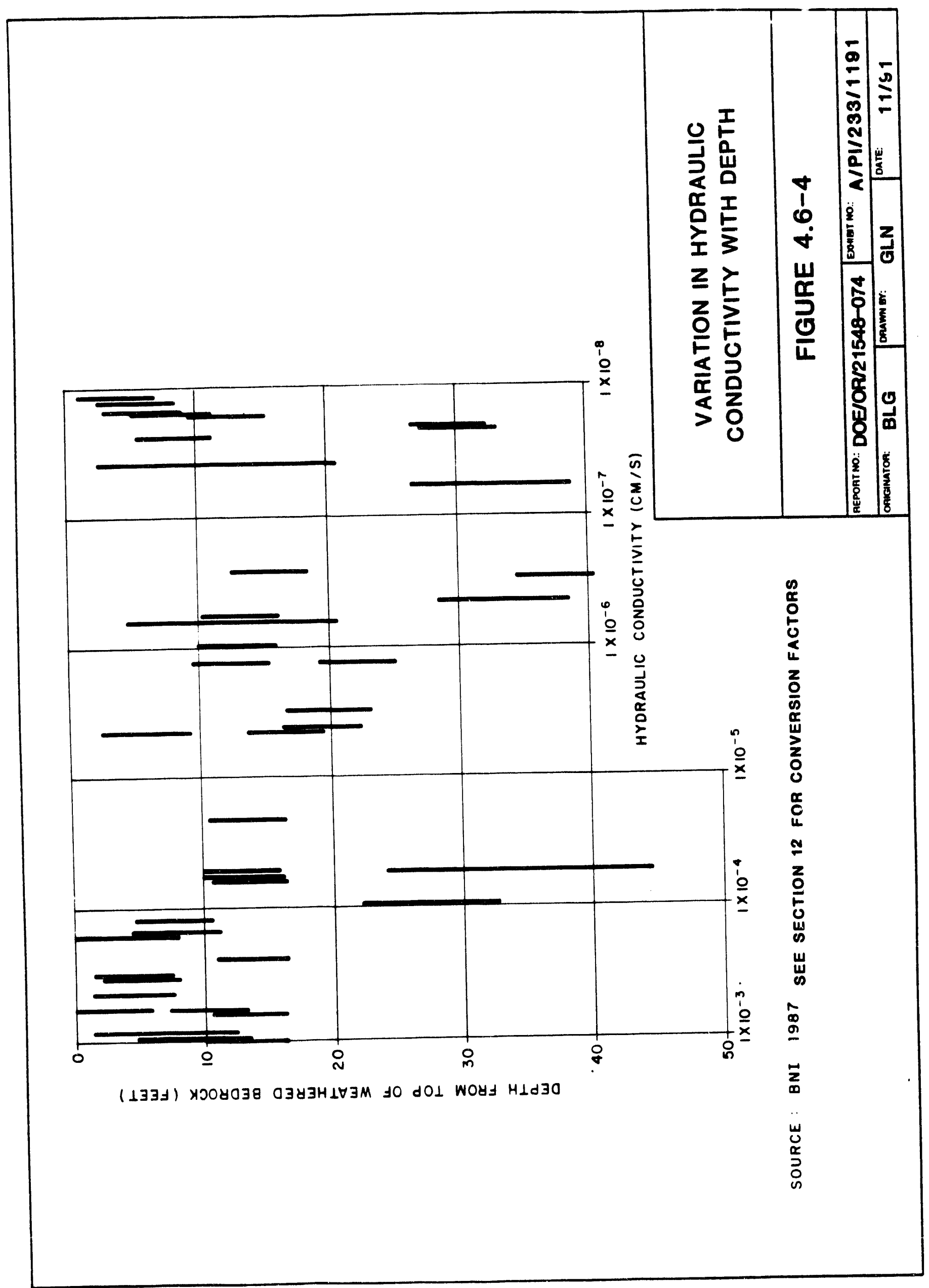




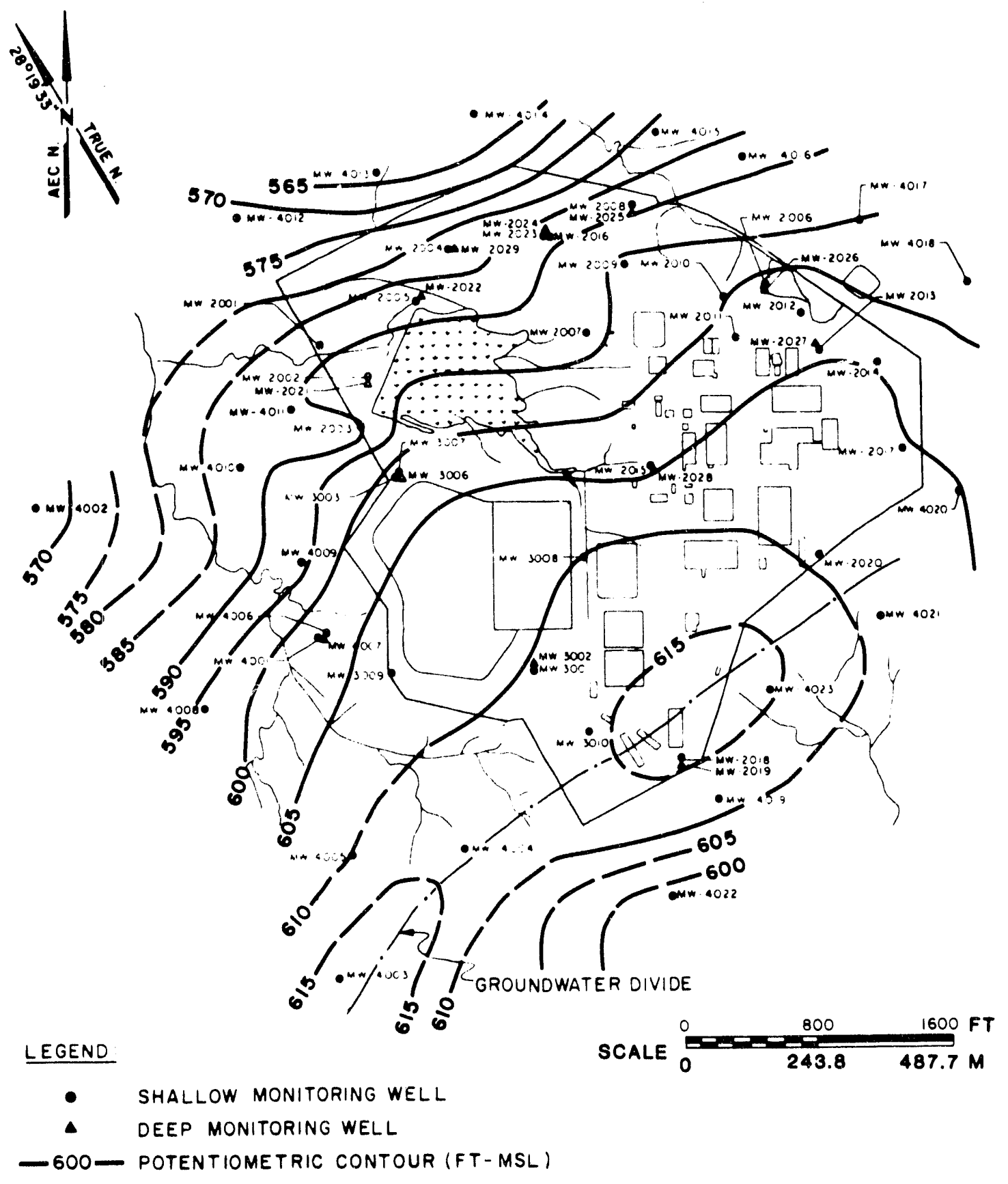

SOURCE: TABLE $4.6-8$

POTENTIOMETRIC SURFACE,

SHALLOW WELLS - JULY 1988

FIGURE 4.6-5

\begin{tabular}{|c|c|c|c|}
\hline AEPOAT NO DOE/O & $548-074$ & EXOHET NO & A/CP/201/1191 \\
\hline OAIGNATOA BLG & orumn or & GLN & $11 / 91$ \\
\hline
\end{tabular}



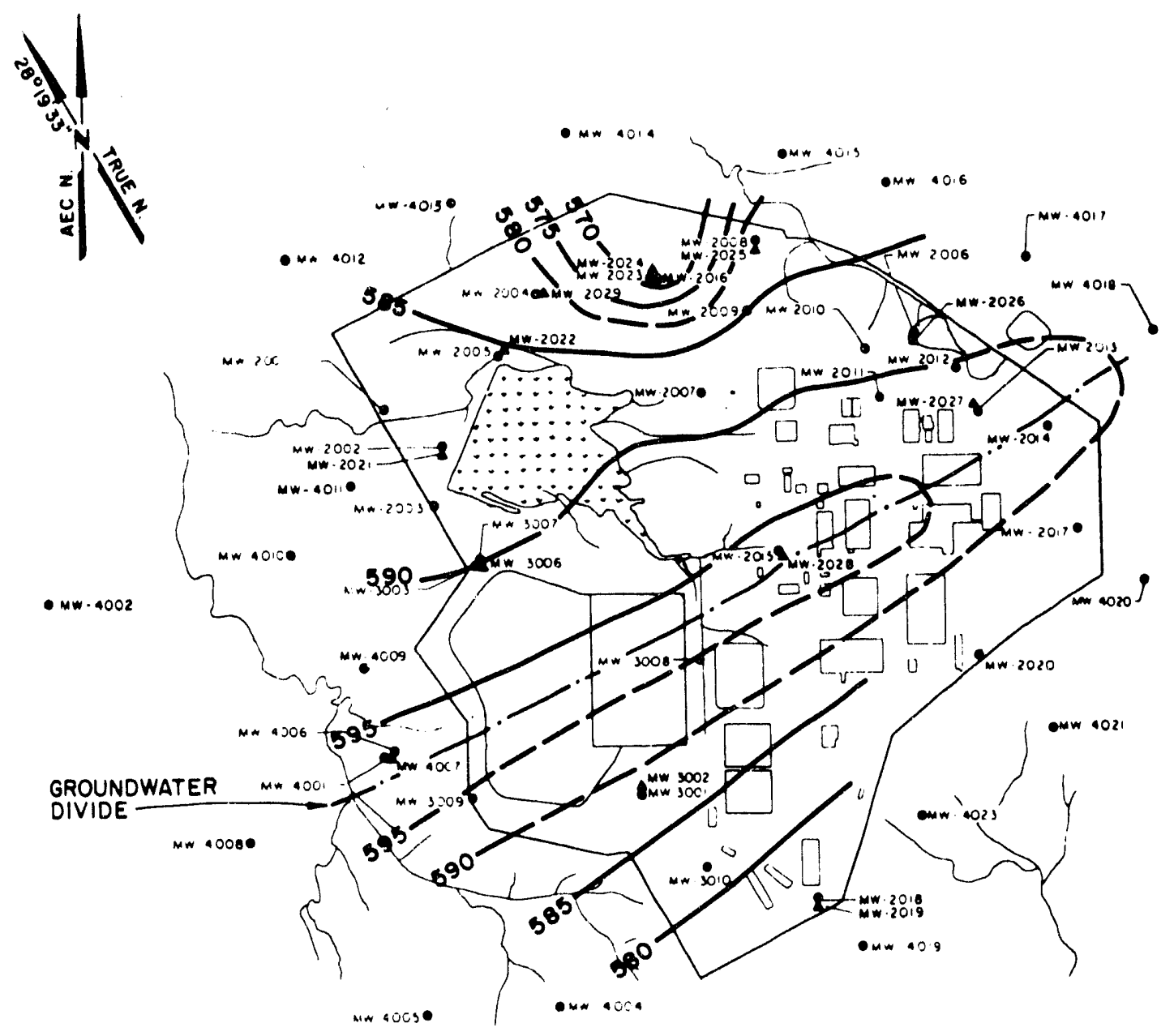

\section{LEGEND:}

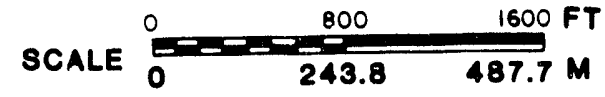

- Shallow monitoring well

- DEEP MONITORING WELL

- 595 - POTENTIOMETRIC CONTOUR (FT - MSL)

SOURCE: TABLE $4.6-8$

POTENTIOMETRIC SURFACE, DEEP WELLS - JULY 1988

FIGURE 4.6-6

\begin{tabular}{|c|c|c|c|c|}
\hline REPOAT NO & DOE/OF & $1548-074$ & EOHEIT NO. & A/CP/202/1191 \\
\hline OAlamató & BLG & DAamer or & GLN & $11 / 91$ \\
\hline
\end{tabular}




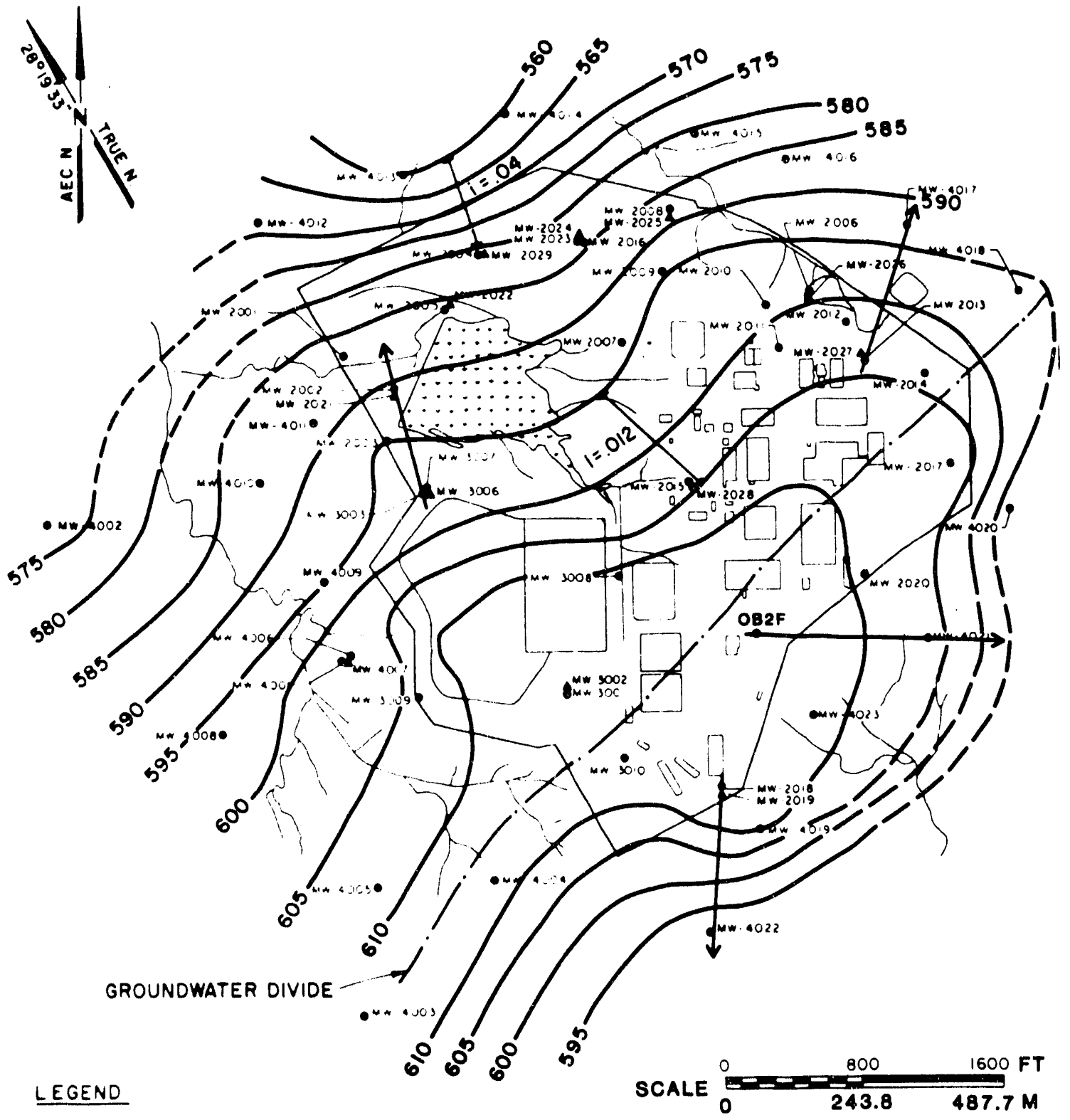

- Shallow monitoring well

- deEP monitoring well

-600- POTENTIOMETRIC CONTOUR (FT - MSL)

SHALLOW GROUNDWATER FLOW PATH

SOURCE: TABLE $4.6-8$

POTENTIOMETRIC SURFACE, SHALLOW WELLS - DECEMBER 1988

FIGURE 4.6-7

\begin{tabular}{|c|c|c|c|c|}
\hline PEPOAT NO. & DOEIO & $1548-074$ & $\begin{array}{l}\text { EDHET to. } \\
\text { E. }\end{array}$ & A/CP/203/1191 \\
\hline ORIGINATOA. & BLG & Doumin or & GLN & $11 / 91$ \\
\hline
\end{tabular}




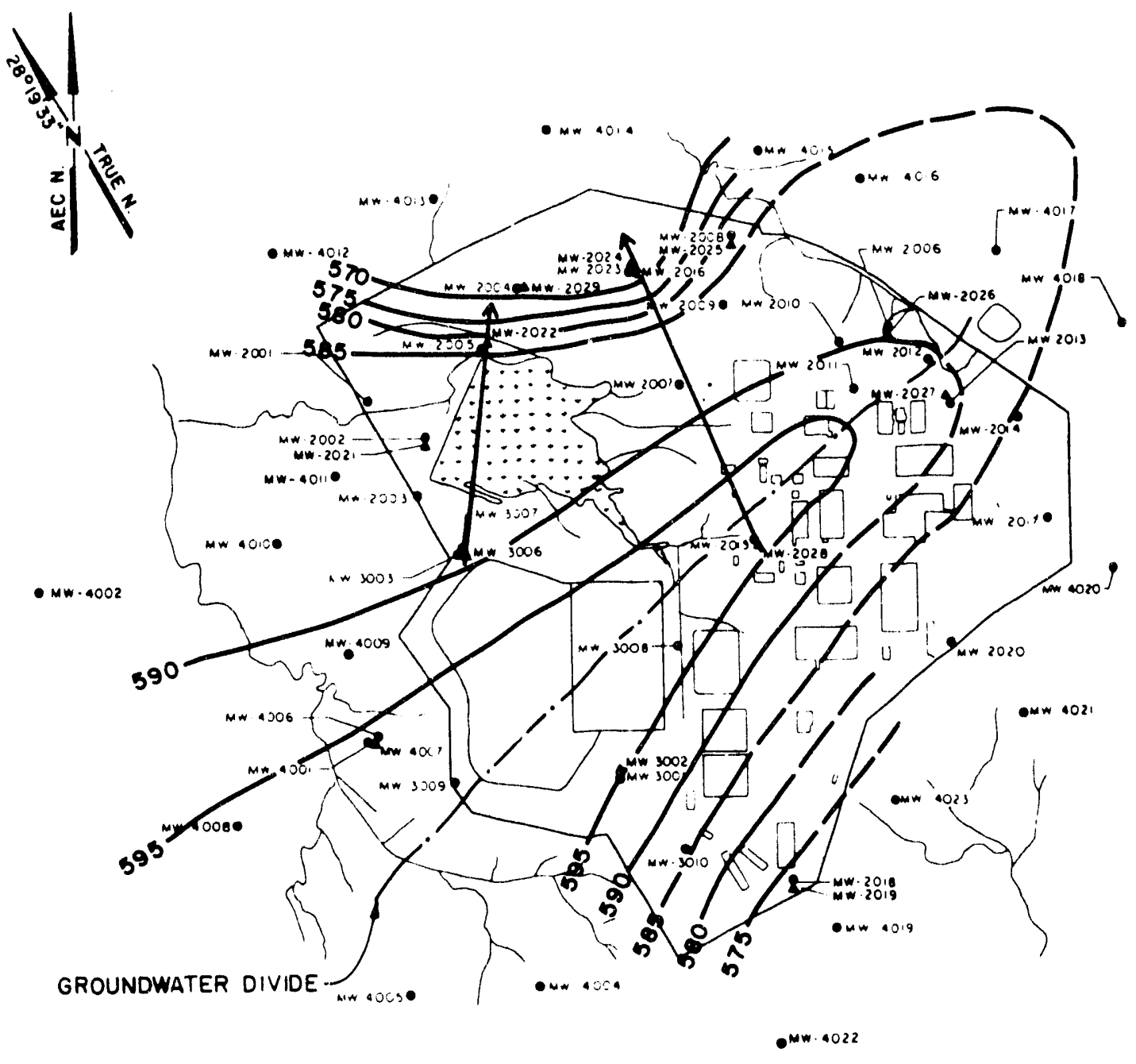

enn . MOS

\section{LEGEND:}

- Shallow monitoring well

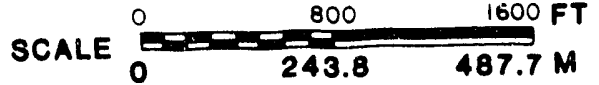

- DEEP MONITORING WELL

- 595 - POTENTIOMETRIC CONTOUR (FT-MSL)

DEEP GROUNDWATER FLOW PATH

SOURCE: TABLE $4.6-8$

\section{POTENTIOMETRIC SURFACE, DEEP WELLS - DECEMBER 1988}

FIGURE 4.6-8

\begin{tabular}{|c|c|c|c|c|}
\hline REPOAT NO: & OE/OF & 548-074 & EOALET NO. & A/CP/204/1191 \\
\hline ORGGMATOO & BLG & Drawn or: & GLN & $11 / 91$ \\
\hline
\end{tabular}




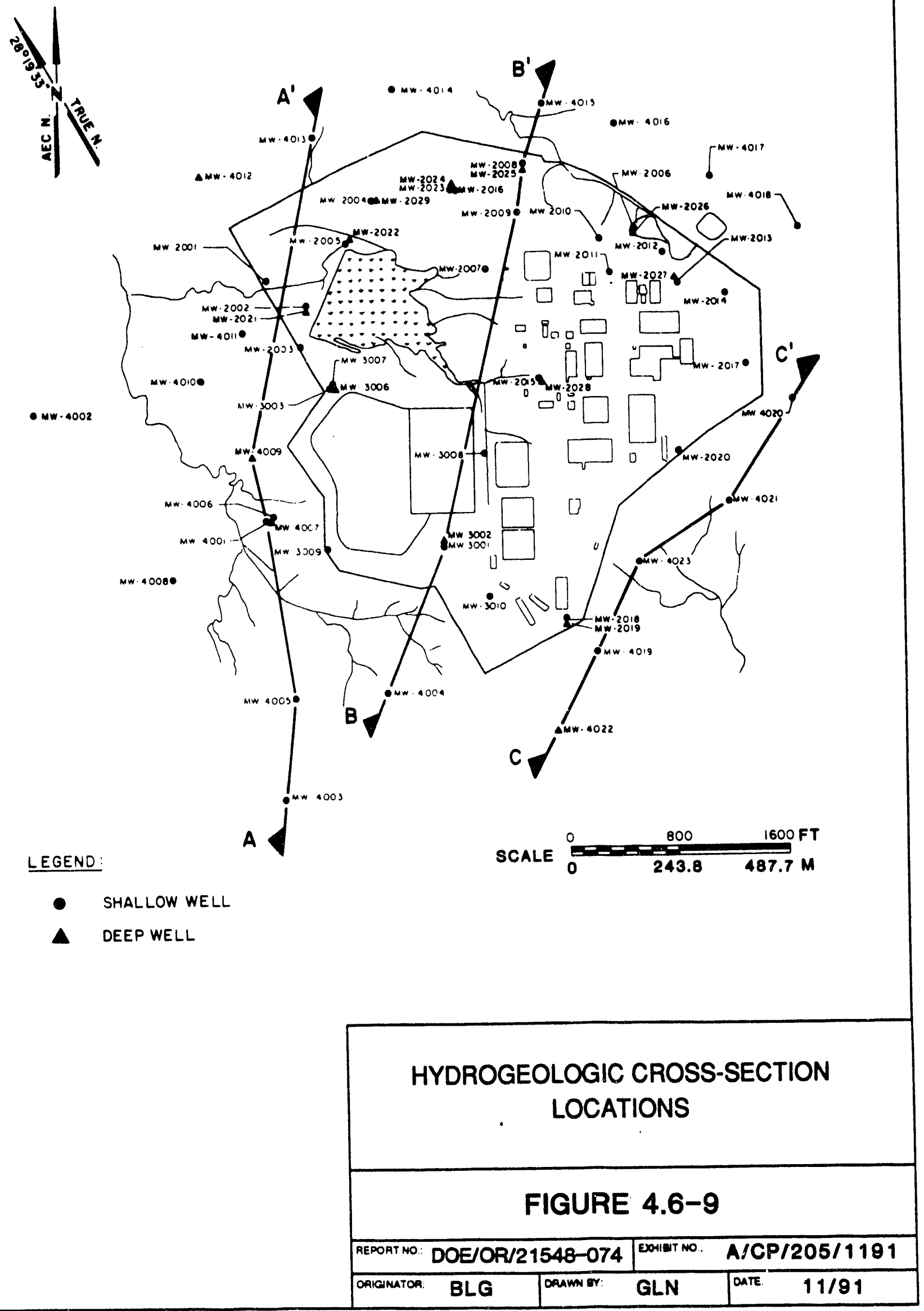




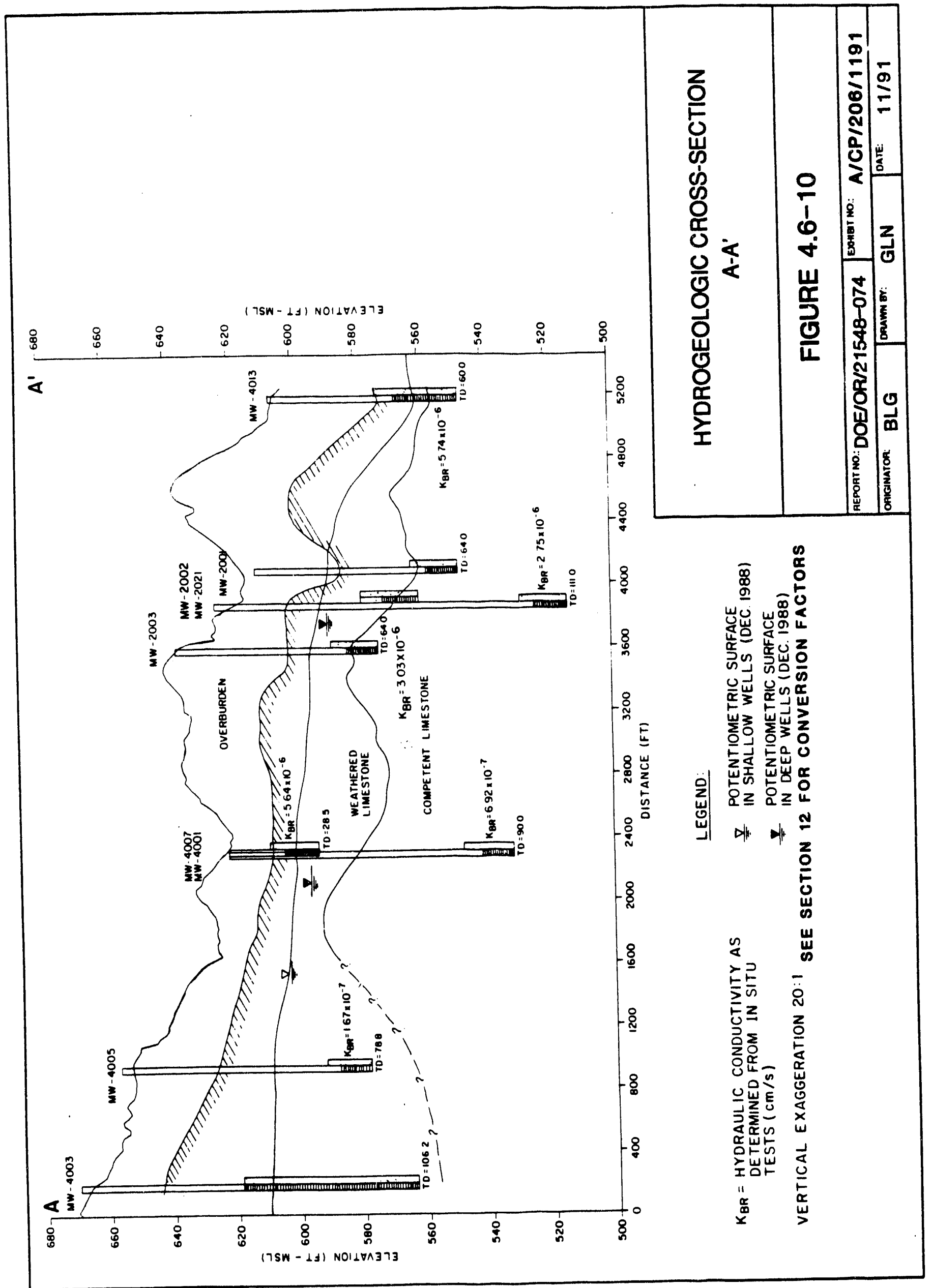




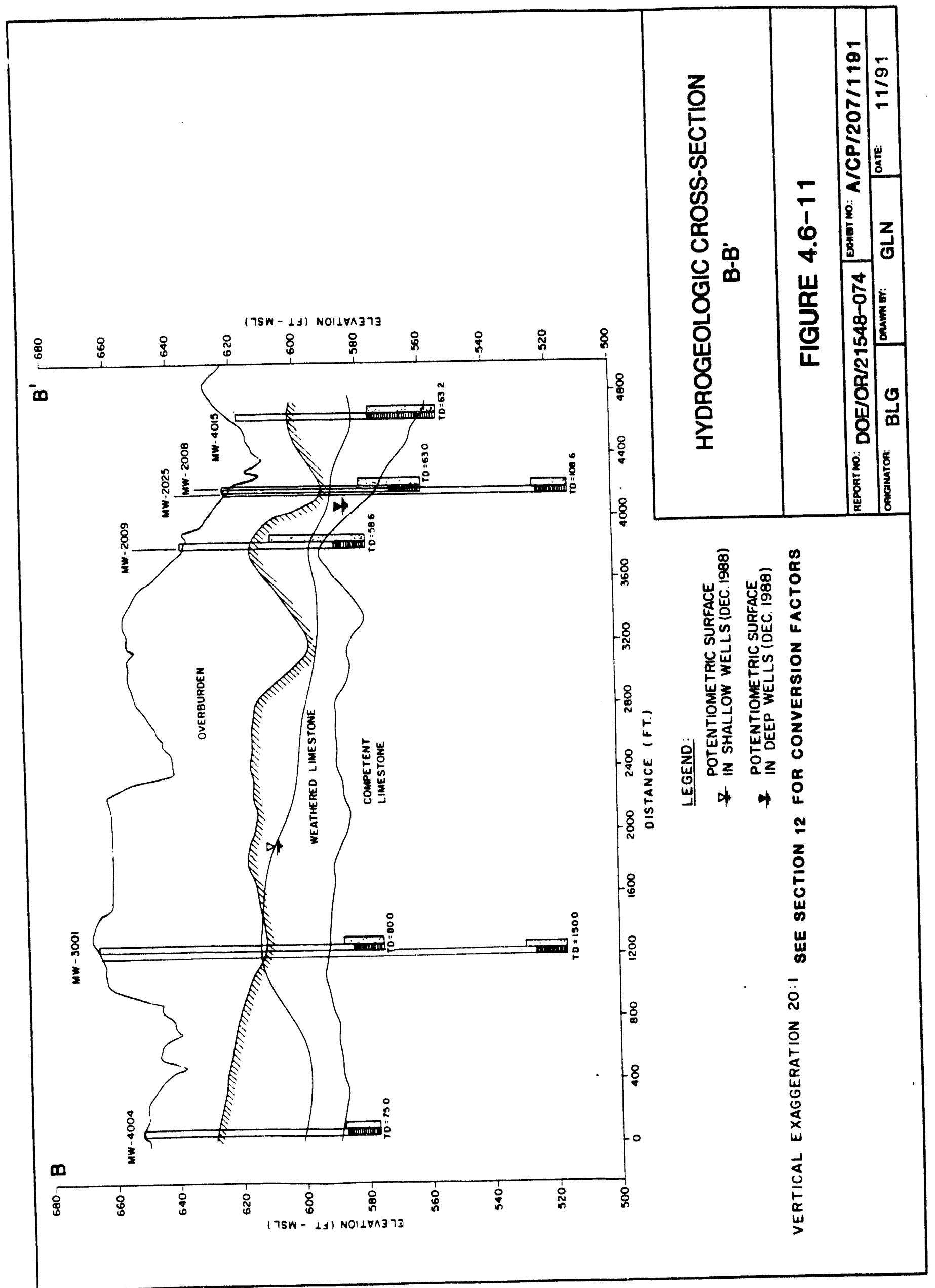



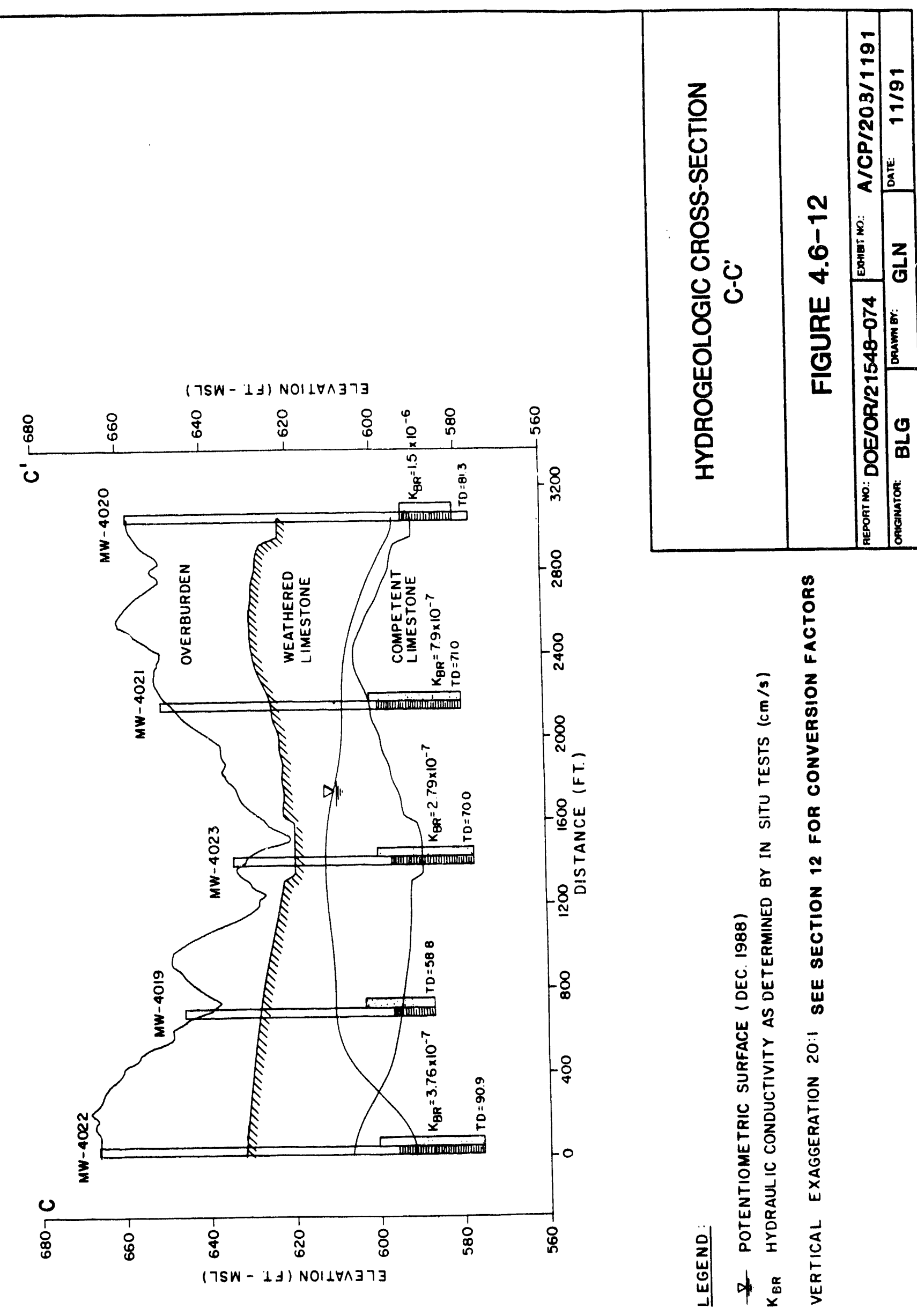


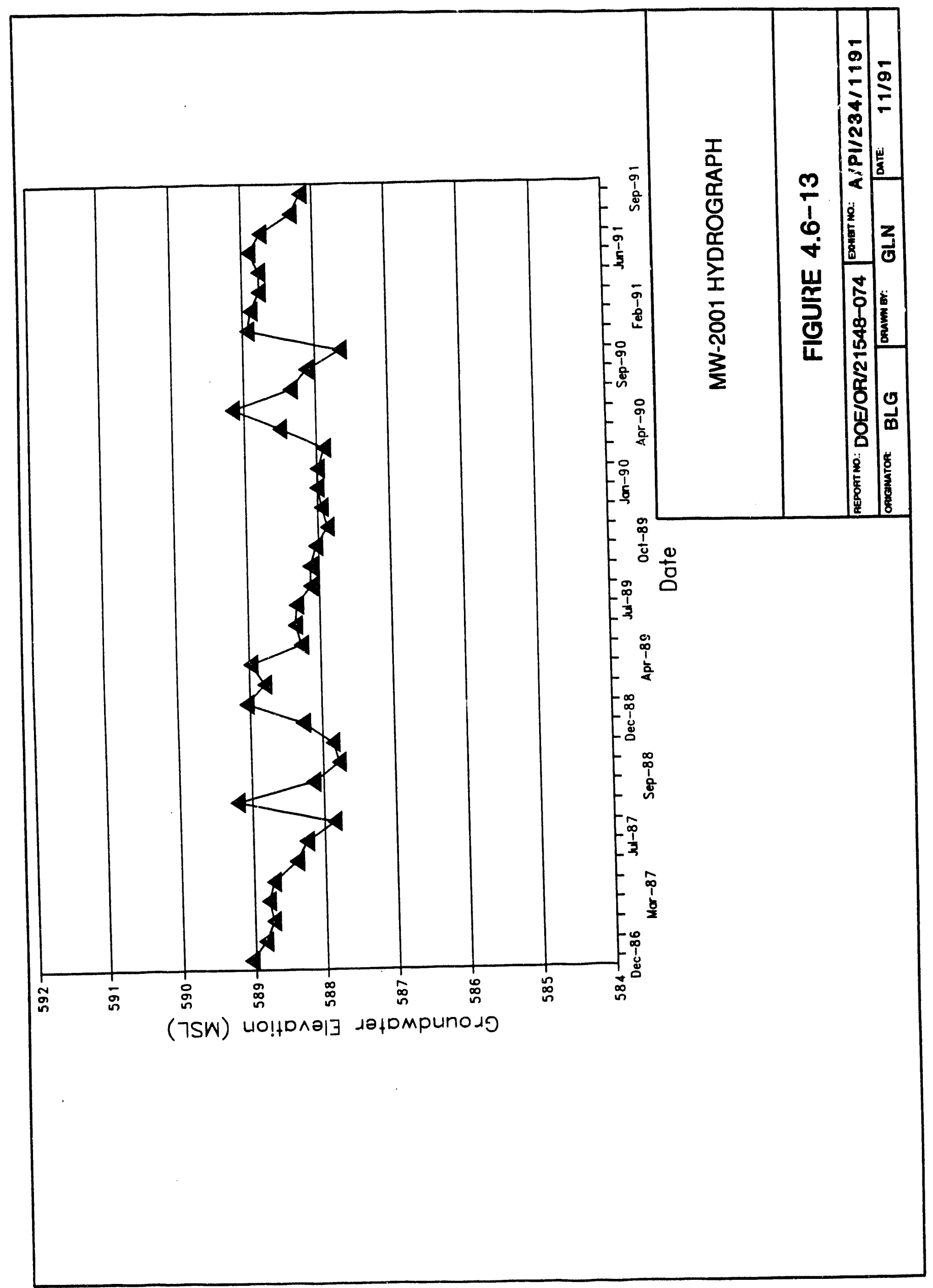




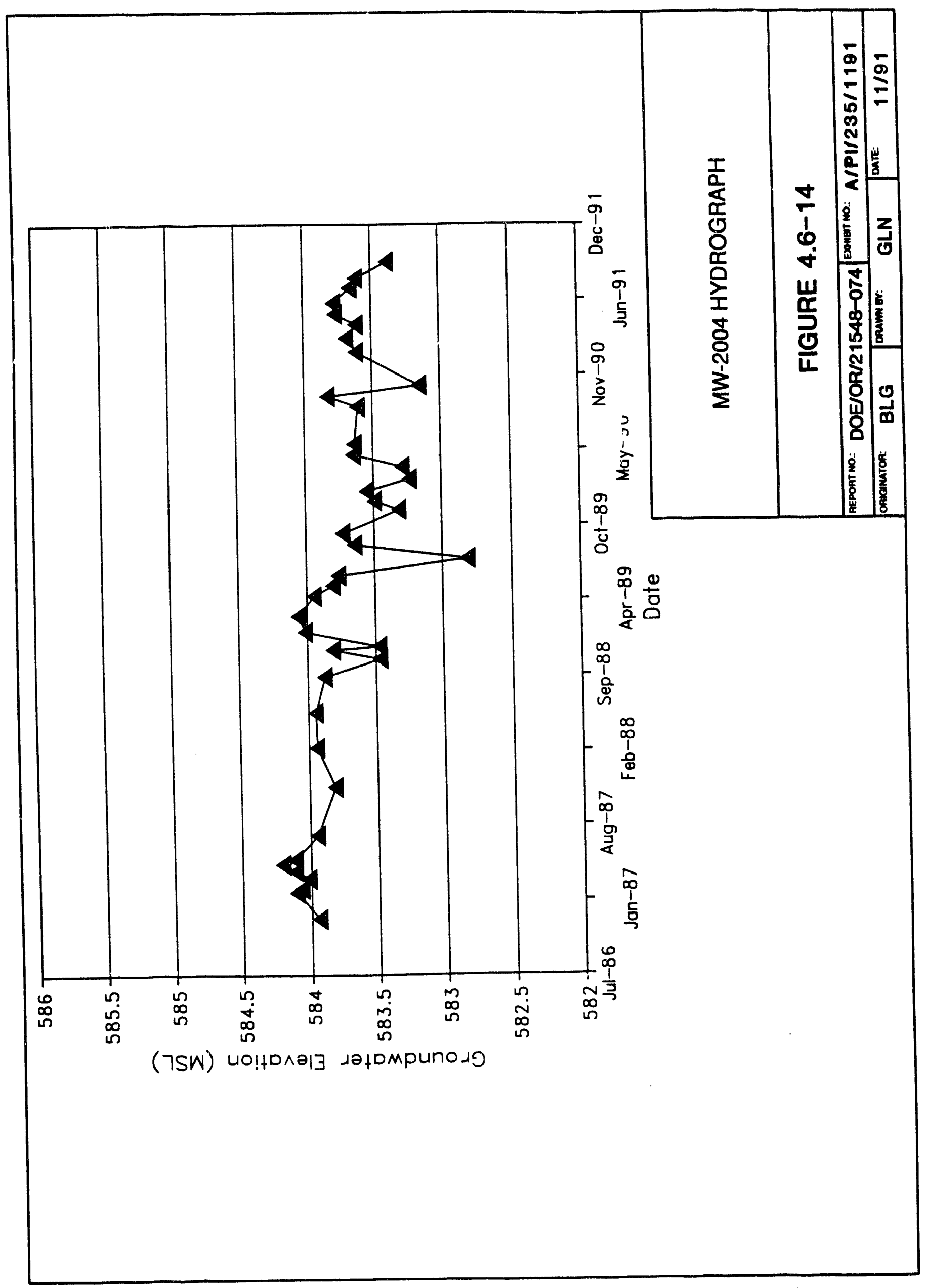




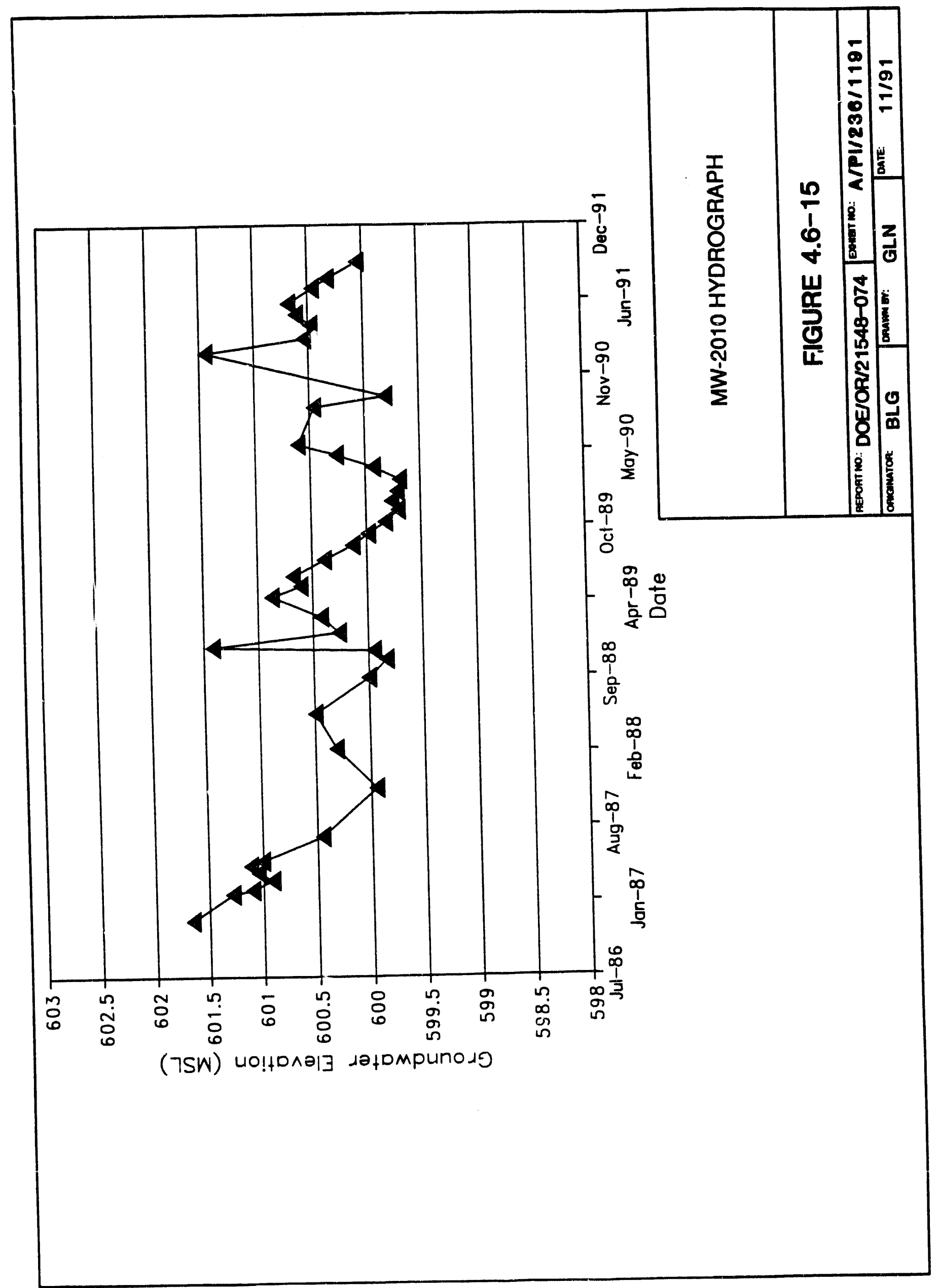




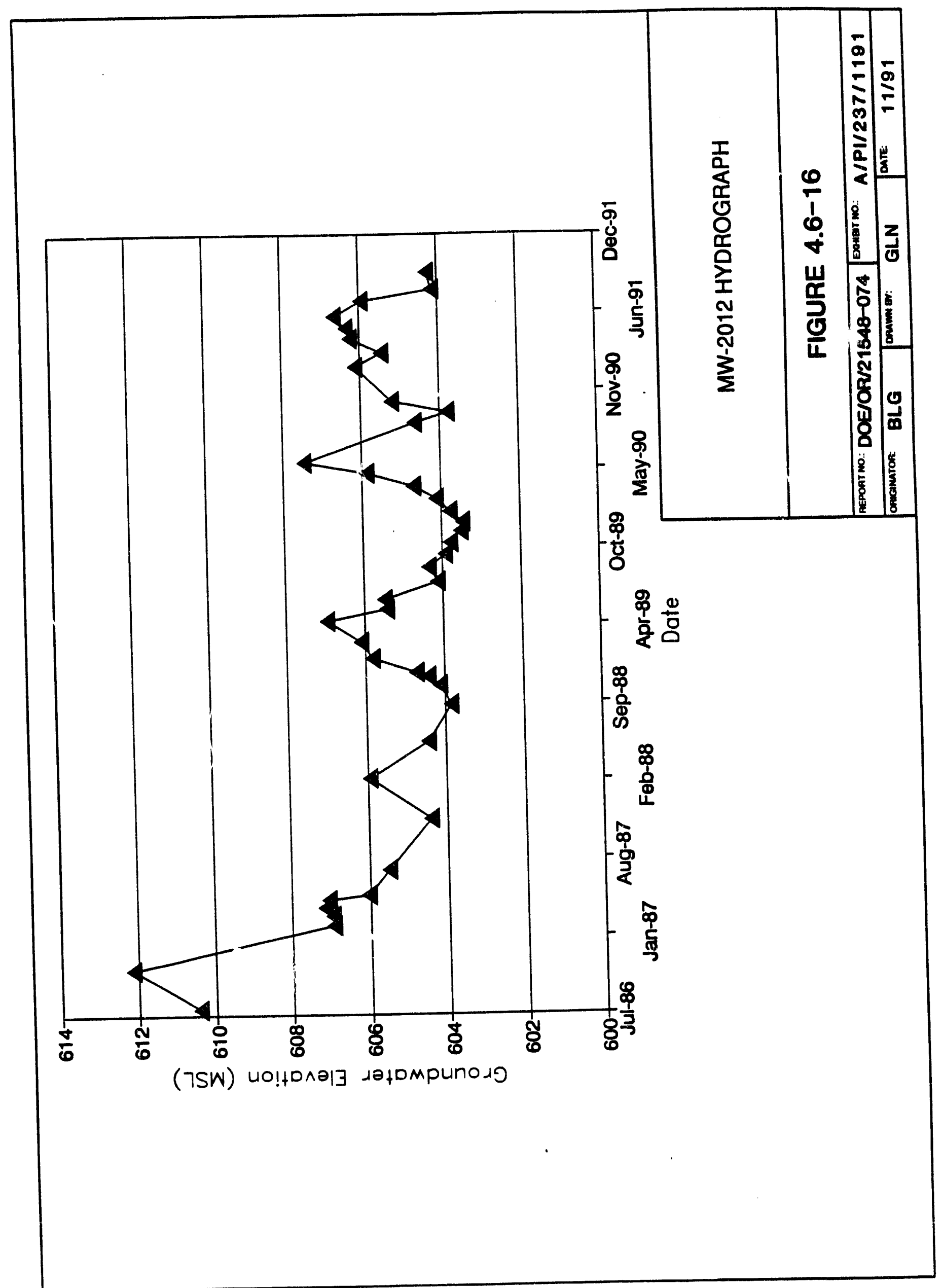




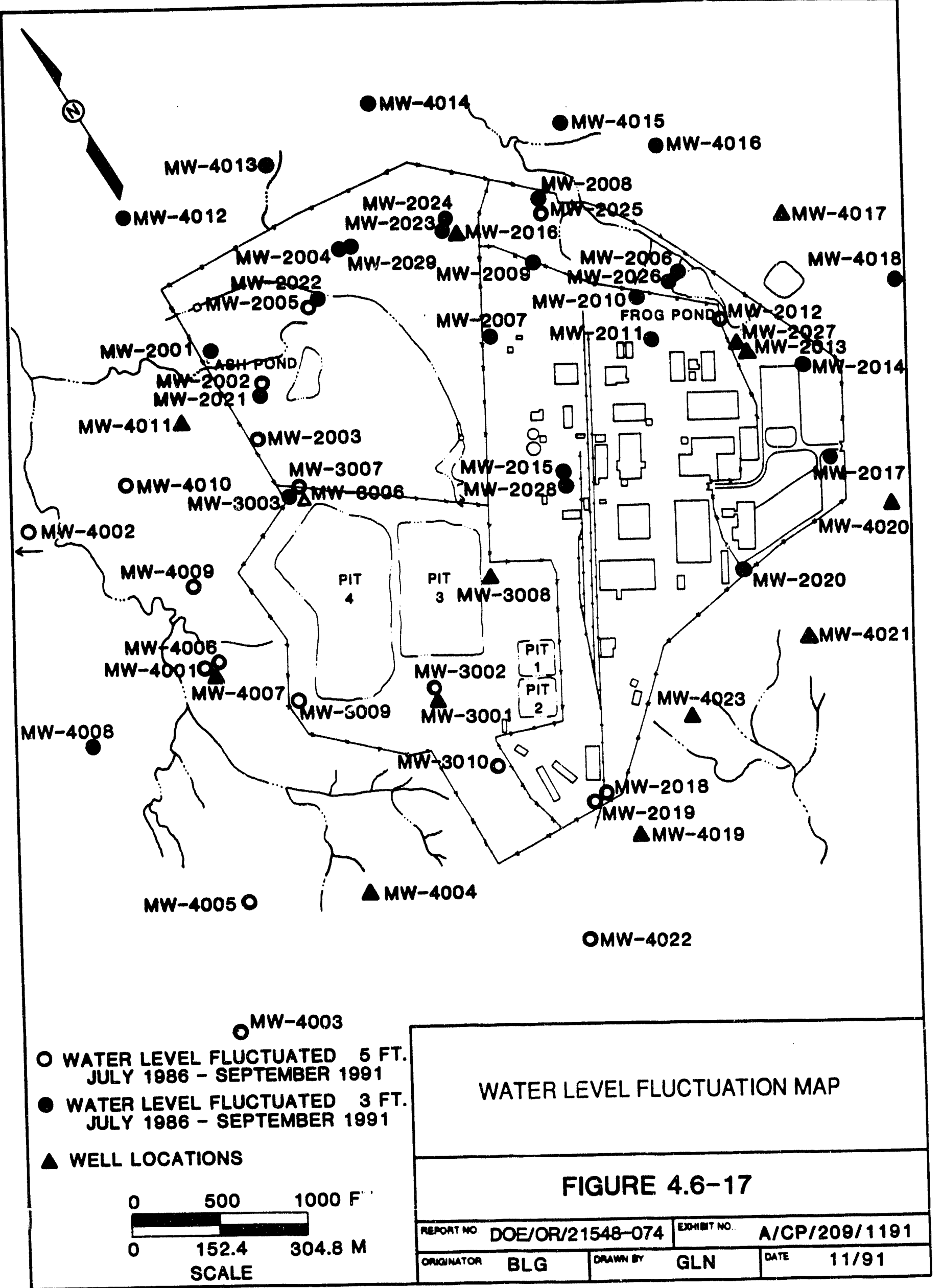




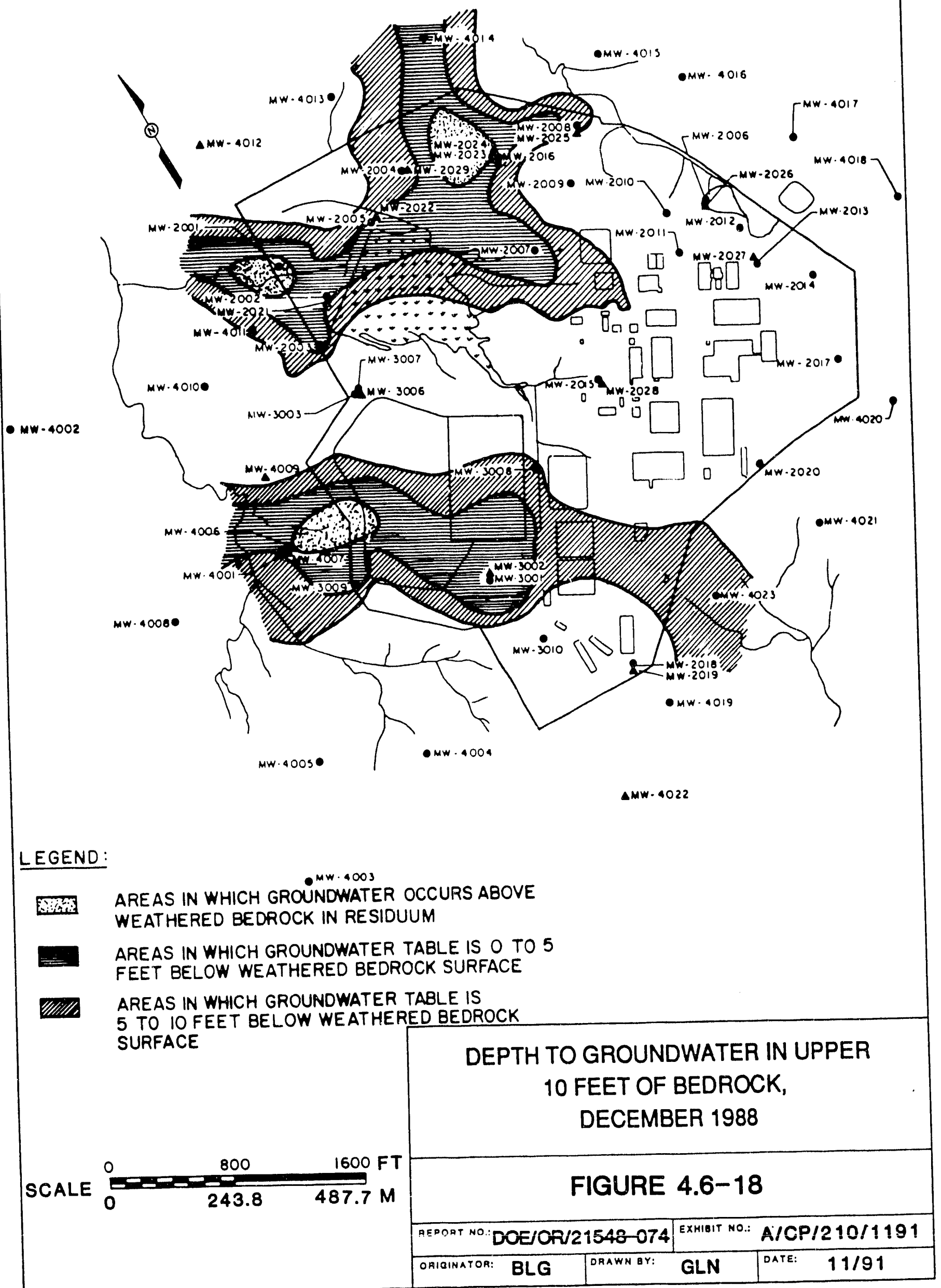




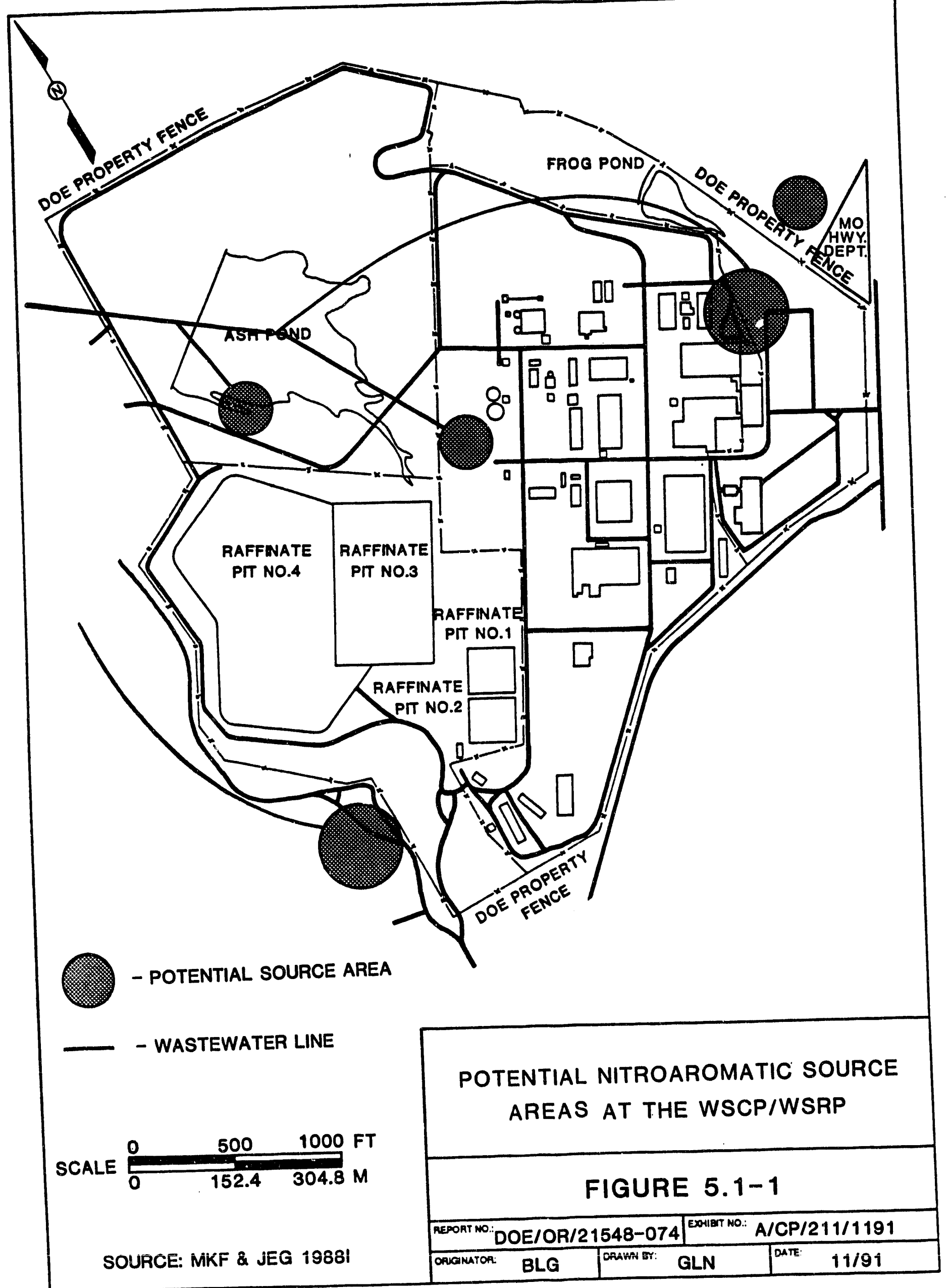




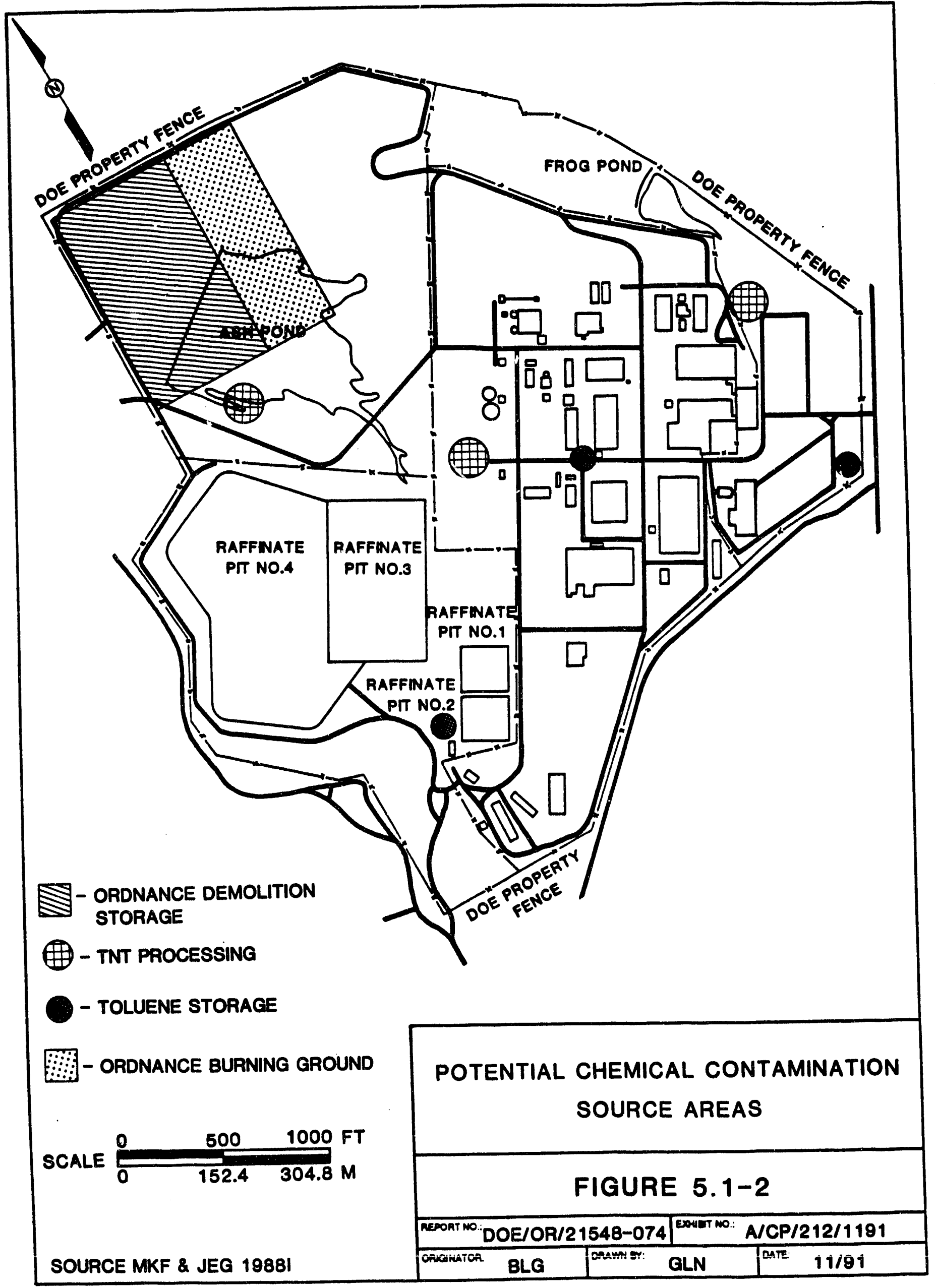




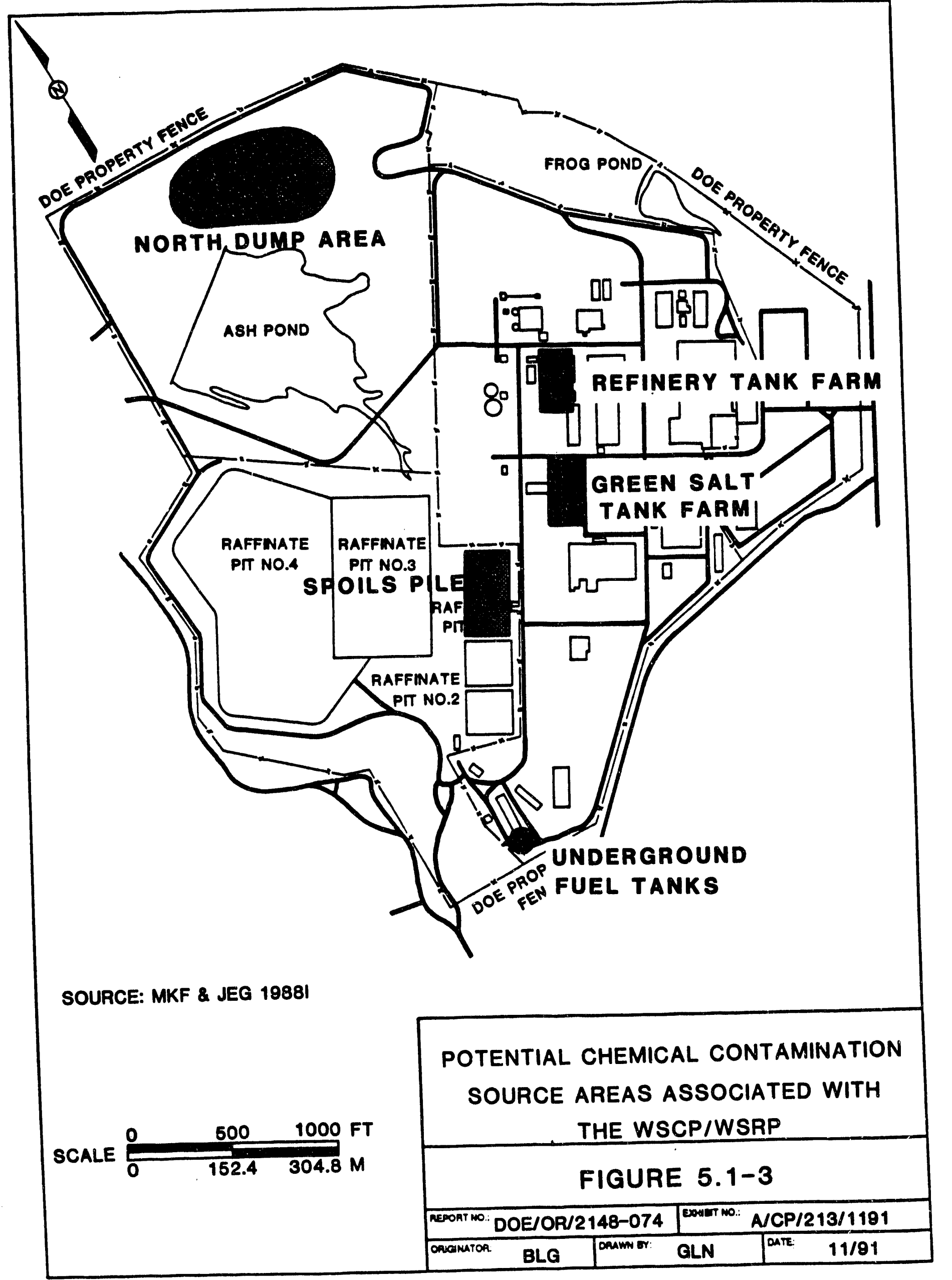




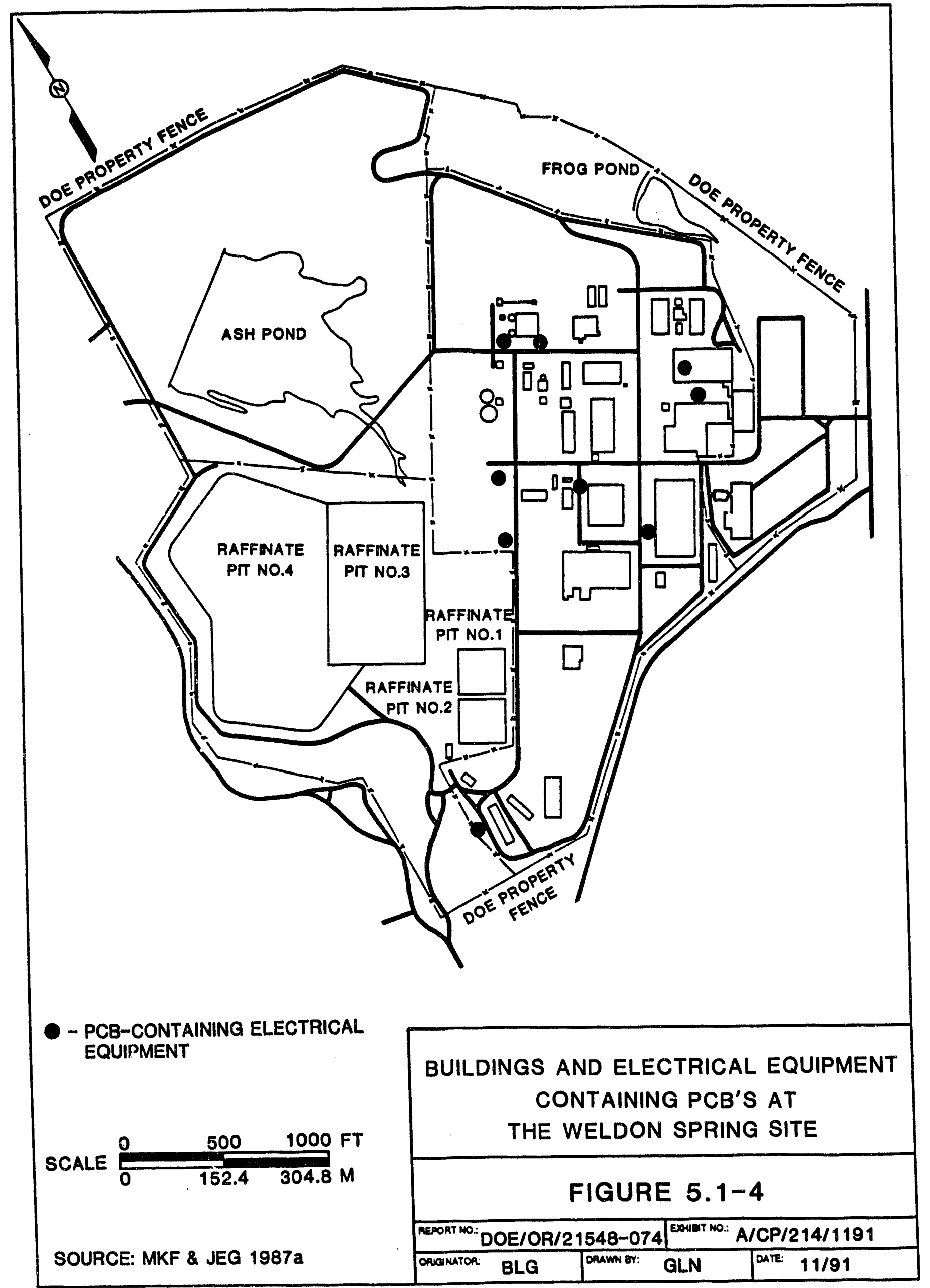




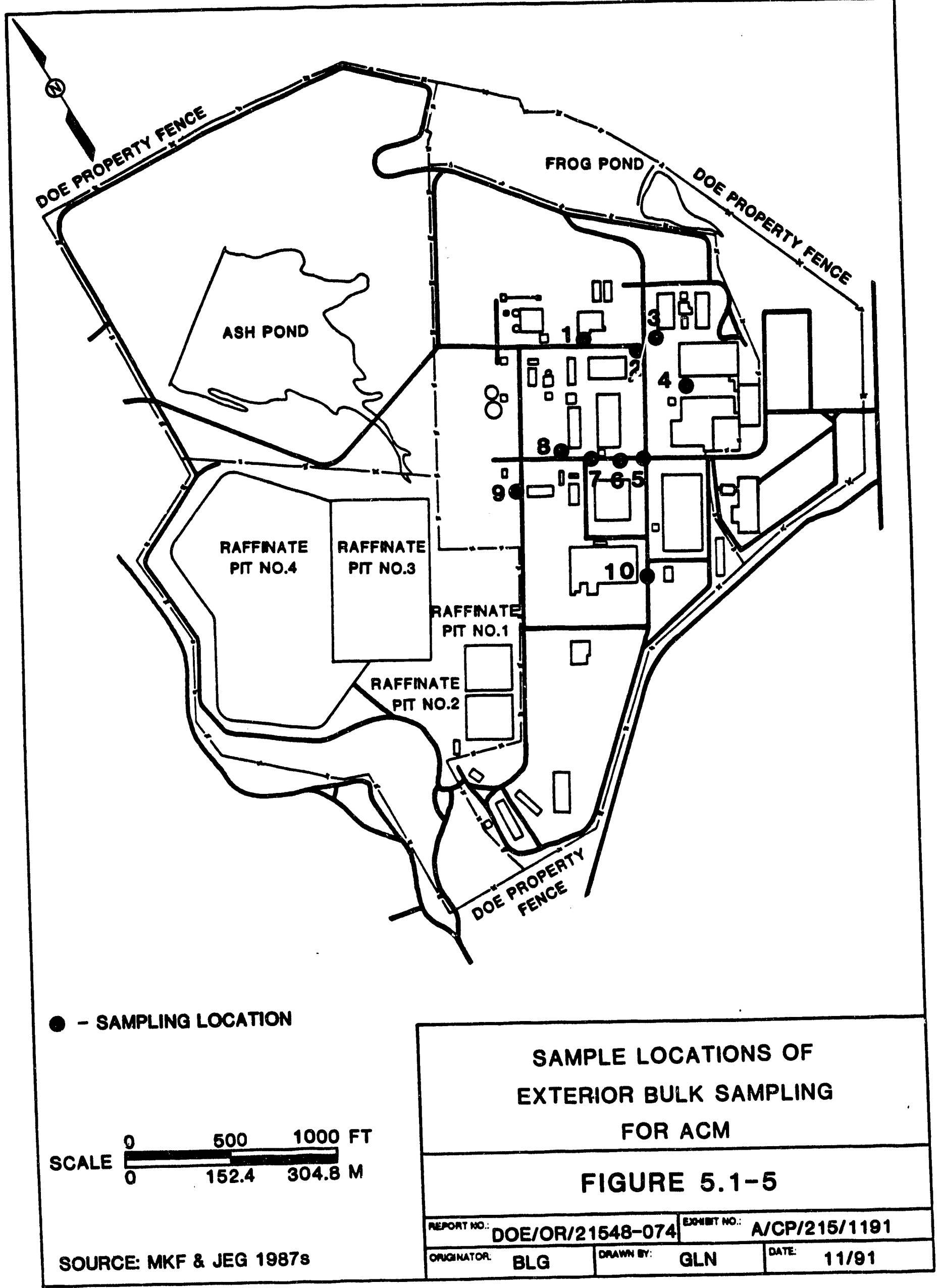




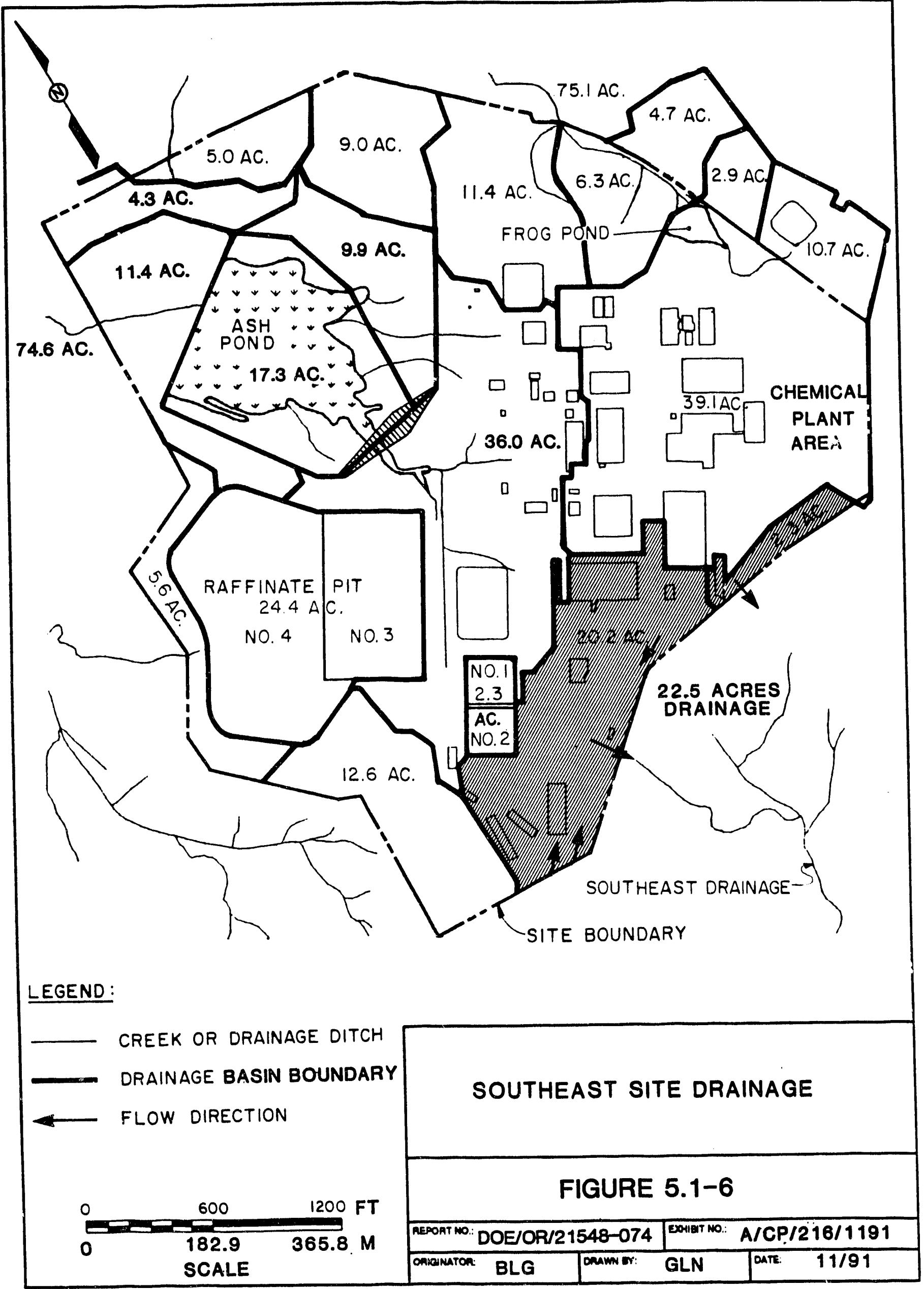




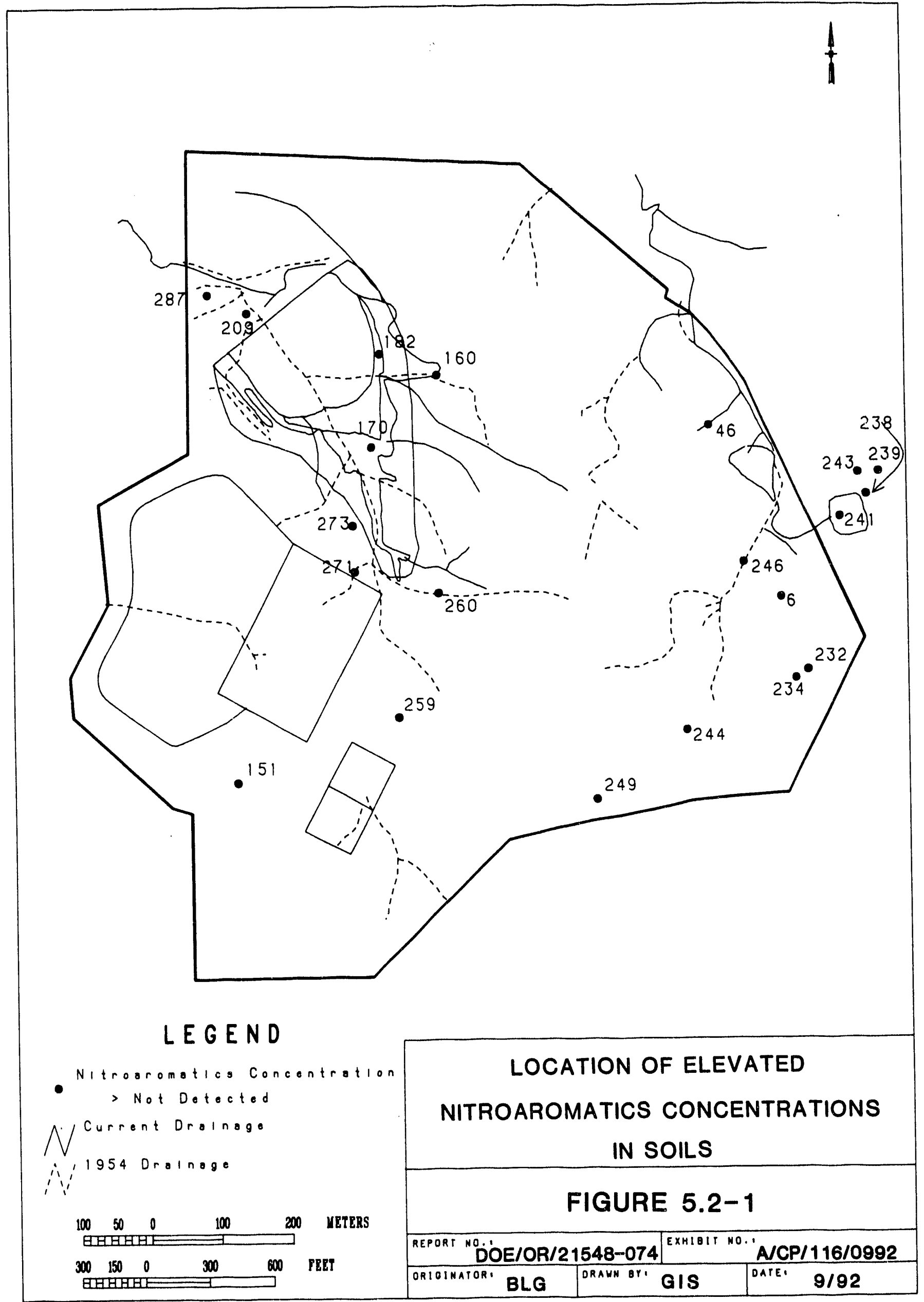




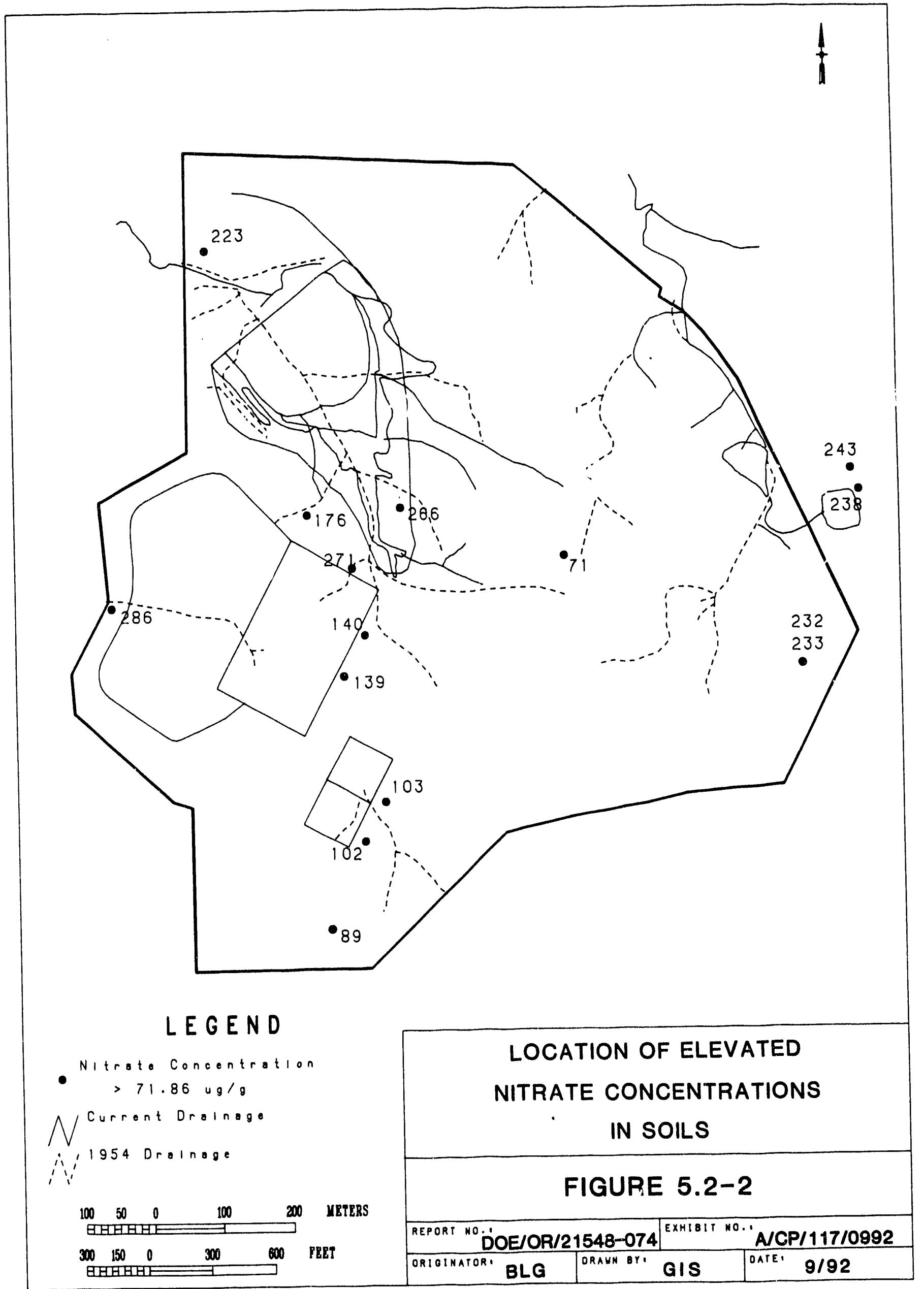




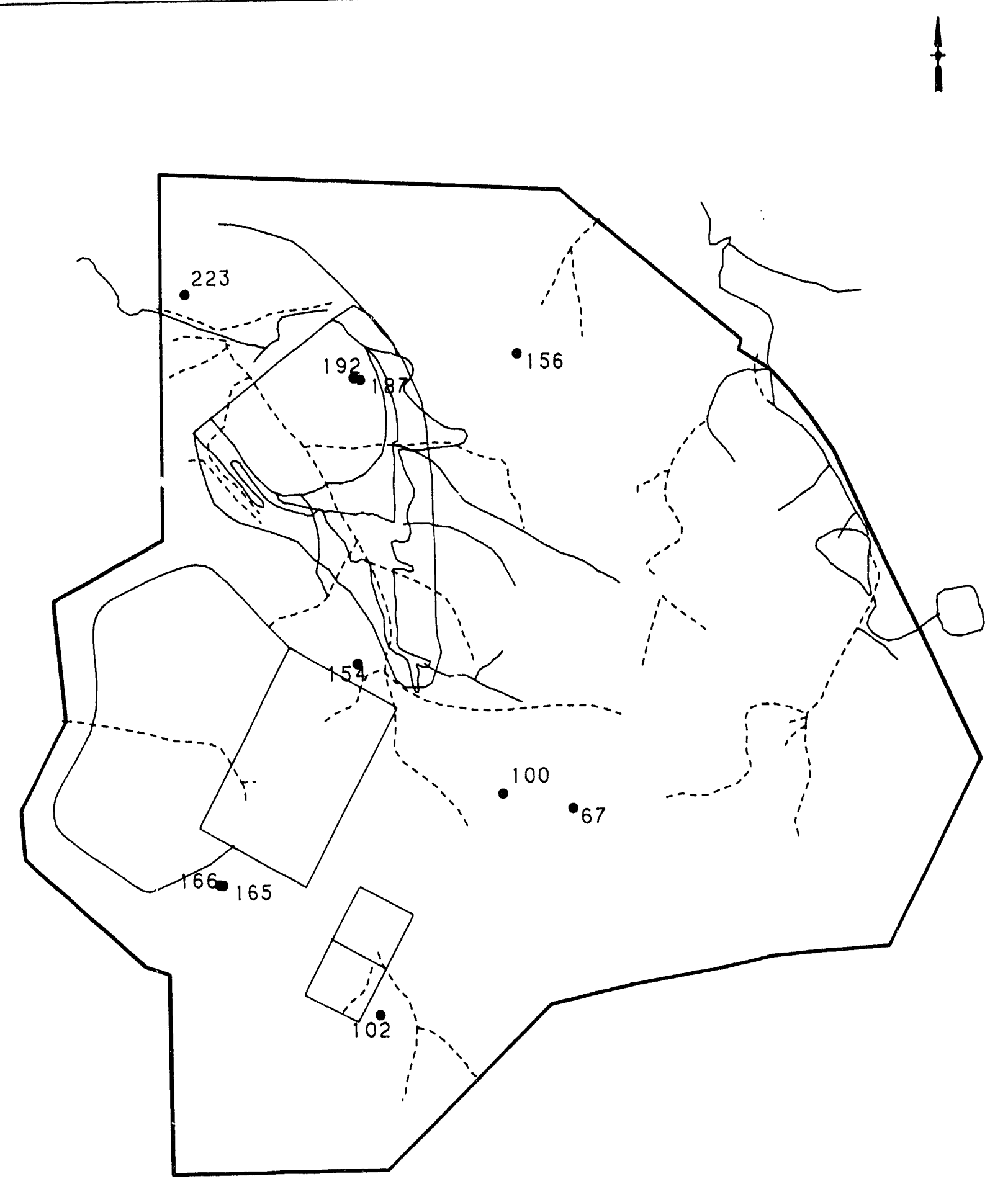

\section{LEGEN D}

Nitrite Concentrotion

- >0.25 ug/g

NCurrent Drolnogo

$\therefore, 1954$ Drolnogo

i'í

\begin{tabular}{|c|c|c|c|c|c|}
\hline 100 & & 0 & 100 & 2000 & VeterS \\
\hline 300 & 150 & 0 & 300 & 600 & PBET \\
\hline
\end{tabular}

\begin{tabular}{|c|c|c|}
\hline \multicolumn{3}{|c|}{$\begin{array}{l}\text { LOCATION OF ELEVATED } \\
\text { NITRITE CONCENTRATIONS } \\
\text { IN SOILS }\end{array}$} \\
\hline \multicolumn{3}{|c|}{ FIGURE 5.2-3 } \\
\hline REPORT NO D'OE/OF & $1548-074]^{E \times 181}$ & A/CP/118/0992 \\
\hline ORIOINATORI BLG & ORAWM BY GIS & $\begin{array}{ll}\text { DATE } & 9 / 92 \\
\end{array}$ \\
\hline
\end{tabular}




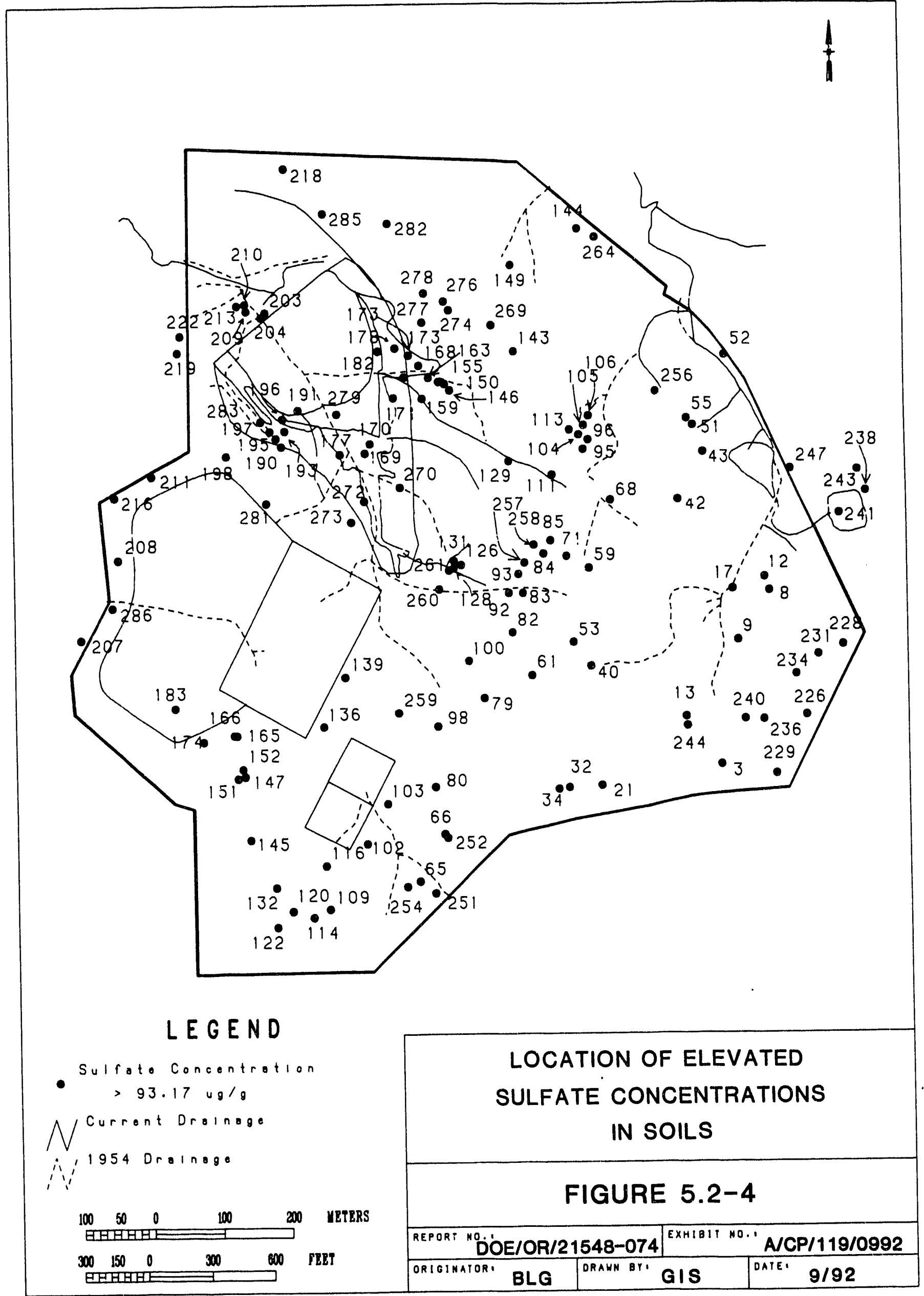




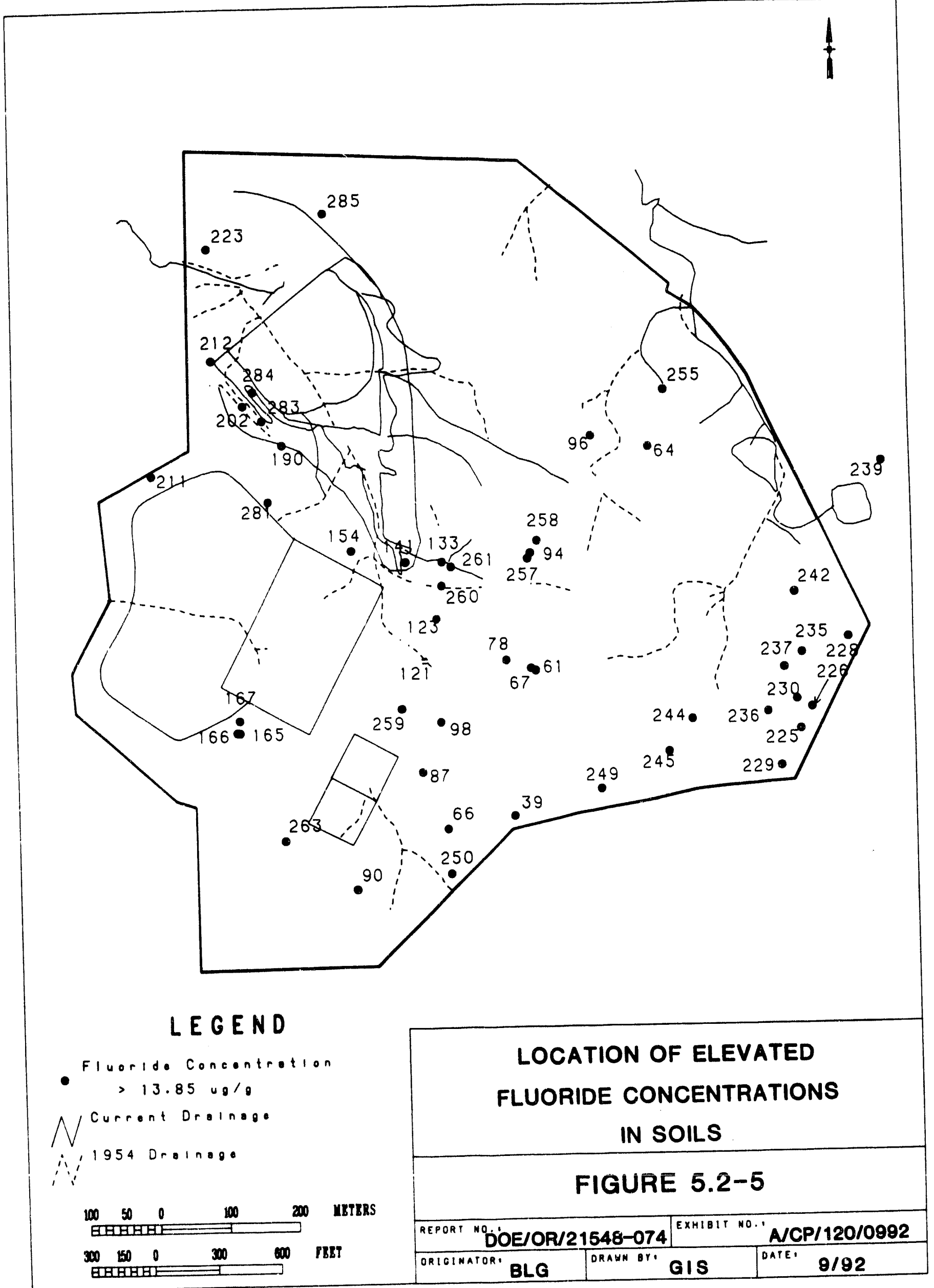




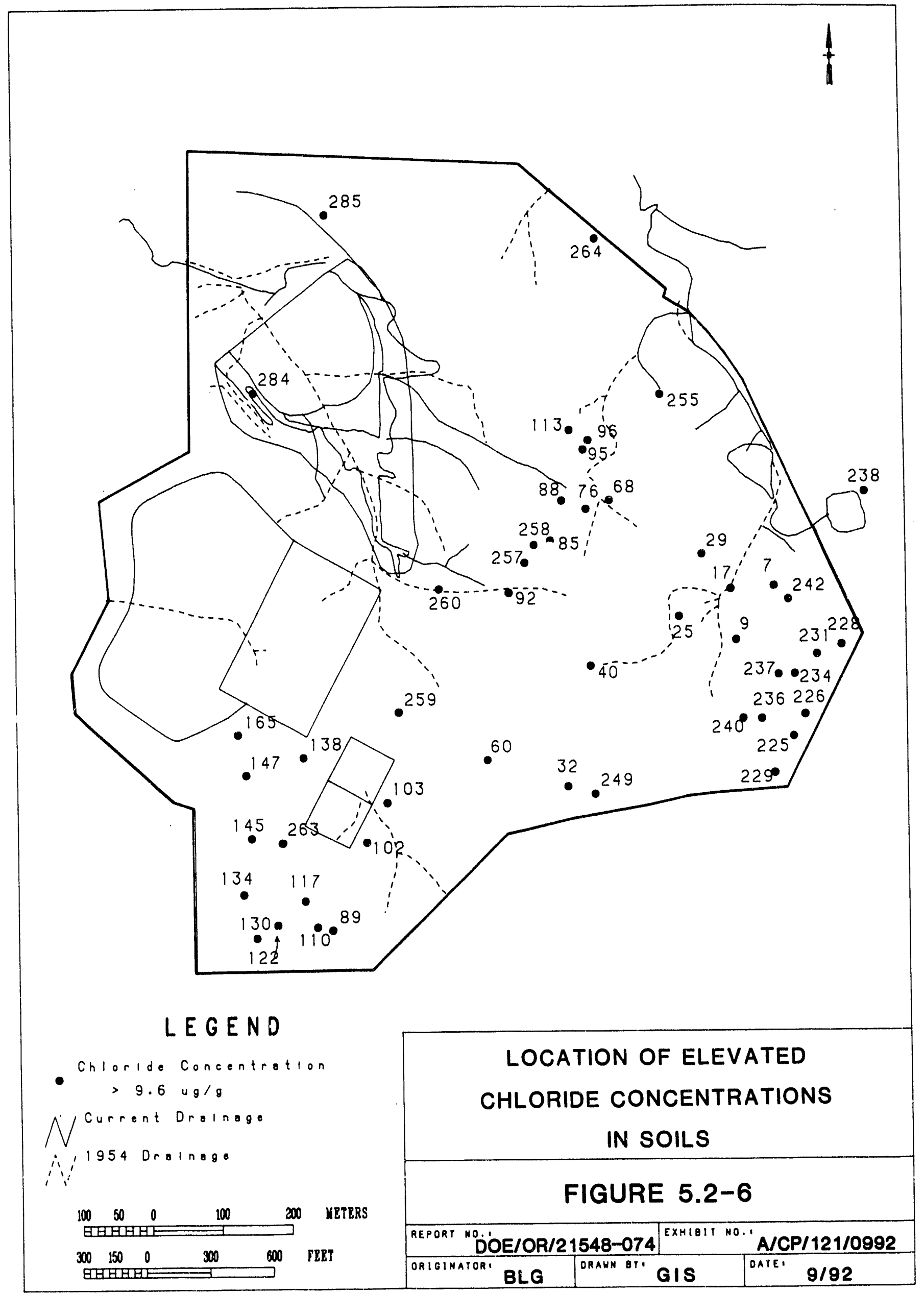




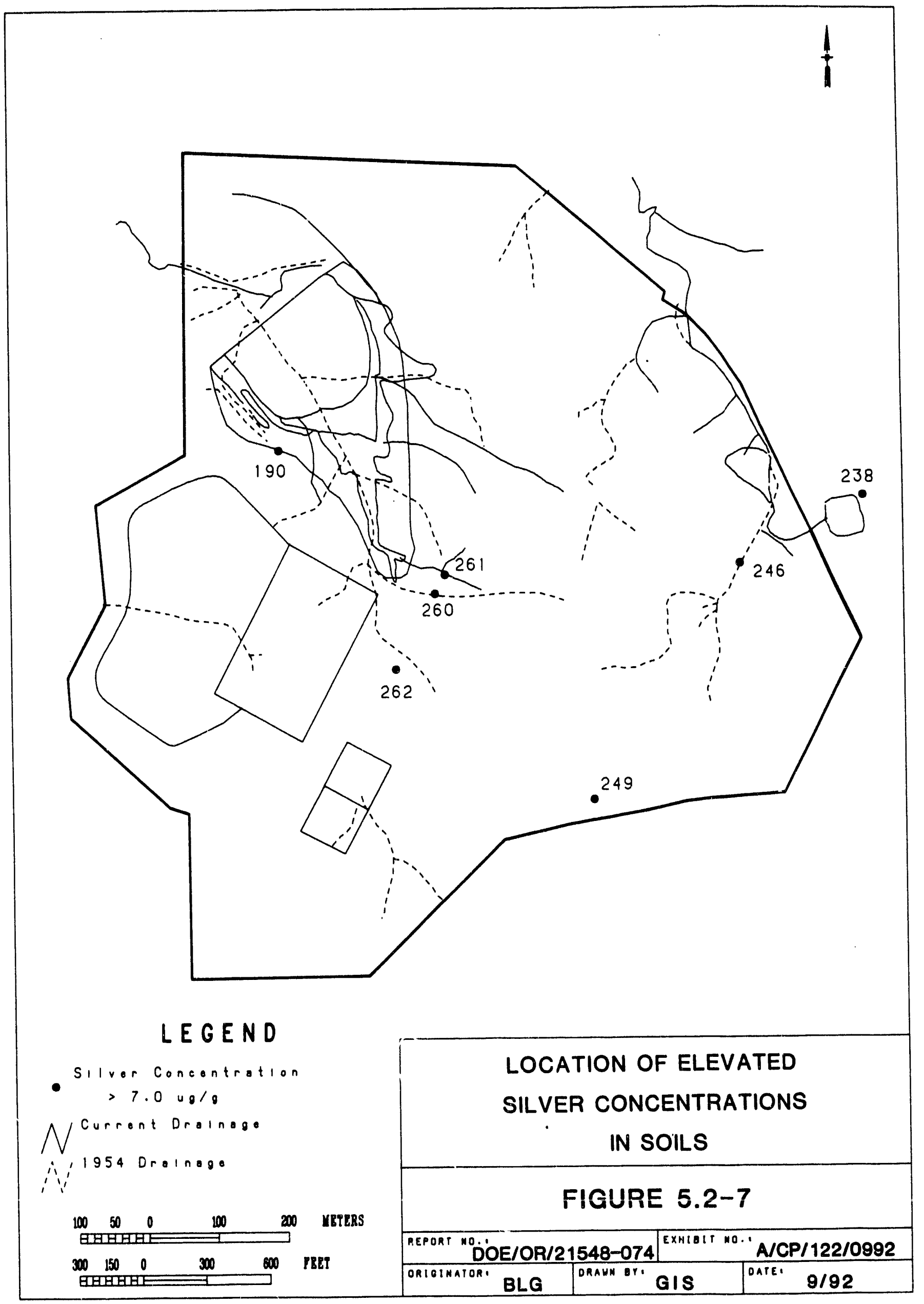




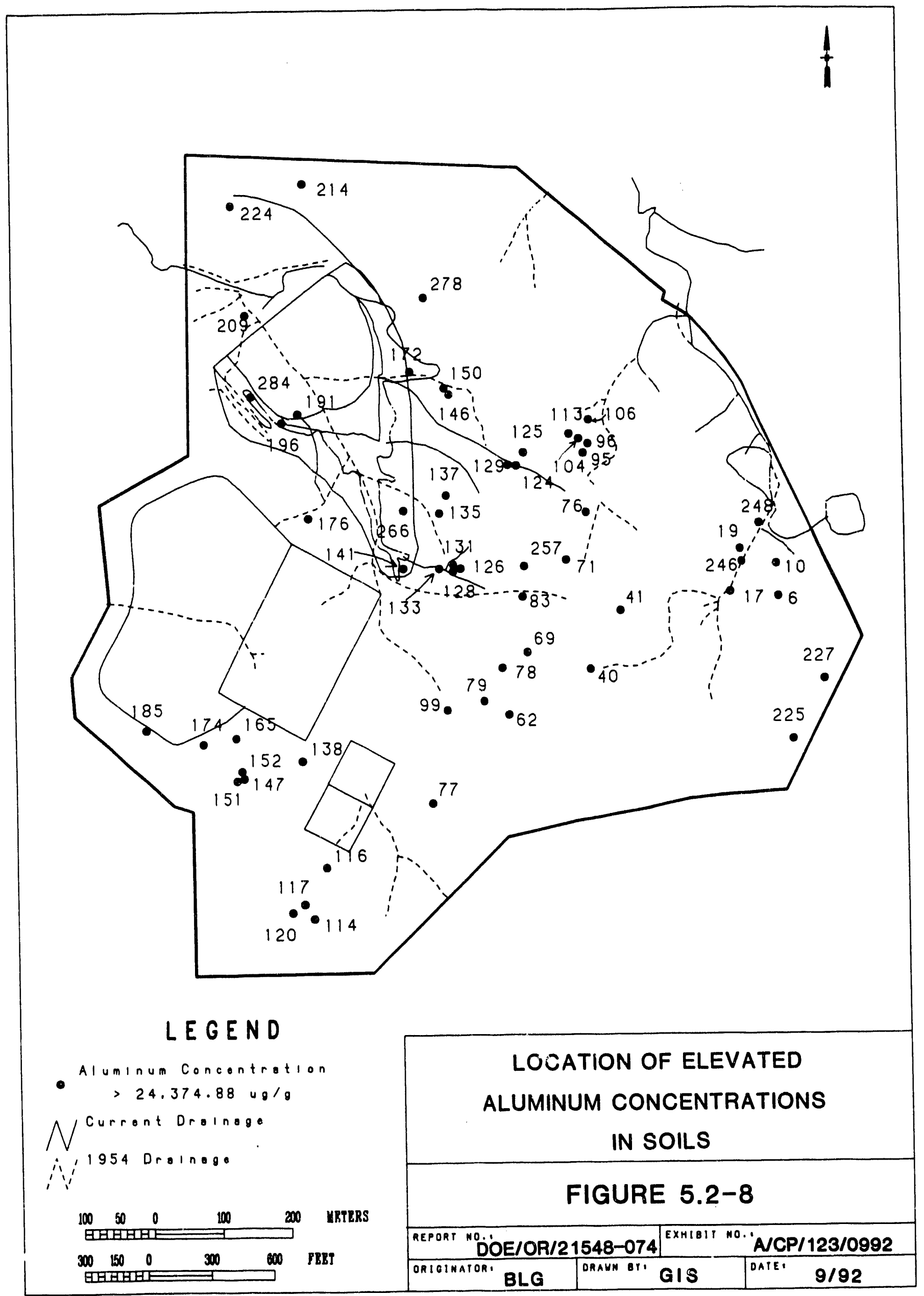




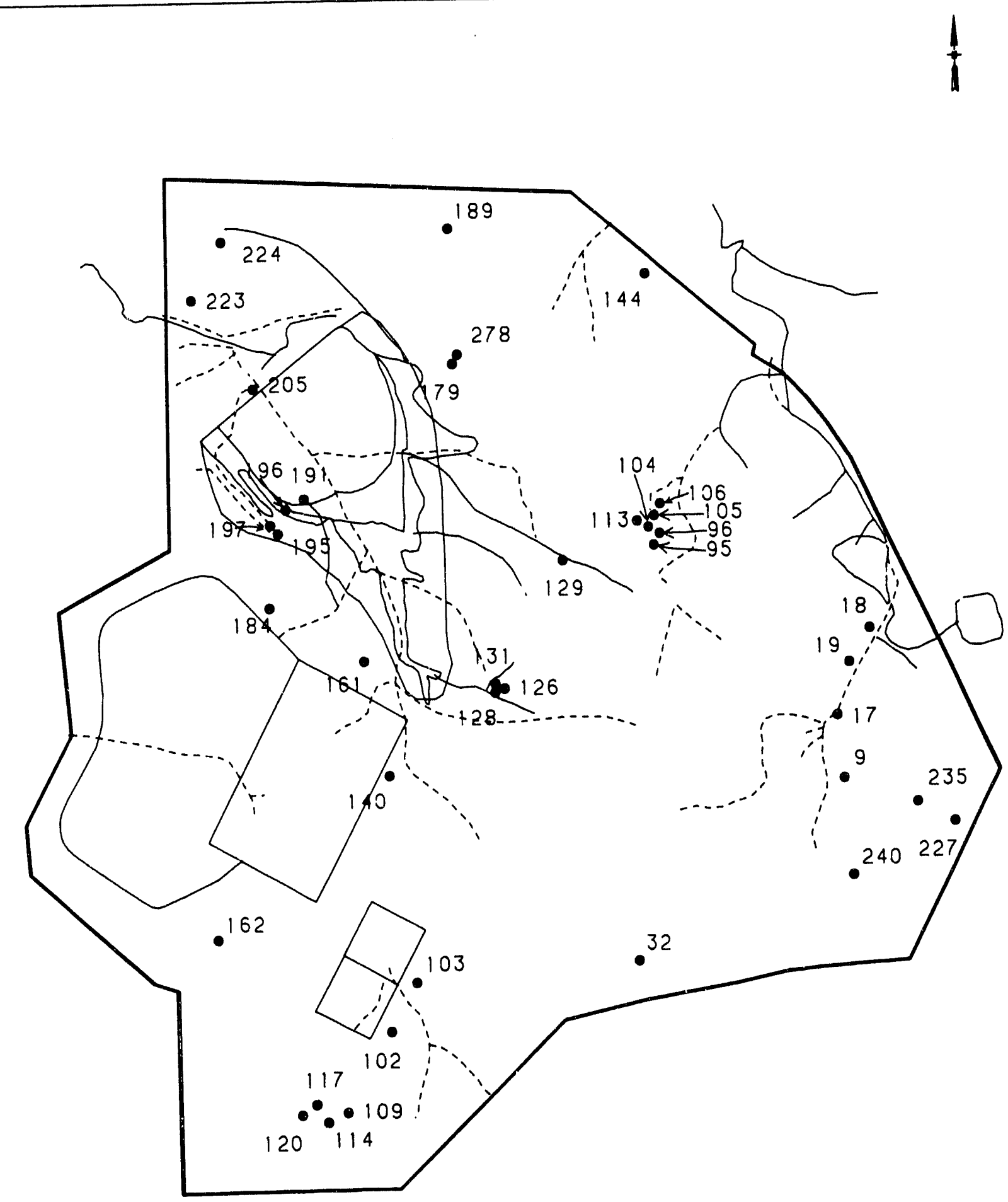

LEGEN D

Arsenic Concentration

- $>25.81 \mathrm{ug} / \mathrm{g}$

NCurrent Dralnogo

$\therefore ; 1954$ Droinoge

$i \because$

\begin{tabular}{|c|c|c|c|c|c|}
\hline 100 & & 0 & $\mathfrak{m}$ & 20 & YBTERS \\
\hline 300 & 150 & 0 & 300 & 600 & PEET \\
\hline
\end{tabular}

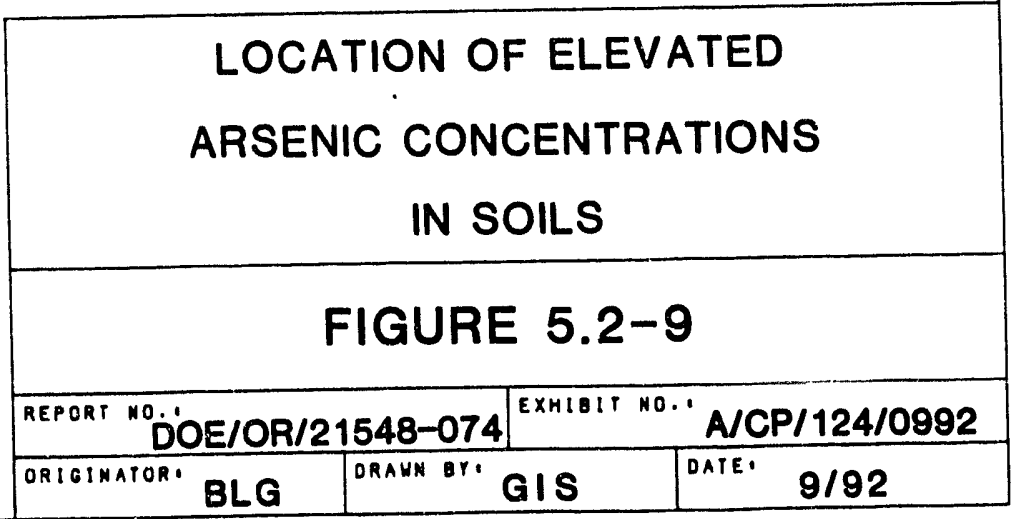




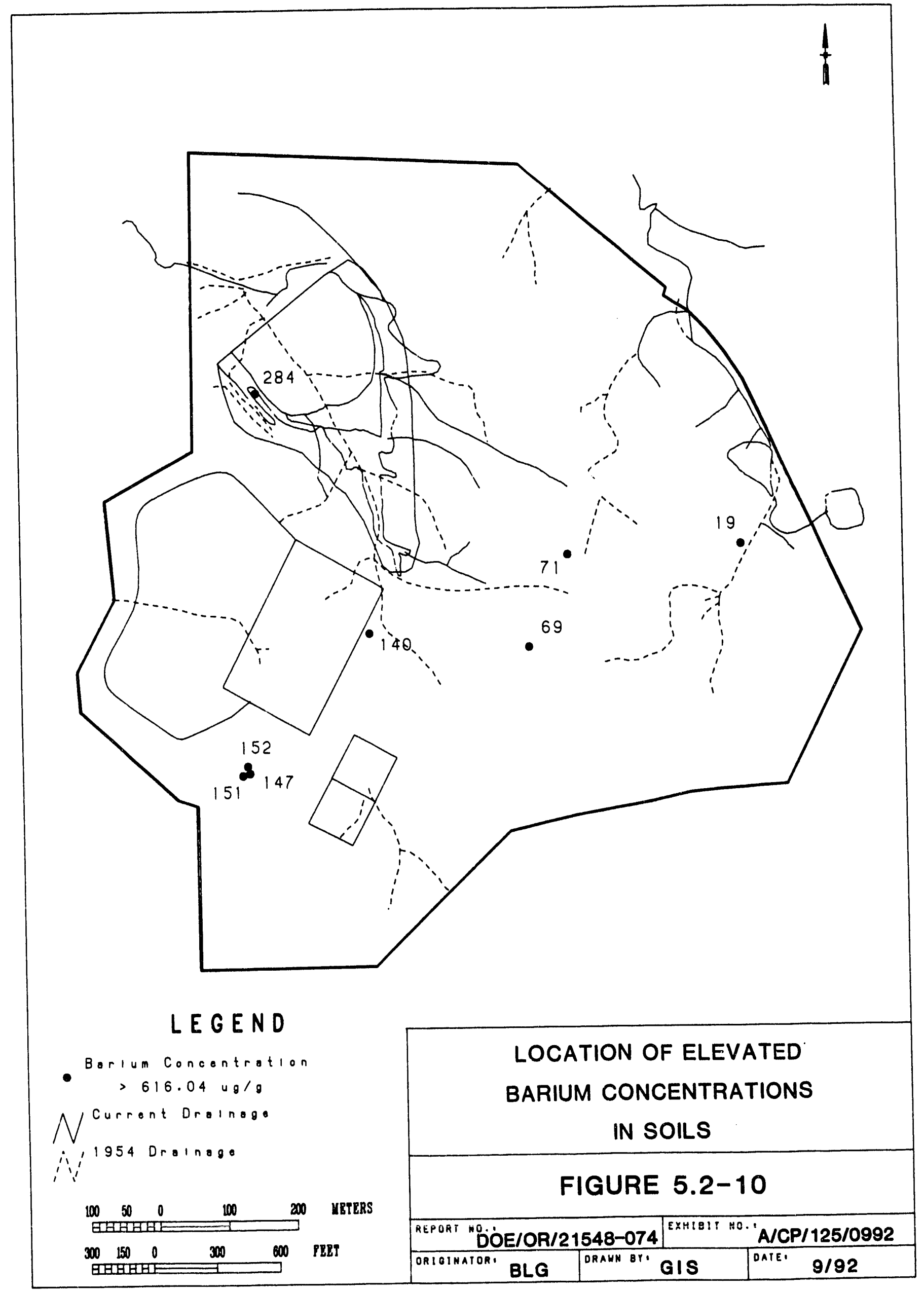




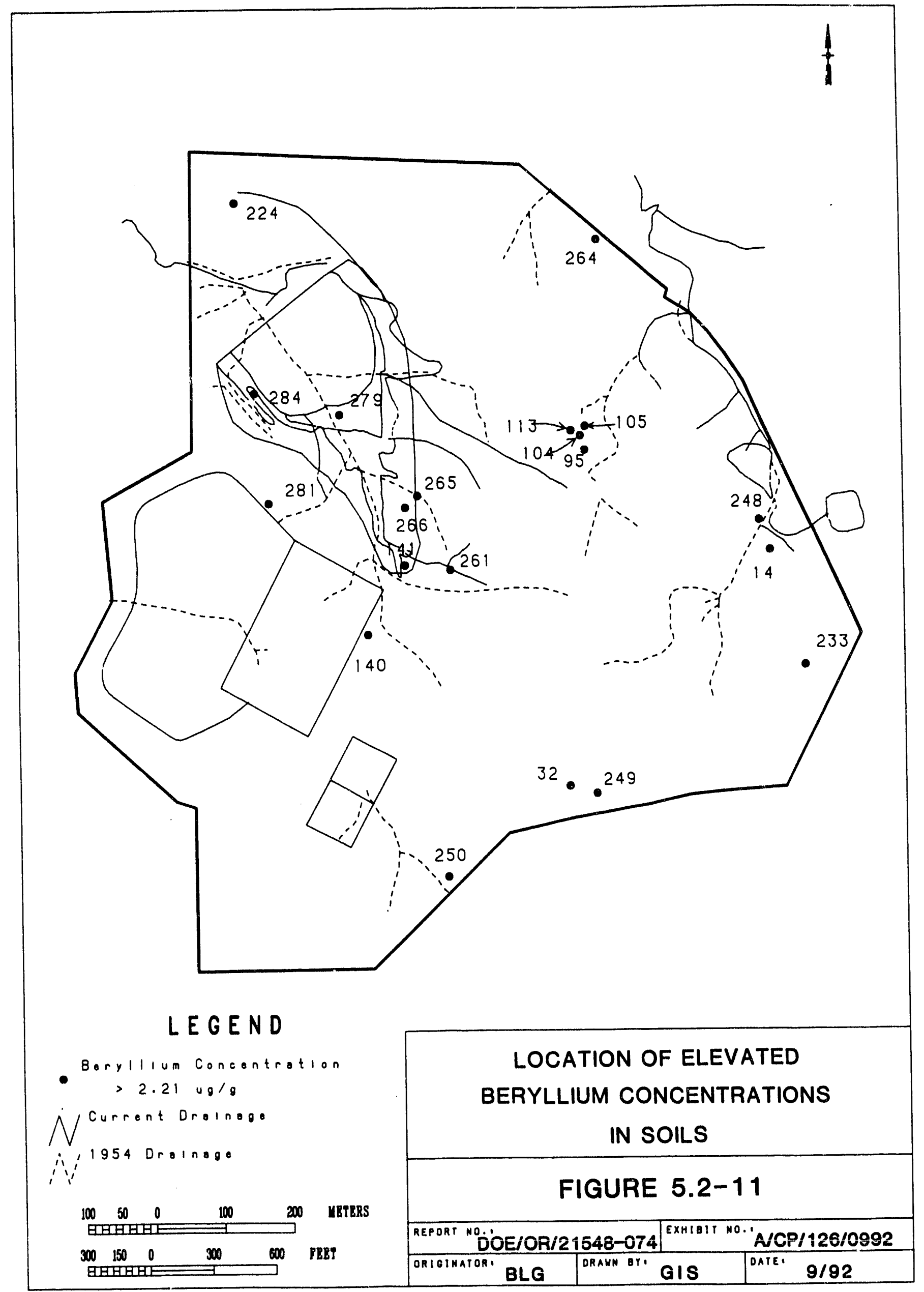




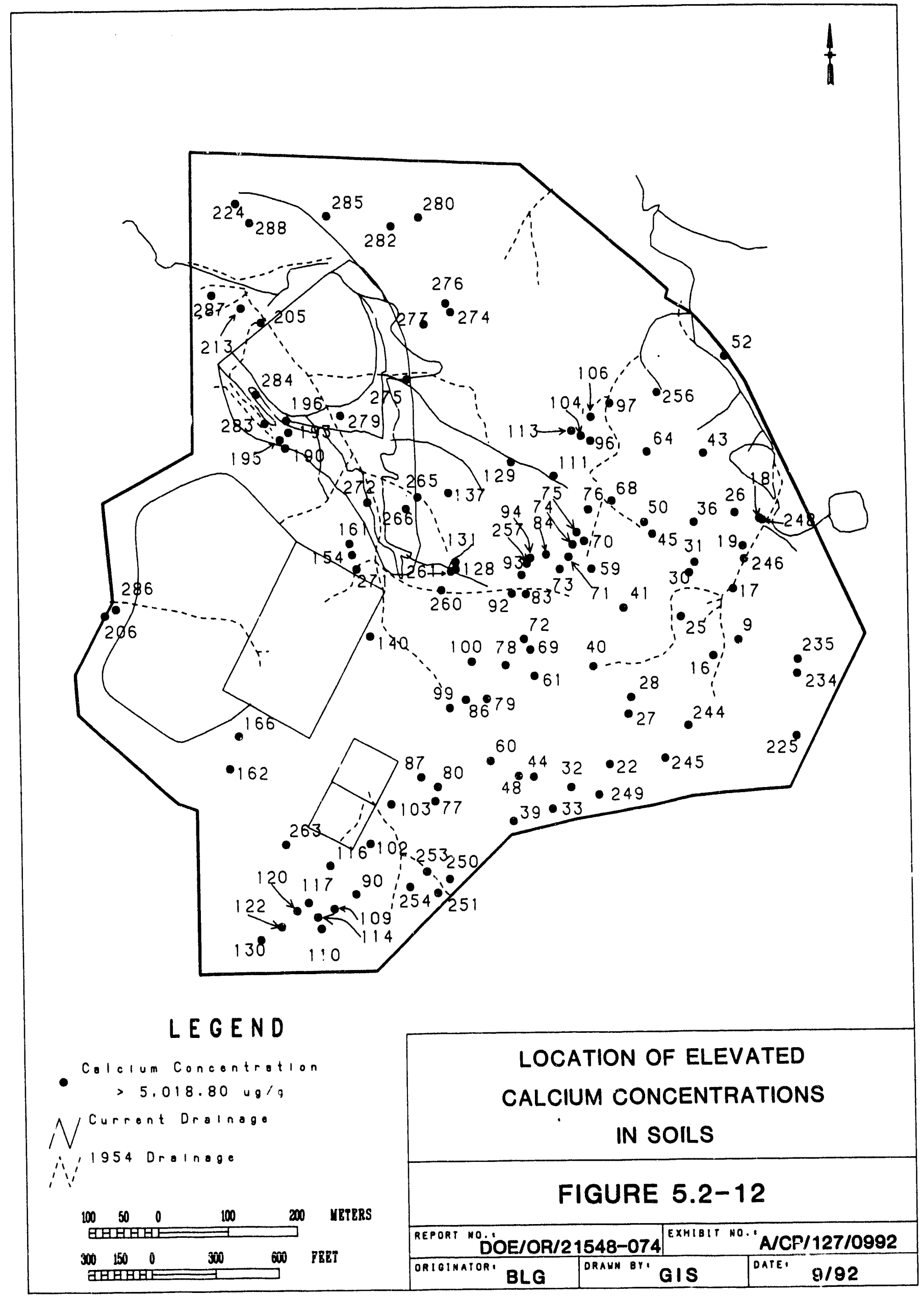




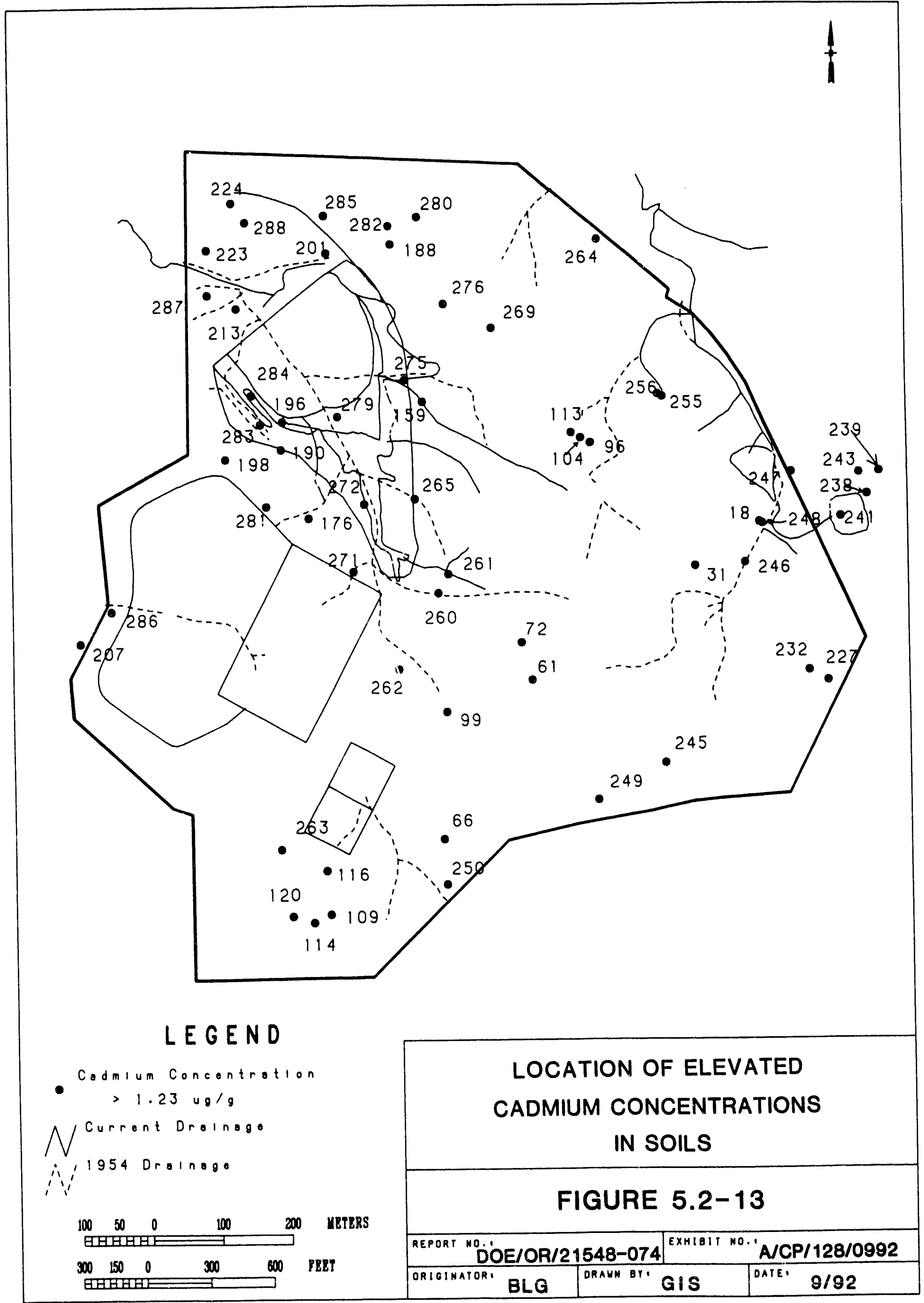




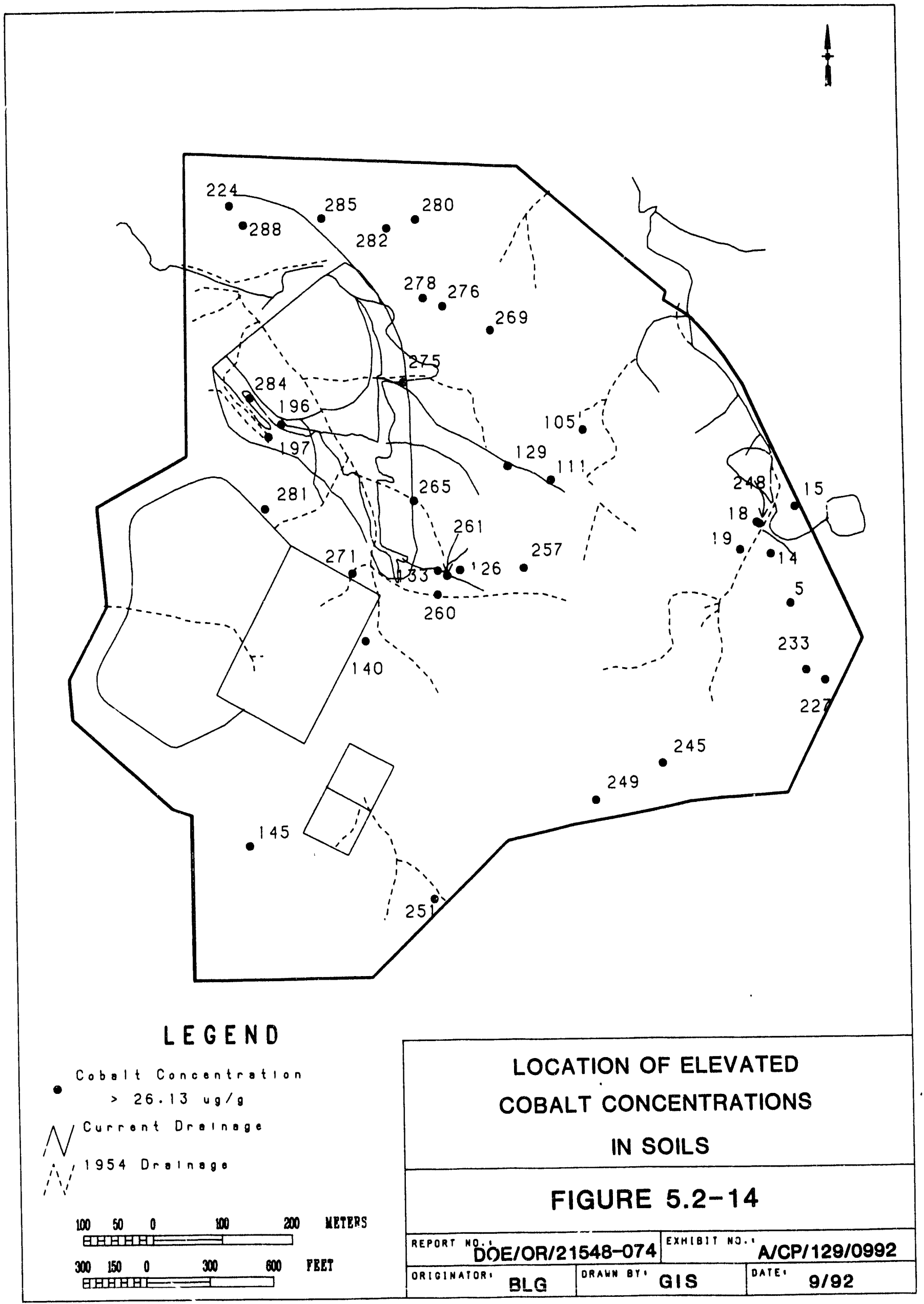




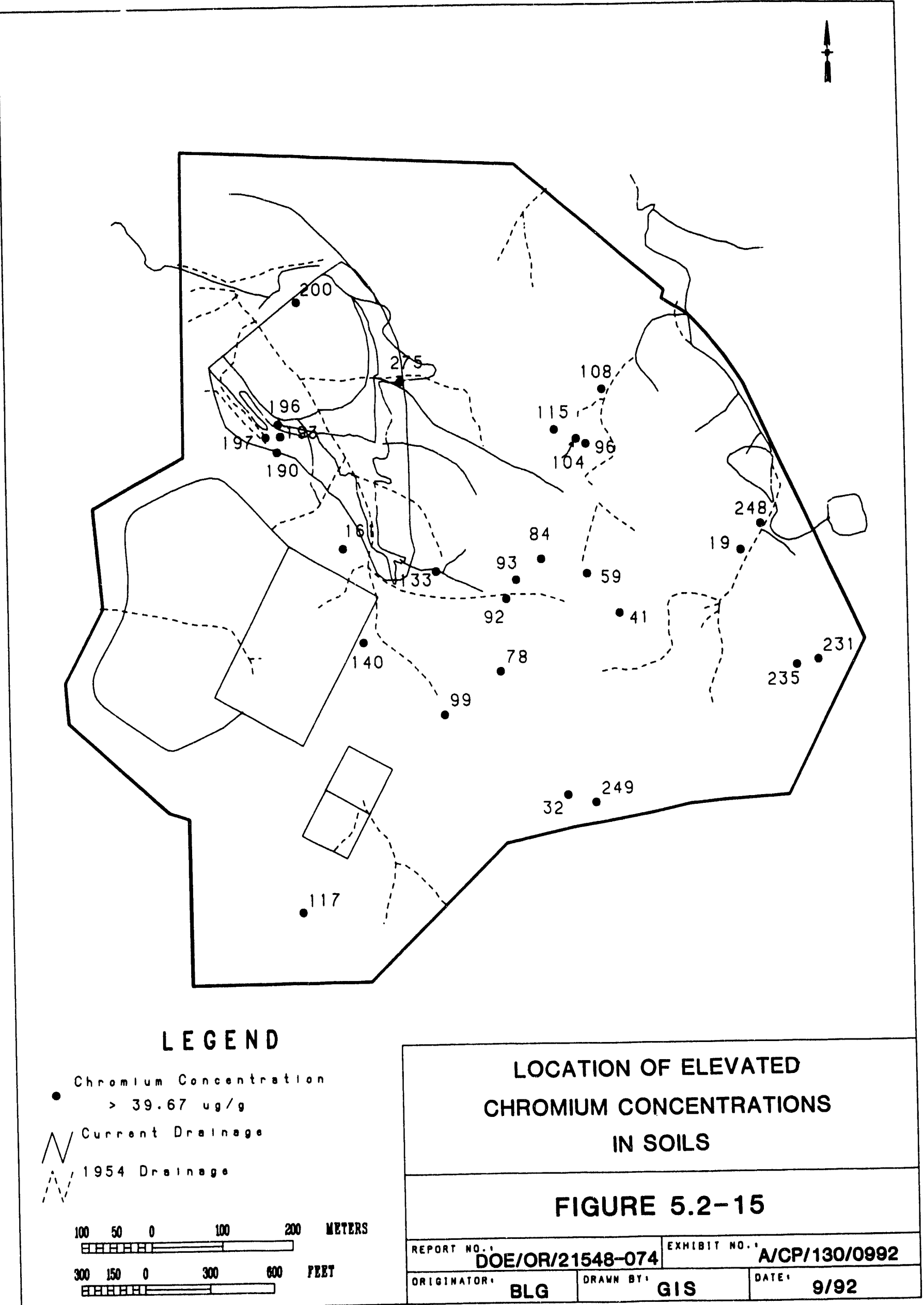




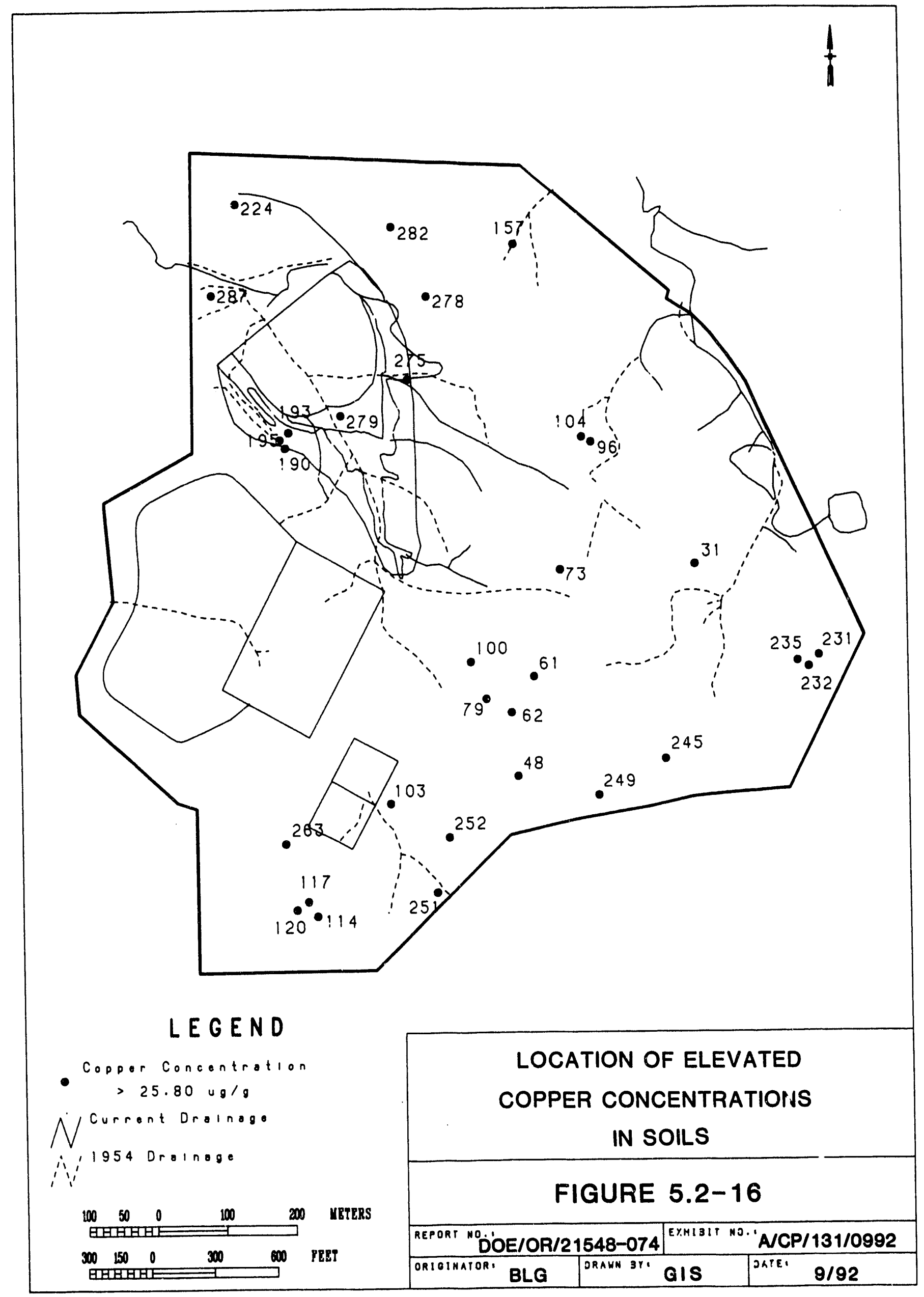




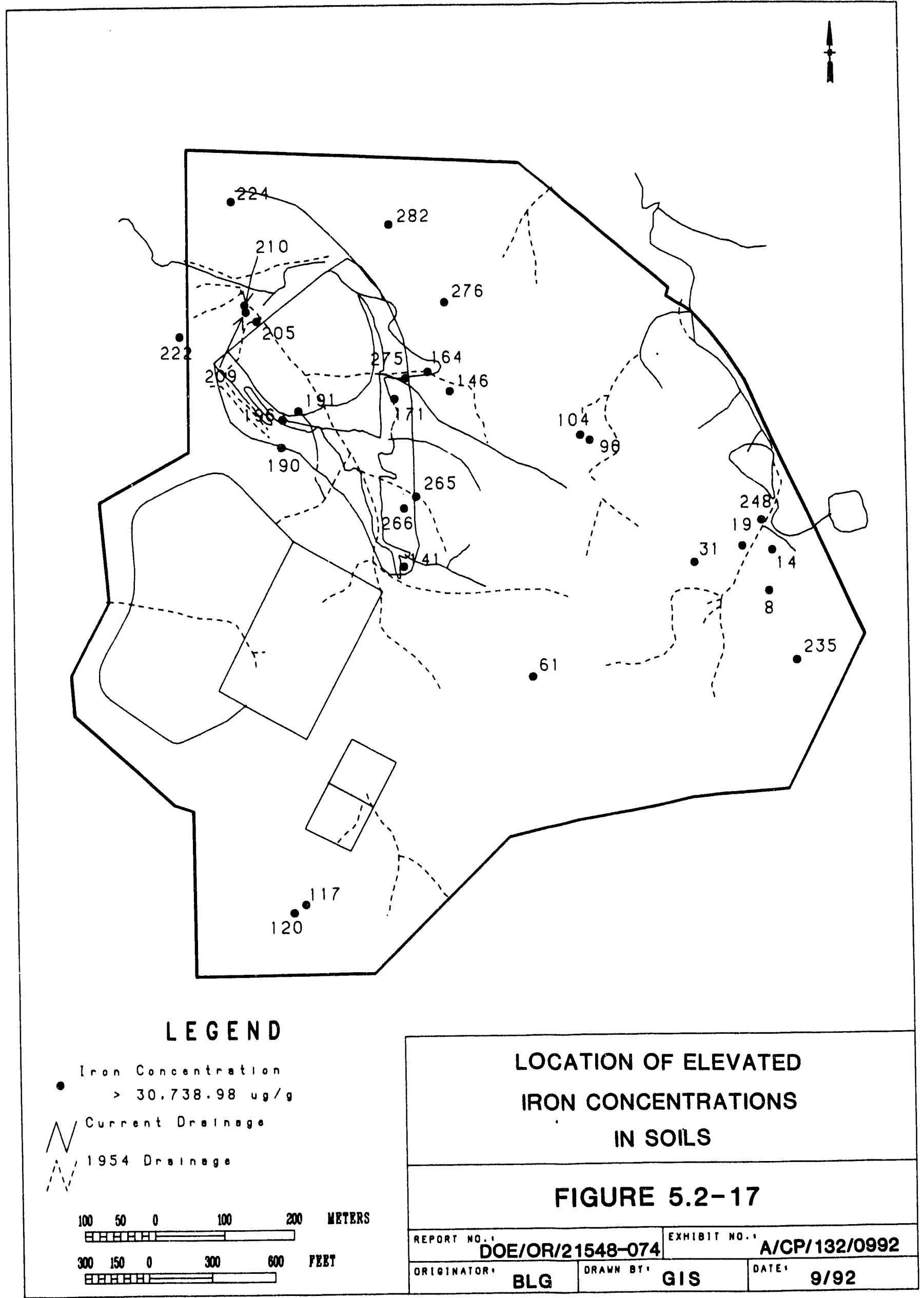




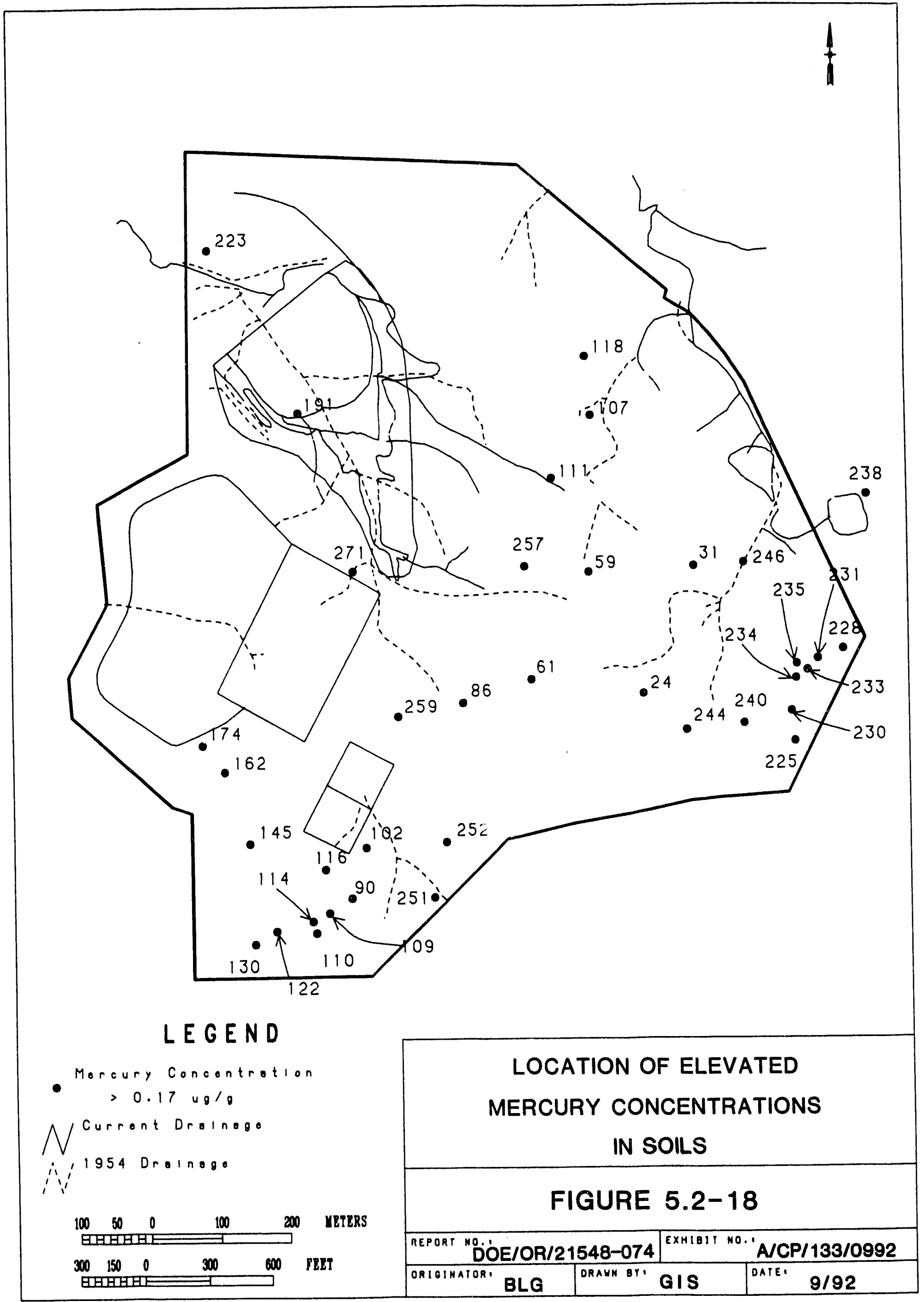




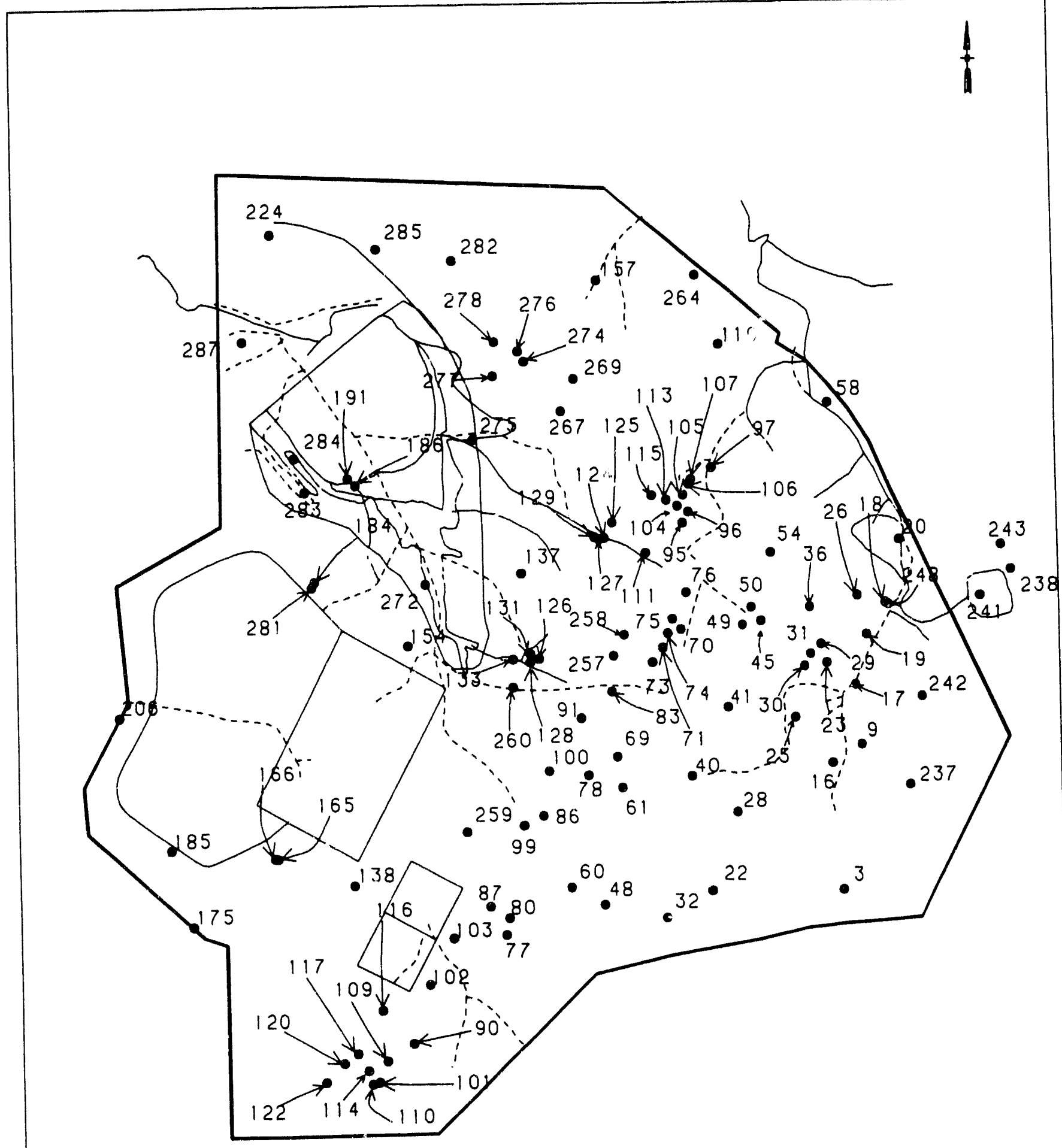

\section{LEGEND}

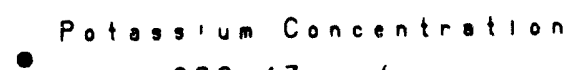

$>982.43 \cup g / 0$

N Current Droln:go

$\therefore, 1954$ Droln:ge

i'í

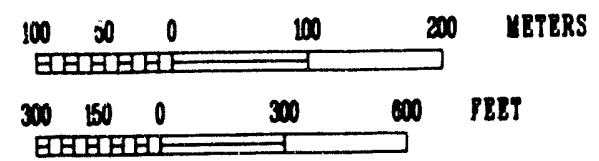

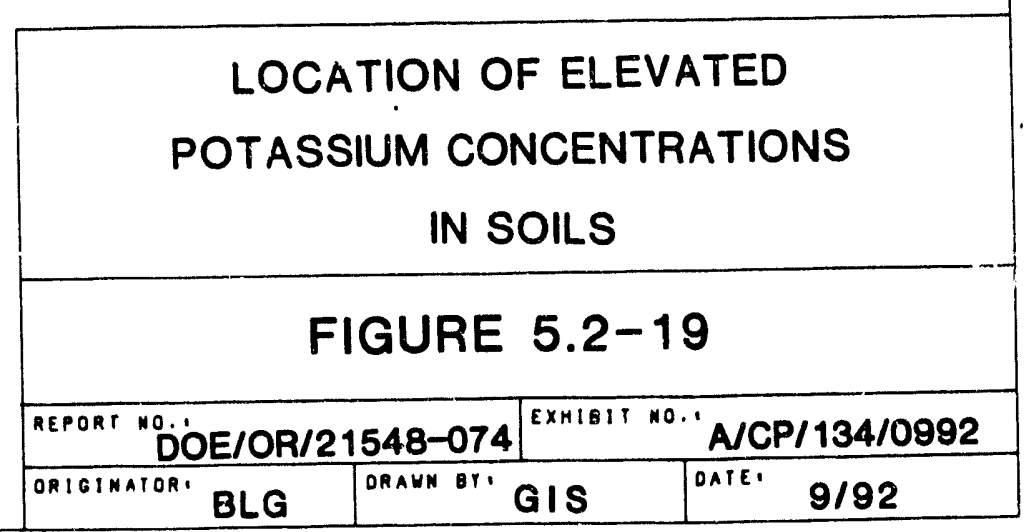




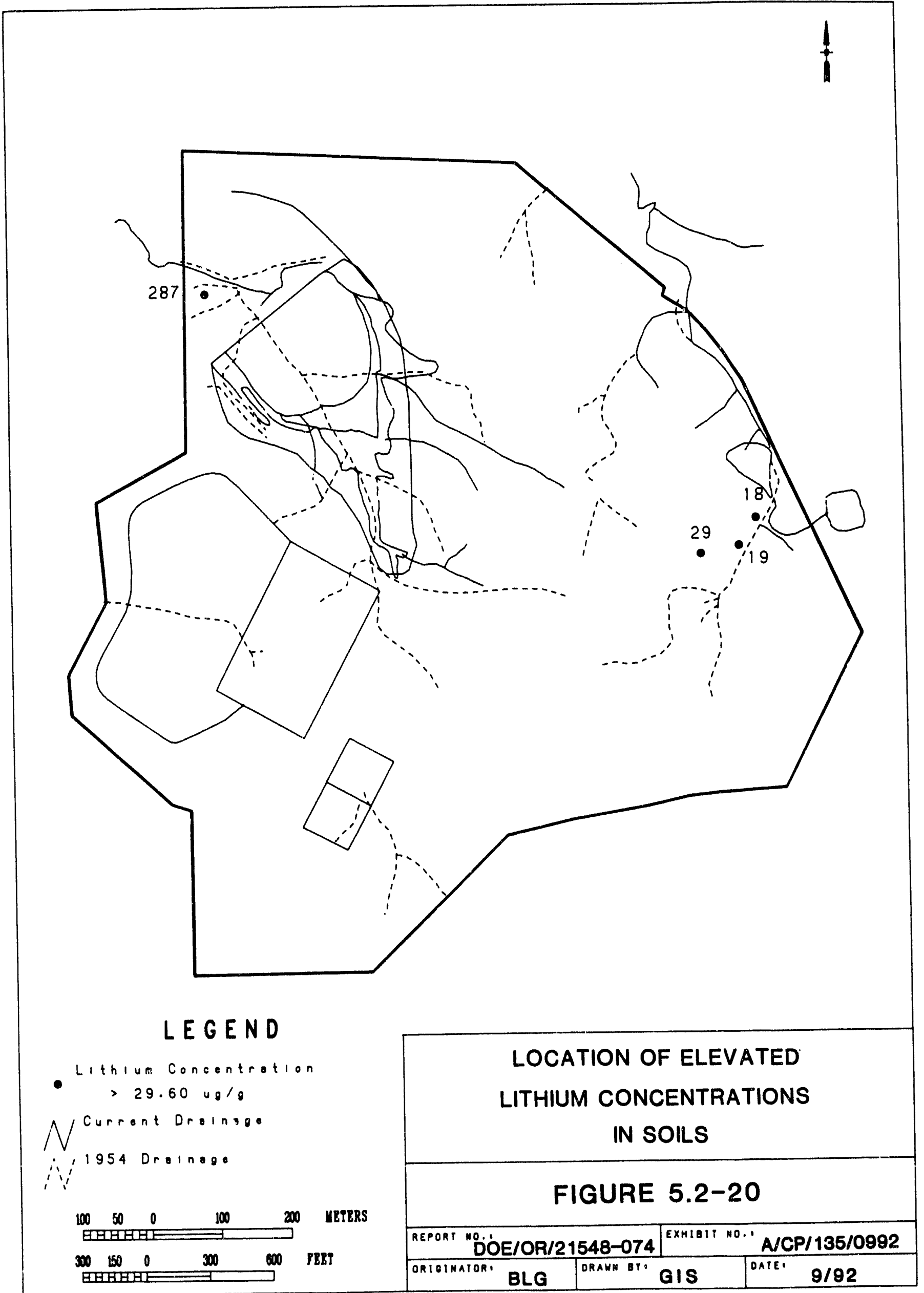




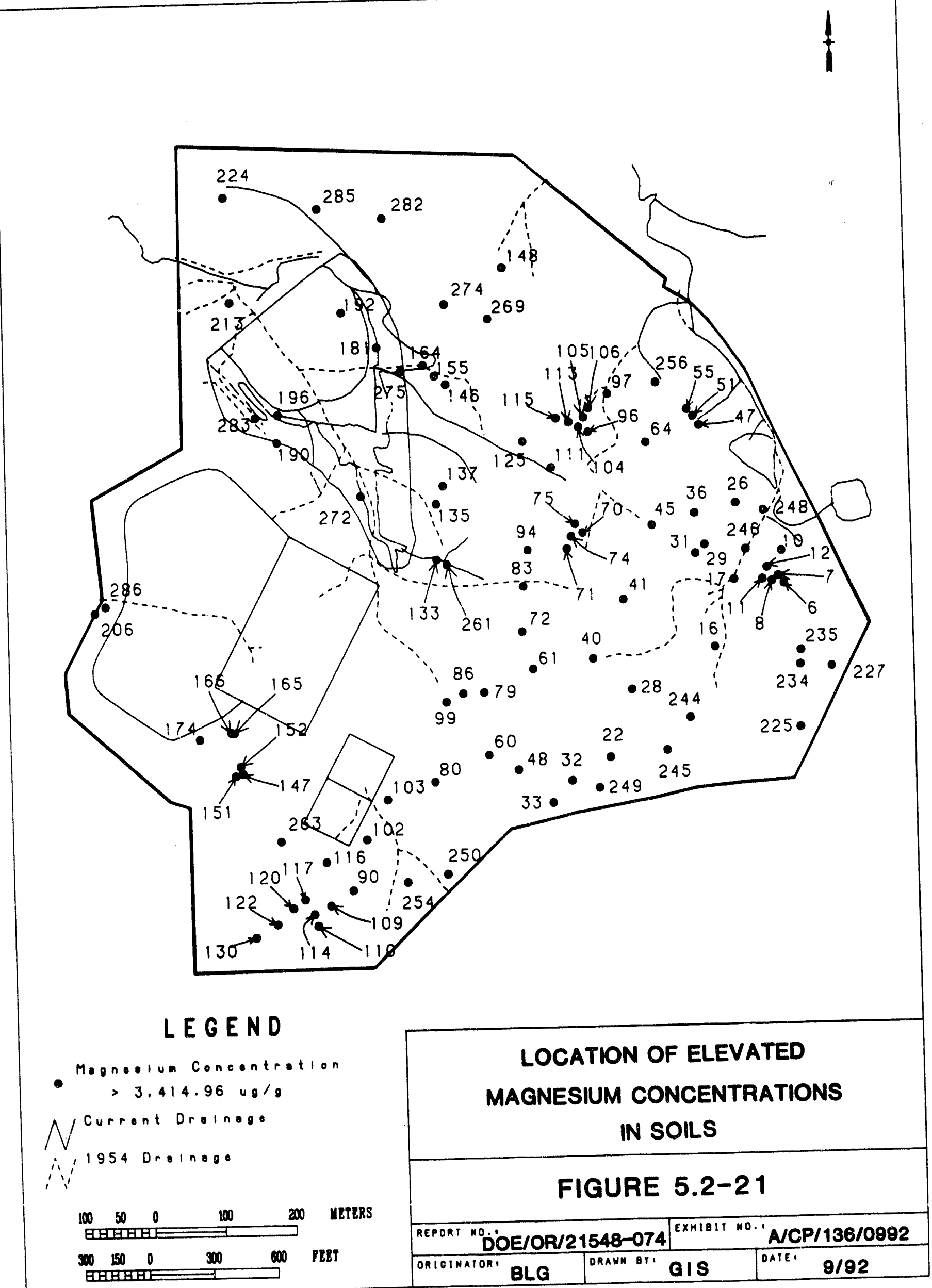




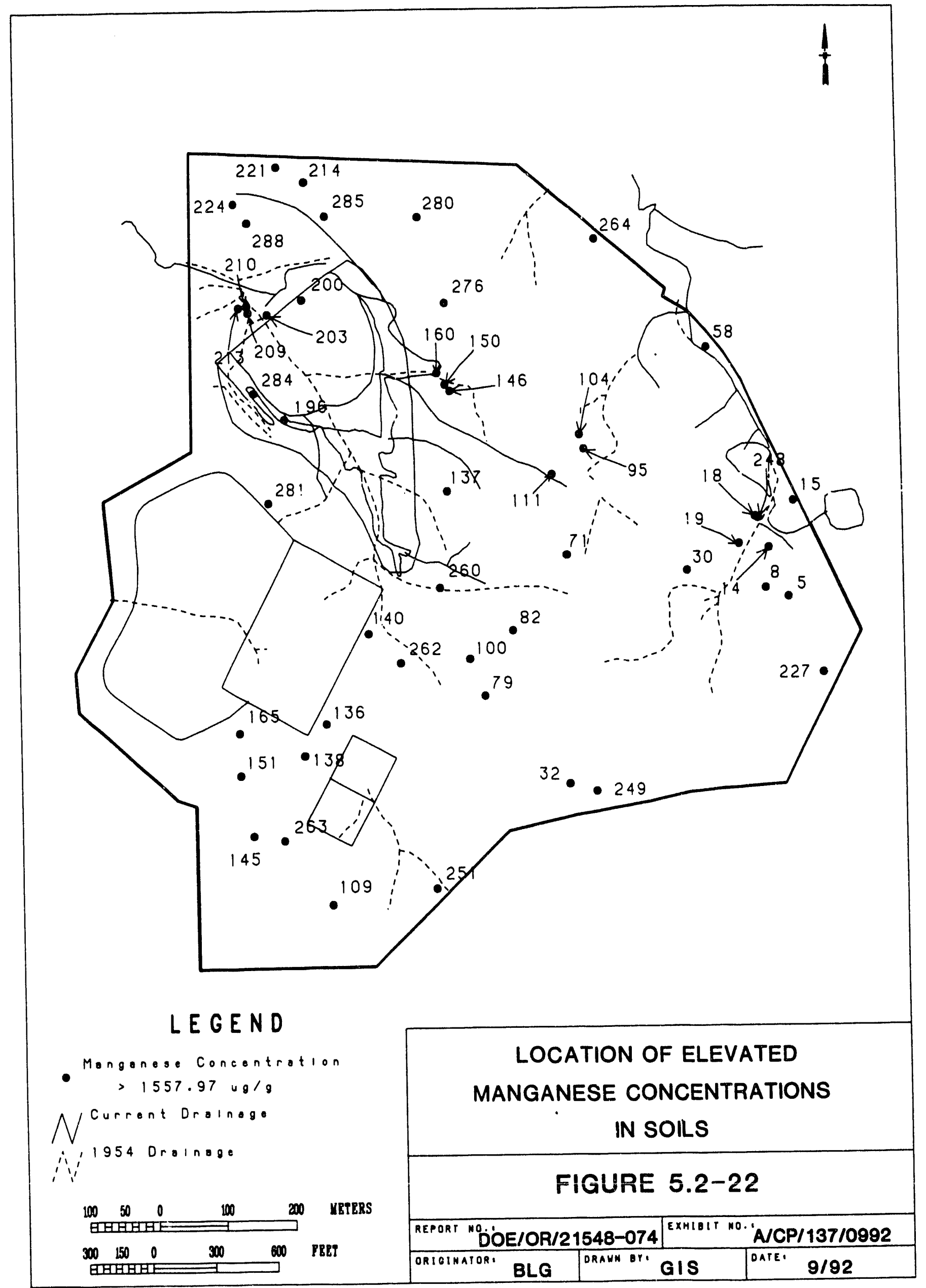




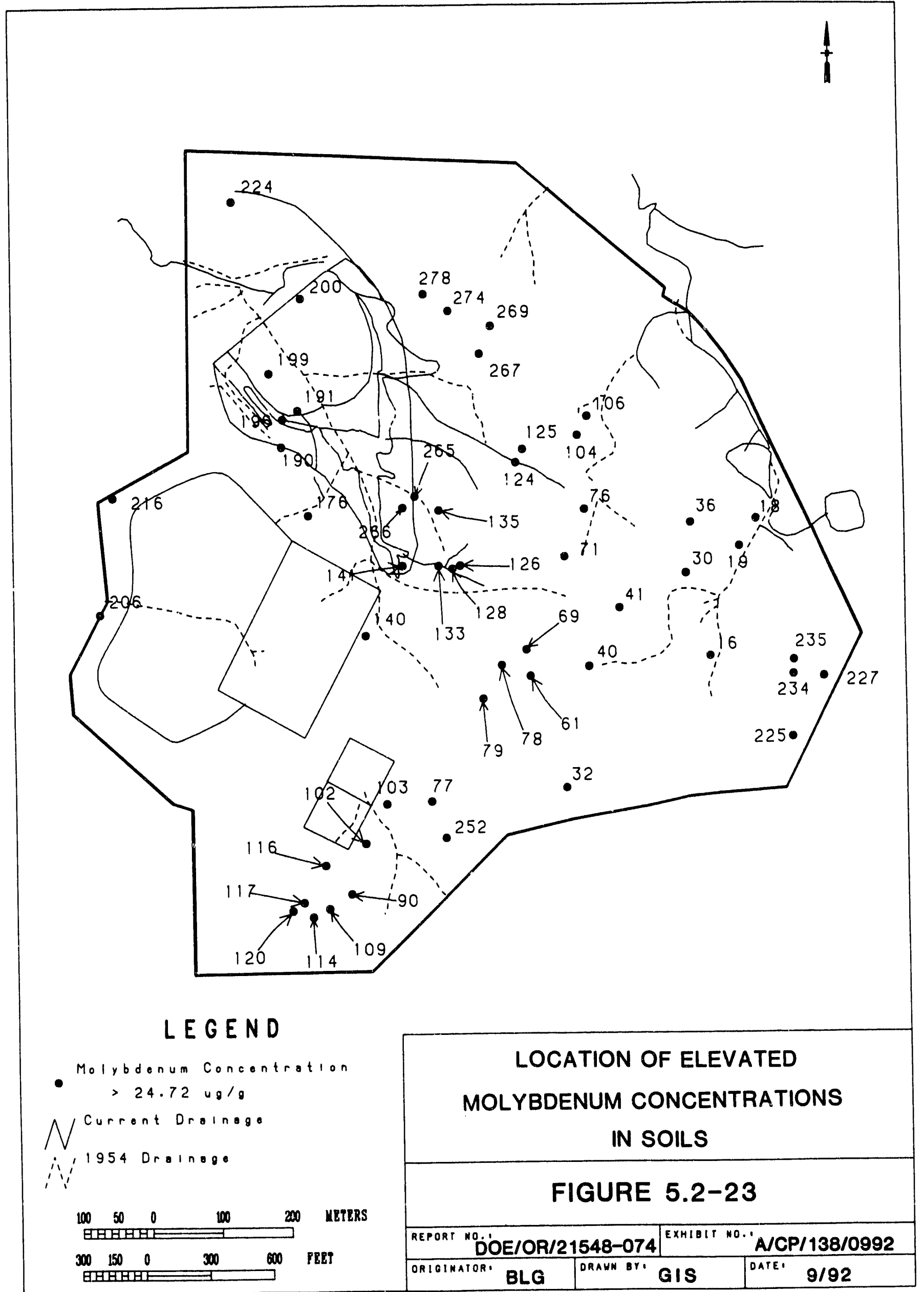




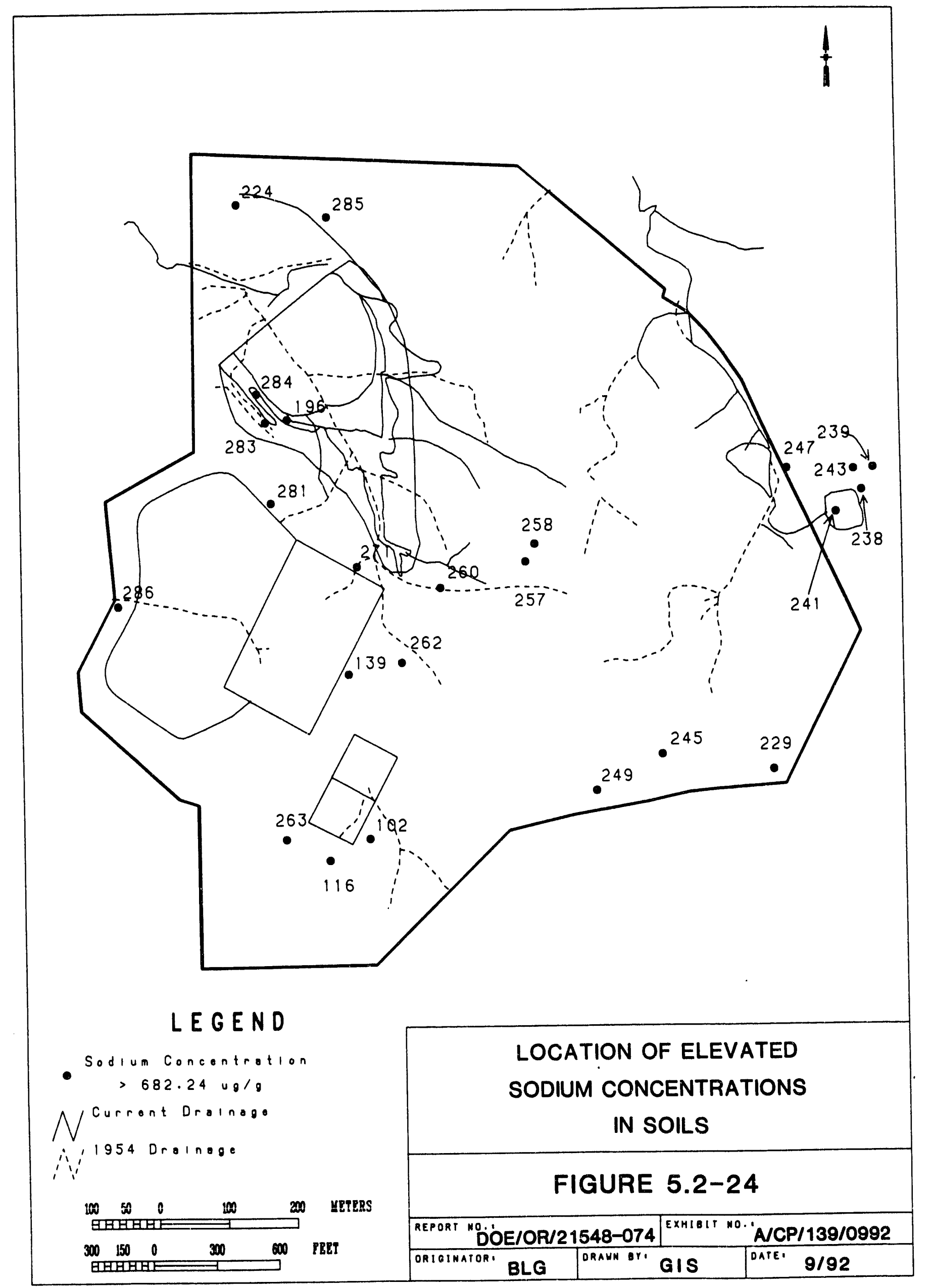




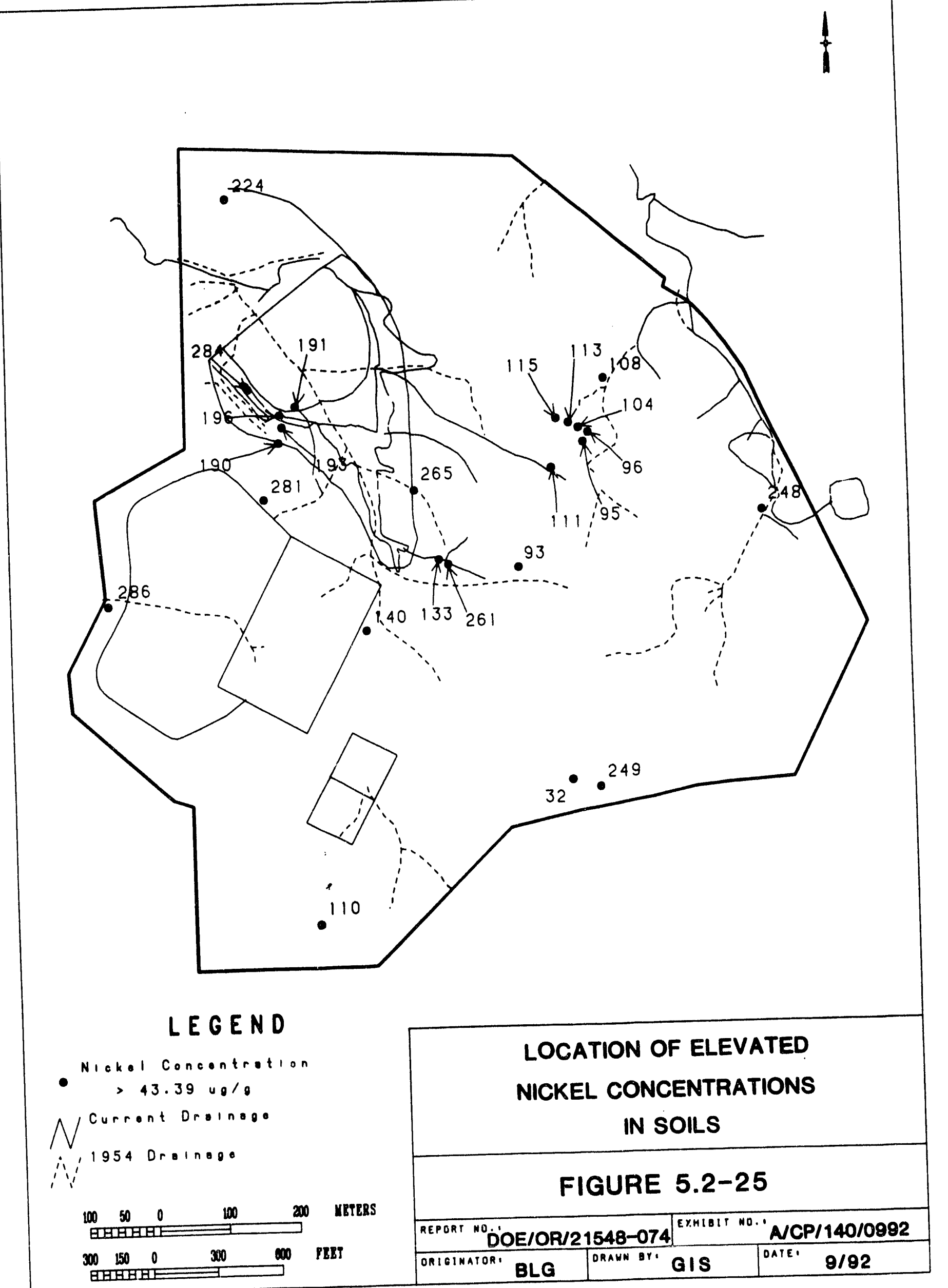




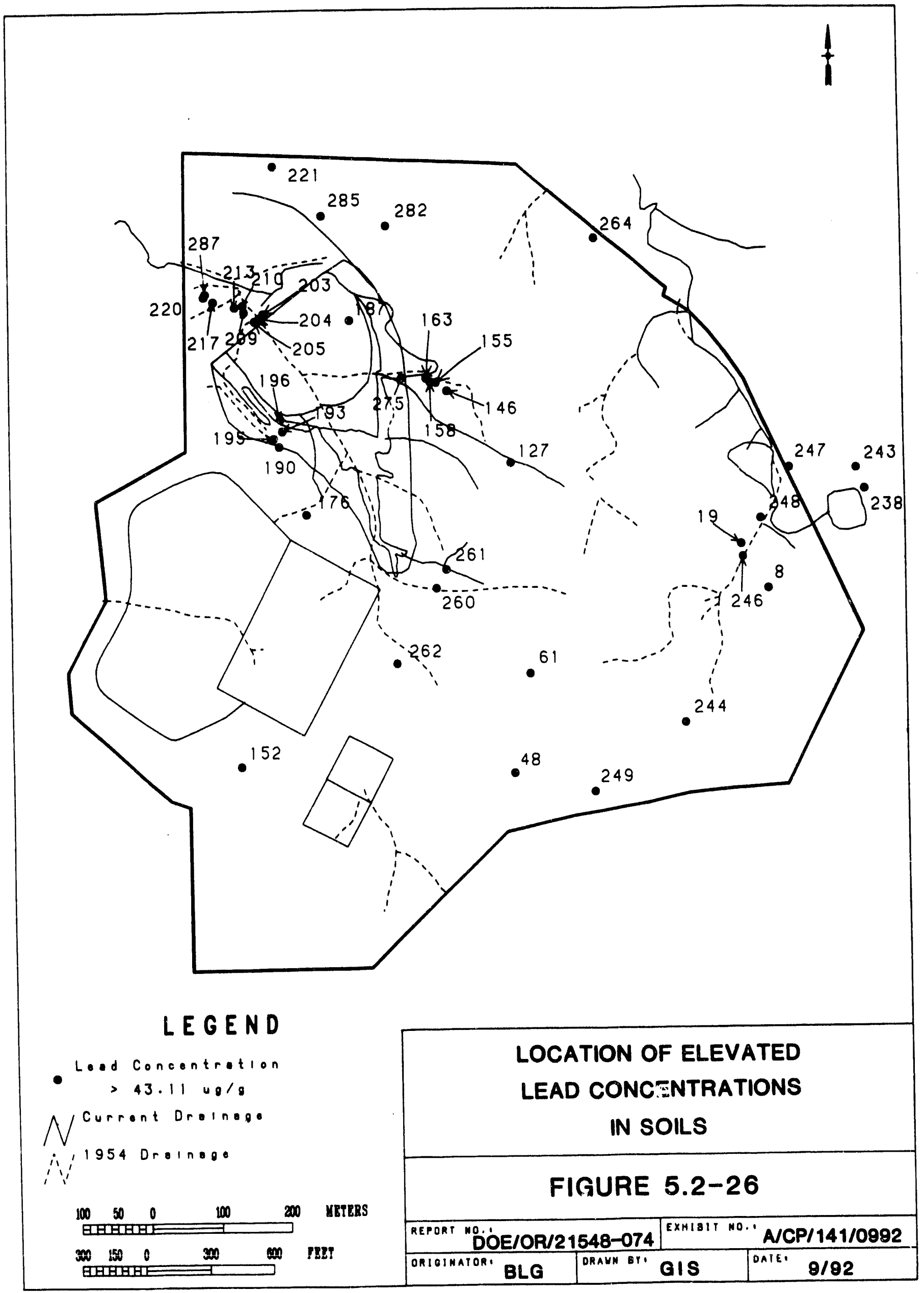




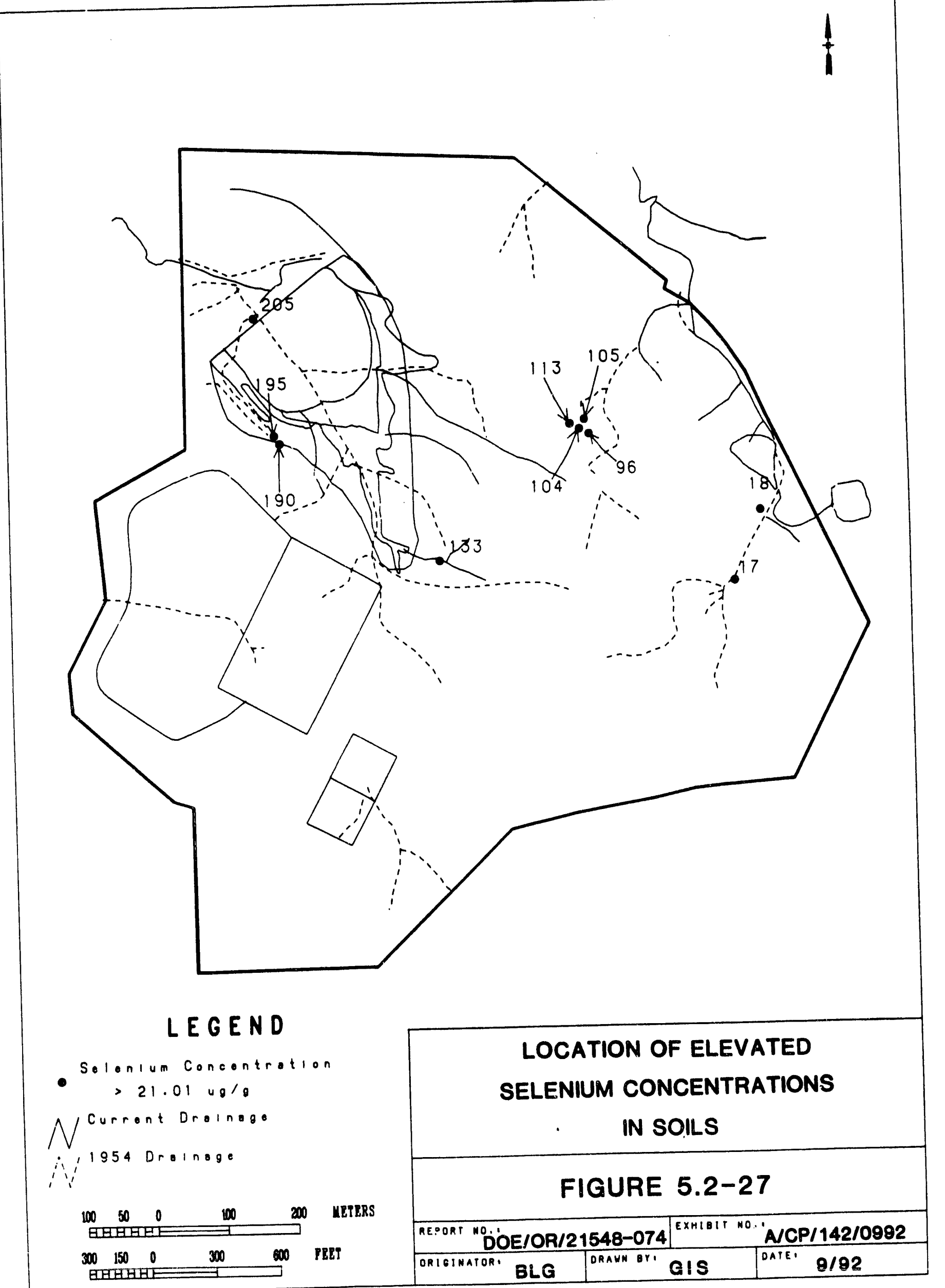




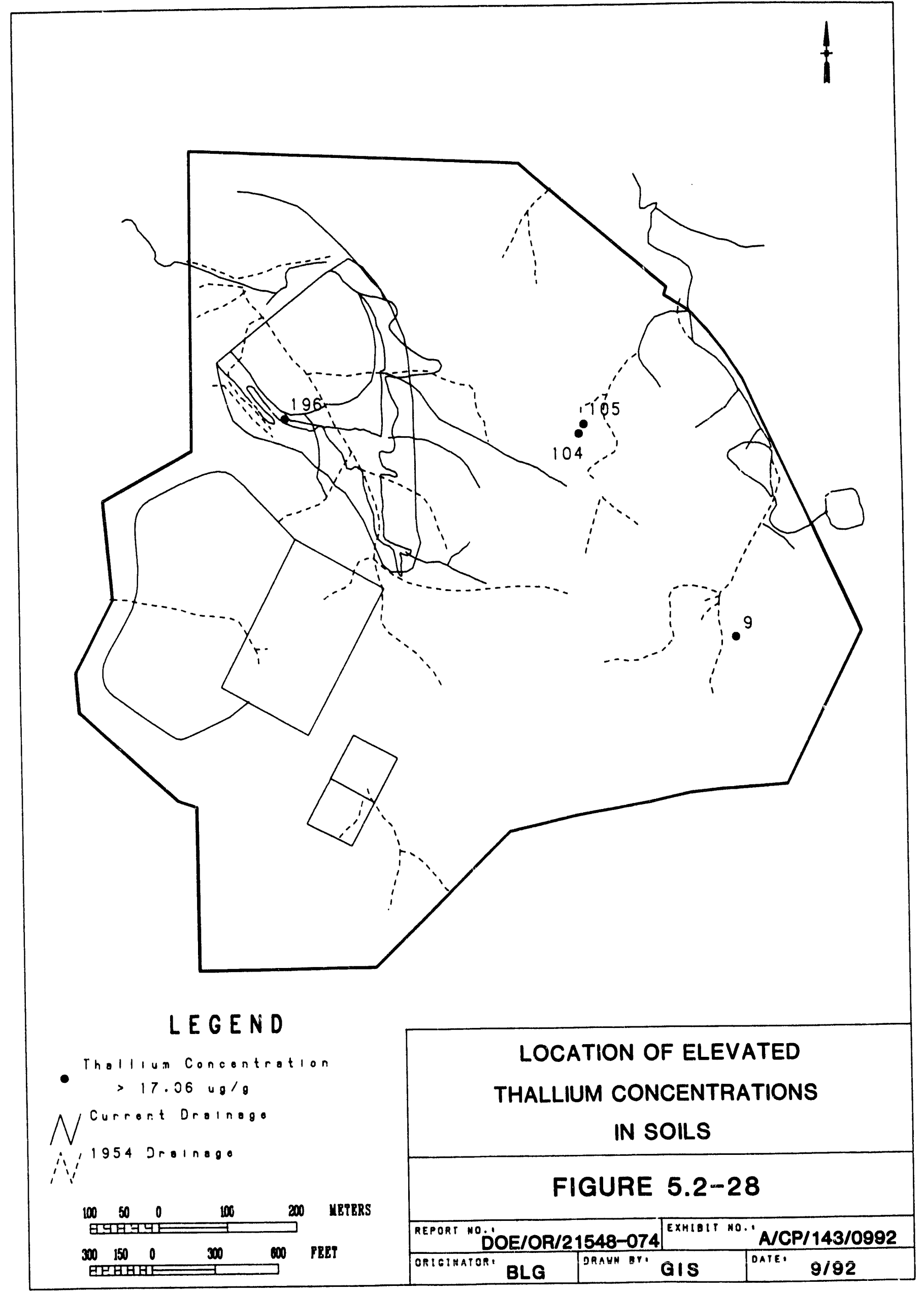




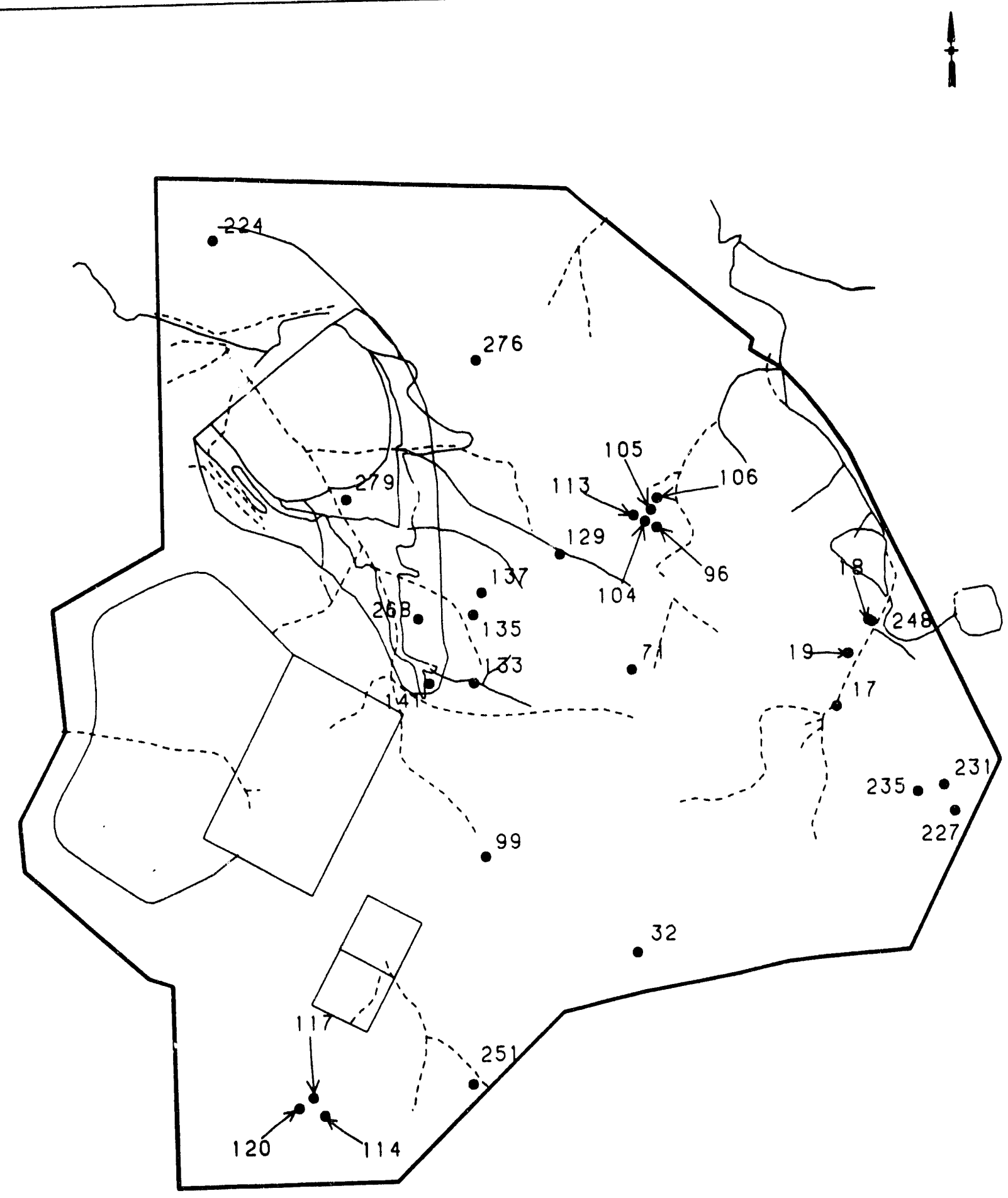

LE GEN D

Vonediun Concentrotion

$>54.8940 / 0$

NCurront Drolnogo

$\therefore, 1954$ Drolnoge

i'

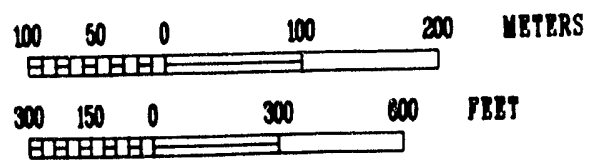

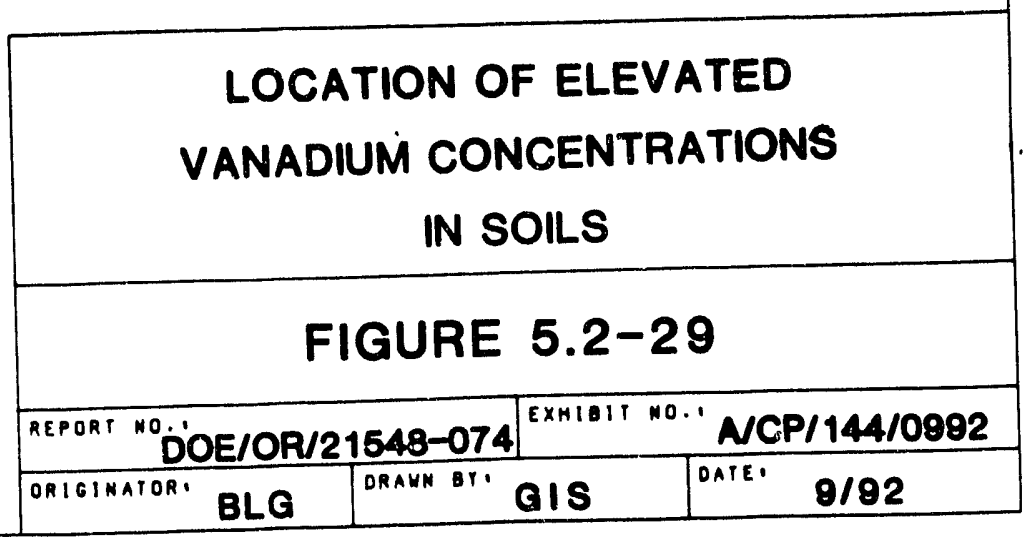




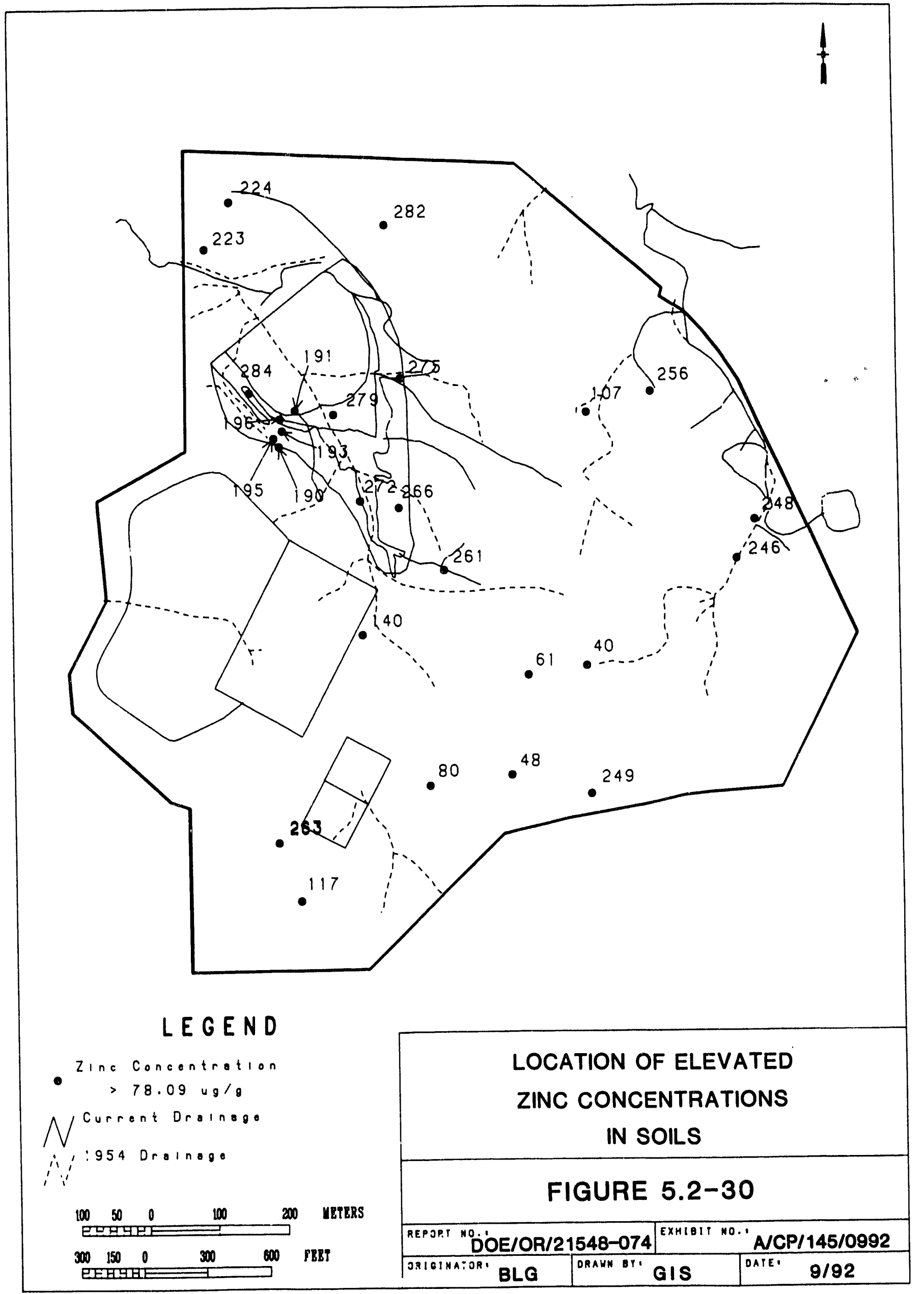




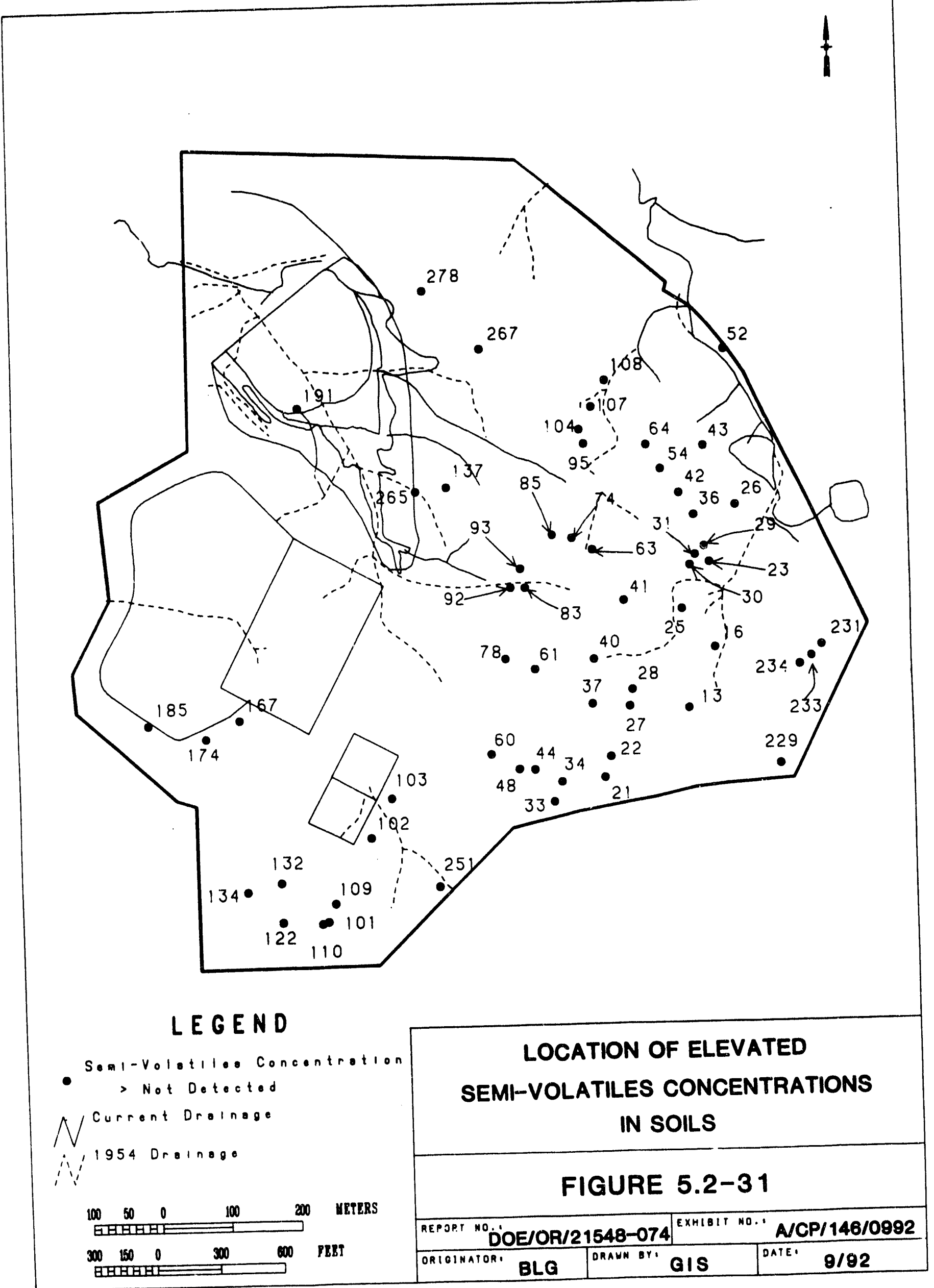




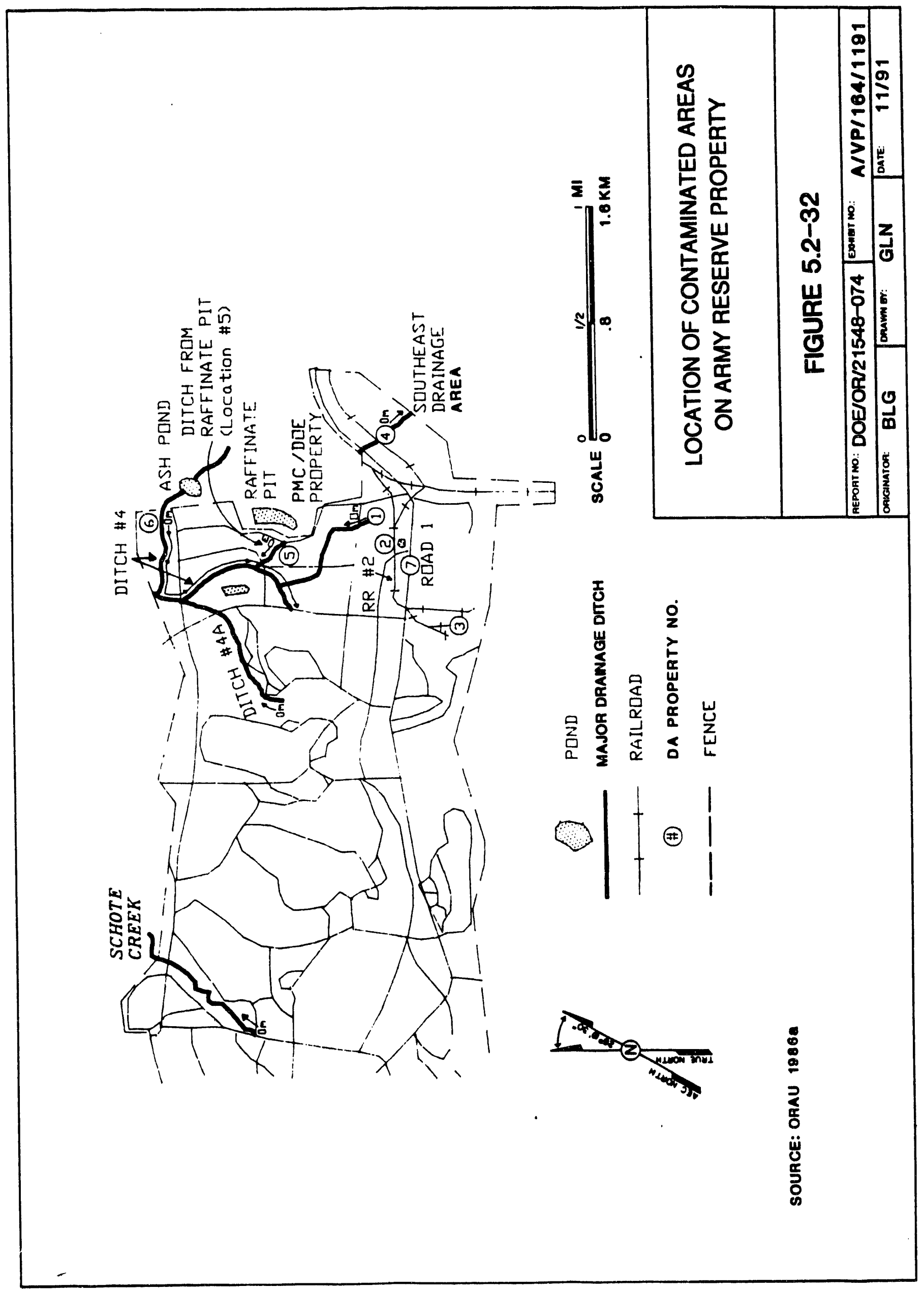




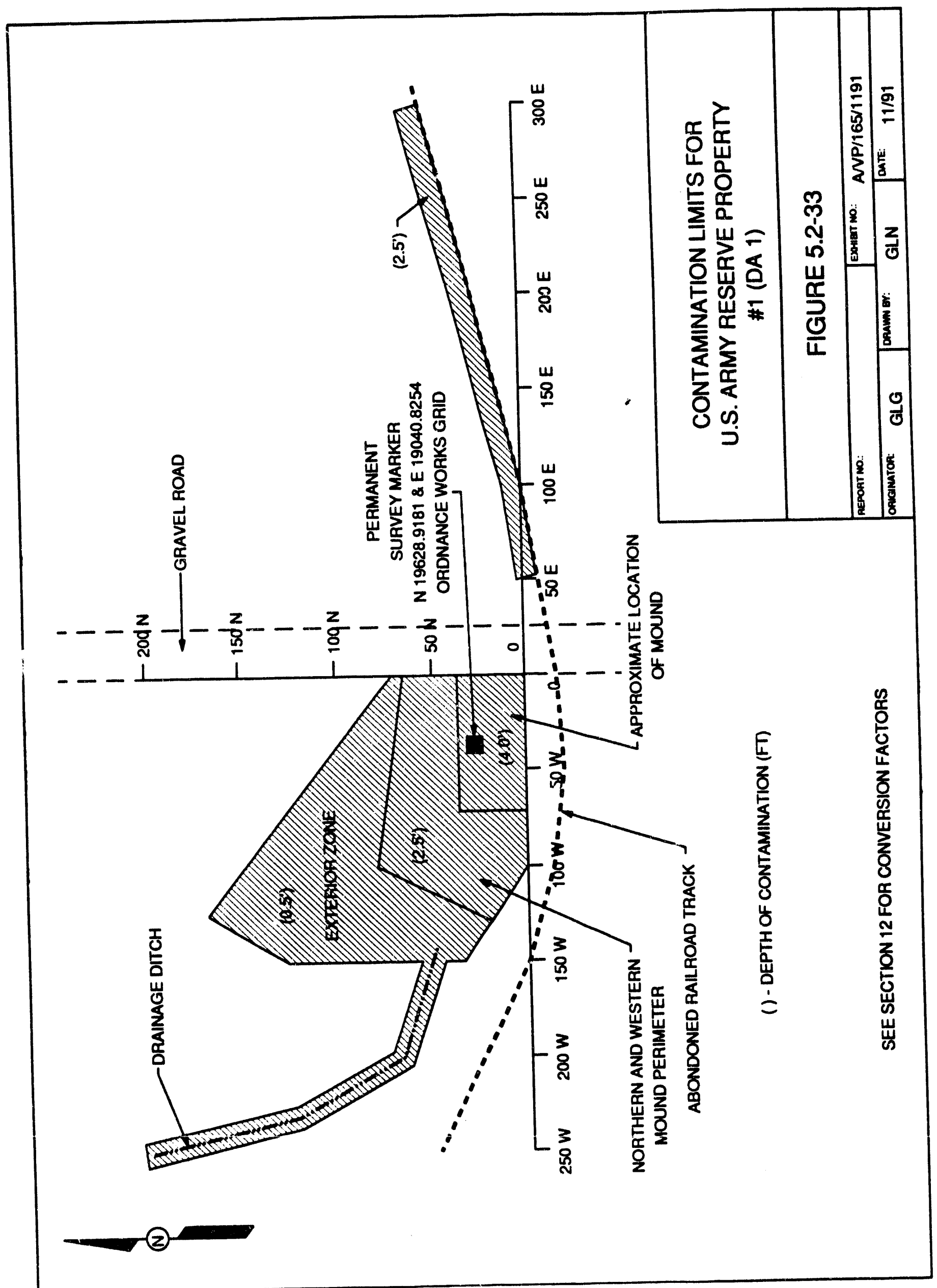




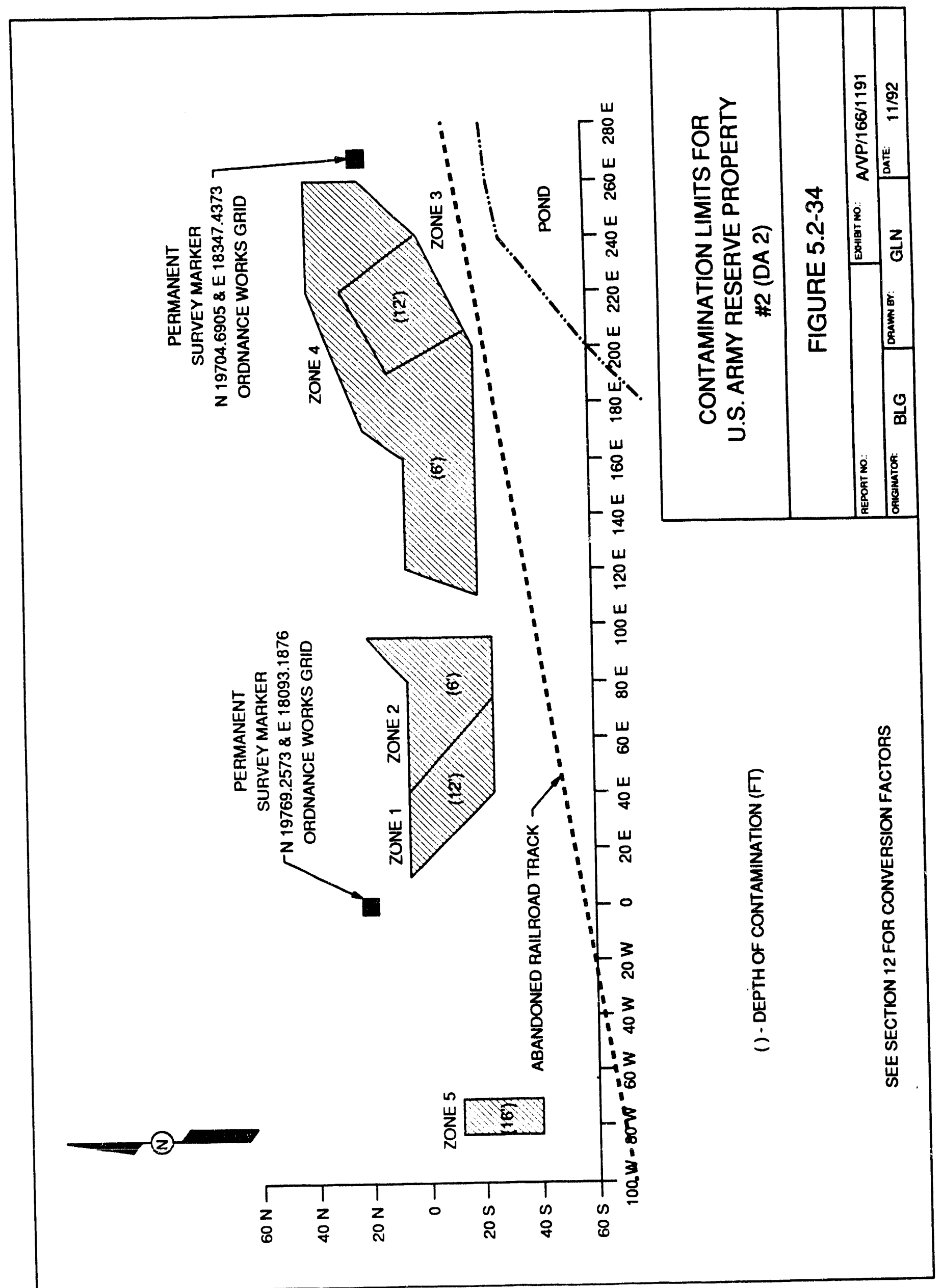



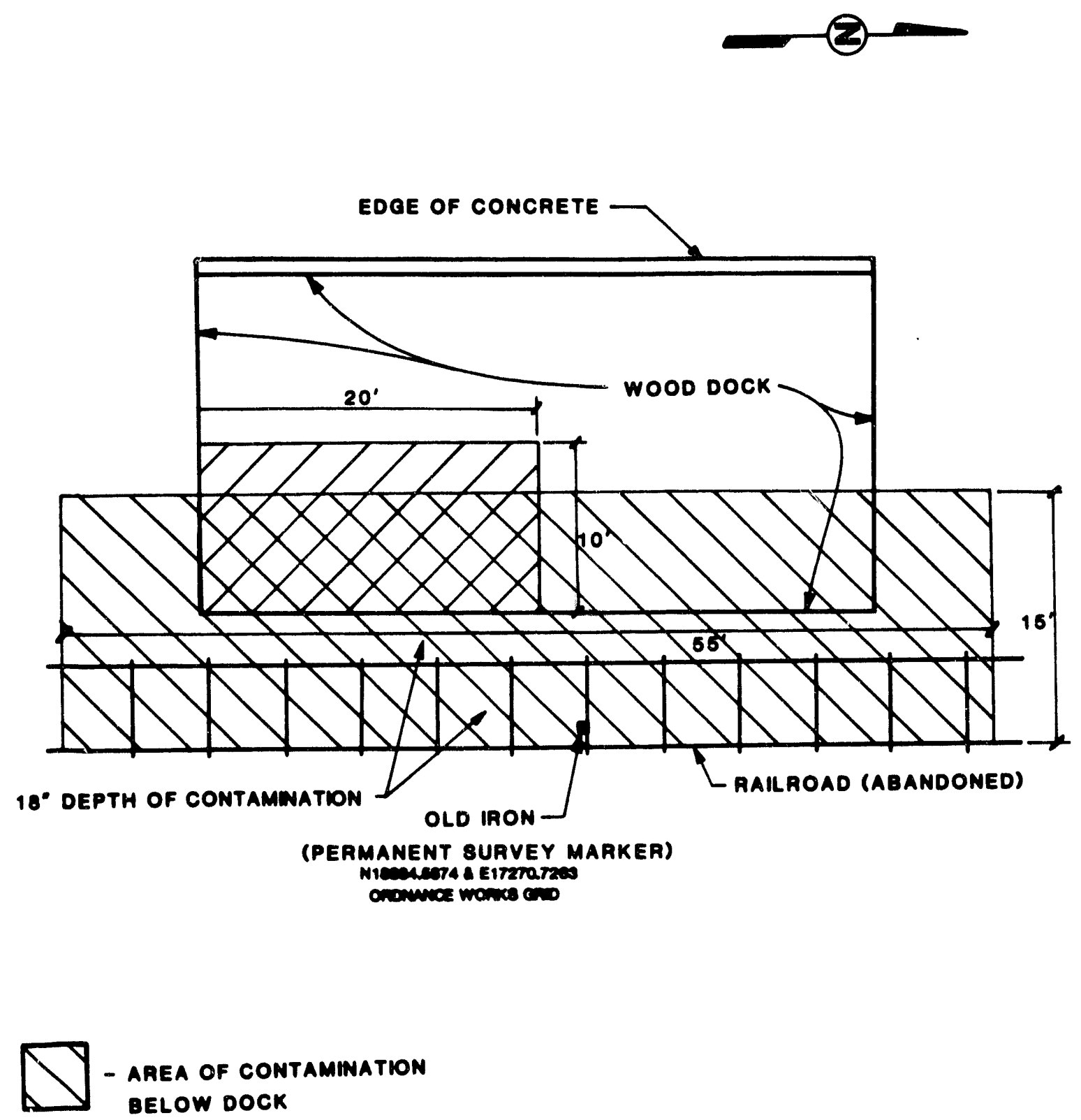

- apea of contamination

BELOW DOCK

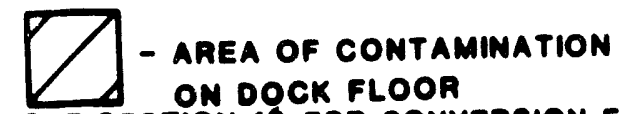

BEE BECTION 12 FOR CONVERBION FACTORS

CONTAMINATION LIMITS FOR

U.S ARMY VICINITY PROPERTY $\# 3$ (DA 3)

FIGURE 5.2-35

\begin{tabular}{|c|c|c|c|c|}
\hline AEPOAT NO & DOE/OI & $1548-074$ & EOHETT NO. & A/VP/ $167 / 1101$ \\
\hline ongematon & BLG & Doum or & GLN & $11 / 91$ \\
\hline
\end{tabular}




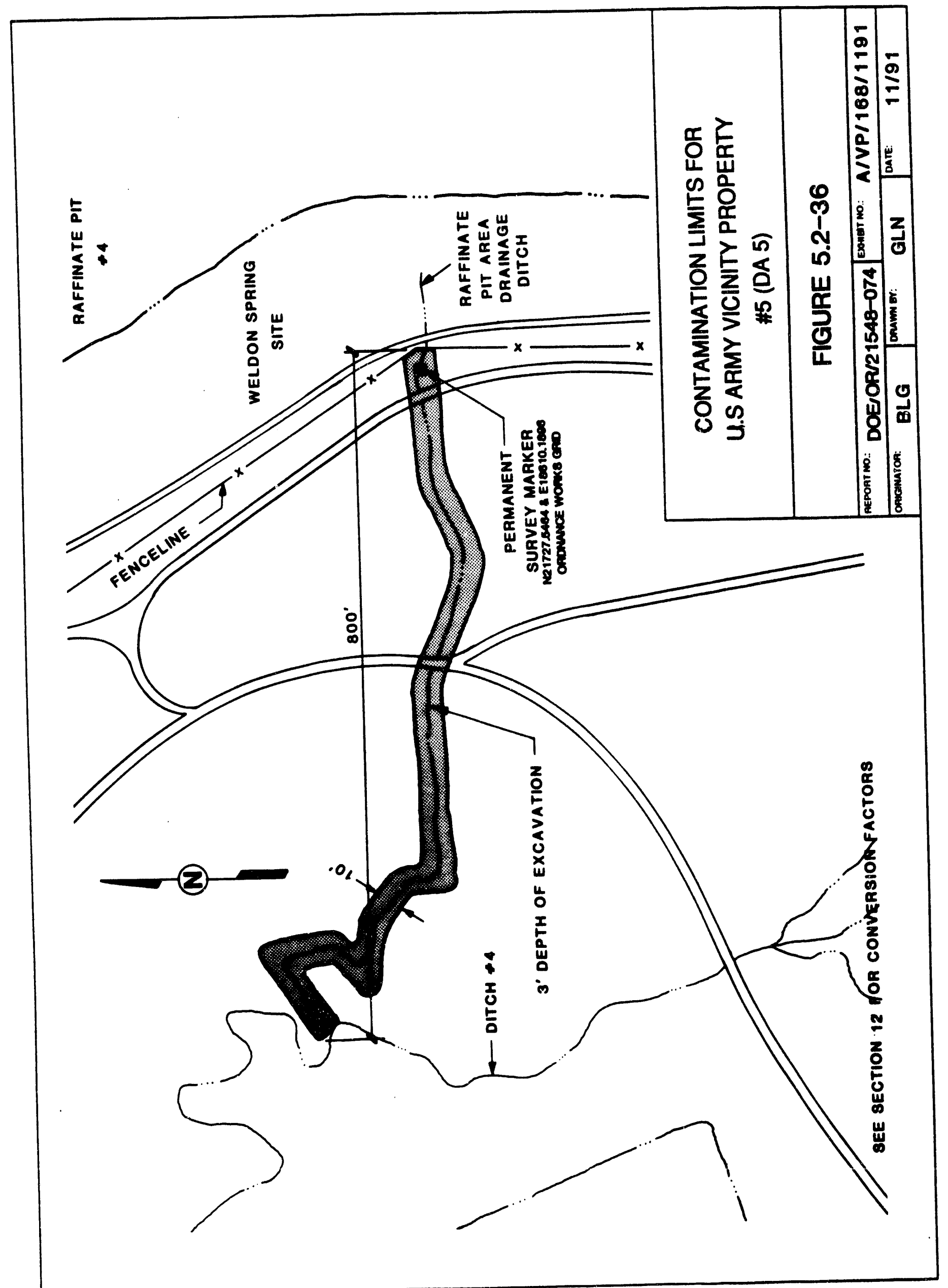




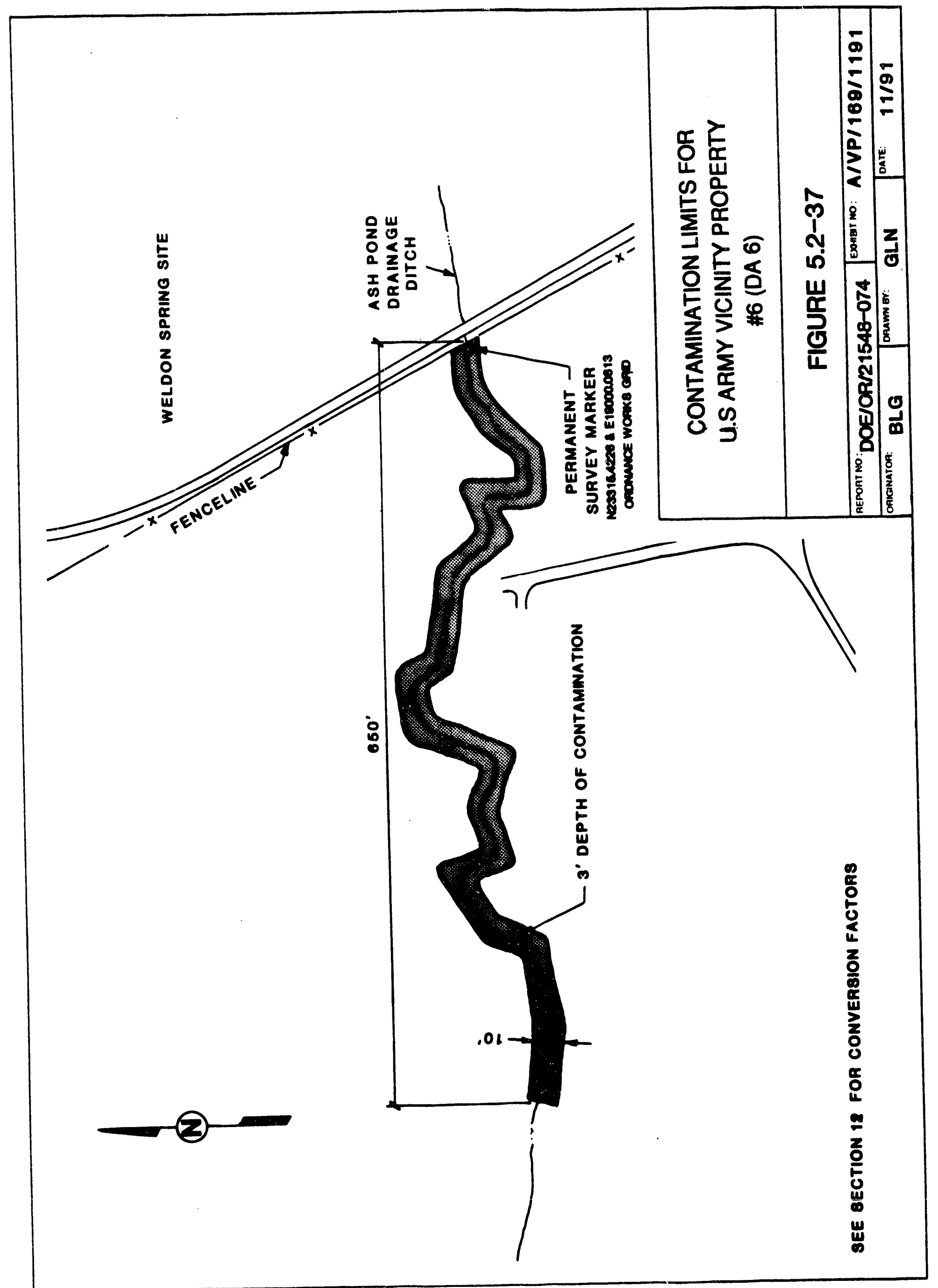




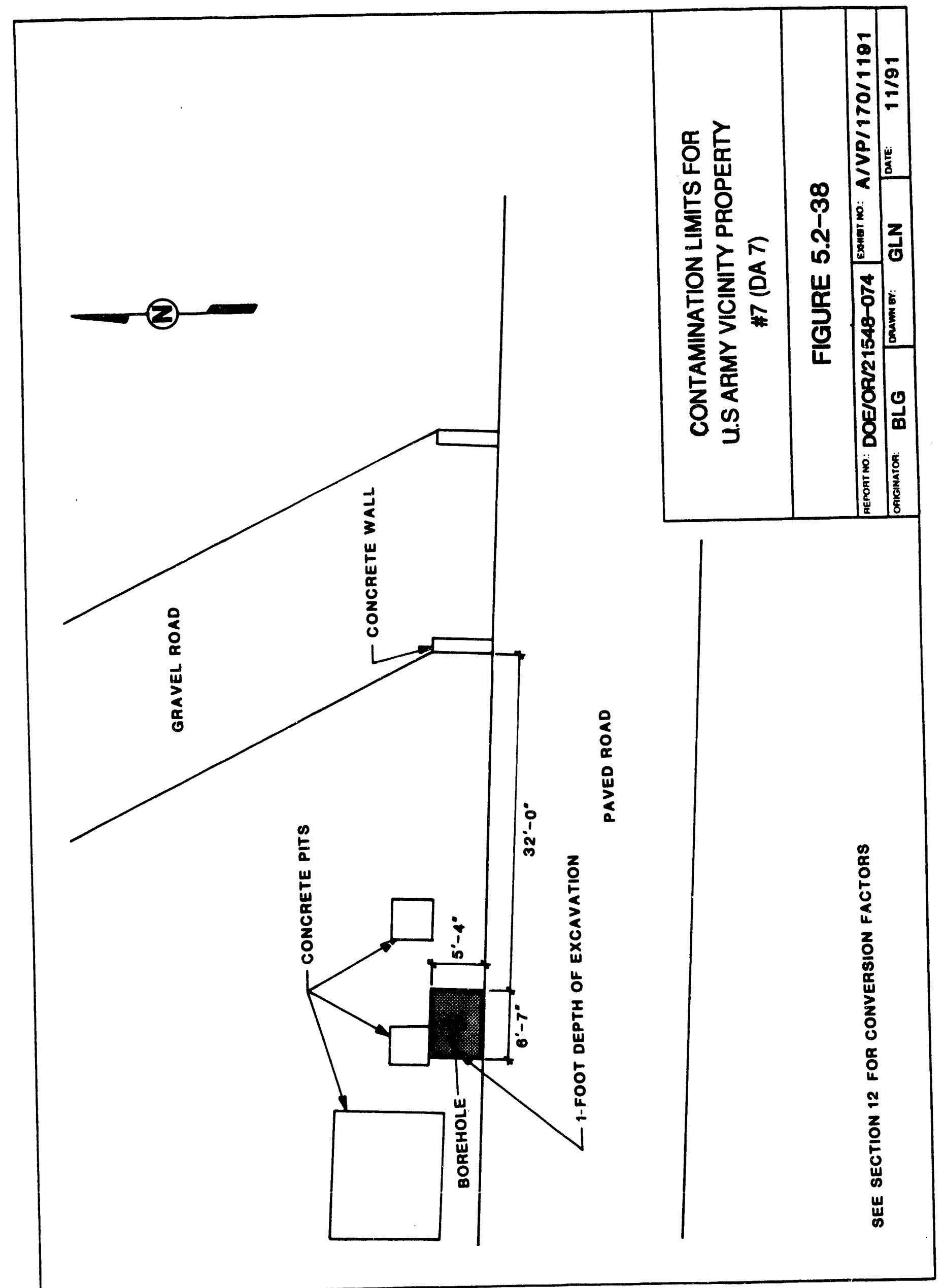




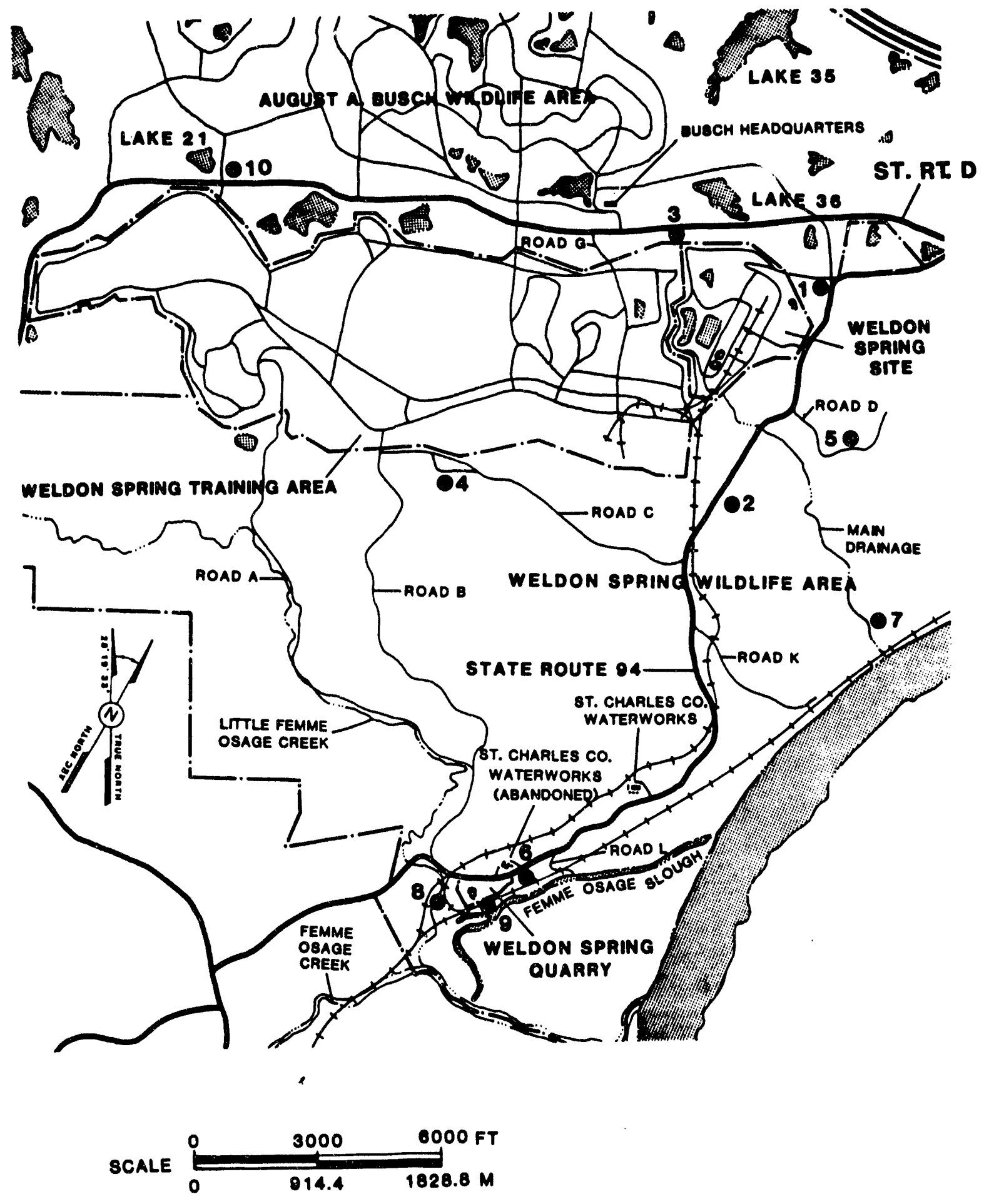

\section{LOCATIONS OF CONTAMINATION IDENTIFIED ON THE WELDON SPRING AND AUGUST BUSCH WILDLIFE AREAS}

FIGURE 5.2-39

\begin{tabular}{|c|c|c|c|}
\hline REPOAT NO & DOE/OR/21548-074 & EXOHBT NO. & A/VP/171/1181 \\
\hline OARGINATOA & Drauwn Br. & GLN & $11 / 91$ \\
\hline
\end{tabular}




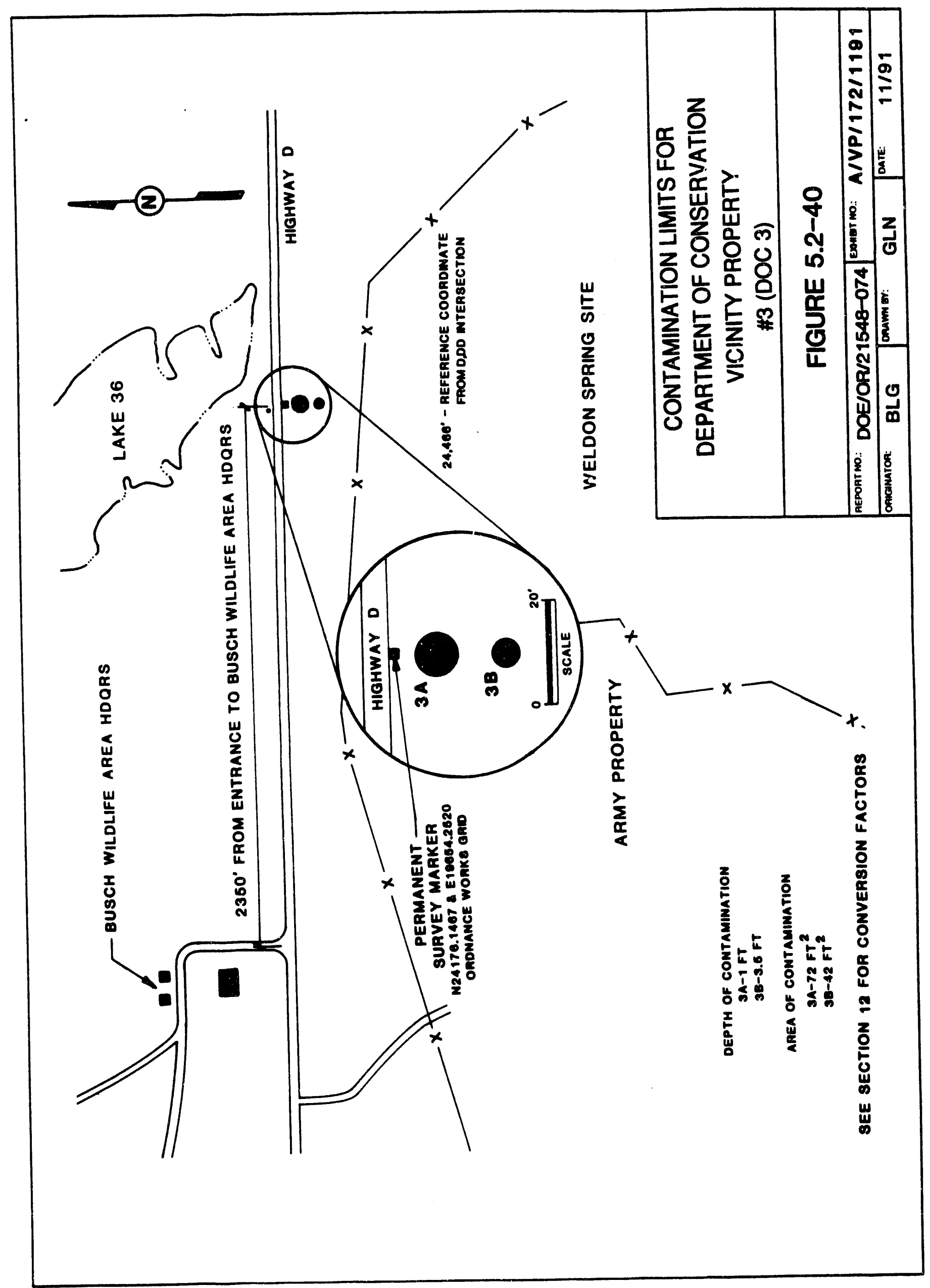



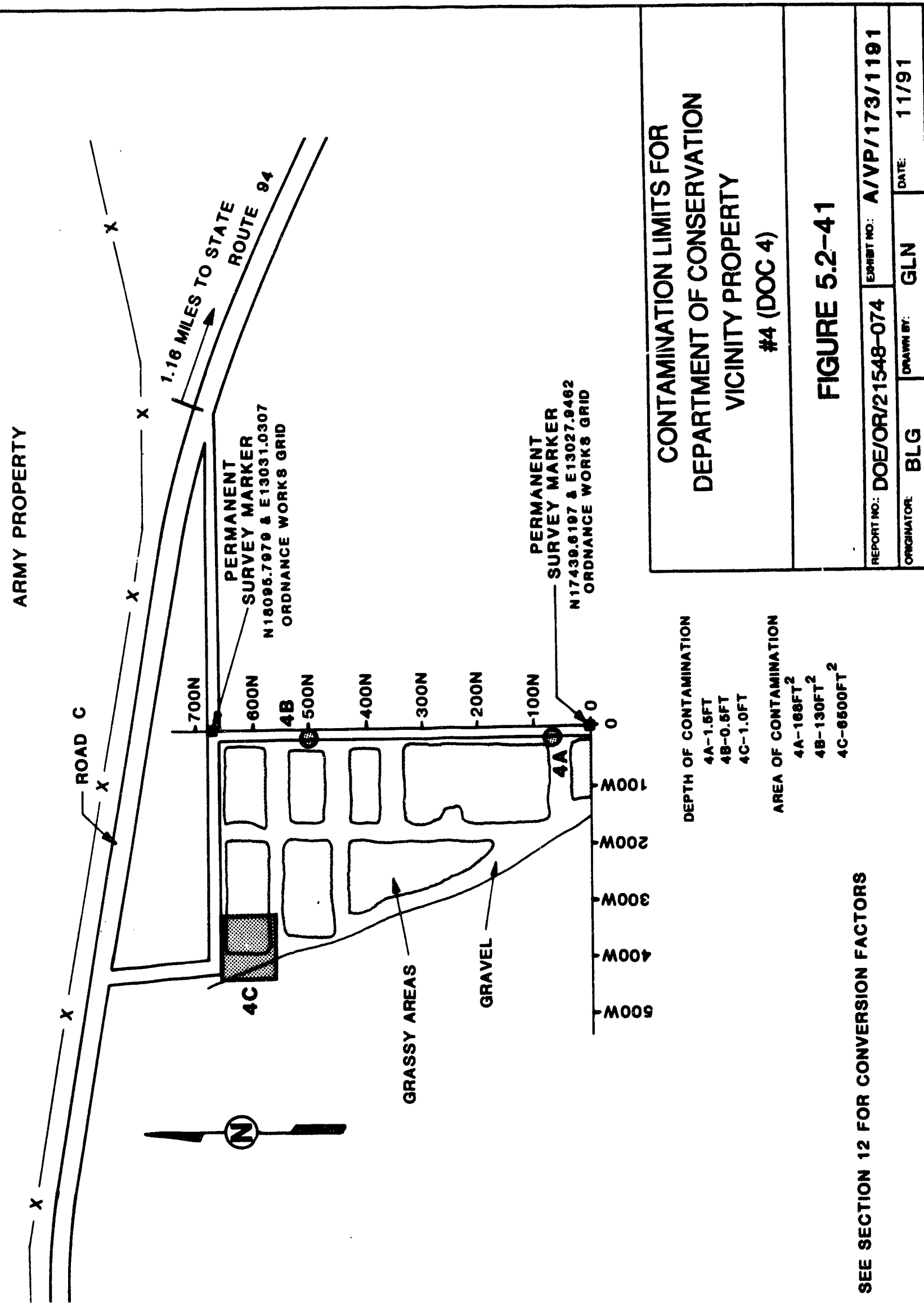

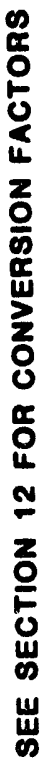




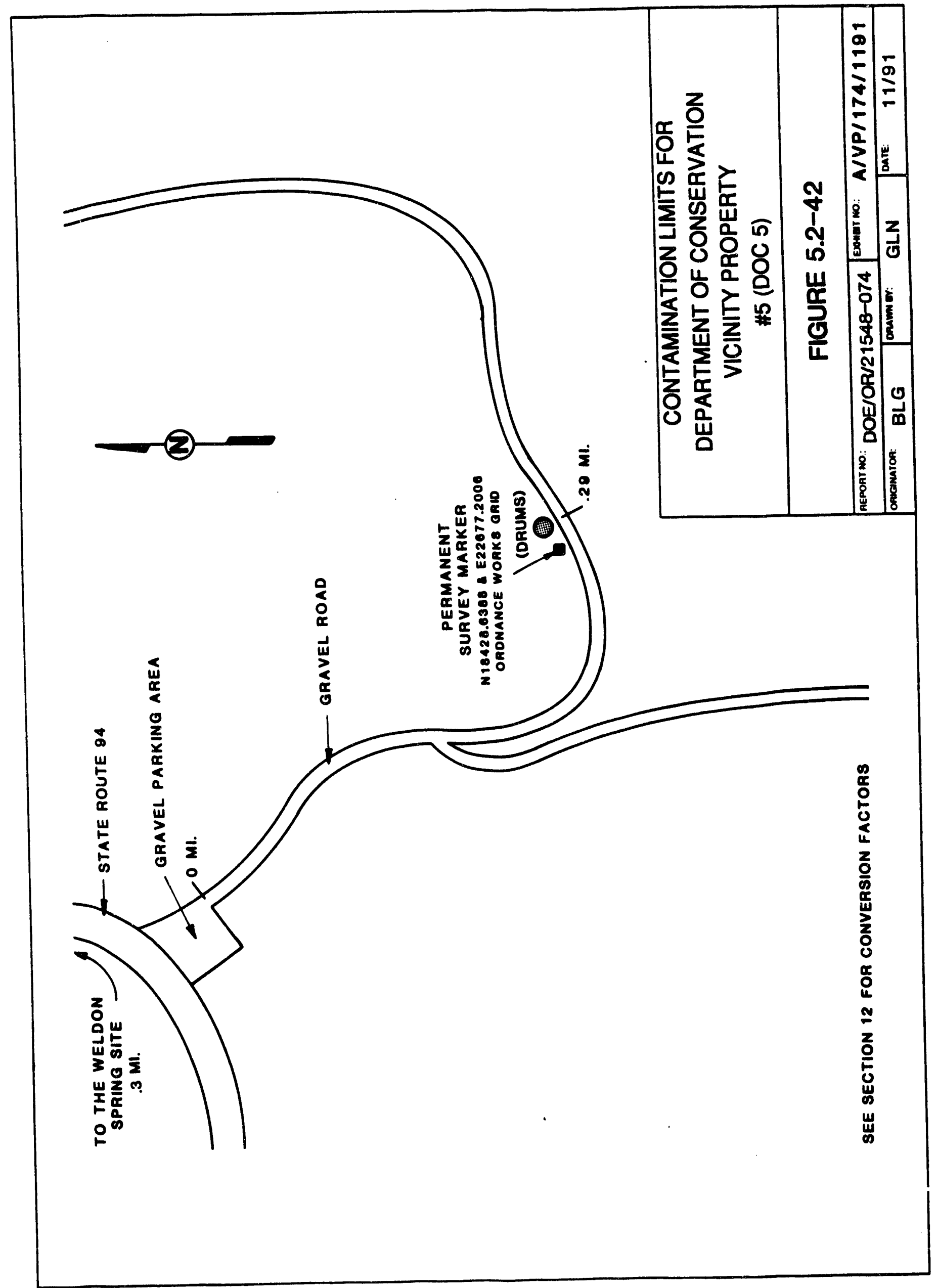




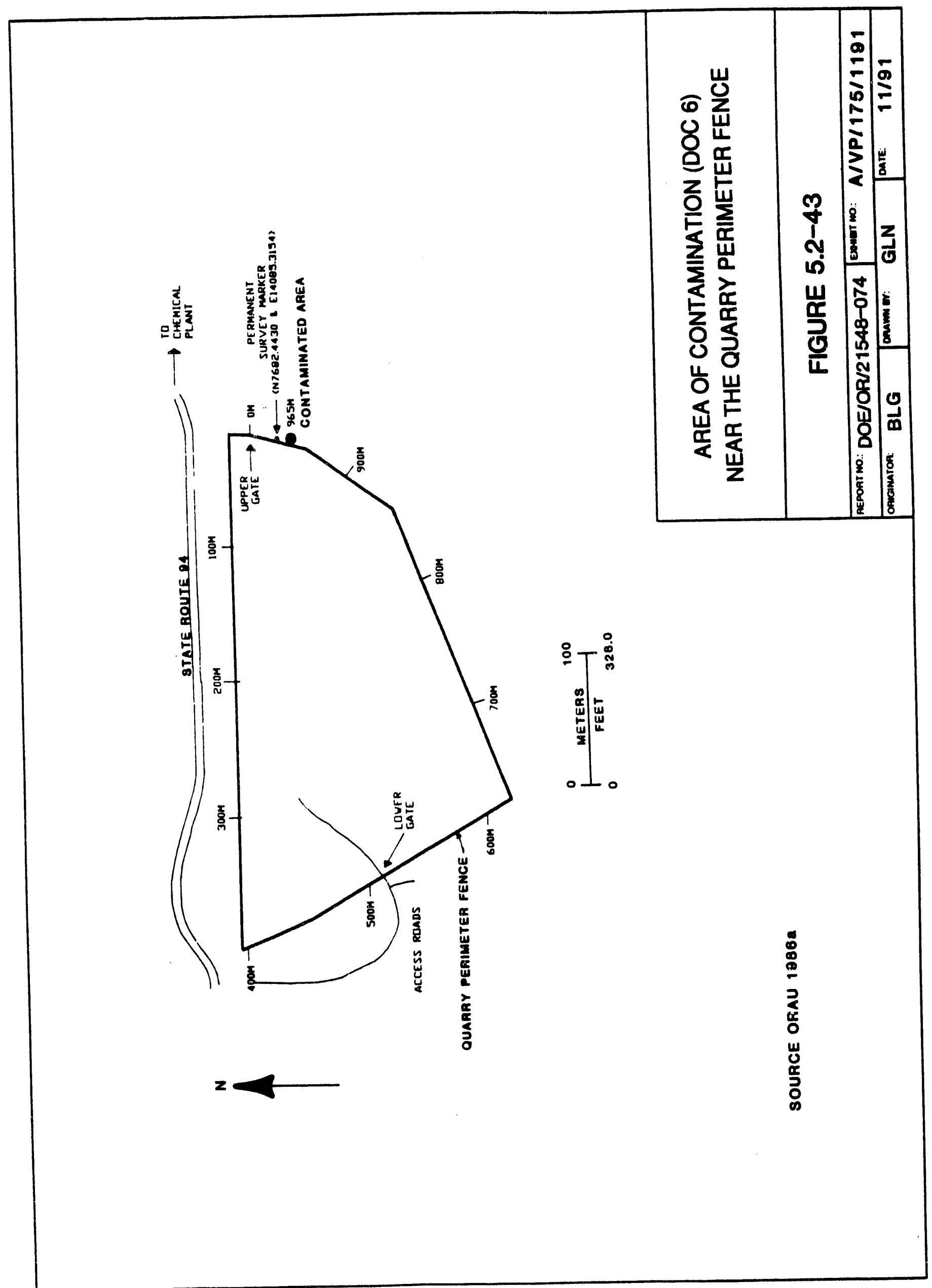




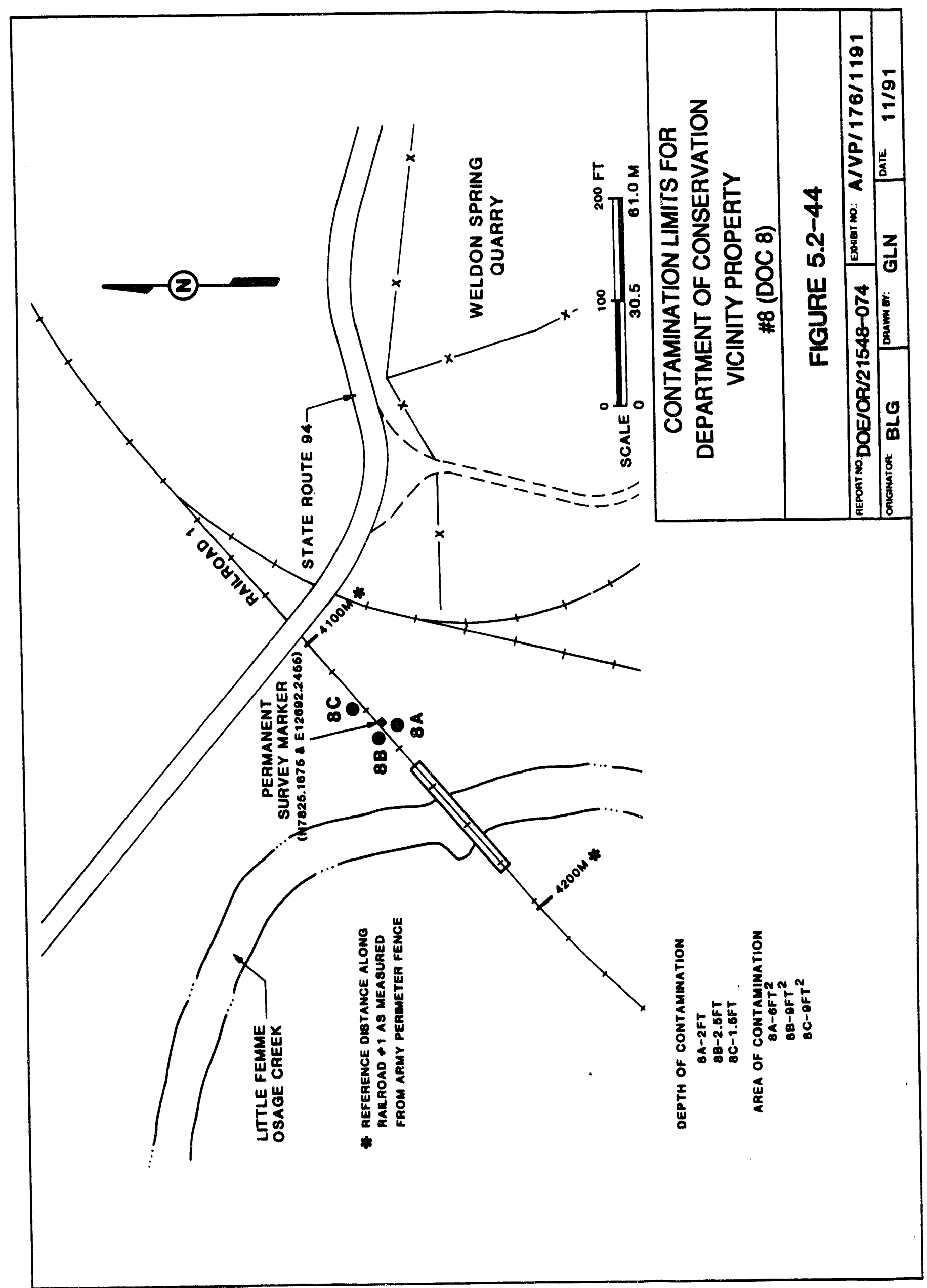




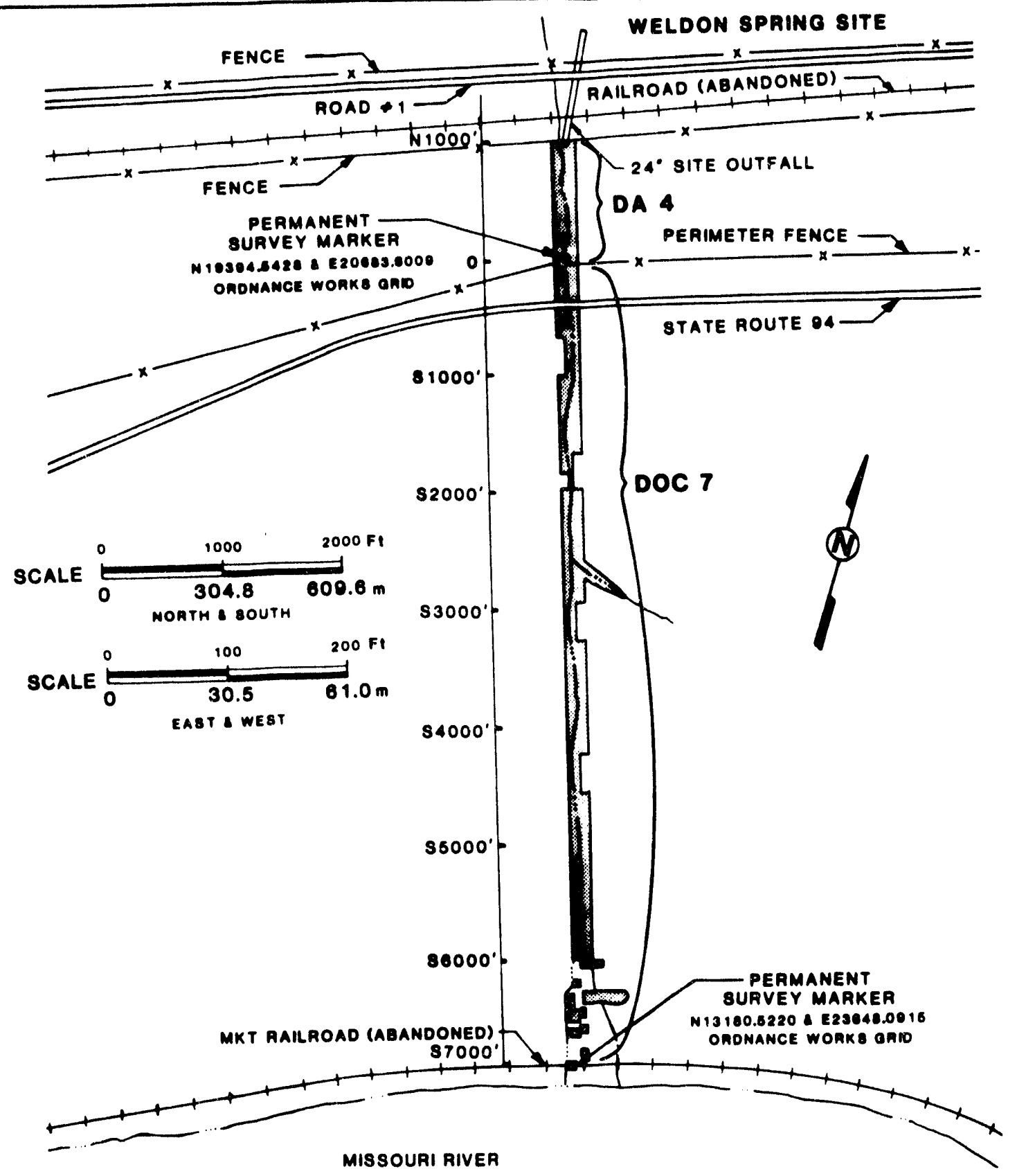

SEE SECTION 12 FOR CONVERBION FACTORS

CONTAMINATION LIMITS FOR THE SOUTHEAST DRAINAGE EASEMENT (SDE)

FIGURE 5.2-45

\begin{tabular}{|c|c|c|c|c|}
\hline AEPOAT NO. & DOE/OR/2 & $1548-074$ & EOHET NO & A/VP/177/1191 \\
\hline OAIGINATOR & BLG & Torawn or & GLN & $11 / 91$ \\
\hline
\end{tabular}




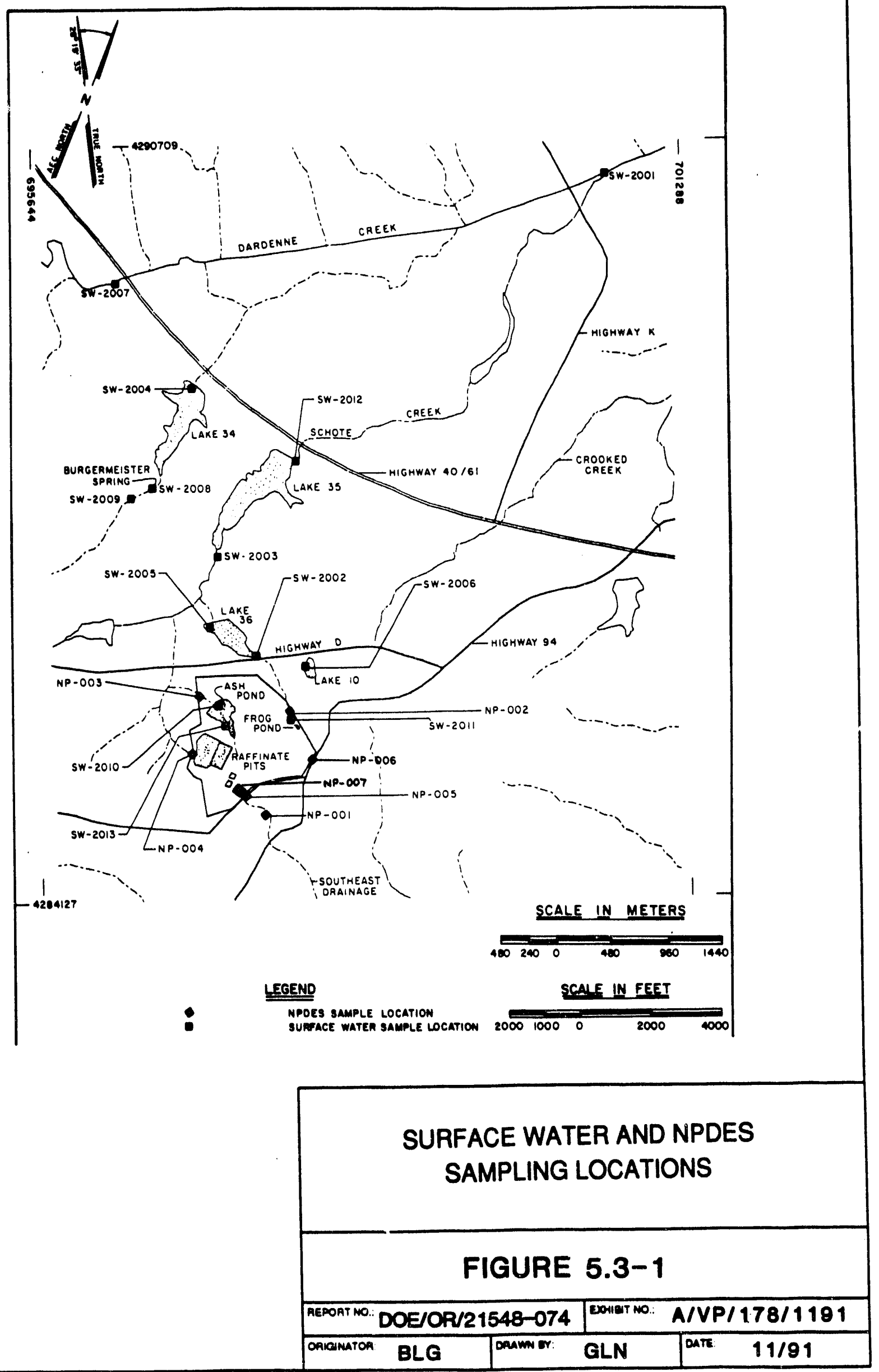




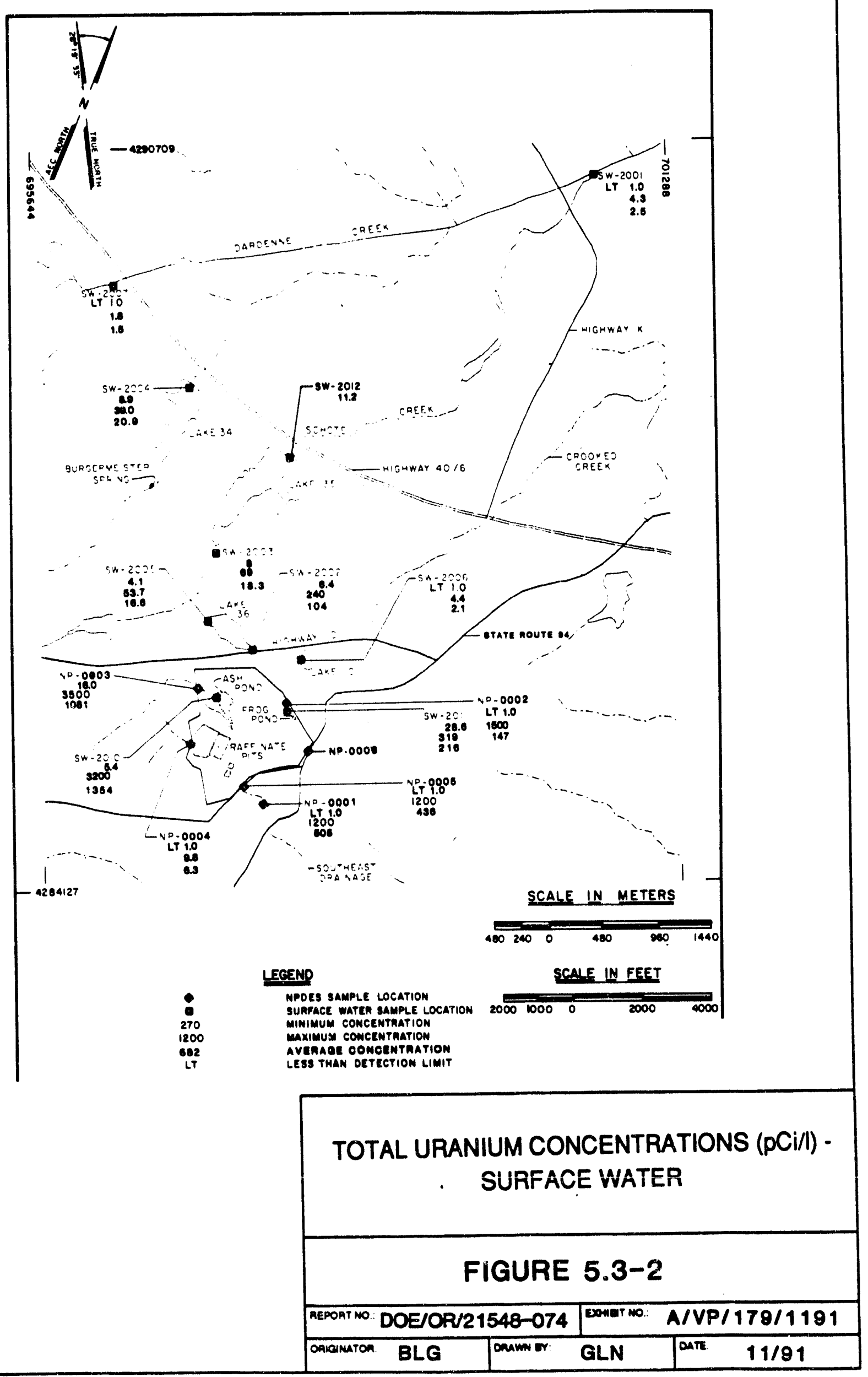




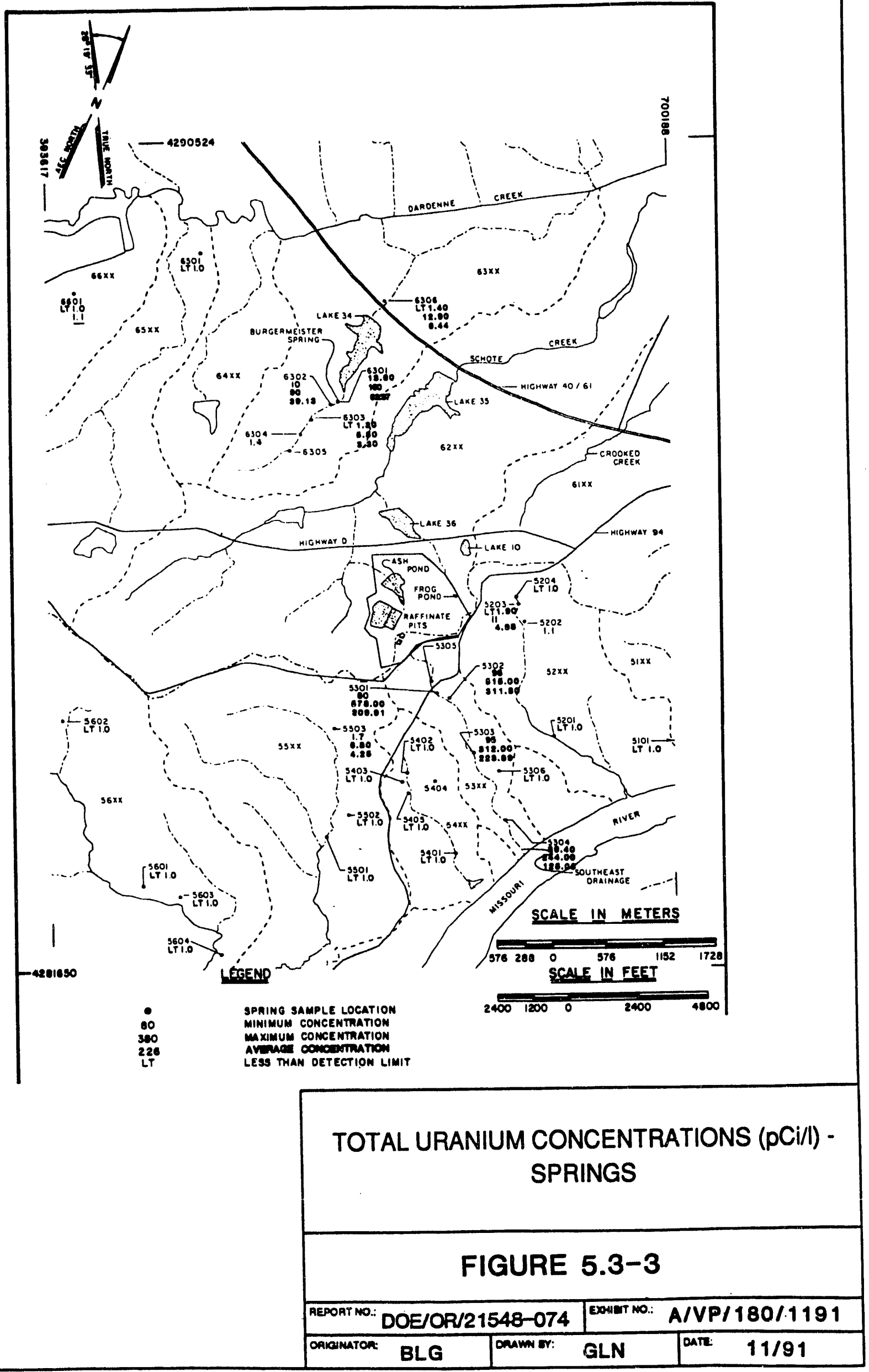




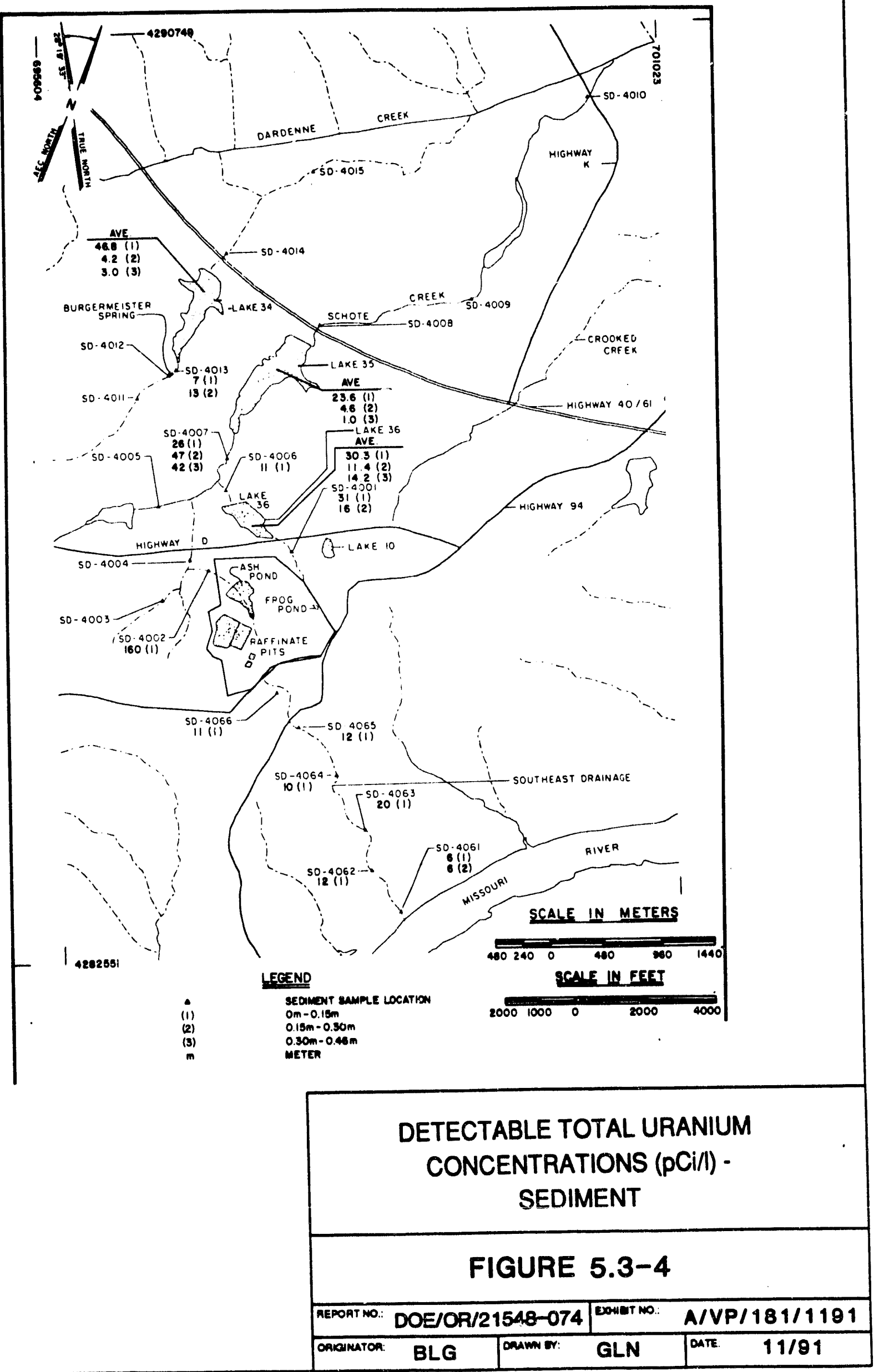




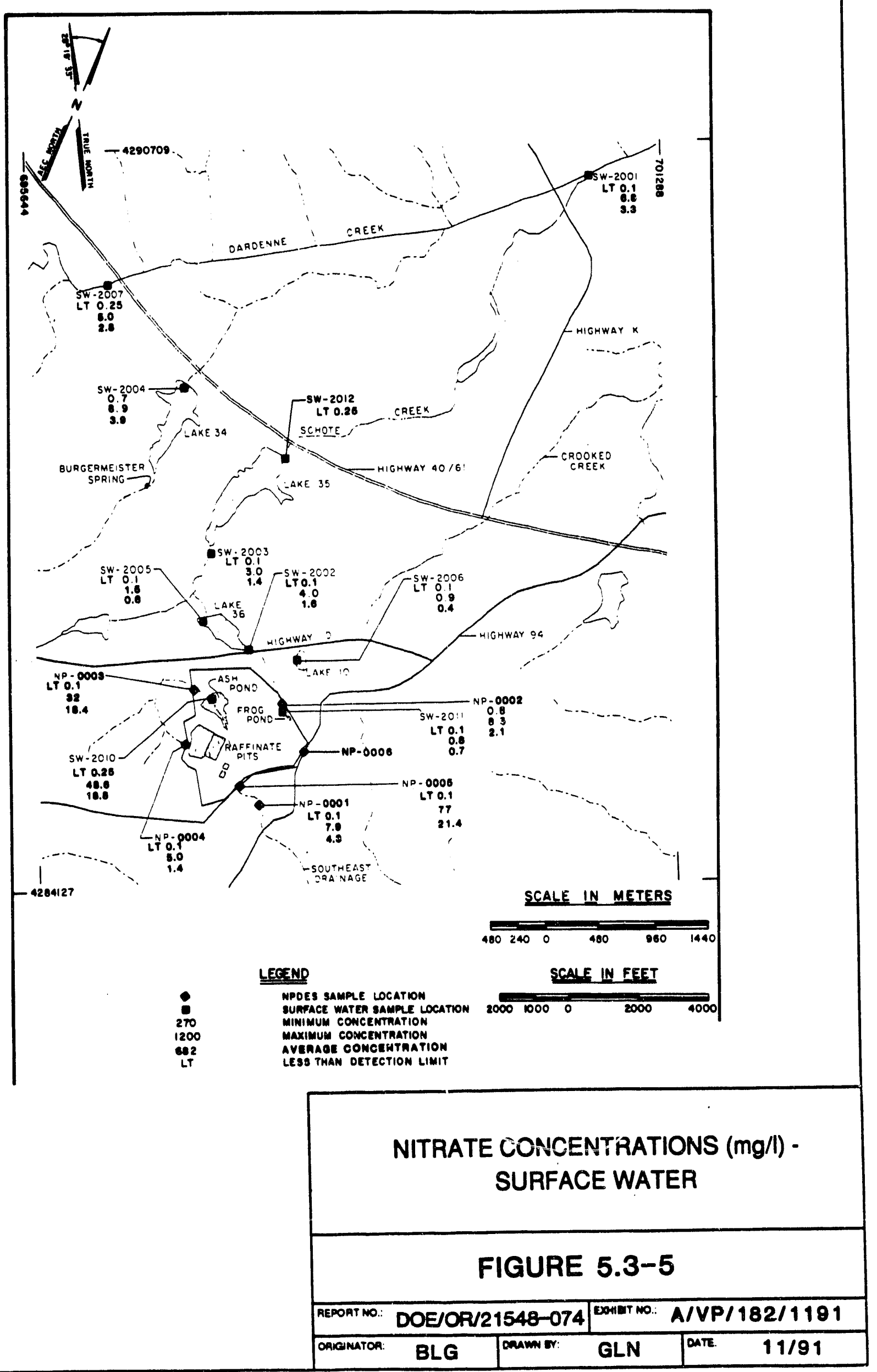




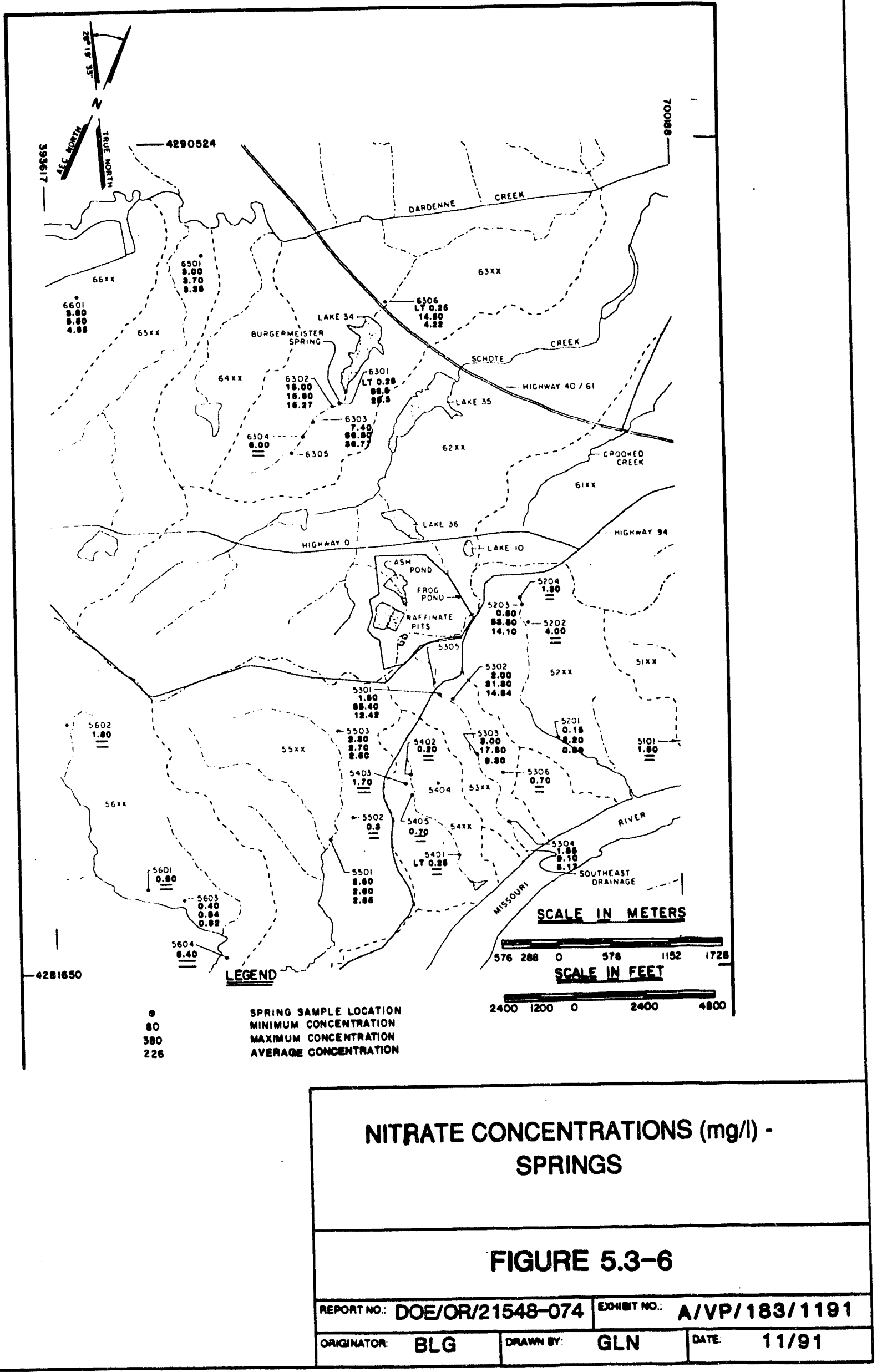




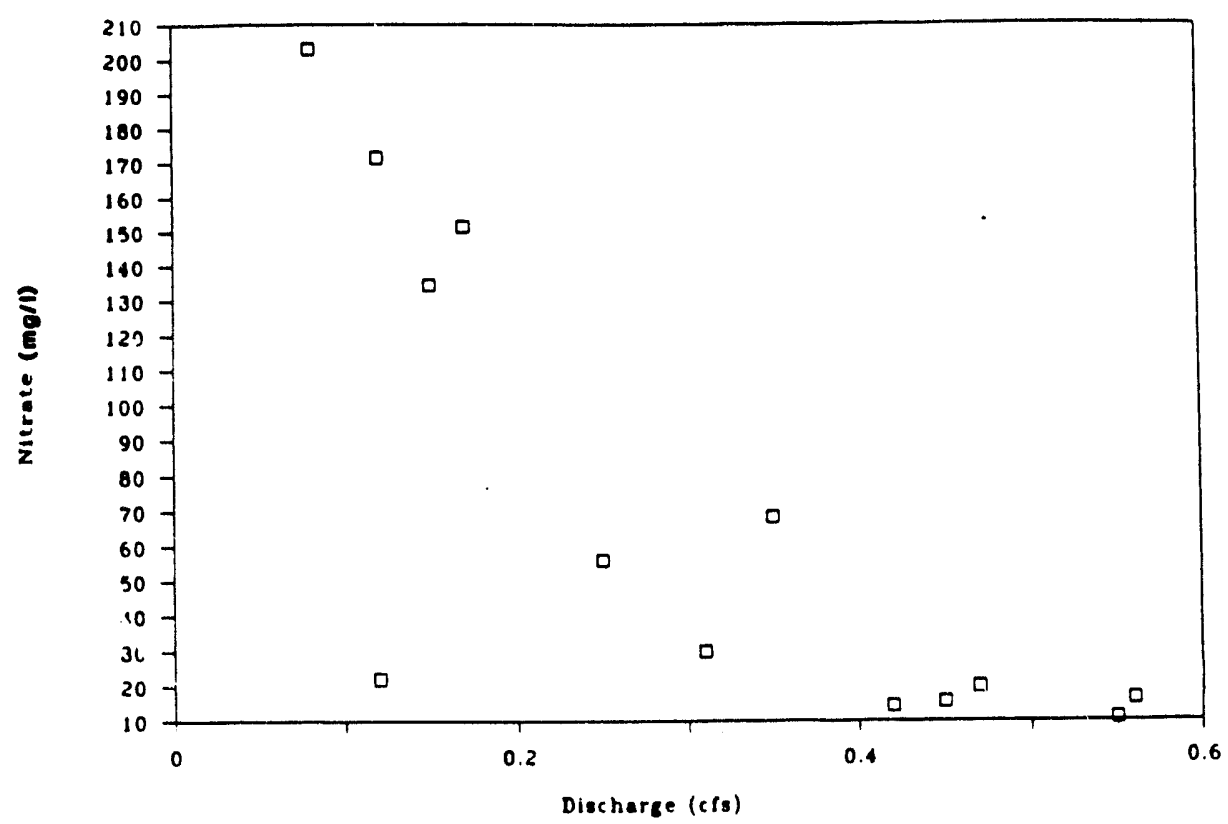

THE CONCENTRATION OF NITRATE AS A FUNCTION OF THE DISCHARGE AT BURGERMEISTER SPRING

FIGURE 5.3-7

REPOAT NOO DOE/OR/21548-074

onicinatón: BLG

Donum or GLN

A/PI/238/1181

DATE $11 / 91$ 


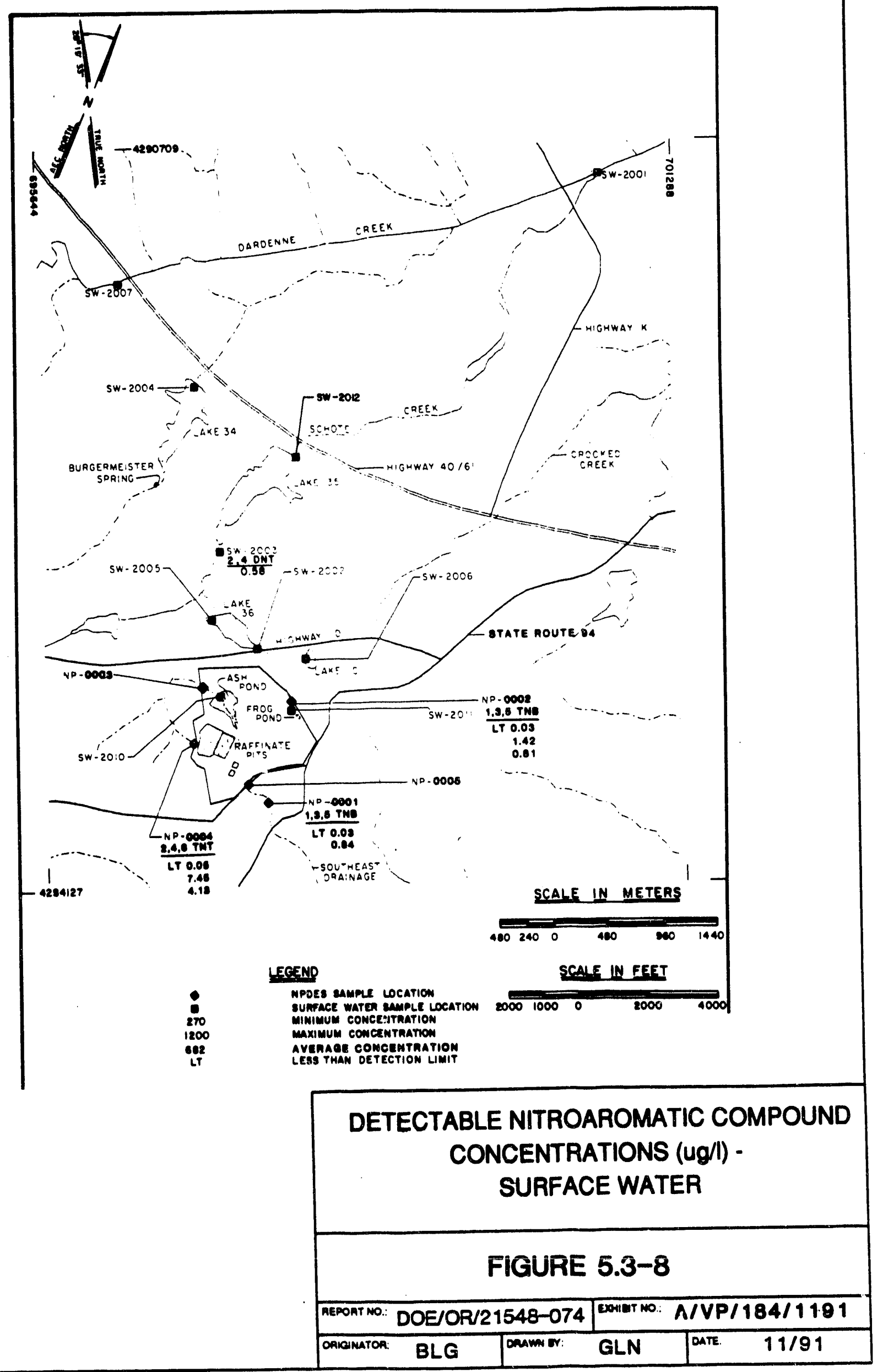




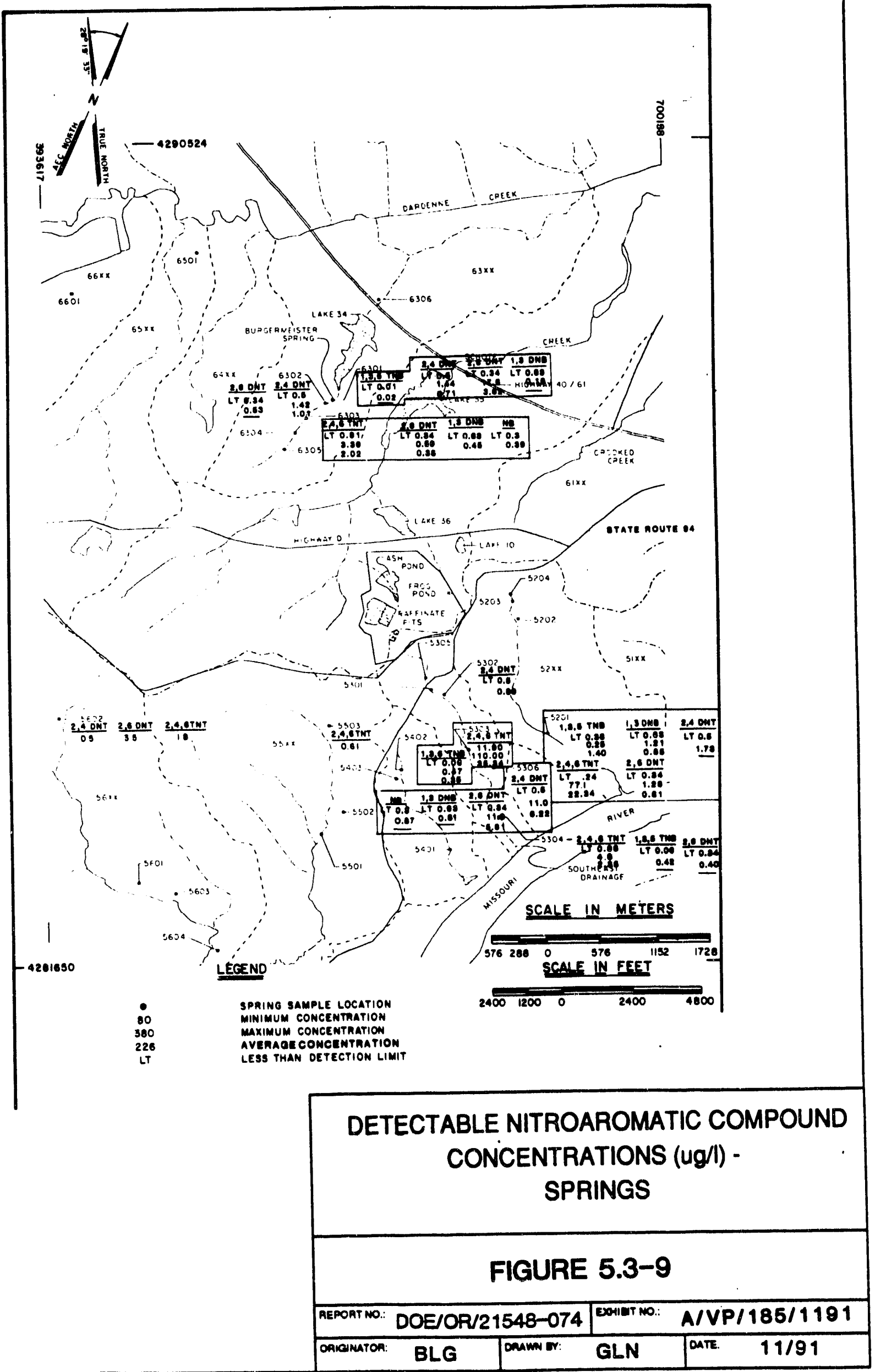




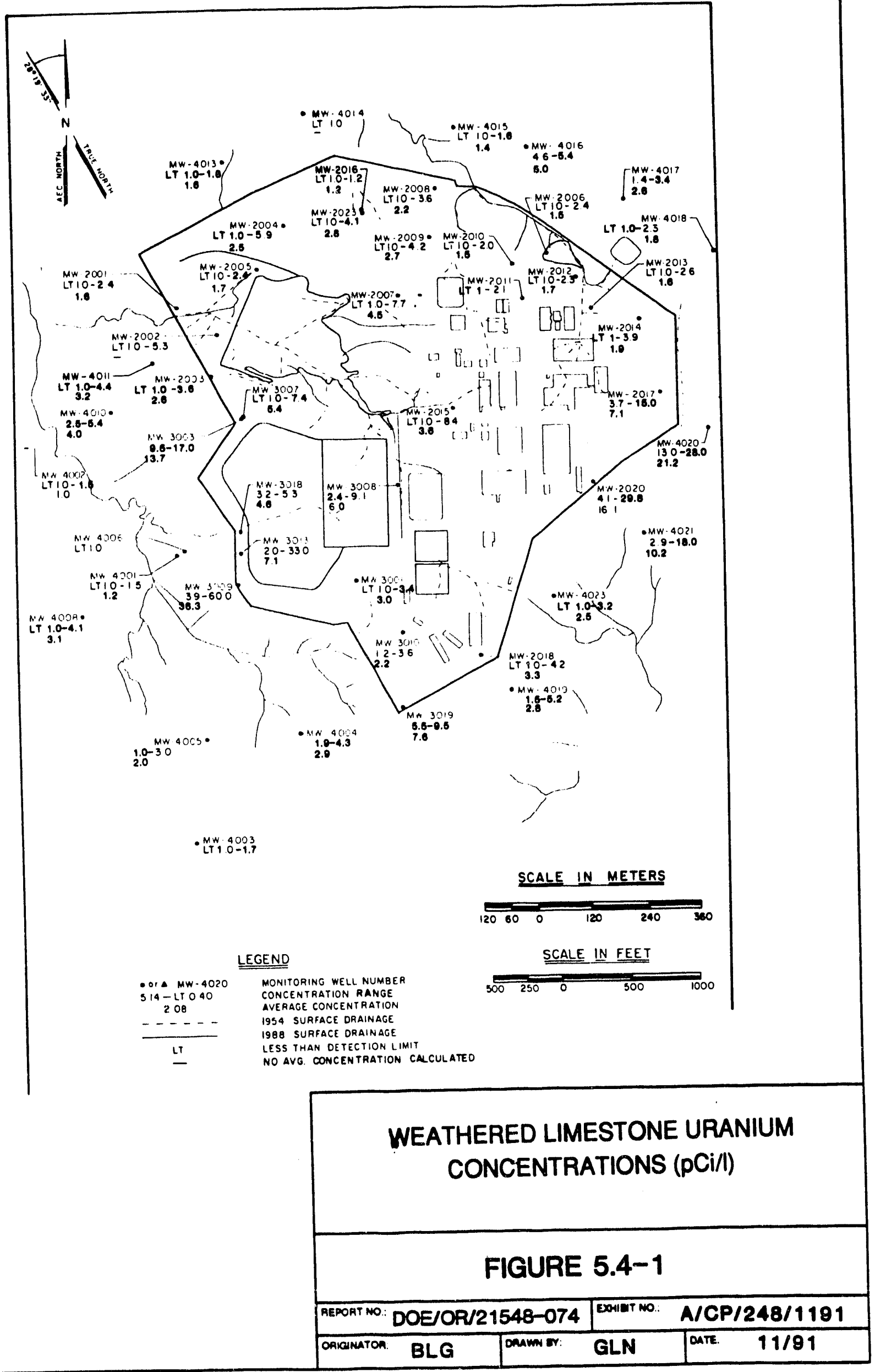




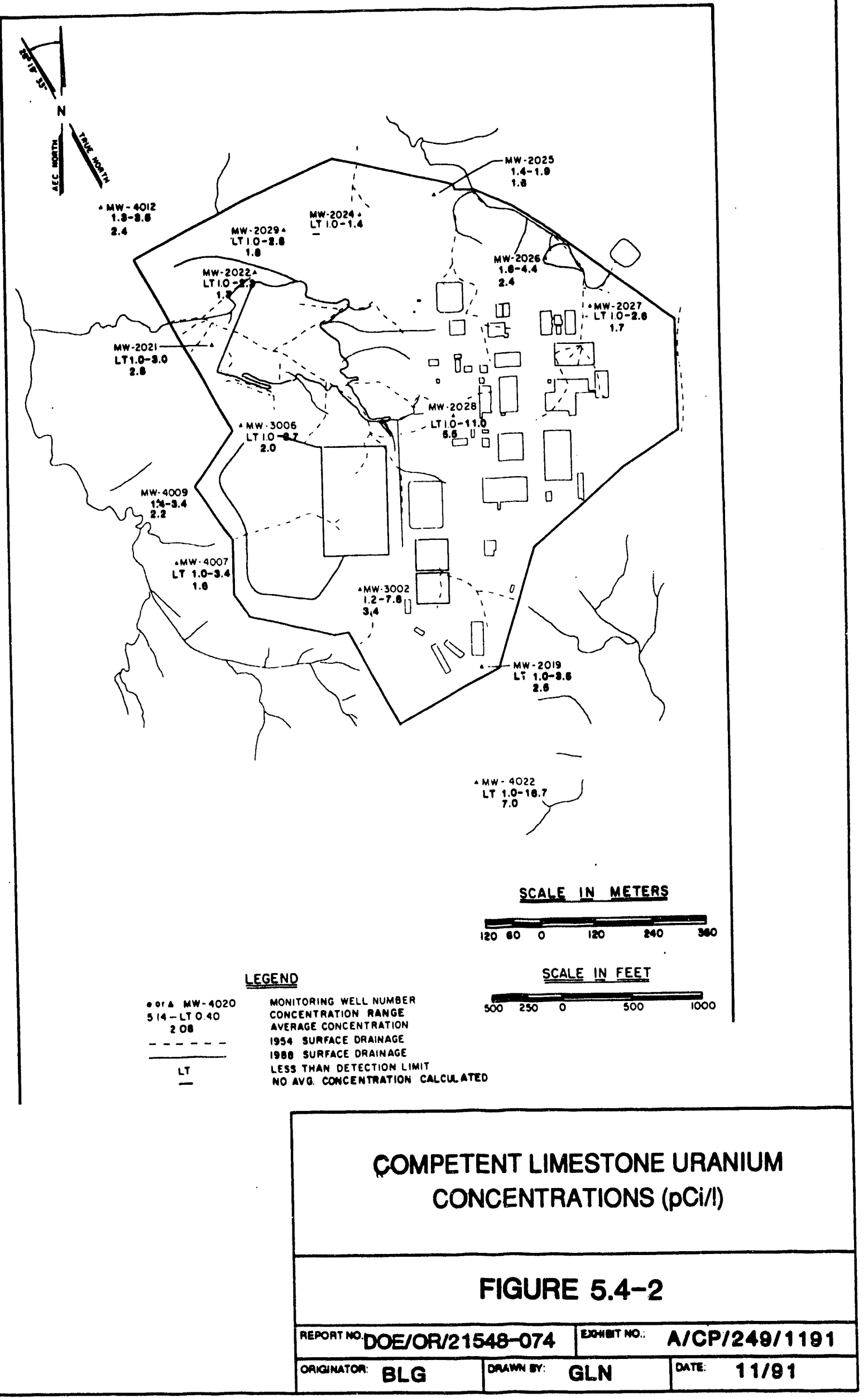




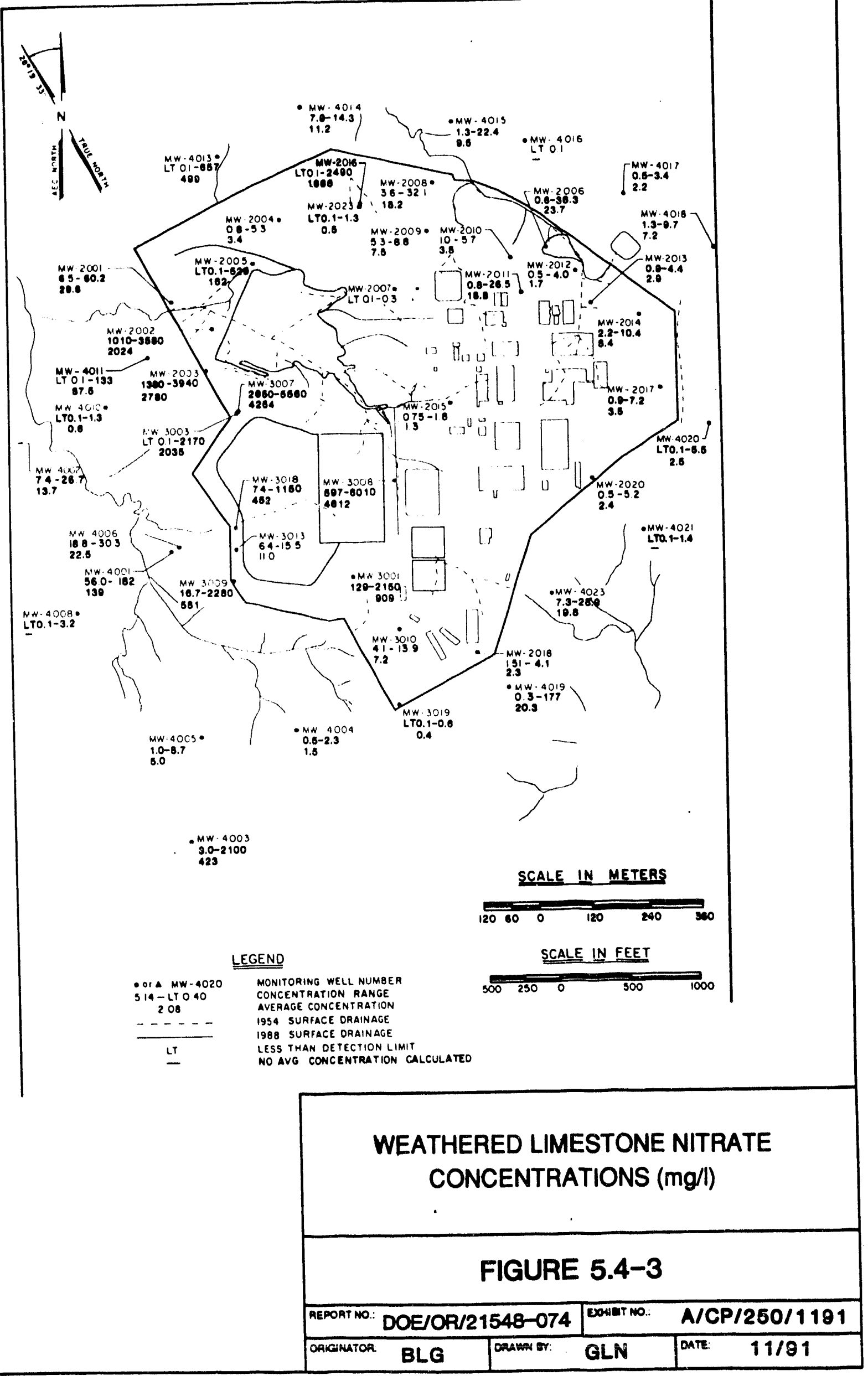




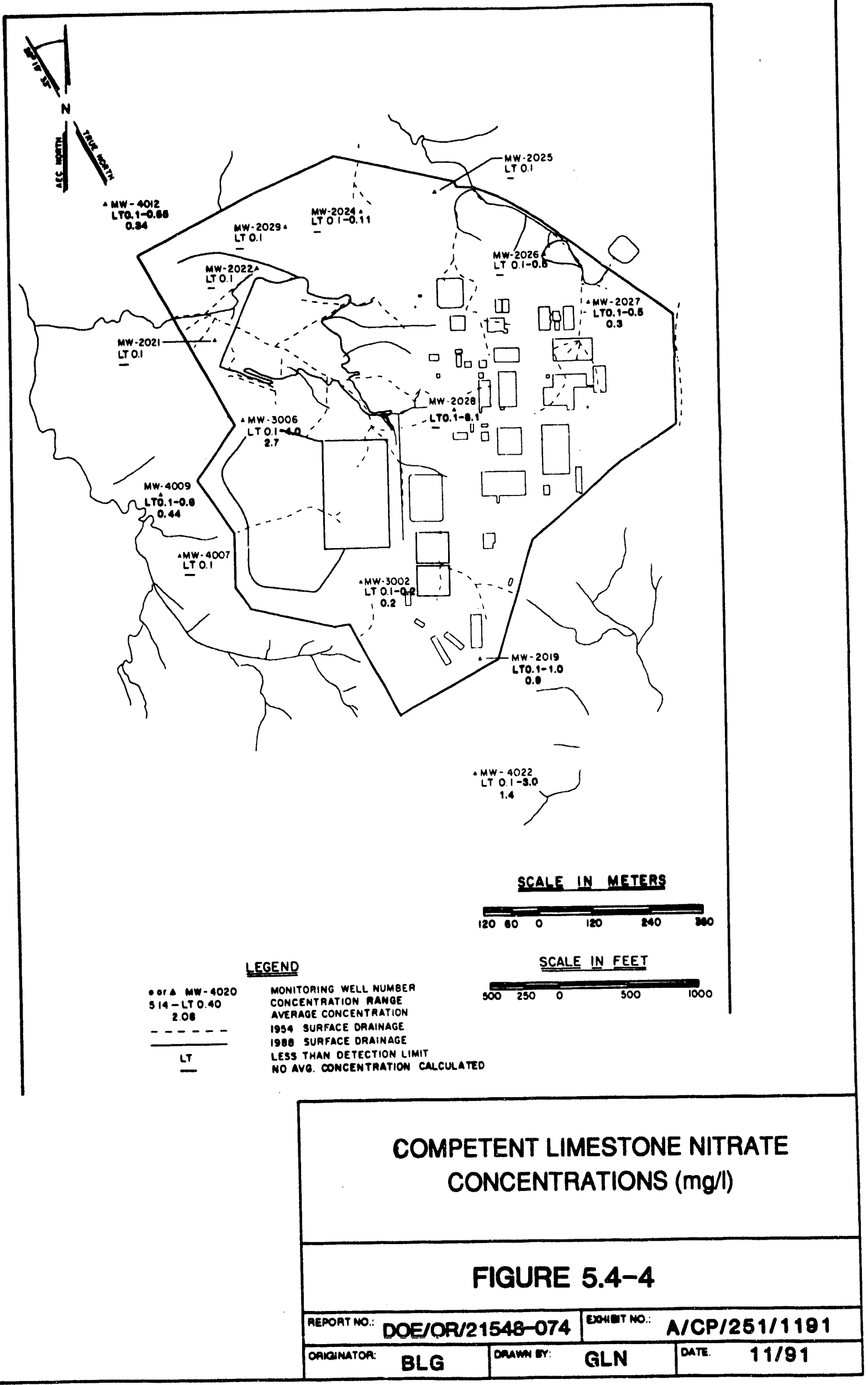




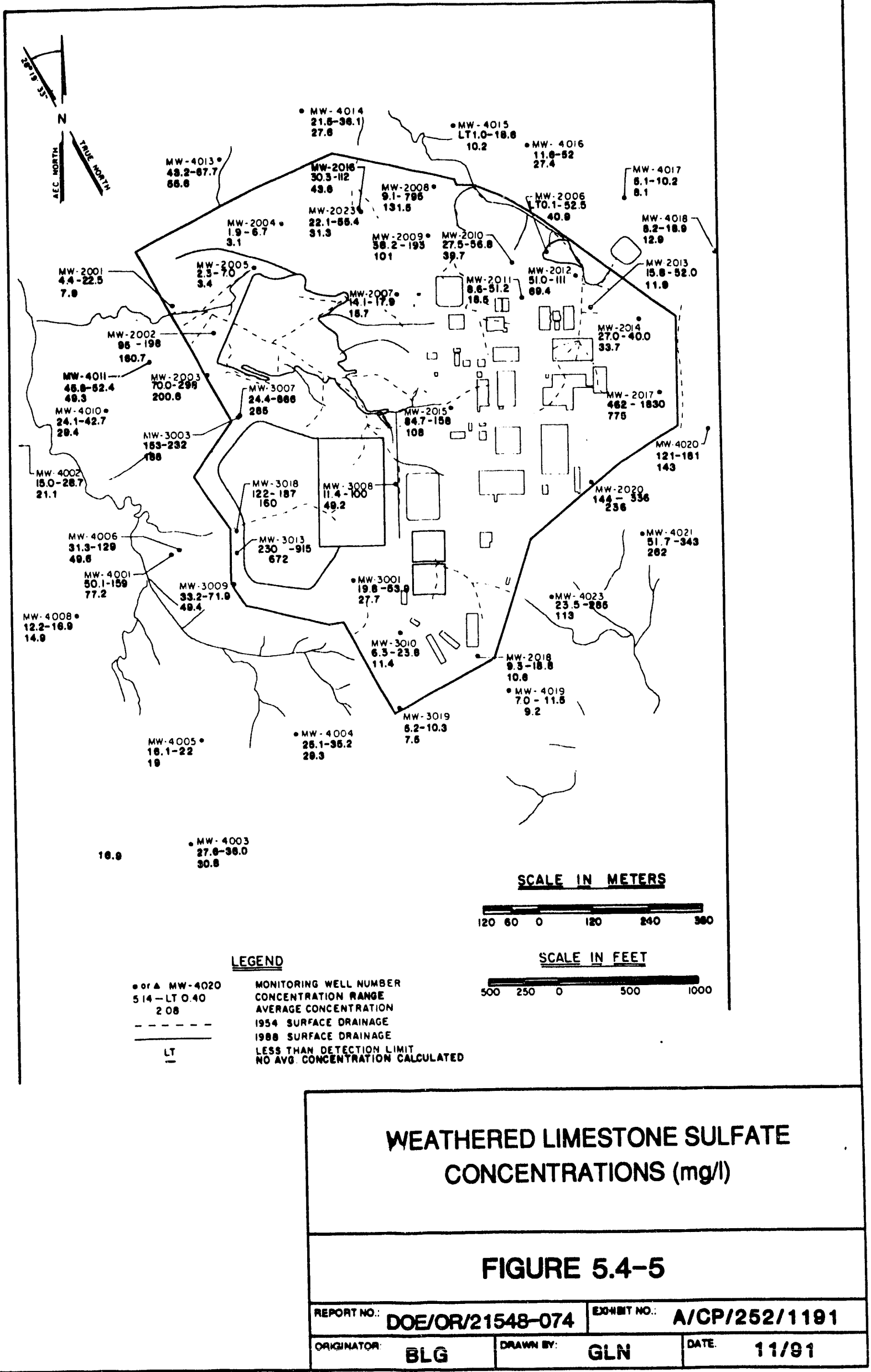




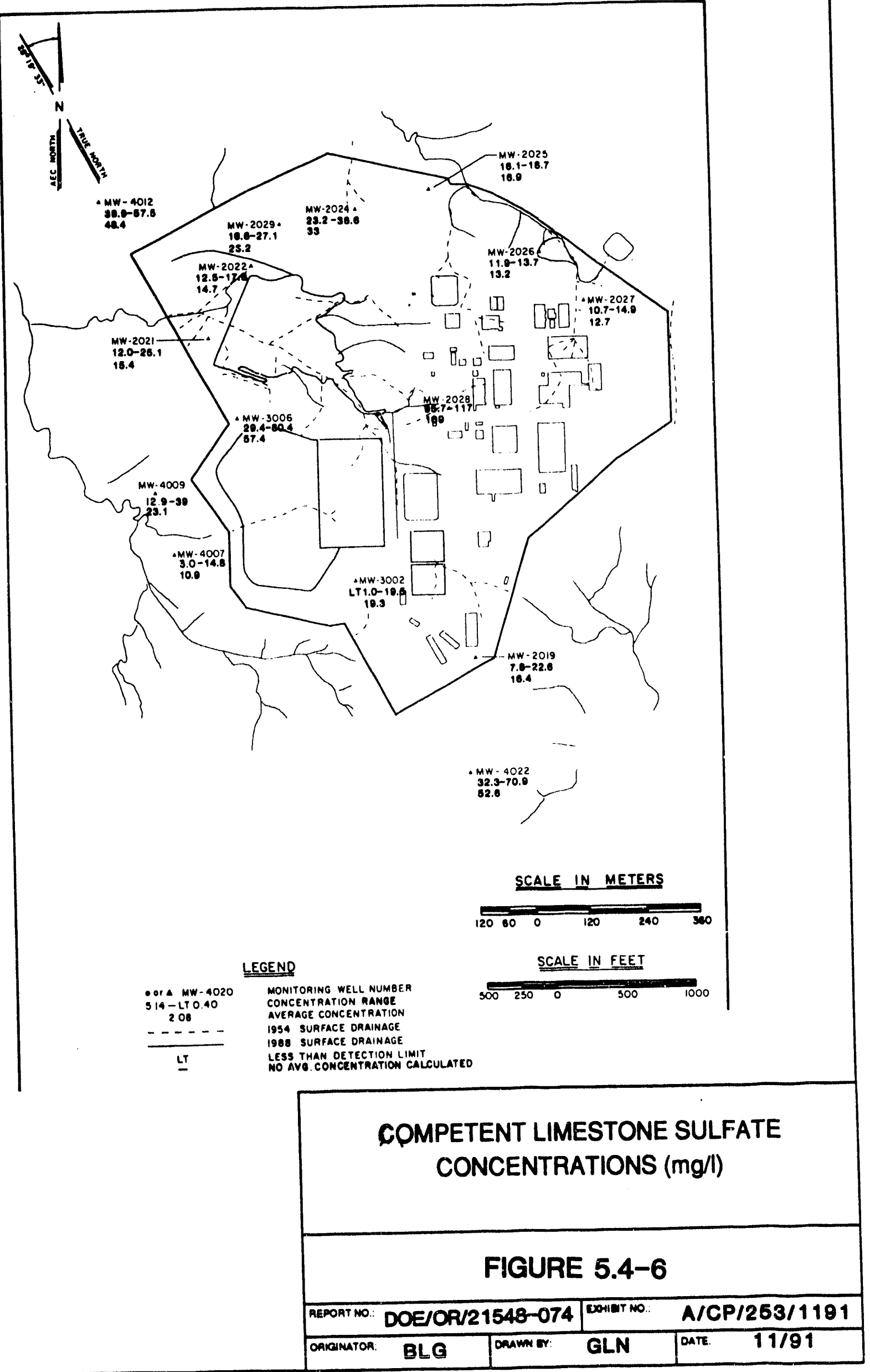




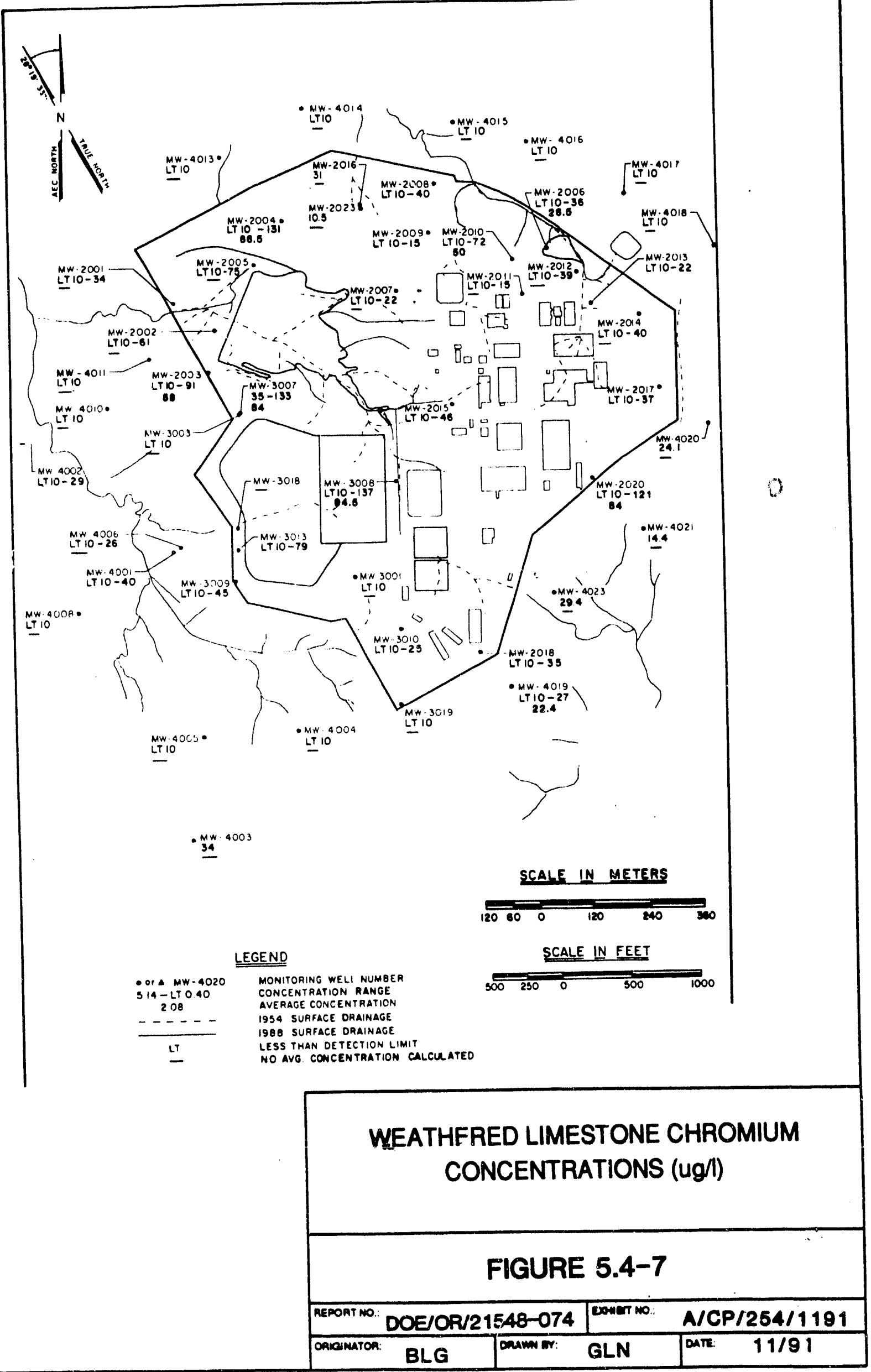




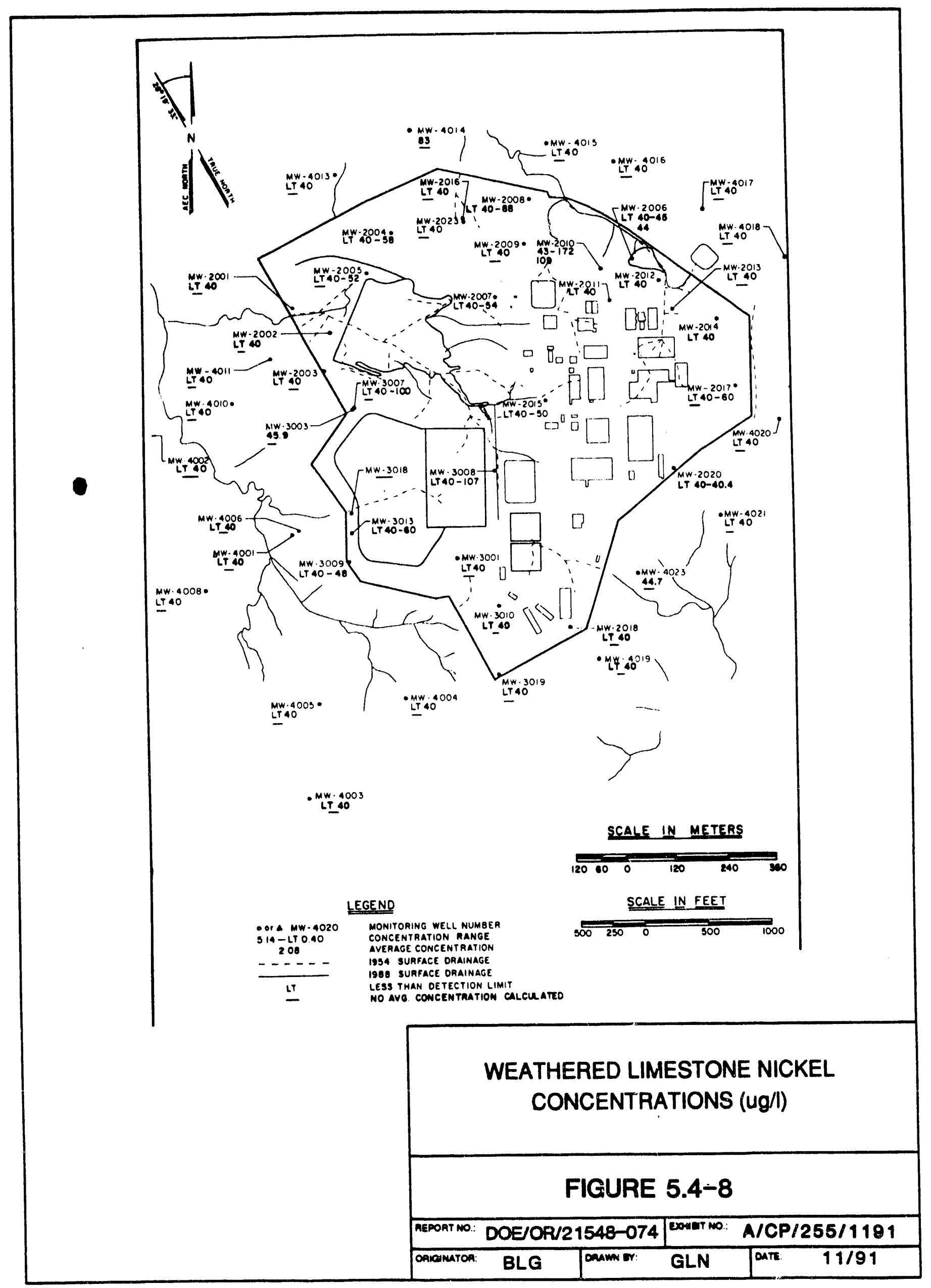




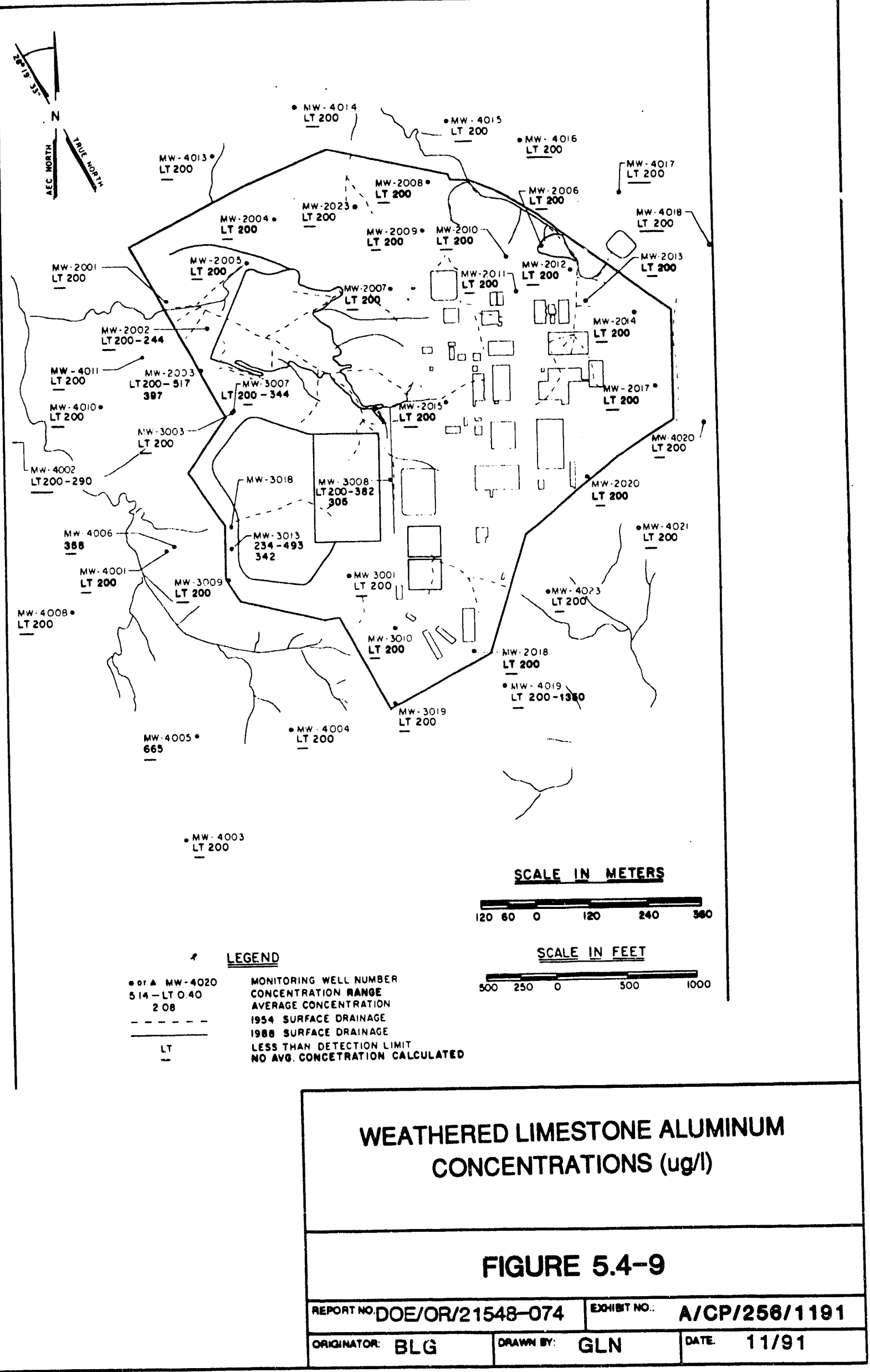




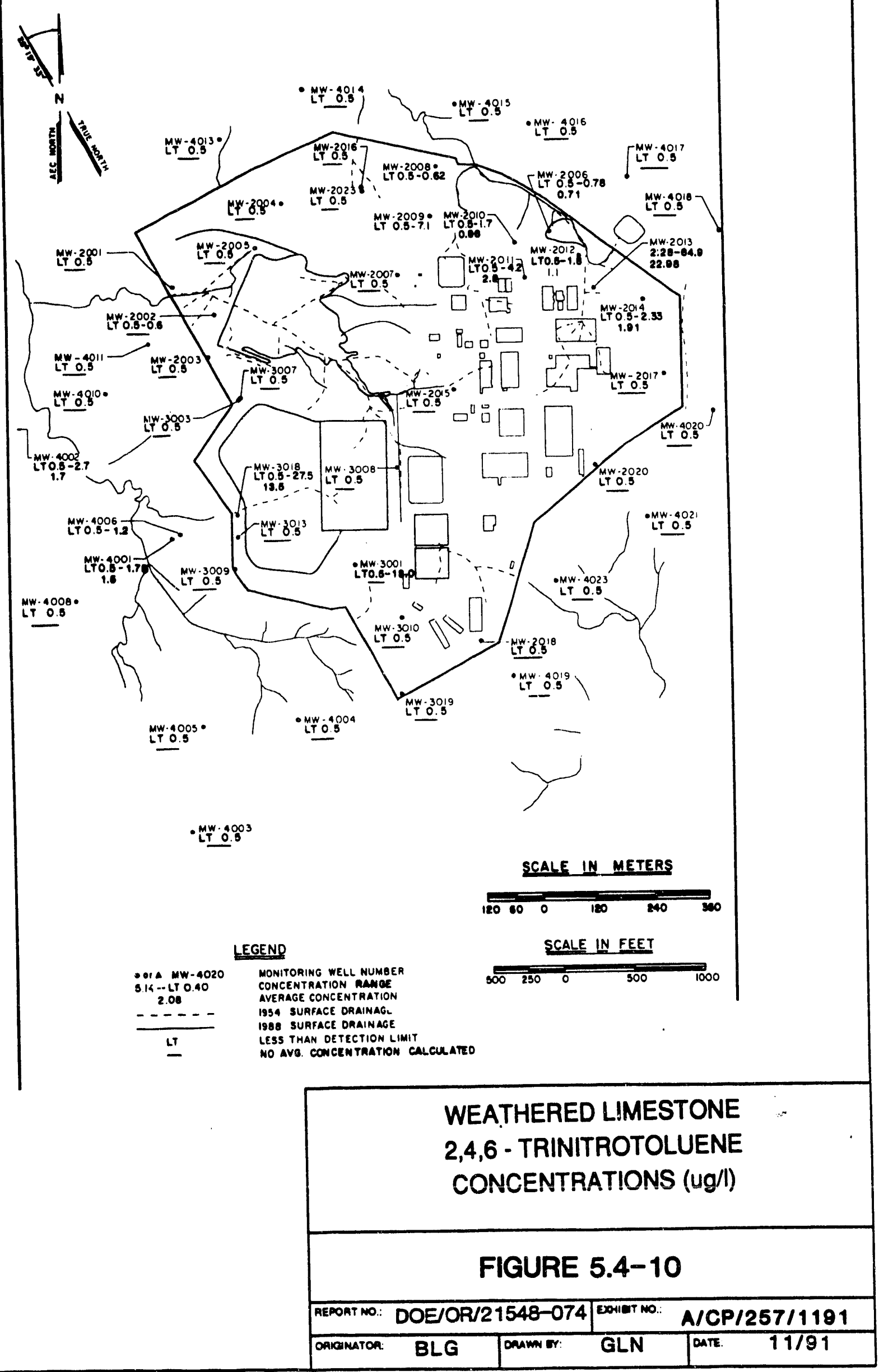




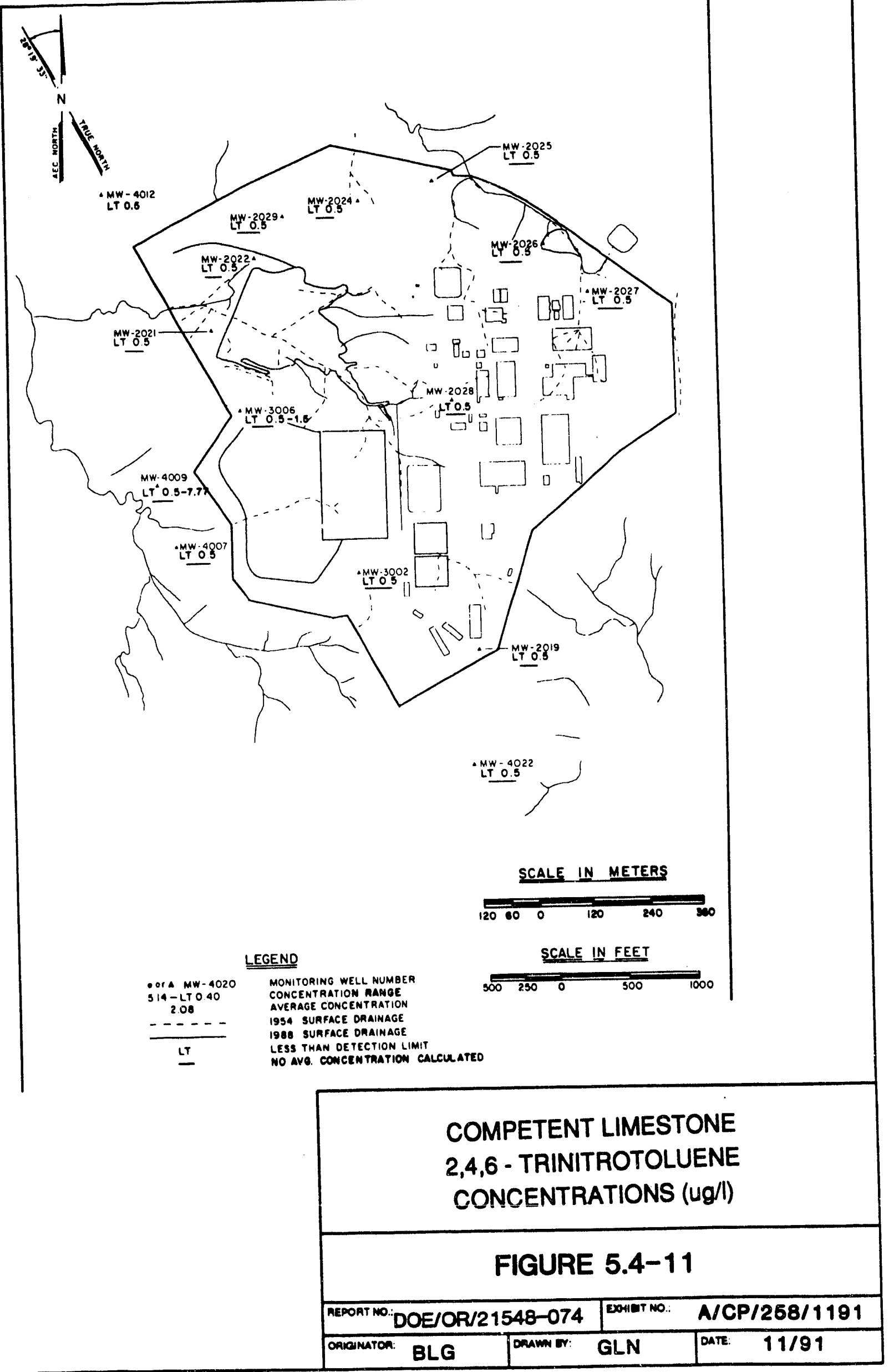




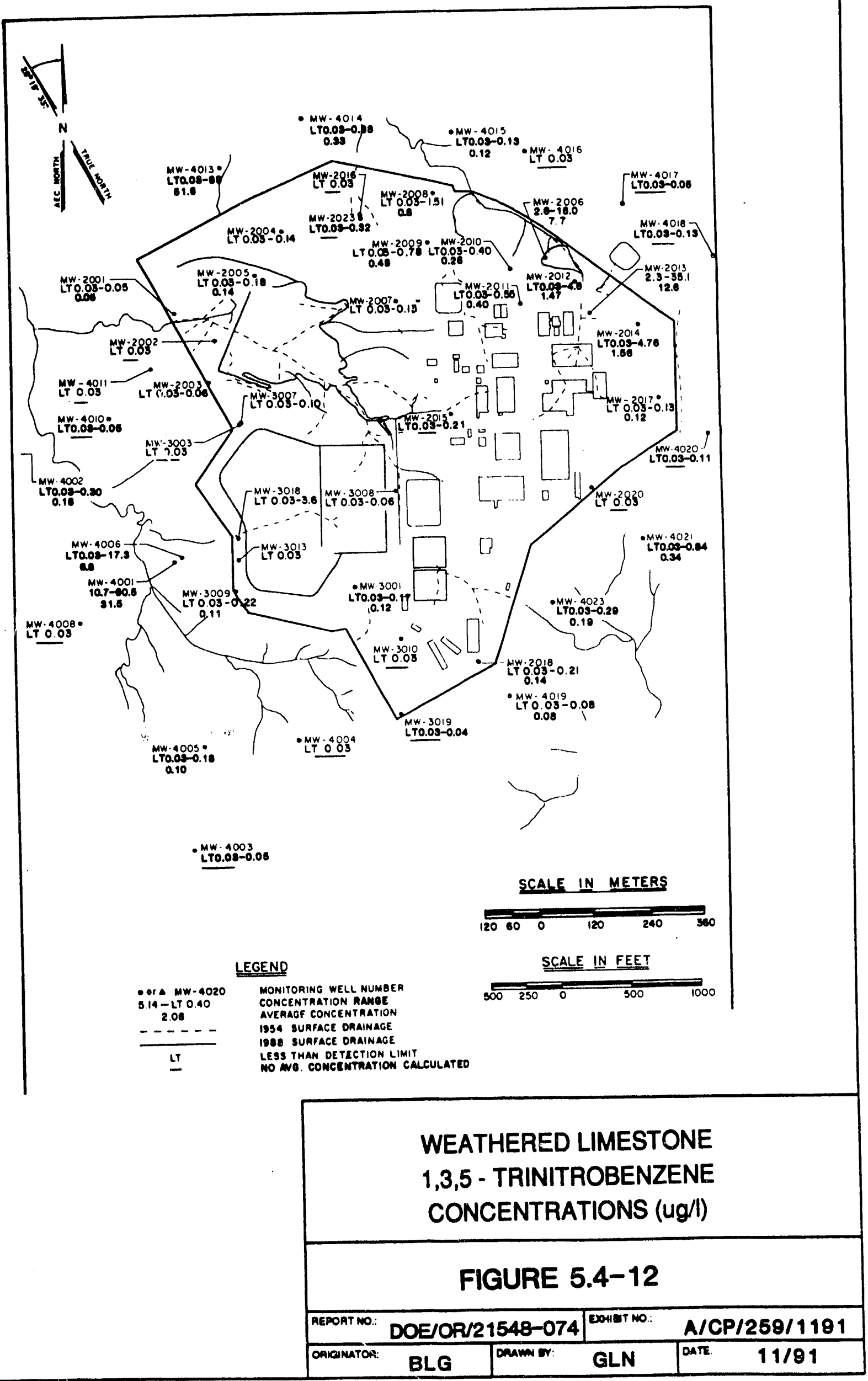




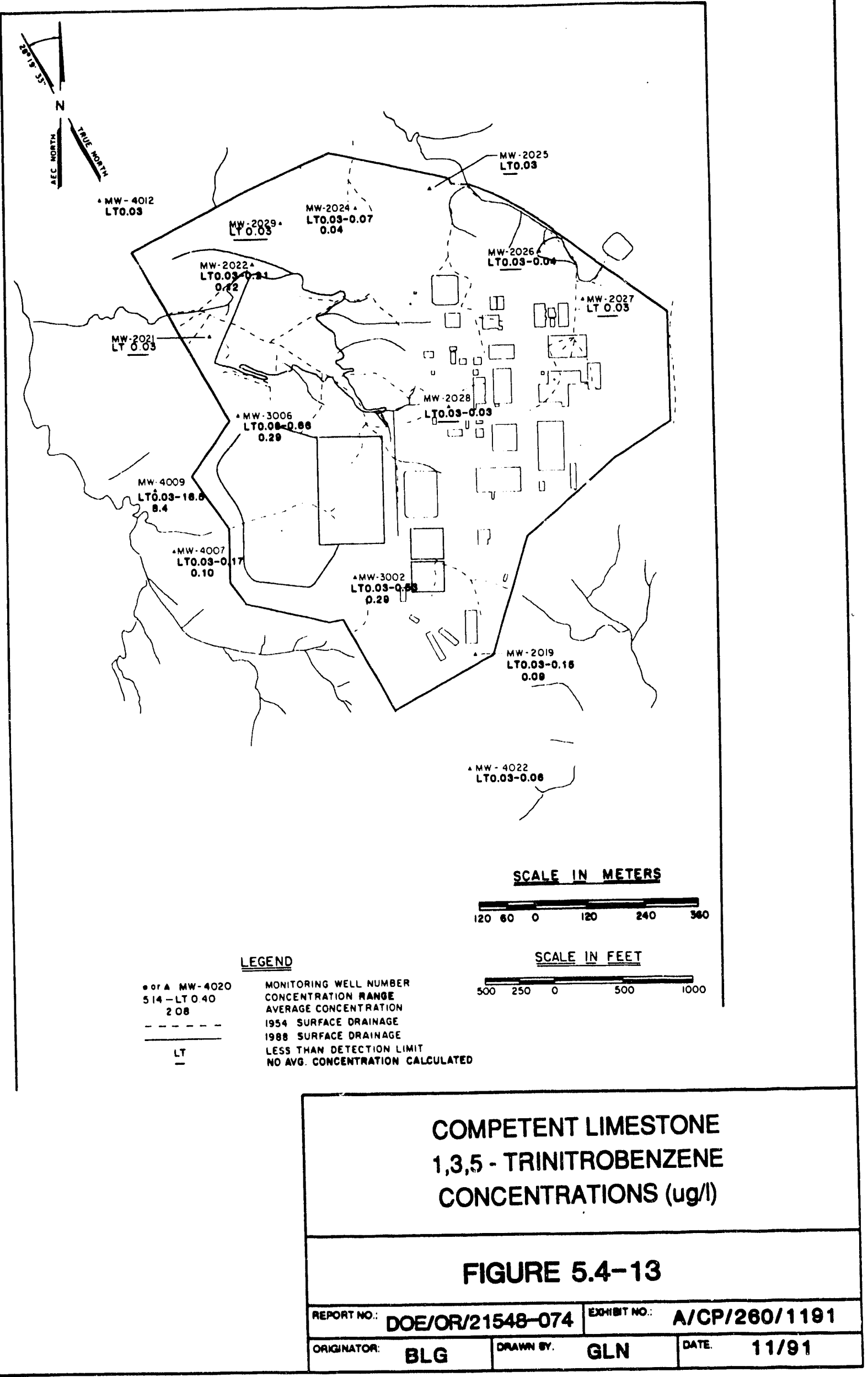




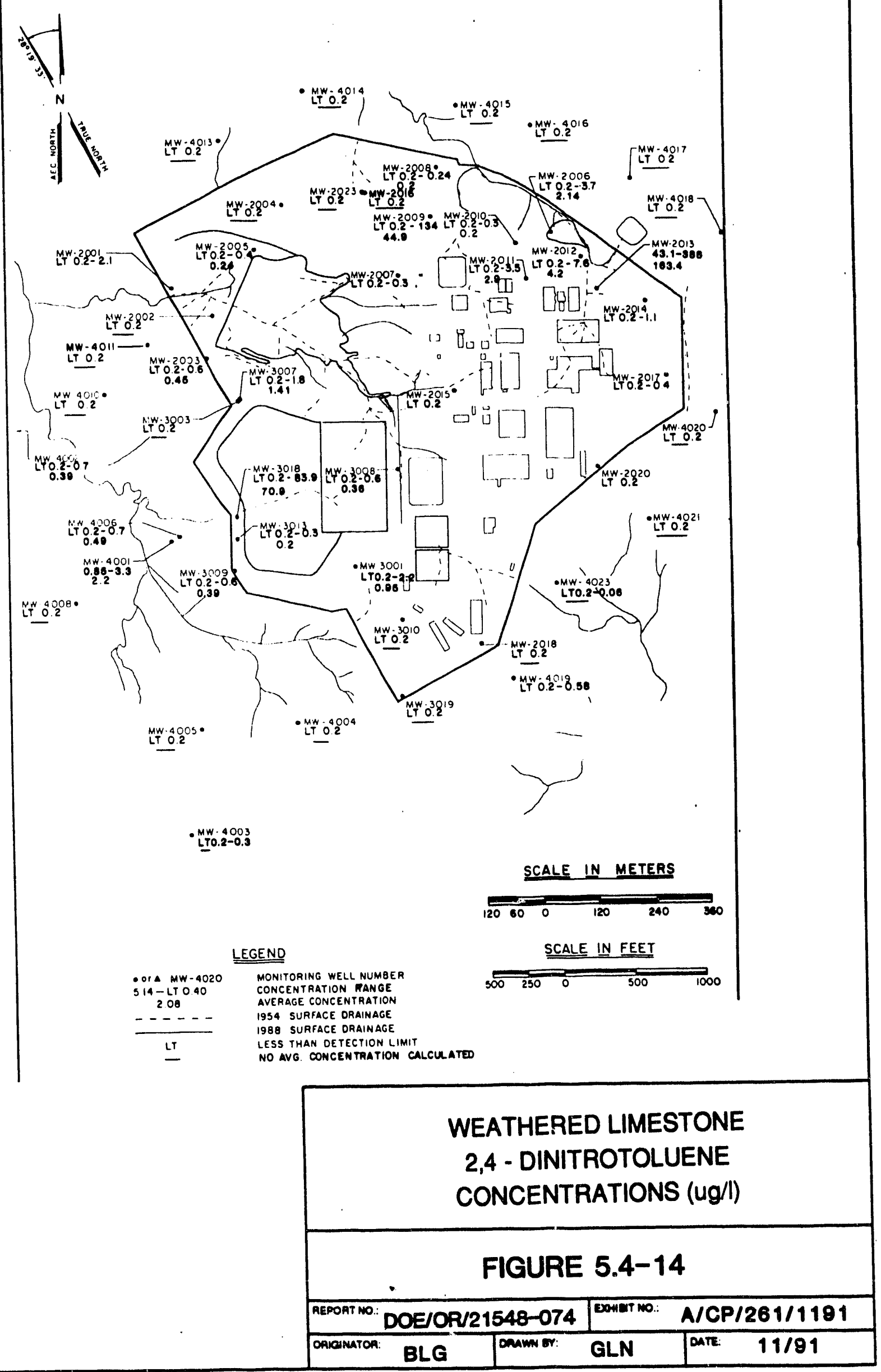




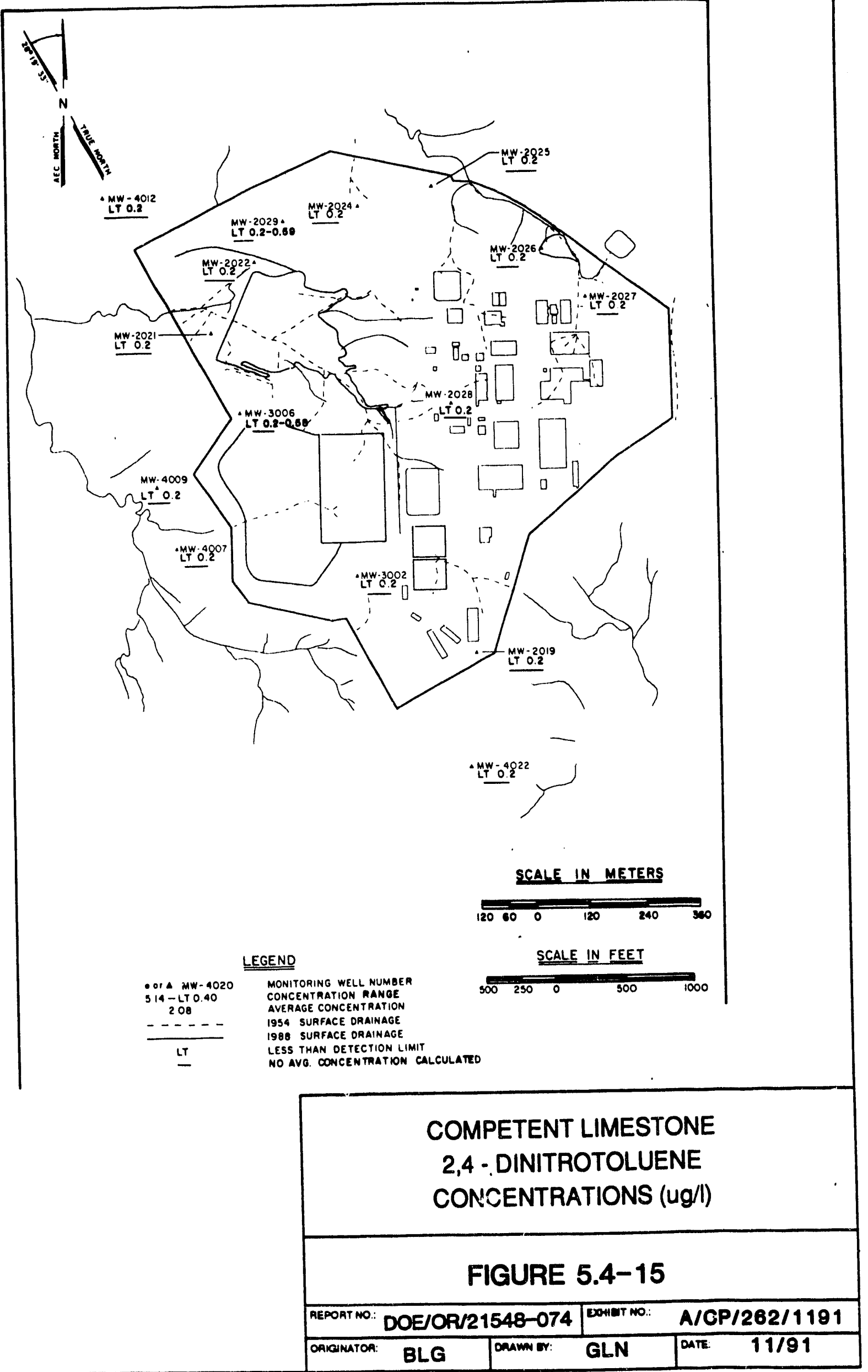




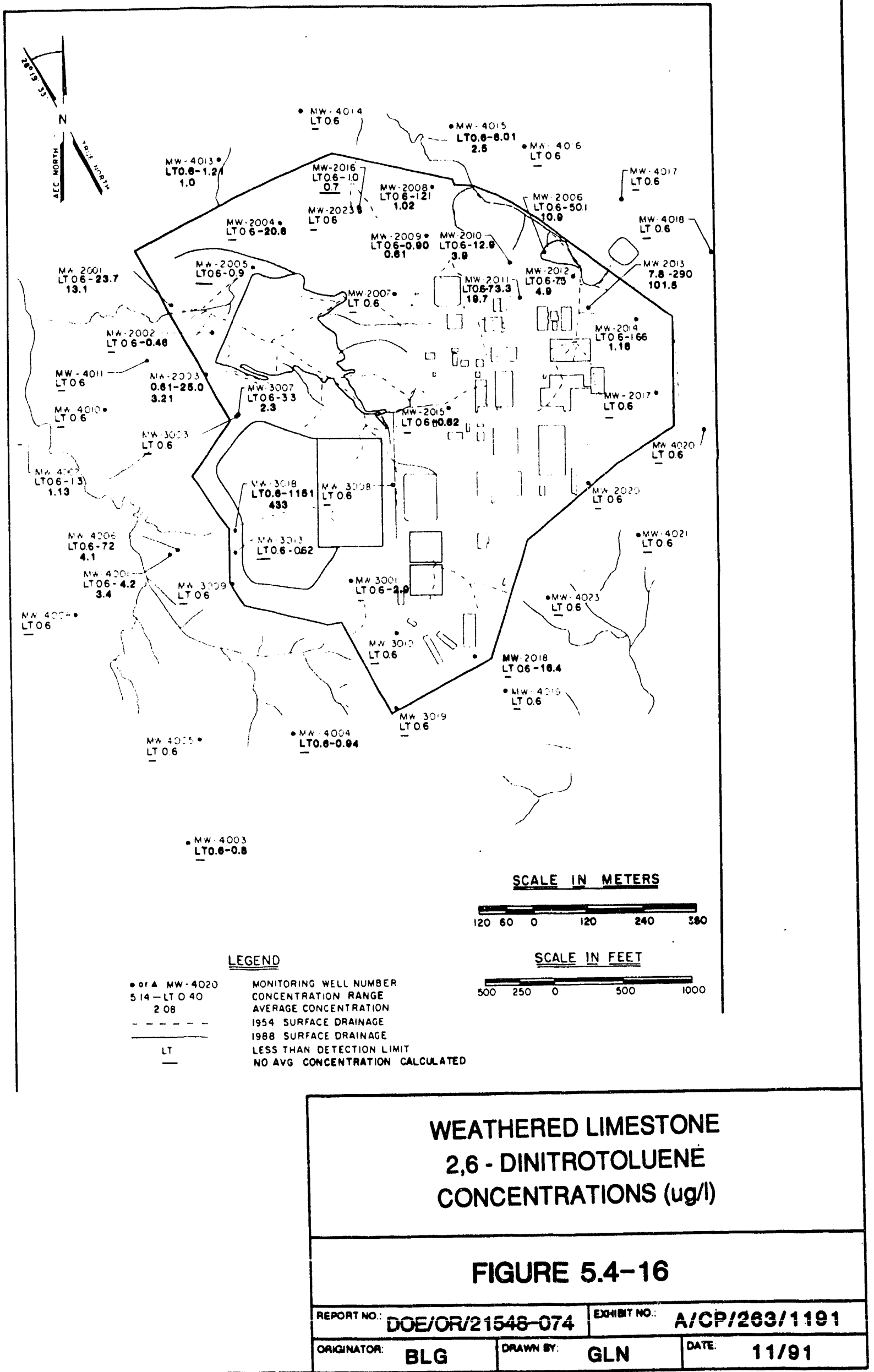




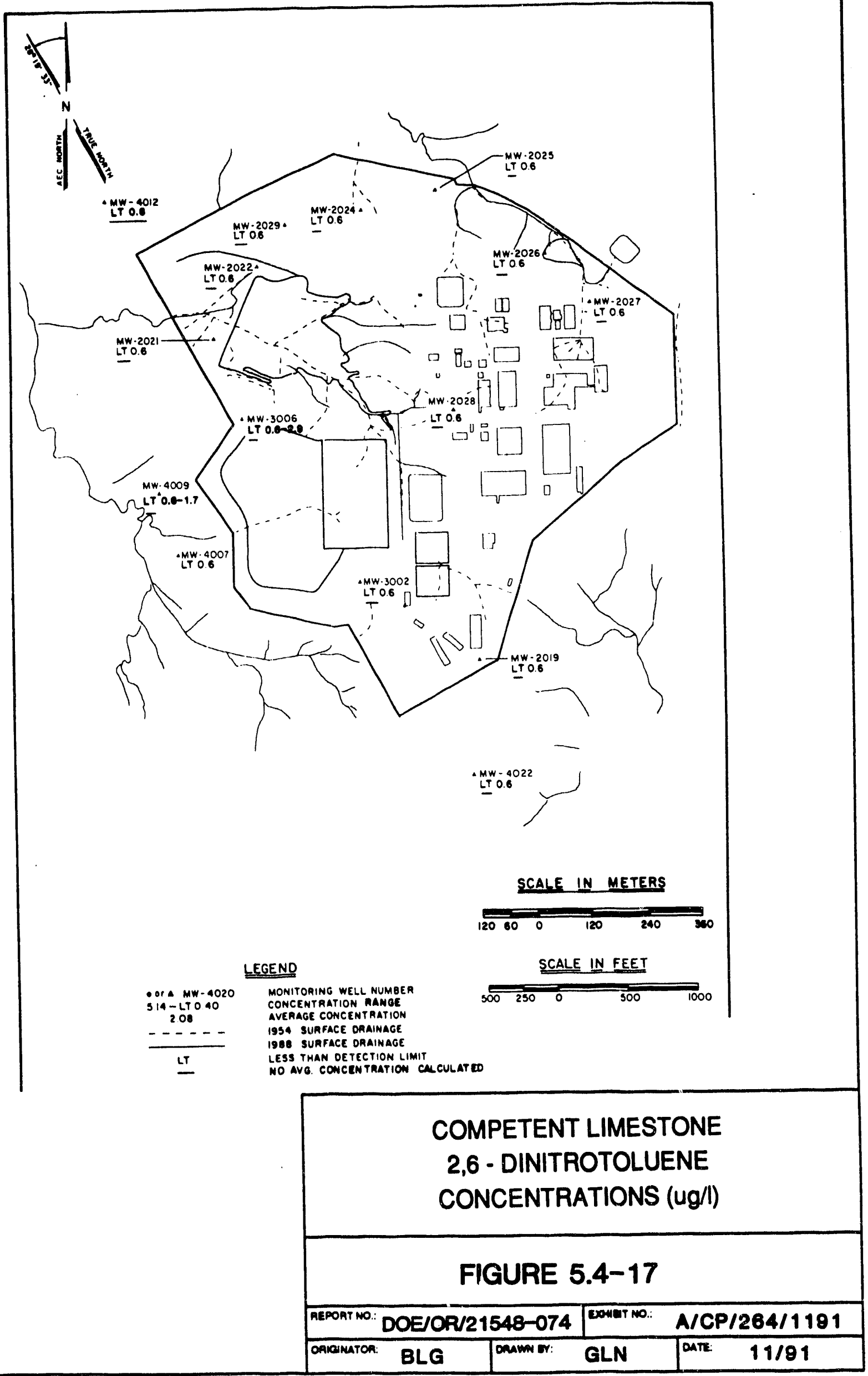




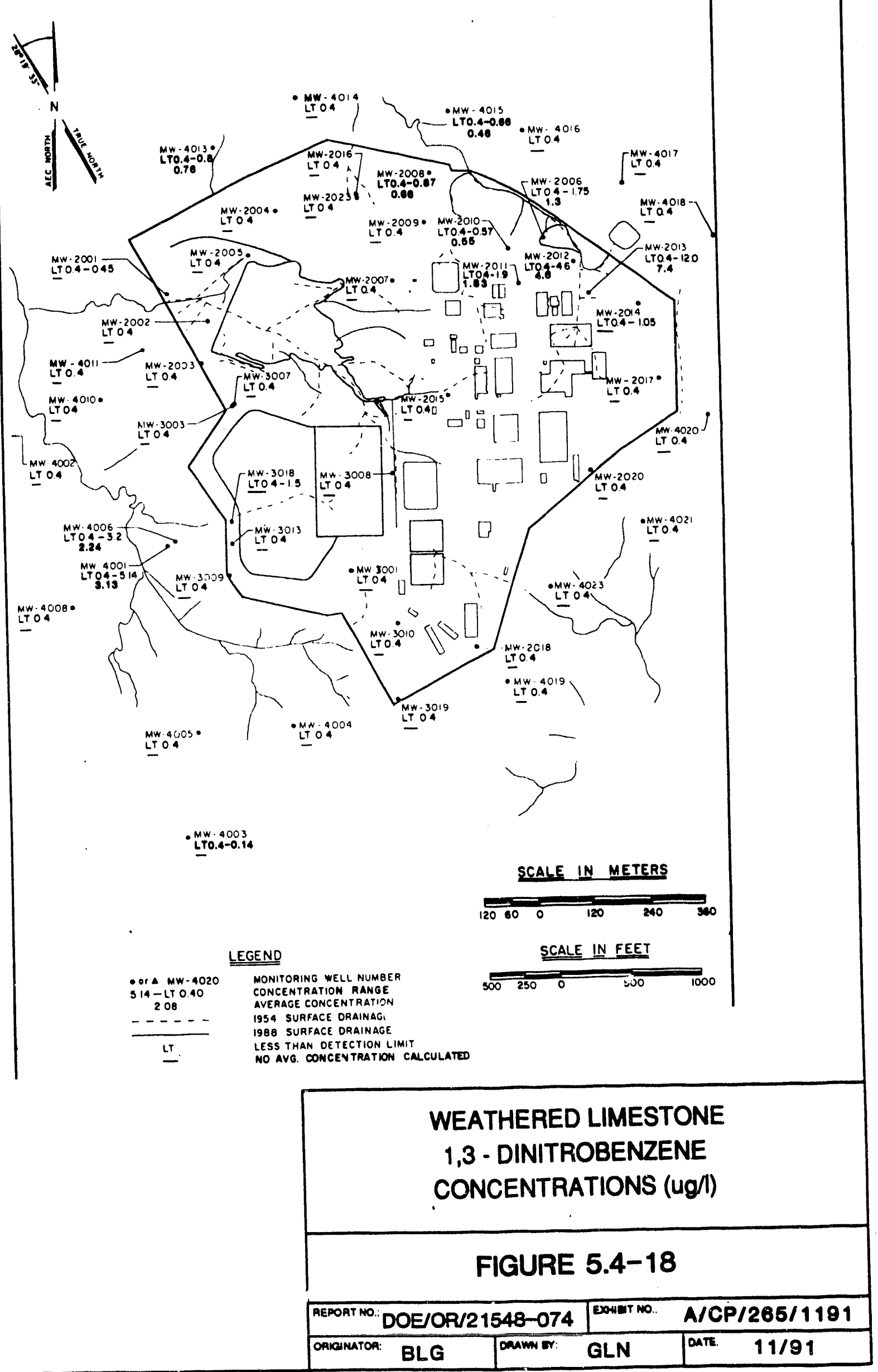




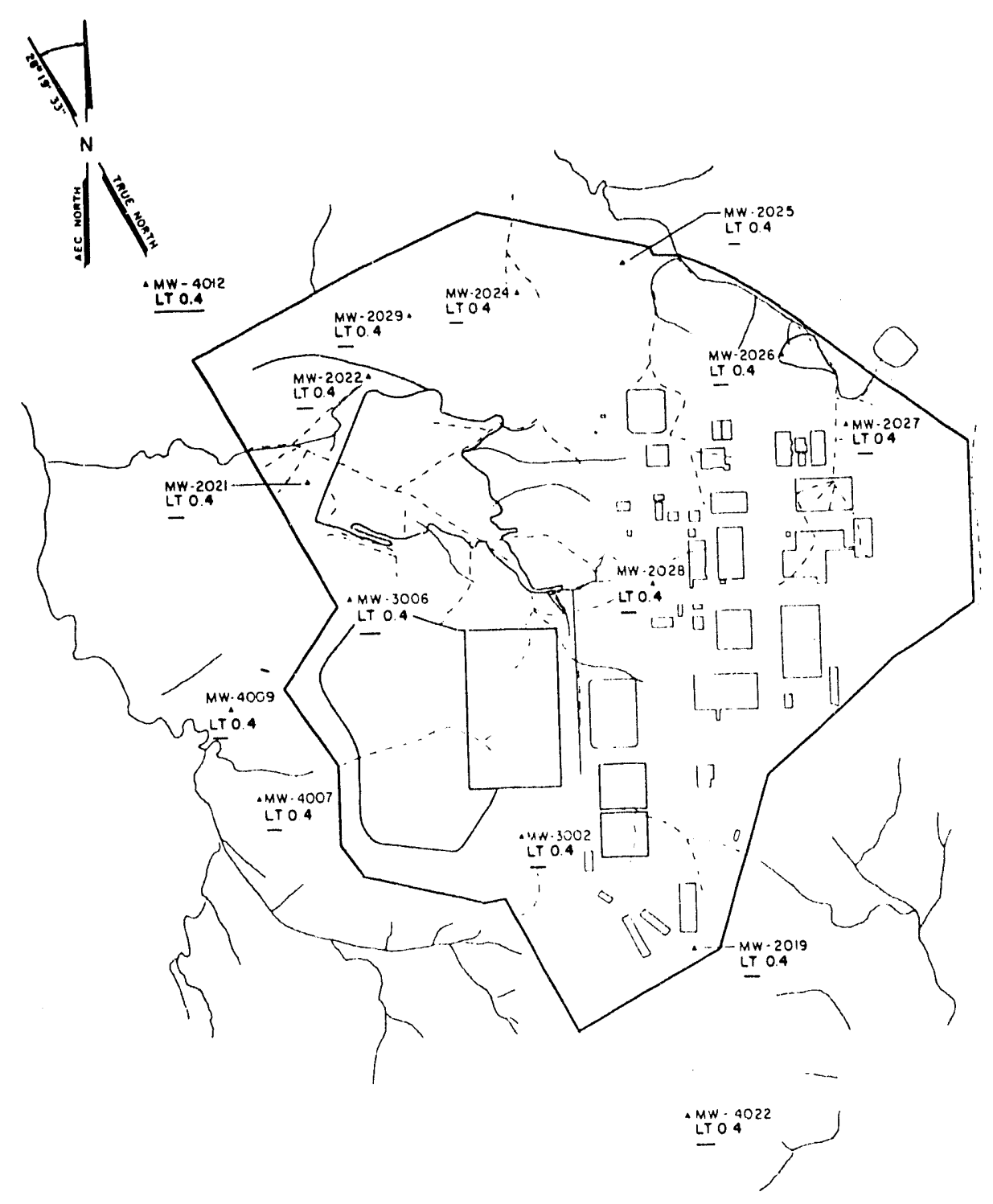

SCALE IN METERS

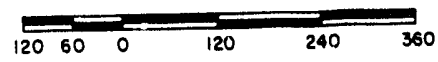

LEGEND

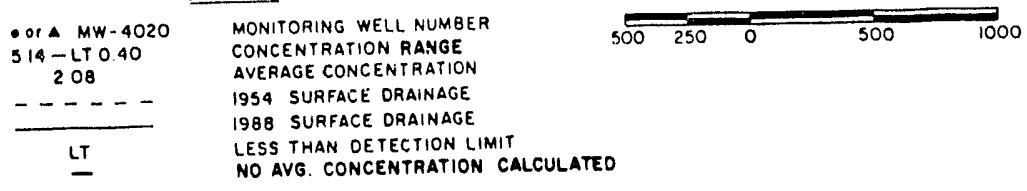

COMPETENT LIMESTONE

1,3 - DINITROBENZENE CONCENTRATIONS (ug/l)

FIGURE 5.4-19

\begin{tabular}{|c|c|c|c|c|c|}
\hline REPOORT NO.: & \multicolumn{2}{|c|}{ DOE/OR/21548-074 } & EXHIBIT NO.: & \multicolumn{2}{|c|}{ AVCP/266/1191 } \\
\hline ORIGIMATOR: & BLG & DRAWN BY: & GLN & & $11 / 91$ \\
\hline
\end{tabular}




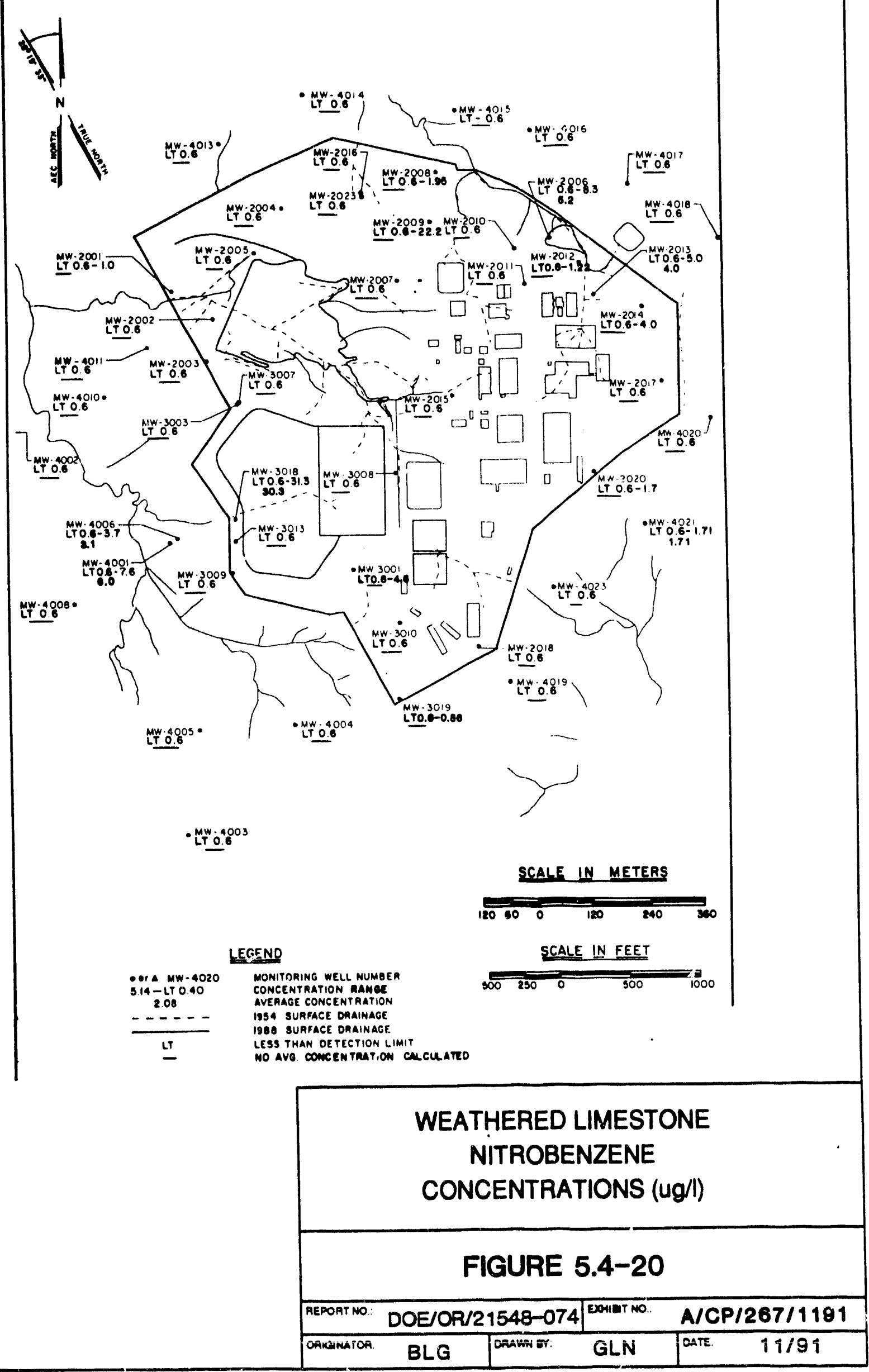



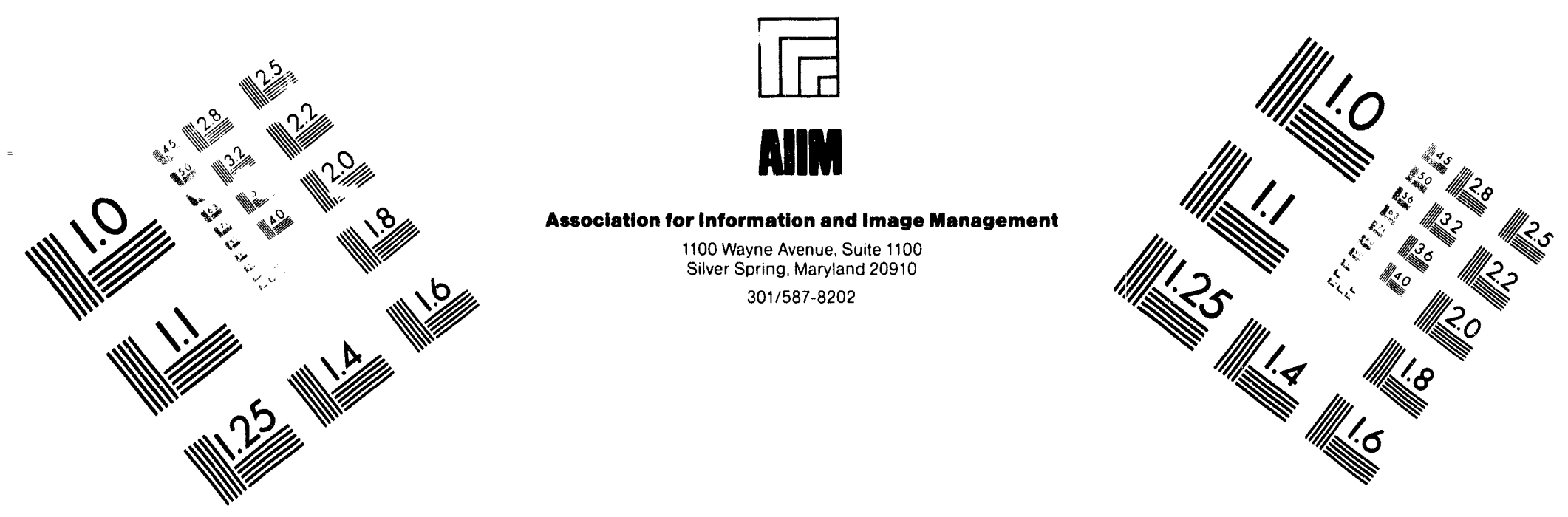

\section{Centimeter}

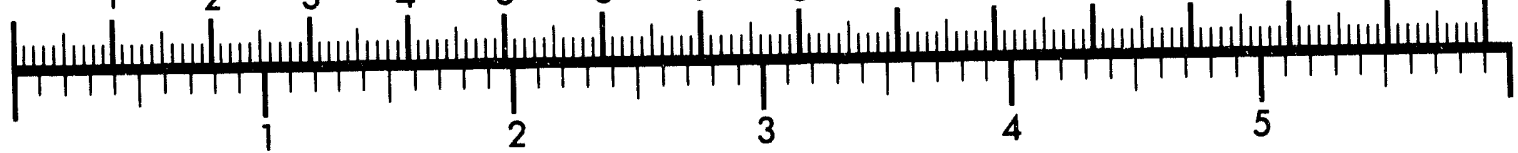

Inches
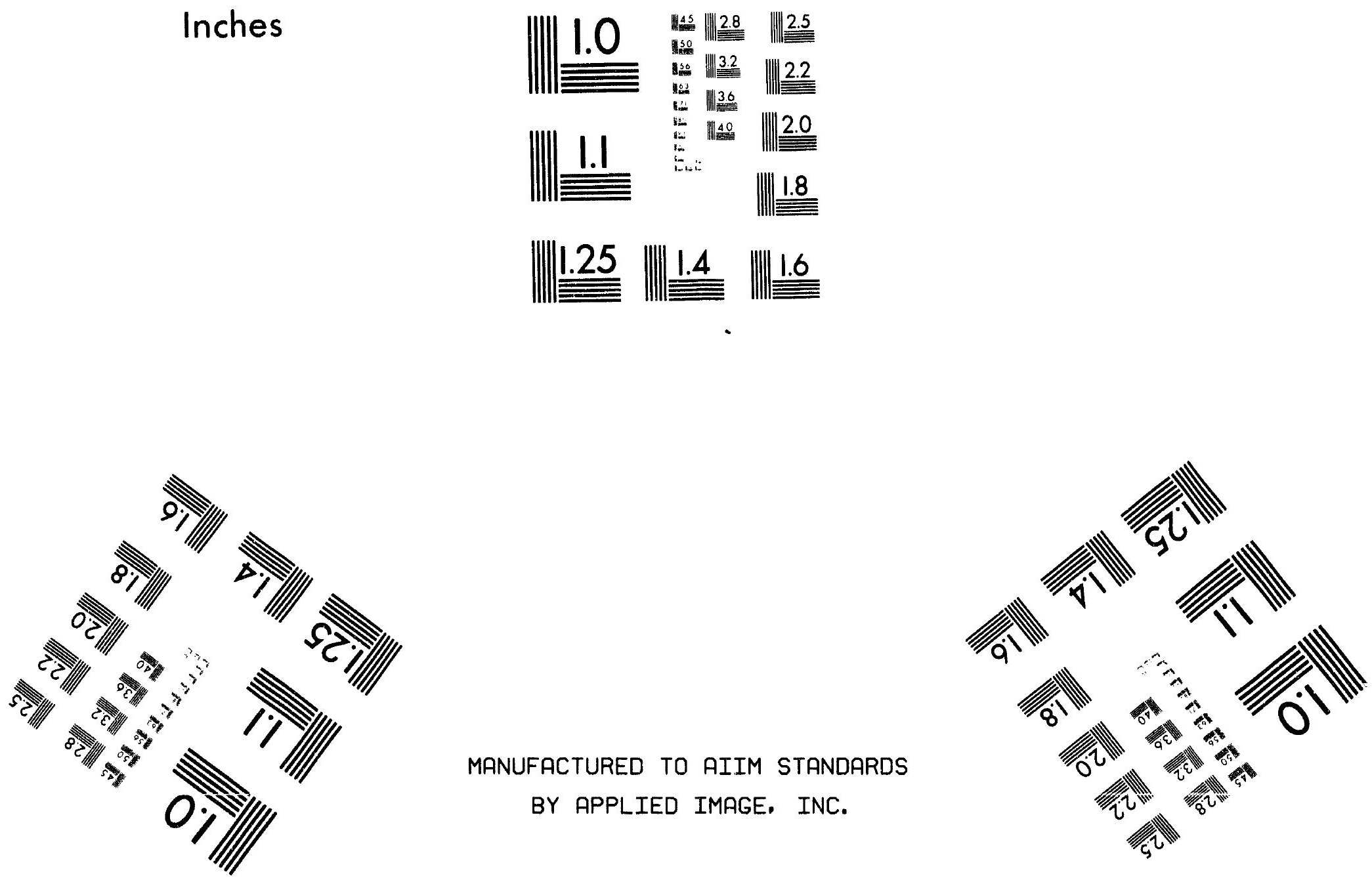

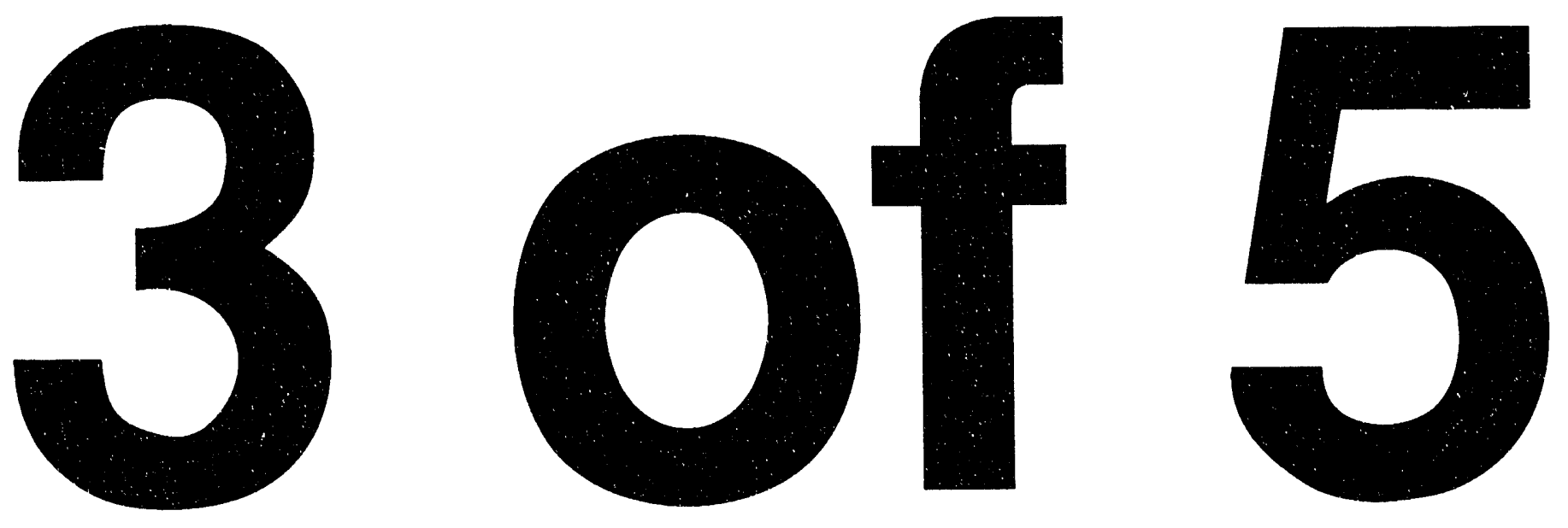


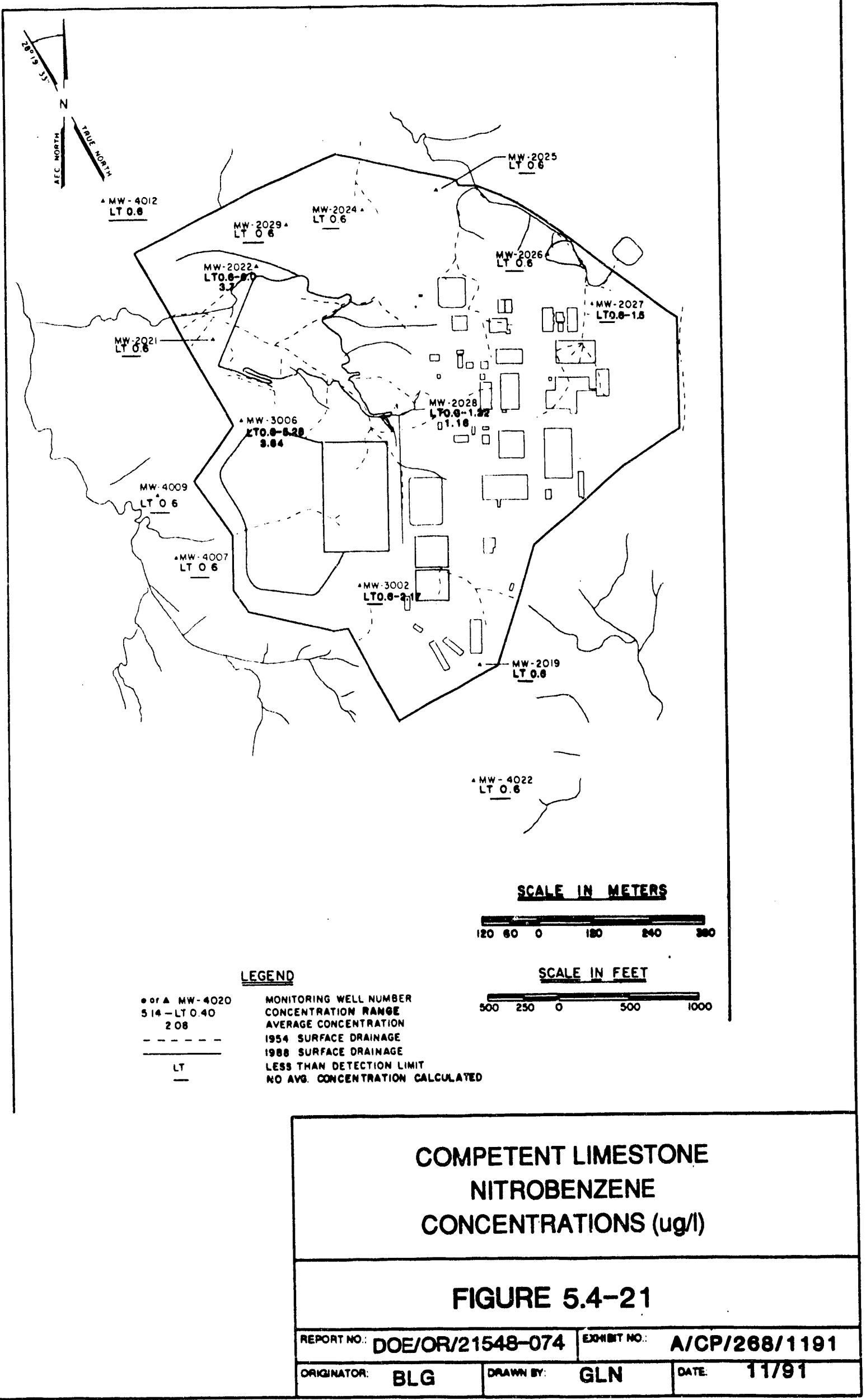




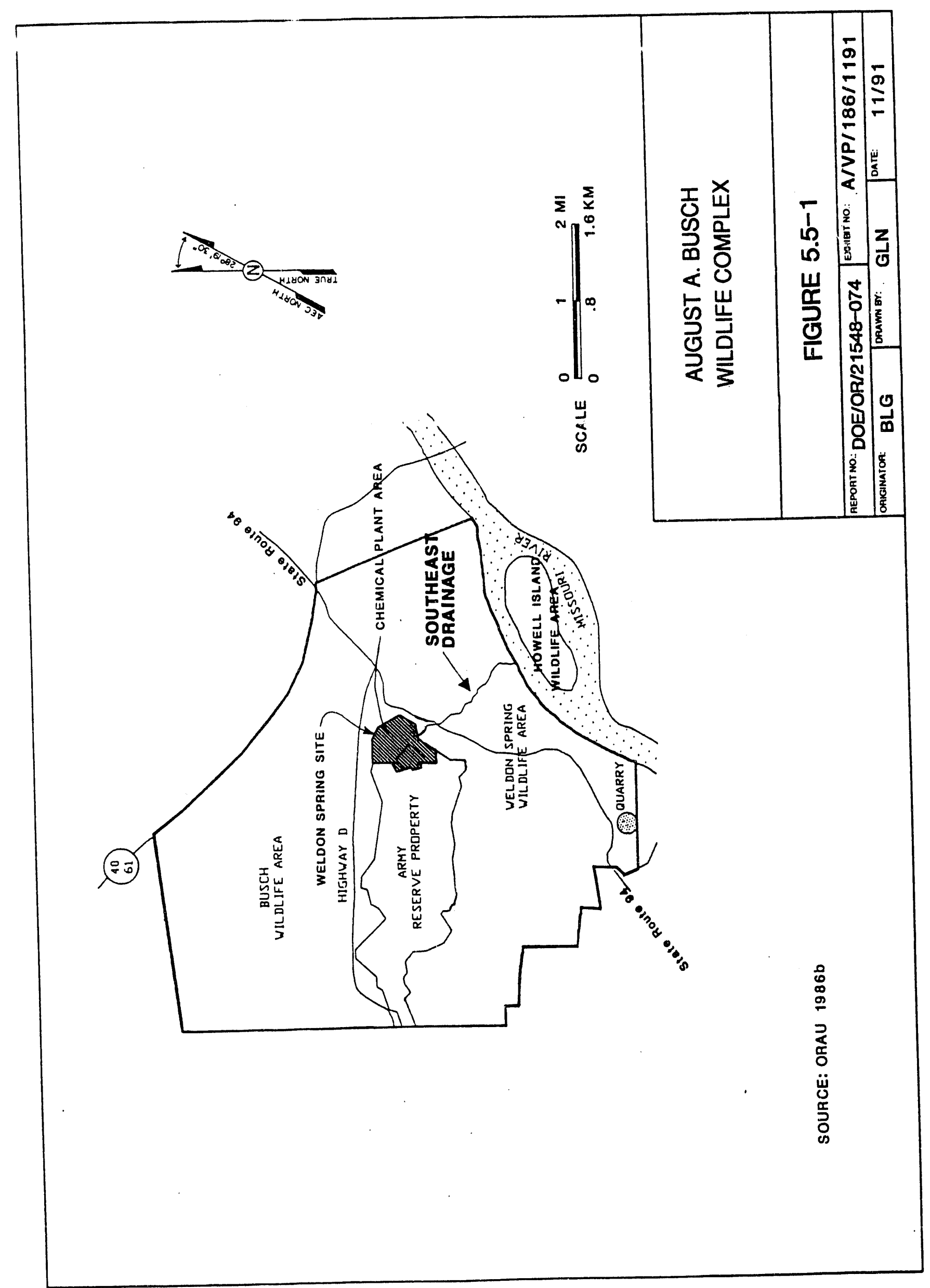




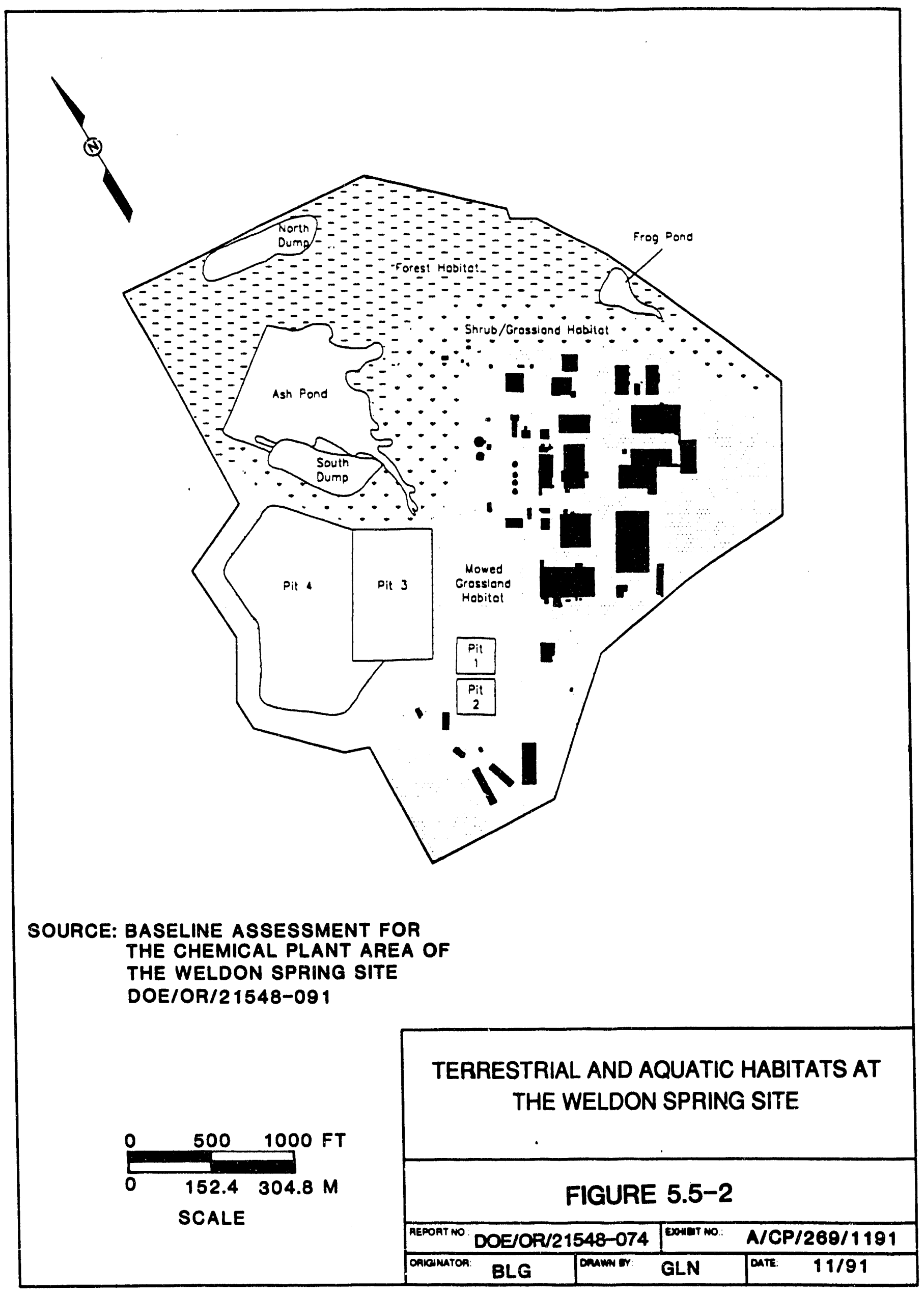




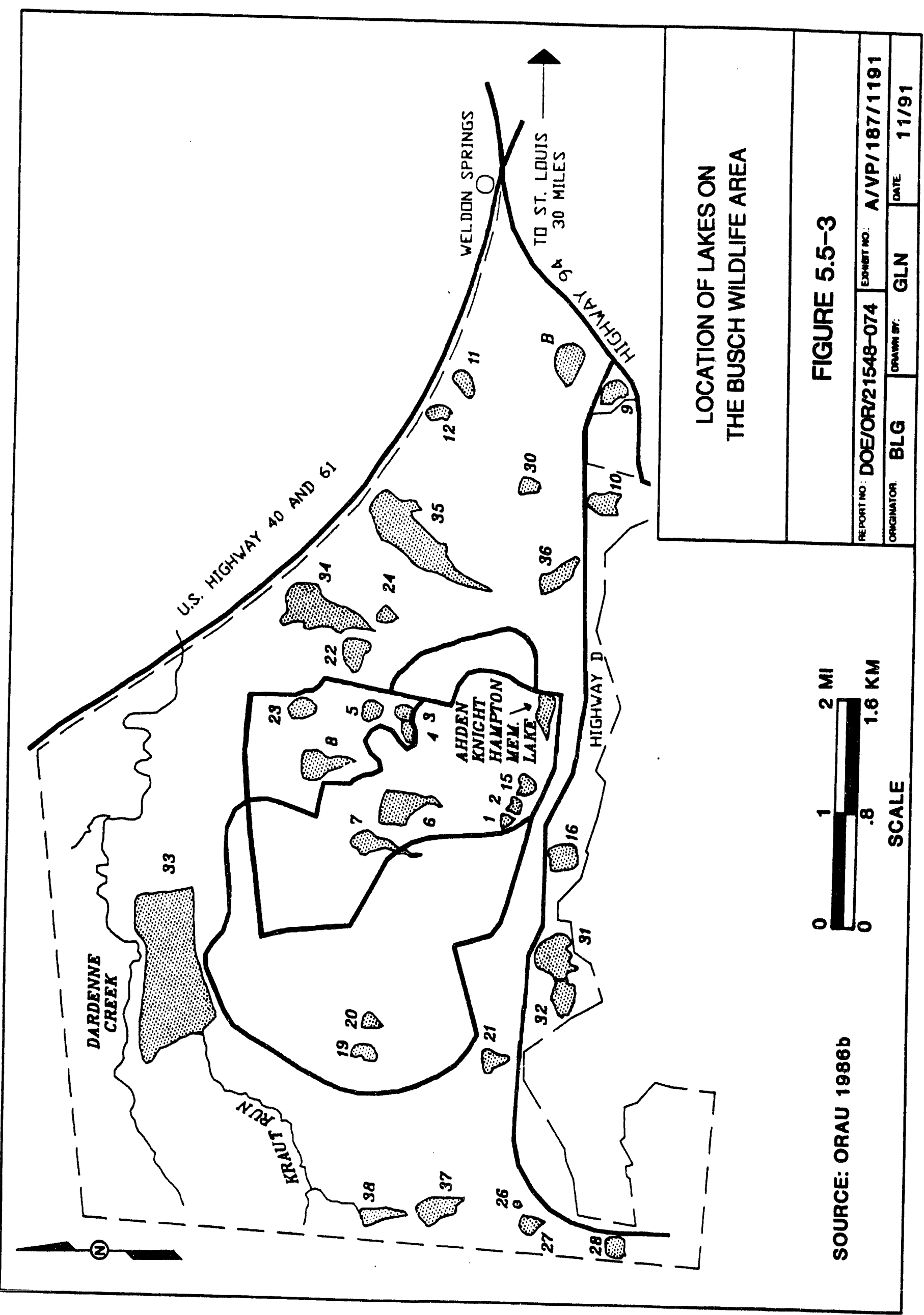




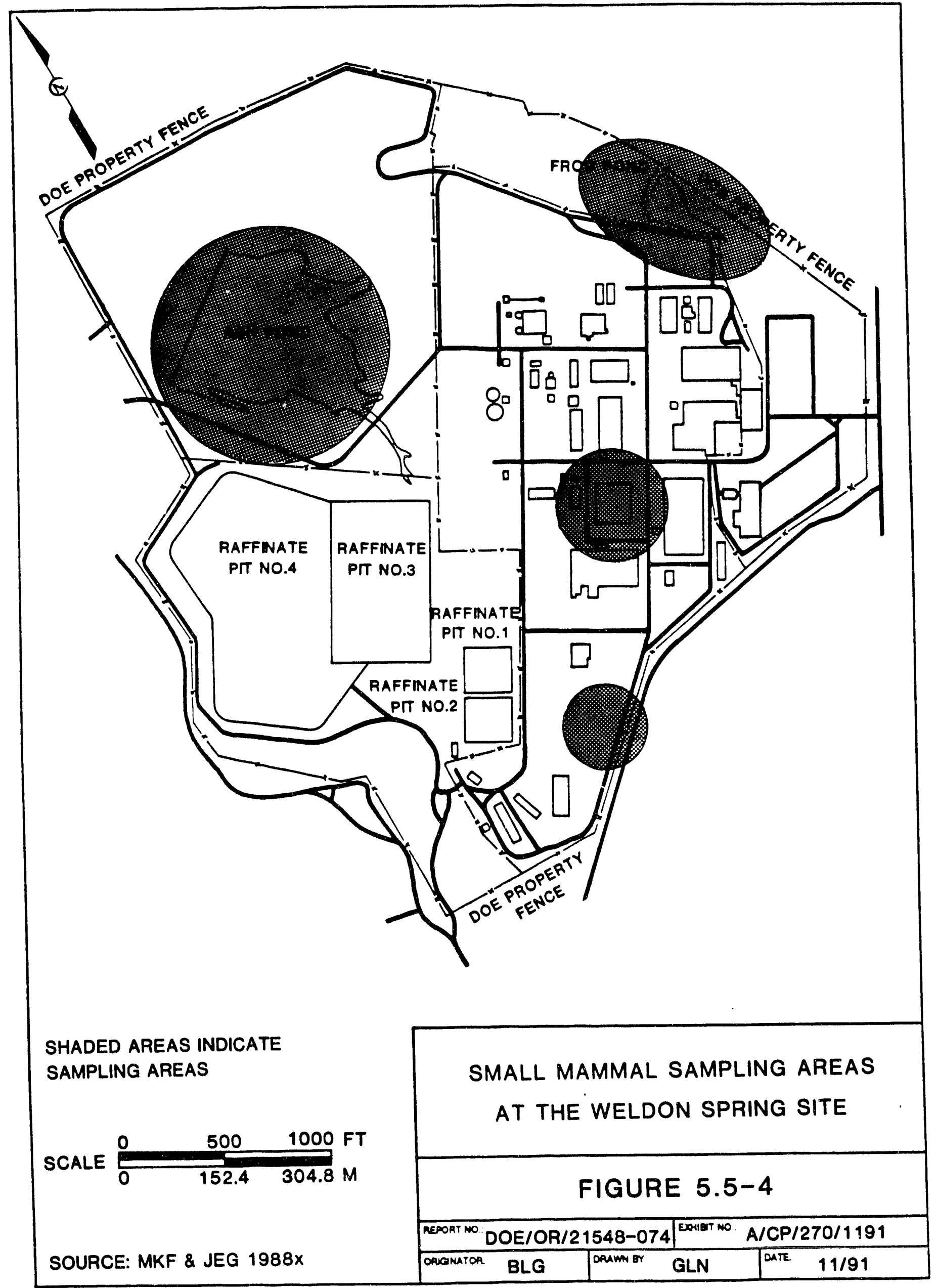




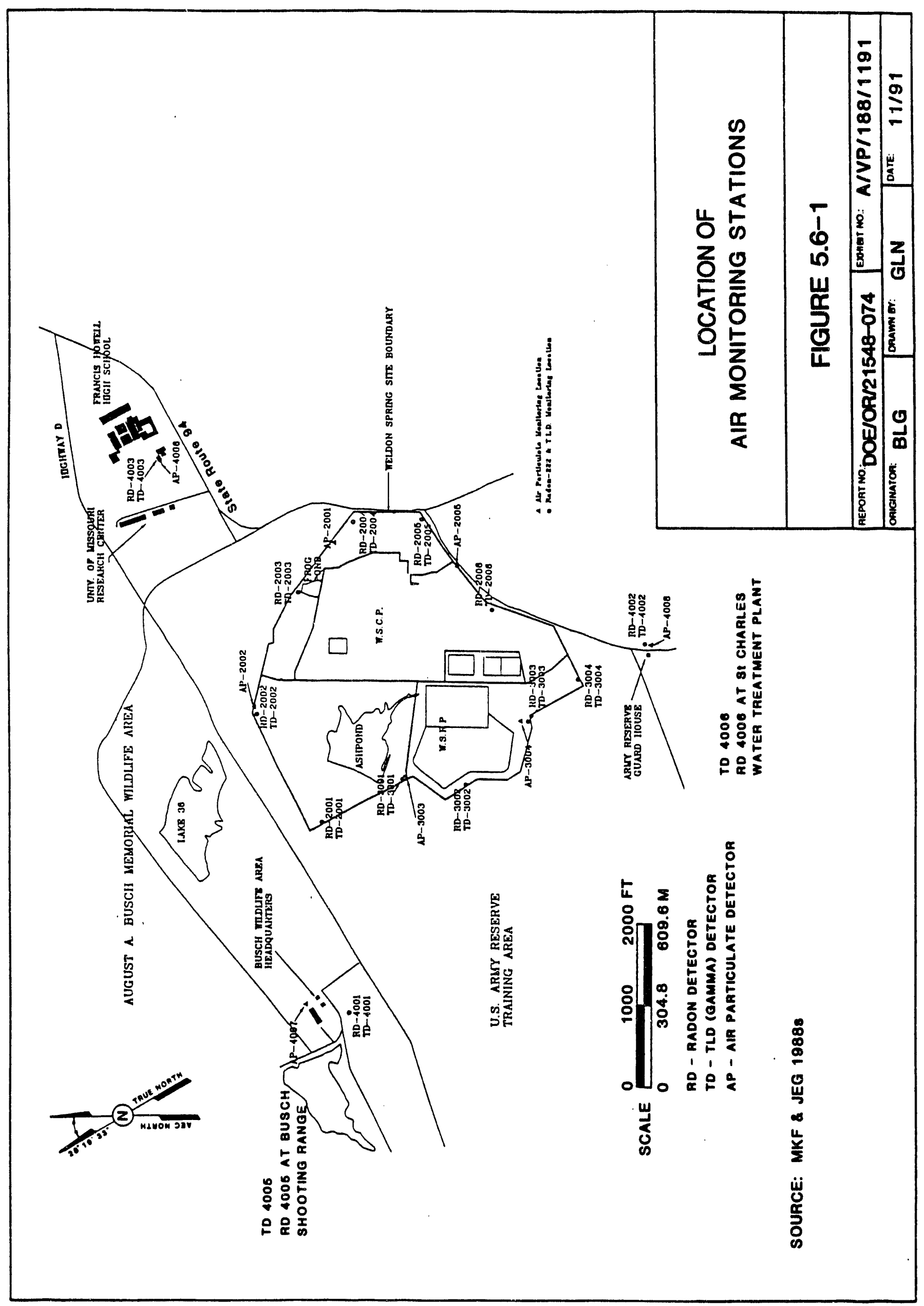




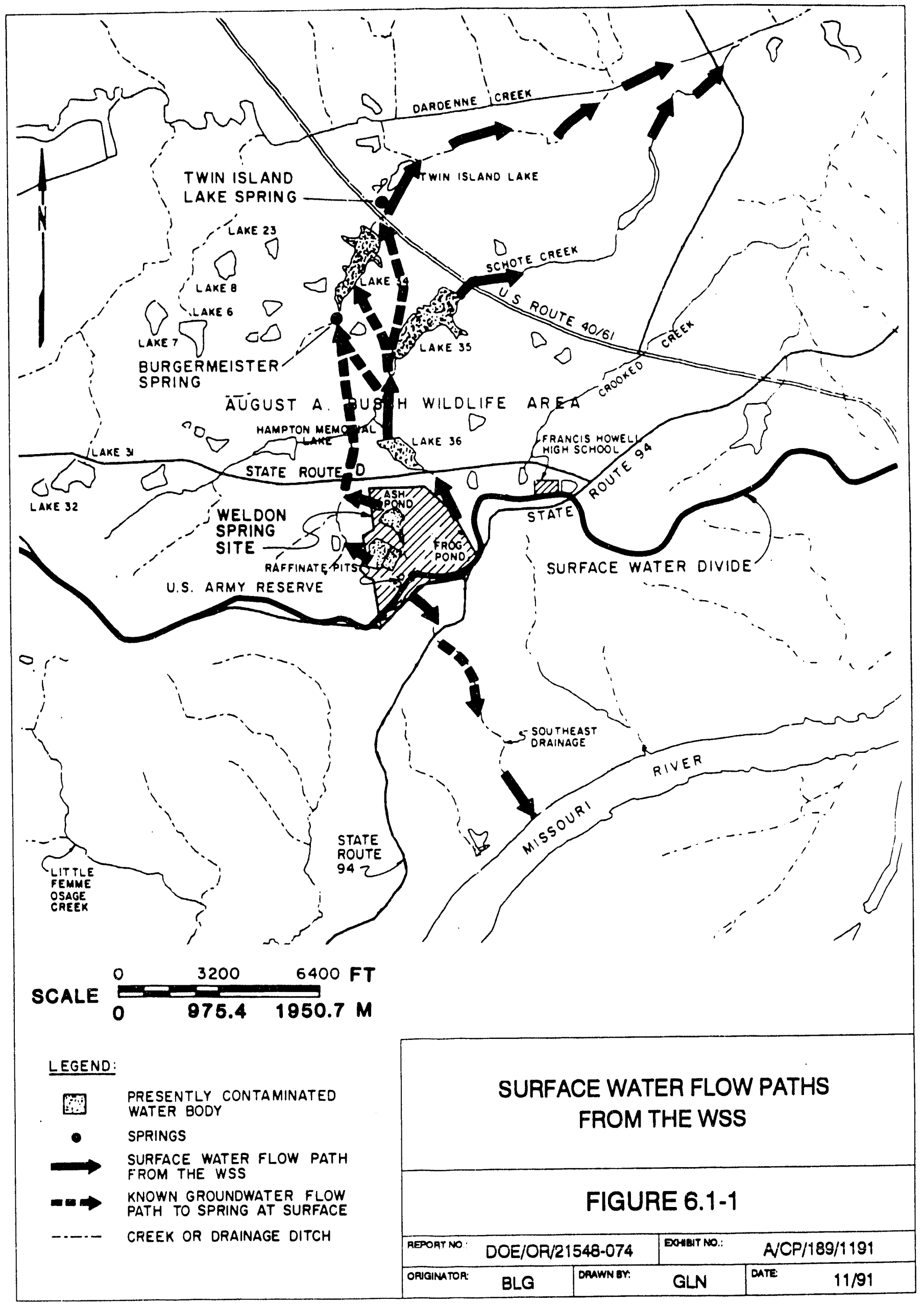




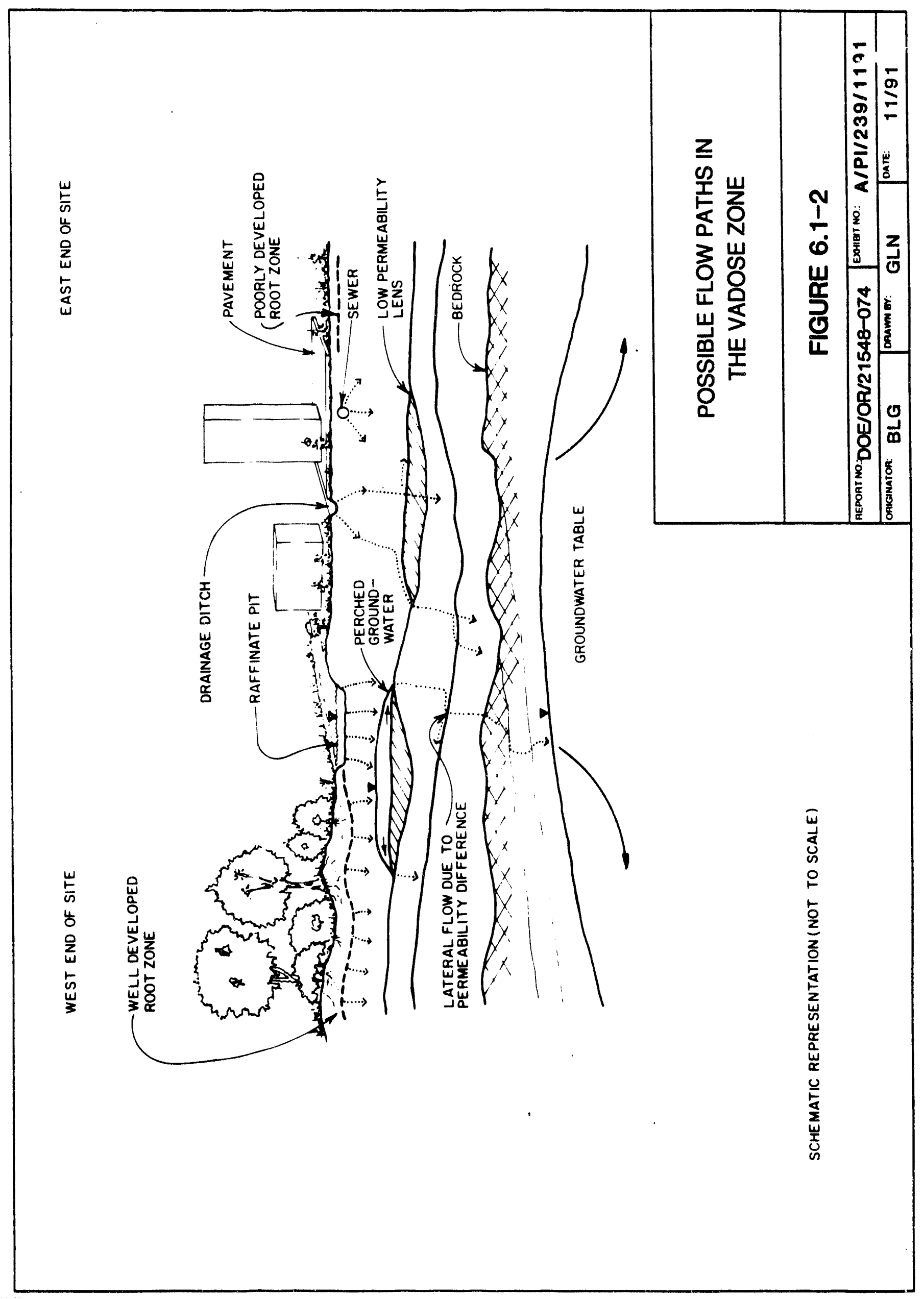



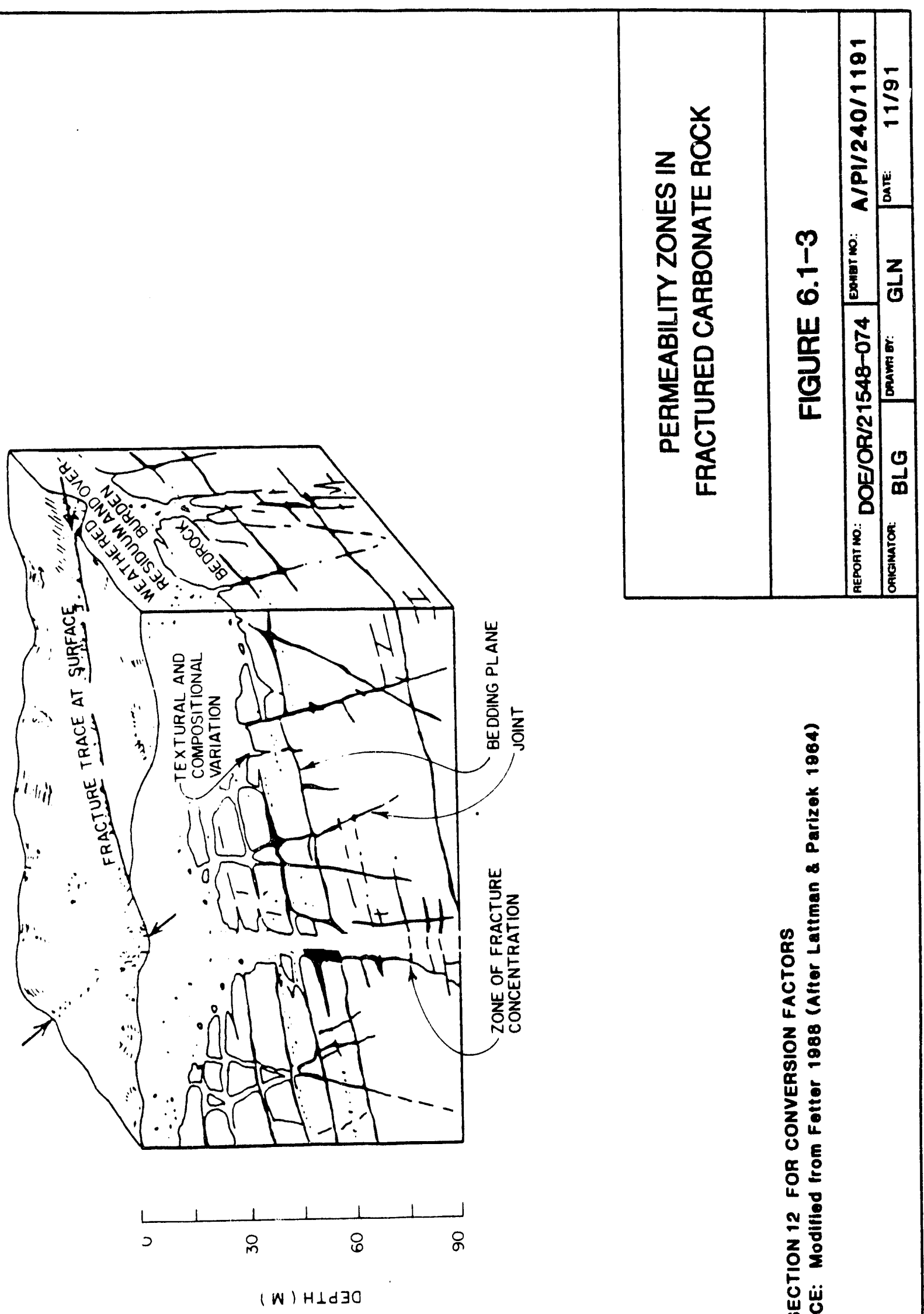


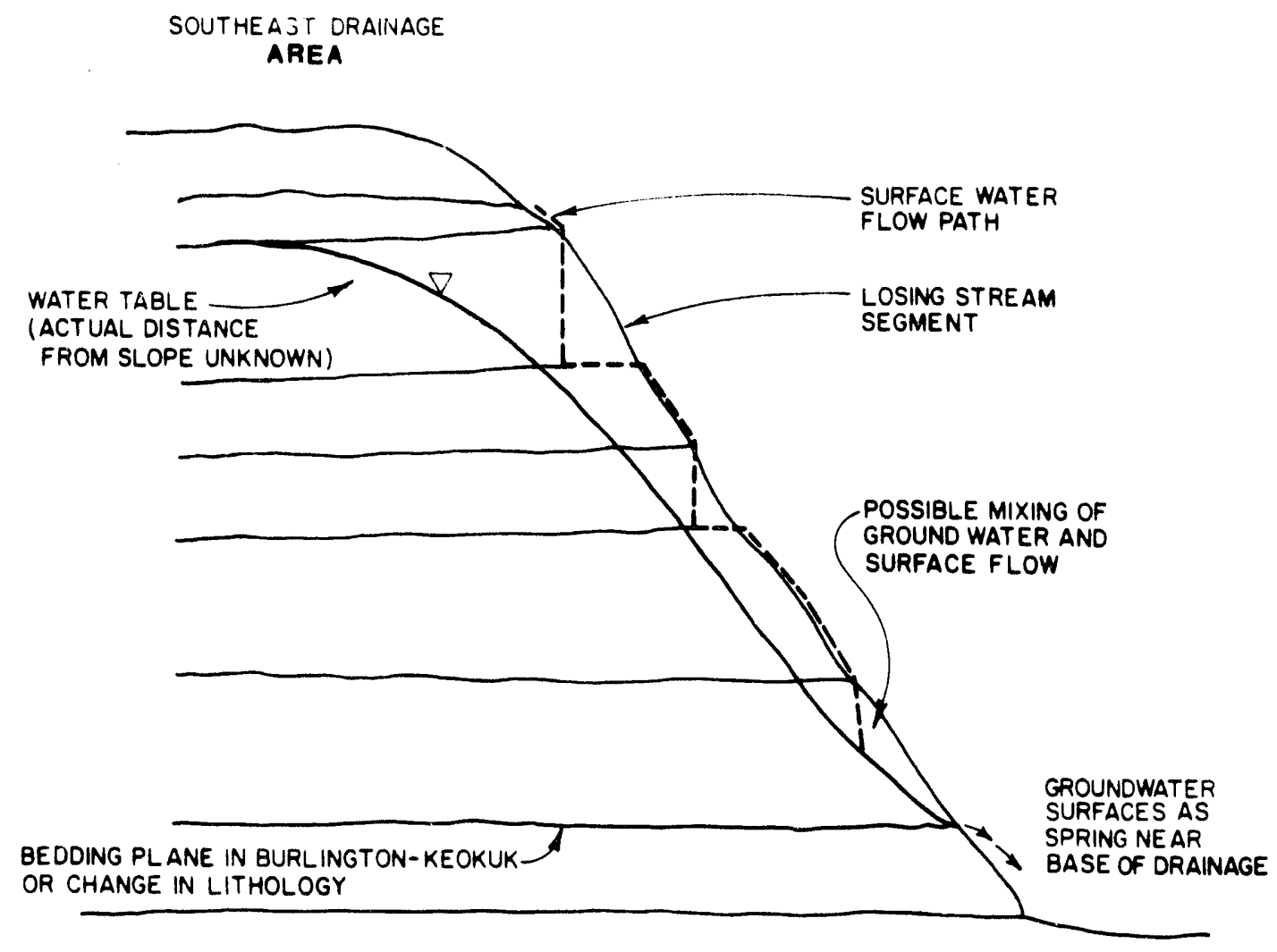

SCHEMATIC SKETCH (NOT TO SCALE)

\section{SURFACE WATER - GROUNDWATER RELATIONSHIP ALONG S.E. DRAINAGE EASEMENT}

FIGURE 6.1-4

\begin{tabular}{|c|c|c|c|}
\hline AEPOAT NO & $D O E / O R / 21548-074$ & EXIEAT NO. & $A / P I / 241 / 1181$ \\
\hline OALGIMATOR & \begin{tabular}{l|l} 
BLG & Daamin or:
\end{tabular} & GLN & $11 / 91$ \\
\hline
\end{tabular}



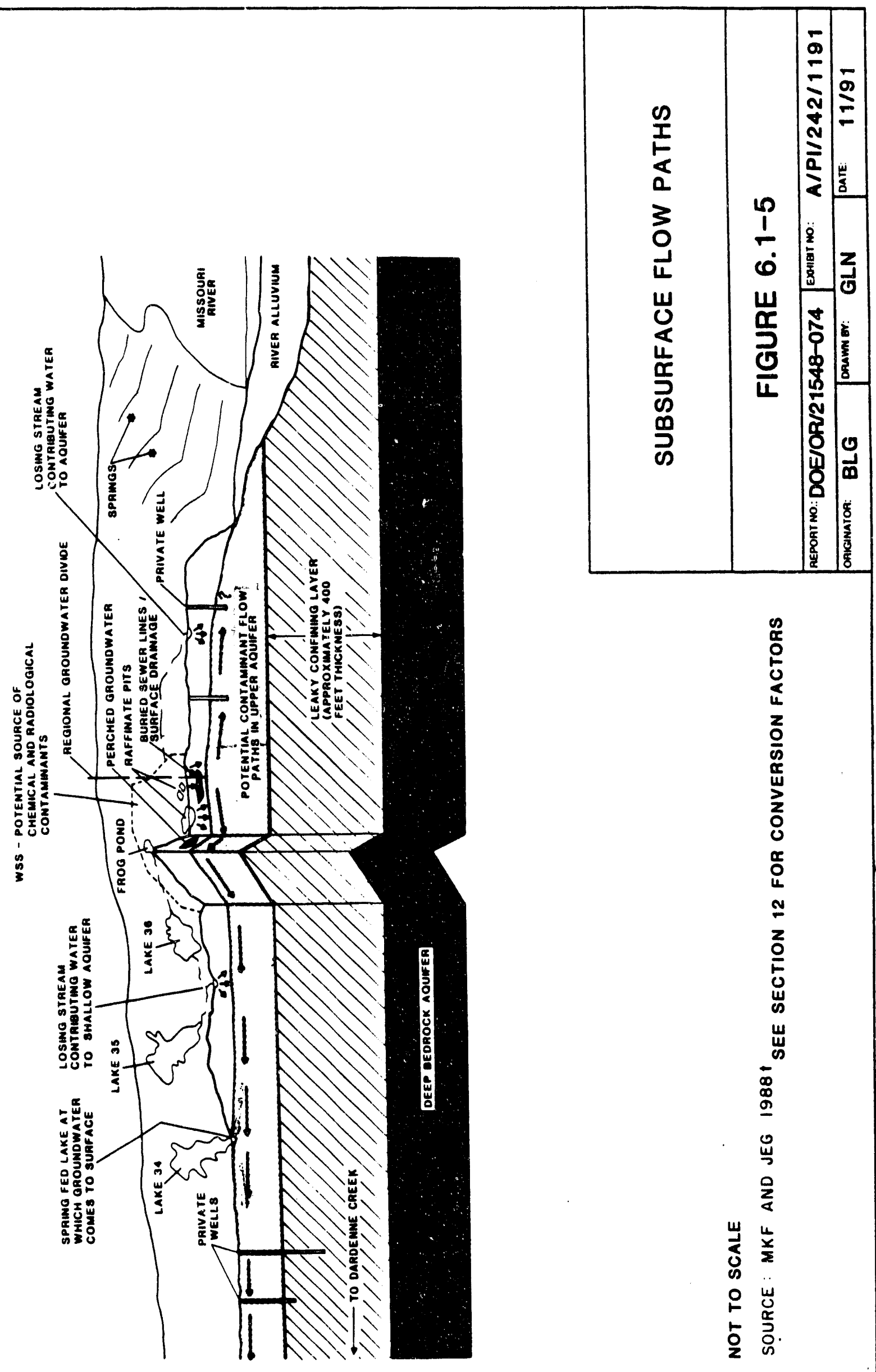


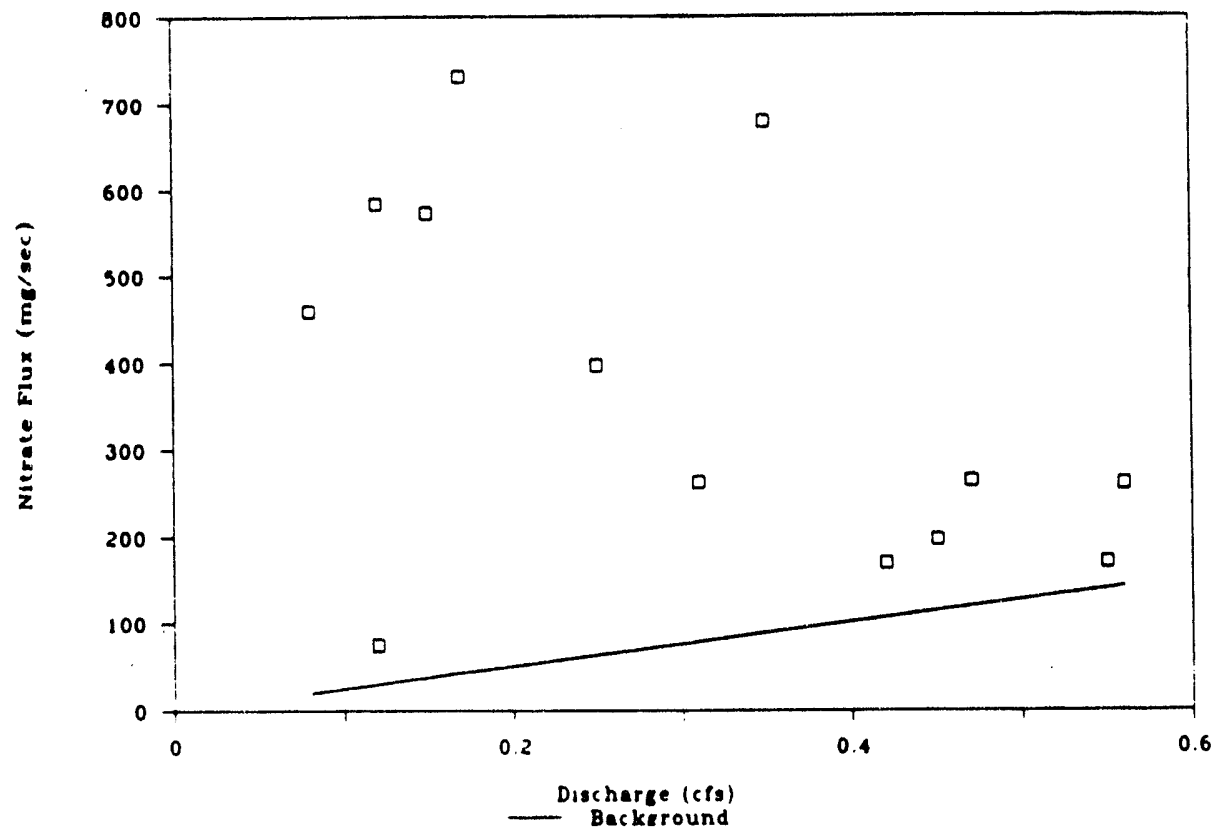

BEE BECTION 12 FOR CONVEABION FACTOR8

NITRATE FLUX AS A FUNCTION OF DISCHARGE AT BUI:GERMEISTER SPRING

FIGURE 6.3-1 REPOAT NO: COE/OR/21548-074 ORIONATOR: BLG onamer ar: GLN A/PI/243/1181 Date: 11/91 


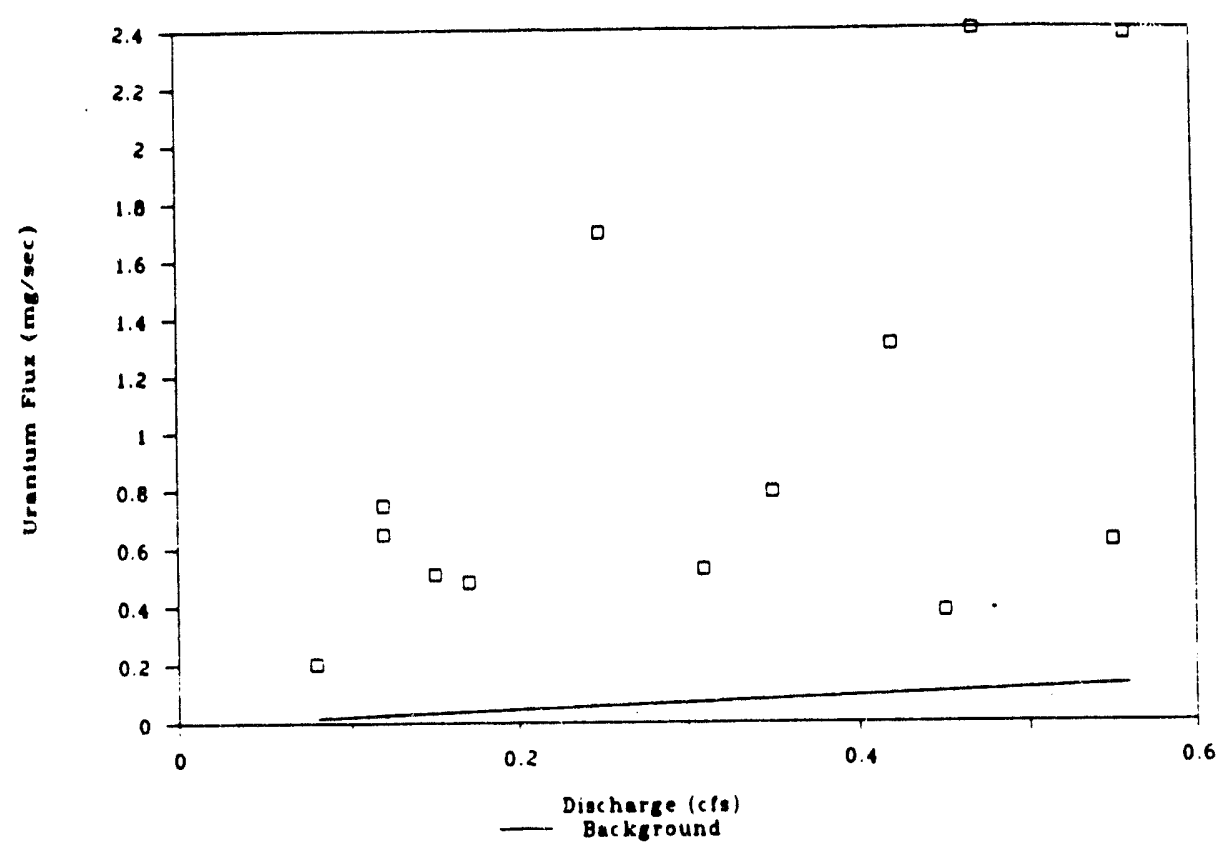

SEE SECTION 12 FOR CONVERBION FACTORS

\section{URANIUM FLUX AS A FUNCTION OF} DISCHARGE AT BURGERMEISTER SPRING

FIGURE 6.3-2

\begin{tabular}{|c|c|c|c|c|}
\hline AEPOAT NO: & DOEJOA & $548-074$ & Eotuet no. & $A / P 1 / 244 / 1101$ \\
\hline DRlamator: & BLG & omanner: & GLN & Dare: $\quad 11 / 91$ \\
\hline
\end{tabular}


REMEDIAL INVESTIGATION REPORT

TABLES 


\section{[IIn!}

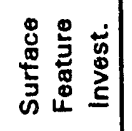

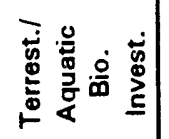

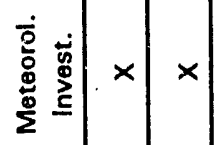

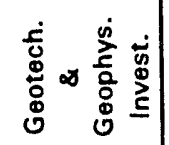

咅毫 $\times \times$

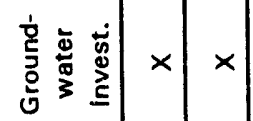

$\times$

$\times \times$

$\times \times \times$

$\times$

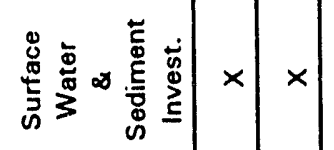

$\times$

$\times x \times$

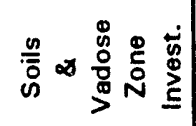

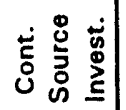

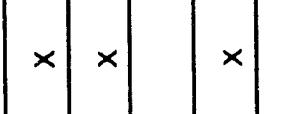

$\times$

$+$

$\times \times x$

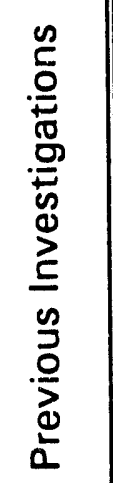
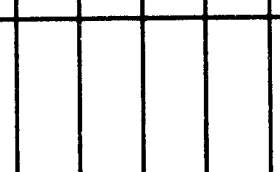


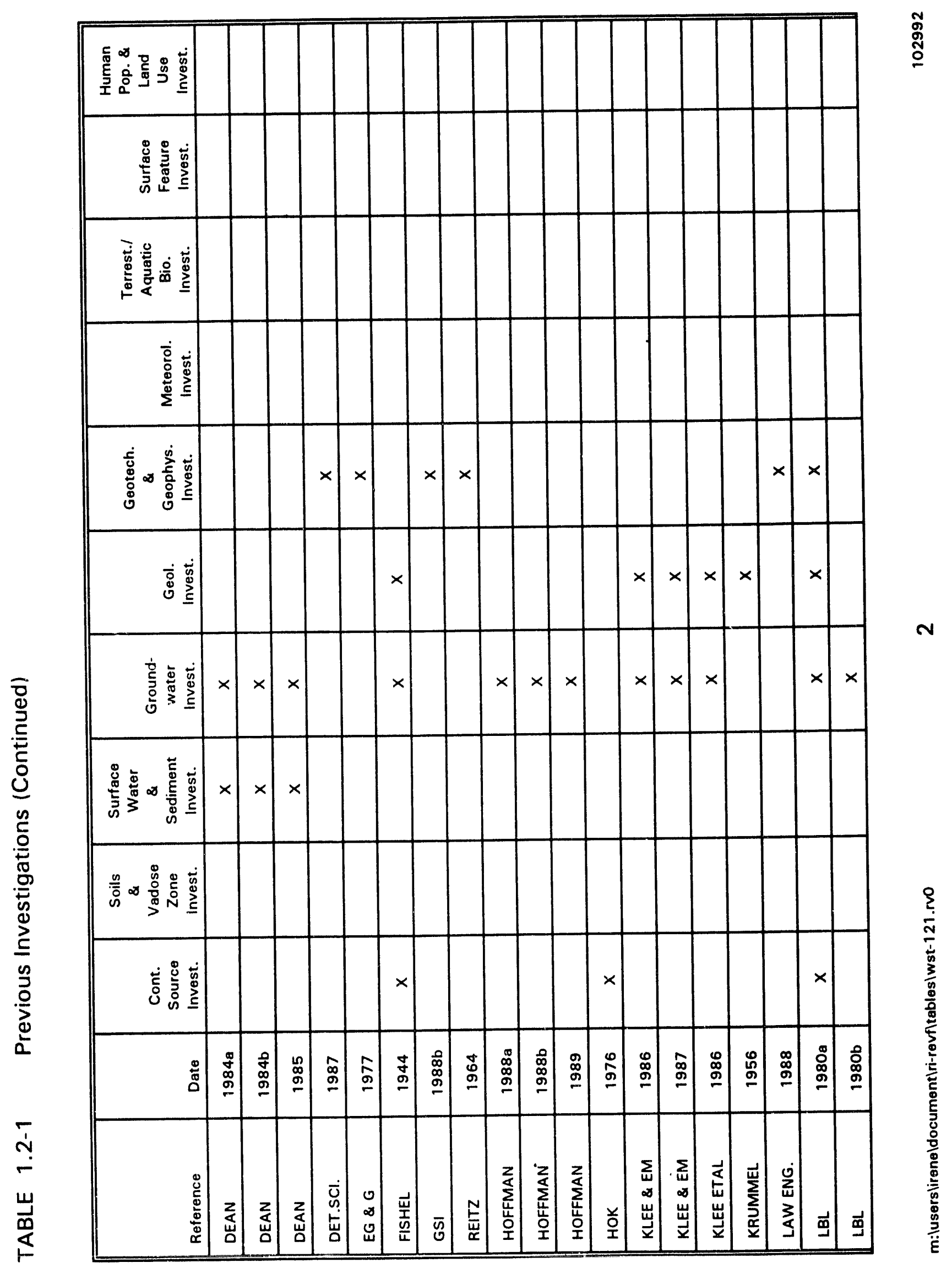




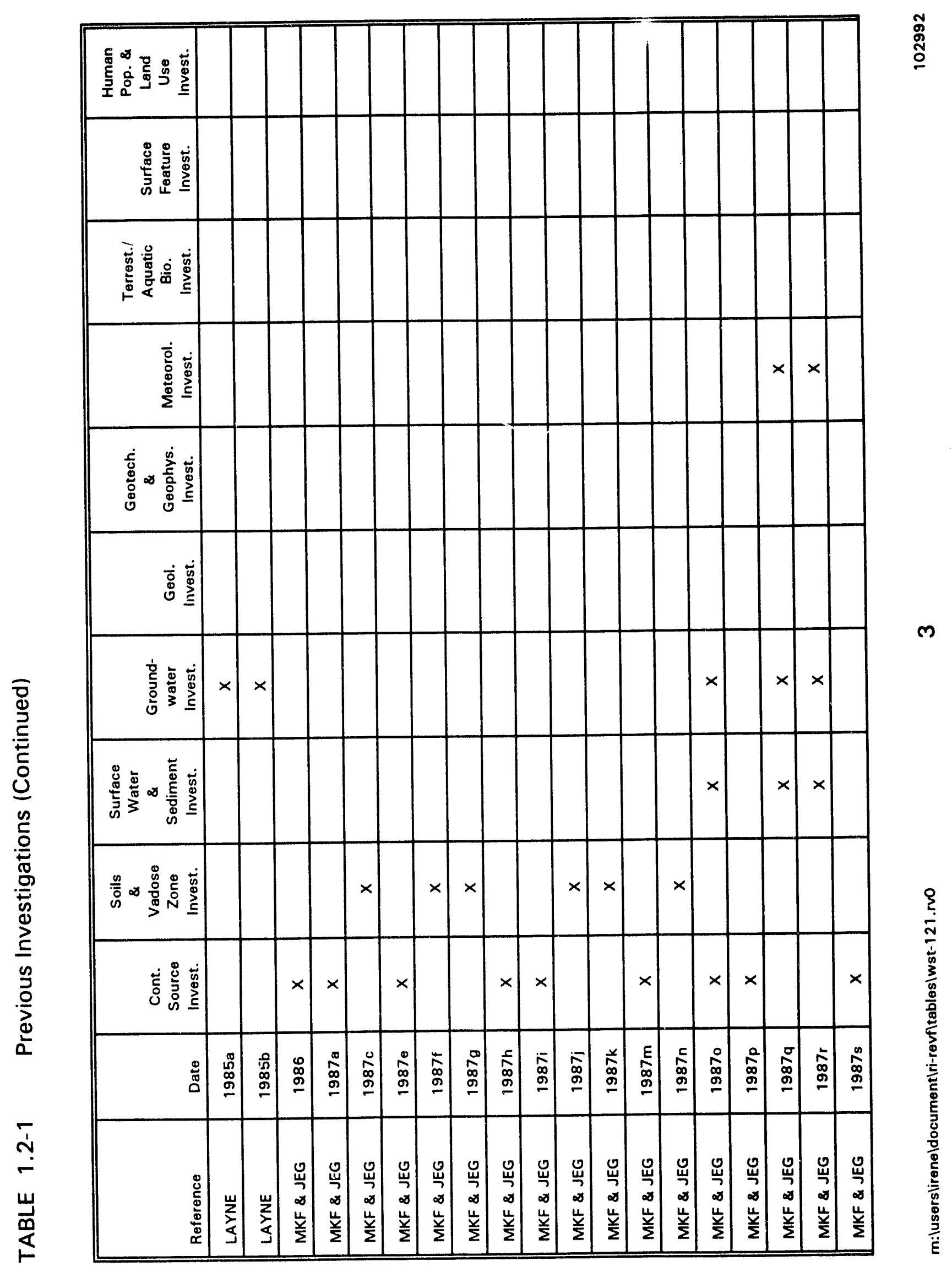




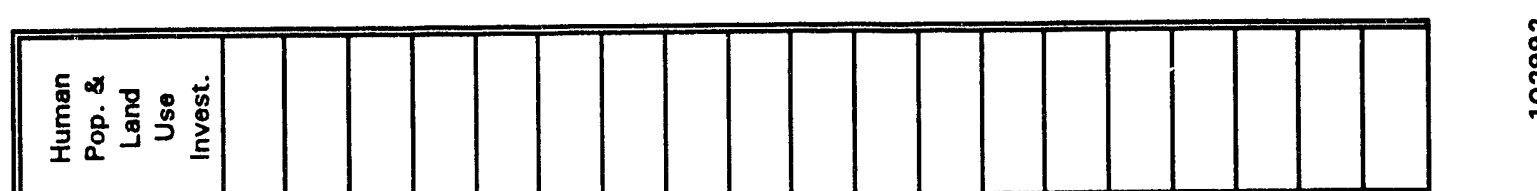

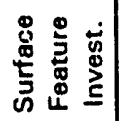

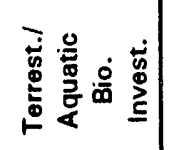

部

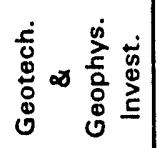

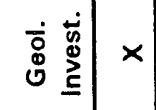

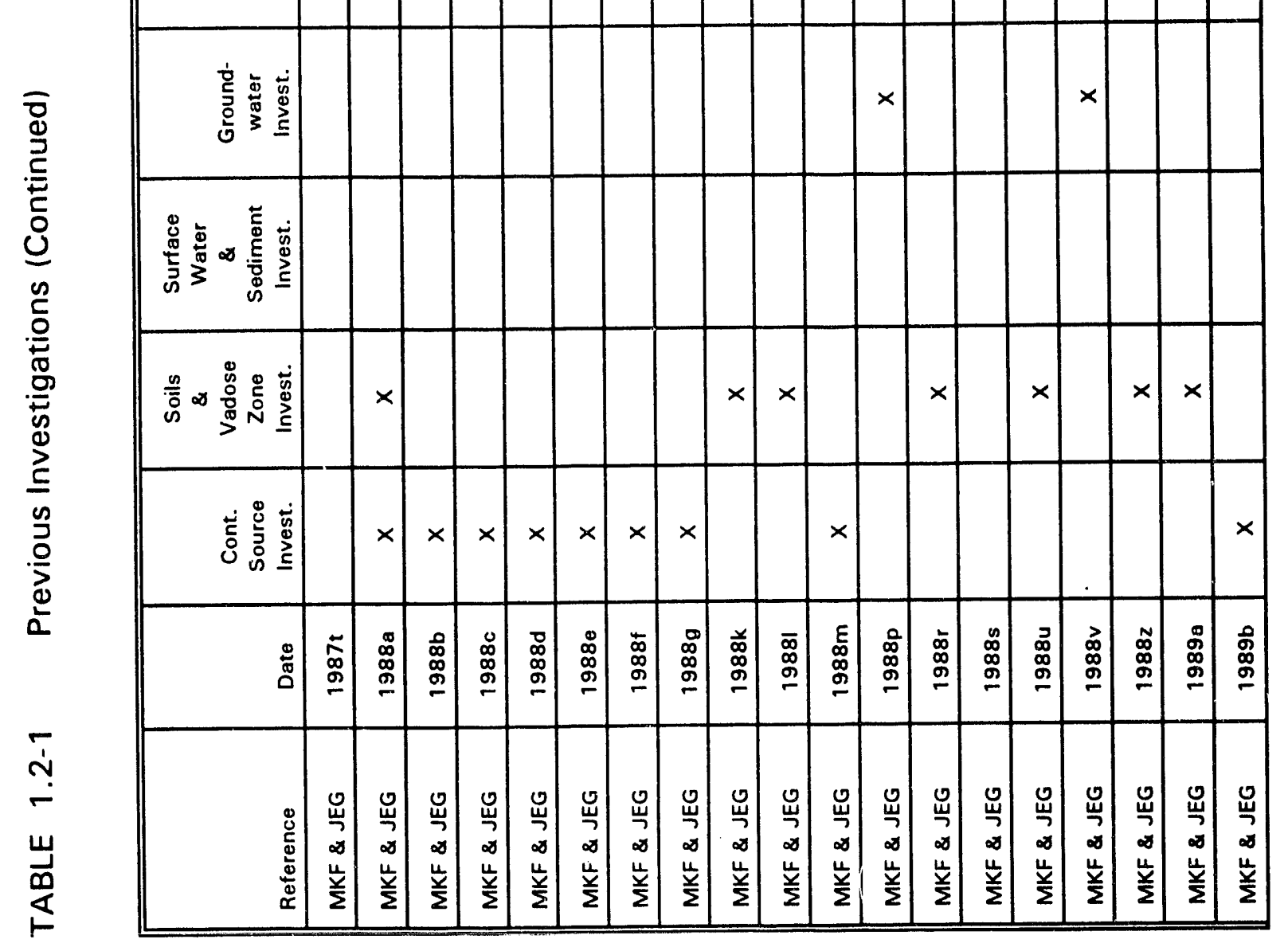

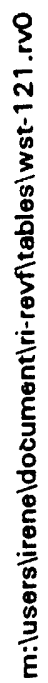




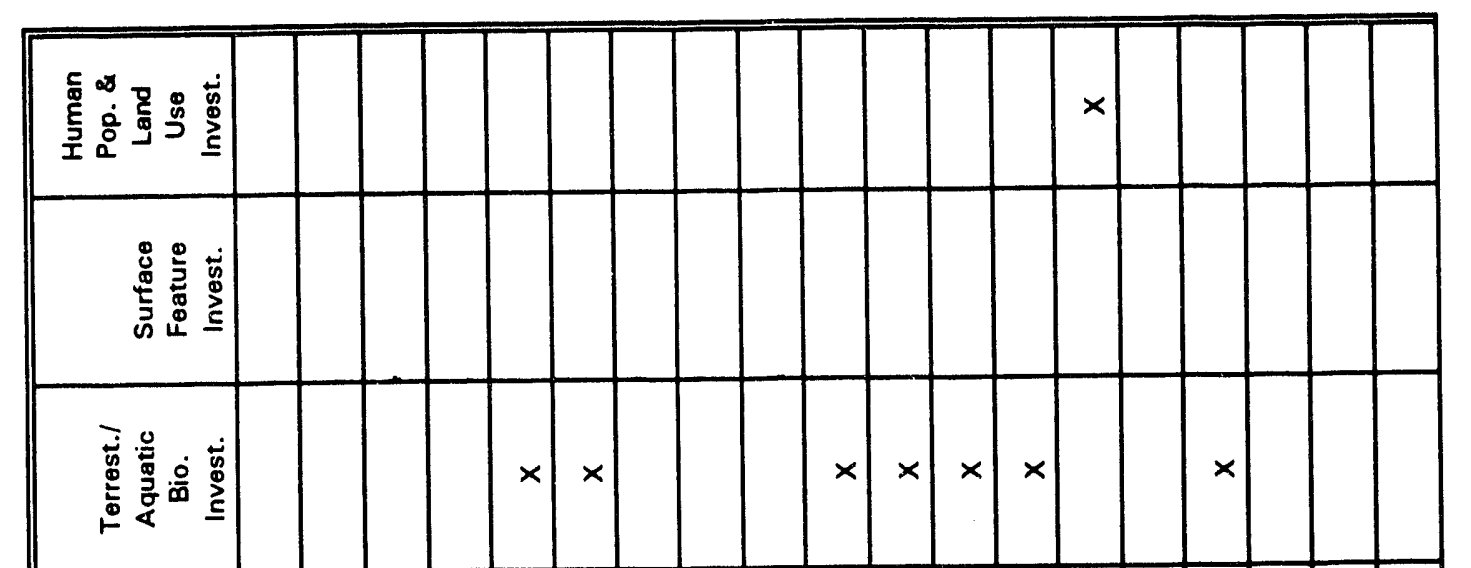

จั

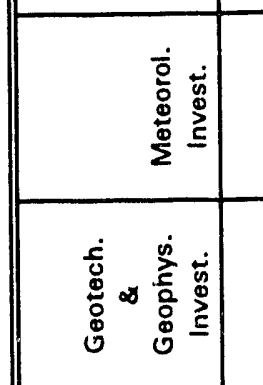

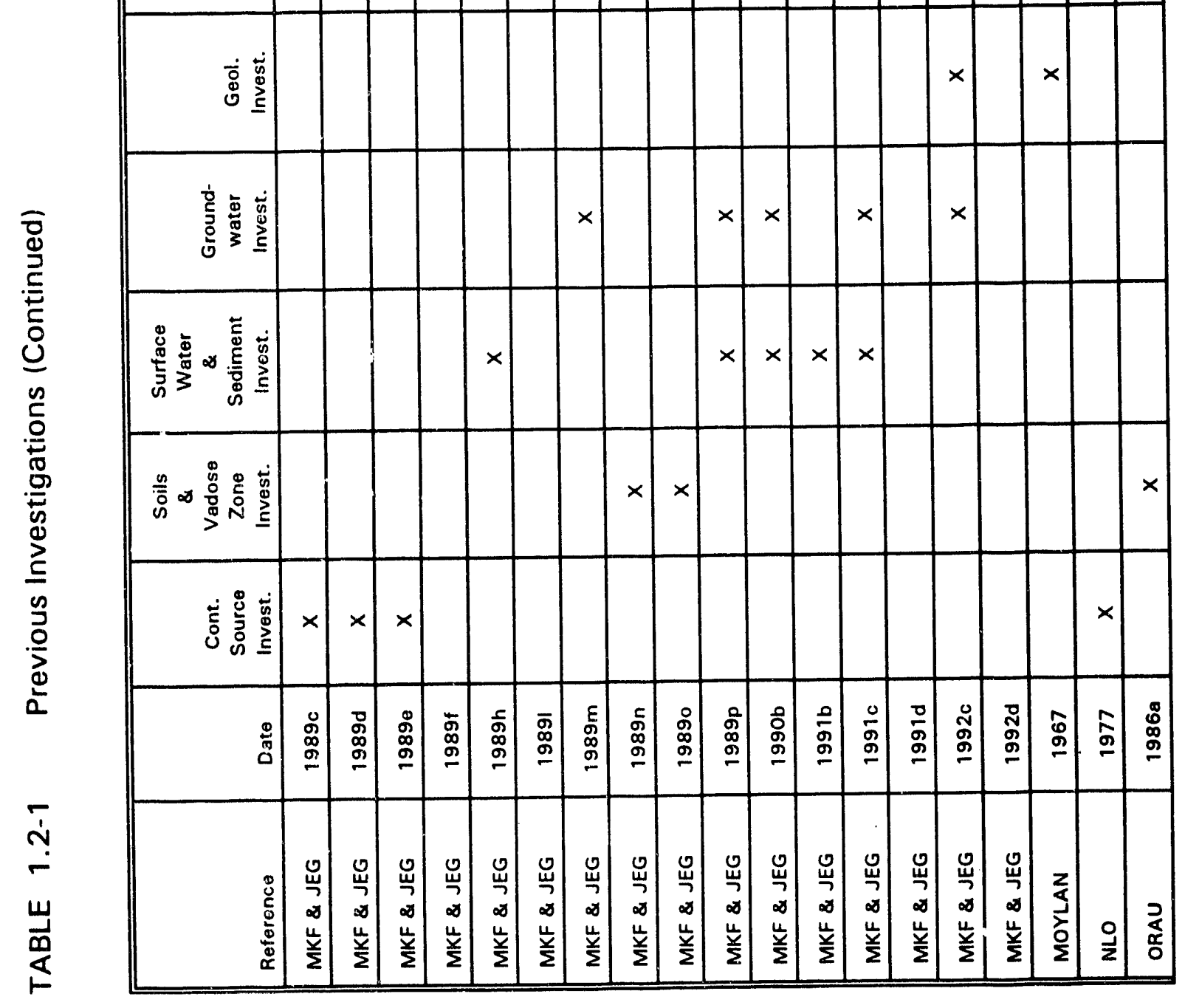

เ

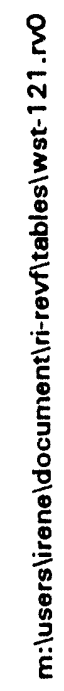




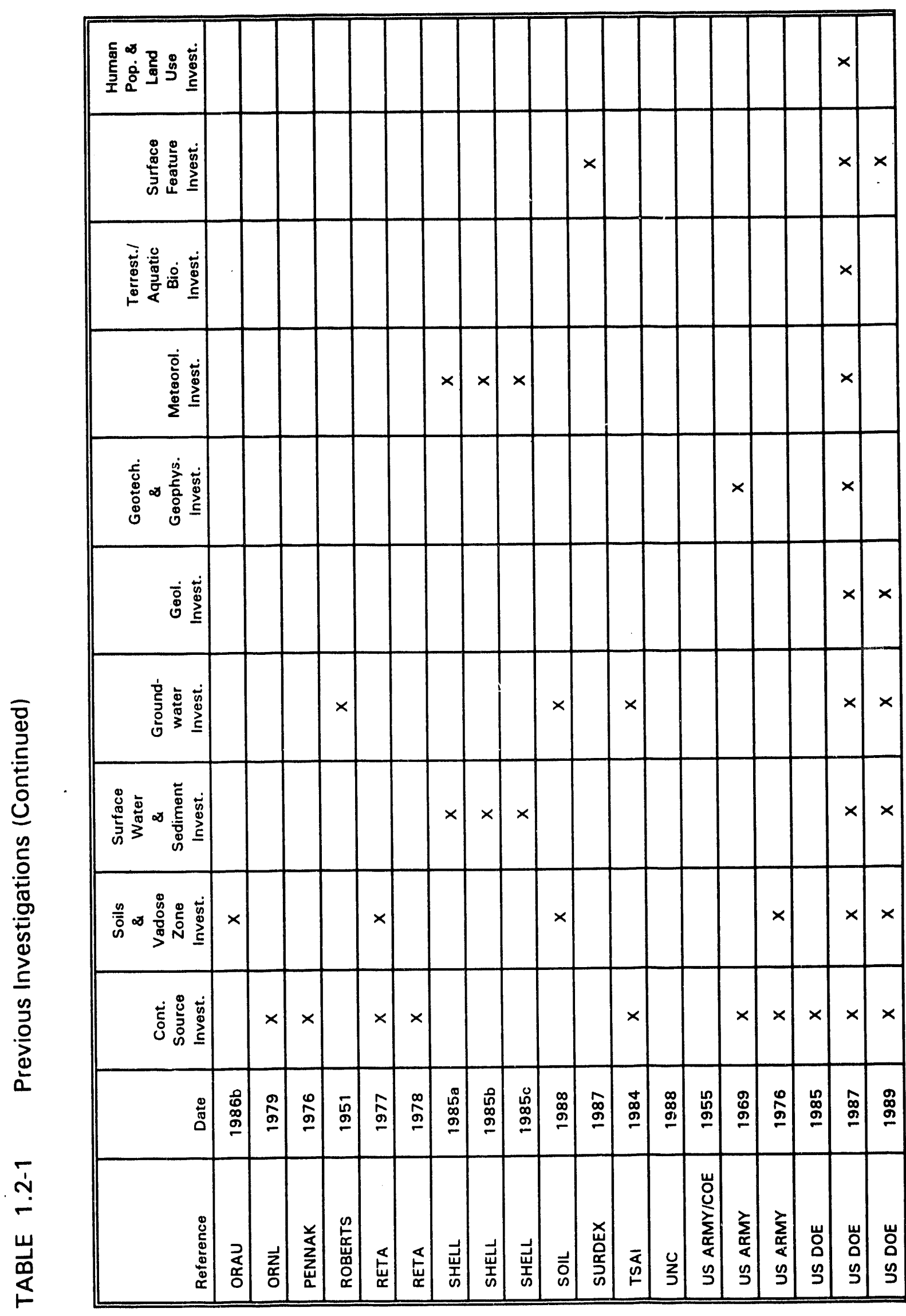




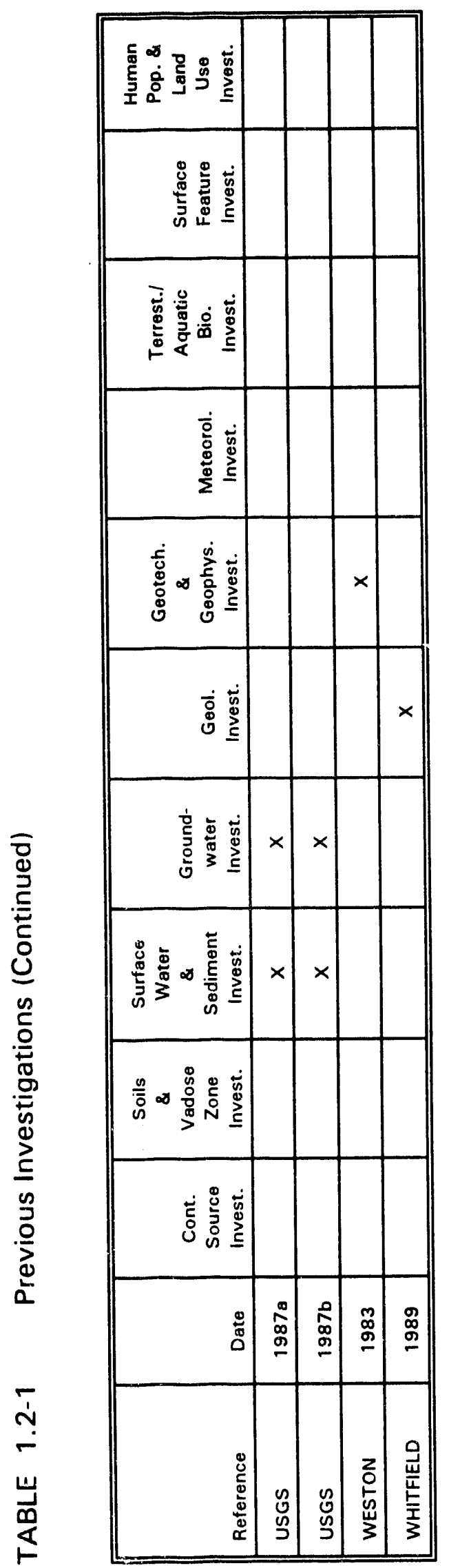

ฟั
ฟิ
o

$N$

ב⿱ 


\section{TABLE 1.2-2 Interim Response Actions (IRAs)}

1 REMOVE AND DISPOSE OF ELECTRICAL TRANSFORMERS AT THE SITE. APPROXIMATELY 49,205 L 113,000 GAL) OF PCB FLUIDS AND FLUSHING SOLUTIONS WERE REMOVED AND TRANSPORTED TO AN OFF-SITE LICENSED INCINERATION FACILITY; THE FLUSHED UNITS WERE THEN TRANSPORTED OFF-SITE TO A LICENSED DISPOSAL FACILITY. ELEVEN NON-PCB TRANSFORMERS WERE ALSO REMOVED. THIS REMOVAL ACTION WAS COMPLETED IN JULY 1988.

2 CONSTRUCT A CONTROL DIKE TO ISOLATE SURFACE WATER FROM CONTACT WITH THE CONTAMINATED AREAS OF THE SOUTH DUMP AND ASH POND. THIS WAS COMPLETED IN APRIL 1989.

3 CONSTRUCT A PAD (MATERIAL STAGING AREA) FOR STORAGE OF SLIGHTLY CONTAMINATED MATERIALS (DEBRIS, DISMANTLED BUILDINGS AND PIPING, ETC.). PHASE I OF THE STAGING AREA WILL CONSIST OF APPROXIMATELY 1.2 ha (3 ac) OF CLEARED GROUND. THE SURFACE WILL BE GRAVEL PAVED, AND THE PAD WILL CONTROL SURFACE RUNOFF USING A RECOMPACTED FINE-GRAINED SOIL LINER AND MEMBRANE LINED SUMP.

4 REMOVE CONTAMINATED SOIL FROM ARMY RESERVE PROPERTY NO. 7. A 3.3-M ${ }^{2}\left(35-\mathrm{FT}^{2}\right)$ AREA WAS EXCAVATED TO A DEPTH OF $30.48 \mathrm{CM}$ (1 FT) AND THE EXCAVATED SOIL (ABOUT $1.2 \mathrm{~m}^{3}\left[1.5 \mathrm{yd}^{3}\right)$ ) DEPOSITED INTO FIVE 55-GALLON DRUMS. THE FIVE DRUMS WERE PLACED IN BUILDING 434 OF THE WSCP FOR INTERIM STORAGE. THE EXCAVATION WAS BACKFILLED WITH SOIL FROM THE ARMY PROPERTY AND GRADED TO THE ORIGINAL ELEVATION. THE AREA WAS RADIOLOGICALLY SURVEYED BY AN INDEPENDENT CONTRACTOR TO CONFIRM THAT THE CLEANUP WAS EFFECTIVE. THIS ACTION WAS COMPLETED ON JANUARY $12,1988$.

5 REMOVE CONTAMINATED SOIL FROM FOUR LOCATIONS ON MISSOURI DEPARTMENT OF CONSERVATION PROPERTY; ONE ON THE BUSCH WILDLIFE AREA, NORTH OF THE RAFFINATE PITS AND CHEMICAL PLANT AREA (NO. 3); AND THREE ON THE WELDON SPRING WILDLIFE AREA, SOUTH OF THE RAFFINATE PITS AND CHEMICAL PLANT AREA (NO. 4, 5, 8). THE EXCAVATED SOIL (APPROXIMATELY $400 \mathrm{~m}^{3}\left[520 \mathrm{yd}^{3}\right.$ ) WILL BE STORED IN A DESIGNATED, CONTROLLED ACCESS AREA AT THE CHEMICAL PLANT SITE UNTIL FINAL DISPOSITION.

6 DISMANTLE ALL OVERHEAD PIPING AT THE CHEMICAL PLANT SITE. TEN THOUSAND LINEAR METERS (33,000 LINEAR FEET) OF OVERHEAD PIPING AND 500 STRUCTURAL SUPPORTS, WHICH HOLD 4,000 LINEAR METERS (13,000 LINEAR FEET) OF ASBESTOS-CONTAINING MATERIAL, WILL BE REMOVED. THE ASBESTOSCONTAINING MATERIAL WILL BE WRAPPED AND DISMANTLED. NONRADIOACTIVE ASBESTOS-CONTAINING MATERIAL WILL BE TRANSPORTED TO AN OFF-SITE LICENSED DISPOSAL FACILITY; RADIOACTIVE ASBESTOS-CONTAINING MATERIAL WILL BE STORED ON SITE UNTIL FINAL DISPOSITION IS DETERMINED. THIS ACTION WAS COMPLETED JUNE 1989.

7 CONSOLIDATE AND REMOVE CONTAINERIZED CHEMICALS AT THE CHEMICAL PLANT SITE. RADIOACTIVELY AND NONRADIOACTIVELY CONTAMINATED MATERIALS WILL BE CONSOLIDATED ON-SITE IN BUILDING 434 FOR FUTURE DISPOSITION. 


\section{TABLE 1.2-2 Interim Response Actions (IRAs) (Continued)}

8 REMOVE ELECTRICAL POLES AND LINES AT THE CHEMICAL PLANT SITE. ALL DE-ENERGIZED EXTERIOR POWER AND TELEPHONE LINES (AN ESTIMATED 46,000 LINEAR METERS [150,000 LINFAR FEET) OF CABLE AND WIRE) AND 300 TIMBER POLES, THEIR CROSS BEAMS, AND SUPPORTS WERE REMOVED. THESE INACTIVE LINES AND POLES WERE DETERIORATING, AND MANY HAD FALLEN OR WERE IN DANGER OF FALLING TO THE GROUND. NONRADIOACTIVE MATERIAL WAS TRANSPORTED OFF-SITE FOR SALVAGE. RADIOACTIVE MATERIAL IS BEING STORED ON SITE ON CONCRETE SLAB 303. THIS REMOVAL ACTION WAS COMPLETED IN APRIL 1988.

9 CONSOLIDATE ALL LOOSE YARD DEBRIS IN AN ON-SITE STAGING AREA UNTIL FINAL DISPOSITION IS MAADE. THE DEBRIS INCLUDES EQUIPMENT, VEHICLES, STRUCTURAL METAL WASTE AND BUILDING RUBBLE WASTE. THIS WASTE IS HIGHLY WEATHERED AND MAY HAVE EITHER FIXED OR REMOVABLE RADIOACTIVE CONTAMINATION. AN ESTIMATED $72,880 \mathrm{~m}^{3}\left(95,160 \mathrm{yd}^{3}\right)$ WILL BE CONSOLICATED.

10 DISMANTLE THE SUPERSTRUCTIIRE OF BUILDING 409 (ADMINISTRATION). THE ASBESTOS INSULATION AND THE PCB-CONTAMINATED MATERIAL WAS REMOVED AND TRANSPORTED OFF-SITE TO LICENSED DISPOSAL FACILITIES. THE RADIOACTIVELY CONTAMINATED MATERIAL HAS BEEN STORED ON-SITE UNTIL FINAL DISPOSITION. INTERNAL EQUIPMENT, WALLS, AND THE SUPERSTRUCTURE WERE DISMANTLED AND TRANSPORTED TO AN OFF-SITE LICENSED DISPOSAL FACILITY OR SALVAGED, WHERE FEASIBLE. THIS ACTION WAS COMPLETED IN MARCH 1989.

11 DISMANTLE THE SUPERSTRUCTURE OF BUILDING 401 (STEAM PLANT). THE NONRADIOACTIVELY CONTAMINATED ASBESTOS TRANSITE PANELS FROM THE BUILDING WAS REMOVED AND TRANSPORTED TO AN OFF-SITE LICENSED DISPOSAL FACILITY. THE RADIOACTIVELY CONTAMINATED MATERIAL HAS BEEN STORED ON-SITE UNTIL FINAL DISPOSITION. STRUCTURAL STEEL WAS DISMANTLED AND TRANSPORTED TO AN OFF-SITE LICENSED DISPOSAL FACILITY OR SALVAGED, WHERE FEASIBLE. THIS ACTION WAS COMPLETED IN DECEMBER 1989.

12 CONSTRUCT A DIKE ON THE SOUTHEAST DRAINAGE CHANNEL. THE DIKE WILL IMPOUND AND CONTROL THE CONTAMINATED WATER CURRENTLY DISCHARGED FROM THE DRAINAGE AREA.

13 REMOVE CONTAMINATED SOIL FROM ARMY RESERVE VICINITY PROPERTIES NOS. 1, 2, AND 3. CONTAMINATED SOIL WILL BE REMOVED AND HAULED TO AN ON-SITE STAGING AREA AT THE CHEMICAL PLANT SITE. THE AFFECTED PROPERTIES WILL BE VERIFIED AND CERTIFIED THAT CLEANUP CRITERIA HAVE BEEN MET, AND THEN WILL BE BACKFILLED, REGRADED, AND RESEEDED.

15 DISMANTLE BUILDINGS 302 (MAGNESIUM BUILDING), 417 (PAINT SHOP), 427 (PRIMARY SEWAGE TREATMENT PLANT), 432 (MAIN SEWER SAMPLER), AND 433 (STORAGE BUILDING), AND SELECTED SEWERS. THESE BUILDINGS WILL BE DISMANTLED TO THE TOP OF FOUNDATION; FOUNDATION AND UNDERGROUND UTILITIES REMOVAL WILL NOT BE INCLUDED. NONRADIOACTIVE CHEMICALLY CONTAMINATED MATERIAL WILL BE TRANSPORTED TO OFF-SITE LICENSED DISPOSAL FACILITIES; RADIOACTIVE MATERIAL WILL BE STORED ON SITE. 
TABLE 1.2-2 Interim Response Actions (IRAs) (Continued)

15 DISMANTLE BUILDINGS 435, 436 (STORAGE BUILDINGS), 437 (RECORDS RETENTION BUILDING), 438 (STORAGE BUILDING), AND UNDERGROUND TANKS. THESE BUILDINGS AND THEIR FOUNDATIONS WILL BE REMOVED TO ALLOW CONSTRUCTION OF THE TEMPORARY STORAGE AREA. NONRADIOACTIVELY AND RADIOACTIVELY MATERIAL WILL BE STORED ON SITE.

15 DISMANTLE BUILDINGS 104 (LIME STORAGE), 412 (ELECTRICAL SUBSTATION), 413 (COOLING TOWER AND PUMP HOUSE), AND 428 (FUEL GAS PLANT). THESE BUILDINGS WILL BE DISMANTLED TO THE TOP OF FOUNDATION; FOUNDATION AND UNDERGROUND UTILITIES REMOVAL WILL NOT BE INCLUDED. NONRADIOACTIVE CHEMICALLY CONTAMINATED MATERIAL WILL BE TRANSPORTED TO OFF-SITE LICENSED DISPOSAL FACILITIES; RADIOACTIVE MATERIAL WILL BE STORED ON SITE.

16 DISMANTLE BUILDING 408 (MAINTENANCE AND STORES). THE BUILDING WILL BE DISMANTLED TO THE TOP OF FOUNDATION; FOUNDATION AND UNDERGROUND UTILITIES REMOVAL WILL NOT BE INCLUDED. NONRADIOACTIVE CHEMICALLY CONTAMINATED MATERIAL WILL BE TRANSPORTED TO OFF-SITE LICENSED DISPOSAL FACILITIES; RADIOACTIVE MATERIAL WILL BE STORED ON SITE.

16 REMOVE THE ON-SITE RAILROAD RAIL, TIES, AND BALLAST. CONTAMINATED MATERIALS WILL BE STOCKPILED ON THE CHEMICAL PLANT PROPERTY UNTIL FINAL DISPOSITION.

16 DISMANTLE BUILDINGS 407 (LABORATORY) AND 431 (LABORATORY SEWER SAMPLER). THESE BUILDINGS WILL BE DISMANTLED TO THE TOP OF FOUNDATION; FOUNDATION AND UNDERGROUND UTILITIES REMOVAL WILL NOT BE INCLUDED. NONRADIOACTIVE CHEMICALLY CONTAMINATED MATERIAL WILL BE TRANSPORTED TO OFF-SITE LICENSED DISPOSAL FACILITIES; RADIOACTIVE MATERIAL WILL BE STORED ON-SITE.

16 DISMANTLE BUILDINGS 410 (SERVICES BUILDING) AND 430 (AMBULANCE GARAGE). THESE BUILDINGS WILL BE DISMANTLED TO THE TOP OF FOUNDATION; FOUNDATION AND UNDERGROUND UTILITIES REMOVAL WILL NOT BE INCLUDED. NONRADIOACTIVE CHEMICALLY CONTAMINATED MATERIAL WILL BE TRANSPORTED TO OFF-SITE LICENSED DISPOSAL FACILITIES; RADIOACTIVE MATERIAL WILL BE STORED ON SITE.

16 DISMANTLE BUILDINGS 109 (WEST DRUM STORAGE), 110 (EAST DRUM STORAGE), 202 (GREEN SALT TANK FARM), 406 (WAREHOUSE), 414 (SALVAGE BUILDING), AND 415 (PROCESS INCINERATOR). THESE BUILDINGS WILL BE DISMANTLED TO THE TOP OF FOUNDATION; FOUNDATION AND UNDERGROUND UTILITIES REMOVAL WILL NOT BE INCLUDED. NONRADIOACTIVE CHEMICALLY CONTAMINATED MATERIAL WILL BE TRANSPORTED TO OFF-SITE LICENSED DISPOSAL FACILITIES; RADIOACTIVE MATERIAL WILL BE STORED ON SITE. 


\section{TABLE 1.2-2 Interim Response Actions (IRAs) (Continued)}

17 DISMANTLE THE WATER TOWER (BUILDING 426) AND SURFACE TANKS (429). THESE STRUCTURES WILL BE DISMANTLED TO THE TOP OF FOUNDATION; FOUNDATION AND UNDERGROUND UTILITIES REMOVAL WILL NOT BE INCLUDED. NONRADIOACTIVELY AND CONTAMINATED MATERIAL AND RADIOACTIVELY CONTAMINATED MATERIAL WILL BE STORED ON SITE.

18 DISMANTLE BUILDING 101 (FEED PREPARATION AND SAMPLING). THIS BUILDING WILL BE DISMANTLED TO THE TOP OF FOUNDATION; FOUNDATION AND UNDERGROUND UTILITIES REMOVAL WILL NOT BE INCLUDED. NONRADIOACTIVE CHEMICALLY CONTAMINATED MATERIAL WILL BE TRANSPORTED TO OFF.SITE LICENSED DISPOSAL FACILITIES; RADIOACTIVE MATERIAL WILL BE STORED ON SITE.

18 DISMANTLE BUILDINGS 102A AND B (REFINERY TANK FARM), 105 (TBP AND ETHER EXTRACTION), AND 106 (PROOF SAMPLER). THESE BUILDINGS WILL BE DISMANTLED TO THE TOP OF FOUNDATION; FOUNDATION AND UNDERGROUND UTILITIES REMOVAL WILL NOT BE INCLUDED. NONRADIOACTIVE CHEMICALLY CONTAMINATED MATERIAL WILL BE TRANSPORTED TO OFF-SITE LICENSED DISPOSAL FACILITIES; RADIOACTIVE MATERIAL WILL BE STORED ON SITE.

18 DISMANTLE BUILDING 103 (DIGESTION AND DENITRATION BUILDING). THIS BUILDING WILL BE DISMANTLED TO THE TOP OF FOUNDATION; FOUNDATION AND UNDERGROUND UTILITIES REMOVAL WILL NOT BE INCLUDED. NONRADIOACTIVE CHEMICALLY CONTAMINATED MATERIAL WILL BE TRANSPORTED TO OFFSITE LICENSED DISPOSAL FACILITIES; RADIOACTIVE MATERIAL WILL BE STORED ON SITE.

18 DISMANTLE BUILDING 108 (NITRIC ACID RECOVERY). THIS BUILDING WILL BE DISMANTLED TO THE TOP OF FOUNDATION; FOUNDATION AND UNDERGROUND UTILITIES REMOVAL. WILL NOT BE INCLUDED. NONRADIOACTIVE CHEMICALLY CONTAMINATED MATERIAL WILL BE TRANSPORTED TO OFF-SITE LICENSED DISPOSAL FACILITIES; RADIOACTIVE MATERIAL WILL BE STORED ON SITE.

18 DISMANTLE BUILDING 201 (GREEN SALT PLANT). THIS BUILDING WILL BE DISMANTLED TO THE TOP OF FOUNDATION; FOUNDATION AND UNDERGROUND UTILITIES REMOVAL WILL NOT BE INCLUDED. NONRADIOACTIVE CHEMICALLY CONTAMINATED MATERIAL WILL BE TRANSPORTED TO OFF-SITE LICENSED DISPOSAL FACILITIES; RADIOACTIVE MATERIAL WILL BE STORED ON SITE.

18 DISMANTLE BUILDINGS 301 (METALS PLANT) AND 303 (CHIP STORAGE). THESE BUILDINGS WILL BE DISMANTLED TO THE TOP OF FOUNDATION; FOUNDATION AND UNDERGROUND UTILITIES REMUVAL WILL. NOT BE INCLUDED. NONRADIOACTIVE CHEMICALLY CONTAMINATED MATERIAL WILL BE TRANSPORTED TO OFF-SITE LICENSED DISPOSAL FACILITIES; RADIOACTIVE MATERIAL WILL BE STORED ON SITE. 
TABLE 1.2-2 Interim Response Actions (IRAs) (Continued)

18 DISMANTLE BUILDINGS 403 (CHEMICAL PILOT PLANT), 404 (METALLURGICAL PILOT PLANT), AND 405A AND $B$ (HILOT PLANTS AUXILLIARY). THESE BUILDINGS WILL BE DISMANTLED TO THE TOP OF FOUNDATION; FOUNDATION AND UNDERGROUND UTILITIES REMOVAL WILL NOT BE INCLUDED. NONRADIOACTIVE CHEMICALLY CONTAMINATED MATERIAL WILL BE TRANSPORTED TO OFF-SITE LICENSED DISPOSAL FACILITIES; RADIOACTIVE MATERIAL WILL BE STORED ON SITE.

19 THIS IRA INITIATES A DECONTAMINATION/TREATMENT FACILITY FOR THE SITE. EARLY TREATMENT PROCESSES WILL BE AIMED AT DECONTAMINATION OF BUILDING MATERIALS. ADDITIONAL PROCESSES (CRUSHING, SHREDDING, ETC.) CAN BE ADDED AS REQUIRED.

20 CONSTRUCT WA:STEWATER TREATMENT PLANT FOR TREATMENT OF THE RAFFINATE PIT WATER AND THE CONTAMINATED WATER ON THE CHEMICAL PLANT PROPERTY. THIS WATER WILL BE COLLECTED, TREATED, AND THEN DISCHARGED AFTER VERIFYING THAT THE EFFLUENT MEETS RELEASE LIMITS TO BE ESTABLISHED IN CONJUNCTION WITH EPA REGION VII AND THE MISSOURI DNR. THE SYSTEM WILL BE DESIGNED WITH TWO TRAINS WHICH CONTINUOUSLY TREAT $440 \mathrm{M}^{3} / D(115,200$ GPD $)$ AND $218 \mathrm{M}^{3} / D$ (57,600 GPD), RESPECTIVELY. THE INITIAL WATER VOLUME TO BE TREATED IS ESTIMATED TO BE 950,000 $M^{3}(251,000,000 \mathrm{GAL})$.

21 CONSTRUCT WASTEWATER TREATMENT PLANT TO TREAT WATER IN THE QUARRY POND, WITHIN THE BULK MATERIAL IN THE QUARRY, AND STORM WATER FALLING ON THE QUARRY DURING EXHUMATION. THIS WATER WILL BE COLLECTED, TREATED, AND DISCHARGED AFTER VERIFYING THAT THE EFFLUENT MEETS RELEASE LIMITS TO BE ESTABLISHED IN CONJUNCTION WITH EPA REGION VII AND THE MISSOURI DNR. THE SYSTEM WILL BE DESIGNED TO TREAT $440 \mathrm{M}^{3} / D(115,200 \mathrm{GPD})$. THE INITIAL VOLUME OF PONDED WATER TO BE TREATED IS ESTIMATED TO $\mathrm{BE} 11,000 \mathrm{M}^{3}(3,000,000 \mathrm{GAL})$. 


\section{TABLE 1.3-1 Chemicals Used in Former Explosives Production Facility and Uranium Feed Materials Processing Plant}

TNT, DNT Praduction (chemicals used at site)

$\begin{array}{ll}\text { Ammonia } & \text { Oloum (sulfuric acid) } \\ \text { Caustic soda } & \text { Toluene } \\ \text { Fuel oil } & \end{array}$

Uranium Processing

Nitric acid

Sodium hydroxide

Sulfuric acid

Sodium carbonate

Phosphate

UNH (uranium amine)

$\mathrm{UO}_{3}$ (uranyl oxide)

Uranium ore concentrate

Lime

Ether

Ethylene glycol

Tributyl phosphate

Ferric nitrate

Paint and catalysts

Anhydrous hydrogen

Ammonia

Green salt (uranium tetrafluoride)

Hydrofluoric acid

Hydrogen gas

Ivitroge., gas

Orange oxide (uranium oxide orange)

Propane

Caustic liquid

Magnesium

Graphite sheets

Diesel fuel

Fuel oil

Gasoline

Hydrogen zeolite

Refrigeration brine

\author{
Sodium zeolite \\ Sulfite \\ Helium \\ Hydraulic oil \\ Uranium metal \\ Laboratory chemicals \\ Perchloric acid \\ Grease \\ Chlorine \\ Chromiuri phosphates \\ Acid (misc.) \\ Benzene \\ Corrosive resistant coating \\ Epoxy paint and catalysts \\ Unspecified flammable materials \\ Hot die lube \\ Linseed oil (boiled) \\ Lubriplate \\ Melcolene \\ Metalube \\ Methylene glycol \\ Methylisobutyl ketone \\ Motor oil \\ Paint \\ Paint solvents \\ Phenoline thinner \\ Polyclad \\ Polyurethane paint \\ Rustbond primer \\ Tar
}

Source: MKF and JEG 19881; reproduced from MKF and JEG 1989a 
TABLE 1.3-2 Chemical Substances Analyzed in Water and Soil Investigations

\begin{tabular}{|c|c|c|c|}
\hline Group & Chemical & Soil & Groundwater \\
\hline \multirow[t]{22}{*}{ Metals } & Aluminum & $x$ & $x$ \\
\hline & Antimony & $x$ & $x$ \\
\hline & Arsenic & $x$ & $x$ \\
\hline & Barium & $x$ & $x$ \\
\hline & Cadmium & $x$ & $x$ \\
\hline & Calcium & $x$ & $x$ \\
\hline & Chromium & $x$ & $x$ \\
\hline & Cobalt & $x$ & $x$ \\
\hline & Copper & $x$ & $x$ \\
\hline & Iron & $x$ & $x$ \\
\hline & Lead & $x$ & $x$ \\
\hline & Lithium & $x$ & $x$ \\
\hline & Magnesium & $x$ & $x$ \\
\hline & Menganese & $x$ & $x$ \\
\hline & Mercury & $x$ & $x$ \\
\hline & Nickel & $x$ & $\mathrm{x}$ \\
\hline & Potassium & $x$ & $x$ \\
\hline & Selenium & $x$ & $x$ \\
\hline & Sodium & $x$ & $x$ \\
\hline & Thallium & $x$ & $x$ \\
\hline & Vanadium & $x$ & $x$ \\
\hline & Zinc & $x$ & $x$ \\
\hline \multirow[t]{5}{*}{ Anions } & Chloride & $x$ & $x$ \\
\hline & Fluoride & $x$ & $x$ \\
\hline & Nitrate & $x$ & $x$ \\
\hline & Sulfate & $x$ & $x$ \\
\hline & Cyanide & -. & $x$ \\
\hline
\end{tabular}

Organics

Nitroaromatics

Radionuclides

Other
Hexane

Polynuclear aromatic

hydrocarbons

Polychlorinated

biphenyls

Pesticides

Phenol

Tributylphosphate

2,4,6-Trinitrotoluene

2,4-Dinitrotoluene

2,6-Dinitrotoluene

Nitrobenzene

1,3,5-Trinitrobenzene

1,3-Dinitrobenzene

Uranium-238

Radium-226

Thorium-230

Thorium-232

Radon (a)

Asbestos ${ }^{(1)}$ $x$
$x$
$x$
$x$
$x$
$x$
$x$
$x$
$x$
$x$
$x$
$x$
$x$
$x$
$x$
$x$
$x$
$x$
$x$
$x$
$x$
$x$
$x$
$x$
$x$
$x$
-

(b)

(b)

..

-.

$x$

$x$

$x$

$x$

$x$

$x$

(d)

(d)

(d)

(d)

$x$

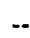

(a)
Surface

Water

-•

-.

(c)

(c)

(c)

(c)

(c)

(c)

$x$
$x$
$x$
$x$
--

-- 
TABLE 1.3-2 Chemical Substances Analyzed in Water and Soil Investigations (Continued)

\begin{tabular}{llll}
\hline Group & Chemical & Soil & Surface \\
Water & Groundwar & Water \\
\hline
\end{tabular}

Source: MKF and JEG 19870 and 19881; reproduced from JEG 1989

(a) Groundwater samples were analyzed for volatile, acid or base-neutral organics.

(b) Analysis was performed in the Phase II soil investigation (MKF and JEG 1989a).

(c) Nitroaromatics were not measured at regular surface water sites but were measured in raffinate pit waters.

(d) Not included in Phase I Chemical Soils Report; reported in UNC 1988.

(o) Radon measurements were made as part of air monitoring program.

(i) In addition to soils, asbestos was measured in air monitoring program. 


\section{TABLE 1.3-3 Primary Contaminants for the Weldon Spring Site}

Radiological Parameters

Gross alpha

Gross beta

Uranium-238

Thorium-230

Thorium-232

Radium-226

Organics

Nitrobenzene

2,4-Dinitrotoluene (2,4 DNT)

2,6-Dinitrotoluene $(2,6$ DNT)
2,4,6-Trinitrotoluene (TNT)

Polychlorinated biphenyls (PCBs)

Inorganics/Metals/Asbestos

\begin{tabular}{lll}
\hline Chromium & Zinc & Sulfate \\
Lithium & Molybdenum & Fluoride \\
Magnesium & Arsenic & Asbestos \\
Nickel & Manganese & Nitrate \\
Vanadium & Barium & \\
Iron & Beryllium & \\
Lead & &
\end{tabular}


TABLE 1.4-1 Laws and Orders Potentially Applicable or Relevant and Appropriate to the Weldon Spring Site

Remedial Action Project

Federal Laws

Archaeological and Historic Preservation Act of 1974

Archaeological Resources Protection Act of 1979

Atomic Energy Act of 1954, as amended

Clean Air Act of 1963, as amended

Clean Water Act, as amended (also referred to as Federal Water Pollution Control Act of 1972, as amended)

Comprehensive Environmental Response, Compensation, and Liability Act of 1980, as amended

Department of Energy Organization Act of 1977

Endangered Species Act of 1973, as amended

Fish and Wildife Coordination Act of 1934, as amended

Hazardous Materials Transportation Act of 1974, as amended

National Environmental Policy Act of 1969, as amended

Noise Control Act of 1972

Noise Pollution and Abatement Act of 1970

Occupational Safety and Health Act of 1970

Safe Drinking Water Act of 1974

Soil and Water Resources Conservation Act of 1977

Solid Waste Disposal Act, as amended by the Resource Conservation and Recovery Act of 1976, as amended by the Hazardous and Solid Waste Amendments of 1984

Superfund Amendments and Reauthorization Act of 1986

Toxic Substance Control Act of 1976

Uranium Mill Tailings Radiation Control Act of 1978 


\section{TABLE 1.4-1 Laws and Orders Potentially Applicable or Relevant and Appropriate to the Weldon Spring Site Remedial Action Project (Continued)}

Executive Orders

Executive Order 11490, Assigning Emergency Proparedness Functions to Federal Departments and Agencies

Executive Order 11514, Protection and Enhancement of Environmental Quality

Executive Order 11738, Providing for Administration of the Clean Air Act and the Federal Weter Pollution Control Act with Respect to Federal Contracts, Grants, or Loans

Executive Order 11807, Occupational Safety and Health Programs for Federal Employees

Executive Order 11988, Floodplain Management

Executive Order 11990, Protection of Wetlands

Executive Order 11991, Relating to the Protection and Enhancement of Environmental Quality

Executive Order 12088, Federal Compliance with Pollution Control Standards

Executive Order 12146, Management of Federal Legal Resources

Executive Order 12480, Superfund Implementing

Department of Energy Orders

Order 1540.1 Materials Transportation and Traffic Management

Order 4240.1H Designation of Major System Acquisition and Major Projects

Order 4320.1A Site Development and Facility Utilization Planning

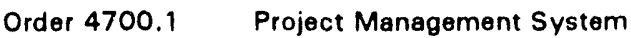

Order 5400.5 Radiation Protection of the Public and the Environment

Order 5440.1C Implementation of the National Environmental Policy Act

Order 5480.1B Environment, Safety, and Health Program for Department of Energy Operation

Order 5480.4 Environmental Protection, Safety, and Health Protection Standards

Order 5480.14 Comprehensive Environmental Response, Compensation, and Liability Act Program

Order 5481.1B Safety Analysis Review System 
TABLE 1.4-1 Laws and Orders Potentially Applicable or Relevant and Appropriate to the Weldon Spring Site Remedial Action Project (Continued)

\begin{tabular}{|c|c|}
\hline & Department of Energy Orders (Continued) \\
\hline Order 5482.1B & Environmental Protection, Safety, and Health Protection Appraisal Program \\
\hline Order 5483.1A & $\begin{array}{l}\text { Occupational Safoty and Health Program for Government-Owned Contractor-Operated } \\
\text { Fecilitios }\end{array}$ \\
\hline Order $\mathbf{5 4 8 4 . 1}$ & $\begin{array}{l}\text { Environmental Protection, Safety, and Health Protection Information Reporting } \\
\text { Requirements }\end{array}$ \\
\hline Order 5000.3 & Unusual Occurrence Reporting System \\
\hline Order 5500.2 & Emergency Planning, Preparedness and Response for Operations \\
\hline Order $5700.6 \mathrm{C}$ & Quality Assurance \\
\hline Order 5820.2 & Radioactive Waste Management \\
\hline
\end{tabular}

Mis: ouri State Environmental Laws

Missouri Clean Water Act

Missouri Clean Air Act

Missouri Hazardous Waste Management Law

Missouri Solid Waste Management Law

Missouri Land Reclamation Act

Governor's Executive Order 82-19 on Flood Plein Management

Missouri 401 Water Quality Certification 


\section{TABLE 2.1-1 Quality Assurance Program Elements}

\section{QA Eloments}

1. Title Page

2. Table of Contents

3. Project Description

4. Project Organization and Responsibility

5. Quality Assurance Objectives for Date Measurement

6. Sampling Procedures

7. Sample and Document Custody

8. Calibration Procedures

9. Analytical Procedures

10. Data Reduction, Validation, and Reporting

11. Internal Quality Control

12. Audits

13 Preventive Maintenance

14. Specific Routine Measures Used to Assess Data (Precision, Accuracy and, and Completeness)

15. Corrective Action

16. Quality Assurance Reports to Management
Information Provided In

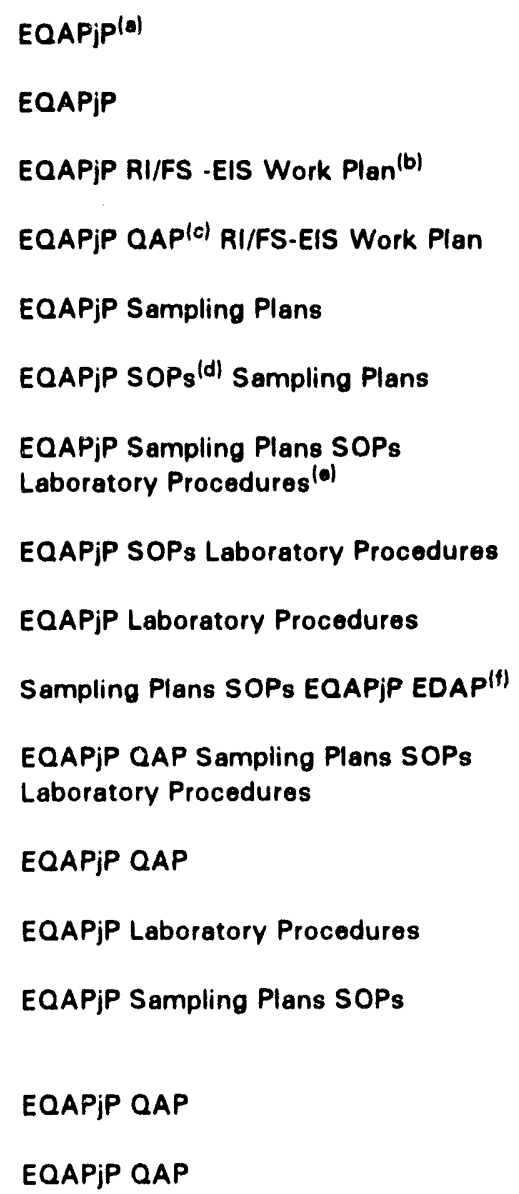

Notes:

(a) Environmental Quality Assurance Project Plan (QAMS 005/80)

(b) Work Plan for the Remedial Investigation and Feasibility Study-Environmental Impact Statement for the Weldon Spring Site, Weldon Spring, Missouri (ORNL 1988)

(c) Quality Assurance Program (NQA-1 1986)

(d) SOPs: Standard Operating Procedures

(a) Laboratory Procedures: Analytical Limits/Detection Methods

(f) Environmental Data Administration Plan 
TABLE 2.3-1 Analytical Methods

\begin{tabular}{|c|c|c|}
\hline Parameter & Water Mothod & Soil/Sediment Method \\
\hline Acidity & EPA 305.1 & EPA 305.1 \\
\hline Alkalinity & EPA 310.1 & EPA 310.1 \\
\hline Asbestos & EPA Methodology & EPA Mothodology \\
\hline Carbonate/Bicarbonate & Standard Methods 203 & Standard Methods 203 \\
\hline Chloride & EPA $325.1 / 325.3$ & EPA $325.1 / 325.3$ \\
\hline Chlorinated Hydrocarbons & EPA 612 & EPA 612 \\
\hline Chlorine, total residual & EPA 330.1 & EPA 330.1 \\
\hline Conductance, specific & EPA 120.1 & EPA 120.1 \\
\hline Corrosivity & EPA 1110 & EPA 1110 \\
\hline Dissolved Oxygen & EPA 360.1 & EPA 360.1 \\
\hline Dissolved Solids & EPA $160.1 / 160.2$ & EPA $160.1 / 160.2$ \\
\hline Fluoride (distillation) & EPA 340.1 & EPA 340.1 \\
\hline Fluoride (electrode) & EPA 340.2 & EPA 340.2 \\
\hline Ignitability & EPA $1010 / 1020160.2$ & EPA $1010 / 1020160.2$ \\
\hline Lithium & Standard Methods 317 & Standard Methods 317 \\
\hline Mercury & EPA 245.1 & EPA 245.1 \\
\hline Metals Only, EP Toxicity & EPA 1310 & EPA 1310 \\
\hline $\begin{array}{l}\text { Metals, total (CLP listing) } \\
\text { ICP metals, Furnace metals }\end{array}$ & EPA 200 series & EPA 7000 series \\
\hline Moisture Content & EPA 160.3 & EPA 160.3 \\
\hline Nitrate & EPA 352.1 & EPA 352.1 \\
\hline Nitrate + Nitrite & EPA 353.2 & EPA 353.2 \\
\hline Nitrite & EPA 354.1 & EPA 354.1 \\
\hline Nitroaromatics & EPA 609 & EPA 609 \\
\hline \multicolumn{3}{|c|}{ Nitrogen series along with chloride, fluoride and sulfate may be analyzed utilizing the ion chromatograph method - EPA 300.0} \\
\hline Nitrogen, ammonia & EPA 350.1 & EPA 350.1 \\
\hline Phenols & EPA $420.1 / 604$ & EPA $420.1 / 604$ \\
\hline \multicolumn{3}{|l|}{ Priority Pollutants } \\
\hline m:luserslireneldocumentlii-1 & Nst-231.rvo & \\
\hline
\end{tabular}


TABLE 2.3-1 Analytical Methods (Continued)

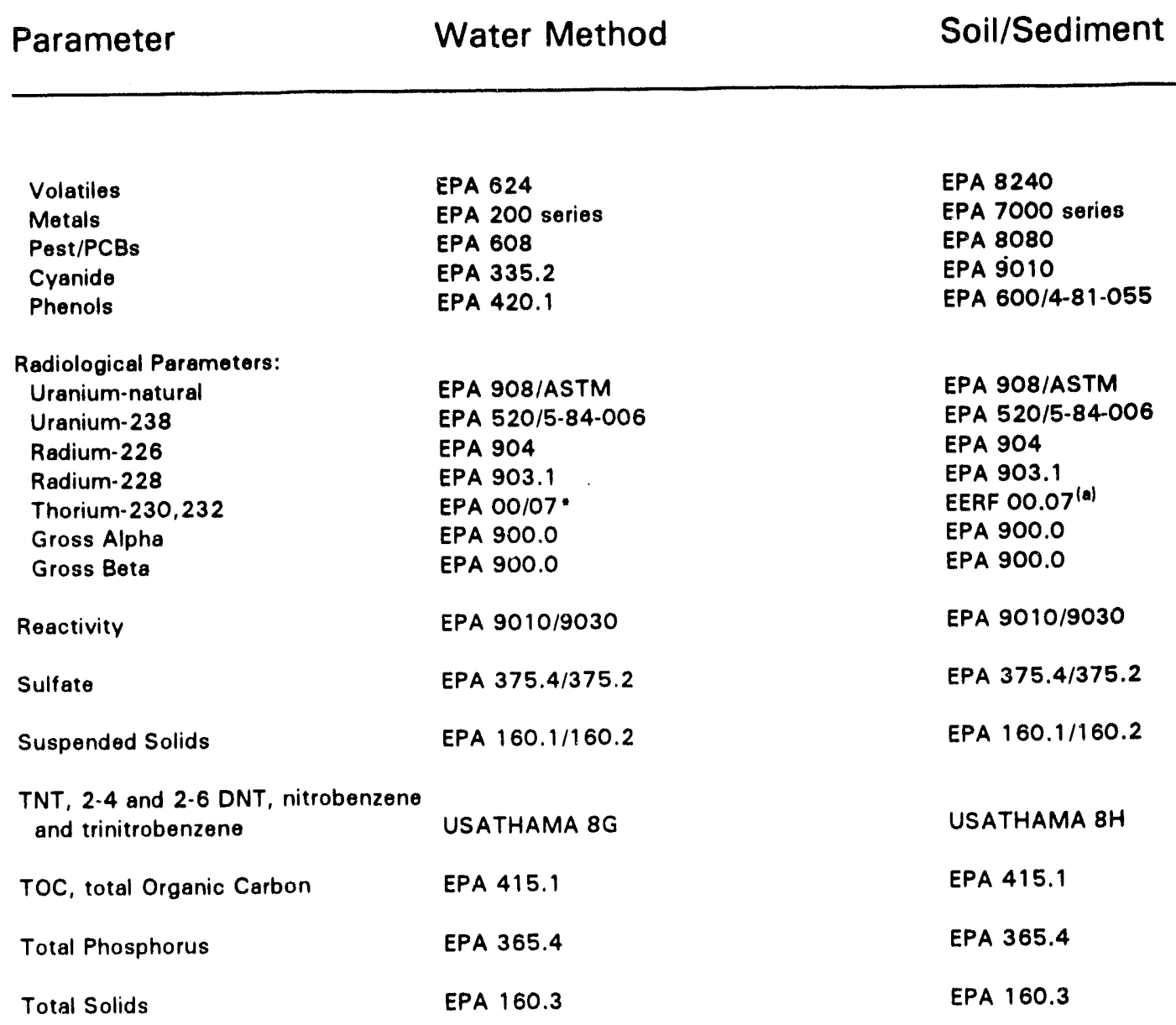

\footnotetext{
(a)EPA Eastern Environmental Radiological Facility m:luserslireneidocumentiri-revfltablesiwst-231.rvo
} 


\section{TABLE 2.5-1 Data Quality Assessments}

\begin{tabular}{|c|c|c|}
\hline Data Characteristic & Record & Criteria \\
\hline Precision & $\begin{array}{l}\text { Lab arid field } \\
\text { duplicates }\end{array}$ & Duplicate QC limits \\
\hline Accuracy & $\begin{array}{l}\text { Lab spiked results } \\
\text { Lab control sample results }\end{array}$ & $\begin{array}{l}\text { Spiked QC limits } \\
\text { Lab control sample QC limits }\end{array}$ \\
\hline Representativeness & Database & Outliers at the $99 \%$ confidence level \\
\hline Completeness & Database & $95 \%$ of acceptable data \\
\hline Comparability & Database & $\begin{array}{l}\text { QC limits at } 99 \% \text { confidence level for } \\
\text { results of duplicate samples from } \\
\text { different labs }\end{array}$ \\
\hline Database Accuracy & Original lab data & $\begin{array}{l}99.5 \% \text { accurate at } 95 \% \text { confidence } \\
\text { level }\end{array}$ \\
\hline
\end{tabular}




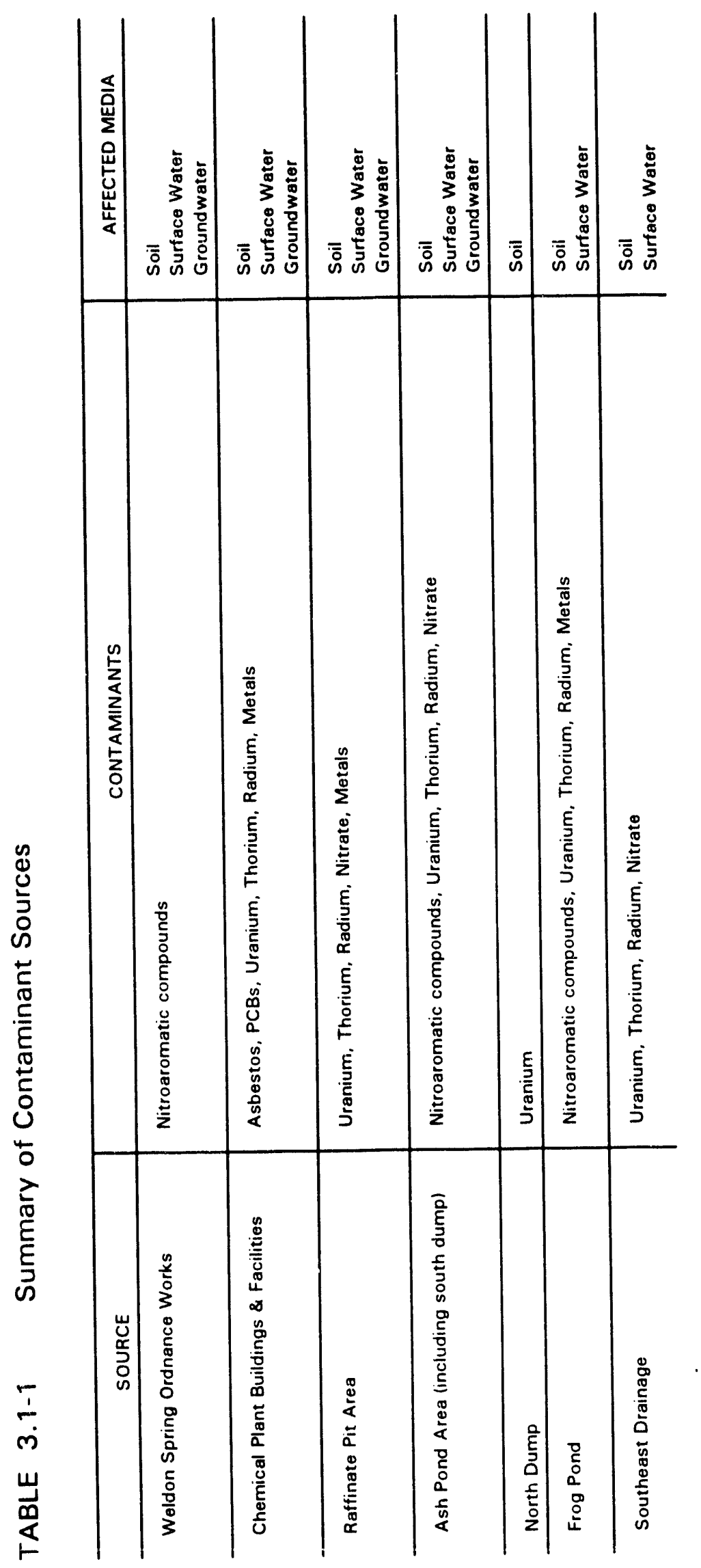

ฟั.

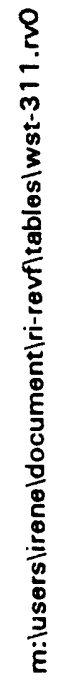




\section{TABLE 3.4-1 Groundwater Sample Analysis and Preservation}

\begin{tabular}{lcc}
\hline Analytical & Sample Preservative \\
Parameter & Sample Container & Used by Lab
\end{tabular}

Total Organic Carbons

Inorganics

Nitrate

Sulfate

Fluoride

Chloride

Total Dissolved Solids

Nitroaromatic Compounds

2,4,6-trinitrotoluene

2,4-dinitrotoluene

2,6-dinitrotoluene

1,3,5-trinitrobenzene

1,3-dinitrobenzene

Nitrobenzene

Radiological Parameters

Natural uranium

Radium-226

Radium-228

Thorium-230

Thorium-232

Gross Alpha

Gross Beta
One 8-oz amber jug

One 250-ml Plastic

One 500-ml polyethylene

plastic jug

One-gallon amber jug

One-gallon polyethylene

plastic jug
$\mathrm{H}_{2} \mathrm{SO}_{4}$ to $\mathrm{pH}<2.0$ and

refrigerated

refrigerated
$\mathrm{HNO}_{3}$ or $\mathrm{HCl}$ to $\mathrm{pH}<2.0$ and
refrigerated 


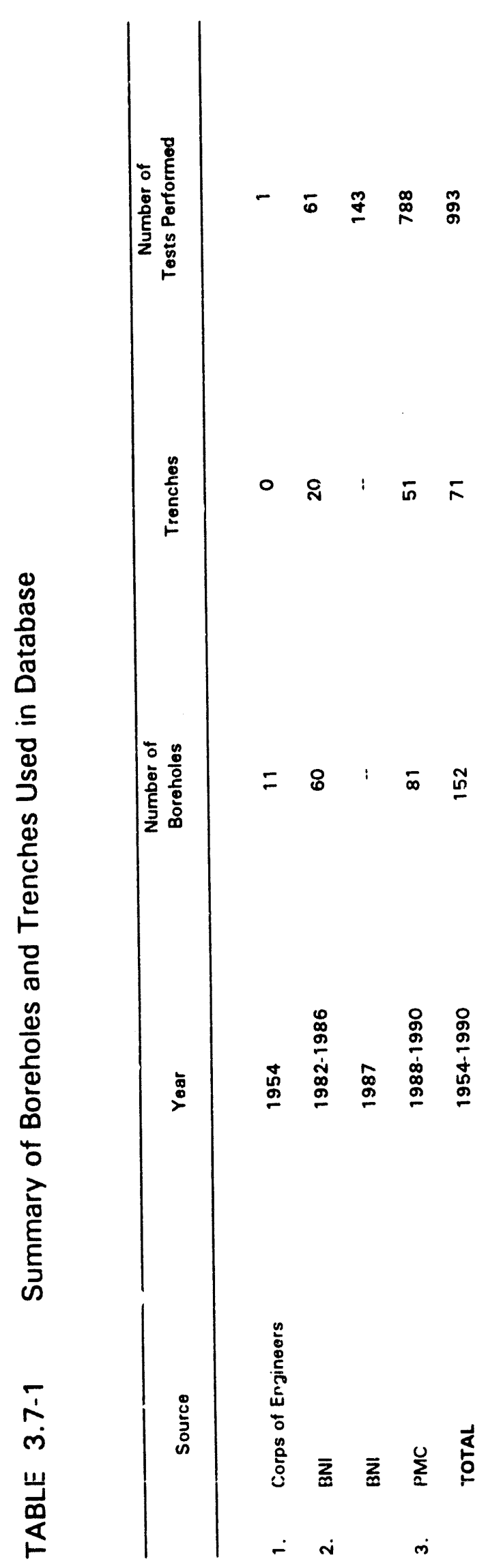

总

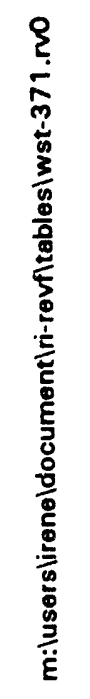




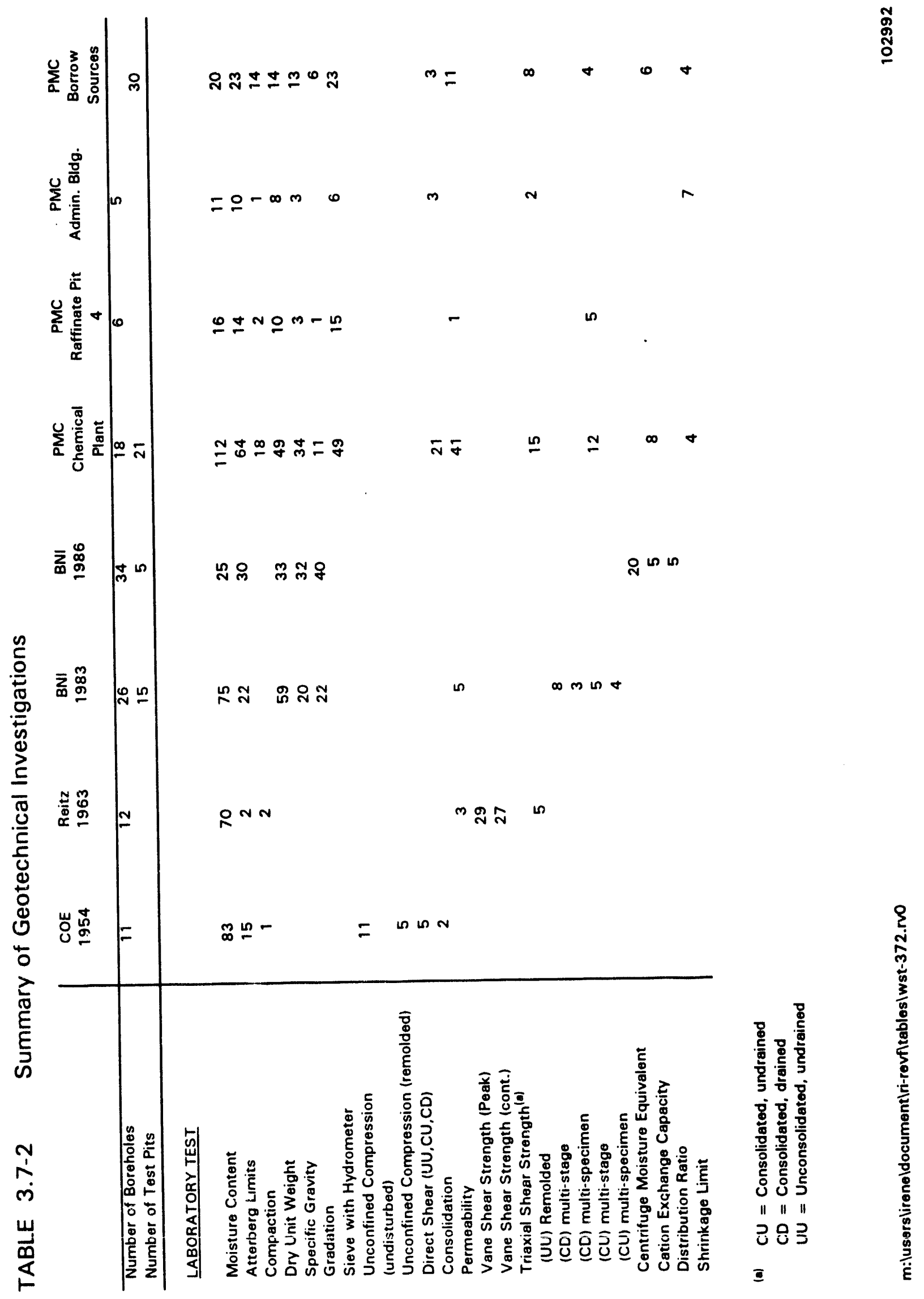


TABLE 4.1-1 Major Buildings, Structures and Facilities at the Chemical Plant

\begin{tabular}{|c|c|}
\hline Building or Area & Name/Function \\
\hline 101 & Sampling Plant \\
\hline 102 & Refinery Tank Farm \\
\hline 103 & Digestion and Denitration \\
\hline 104 & Lime Storage \\
\hline 105 & Ether Extraction \\
\hline 106 & Refinery Sewer Sampler \\
\hline 108 & Nitric Acid Plant \\
\hline 109 & West Drum Storage \\
\hline 110 & East Drum Storage \\
\hline 201 & Green Salt Building \\
\hline 202 (A\&B) & Green Salt Tank Farm \\
\hline 301 & $\begin{array}{l}\text { Metals Building; Concrete Pad Storage Area and Drum Packaging Stations on South Side } \\
\text { of Building }\end{array}$ \\
\hline 302 & Magnesium Building \\
\hline 303 & Foundation (only remaining structure) \\
\hline 401 & $\begin{array}{l}\text { Steam Plant: Coal Conveyor and Coal Yard North of Building 401; Smokestacks Wesx of } \\
\text { Building } 401\end{array}$ \\
\hline 403 & Chemical Pilot Plant and Filter and Substation North of Buildings 403 and 404 \\
\hline 404 & Metal Pilot Plant \\
\hline $405(A \& B)$ & Pilot Plant Maintenance Building \\
\hline 406 & Warehouse \\
\hline 407 & Laboratory \\
\hline 408 & Maintenence and Stores \\
\hline 409 & Administration Building \\
\hline 410 & Services Building \\
\hline 412 & Electrical Substation \\
\hline 413 & Cooling Tower and Pump House \\
\hline 414 & Salvage Building \\
\hline 415 & Process Incinerator \\
\hline 417 & Paint Shop \\
\hline 426 & Water Tower \\
\hline 427 & Primary Sewage Treatment Plant \\
\hline 428 & Fuel Gas Plant \\
\hline 429 & Reserve Water Facilities \\
\hline 430 & Ambulance Garage \\
\hline 431 & Laboratory Sewer Sampler \\
\hline 432 & Main Sewer Sampler \\
\hline 433.436 & Storage Buildings \\
\hline 437 & Records Retention Building \\
\hline 438 & Storage Building \\
\hline 439 & Fire Training Building \\
\hline 441 & Cylinder Storage \\
\hline 443 & Fire Training Storage Building \\
\hline
\end{tabular}

Source:

ORNL 1988 


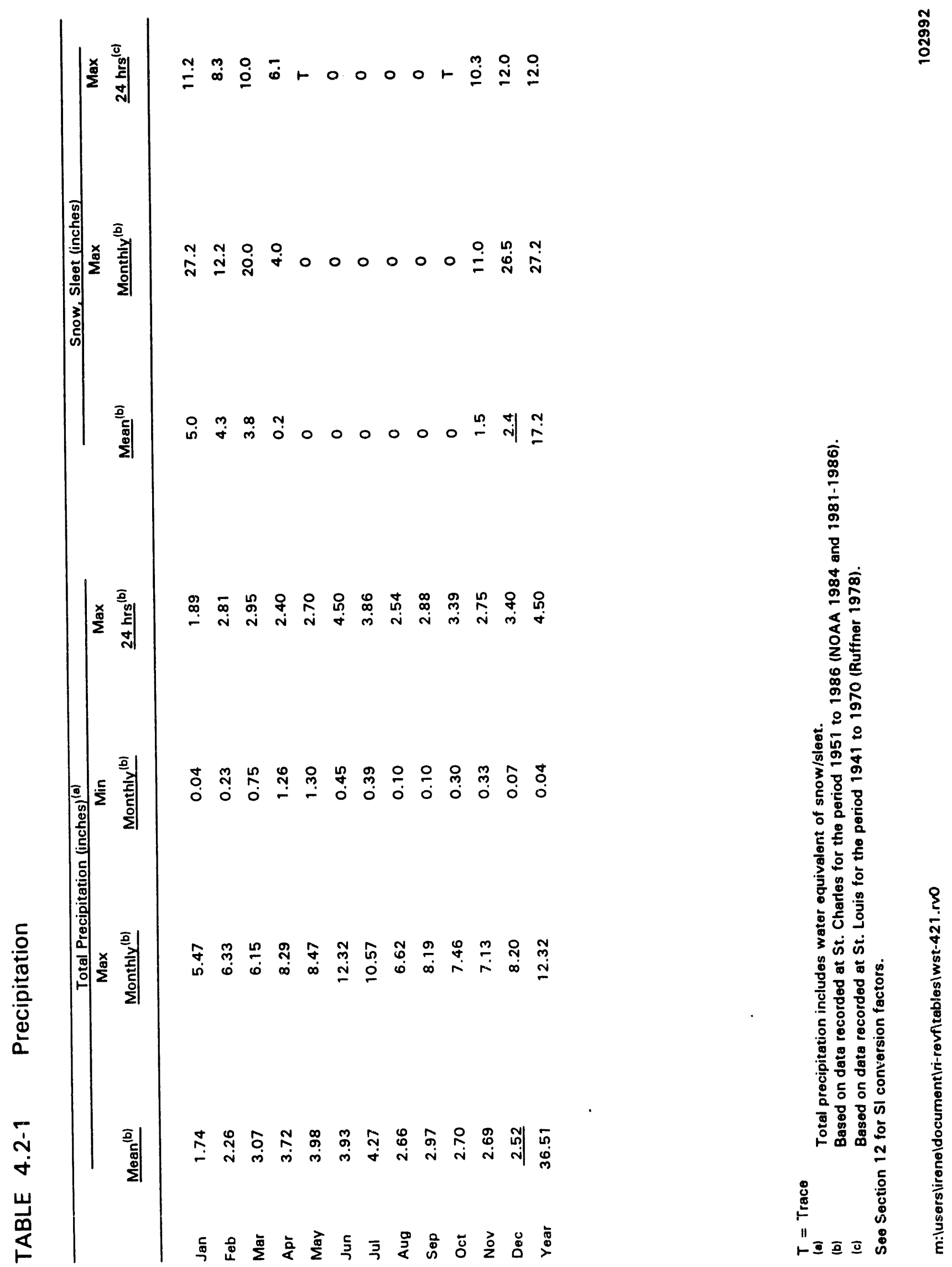




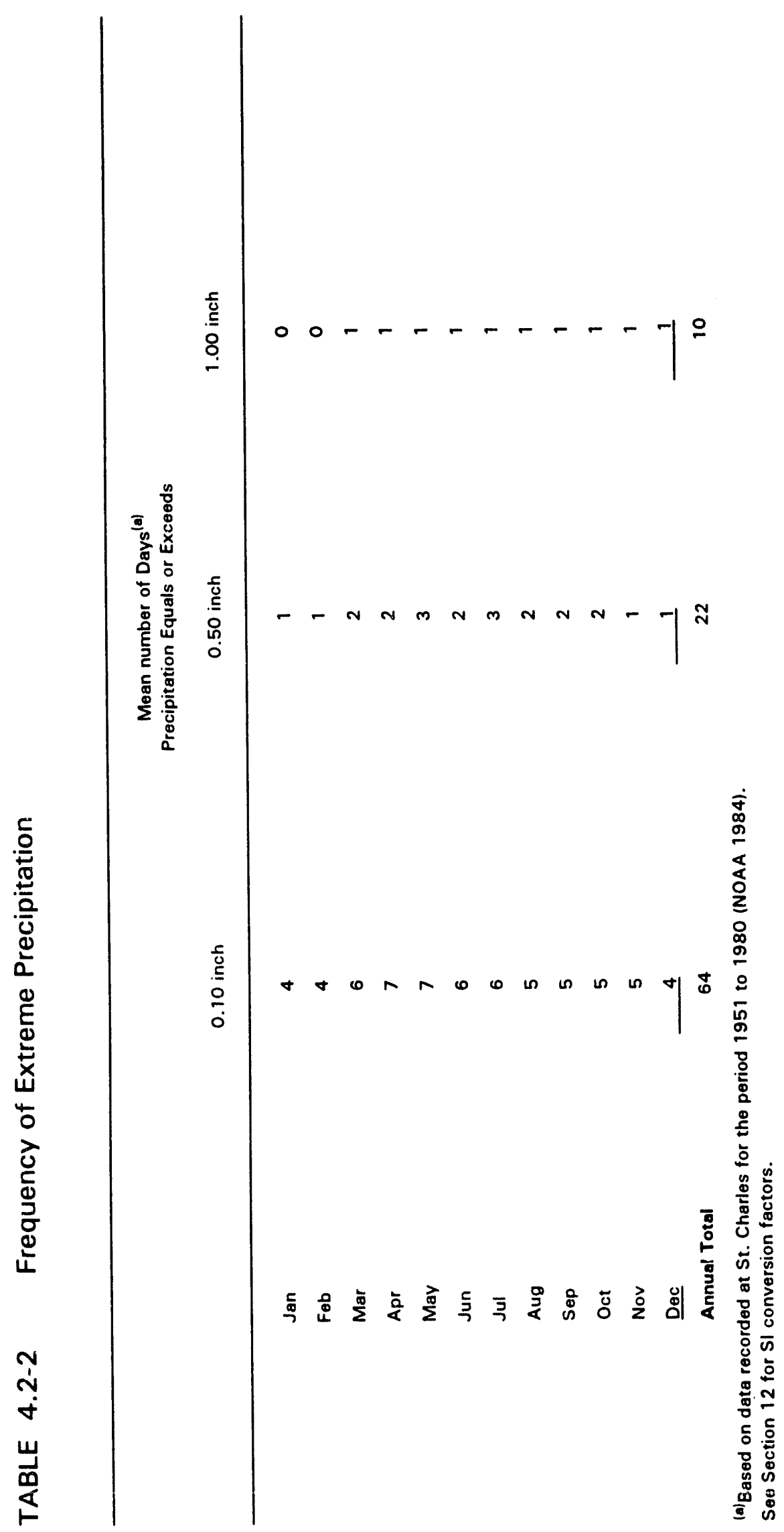

จ
ญิ
으 


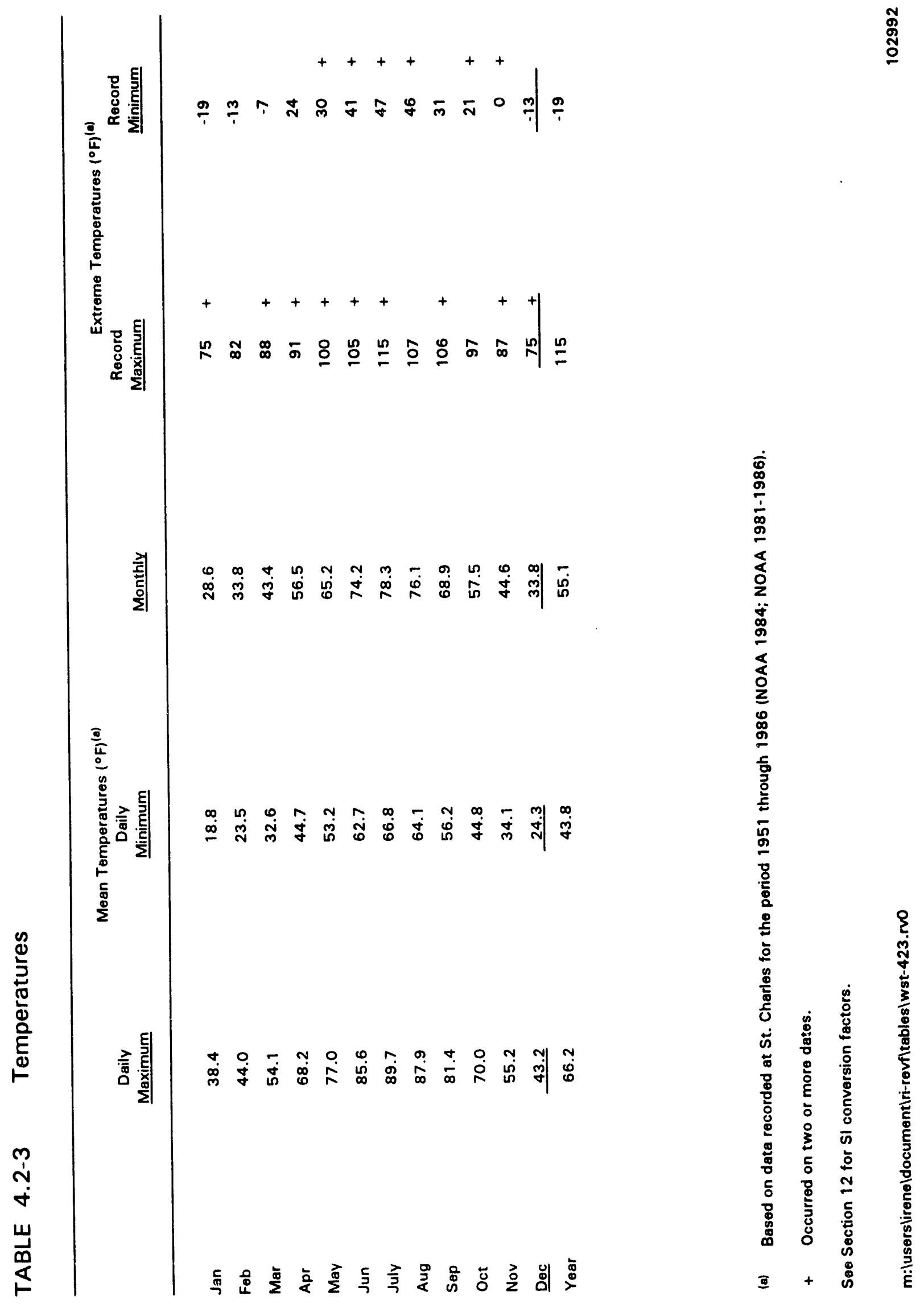


TABLE 4.2-4 Frequency of Temperature Extremes ${ }^{(a)}$

\begin{tabular}{|c|c|c|c|c|}
\hline \multicolumn{5}{|c|}{ Mean Number of Days } \\
\hline & $\begin{array}{c}\text { Maximum is } \\
90^{\circ} \mathrm{F} \\
\text { and above }\end{array}$ & $\begin{array}{l}\text { Maximum is } \\
32^{\circ} \mathrm{F} \\
\text { and below }\end{array}$ & $\begin{array}{l}\text { Minimum is } \\
32^{\circ} \mathrm{F} \\
\text { and below }\end{array}$ & $\begin{array}{c}\text { Minimum is } \\
\text { O०F } \\
\text { and below }\end{array}$ \\
\hline Jan & 0 & 11 & 27 & 3 \\
\hline Fob & 0 & 6 & 23 & 1 \\
\hline Mar & 0 & 1 & 16 & 0 \\
\hline Apr & 0 & 0 & 3 & 0 \\
\hline May & 2 & 0 & 0 & 0 \\
\hline Jun & 10 & 0 & 0 & 0 \\
\hline July & 16 & 0 & 0 & 0 \\
\hline Aug & 14 & 0 & 0 & 0 \\
\hline Sep & 6 & 0 & 0 & 0 \\
\hline Oct & 1 & 0 & 3 & 0 \\
\hline Nov & 0 & 1 & 14 & 0 \\
\hline Dec & 0 & 6 & 25 & 1 \\
\hline Year & 49 & 25 & 111 & 5 \\
\hline
\end{tabular}

(a) Based on date recorded at St. Charles for the period 1951 through 1980 (NOAA 1984).

See Section 12 for SI conversion factors.

m:luserslireneldocument|ri-revf|tables|wst-424.rvo 
TABLE 4.2-5 Relative Humidity and Heat Stress Factors

\begin{tabular}{|c|c|c|c|c|c|}
\hline & \multirow[b]{2}{*}{ Midnight } & \multicolumn{2}{|c|}{ Relative Humidity $(\%)^{(a)}$} & \multirow[b]{2}{*}{$6: 00 \mathrm{pm}$} & \multirow{2}{*}{$\begin{array}{l}\text { Heat } \\
\text { Stress } \\
\text { Factor }^{(b)}\end{array}$} \\
\hline & & 6:00 am & Noon & & \\
\hline Jan & 76 & 81 & 64 & 68 & $\cdot$ \\
\hline Feb & 75 & 79 & 60 & 63 & - \\
\hline Mar & 73 & 80 & 57 & 58 & - \\
\hline Apr & 70 & 77 & 54 & 53 & - \\
\hline May & 75 & 82 & 56 & 55 & 0 \\
\hline Jun & 78 & 84 & 57 & 56 & $f$ \\
\hline July & 78 & 86 & 57 & 56 & $f$ \\
\hline Aug & 80 & 89 & 57 & 58 & $f$ \\
\hline Sep & 83 & 91 & 60 & 63 & 0 \\
\hline Oct & 76 & 84 & 55 & 60 & $\cdot$ \\
\hline Nov & 78 & 84 & 62 & 68 & - \\
\hline Dec & 81 & 85 & 69 & 74 & - \\
\hline
\end{tabular}

(a) Relative humidity based on data recorded at St. Louis for the period 1941 to 1970 (Ruffner 1978).

(b) Heat Stress Factors for St. Louis (Rudloff 1981):

Stress occurs occasionally and only for a short period during the daytime, generally in the afternoon.

$f$ Stress occurs frequently; often for several hours in the daytime. 
TABLE 4.2-6 Wind Speed and Direction ${ }^{(\mathrm{a})}$

\begin{tabular}{lcccc}
\hline & $\begin{array}{c}\text { Mean } \\
\text { Wind Speed } \\
(\mathrm{mph})\end{array}$ & $\begin{array}{c}\text { Prevailing } \\
\text { Direction }\end{array}$ & $\begin{array}{c}\text { Fastest Milo } \\
\text { of Wind } \\
(\mathrm{mph})\end{array}$ & Direction \\
\hline Jan & 10.2 & $\mathrm{NW}$ & 39 & $\mathrm{~W}$ \\
Fob & 10.8 & $\mathrm{NW}$ & 46 & $\mathrm{NW}$ \\
Mar & 11.7 & WNW & 45 & $\mathrm{NE}$ \\
Apr & 11.4 & WNW & 45 & $\mathrm{~W}$ \\
May & 9.5 & $\mathrm{~S}$ & 42 & $\mathrm{SE}$ \\
Jun & 8.6 & $\mathrm{~S}$ & 60 & $\mathrm{SE}$ \\
July & 7.7 & $\mathrm{~S}$ & 40 & $\mathrm{NW}$ \\
Aug & 7.4 & $\mathrm{~S}$ & 38 & $\mathrm{NW}$ \\
Sop & 8.0 & $\mathrm{~S}$ & 39 & $\mathrm{SW}$ \\
Oct & 8.5 & $\mathrm{~S}$ & 48 & $\mathrm{SW}$ \\
Nov & 9.9 & $\mathrm{~S}$ & 41 & $\mathrm{~S}$ \\
Dec & 10.2 & WNW & 44 & $\mathrm{~W}$ \\
Yoar & 9.5 & $\mathrm{~S}$ & 60 & $\mathrm{SE}$
\end{tabular}

(a) Based on data recorded at St. Louis for the period 1941 to 1970 (Ruffner 1978).

See Section 12 for SI conversion factors. 
TABLE 4.2-7 Precipitation Recorded at the Weldon Spring Site

\begin{tabular}{|c|c|c|c|c|c|c|}
\hline \multicolumn{7}{|c|}{ Precipitation (inches) (e) $^{(a)}$} \\
\hline Month & 1983 & 1984 & 1985 & 1986 & 1987 & 1988 \\
\hline Jan & NA & 0.83 & 0.08 & NA & NA & 0.99 \\
\hline Fob & NA & 2.48 & 5.43 & NA & 0.39 & 2.35 \\
\hline Mar & NA & 7.99 & 5.55 & NA & 3.58 & 3.02 \\
\hline Apr & 3.35 & 9.84 & 3.31 & NA & 2.32 & 1.67 \\
\hline May & 6.89 & 5.63 & 2.40 & NA & 1.88 & 1.33 \\
\hline Jun & 425 & 1.18 & 6.81 & NA & 1.94 & 1.75 \\
\hline Jul & 3.43 & 1.14 & 3.46 & NA & 5.05 & 6.56 \\
\hline Aug & 5.47 & 1.93 & 4.65 & NA & 1.20 & 2.13 \\
\hline Sep & $0.04^{\circ}$ & $2.24^{\circ}$ & 0.94 & NA & 2.56 & 1.15 \\
\hline Oct & 3.31 & 6.14 & 2.64 & NA & 2.19 & 3.24 \\
\hline Nov & 4.69 & 4.96 & 9.96 & NA & 4.40 & 6.54 \\
\hline$\underline{\text { Dec }}$ & $\underline{2.05}$ & $\underline{6.02}$ & $\underline{2.40}$ & NA & 1.43 & 1.34 \\
\hline Yoar & .. & 50.39 & 47.64 & -- & -. & 32.07 \\
\hline
\end{tabular}

(a) 1983-1985 data recorded at the raffinate pits (BNI 1986a) and 1987-1988 data recorded at the chernical plant (MKF and JEG 1989f).

NA Not available

- Partial data

-. Incomplete

See Section 12 for SI conversion factors.

m:luserslireneldocument|ri-revfltables $\mid w s t-427 . r v 0$ 
TABLE 4.2-8 Temperatures Recorded at the Weldon Spring Site

\begin{tabular}{|c|c|c|c|c|}
\hline \multicolumn{4}{|c|}{ MONTHLY AVERAGE TEMPERATURE $\left({ }^{\circ} \mathrm{F}\right)^{(0)}$} & \\
\hline Jan & NA & 32.7 & 22.8 & \\
\hline Feb & NA & 44.2 & 30.2 & \\
\hline Mar & NA & 36.1 & 48.9 & \\
\hline Apr & 50.5 & 53.1 & 58.5 & \\
\hline May & 63.1 & 62.1 & 68.0 & \\
\hline Jun & 75.4 & 76.3 & 70.2 & \\
\hline Jul & 82.9 & 76.3 & 76.8 & \\
\hline Aug & 82.6 & 77.5 & 71.1 & \\
\hline Sep & 71.4 & $73.4^{\circ}$ & 68.2 & \\
\hline Oct & 61.2 & 60.3 & 60.8 & \\
\hline Nov & 50.9 & 44.4 & 45.0 & \\
\hline$\underline{\text { Dec }}$ & $\underline{24.1}$ & 41.4 & $\underline{27.0}$ & \\
\hline Year & .. & 55.8 & 53.7 & \\
\hline
\end{tabular}

(a) Based on data recorded at the raffinate pits (Shell Engineering, Inc. 1985a).

NA Not available

- Partial data

-. Incomplete

See Section 12 for SI conversion factors.

m:luserslireneldocument|ri-revfltablesiwst-428.rvo 


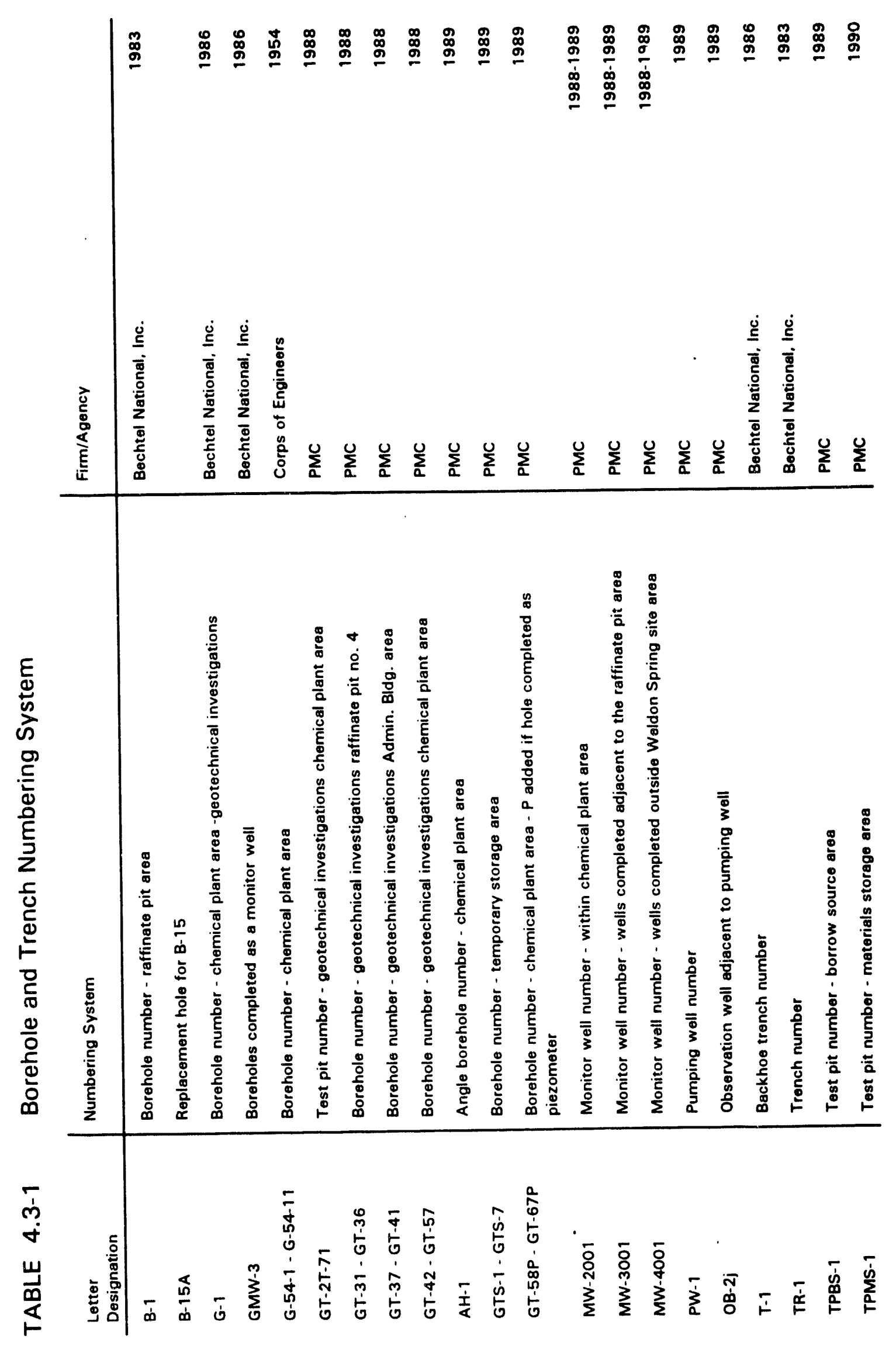

จั้

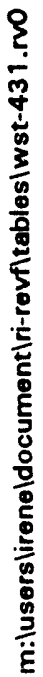


TABLE 4.3-2 Boreholes and Trenches Used in the Geologic Database

\begin{tabular}{|c|c|c|c|c|c|c|}
\hline $\begin{array}{l}\text { WSSRAP } \\
\text { ID }\end{array}$ & Old ID & $\begin{array}{l}\text { Northing } \\
\text { (ft) }\end{array}$ & $\begin{array}{l}\text { Westing } \\
(\mathrm{ft})\end{array}$ & $\begin{array}{l}\text { Ground } \\
\text { Elovation } \\
\text { (ft) }\end{array}$ & $\begin{array}{l}\text { Depth } \\
\text { To Filter } \\
\text { Top (ft) }\end{array}$ & $\begin{array}{c}\text { Total } \\
\text { Depth } \\
\text { (ft) }\end{array}$ \\
\hline B-1 & & 99507.3 & 52283.3 & 638.9 & 17.5 & 21.5 \\
\hline B-10 & & 99257.8 & 52044.6 & 665.8 & 22.3 & 25.6 \\
\hline B-12 & & 100003.4 & 51968.9 & 663.6 & 27.8 & 30.0 \\
\hline B.13 & & 99890.2 & 51545.7 & 663.8 & 0.0 & 27.0 \\
\hline B-14 & & 99236.9 & 50965.7 & 653.5 & 13.7 & 21.8 \\
\hline B-15 & & 99420.6 & 51025.4 & 663.9 & 0.0 & 30.0 \\
\hline B-15A & & 99410.5 & 51021.6 & 663.4 & 24.3 & 37.0 \\
\hline B-18 & & 99218.8 & 50750.8 & 658.8 & 22.3 & 24.4 \\
\hline B-19 & & 99596.7 & 50805.6 & 645.4 & 0.0 & 21.5 \\
\hline B-20 & & 99597.6 & 50956.6 & 643.8 & 27.8 & 29.5 \\
\hline B-22 & & 99931.7 & 51266.7 & 647.4 & 12.0 & 15.0 \\
\hline B-5 & & 99235.3 & 50975.6 & 653.3 & 18.0 & 21.5 \\
\hline B-6 & & 99050.0 & 51224.3 & 663.7 & 17.7 & 21.5 \\
\hline B-7 & & 98764.4 & 51596.9 & 658.2 & 20.1 & 22.8 \\
\hline B-8 & & 98750.8 & 51969.1 & 646.7 & 26.2 & 33.0 \\
\hline G-1 & & 98473.2 & 50581.0 & 668.0 & 0.0 & 84.0 \\
\hline G-13 & & 99521.0 & 50517.0 & 654.7 & 0.0 & 71.0 \\
\hline G-14 & & 99199.0 & 49935.0 & 655.8 & 0.0 & 76.4 \\
\hline G-15 & & 98924.0 & 50447.0 & 658.0 & 0.0 & 75.5 \\
\hline G-16 & & - $\quad 98051.0$ & 51007.0 & 656.7 & 0.0 & 80.4 \\
\hline G-18 & & 101350.0 & 52551.0 & 633.8 & 0.0 & 79.0 \\
\hline G-19 & & 101700.0 & 51950.0 & 619.4 & 0.0 & 66.0 \\
\hline G-2 & & 100449.9 & 50577.3 & 657.9 & 0.0 & 64.0 \\
\hline G.20 & & 101850.0 & 50950.0 & 630.3 & 0.0 & 66.0 \\
\hline G.21 & & 101336.0 & 52116.0 & 638.7 & 0.0 & 74.5 \\
\hline$G-2 A$ & & 100440.0 & 50578.1 & 658.0 & 0.0 & 83.0 \\
\hline G-3 & & 101195.3 & 50948.6 & 654.0 & 0.0 & 88.2 \\
\hline G.4 & & 101140.6 & 51295.6 & 644.0 & 0.0 & 81.0 \\
\hline G.5 & & 100650.0 & 51250.0 & 635.9 & 0.0 & 73.0 \\
\hline G-54-1 & & 99718.0 & 49700.0 & 657.0 & 0.0 & 28.8 \\
\hline G-54-10 & & 100681.0 & 50603.0 & 661.6 & 0.0 & 67.7 \\
\hline G-54-11 & & 100761.0 & 50601.0 & 659.6 & 0.0 & 64.0 \\
\hline G.54-2 & & 100606.0 & 50167.0 & 659.0 & 0.0 & 46.5 \\
\hline G-54-3 & & 101491.0 & 50635.0 & 630.0 & 0.0 & 24.5 \\
\hline G-54-4 & & 101103.0 & 49872.0 & 640.3 & 0.0 & 26.8 \\
\hline G-54-5 & & 100388.0 & 49872.0 & 656.0 & 0.0 & 36.4 \\
\hline G.54-6 & & 99250.0 & 50583.0 & 660.0 & 0.0 & 47.0 \\
\hline G-54-7 & & 99918.0 & 50370.0 & 664.5 & 0.0 & 52.5 \\
\hline G-54-8 & & 100810.0 & 50838.0 & 654.4 & 0.0 & 63.6 \\
\hline G-54-9 & & 100732.0 & 50510.0 & 659.6 & 0.0 & 65.4 \\
\hline G-6 & & 100450.0 & 51150.0 & 639.7 & 0.0 & 67.0 \\
\hline G-7 & & 101200.0 & 50450.0 & 633.3 & 0.0 & 64.0 \\
\hline G.8 & & 100450.0 & 49900.0 & 655.3 & 0.0 & 75.0 \\
\hline G-9 & & 100065.0 & 49905.0 & 656.0 & 0.0 & 76.0 \\
\hline GT-2T-71 & & 100381.3 & 49131.3 & 654.7 & 0.0 & 12.0 \\
\hline GT-2T-72 & & 100864.2 & 49426.0 & 639.4 & 0.0 & 12.0 \\
\hline GT-2T-73 & & 99346.1 & 49908.1 & 656.2 & 0.0 & 12.0 \\
\hline GT-2T-74 & & 100002.3 & 49862.6 & 655.7 & 0.0 & 12.0 \\
\hline GT-2T-75 & & 100970.4 & 50218.3 & 650.0 & 0.0 & 12.0 \\
\hline
\end{tabular}

(a) Atomic Energy Commission Coordinate System See Section 12 for SI conversion factors.

$\mathrm{m}$ :|userslireneldocument|ri-revfltablesiwst-432.rvo 
TABLE 4.3-2 Boreholes and Trenches Used in the Geologic Database (Continued)

\begin{tabular}{|c|c|c|c|c|c|c|}
\hline $\begin{array}{l}\text { WSSRAP } \\
\text { ID }\end{array}$ & Old ID & ${ }_{(f t)}^{\text {Northing }}{ }^{(a)}$ & $\begin{array}{l}\text { Westing } \\
\text { (ft) }\end{array}$ & $\begin{array}{l}\text { Ground } \\
\text { Elevation } \\
(f t)\end{array}$ & $\begin{array}{l}\text { Depth } \\
\text { To Filter } \\
\text { Top (ft) }\end{array}$ & $\begin{array}{l}\text { Total } \\
\text { Depth } \\
\text { (ft) }\end{array}$ \\
\hline GT-2T-76 & & 99071.0 & 50508.2 & 660.6 & 0.0 & 12.0 \\
\hline GT-2T-77 & & 99739.0 & 50636.7 & 649.9 & 0.0 & 12.0 \\
\hline GT-2T-78 & & 100495.3 & 50768.8 & 657.6 & 0.0 & 12.0 \\
\hline GT-2T-79 & & 101256.9 & 51188.4 & 650.3 & 0.0 & 12.0 \\
\hline GT-2T-80 & & 98894.6 & 51404.7 & 661.1 & 0.0 & 12.0 \\
\hline GT-2T-81 & & 98818.2 & 51869.6 & 664.7 & 0.0 & 12.0 \\
\hline GT-2T-82 & & 100196.1 & 52173.4 & 638.3 & 0.0 & 11.0 \\
\hline GT-31 & & 99119.3 & 52130.8 & 632.5 & 4.0 & 34.0 \\
\hline GT-32 & & 99939.8 & 52126.5 & 641.3 & 3.0 & 24.0 \\
\hline GT-33 & & 99901.6 & 52079.0 & 664.6 & 4.0 & 51.0 \\
\hline GT.34 & & 99546.3 & 52202.1 & 663.8 & 4.0 & 54.5 \\
\hline GT-35 & & 99115.1 & 52039.3 & 664.3 & 4.0 & 54.0 \\
\hline GT-36 & & 98864.4 & 52006.5 & 663.2 & 3.5 & 47.8 \\
\hline GT-37 & GT-2B37 & 99800.0 & 49522.0 & 657.0 & 0.0 & 24.0 \\
\hline GT-38 & GT-2B38 & 99700.0 & 49570.0 & 657.7 & 0.0 & 26.5 \\
\hline GT-39 & GT-2B39 & 99900.0 & 49470.0 & 656.7 & 0.0 & 22.5 \\
\hline GT.40 & GT-2B40 & 99700.0 & 49470.0 & 657.4 & 0.0 & 23.0 \\
\hline GT-41 & GT-2B41 & 99900.0 & 49570.0 & 55.9 & 0.0 & 21.5 \\
\hline GT-42 & & 101206.0 & 50804.6 & 648.0 & 0.0 & 57.0 \\
\hline GT-43 & & 100736.6 & 50786.1 & 654.2 & 0.0 & 78.5 \\
\hline GT-44 & & 101030.8 & 49875.6 & 643.3 & 0.0 & 49.5 \\
\hline GT-45 & & 100775.2 & 49344.2 & 645.0 & 0.0 & 41.5 \\
\hline GT-46 & & 100364.8 & 49340.0 & 651.8 & 0.0 & 40.5 \\
\hline GT-47 & & 100024.8 & 50128.9 & 660.3 & 0.0 & 55.5 \\
\hline GT- 48 & & 99353.0 & 50568.1 & 657.7 & 0.0 & 53.6 \\
\hline GT.49 & & 98517.3 & 50560.8 & 665.3 & 0.0 & 29.5 \\
\hline GT-50 & & 99223.0 & 50905.0 & 653.8 & 0.0 & 55.5 \\
\hline GT-51 & & 99725.9 & 50758.5 & 646.1 & 0.0 & 49.9 \\
\hline GT-52 & & 100577.0 & 50335.0 & 658.8 & 0.0 & 70.2 \\
\hline GT-53 & & 100284.0 & 50781.0 & 655.5 & 0.0 & 42.5 \\
\hline GT-54 & & 100618.0 & 49635.0 & 652.4 & 0.0 & 50.0 \\
\hline GT-55 & & 100490.0 & 49937.0 & 655.6 & 0.0 & 53.0 \\
\hline GT-56 & & 99689.0 & 49971.0 & 657.1 & 0.0 & 43.0 \\
\hline GT-57 & & 100017.0 & 49739.0 & 654.7 & 0.0 & 36.5 \\
\hline $\mathrm{AH}-1$ & & 100017.0 & 49739.0 & 654.7 & 0.0 & 151.6 \\
\hline $\mathrm{AH}-2$ & & 100284.0 & 50781.0 & 655.5 & 0.0 & 165.0 \\
\hline GTS-1 & & 98670.0 & 50978.0 & 665.3 & 0.0 & 39.8 \\
\hline GTS-2 & & 98165.0 & 50800.0 & 665.8 & 0.0 & 37.3 \\
\hline GTS-3 & & 98110.0 & 51075.0 & 654.0 & 0.0 & 31.5 \\
\hline GTS-4 & & 98475.0 & 51250.0 & 651.5 & 0.0 & 27.5 \\
\hline GTS-5 & & 98607.7 & 51245.8 & 662.6 & 0.0 & 37.0 \\
\hline GTS- 6 & & 98933.0 & 51035.7 & 665.3 & 0.0 & 47.0 \\
\hline GTS-7 & & 98771.0 & 51381.8 & 662.1 & 0.0 & 44.5 \\
\hline GT-58P & & 100952.3 & 50055.2 & 653.0 & 30.5 & 60.9 \\
\hline GT-59 & & 99905.7 & 49963.8 & 657.0 & 0.0 & 27.0 \\
\hline
\end{tabular}

(a) A tomic Energy Commission Coordinate System

m:lusers lireneldocument|ri-revf|tables|wst-432.rvo 
TABLE 4.3-2 Boreholes and Trenches Used in the Geologic Database (Continued)

\begin{tabular}{|c|c|c|c|c|c|c|}
\hline $\begin{array}{l}\text { WSSRAP } \\
\text { ID }\end{array}$ & Old ID & $\begin{array}{l}\text { Northing } \\
(\mathrm{ft})\end{array}$ & $\begin{array}{l}\text { Westing } \\
\text { (ft) }\end{array}$ & $\begin{array}{l}\text { Ground } \\
\text { Elevation } \\
\text { (ft) }\end{array}$ & $\begin{array}{l}\text { Depth } \\
\text { To Filter } \\
\text { Top (ft) }\end{array}$ & $\begin{array}{c}\text { Total } \\
\text { Depth } \\
\text { (ft) }\end{array}$ \\
\hline G $P-60 P$ & & 100388.4 & 50260.4 & 660.6 & 58.2 & 67.5 \\
\hline GT-61 & & 100609.3 & 50679.2 & 658.1 & 0.0 & 59.5 \\
\hline GT-62 & & 100062.4 & 50630.9 & 657.3 & 0.0 & 43.5 \\
\hline GT-63P & & 98626.1 & 51472.0 & 656.9 & 35.2 & 51.0 \\
\hline GT-64P & & 100053.1 & 52053.3 & 640.9 & 22.0 & 57.0 \\
\hline GT-65P & & 101533.0 & 51161.1 & 634.9 & 48.0 & 60.0 \\
\hline GT-66P & & 100814.7 & 49527.9 & 644.7 & 23.1 & 47.5 \\
\hline GT.67P & & 100196.8 & 49036.8 & 657.7 & 34.5 & 60.0 \\
\hline MW-2001 & GMW-1 & 100857.7 & 52544.3 & 611.8 & 31.6 & 64.0 \\
\hline$M W-2002$ & $G M W-2 / 2 A$ & 100657.3 & 52249.8 & 623.8 & 31.7 & 64.0 \\
\hline MW-2003 & GMW-3 & 100353.6 & 52299.1 & 637.0 & 41.5 & 64.0 \\
\hline MW-2004 & GMW.4 & 101449.1 & 51753.4 & 642.9 & 54.3 & 77.0 \\
\hline MW-2005 & GMW-5 & 101131.4 & 51951.6 & 635.6 & 50.0 & 81.0 \\
\hline MW-2006 & GMW-6 & 101227.2 & 49849.7 & 634.1 & 27.0 & 71.0 \\
\hline$M W-2007$ & GMW-7 & 100930.3 & 50932.1 & 651.9 & 62.3 & 99.0 \\
\hline$M W-2008$ & GMW-8 & 101707.4 & 50654.1 & 622.8 & 34.0 & 63.0 \\
\hline MW-2009 & GMW-9 & 101351.5 & 50699.1 & 636.3 & 27.2 & 58.6 \\
\hline MW-2010 & GMW-10 & 101151.5 & 50100.1 & 643.0 & 37.2 & 64.0 \\
\hline$M W-2011$ & GMW-11 & 100898.2 & 50032.5 & 653.3 & 36.6 & 79.0 \\
\hline MW-2012 & GMW-12 & 101052.2 & 49641.6 & 634.9 & 29.3 & 69.5 \\
\hline$M W-2013$ & GMW-13 & 100820.7 & 49538.3 & 645.4 & 31.3 & 75.0 \\
\hline$M W-2014$ & GMW-14 & 100736.9 & 49189.9 & 647.4 & 37.0 & 64.0 \\
\hline$M W-2015$ & GMW-15 & 100099.7 & 50550.5 & 657.4 & 47.3 & 86.0 \\
\hline$M W \cdot 2016$ & B.3 & 101532.6 & 51176.7 & 635.7 & 62.7 & 150.5 \\
\hline MW-2017 & GMW-17 & 100201.0 & 49049.9 & 657.7 & 30.0 & 69.0 \\
\hline MW-2018 & GMW-18 & 98296.9 & 50381.6 & 661.5 & 37.4 & 69.0 \\
\hline MW-2019 & & 98287.4 & 50391.3 & 661.5 & 103.0 & 116.3 \\
\hline MW-2020 & B.4 & 99548.3 & 49549.1 & 655.5 & 36.5 & 119.6 \\
\hline MW-2021 & & 100634.9 & 52263.2 & 624.6 & 96.3 & 111.0 \\
\hline MW-2022 & & 101154.8 & 51945.6 & 636.1 & 112.0 & 128.0 \\
\hline$M W-2023$ & & 101551.0 & 51140.9 & 635.7 & 68.5 & 91.8 \\
\hline MW. 2024 & & 101552.0 & 51186.0 & 635.0 & 0.0 & 149.6 \\
\hline MW-2025 & & 101688.3 & 50648.9 & 622.3 & 94.0 & 108.6 \\
\hline MW-2026 & & 101215.3 & 49861.1 & 631.0 & 105.5 & 118.0 \\
\hline MW-2027 & & 100858.0 & 9546.5 & 646.6 & 105.5 & 120.5 \\
\hline$M W \cdot 2028$ & & 100092.5 & 50543.0 & 657.8 & 116.0 & 131.2 \\
\hline MW-2029 & & 101452.6 & 51734.1 & 643.4 & 89.0 & 101.3 \\
\hline MW-3001 & & 98852.6 & 51262.4 & 664.3 & 52.7 & 90.0 \\
\hline$M W-3002 B$ & & 98856.6 & 51245.6 & 664.7 & 134.0 & 150.0 \\
\hline MW-3003 & & 100046.0 & 52094.5 & 644.3 & 75.7 & 89.5 \\
\hline MW-3006 & & 100048.7 & 52070.6 & 645.9 & 120.0 & 135.5 \\
\hline$M W-3007$ & B.17 & 100043.4 & 52082.1 & 645.5 & 39.0 & 99.1 \\
\hline MW-3008 & B. $19 A$ & 99546.4 & 50954.3 & 645.1 & 39.0 & 101.0 \\
\hline MW-3009 & $8-21$ & 98832.5 & 52123.2 & 644.3 & 45.0 & 99.4 \\
\hline MW-3010 & B-23 & 98471.5 & 50936.4 & 665.0 & 52.5 & 90.7 \\
\hline \multicolumn{7}{|c|}{ (a) Atomic Energy Commission Coordinate System } \\
\hline m:luserslire & neldocumen & tablesiwst-4 & 3 & & & 1029 \\
\hline
\end{tabular}


TABLE 4.3-2 $\begin{aligned} & \text { Boreholes and Trenches Used in the Geologic Database } \\ & \text { (Continued) }\end{aligned}$

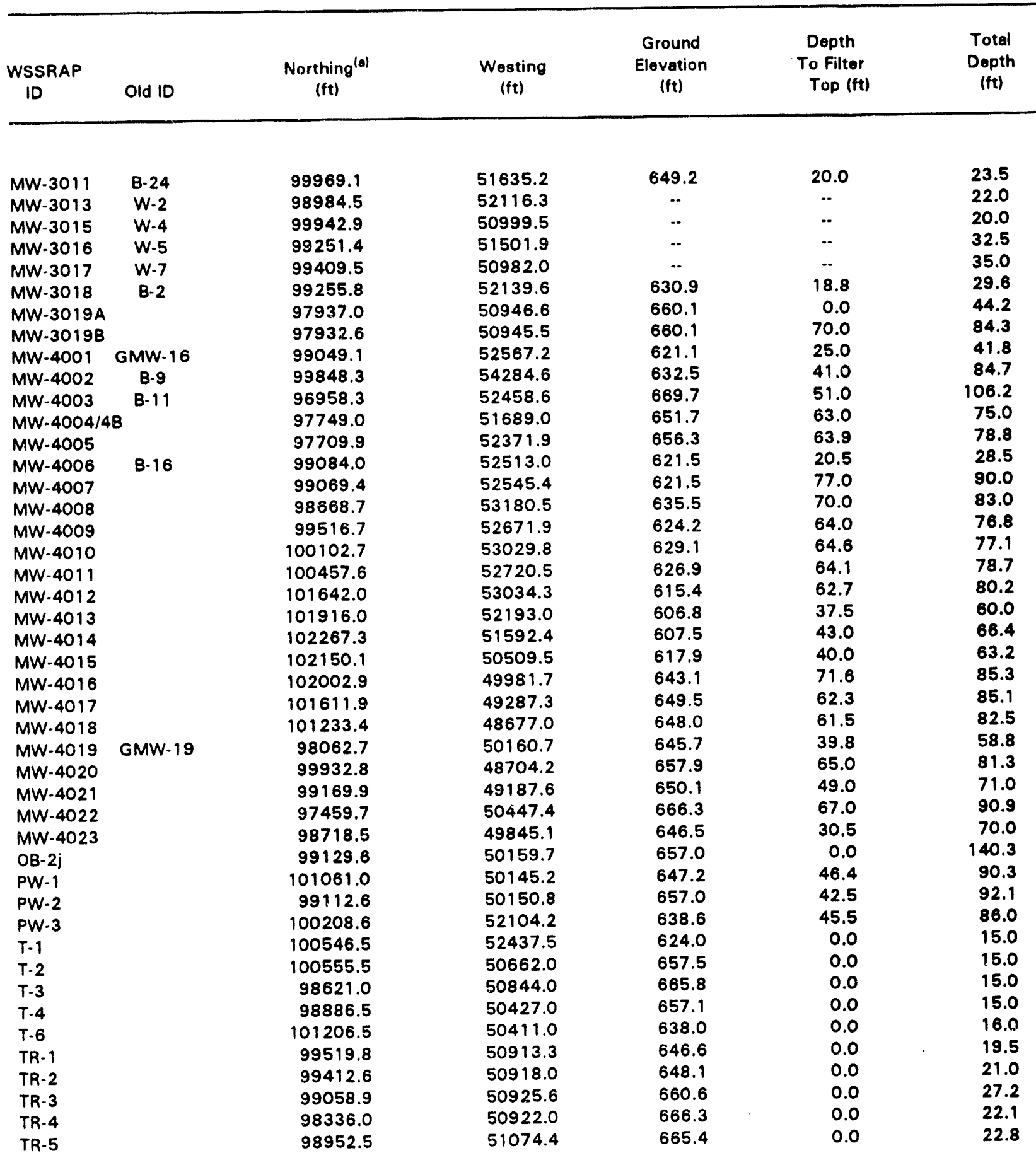

(a) Atomic Energy Commission Coordinate System

m:luserslireneldocument|ri-revf|tables|wst-432.rvo 
TABLE 4.3-2 $\begin{aligned} & \text { Boreholes and Trenches Used in the Geologic Database } \\ & \text { (Continued) }\end{aligned}$

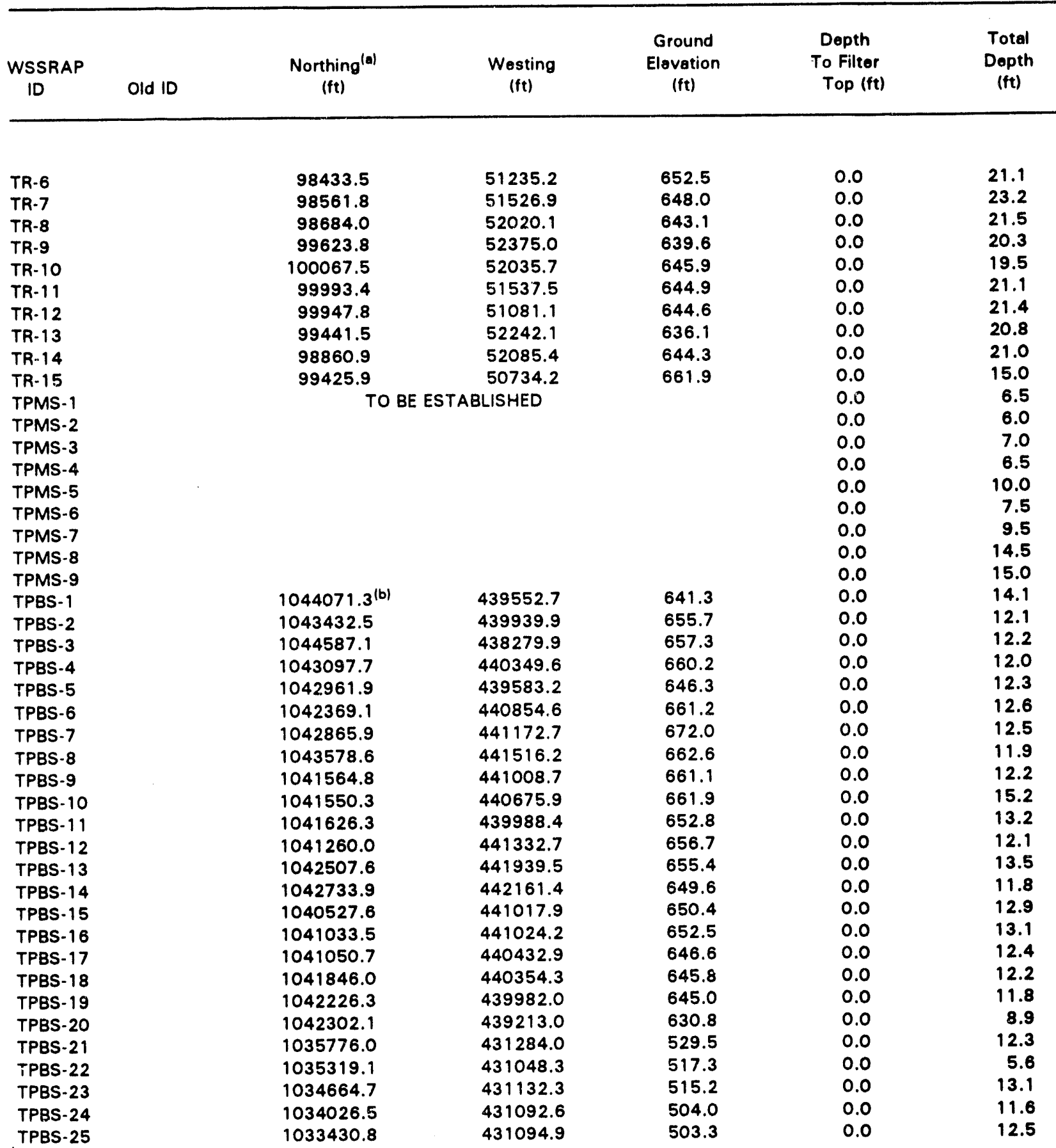

\footnotetext{
(a) Atomic Energy Commission Coordinate System

(b) Missouri State Plane Coordinates

m:luserslireneldocument|ri-revfltables|wst-432.rvo
} 
TABLE 4.3-2 Boreholes and Trenches Used in the Geologic Database (Continued)

\begin{tabular}{|c|c|c|c|c|c|c|}
\hline $\begin{array}{l}\text { WSSRAP } \\
\text { ID }\end{array}$ & Old ID & $\begin{array}{c}\text { Northing } \\
(f t)\end{array}$ & $\begin{array}{l}\text { Westing } \\
\text { (ft) }\end{array}$ & $\begin{array}{l}\text { Ground } \\
\text { Elevetion } \\
\text { (ft) }\end{array}$ & $\begin{array}{l}\text { Depth } \\
\text { To Filtor } \\
\text { Top (ft) }\end{array}$ & $\begin{array}{l}\text { Total } \\
\text { Depth } \\
\text { (ft) }\end{array}$ \\
\hline TPBS-26 & & 1032969.4 & 431062.8 & 497.7 & 0.0 & 13.4 \\
\hline TPBS-27 & & 1032350.8 & 431030.4 & 488.7 & 0.0 & 12.5 \\
\hline TPBS- 28 & & 1031686.4 & 430597.9 & 510.5 & 0.0 & 12.4 \\
\hline TPBS-29 & & 1031532.0 & 430195.4 & 511.9 & 0.0 & 12.0 \\
\hline TPBS-30 & & 1031340.5 & 429726.5 & 514.3 & 0.0 & 12.4 \\
\hline
\end{tabular}

\footnotetext{
(a) Atomic Energy Commission Coordinate System

(b) Missouri State Plane Coordinates

m:luserslireneldocument|ri-revfltablesiwst-432.rvo
} 


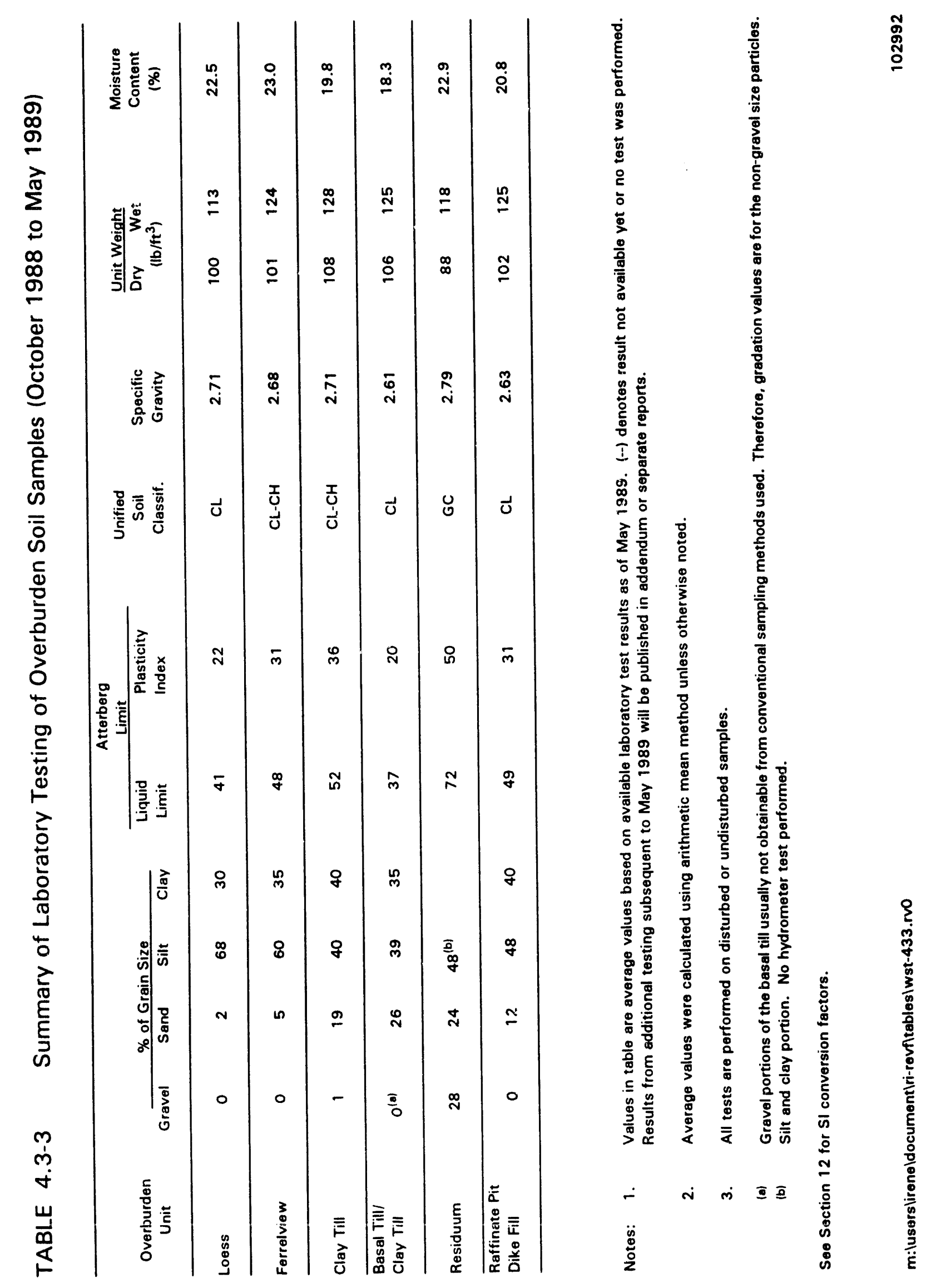




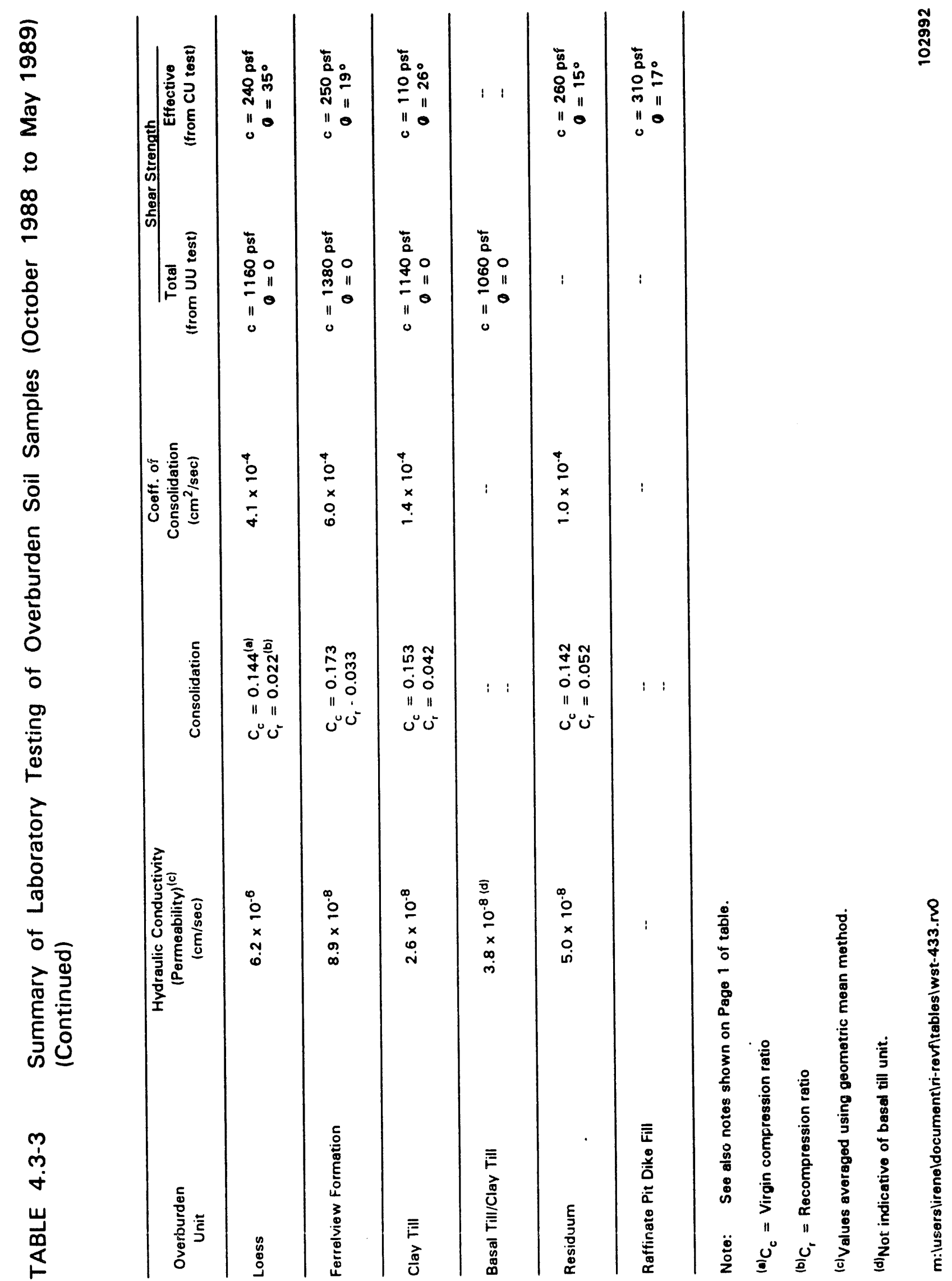




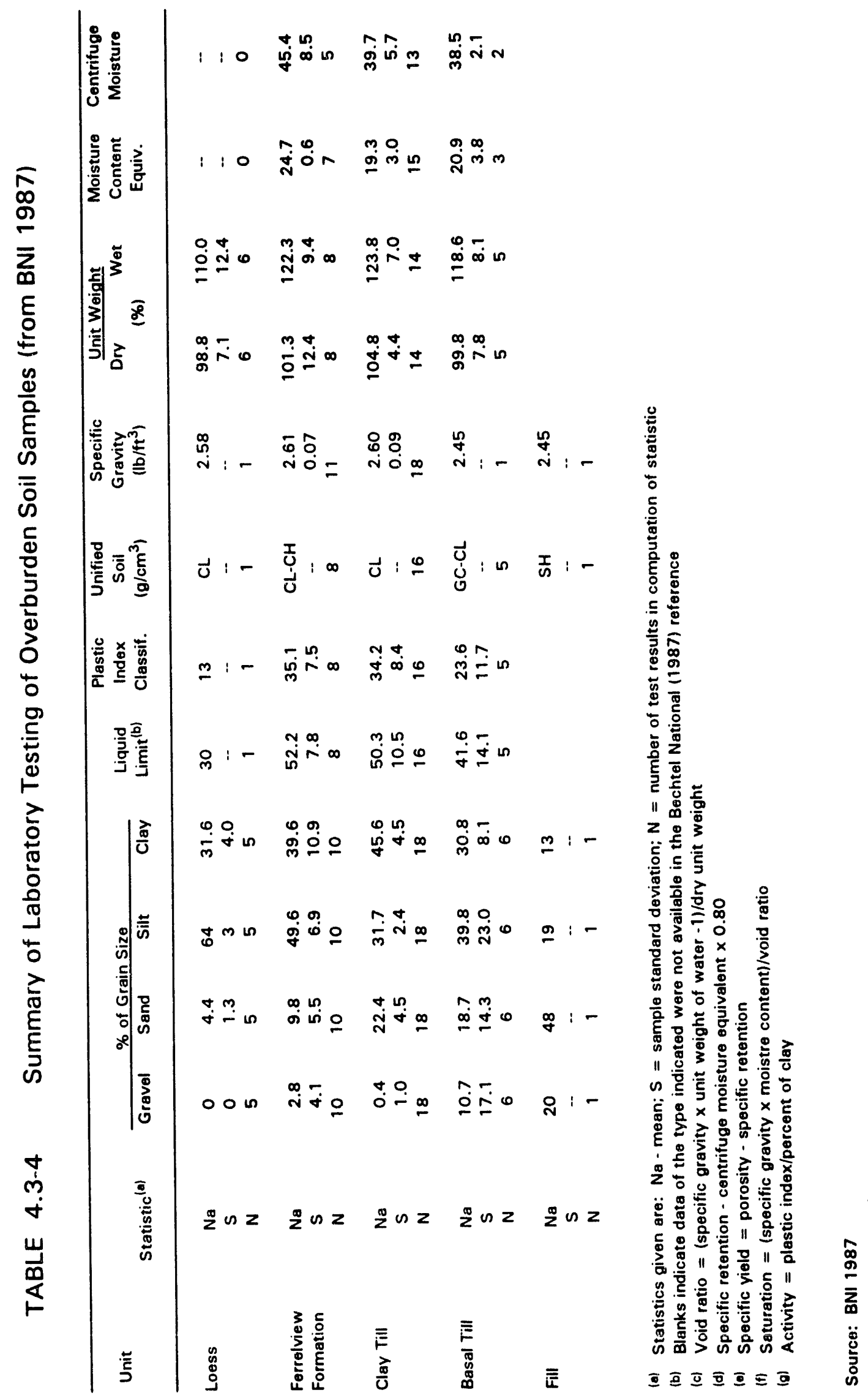




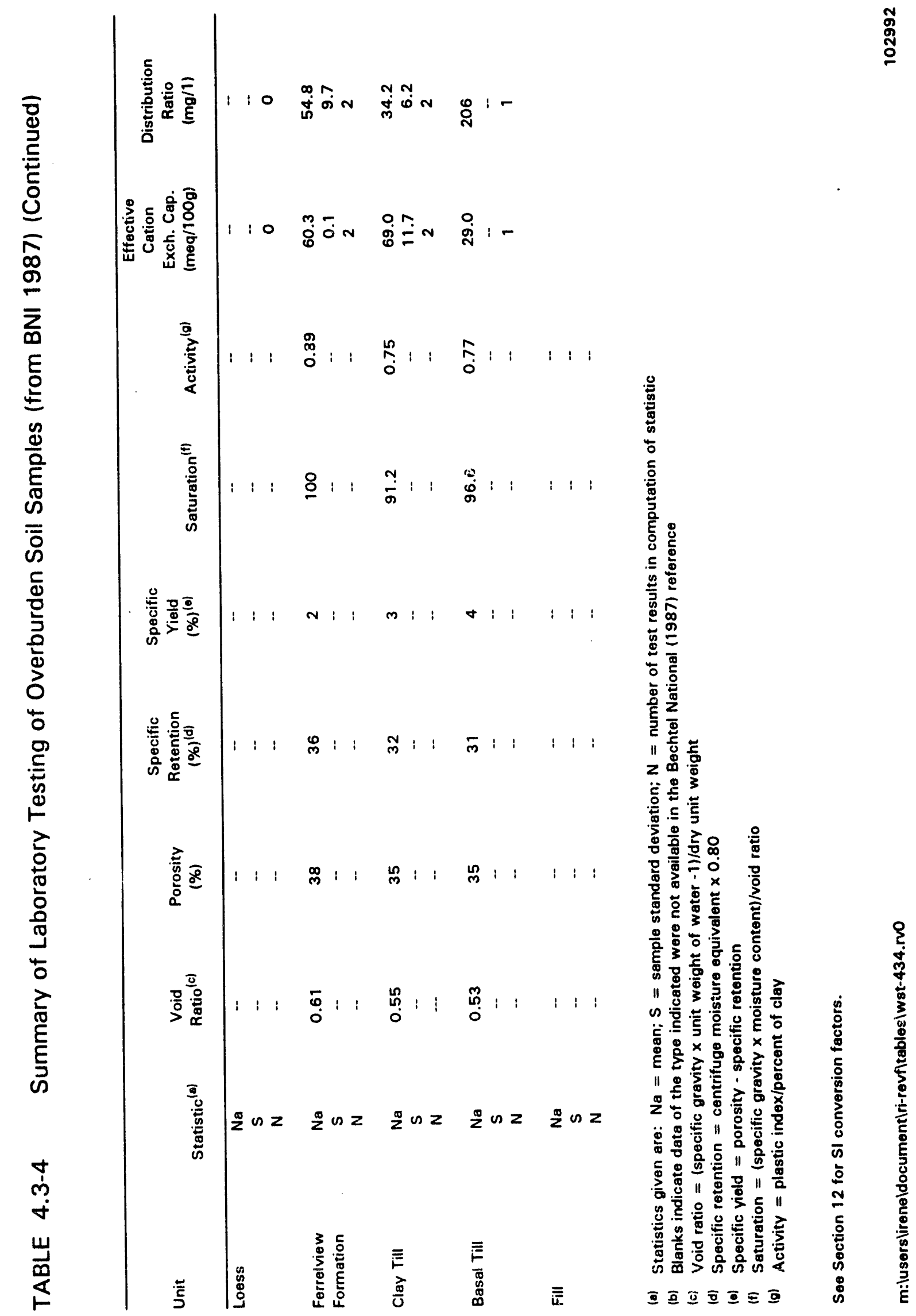


TABLE 4.3-5 Summary of Rock Quality Designation (RQD)

\begin{tabular}{lcccccc}
\hline & \multicolumn{5}{c}{ (Percentage of Core Runs in Given Range) } \\
\cline { 2 - 5 } Stratigraphic Unit & Very Poor & Poor & Fair & Good & Excellent $^{(\mathrm{n})}$ \\
\hline Weathered Limestone & 41 & 32 & 21 & 5 & 1 \\
Competent Limestone & 9 & 12 & 44 & 25 & 10
\end{tabular}

Note: ${ }^{(\theta)}$ RQD designation are defined as follows:

$\begin{array}{lr}\text { Very Poor } & 0-25 \% \\ \text { Poor } & 26-50 \% \\ \text { Fair } & 51-75 \% \\ \text { Good } & 76-90 \% \\ \text { Excellent } & 91-100 \%\end{array}$

m:luserslireneldocument $|r i-r e v f| t a b l e s \mid w s t-435 . r v o$ 


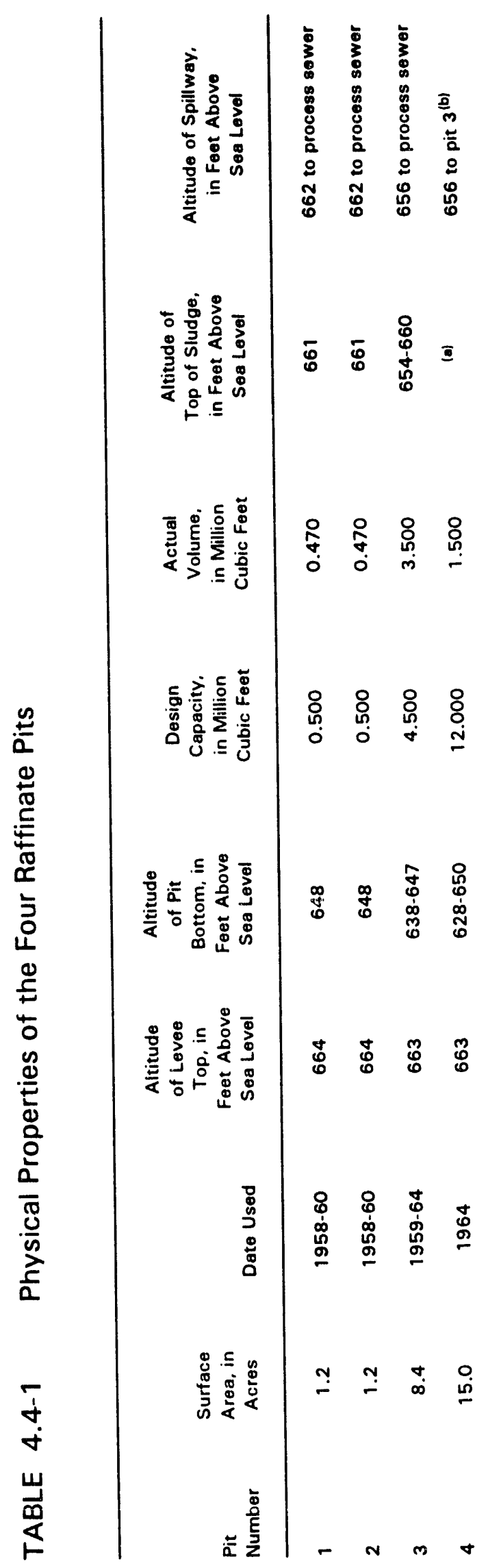

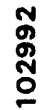

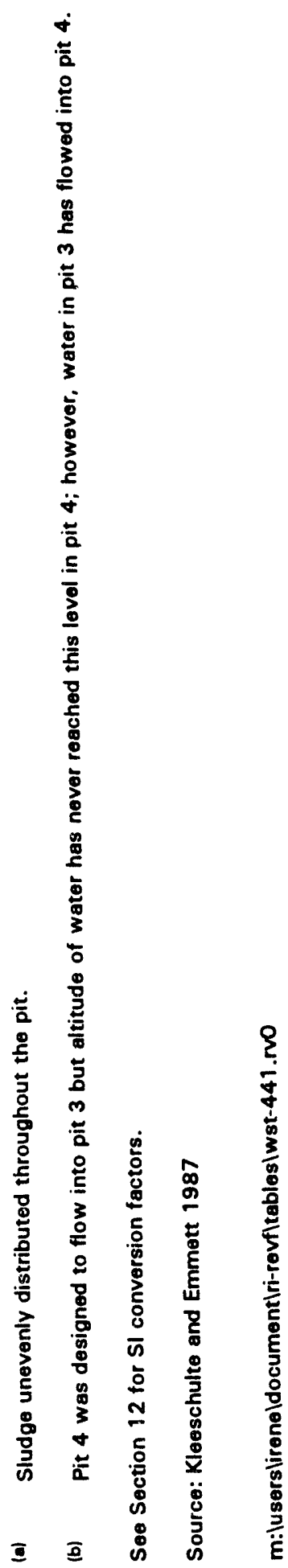




\section{TABLE 4.4-2 Drainage Areas of Surface Streams in the Vicinity of the Weldon Spring Site}

A. Schote Creak Drainage Basin

1. Frog Pond $\quad 52.7$

2. Lake 36

3. Unnamed tributary that drains Lake 36

at junction with Schote Creak

4. Ash Pond

60.1

5. Raffinate pits $1,2,3$ and 4 (closed basins)

6. Unnamed tributary containing Ash

Pond and raffinate pits areas

7. Schote Creek above junction with unnamed tributary containing Ash Pond and raffinate pit areas

8. Schote Creek at junction with

9. Lake 35

10. Schote Creek at junction with

Dardenne Creek

B. Unnamed Tributary of Dardenne Creek

1. At Burgermeister Spring $\quad 356$

352
622

$\begin{array}{lr}\text { 3. At junction with Dardenne Creek } & 1,358\end{array}$

$\begin{array}{lr}\text { C. Southeast Drainage } & 373\end{array}$

See Section 12 for SI conversion factors.

Sources: Surdex Corporation 1987 and USGS 1972 and 1982. 


\section{TABLE 4.4-3 Average Measured Flow Rate from NPDES Discharge Points in}

1987 and Estimated Average Annual Runoff

\begin{tabular}{|c|c|c|c|c|}
\hline $\begin{array}{l}\text { Discharge } \\
\text { Point }\end{array}$ & $\begin{array}{c}\text { Drainage } \\
\text { Area } \\
\text { (Acres) }\end{array}$ & $\begin{array}{c}\text { Average } \\
\text { Measured } \\
\text { Flow Rate }(1987) \\
\text { (gpm) }\end{array}$ & $\begin{array}{l}\text { Percent of } \\
\text { Precipitation } \\
\text { as Runoff }\end{array}$ & $\begin{array}{c}\text { Estimated Average } \\
\text { Annnual Runoff } \\
\text { Volume } \\
\text { (Mgal/yr) }\end{array}$ \\
\hline NP-0001/NP-0005 & 20.2 & 40 & 60 & 11.8 \\
\hline NP.0002 & 63.6 & 50 & 65 & 40.3 \\
\hline NP-0003 & 66.8 & 200 & 20 & 13.1 \\
\hline NP-0004 & 5.6 & 25 & 50 & 2.7 \\
\hline
\end{tabular}

(a) Flows were measured when there was sufficient precipitation to cause consistent runoff

See Section 12 for SI conversion factors.

Source: $\quad$ MKF and JEG 1988s (with modified drainage areas of NP-0002 and NP-0003) 


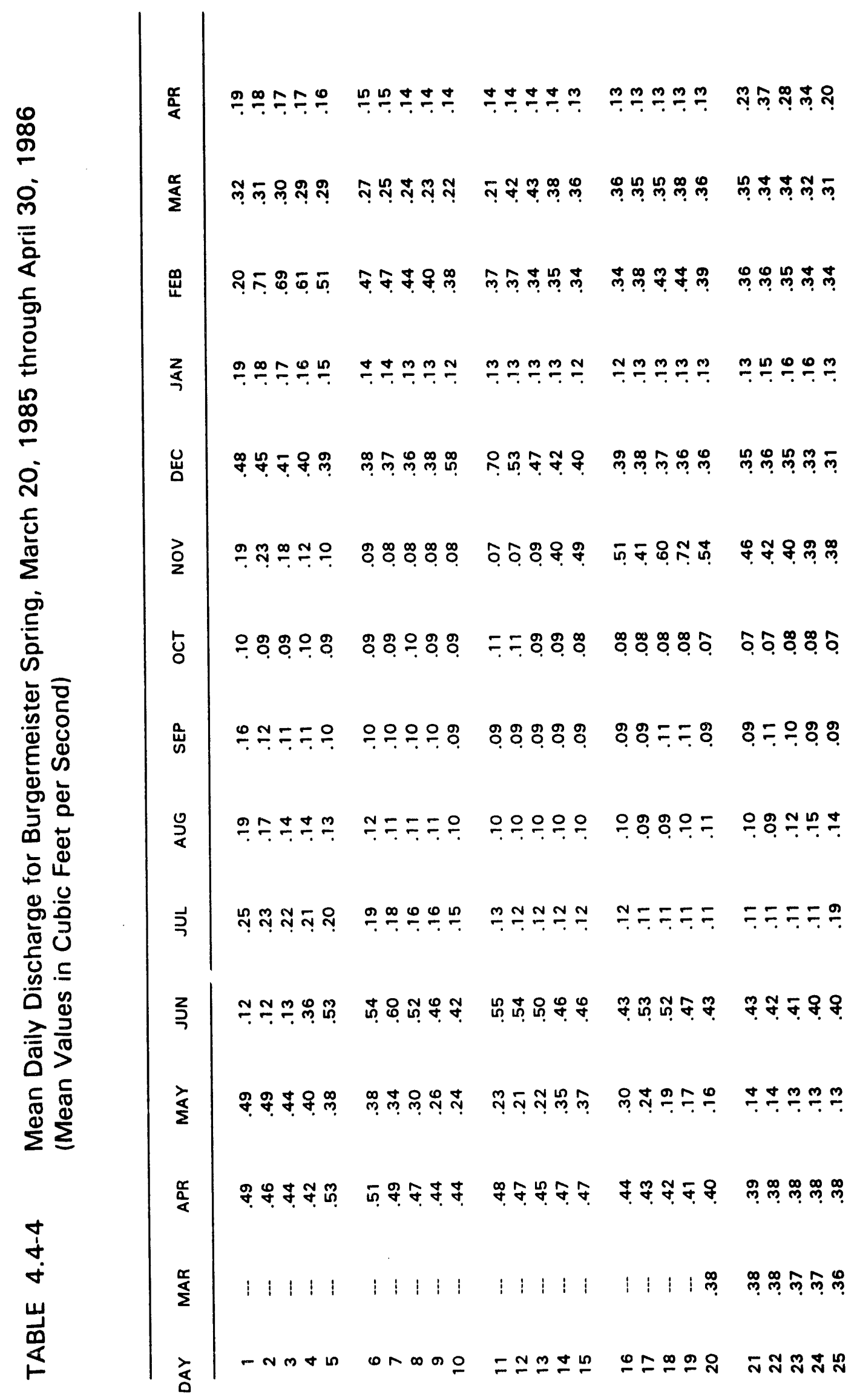




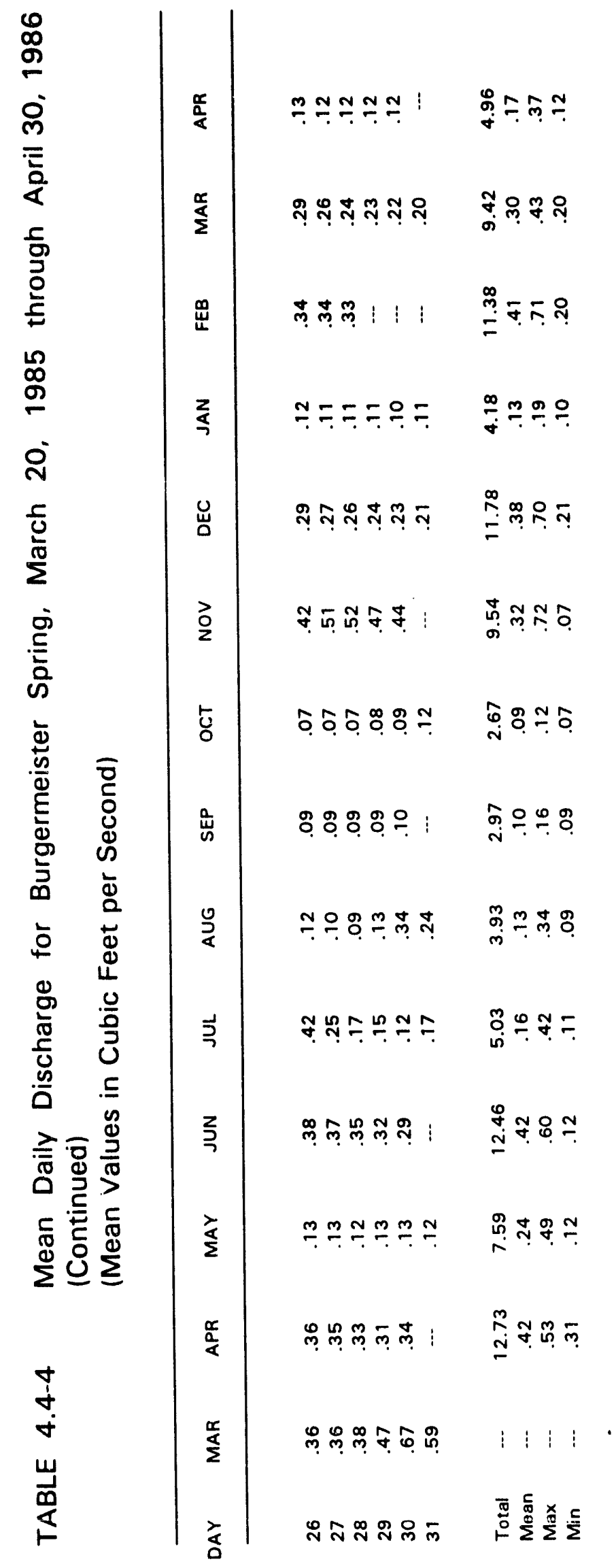


$\stackrel{\circ}{\circ}$

요

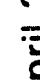

웅

독

$\equiv$

$\stackrel{\infty}{\infty}$

$\stackrel{\infty}{\circ}$

จ

竞

흠

के

3

힌

을

过.

品

츰

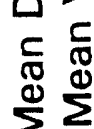

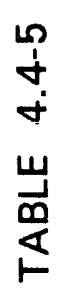

产

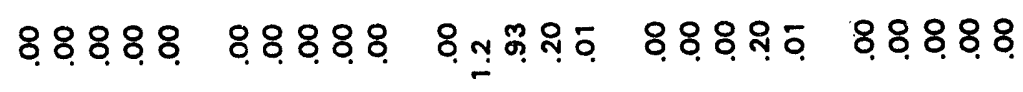

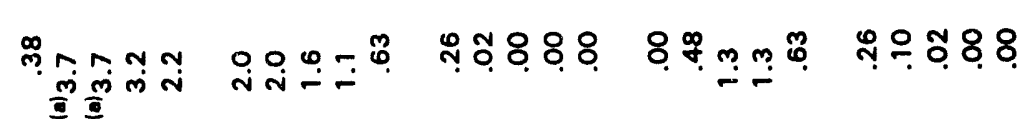

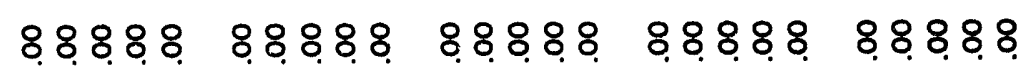

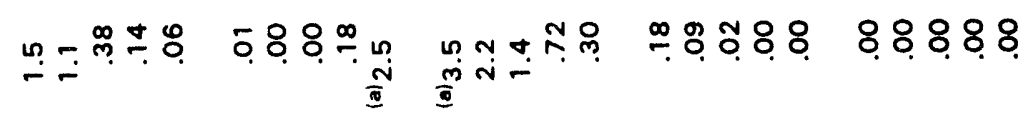

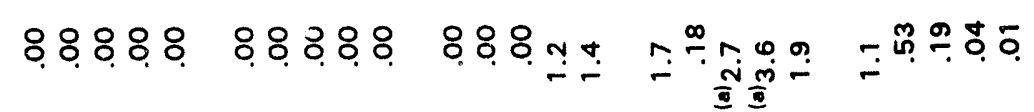

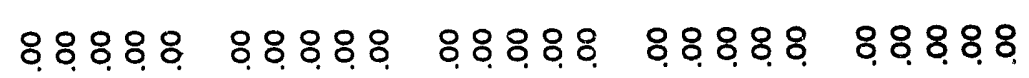

ţ

岃

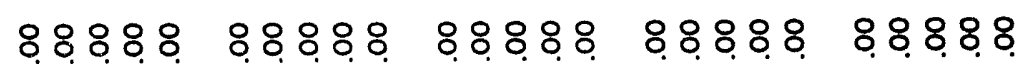

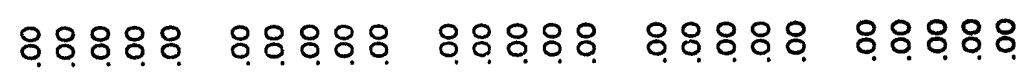

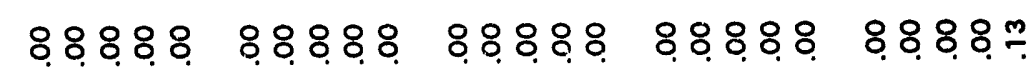

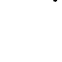

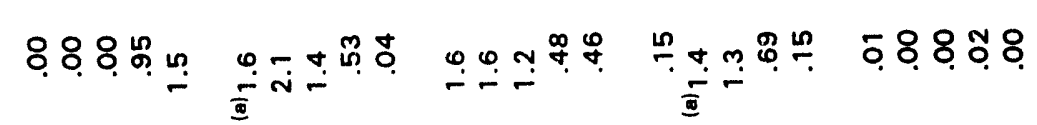

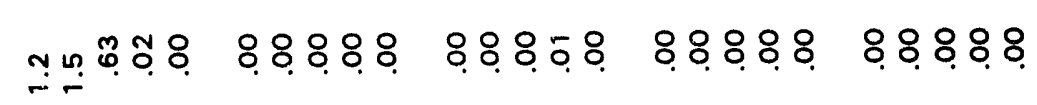
-

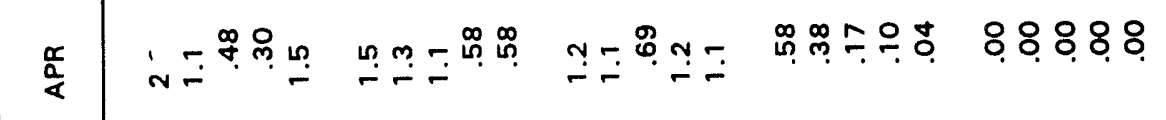

$\frac{\alpha}{\Sigma}$

$8 \quad 8.8 \div 8$

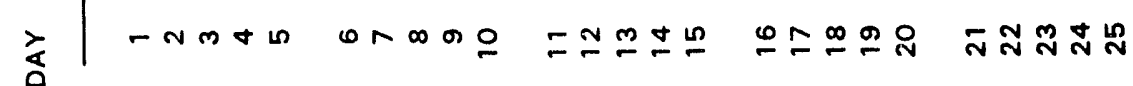




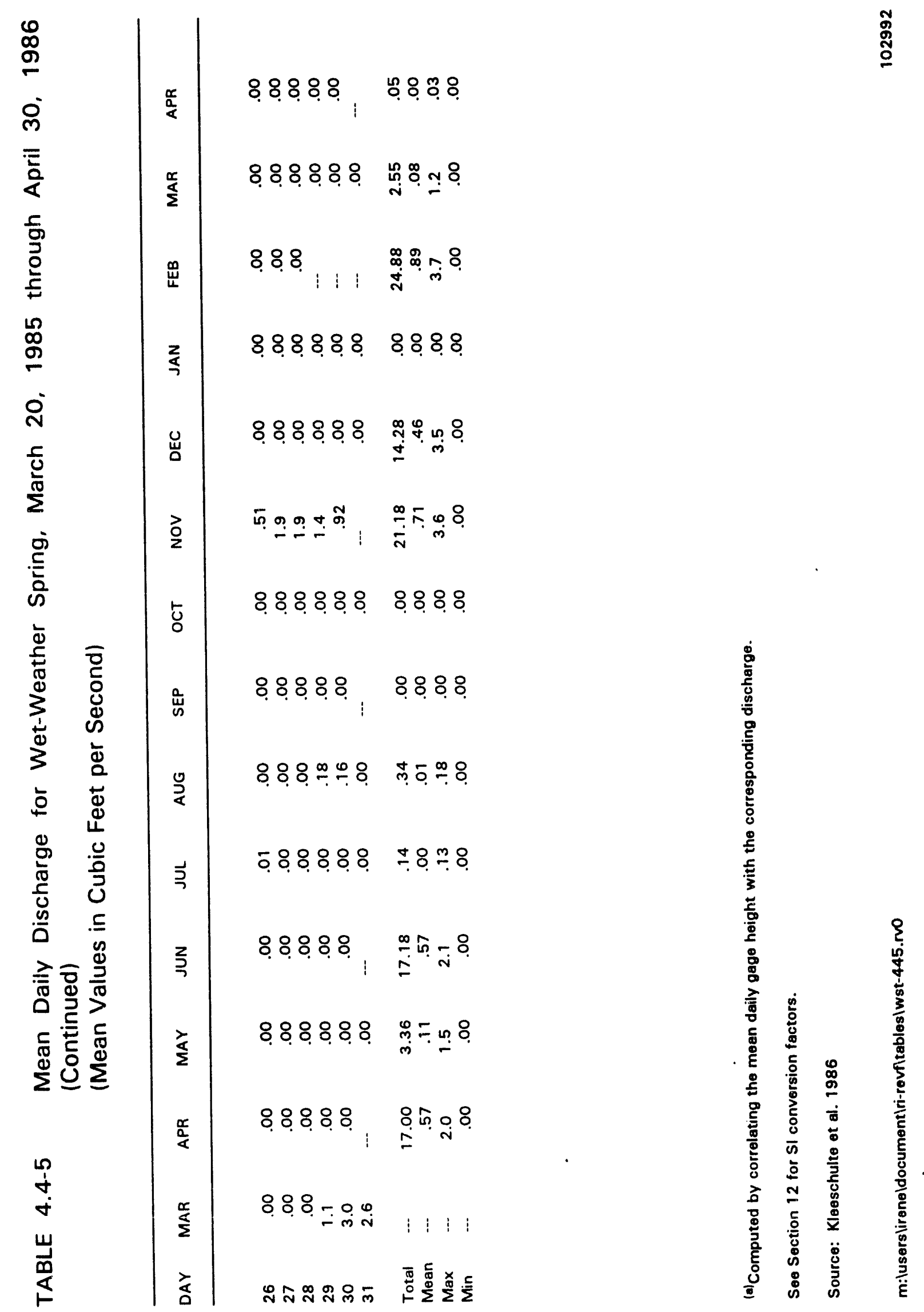


TABLE $\quad$ 4.5-1 Lysimeter Installations

\begin{tabular}{|c|c|c|c|}
\hline \multirow{2}{*}{$\begin{array}{l}\text { Lysimeter } \\
\text { Number }\end{array}$} & \multicolumn{2}{|c|}{$\begin{array}{c}\text { Depth } \\
\text { Interval } \\
\text { (ft.) }\end{array}$} & \multirow[t]{2}{*}{$\begin{array}{l}\text { Monitoring } \\
\text { Zone }\end{array}$} \\
\hline & 10.0 & - $\quad 12.0$ & \\
\hline 3602 & 17.8 & $-\quad 19.8$ & $\begin{array}{l}\text { moist clay layer below } 19 \text { foet natural fracture at } 19.3 \\
\text { feet }\end{array}$ \\
\hline 3603 & 23.75 & - $\quad 25.75$ & chert layer \\
\hline 3604 & 5.0 & 7.0 & silt layer above clay layer \\
\hline 3605 & 11.0 & $-\quad 13.0$ & silt layer above clay layer \\
\hline 3606 & 14.5 & - $\quad 16.5$ & silt layer above clay layer \\
\hline 3607 & 23.0 & $-\quad 25.0$ & chert/clay \\
\hline 3608 & 3.5 & 5.5 & clayey silt \\
\hline 3609 & 22.25 & $-\quad 24.25$ & silt \\
\hline 3610 & 27.3 & - $\quad 29.3$ & silty clay \\
\hline
\end{tabular}

\footnotetext{
(a) Lysimeter locations are shown in Figure 4.5-3

See Section 12 for SI conversion factors.

Source: UNC 1988

m:luserslireneldocument|ri-revfltablesiwst-451.rvo
} 


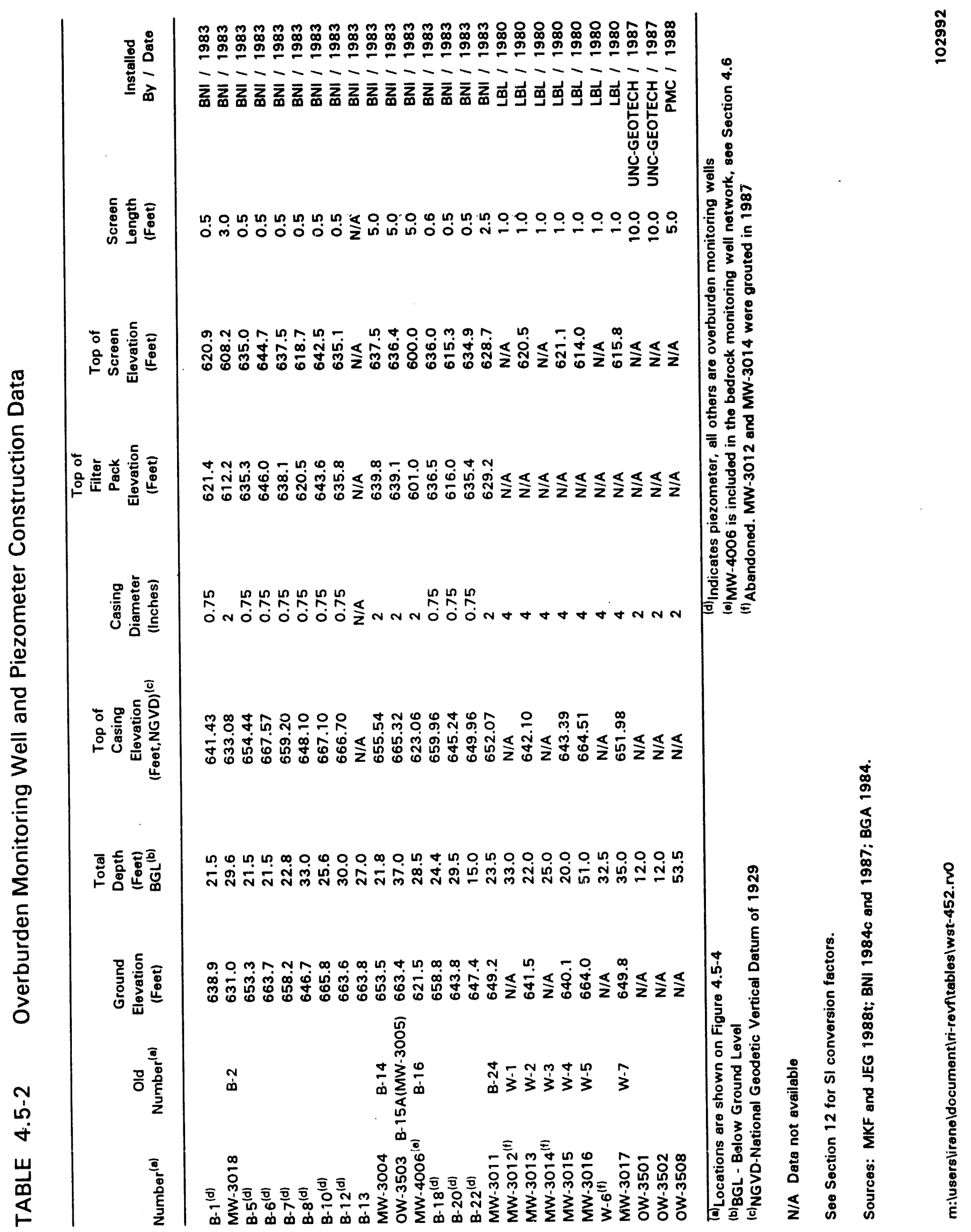



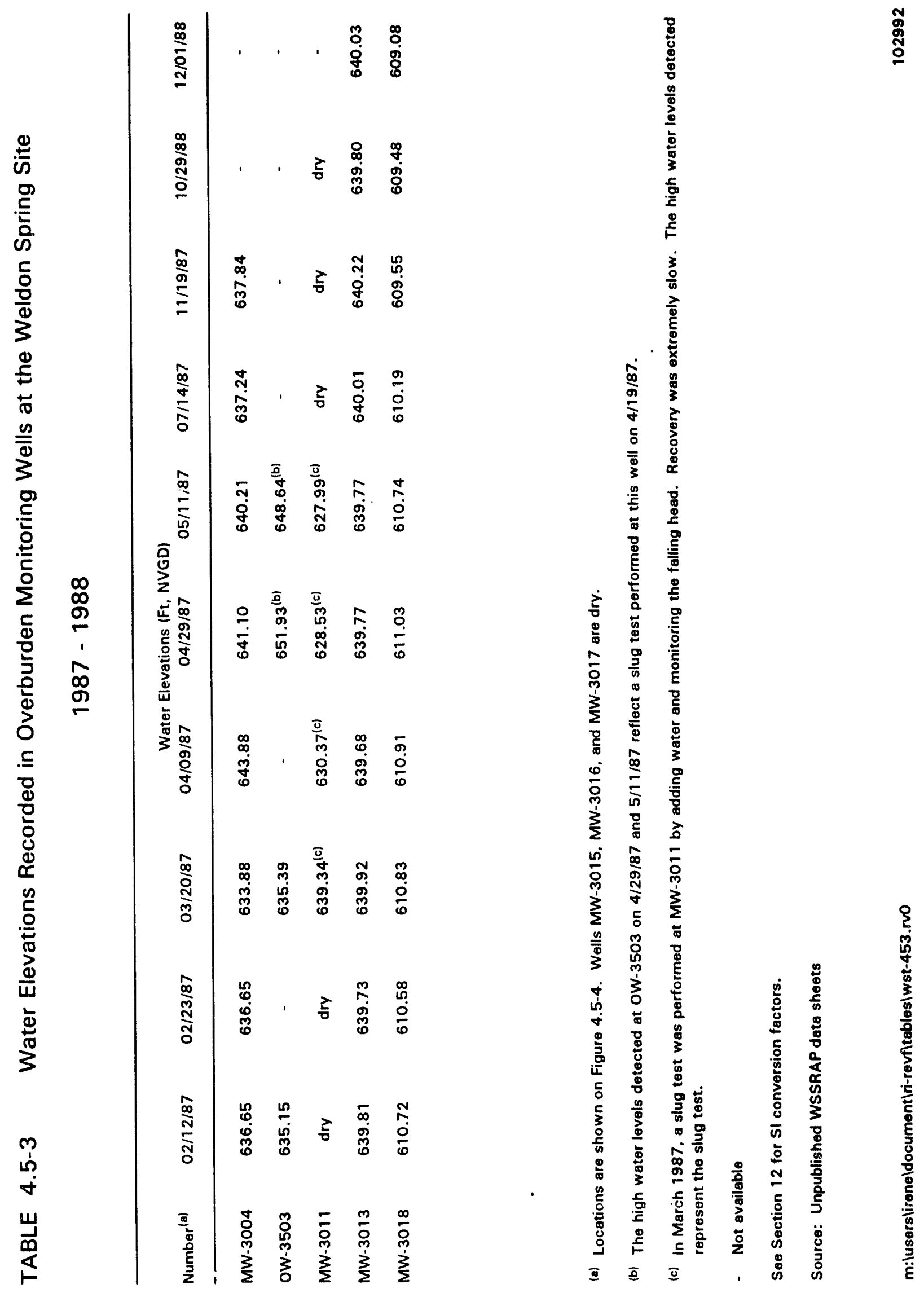


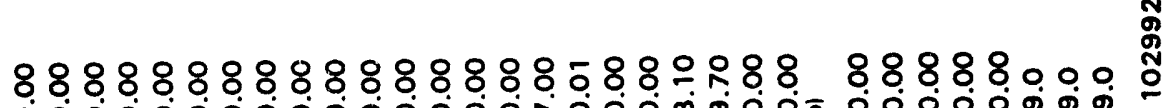

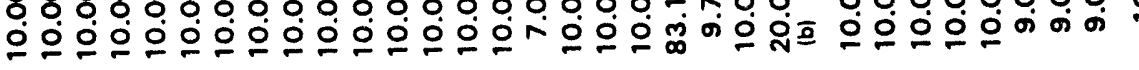

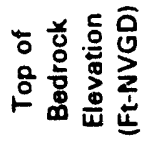

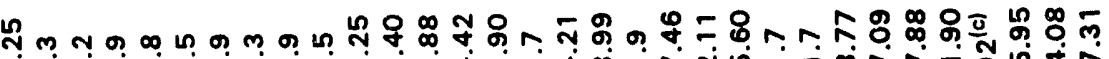

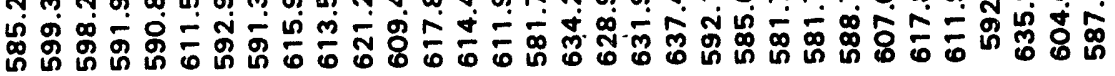

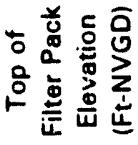

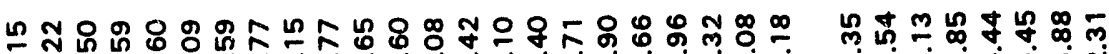

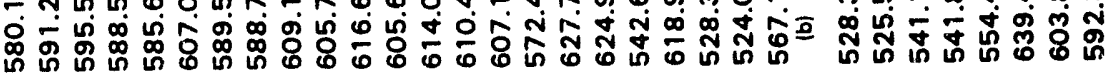

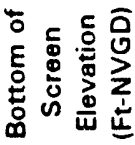

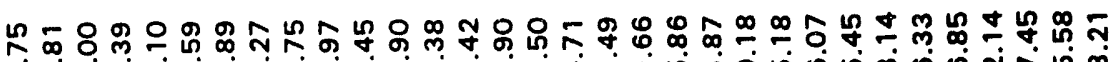
ن

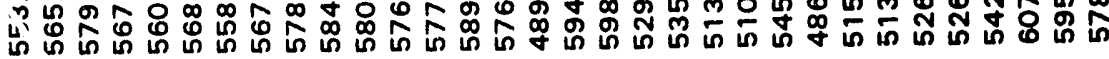

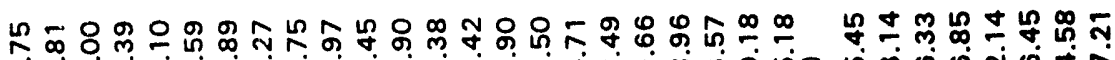

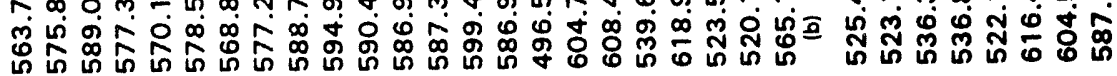

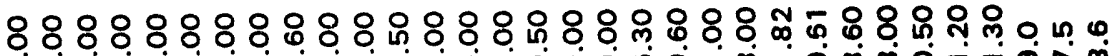

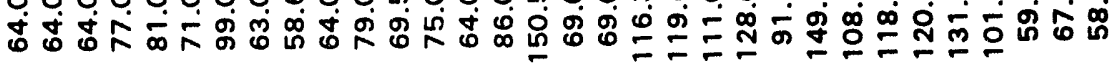

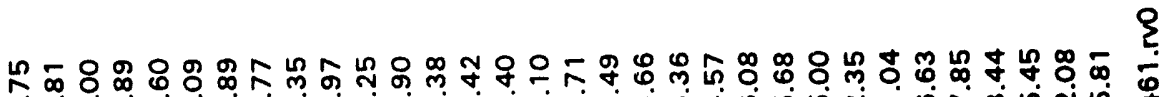

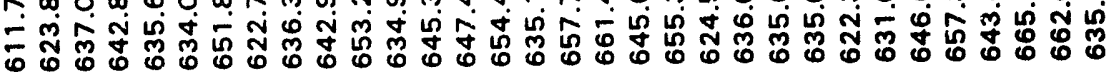

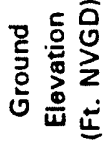

(a)

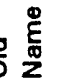

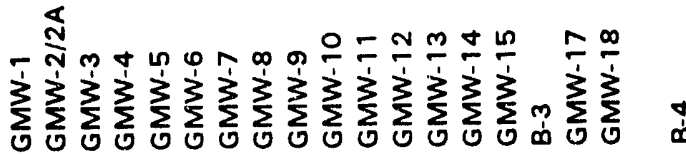

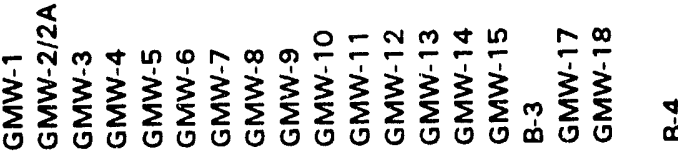

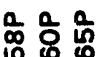

它它它

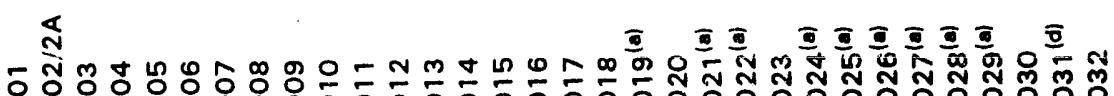

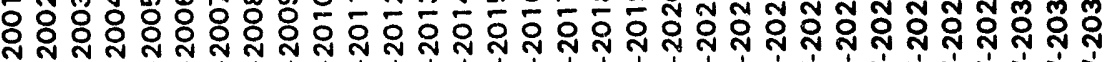

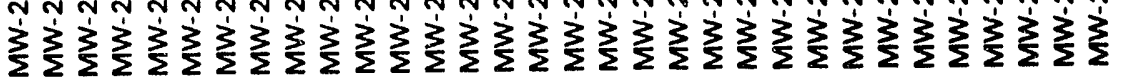




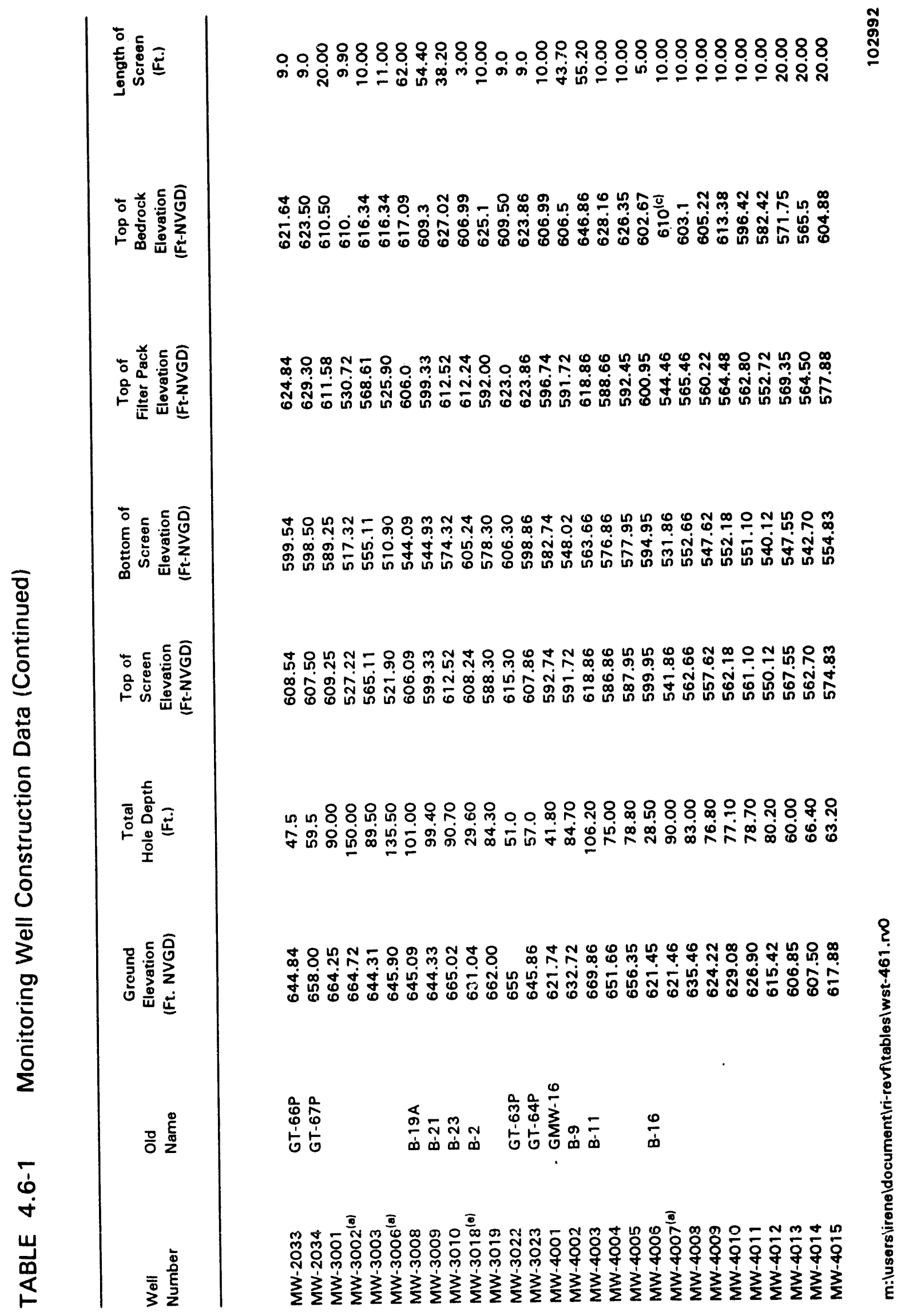




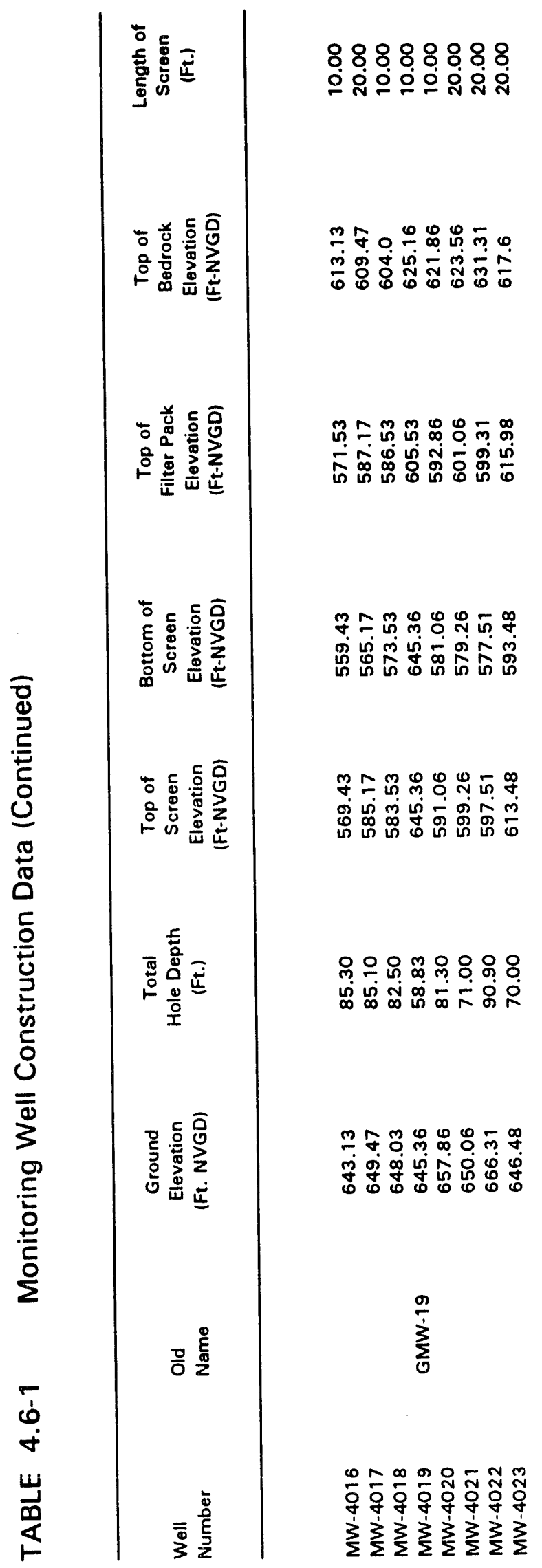

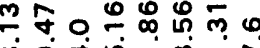

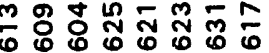

กำ

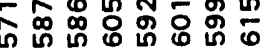

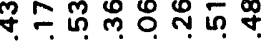

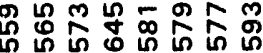

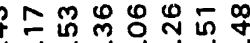

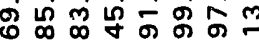

응요 잉용요

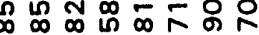

于 $000 \%$

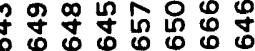

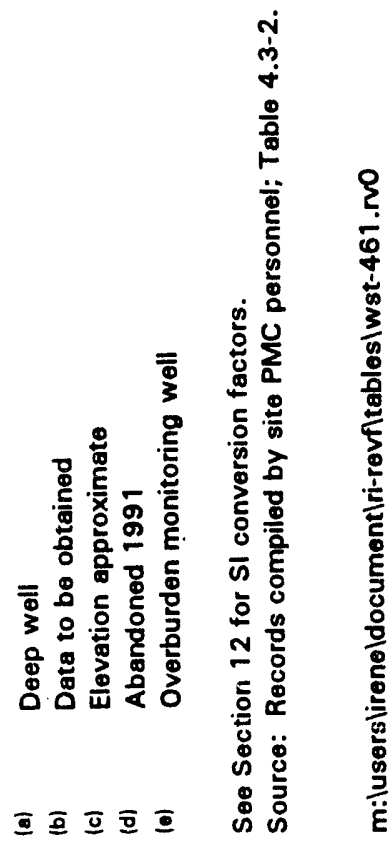


TABLE 4.6-2 Hydraulic Conductivity in Bedrock as Determined by Packer Tests

\begin{tabular}{|c|c|c|c|c|}
\hline Borehole or Well & $\begin{array}{l}\text { Test Interval } \\
\text { Depths Below } \\
\text { Top of Bedrock }\end{array}$ & $\begin{array}{l}\text { Strati- } \\
\text { graphic } \\
\text { Unit }^{(b)}\end{array}$ & $\begin{array}{c}\text { Depth to } \\
\text { Groundwater } \\
\text { from } \\
\text { TOB }(f t)^{(c)}\end{array}$ & $\begin{array}{r}\text { Hydraulic } \\
\text { Conductivity } \\
(\mathrm{cm} / \mathrm{s})^{(d)}\end{array}$ \\
\hline
\end{tabular}

[From 0 to 16.5 Feet Below Top of Bedrock]

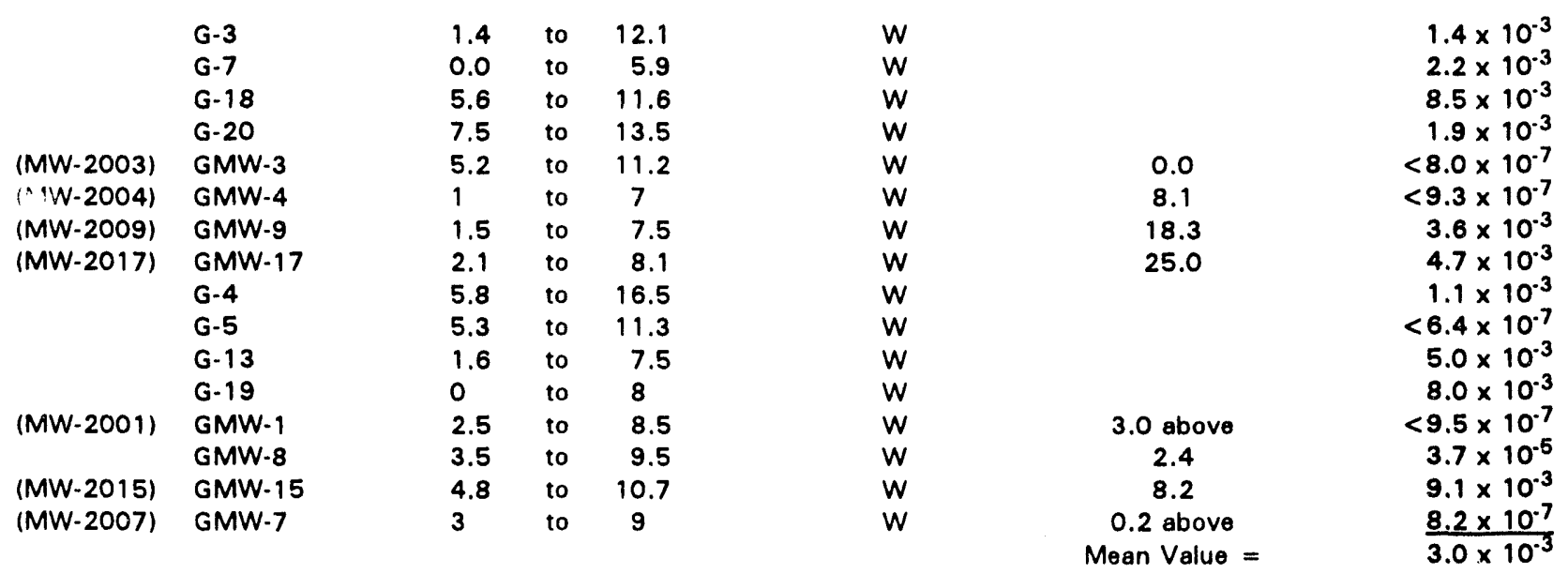

[From 9 to 25.1 Feet Below Top of Bedrock]

$\begin{array}{llcll} & \text { G-1 } & 8.7 & \text { to } & 19.4 \\ & \text { G-2A } & 11.7 & \text { to } & 22.4 \\ & \text { G-5 } & 10 & \text { to } & 16 \\ & \text { G-15 } & 10 & \text { to } & 16.3 \\ & \text { G-16 } & 11 & \text { to } & 16.3 \\ & \text { G-20 } & 10.5 & \text { to } & 16.5 \\ \text { (MW-2002) } & \text { GMW-2A } & 10.7 & \text { to } & 16.7 \\ \text { (MW-2005) } & \text { GMW-4 } & 2.6 & \text { to } & 21 \\ \text { (MW-2006) } & \text { GMW-6 } & 19.2 & \text { to } & 25.1 \\ & \text { GMW-6 } & 10.2 & \text { to } & 16.1 \\ \text { (MW-2008) } & \text { GMW-8 } & 16.4 & \text { to } & 22.4 \\ \text { (MW-2013) } & \text { GMW-13 } & 12.5 & \text { to } & 18.5 \\ \text { (MW-2010) } & \text { GMW-10 } & 10.5 & \text { to } & 16.5 \\ \text { (MW-2014) } & \text { GMW-14 } & 10 & \text { to } & 16 \\ \text { (MW-2015) } & \text { GMW-15 } & 16.8 & \text { to } & 23.2 \\ \text { (MW-2018) } & \text { GMW-18 } & 9.5 & \text { to } & 15.5 \\ & \text { G-19 } & 9.5 & \text { to } & 15.5 \\ & \text { G.21 } & 4.5 & \text { to } & 20.5\end{array}$

6.1

3.0

11.2

2.4

13.4

13.1

10.3

8.2

18.0

Mean Value $=$
$1.0 \times 10^{-3}$

$3.5 \times 10^{.4}$ $1.3 \times 10^{-6}$

$2.4 \times 10^{-4}$

$6.1 \times 10^{-3}$

$6.9 \times 10^{-4}$

$2.1 \times 10^{-4}$

$<3.9 \times 10^{-7}$

$8.9 \times 10^{.5}$

$2.6 \times 10^{-6}$

$3.9 \times 10^{-5}$

$5.9 \times 10^{.6}$

$2.1 \times 10^{-3}$

$2.8 \times 10^{-4}$

$5.2 \times 10^{-5}$

$8.9 \times 10^{-5}$ $<7.8 \times 10^{-7}$ $\frac{2.1 \times 10^{-6}}{6.5 \times 10^{-4}}$ 
TABLE 4.6-2 Hydraulic Conductivity in Bedrock as Determined by Packer Tests (Continued)

\begin{tabular}{|c|c|c|c|c|}
\hline Borehole or Well & $\begin{array}{c}\text { Test Interval } \\
\text { Depths Below } \\
\text { Top of Bedrock }\end{array}$ & $\begin{array}{l}\text { Strati- } \\
\text { graphic } \\
\text { Unit }^{(b)}\end{array}$ & $\begin{array}{l}\text { Depth to } \\
\text { Groundwater } \\
\text { from } \\
\text { TOB(ft) }\end{array}$ & $\begin{array}{r}\text { Hydraulic } \\
\text { Conductivity } \\
(\mathrm{cm} / \mathrm{s})^{(\mathrm{d})}\end{array}$ \\
\hline
\end{tabular}

[From 18 to 40 Feet Below Top of Bedrock]

\begin{tabular}{|c|c|c|c|c|c|c|c|}
\hline & G-1 & 24.3 & to & 45.3 & W & & $2.8 \times 10^{-4}$ \\
\hline & G-2A & 22.2 & to & 32.9 & C & & $1.2 \times 10^{-4}$ \\
\hline & G-9 & 28.5 & to & 38.5 & C & & $3.6 \times 10^{-6}$ \\
\hline & G-14 & 26.5 & to & 38.9 & $W / C$ & & $<3.5 \times 10^{-7}$ \\
\hline (MW-2001) & GMW-2A & 27.3 & to & 33.3 & $W / C$ & 3.0 above & $<6.8 \times 10^{-7}$ \\
\hline (MW-2012) & GMW-12 & 26.5 & to & 32.5 & $w$ & 3.9 & $1.7 \times 10^{-7}$ \\
\hline (MW-2013) & GMW-13 & 34.5 & to & 40.5 & C & $\begin{array}{c}13.4 \\
\text { Mean Value }\end{array}$ & $\frac{5.2 \times 10^{-6}}{5.9 \times 10^{-6}}$ \\
\hline
\end{tabular}

(a) Depth of test interval below top of bedrock was determined by information in Table 4.6-1 and BNI 1987.

(b) $W=$ Weathered Limestone; $C=$ Competent Limestone

(c) Based on water levels obtained July 1987; water levels available for monitoring wells only.

(d) Values shown as "less than" were assumed to be at value for purpose of calculating means.

See Section 12 for SI conversion factors.

m:lusers lireneldocument $|r i-r e v f| t a b l e s i w s t-462 . r v o$ 
TABLE 4.6-3 Hydraulic Conductivity Values in Shallow Wells from In Situ Tests

\begin{tabular}{|c|c|c|c|c|}
\hline Well No. & $\begin{array}{l}\text { Test Interval } \\
\text { Elevation } \\
\text { (Ft NVGD) }\end{array}$ & $\begin{array}{l}\text { Top of } \\
\text { Competent } \\
\text { Limestone } \\
\text { Elevation } \\
\text { (Ft NVGD) }\end{array}$ & $\begin{array}{c}\text { Interval } \\
\text { Tested }^{(a)} \\
\text { Below TOB } \\
\text { (Ft NVGD) }\end{array}$ & $\begin{array}{c}\text { Mean } \\
\text { Hydraulic } \\
\text { Conductivity } \\
\text { (cm/sec) }\end{array}$ \\
\hline
\end{tabular}

\begin{tabular}{|c|c|c|c|}
\hline MW 2002 (GMW-2) & 59 & 565 & 572.7 \\
\hline MW 2005 (GMW-5) & 585 & 560 & 568.70 \\
\hline MW 2006 (GMW-6) & 607 & 568.6 & 582 \\
\hline MW 2008 (GMW-8) & 588.8 & 567.3 & 571.5 \\
\hline MW 2013 (GMW-13) & 614.1 & 577.4 & 585.4 \\
\hline $\begin{array}{l}\text { MW } 2015 \\
\text { MW } 2018 \text { (GMW-18) }\end{array}$ & 607.1 & 577.4 & $\underset{(c)}{587.0}$ \\
\hline$M W-2023$ & $\begin{array}{l}567.18 \\
545.18\end{array}$ & - & 570.7 \\
\hline MW 3003 & & & (c) \\
\hline $\begin{array}{l}\text { MW } 3019 \\
\text { MW } 4001 \text { (GMW-16) }\end{array}$ & 592 & 578.3 & $\underset{(c)}{581.8}$ \\
\hline $\begin{array}{l}\text { MW } 4004 \\
\text { MW } 4005\end{array}$ & 588.7 & 576.9 & $\underset{(c)}{588.7}$ \\
\hline MW 4008 & 565.5 & 552.7 & 578.1 \\
\hline MW 4009 & 560.2 & - $\quad 547.6$ & 569.6 \\
\hline MW 4010 & 564.5 & 552.2 & 558.3 \\
\hline MW 4011 & 562.8 & 551.1 & 568.2 \\
\hline MW 4012 & 552.7 & 540.1 & 554.7 \\
\hline MW 4013 & 569.3 & 547.6 & 555.2 \\
\hline MW 4014 & 564.5 & 542.7 & 553.3 \\
\hline MW 4015 & 577.9 & 554.8 & 561.4 \\
\hline MW 4016 & 571.5 & 559.4 & 568.9 \\
\hline MW 4017 & 587.2 & 565.2 & 571.7 \\
\hline MW 4018 & 586.6 & - 573.6 & 575.0 \\
\hline MW 4020 & 592.9 & - 581.1 & 590.4 \\
\hline MW 4021 & 601.1 & - $\quad 579.3$ & 582.1 \\
\hline MW 4022 & 599.3 & 577.5 & 606.1 \\
\hline MW 4023 & 616.0 & - $\quad 593.5$ & 587.5 \\
\hline
\end{tabular}

0 to 32.3

0 to 15

0 to 38.4

0 to 21.4

3.8 to 40.5

4.8 to 35.0

4.9 to 30.5

16 to 38

47.7 to 61.2

33 to 47

10.3 to 24.3

39.5 to 51.3

33.9 to 48.4

45.8 to 58.6

45 to 57.6

48.9 to 61.2

33.6 to 45.3

29.7 to 42.3

2.4 to 24.2

7.9 to 29.7

27 to 50.1

41.6 to 53.7

22.3 to 44.3

21.5 to 34.5

29 to 40.8

22.5 to 44.3

32 to 53.8

2.5 to 25

Mean
$7.5 \times 10^{.5}$

$2.1 \times 10^{-5}$

$6.8 \times 10^{-5}$

$5.9 \times 10^{-5}$

$1.7 \times 10^{-5}$

$1.2 \times 10^{-4}$

$6.6 \times 10^{.5}$

$9.1 \times 10^{-6}$

$5.8 \times 10^{-6}$

$5.4 \times 10^{-5}$

$5.0 \times 10^{-5}$

$6.3 \times 10^{-6}$

$4.2 \times 10^{-6}$

$1.0 \times 10^{-5}$

$6.7 \times 10^{-6}$

$5.0 \times 10^{.6}$

$1.7 \times 10^{-6}$

$4.5 \times 10^{.6}$

$5.7 \times 10^{-5}$

$1.0 \times 10^{-3}$

$2.9 \times 10^{-5}$

$4.1 \times 10^{-5}$

$3.0 \times 10^{.5}$

$3.0 \times 10^{-5}$

$2.3 \times 10^{.5}$

$3.9 \times 10^{-5}$

$2.3 \times 10^{.5}$

$7.8 \times 10^{.5}$

$6.37 \times 10^{-6}$

(a) Interval tested equals screen length plus filter pack

(b) TOB - Top of Weathered Limestone Unit

(c) Top of Competent Limestone below bottom of well

See Section 12 for SI conversion factors.

Source: Site PMC Personnel 


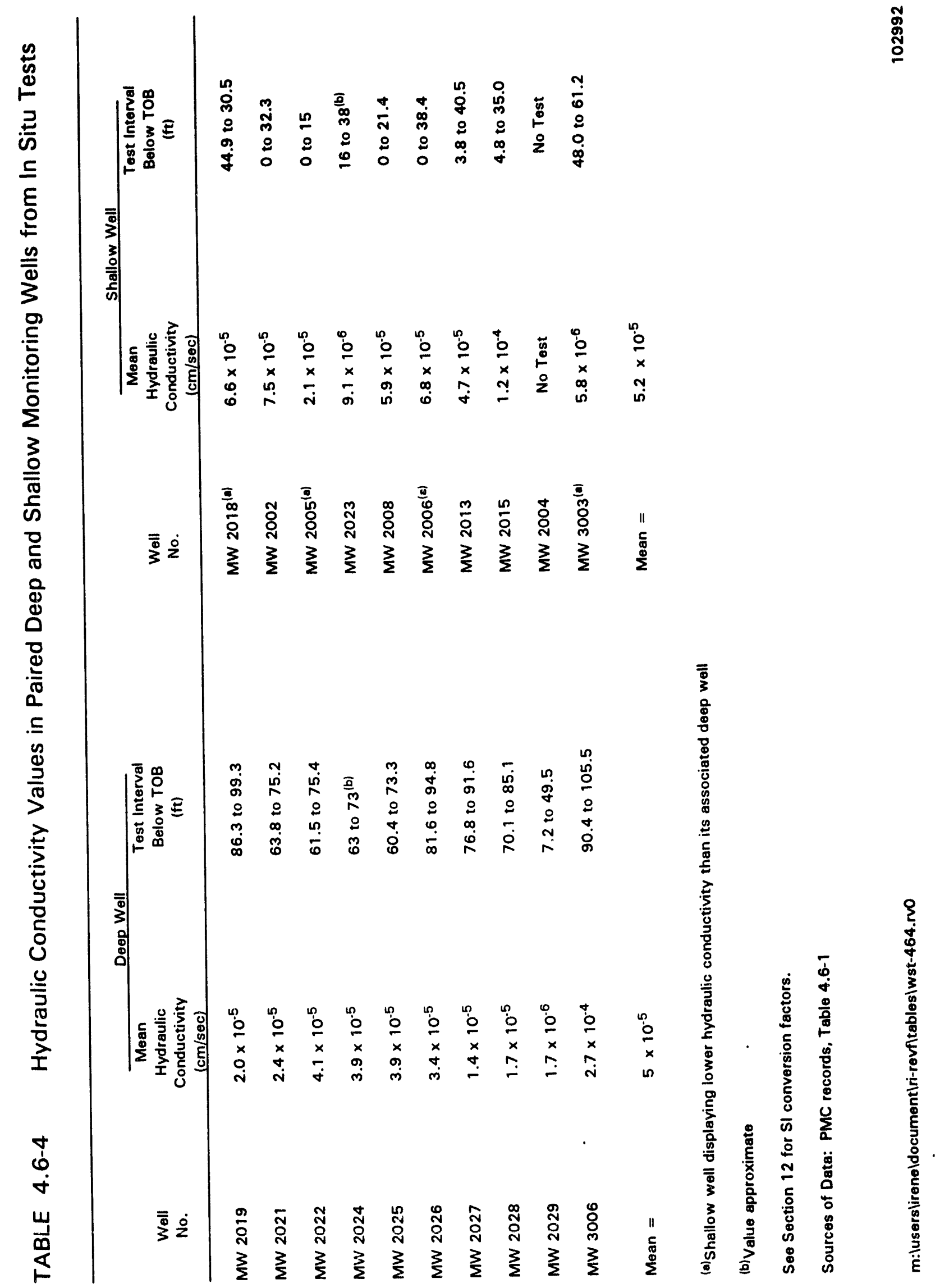




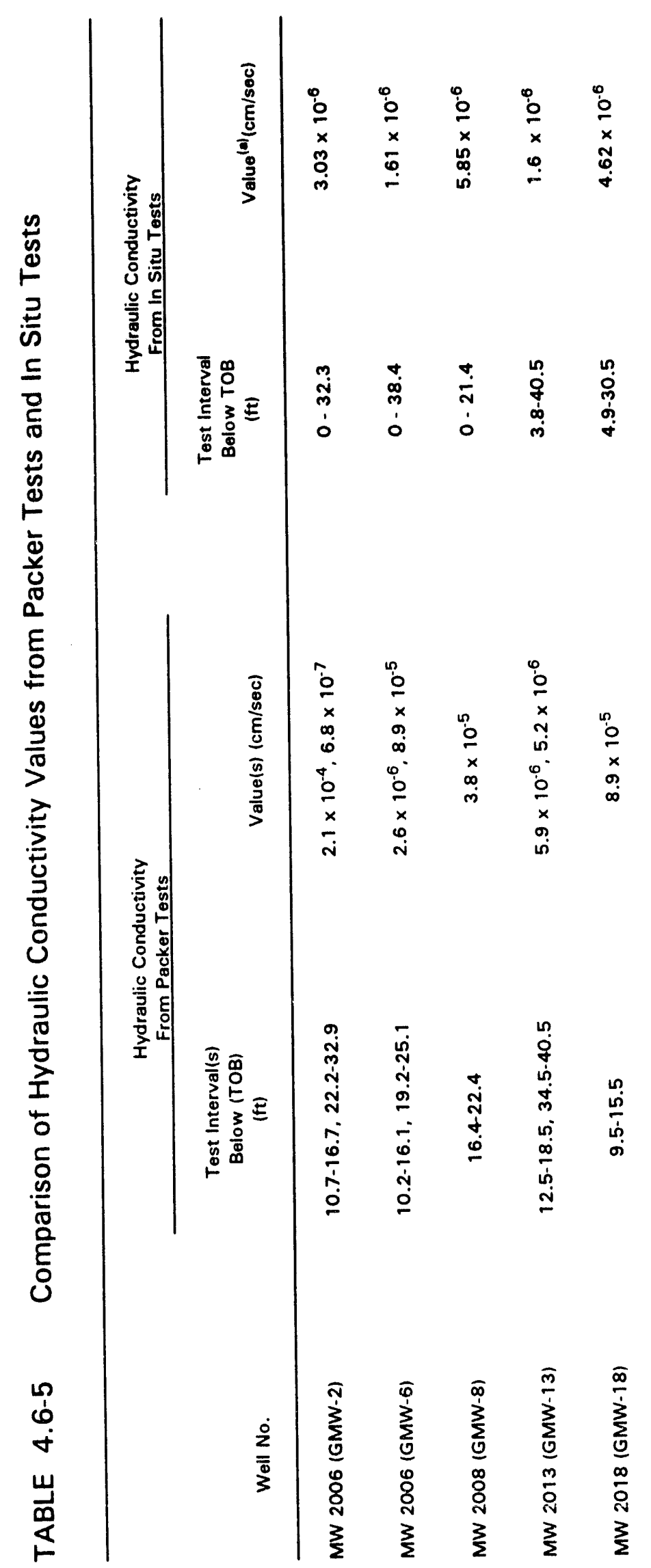

ม
ฟิ
0

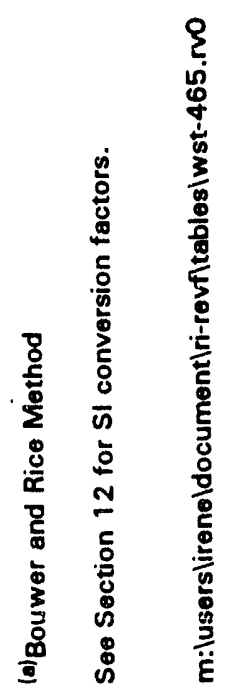




\section{TABLE 4.6-6 Angled Borehole Packer Test Results}

\begin{tabular}{|c|c|c|}
\hline Drill Hole & $\begin{array}{l}\text { Depth from } \\
\text { Top of Bedrock (ft) }\end{array}$ & $\begin{array}{c}\text { Average Hydraulic } \\
\text { Conductivity }(\mathrm{cm} / \mathrm{sec})^{(a)}\end{array}$ \\
\hline \multirow[t]{7}{*}{$A H-1$} & $3.8-12.6$ & $9.3 \times 10^{.4}(\mathrm{~b})$ \\
\hline & $12.4 \cdot 21.3$ & $6.1 \times 10^{-4}$ \\
\hline & $20.6 \cdot 29.5$ & 0 \\
\hline & $29.2 \cdot 46.8$ & $<1.16 \times 10^{.5(c)}$ \\
\hline & $46.7-64.1$ & $<3.60 \times 10^{-7}(\mathrm{c})$ \\
\hline & $64.0 \cdot 81.5$ & $8.4 \times 10^{.6}$ \\
\hline & $79.7 \cdot 100.6$ & $<6.60 \times 10^{.8|c|}$ \\
\hline \multirow[t]{8}{*}{$\mathrm{AH}-2$} & & \\
\hline & $4.2 \cdot 12.7$ & $8.7 \times 10^{-4}$ \\
\hline & $11.5 \cdot 21.4$ & $1.7 \times 10^{-4}$ \\
\hline & $21.0 \cdot 30.0$ & $4.0 \times 10^{.7}$ \\
\hline & $29.9 \cdot 47.3$ & $<8.4 \times 10^{.6(c)}$ \\
\hline & $47.2 \cdot 74.7$ & $<1.9 \times 10^{.6(c)}$ \\
\hline & $64.6 \cdot 82.0$ & $1.0 \times 10^{.6}$ \\
\hline & $81.8-100.7$ & $2.1 \times 10^{-5}$ \\
\hline
\end{tabular}

(a) Average of three tests, except where noted otherwise.

(b) Average of four tests.

(c) Value obtained from one test at - - essure 70 to $90 \mathrm{psi}$. Two tests at lower pressure vielded indicated $K=0$; therefore, the average value is listed as less than the high-pressure test value.

See Section 12 for SI conversion factors.

Source of Data: Drill logs; PMC records 


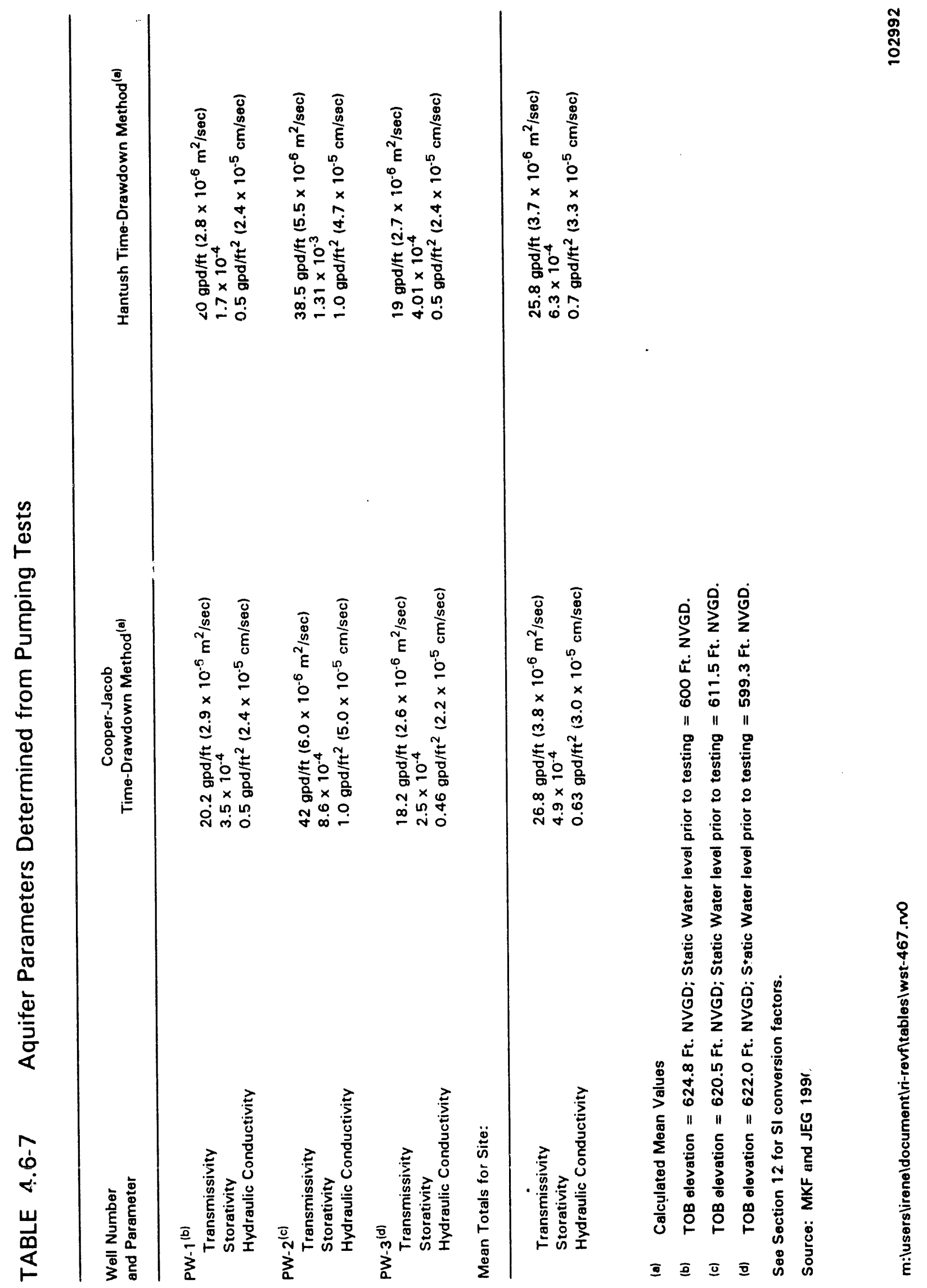




\section{TABLE 4.6-8 Water Level Elevations in Shallow and Deep Monitoring Wells}

\begin{tabular}{|c|c|c|}
\hline \multirow[t]{2}{*}{ WELL NUMBER } & \multicolumn{2}{|c|}{ WATER LEVEL ELEVATION (ft) } \\
\hline & July 23, 1988 & December 1,1988 \\
\hline \multicolumn{3}{|l|}{ Shallow Wells } \\
\hline MW-2001 & 588.03 & 588.26 \\
\hline$M W-2002 / 2 A$ & 593.05 & 592.89 \\
\hline$M W-2003$ & 597.78 & 597.58 \\
\hline$M W-2004$ & 583.69 & 583.47 \\
\hline MW-2005 & 587.85 & 587.57 \\
\hline MW-2006 & 599.58 & 599.62 \\
\hline MW-2007 & 592.89 & 592.65 \\
\hline MW-2008 & 588.57 & 588.08 \\
\hline MW-2009 & 597.39 & 595.88 \\
\hline$M W-2010$ & 599.96 & 601.41 \\
\hline MW-2011 & 600.79 & 600.80 \\
\hline MW-2012 & 604.38 & 604.72 \\
\hline$M W-2013$ & 604.24 & 604.94 \\
\hline MW-2014 & 603.93 & 603.21 \\
\hline$M W-2015$ & 600.62 & 603.37 \\
\hline$M W-2016$ & (a) & (a) \\
\hline$M W-2017$ & 605.22 & 618.76 \\
\hline$M W-2018$ & 614.93 & 610.60 \\
\hline$M W-2019$ & 575.68 & 574.05 \\
\hline MW-2020 & (a) & (a) \\
\hline$M W-2023$ & 568.26 & 584.93 \\
\hline MW-3001 & 612.51 & (a) \\
\hline MW-3003 & 597.77 & 597.72 \\
\hline$M W-3008$ & 610.37 & (a) \\
\hline MW-3009 & 606.71 & 605.63 \\
\hline MW-3010 & 612.13 & (a) \\
\hline$M W-4001$ & 602.09 & 603.39 \\
\hline$M W-4002$ & 569.06 & 572.60 \\
\hline$M W-4003$ & 612.99 & 611.20 \\
\hline$M W-4005$ & 609.51 & 608.54 \\
\hline$M W-4008$ & 597.14 & 596.86 \\
\hline$M W-4009$ & 594.14 & 594.04 \\
\hline MW-4010 & 589.10 & 589.08 \\
\hline$M 1 \%-4011$ & 590.02 & 589.92 \\
\hline
\end{tabular}


TABLE 4.6-8 Water Level Elevations in Shallow and Deep Monitoring Wells (Continued)

\begin{tabular}{|c|c|c|}
\hline WELL NUMBER & $\begin{array}{r}\text { WAT } \\
\text { July 23, } 1988\end{array}$ & Decemoer 1, 1988 \\
\hline \multicolumn{3}{|l|}{ Shallow Wells } \\
\hline$M W-4012$ & 570.49 & 570.99 \\
\hline$M W-4013$ & 560.51 & 560.50 \\
\hline MW-4014 & 561.37 & 561.36 \\
\hline$M W-4015$ & 581.41 & 581.13 \\
\hline$M W-4016$ & 589.71 & 589.43 \\
\hline$M W-4017$ & 593.31 & 591.42 \\
\hline$M W-4018$ & 597.70 & 597.25 \\
\hline$M W-4019$ & 611.77 & 611.10 \\
\hline MW-4020 & 605.10 & 594.13 \\
\hline$M W-4021$ & 607.75 & 607.32 \\
\hline$M W-4022$ & 596.74 & 592.83 \\
\hline$M W-4023$ & 614.77 & 612.48 \\
\hline \multicolumn{3}{|l|}{ Deep Wells } \\
\hline MW-2021 & 588.68 & 589.00 \\
\hline MW-2022 & 585.61 & 585.66 \\
\hline MW-2024 & 567.67 & 568.05 \\
\hline MW-2025 & 583.81 & 583.40 \\
\hline MW-2026 & 587.40 & 587.22 \\
\hline$M W-2027$ & 594.32 & 592.00 \\
\hline MW-2028 & 595.48 & 595.27 \\
\hline MW-2029 & 582.55 & 582.50 \\
\hline MW-3002 & (a) & (a) \\
\hline MW-3006 & 590.09 & 590.30 \\
\hline$M W-4007$ & 595.79 & 595.61 \\
\hline \multicolumn{3}{|l|}{ (a) No measurement } \\
\hline See Section 12 for $S$ & & $\cdot$ \\
\hline
\end{tabular}




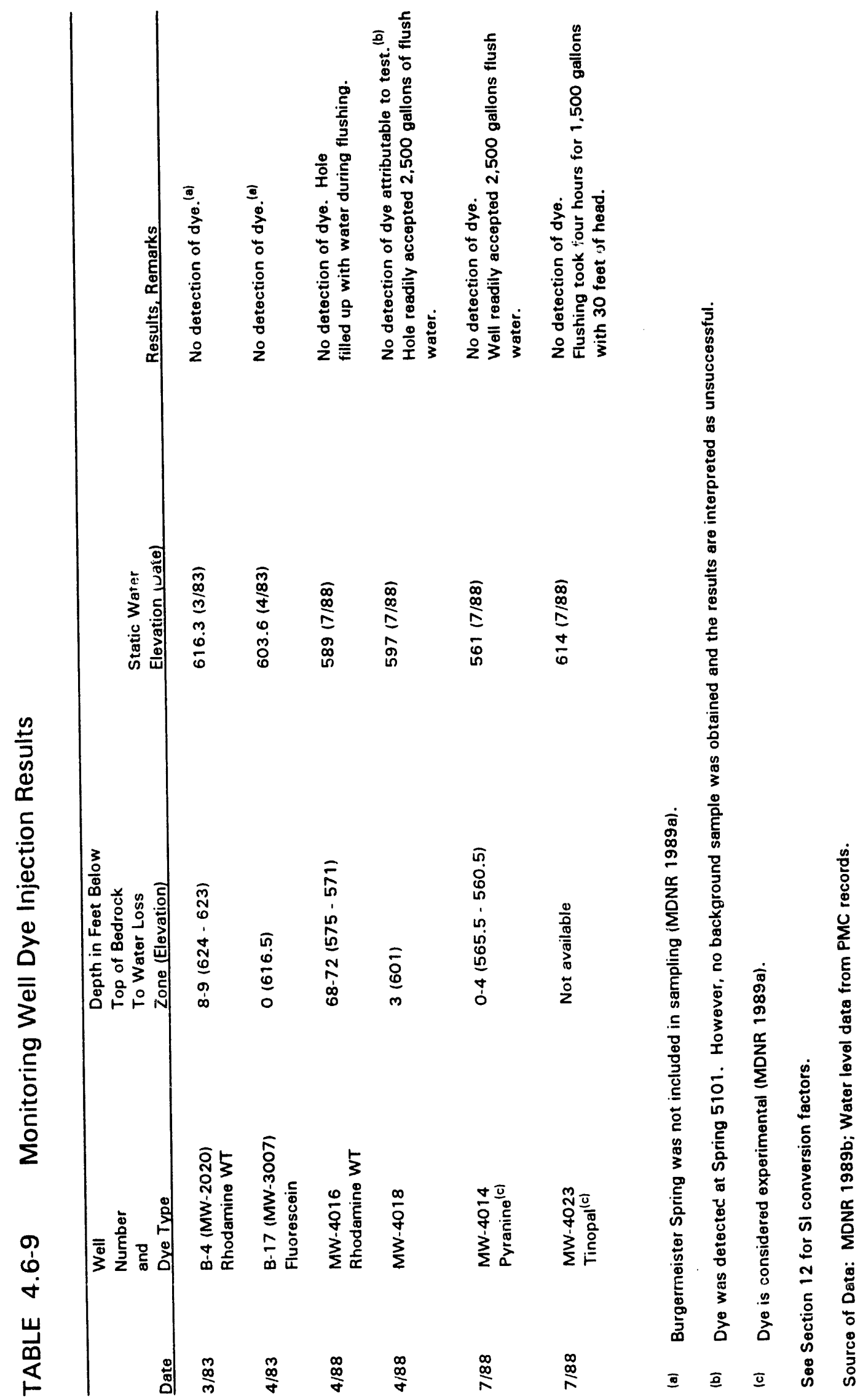

\%
ูํ
ㅇ

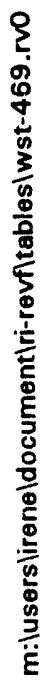




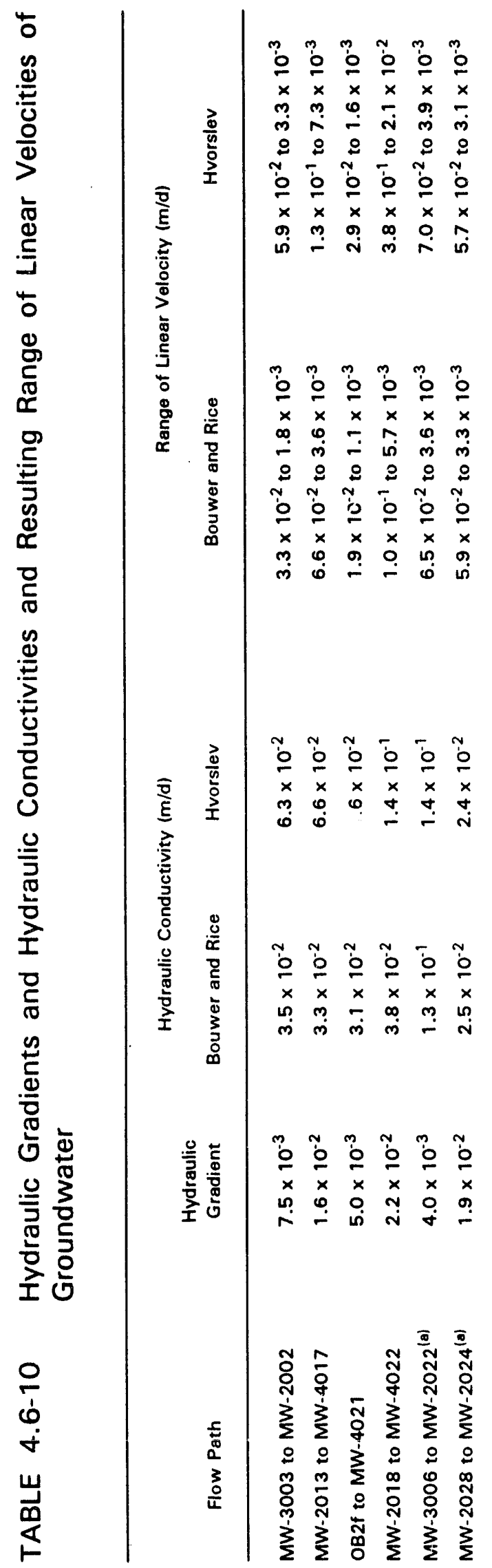




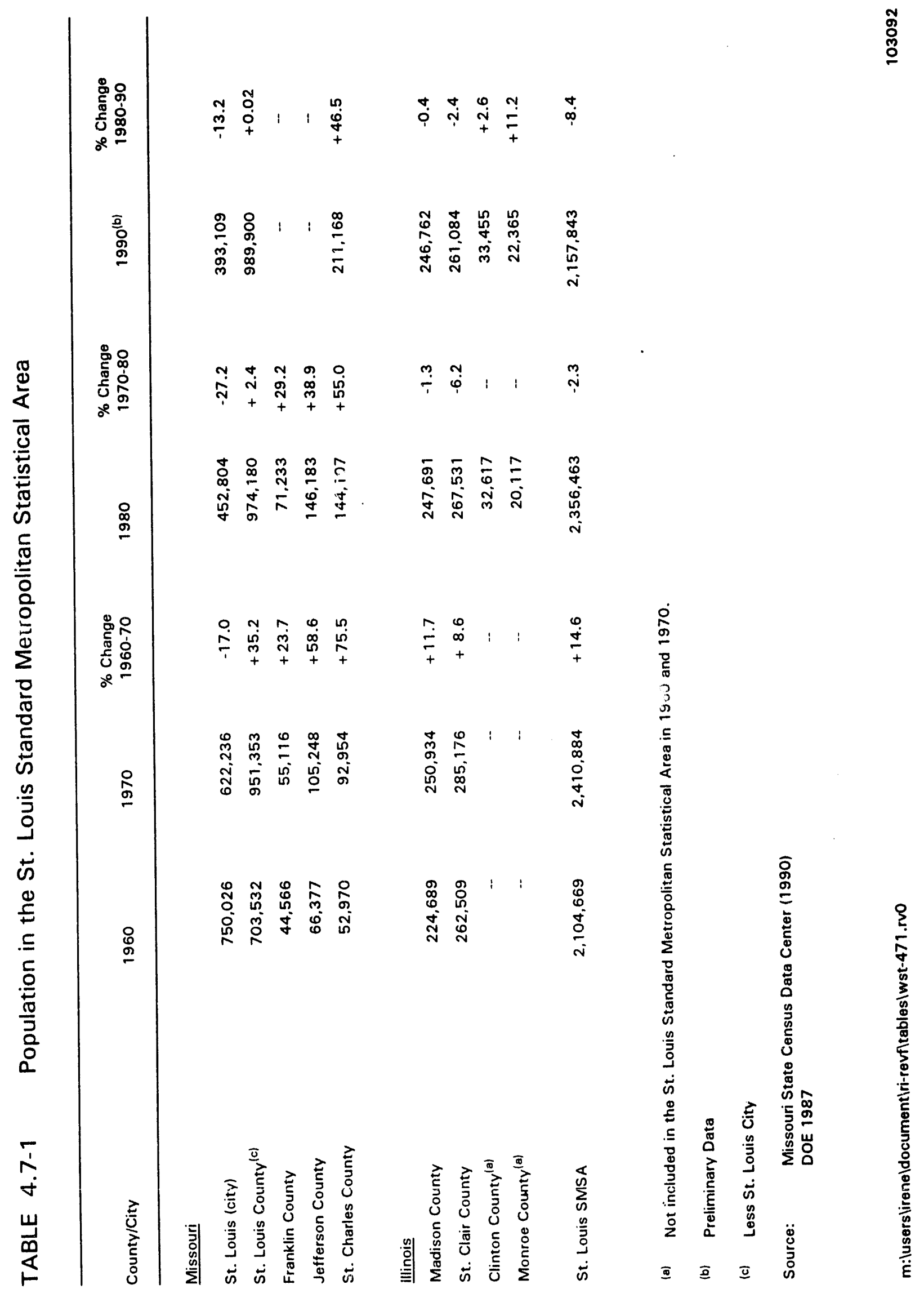




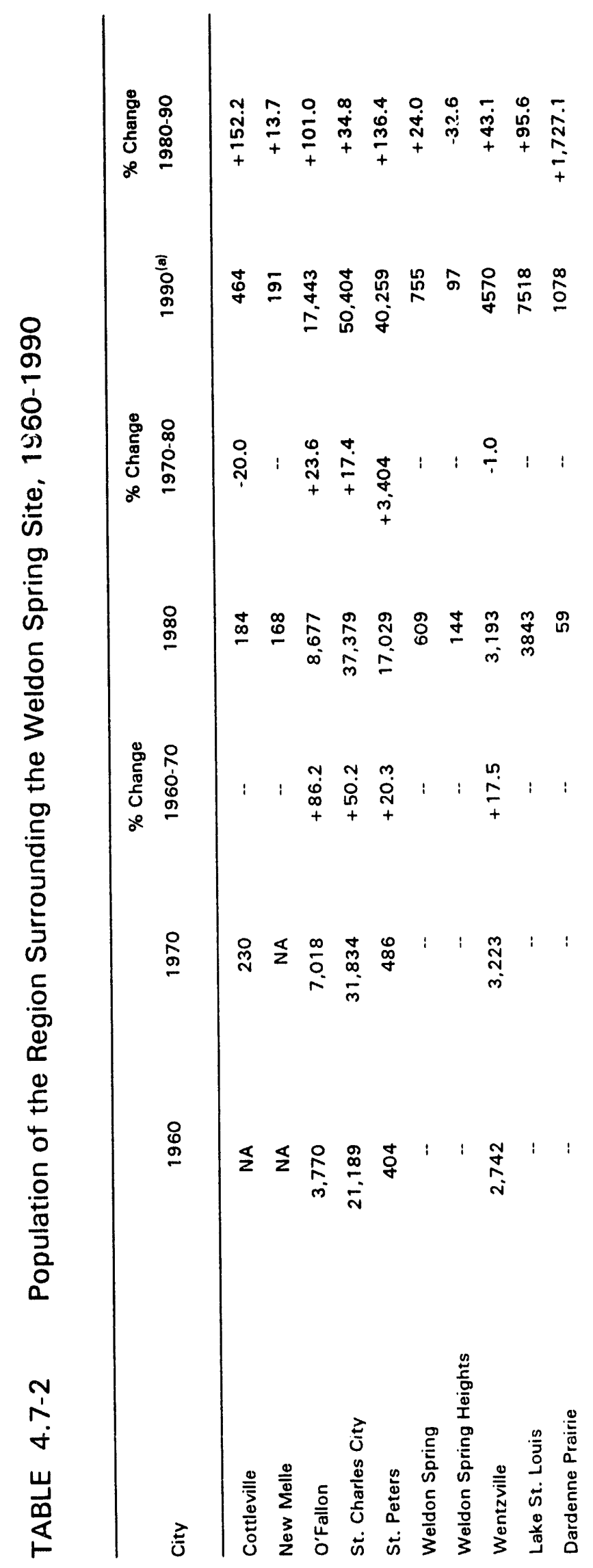




\section{TABLE 5.1-1 Potential Sources of Contaminants at the Weldon Spring Site}

Nitrates

Nitric acid recovery plant (Area 100)

Digestion and denitration plant (Building 103)

Refinery tank farm (Area 102)

Spills from above areas

Process line and sewer leaks

Past management practices

Raffinate pits sludge

Nitric acid used in production of TNT/DNT

Spills and poor waste management prectices during the

World War II production effort

Sulfates

Raffinate pits sludges which contain sulfates Sulfuric acid used in production of TNT/DNT

Fluoride

Green salt plant (Building 201)

Green salt farm (Area 202)

Metal pilot plant (Building 404)

Raffinate pits sludge

Motals

Metals plant (3uilding 3011

Magnesium building (Building 302)

Metal pilot plant (Building 404)

Raffinate pits water

Raffinate pits water

Raffinate pits water

Metals plant (Building 301) tanks

Ordnance production - toluene storage tanks

Building 105-tributyl phosphate separation

Source: MKF and JEG 1987o

Magnesium Magnesium Lithium Chromium Nickel Vanadium 


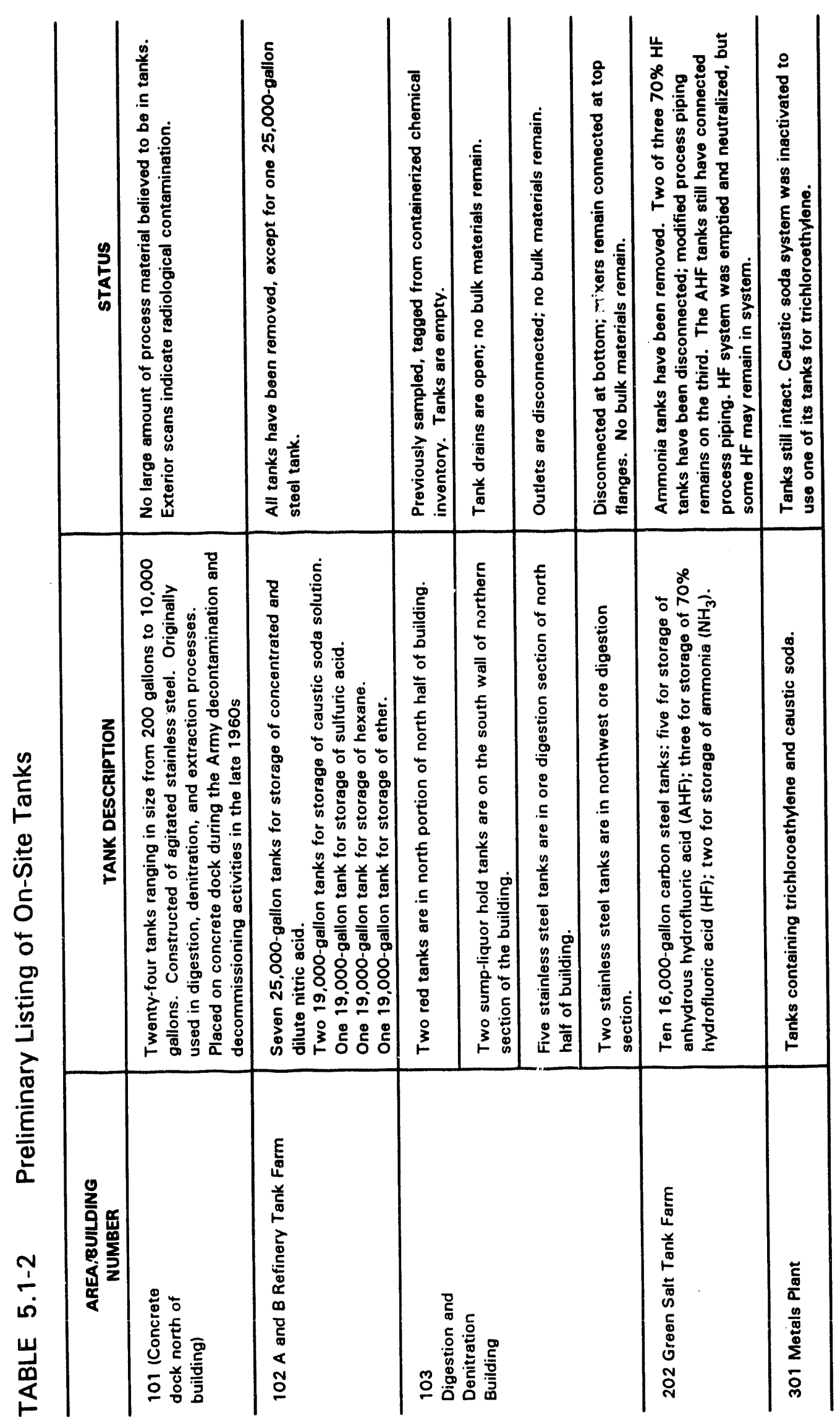

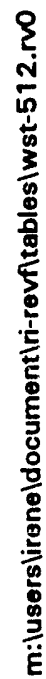




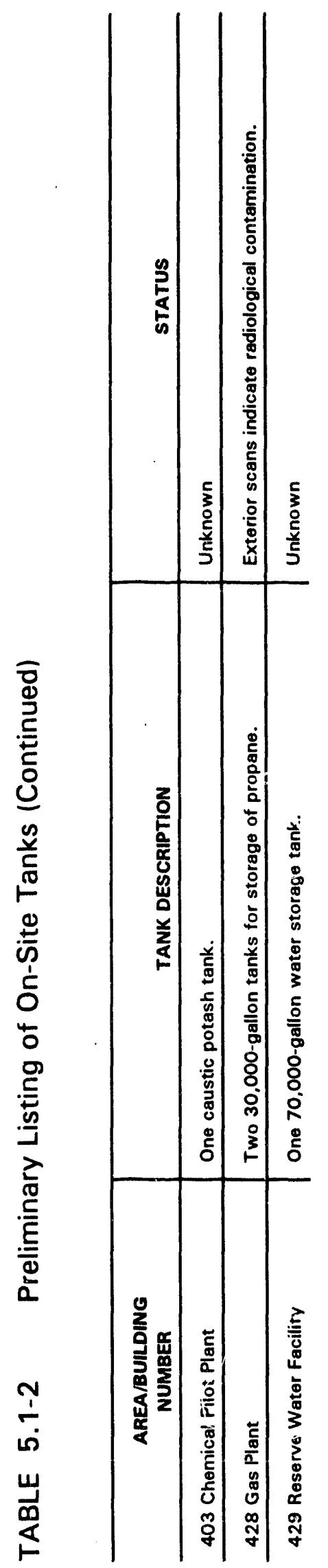

ํ
ํ

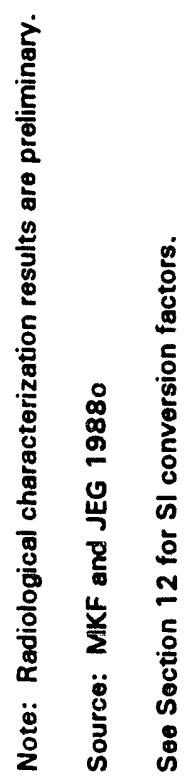

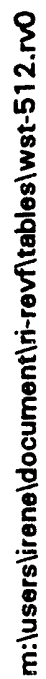




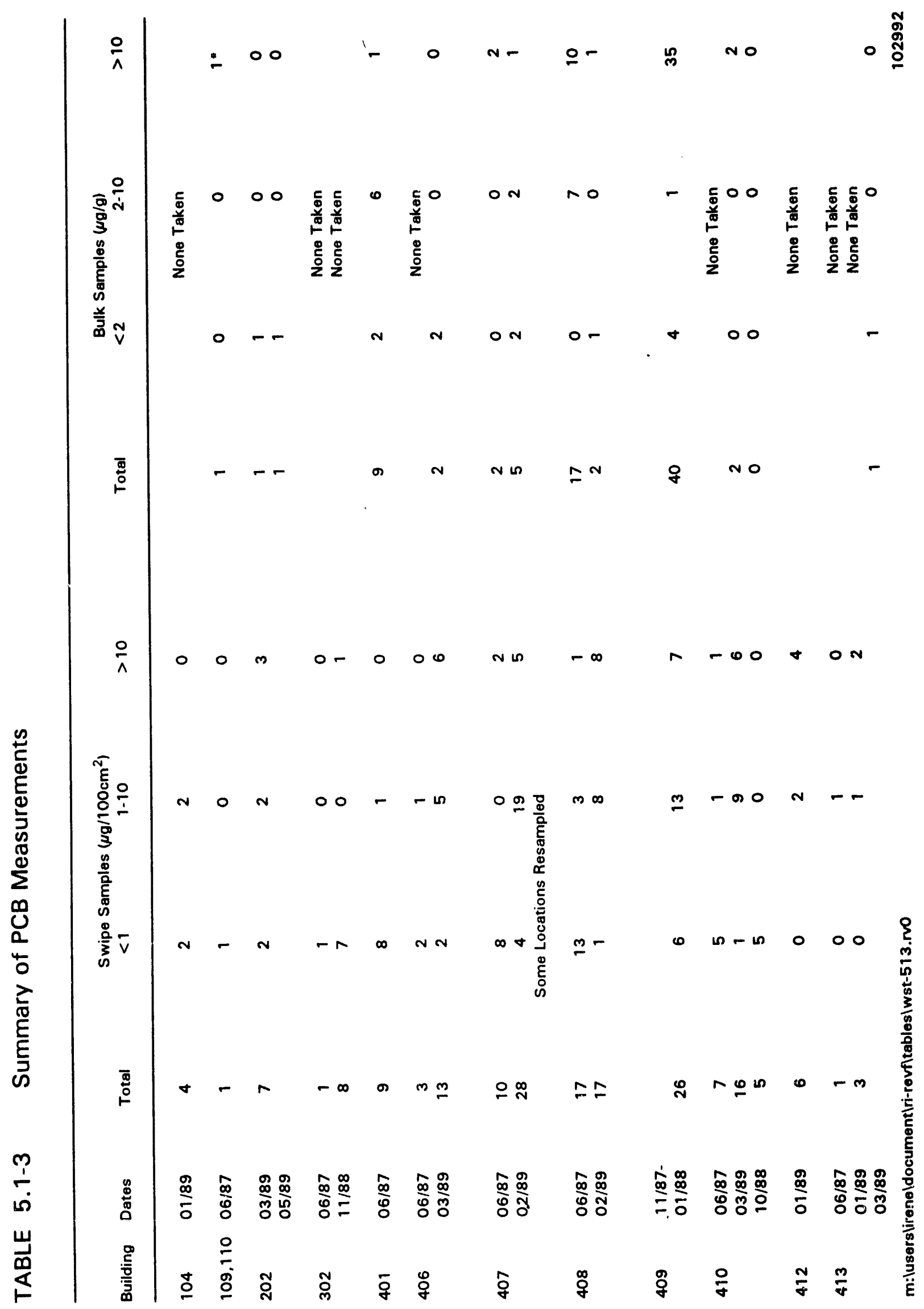




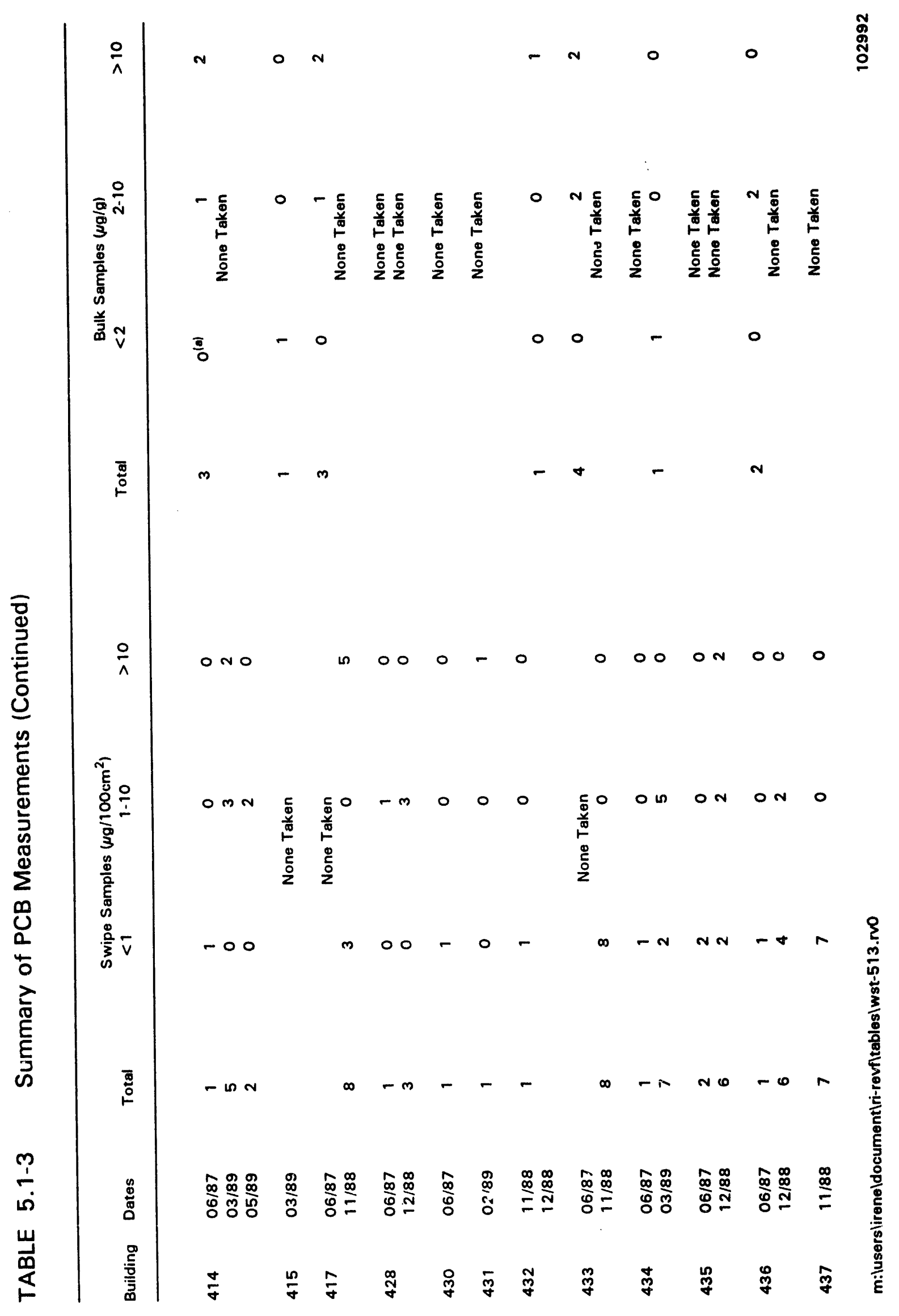




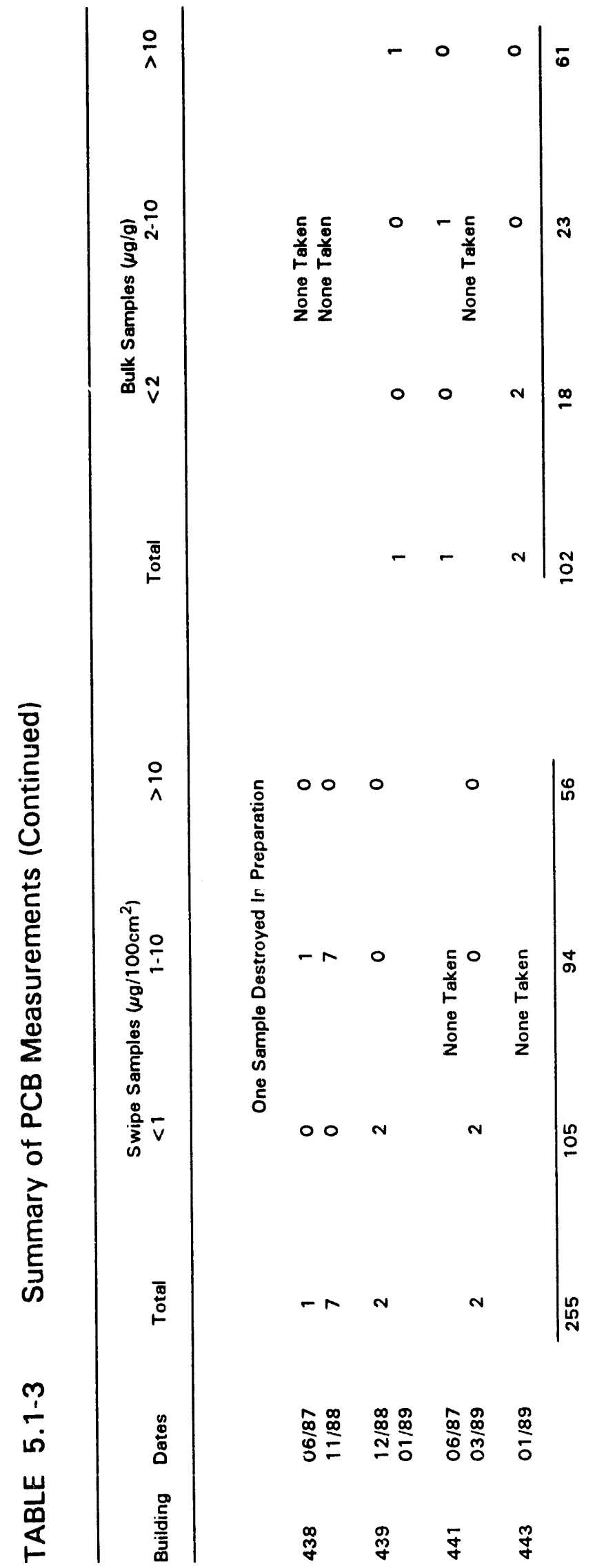

ฆั

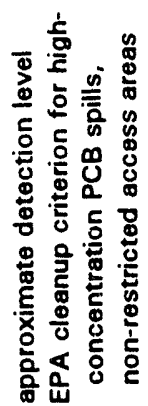

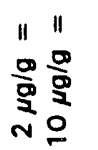



TABLE 5.1-4 Asbestos C ontent of Pipe Insulation on Overhead Utilities (All
Samples Collected on 11/17-18/86)

\begin{tabular}{|c|c|c|c|}
\hline Location & $\begin{array}{l}\text { Location No. } \\
\text { Fig. } 5.1-5\end{array}$ & Pipe Type & Results \\
\hline $\begin{array}{l}\text { NW Corner } \\
\text { Bldg. } 105\end{array}$ & 1 & $\begin{array}{l}\text { Steam } \\
\text { Ethylene Glycol } \\
\text { Ethylene Glycol } \\
\text { Ethylene Glycol } \\
\text { Ethylene Glycol (elbow) } \\
\text { Ethylene Glycol } \\
\text { Ethylene Glycol }\end{array}$ & $\begin{array}{l}40-50 \% \\
\text { Not Analyzed } \\
\text { Trace } \\
\text { Not Analyzed } \\
0-30 \% \\
5-10 \% \\
\text { Not Analyzed }\end{array}$ \\
\hline $\begin{array}{l}\text { NE Corner } \\
\text { Bldg. } 105\end{array}$ & 2 & $\begin{array}{l}\text { Steam } \\
\text { Process } \\
\text { Process } \\
\text { Process } \\
\text { Process } \\
\text { Process } \\
\text { Ethylene Glycol } \\
\text { Ethylene Glycol }\end{array}$ & $\begin{array}{l}45-50 \% \\
40 \% \\
20-30 \% \\
\text { Not Analyzed } \\
30 \% \\
\text { Not Analyzed } \\
\text { Not Analyzed } \\
\text { Not Analyzed }\end{array}$ \\
\hline $\begin{array}{l}\text { SW Corner } \\
\text { Bldg. } 403\end{array}$ & 3 & $\begin{array}{l}\text { Steam } \\
\text { Ethylene Glycol } \\
\text { Ethylene Glycol } \\
\text { Process } \\
\text { Process } \\
\text { Process } \\
\text { Process }\end{array}$ & $\begin{array}{l}35-40 \% \\
\text { Not Analyzed } \\
1-2 \% \\
40-50 \% \\
10-20 \% \\
1-2 \% \\
10-20 \%\end{array}$ \\
\hline Bldg. $407-410$ & 4 & $\begin{array}{l}\text { Ethylene Glycol } \\
\text { Ethylene Glycol } \\
\text { Process }\end{array}$ & $\begin{array}{l}\text { Not Analyzed } \\
\text { Not Analyzed } \\
20-30 \%\end{array}$ \\
\hline $\begin{array}{l}\text { NE Corner } \\
\text { Bldg. } 201\end{array}$ & 5 & $\begin{array}{l}\text { Raffinate } \\
\text { Raffinate } \\
\text { Steam }\end{array}$ & $\begin{array}{l}25-30 \% \\
20 \% \\
20-30 \%\end{array}$ \\
\hline $\begin{array}{l}\text { N. Center } \\
\text { Bldg. } 201\end{array}$ & 6 & $\begin{array}{l}\text { Process } \\
\text { Raffinate } \\
\text { Raffinate } \\
\text { Steam (elbow) } \\
\text { Steam (elbow) }\end{array}$ & $\begin{array}{l}25-30 \% \\
40-60 \% \\
20-30 \% \\
\text { Trace } \\
40-60 \%\end{array}$ \\
\hline $\begin{array}{l}\text { NE Corner } \\
\text { Bldg. } 201\end{array}$ & 7 & Raffinate & $20-30 \%$ \\
\hline $\begin{array}{l}\text { SW Corner } \\
\text { Bldg. } 406\end{array}$ & 8 & Steam & $25-30 \%$ \\
\hline $\begin{array}{l}\text { W. Center } \\
\text { Bldy. } 414\end{array}$ & 9 & $\begin{array}{l}\text { Raffinate } \\
\text { Raffinate } \\
\text { Raffinate } \\
\text { Raffinate (elbow) }\end{array}$ & $\begin{array}{l}50-60 \% \\
20-30 \% \\
30-50 \% \\
\text { Not Analyzor! }\end{array}$ \\
\hline $\begin{array}{l}\text { E. Center } \\
\text { Bldg. } 301\end{array}$ & 10 & $\begin{array}{l}\text { Steam } \\
\text { Steam } \\
\text { Stjam }\end{array}$ & $\begin{array}{l}1-2 \% \\
45-50 \% \\
40-50 \%\end{array}$ \\
\hline
\end{tabular}

Source: MKF and JEG 1987s 

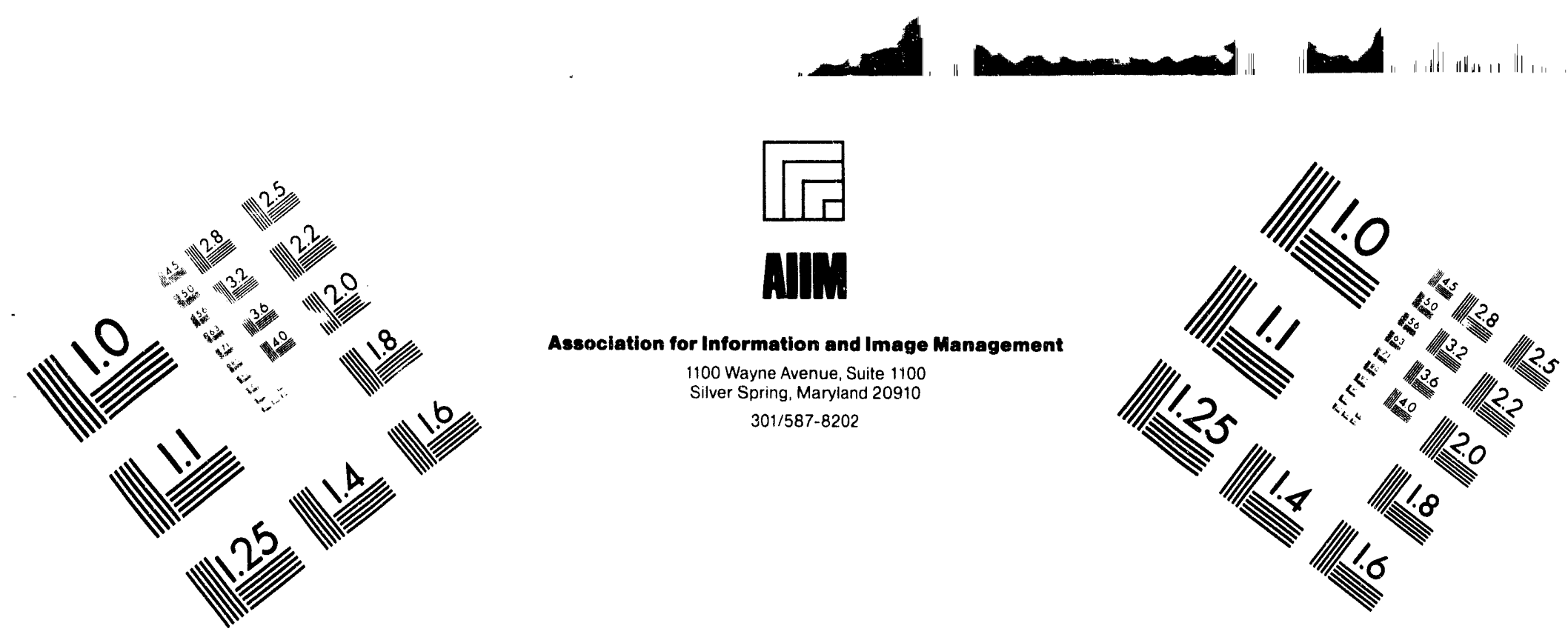

\section{Centimeter}

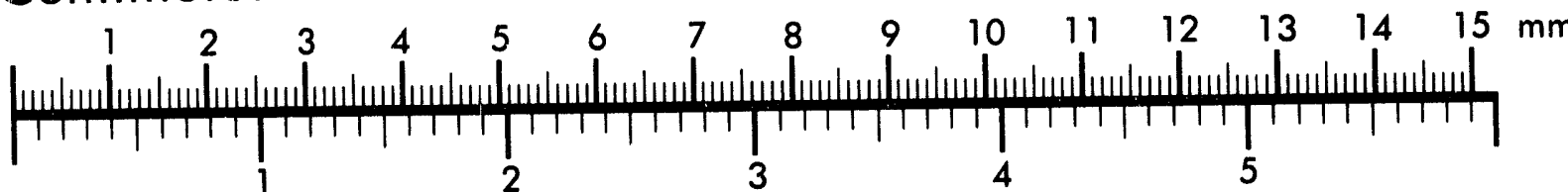

Inches
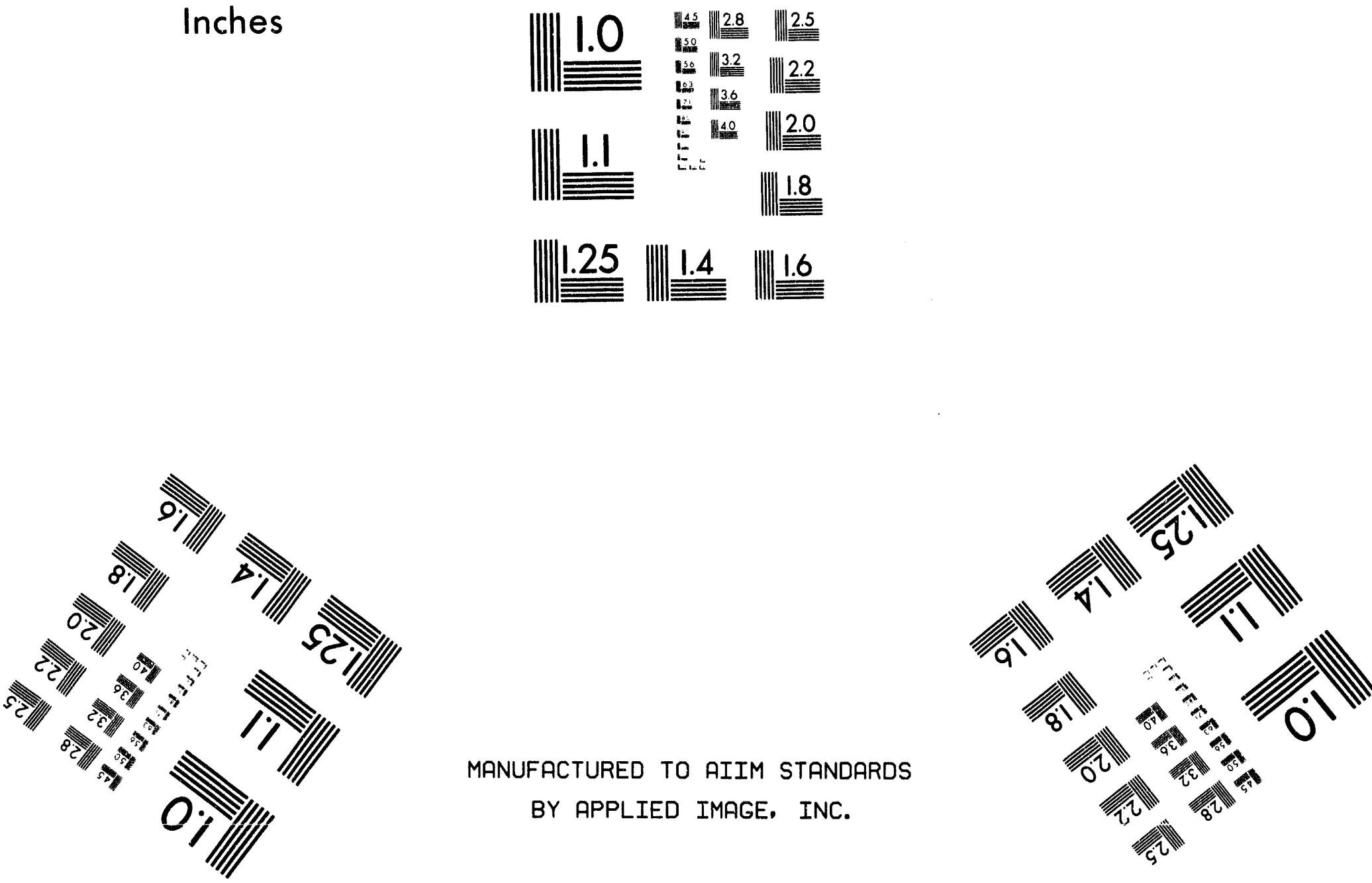

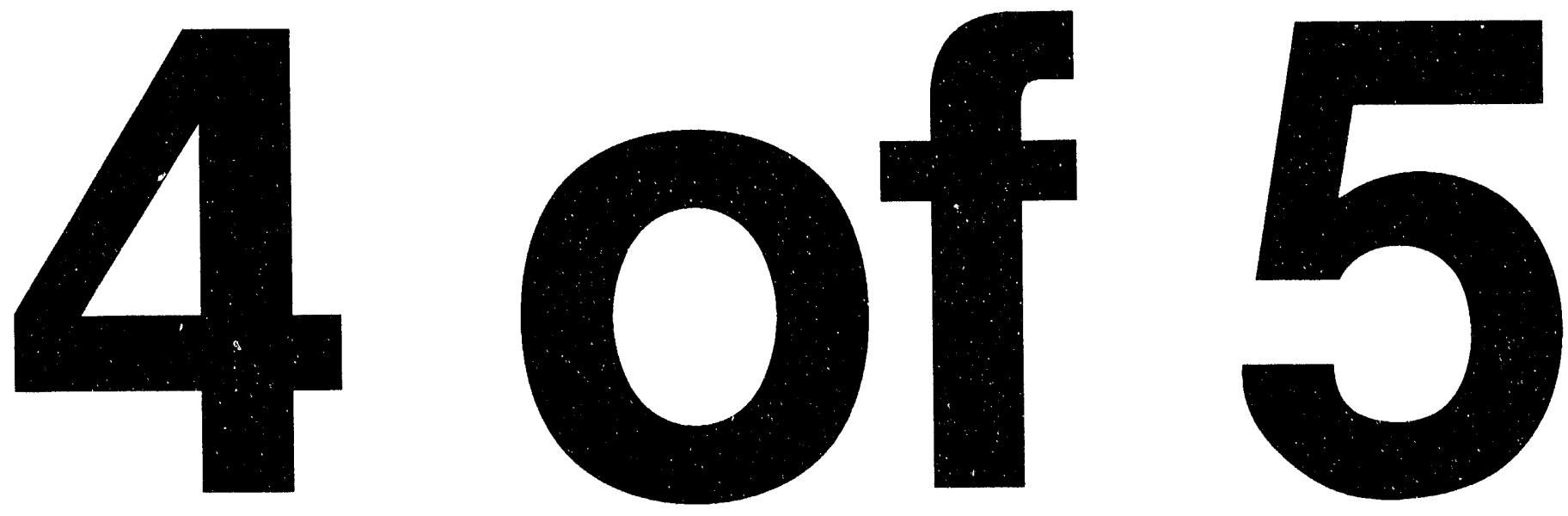


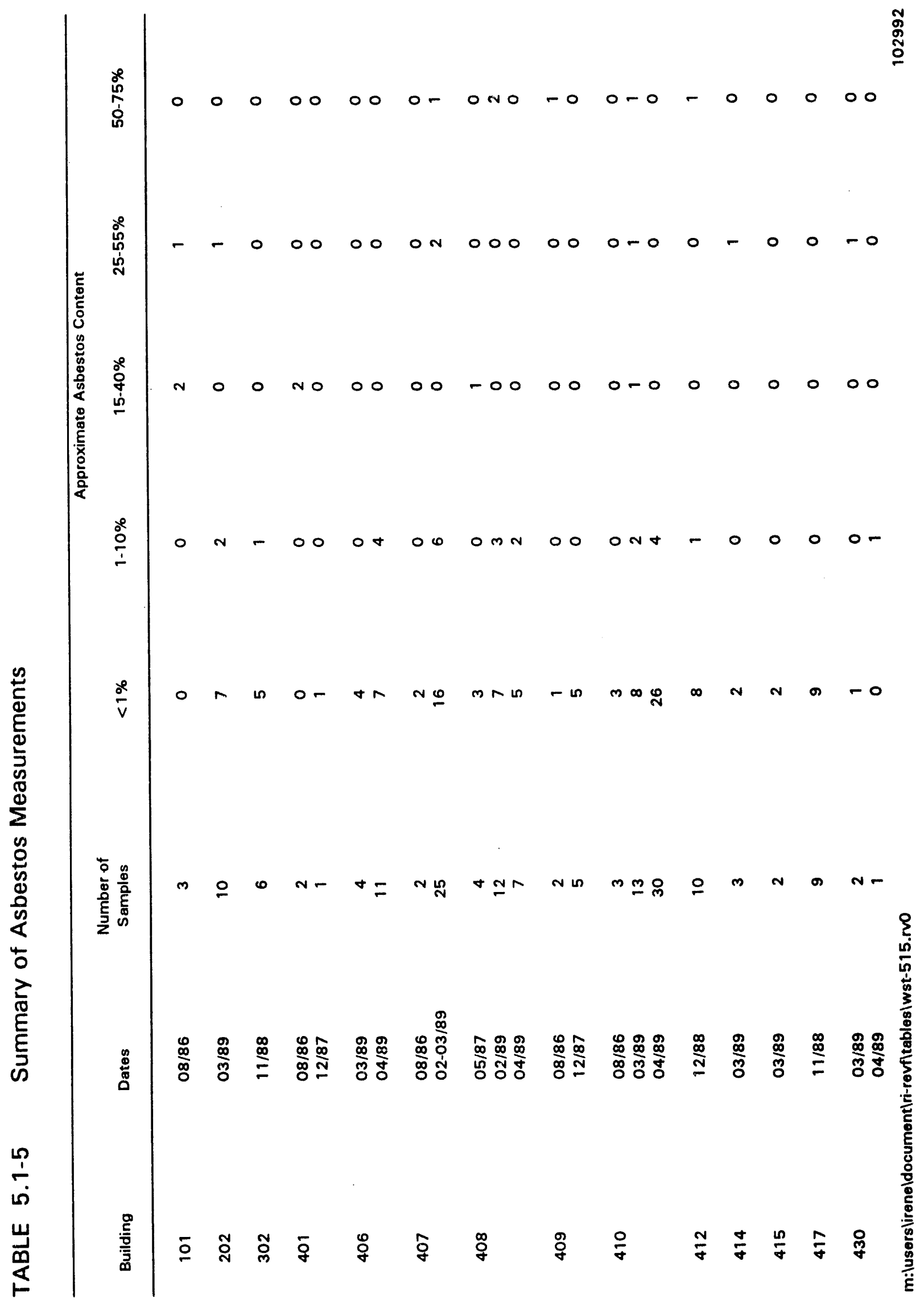



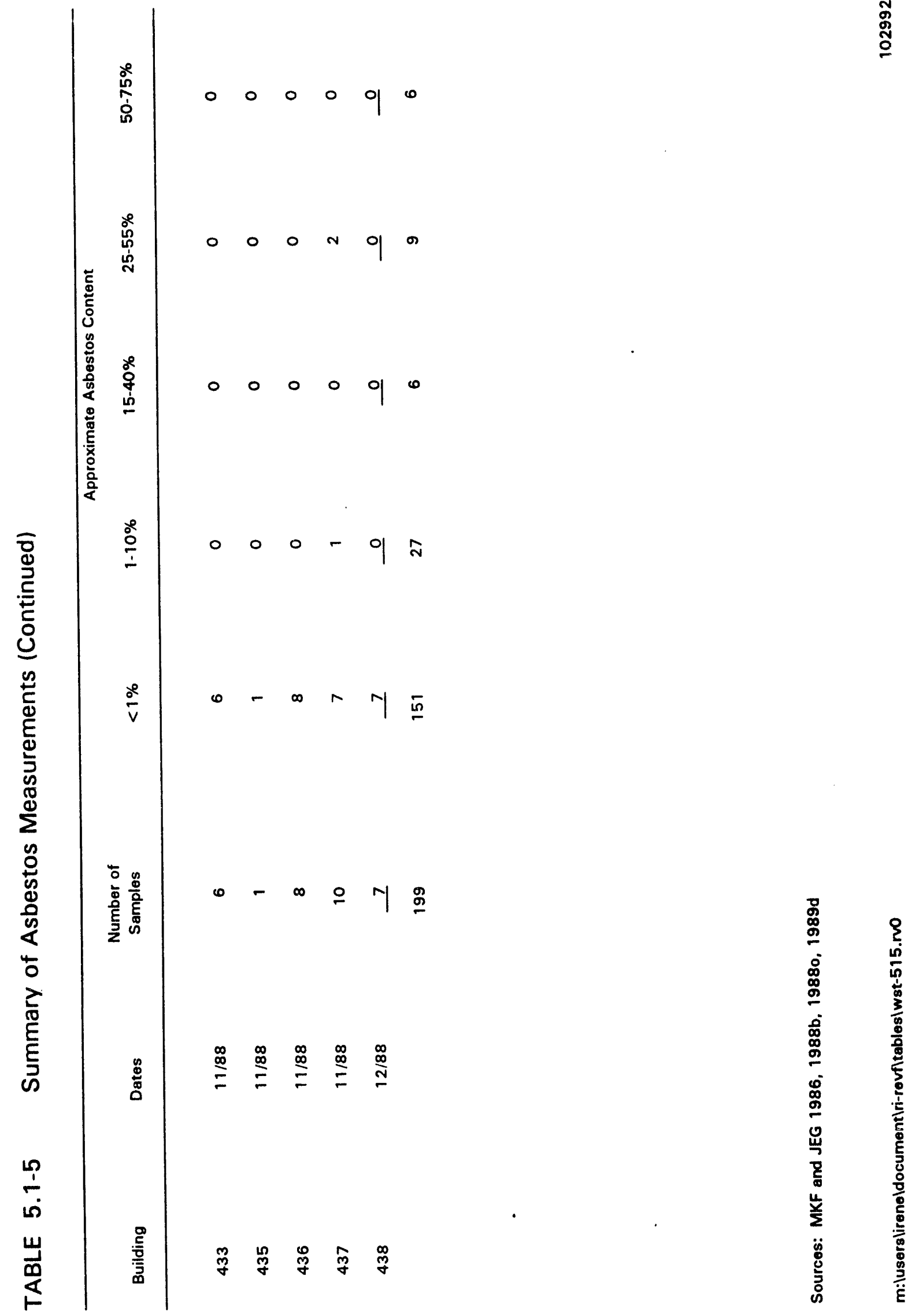


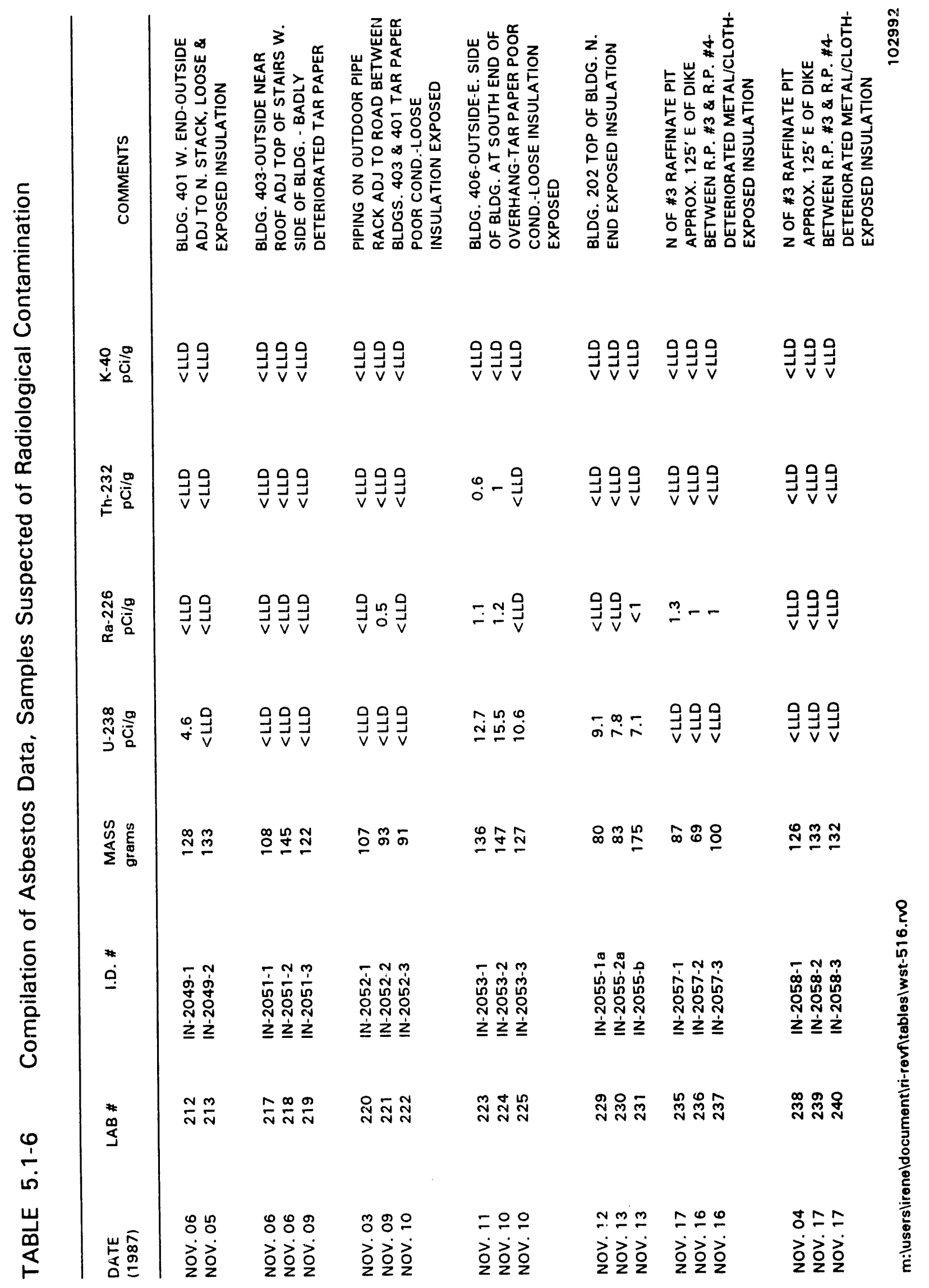




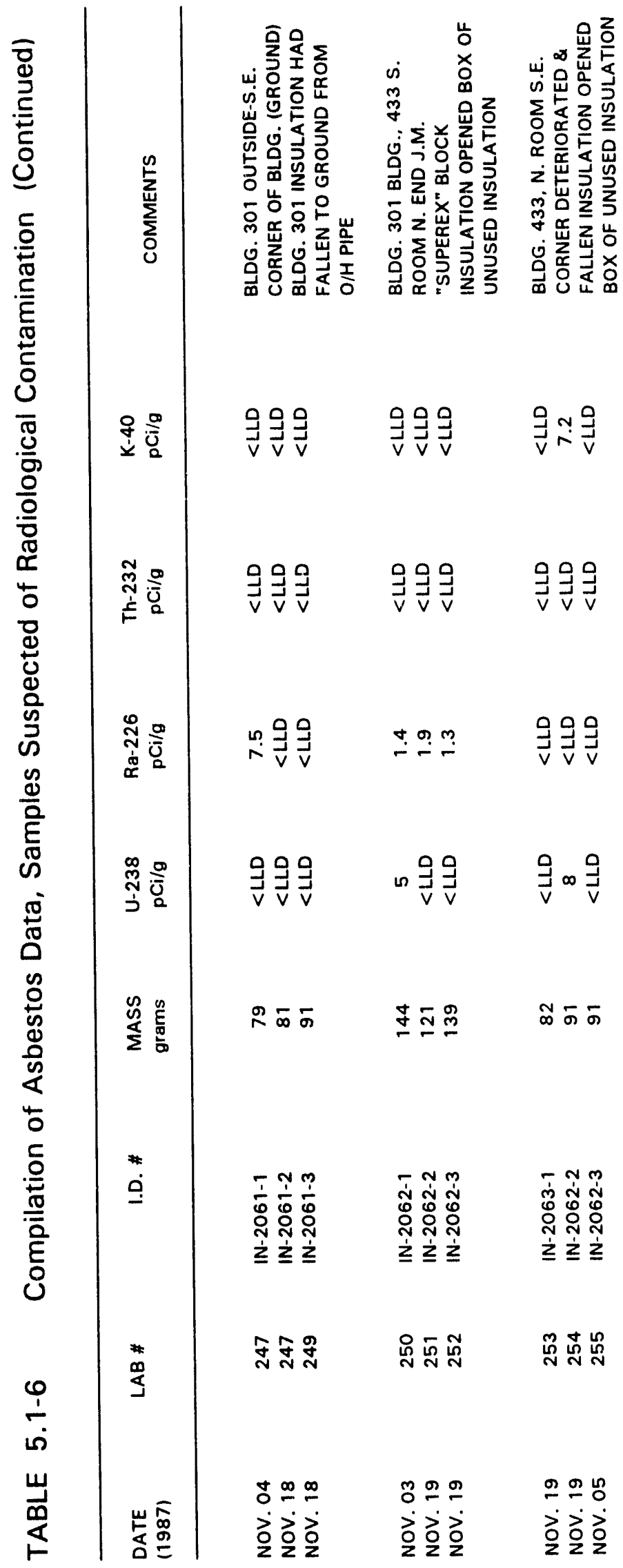

ฆั

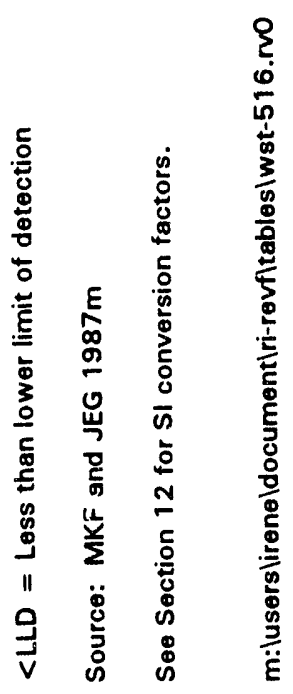




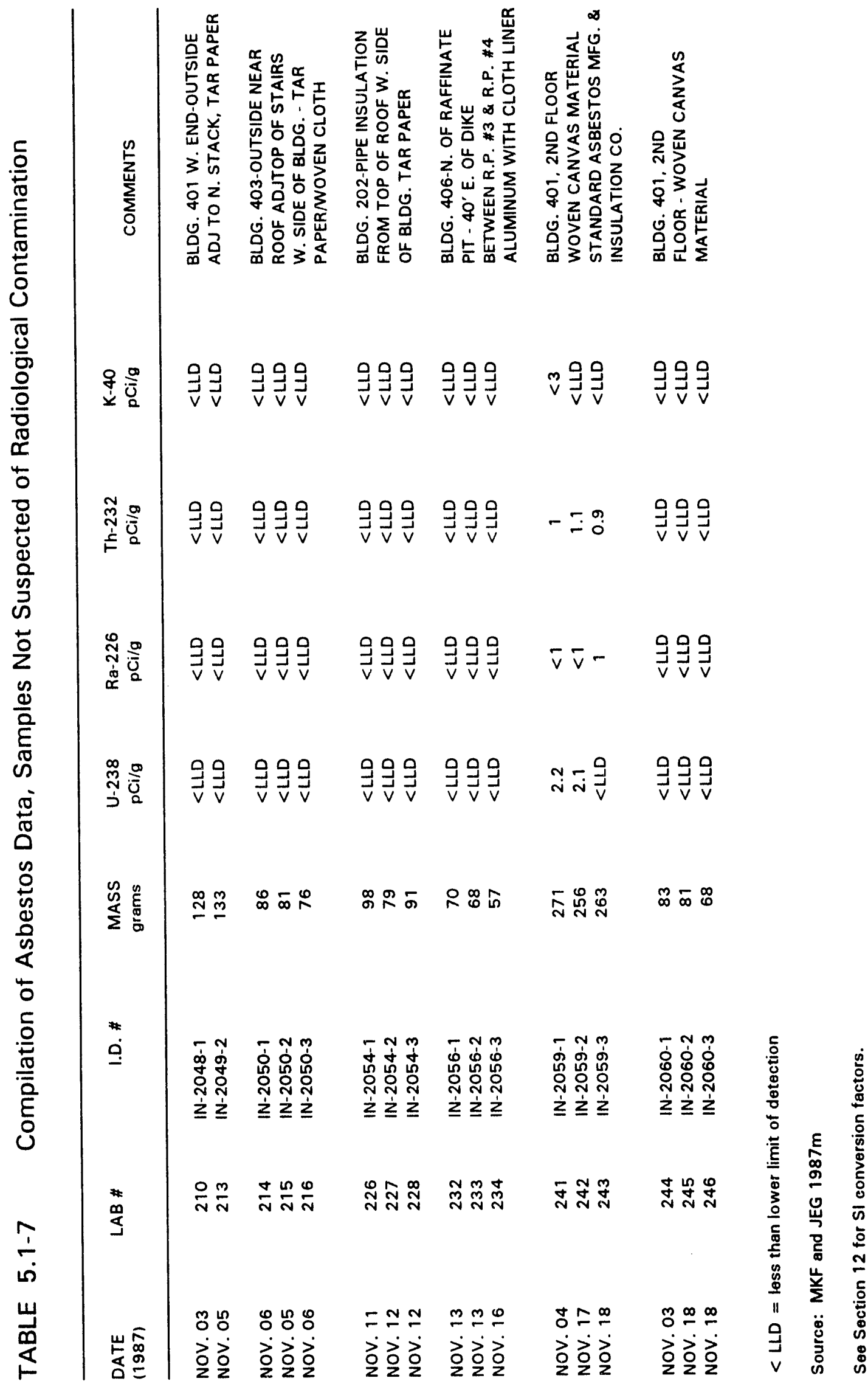



$\begin{array}{ll}\text { WABLE 5.1-8 } & \begin{array}{l}\text { Weldon Spring Raffinate Pits Sludge Sample Radionuclide } \\ \text { Activities }\end{array}\end{array}$

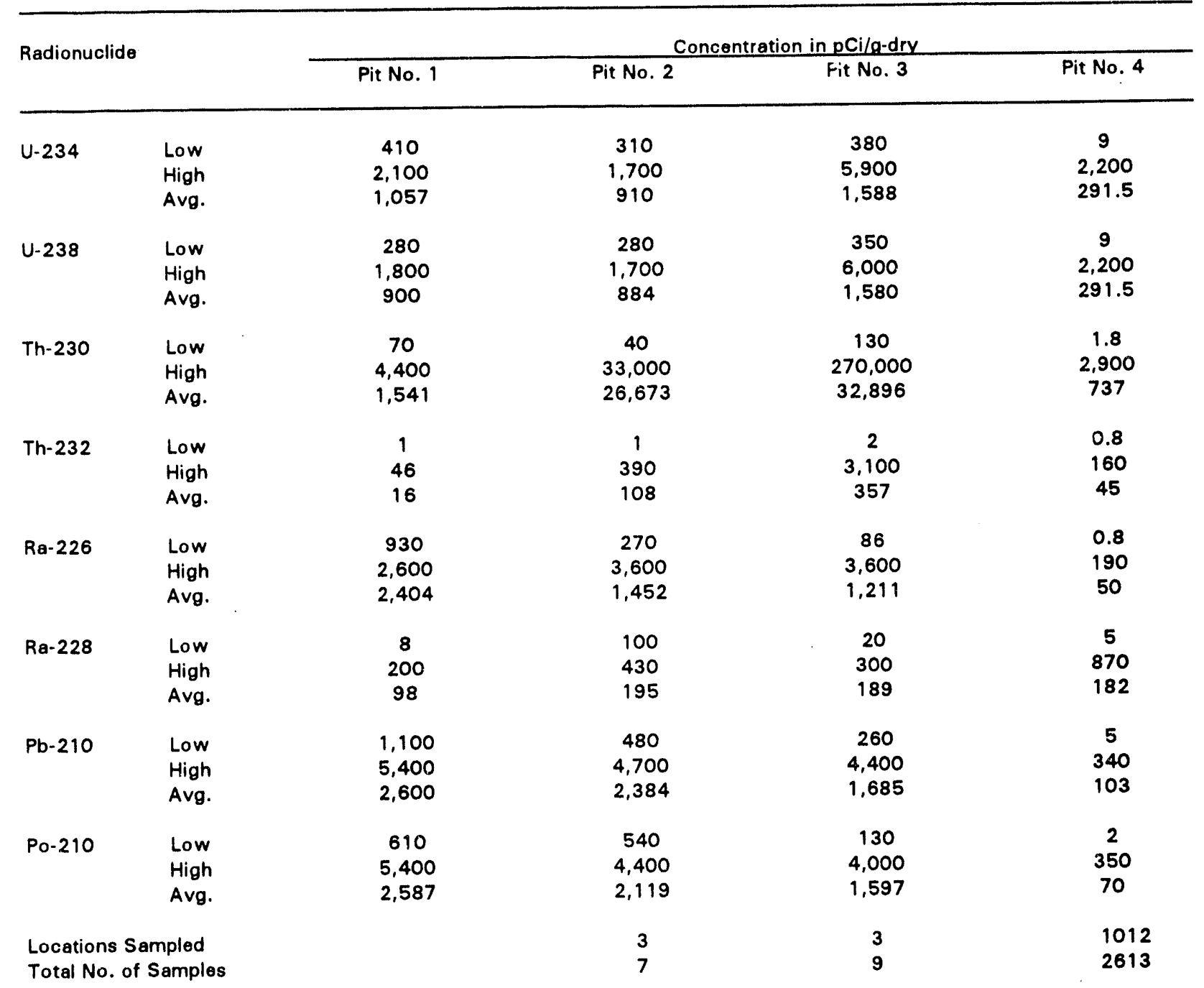




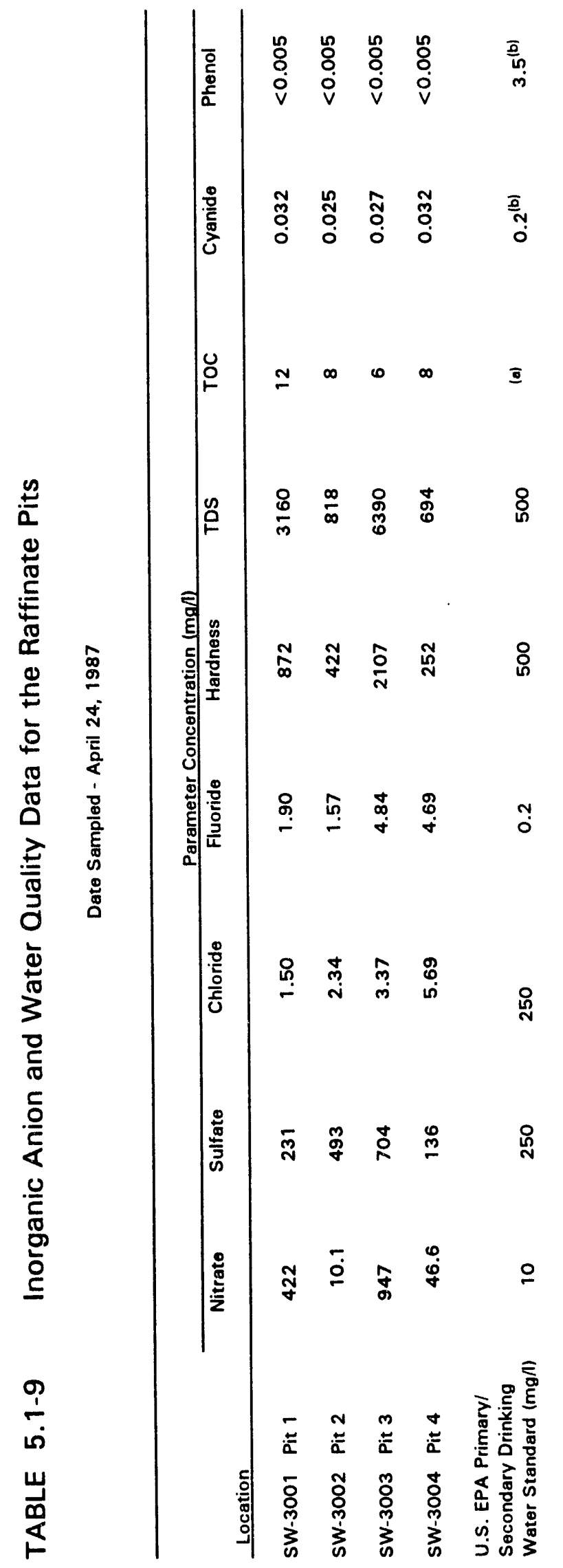

กั 


\section{TABLE 5.1-10 Historical Concentrations of Inorganic Anions in Raffinate Pit Water and Sludge}

\begin{tabular}{|c|c|c|c|c|c|c|}
\hline Compound & 1987 & $1984^{(a)}$ & $\begin{array}{l}\text { Water } \\
(\mathrm{mg} / \mathrm{l}) \\
1983^{(a)}\end{array}$ & $1979^{(a)}$ & $1967^{(a)}$ & $\begin{array}{c}\text { Sludge } \\
\text { (mg/kg dry) } \\
1983\end{array}$ \\
\hline
\end{tabular}

Pit 1

\begin{tabular}{|c|c|c|c|c|c|c|}
\hline Nitrate (as N) & 422 & 652 & 697 & $--\cdot$ & 5625 & 11,250 \\
\hline Sulfate & 231 & 400 & 100 & $\cdots$ & 2300 & 400 \\
\hline Fluoride & 1.9 & 2.5 & 1.1 & --- & --- & 23,000 \\
\hline Chloride & 1.5 & 17 & 15 & $\cdots$ & 210 & 670 \\
\hline \multicolumn{7}{|l|}{ Pit 2} \\
\hline Nitrate (as N) & 10 & 204 & -- & $-\cdot$ & 8550 & 4050 \\
\hline Sulfate & 493 & 990 & 460 & $\cdots$ & 3300 & 400 \\
\hline Fluoride & 2.3 & 2.7 & 1 & $\cdots$ & $-\cdot$ & 2500 \\
\hline Chloride & 1.6 & 5.7 & 6 & $\cdots$ & 50 & 230 \\
\hline \multicolumn{7}{|l|}{ Pit 3} \\
\hline Nitrate (as N) & 947 & 1890 & 1485 & 2925 & 8325 & 4950 \\
\hline Sulfate & 704 & 640 & 268 & 6203 & 2200 & 370 \\
\hline Fluoride & 4.8 & 8.9 & 2.7 & 6 & --- & 10,700 \\
\hline Chloride & 3.4 & 25 & 20 & 37 & 90 & 300 \\
\hline \multicolumn{7}{|l|}{ Pit 4} \\
\hline Nitrate (as N) & 47 & 92 & 99 & 126 & 4725 & 495 \\
\hline Sulfate & 136 & 150 & 70 & 140 & 2200 & 270 \\
\hline Fluoride & 4.7 & 7.8 & 5.8 & 13 & --- & 64,300 \\
\hline Chloride & 5.7 & 7.720 & 7 & 10 & 90 & 50 \\
\hline
\end{tabular}

\section{(a) - DOE 1987}

Source: MKF and JEG 19870

m:lusers \ireneldocument|ri-revf|tables $\mid w s t-5110 . r v o$ 


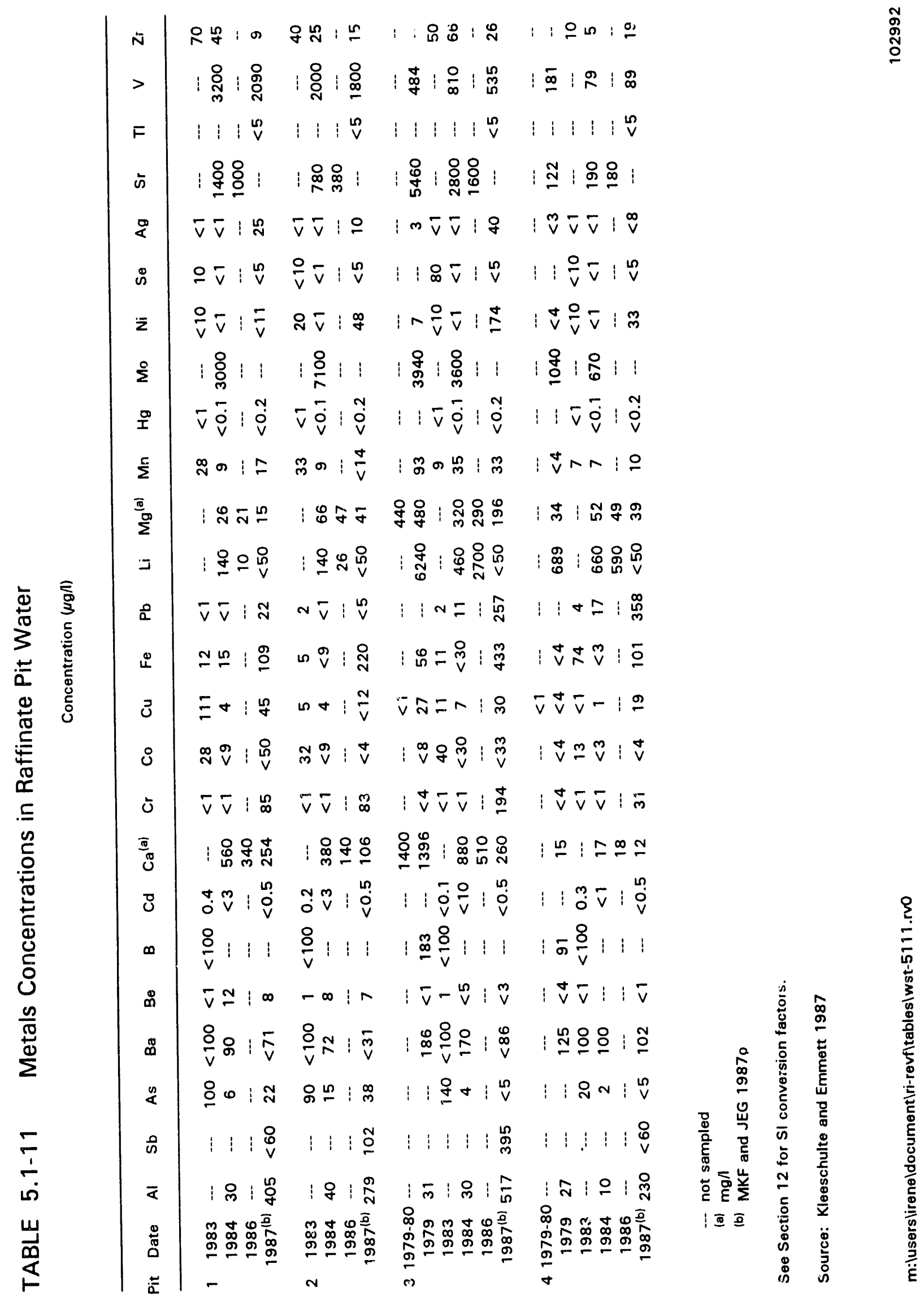




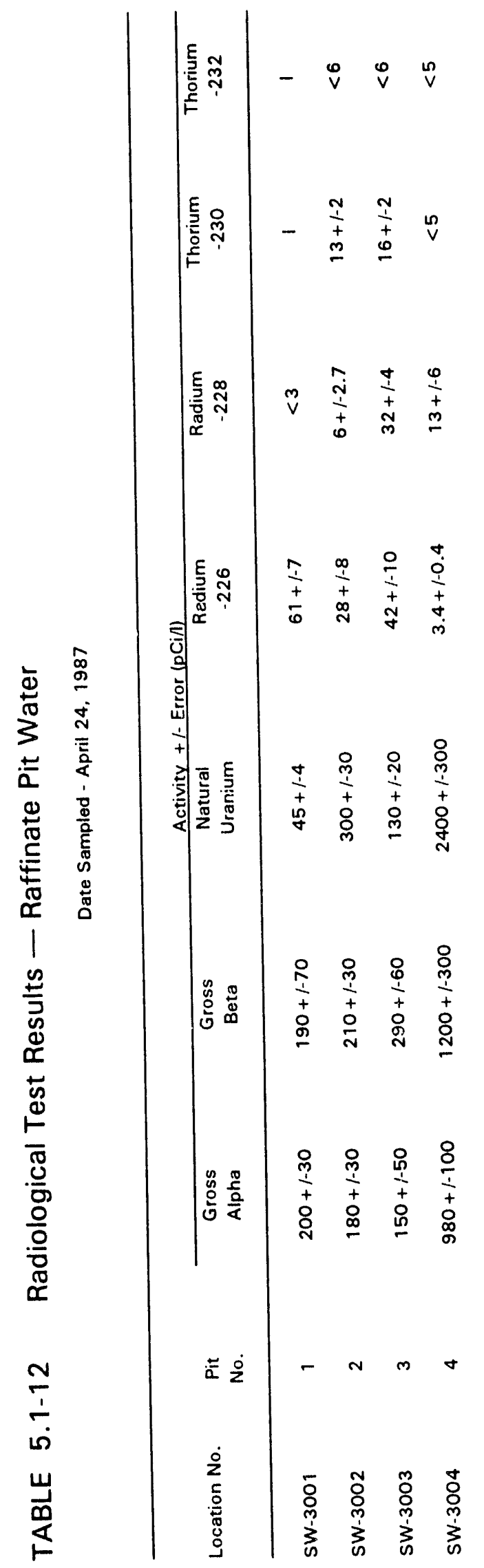

ฟั

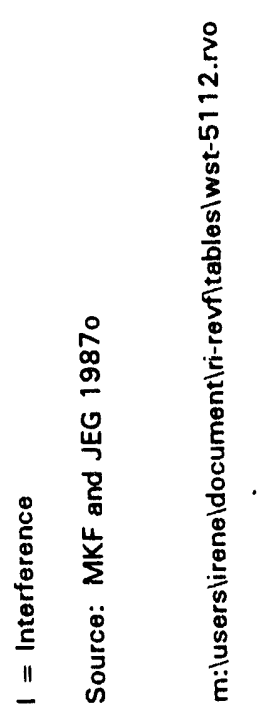


TABLE 5.1-13 Summary of the Characteristics of the Raffinate Pits

Physical Characteristics

\begin{tabular}{cccccc}
\hline $\begin{array}{c}\text { Pit } \\
\text { Number }\end{array}$ & $\begin{array}{c}\text { Construction } \\
\text { Date }\end{array}$ & $\begin{array}{c}\text { Pit } \\
\text { Volume } \\
\left(y d^{3}\right)\end{array}$ & $\begin{array}{c}\text { Percent } \\
\text { Filled }\end{array}$ & $\begin{array}{c}\text { Surface Water (a) } \\
\text { Volume } \\
\left(\mathrm{m}^{3}\right)\end{array}$ & $\begin{array}{c}\text { Surface } \\
\text { Area } \\
\text { (hectares) }\end{array}$ \\
\hline Pit 1 & 1958 & 18,500 & 97 & $<5,000$ & 0.49 \\
Pit 2 & 1958 & 18,500 & 103 & $<5,000$ & 0.49 \\
Pit 3 & 1959 & 166,700 & 78 & 19,000 & 3.4 \\
Pit 4 & 1964 & 444,400 & 7 & 57,000 & 6.1
\end{tabular}

Waste Characteristics

\begin{tabular}{lccrr}
\hline $\begin{array}{c}\text { Pit } \\
\text { Number }\end{array}$ & $\begin{array}{c}\text { Waste } \\
\text { Volume } \\
\left(\mathrm{yd}^{3}\right)\end{array}$ & $\begin{array}{c}\text { Weight } \\
\text { Percent } \\
\text { Solids }\end{array}$ & $\begin{array}{c}\text { Wet } \\
\text { Bulk Density } \\
\left(\mathrm{g} / \mathrm{cm}^{3}\right)\end{array}$ & $\begin{array}{c}\text { Solids } \\
\text { Weight } \\
\text { (MT) }\end{array}$ \\
\hline Pit 1 & 17,900 & 27.6 & 1.19 & 5,900 \\
Pit 2 & 19,000 & 29.4 & 1.22 & 6,800 \\
Pit 3 & 129,200 & 27.3 & 1.20 & 42,000 \\
Pit 4 & 30,200 & 25.3 & 1.18 & 9,010
\end{tabular}

(a) Surface water volume varies with season

See Section 12 for SI conversion factors.

Source: DOE 1987 


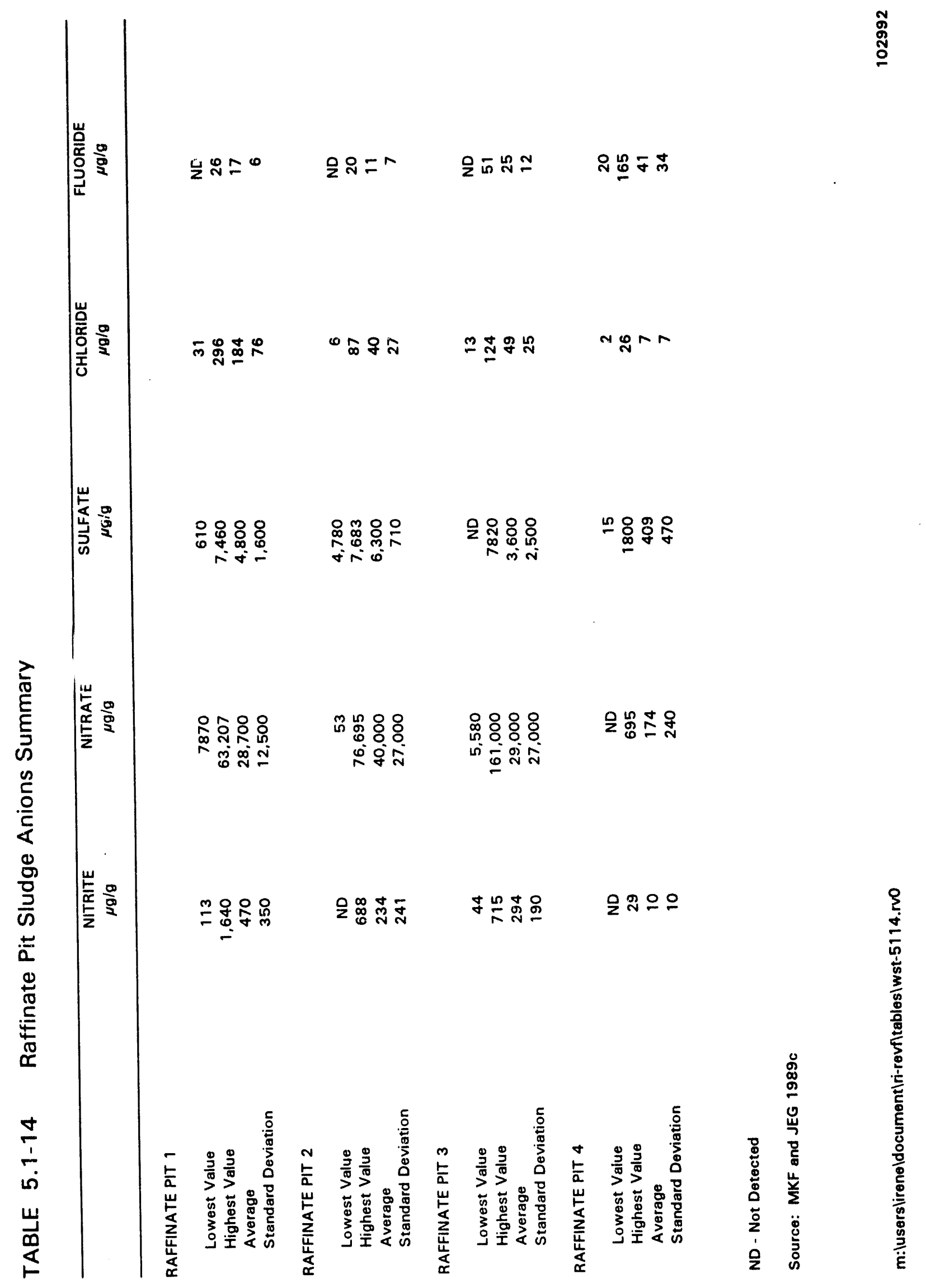




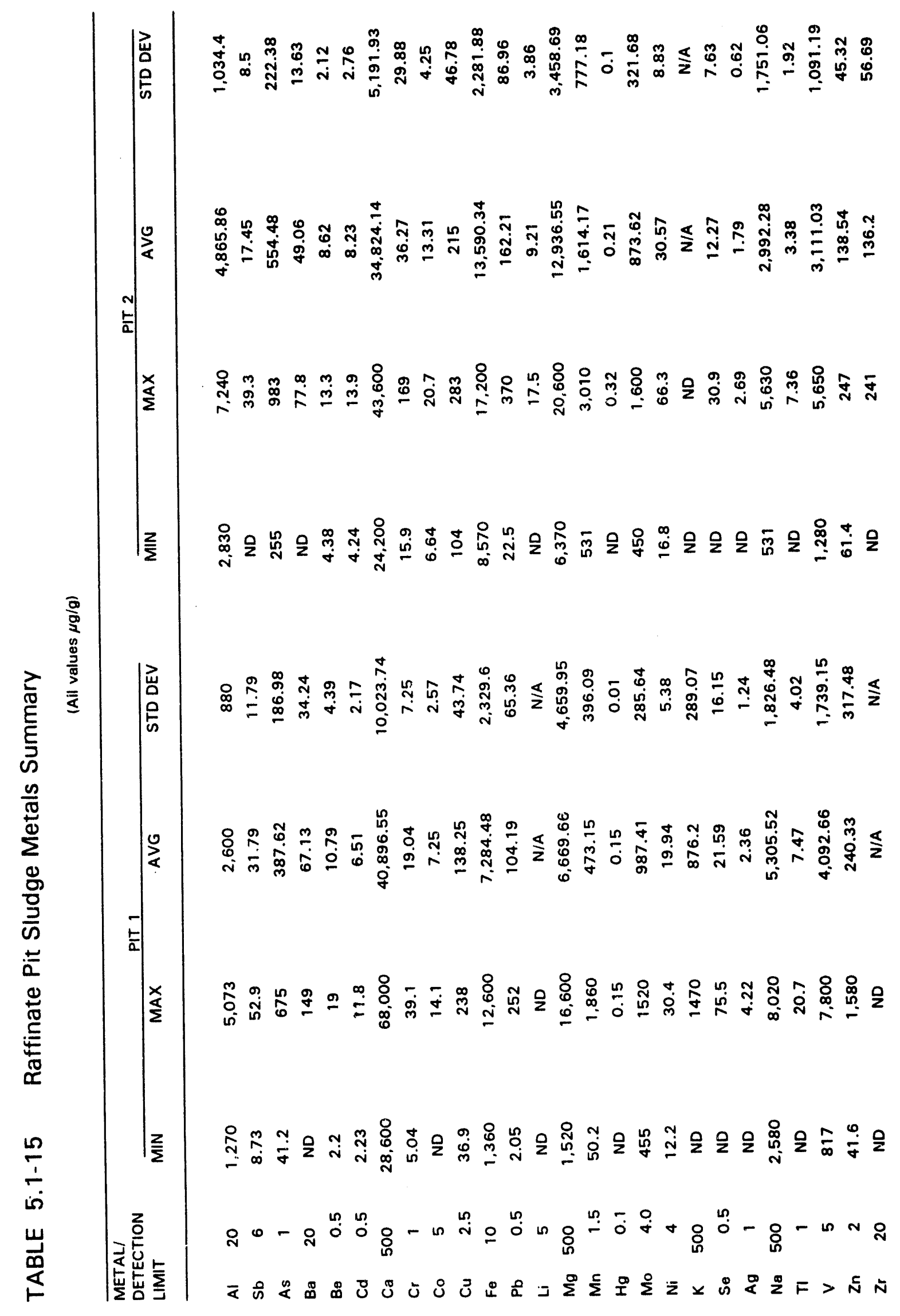




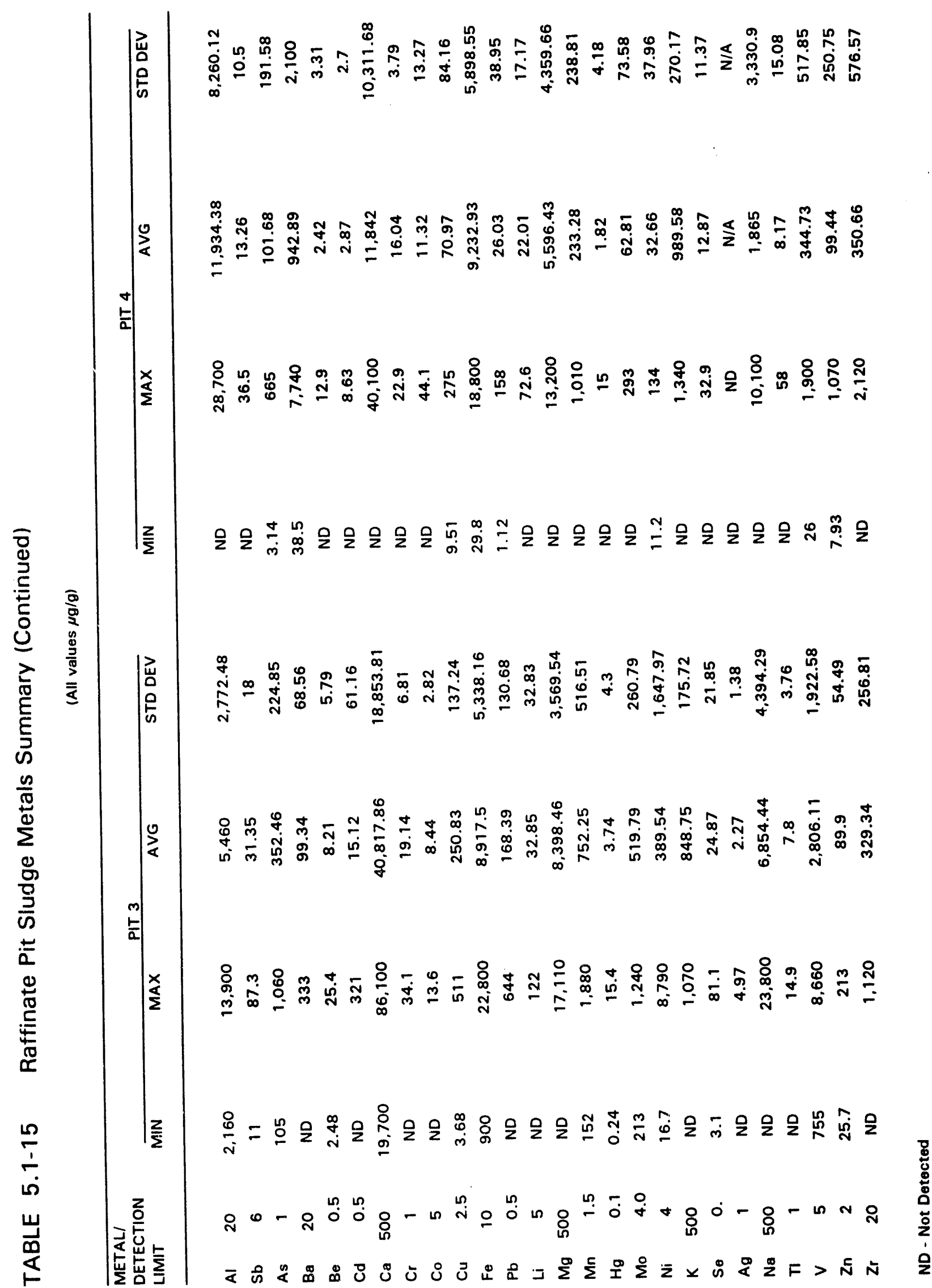




\section{TABLE 5.1-16 Raffinate Pit Radionuclide Contents Determined from the WSSRAP Project Management Contractor Study 1988}

\begin{tabular}{|c|c|c|c|c|c|}
\hline \multirow[b]{2}{*}{ Radionuclide } & \multicolumn{5}{|c|}{ Concentrations $\left(\mathrm{pCi} / \mathrm{g}-\right.$ wet $^{(\mathrm{a})}$} \\
\hline & & Pit 1 & Pit 2 & Pit 3 & Pit 4 \\
\hline \multirow[t]{4}{*}{ Total Uranium } & low & 620 & 340 & 110 & 10 \\
\hline & high & 1200 & 680 & 1100 & 3400 \\
\hline & avg. & 840 & 540 & 600 & 570 \\
\hline & std. & 190 & 140 & 220 & 790 \\
\hline \multirow[t]{4}{*}{ Thorium-230 } & low & 12000 & 22000 & 3300 & 8 \\
\hline & high & 34000 & 33000 & 28000 & 6800 \\
\hline & avg. & 27000 & 27000 & 17000 & 2500 \\
\hline & std. & 6200 & 3600 & 5700 & 2300 \\
\hline \multirow[t]{4}{*}{ Radium-226 } & low & 140 & 300 & 18 & 1 \\
\hline & high & 1700 & 900 & 610 & 200 \\
\hline & avg. & 840 & 540 & 320 & 72 \\
\hline & std. & 420 & 200 & 150 & 58 \\
\hline \multirow[t]{4}{*}{ Thorium-232 $2^{(b)}$} & low & & & & 4 \\
\hline & high & & & & 1400 \\
\hline & avg. & & & & 320 \\
\hline & std. & & & & 340 \\
\hline \multirow[t]{4}{*}{ Radium-228 ${ }^{(c)}$} & low & 19 & 56 & 9 & 4 \\
\hline & high & 110 & 170 & 160 & 1400 \\
\hline & avg. & 61 & 130 & 64 & 230 \\
\hline & std. & 23 & 40 & 41 & 310 \\
\hline \multirow[t]{4}{*}{ Thorium-228 ${ }^{(c)}$} & low & 18 & 47 & 18 & 3 \\
\hline & high & 120 & 160 & 200 & 1100 \\
\hline & avg. & 60 & 100 & 91 & 300 \\
\hline & std. & 35 & 37 & 44 & 310 \\
\hline Locations Sampled & & 3 & 3 & 9 & 19 \\
\hline Total No. Samples & & 9 & 5 & 41 & 22 \\
\hline
\end{tabular}

\footnotetext{
(a) This table includes results of samples collected during the second phase of sampling.
(b) The Th-232 values for pits 1, 2, and 3 are not reported because they are considered erroneous due to interferences during laboratory analysis caused by high Th- 230 concentrations (see Section 3.3 .5 of DOE/OR/21548-062, Rev. 0).
(c) The analysis date for these samples was August 1988 for pit 3, September 1988 for pit 4 , and October 1988 for pit 1 and pit 2.

Source: MKF and JEG 1989e 
TABLE 5.1-17 Total Activity Inventory of Ra-226 per Pit at the Time of Sampling and at 200 Years and 1000 Years after Sampling

\begin{tabular}{lccr} 
& Ra-226 (Ci) & 1000 Years \\
\hline & Mid 1988 & 200 Years & 160 \\
\hline Pit 1 & 14 & 48 & 170 \\
Pit 2 & 10 & 48 & 740 \\
Pit 3 & 39 & 210 & 26 \\
\hline
\end{tabular}


TABLE 5.1-18 Average Concentration of Ra-226 per Pit at the Time of Sampling and at 200 Years and 1000 Years after Sampling

Ra-226 Concentration (pCi/g)

\begin{tabular}{lccc}
\hline & Mid 1988 & 200 Years & 1000 Years \\
\hline Pit 1 & 840 & 3000 & 10000 \\
Pit 2 & 540 & 2700 & 10000 \\
Pit 3 & 320 & 1700 & 6200 \\
Pit 4 & 76 & 290 & 1000 \\
\hline
\end{tabular}




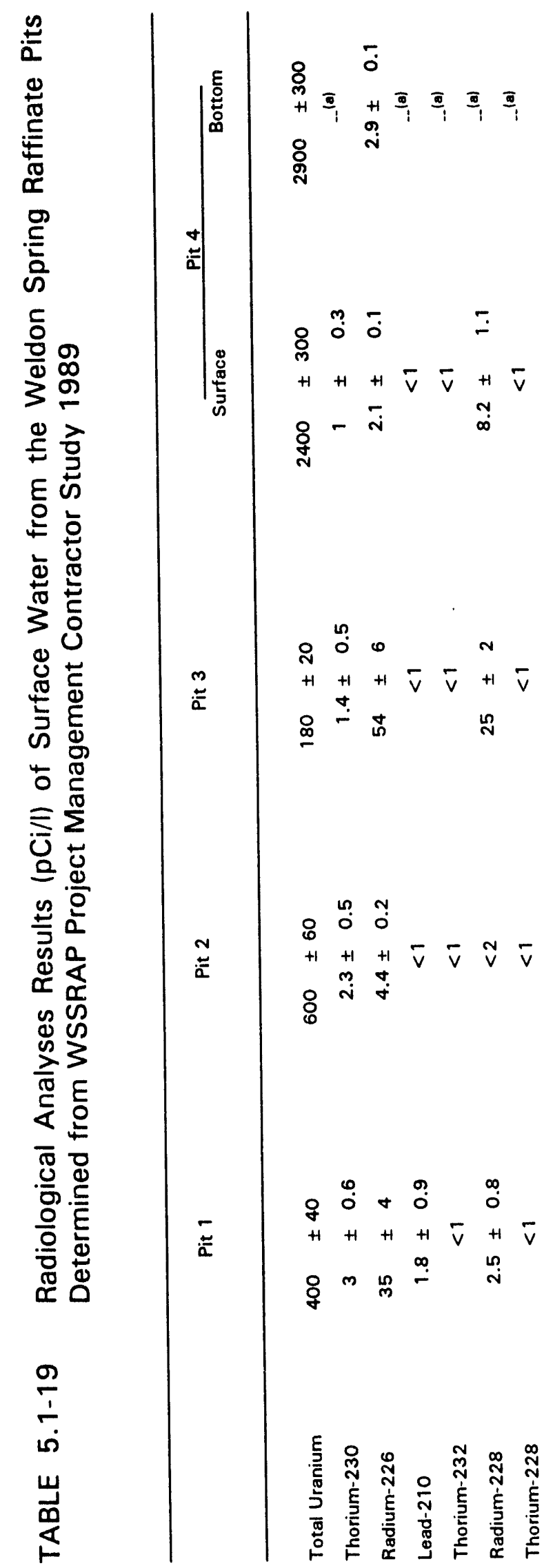


TABLE 5.1-20 Raffinate Pit Water Data - Metals

(All values $\mu \mathrm{g} /$ )

\begin{tabular}{|c|c|c|c|c|c|c|}
\hline \multirow{2}{*}{\multicolumn{2}{|c|}{$\begin{array}{l}\text { METAL/ } \\
\text { DETECTION } \\
\text { LIMIT }\end{array}$}} & \multicolumn{5}{|c|}{ TOTAL } \\
\hline & & $\begin{array}{c}\text { PIT 1 } \\
\text { SW-3001-031789 }\end{array}$ & $\begin{array}{c}\text { PIT 2 } \\
\text { SW.3002.031789 }\end{array}$ & $\begin{array}{c}\text { PIT 3 } \\
\text { sW.3003-031789 }\end{array}$ & $\begin{array}{c}\text { PIT } 4 \\
\text { SW-3004-031789 }\end{array}$ & $\begin{array}{l}\text { PIT } 4 \text { (BOTTOM) } \\
\text { SW-3004.031789 }\end{array}$ \\
\hline Al & 200 & ND & ND & ND & 496.2 & 510.2 \\
\hline Sb & 60 & ND & ND & 65.1 & ND & ND \\
\hline As & 10 & 12.7 & 111 & ND & 4.49 & ND \\
\hline $\mathrm{Ba}$ & 200 & ND & ND & ND & ND & ND \\
\hline Be & 5 & ND & ND & ND & ND & ND \\
\hline $\mathrm{Cd}$ & 5 & ND & ND & ND & ND & ND \\
\hline $\mathrm{Ca}$ & 5000 & 104400 & 26640 & 83720 & 10240 & 10020 \\
\hline $\mathrm{Cr}$ & 10 & ND & ND & ND & ND & ND \\
\hline Co & 50 & ND & ND & ND & ND & ND \\
\hline $\mathrm{Cu}$ & 25 & ND & ND & ND & ND & ND \\
\hline Fo & 100 & ND & ND & ND & 456.9 & 421.2 \\
\hline $\mathrm{Pb}$ & 5 & ND & ND & ND & ND & ND \\
\hline $\mathrm{Li}$ & 50 & 257.4 & 185.9 & 4462 & 492.9 & 496.7 \\
\hline $\mathrm{Mg}$ & 5000 & 26020 & 37130 & 63820 & 33720 & 34310 \\
\hline Mn & 15 & ND & 26 & ND & 15.9 & 17.5 \\
\hline $\mathrm{Hg}$ & 0.2 & ND & 0.29 & ND & ND & ND \\
\hline Mo & 4.0 & 2305 & 2743 & 3947 & 693.4 & 705.1 \\
\hline $\mathrm{Ni}$ & 40 & ND & ND & ND & ND & ND \\
\hline$k$ & 5000 & 34050 & 17450 & 102300 & 16600 & 16600 \\
\hline Se & 5 & 14.7 & ND & 220 & 7.46 & 7.88 \\
\hline $\mathrm{Ag}_{\mathrm{g}}$ & 10 & ND & ND & ND & ND & ND \\
\hline $\mathrm{Na}$ & 5000 & 71980 & 134700 & 222400 & 164100 & 70360 \\
\hline$T I$ & 10 & ND & ND & ND & ND & ND \\
\hline $\mathrm{v}$ & 50 & 1360 & 747.9 & ND & ND & ND \\
\hline $\mathrm{Zn}$ & 20 & ND & ND & 26.8 & 59.6 & ND \\
\hline
\end{tabular}

ND - Not Detected

Source: MKF and JEG 1989c

m:lusers $\backslash$ ireneldocument $|r i-r e v f| t a b l e s \mid w s t-5120 . r v o$

102992 
TABLE 5.1-20 Raffinate Pit Water Data - Metals (Continued)

(All Values $\mu \mathrm{g} /$ )

\begin{tabular}{|c|c|c|c|c|c|c|}
\hline \multirow{2}{*}{\multicolumn{2}{|c|}{$\begin{array}{l}\text { METAL/ } \\
\text { DETECTION } \\
\text { LIMIT }\end{array}$}} & \multirow{3}{*}{$\begin{array}{c}\begin{array}{c}\text { PIT 1 } \\
\text { sW.3001.031 }\end{array} \\
\text { ND }\end{array}$} & \multicolumn{3}{|c|}{ FILTERED } & \multirow{3}{*}{$\begin{array}{r}\begin{array}{r}\text { PIT } 4 \text { (800तоM) } \\
\text { sw.3004001780 }\end{array} \\
218.7\end{array}$} \\
\hline & & & $\begin{array}{c}\text { PIT 2 } \\
\text { SW-3002.001780 }\end{array}$ & $\begin{array}{c}\text { PIT 3 } \\
\text { sw.-3000.091780 }\end{array}$ & $\begin{array}{c}\text { PIT } 4 \\
\text { sW-3004. }\end{array}$ & \\
\hline Al & 200 & & ND & ND & ND & \\
\hline Sb & 60 & ND & ND & 81.3 & ND & ND \\
\hline As & 10 & 12.2 & 119 & ND & ND & ND \\
\hline $\mathrm{Ba}$ & 200 & ND & ND & ND & ND & ND \\
\hline $\mathrm{Be}$ & 5 & ND & ND & ND & ND & ND \\
\hline $\mathrm{Cd}$ & 5 & ND & ND & ND & ND & ND \\
\hline $\mathrm{Ca}$ & 5000 & ND & 27960 & 46100 & 10530 & 19500 \\
\hline $\mathrm{Cr}$ & 10 & ND & ND & ND & ND & ND \\
\hline Co & 50 & ND & ND & ND & ND & ND \\
\hline $\mathrm{Cu}$ & 25 & ND & ND & ND & ND & ND \\
\hline $\mathrm{Fe}$ & 100 & ND & 171.4 & 153.4 & 132 & 139.4 \\
\hline $\mathrm{Pb}$ & 5 & ND & ND & ND & ND & ND \\
\hline $\mathrm{Li}$ & 50 & ND & 181.5 & 4334 & 465.3 & 542.8 \\
\hline $\mathrm{Mg}$ & 5000 & ND & 38420 & 34850 & 33420 & 39880 \\
\hline Mn & 15 & ND & ND & ND & ND & ND \\
\hline $\mathrm{Hg}$ & 0.2 & ND & ND & ND & ND & ND \\
\hline Mo & 4.0 & 470.8 & 2851 & 4052 & 716.8 & 769.5 \\
\hline $\mathrm{Ni}$ & 40 & ND & ND & ND & 49.1 & ND \\
\hline K & 5000 & ND & 15590 & 107300 & 22320 & 14450 \\
\hline Se & 5 & 18 & ND & 199 & 10.2 & 10.4 \\
\hline$A g$ & 10 & ND & ND & ND & ND & ND \\
\hline $\mathrm{Na}$ & 5000 & 70360 & 139900 & 116100 & 16100 & 185200 \\
\hline $\mathrm{TI}$ & 10 & ND & ND & ND & ND & ND \\
\hline v & 50 & 266.7 & 775.4 & ND & ND & ND \\
\hline $\mathrm{Zn}$ & 20 & ND & ND & ND & 41.5 & 31.8 \\
\hline
\end{tabular}

ND - Not Detected

Source: MKF and JEG 1989c

m:luserslireneldocument|ri-revfltables|wst-5120.rvo

102992 


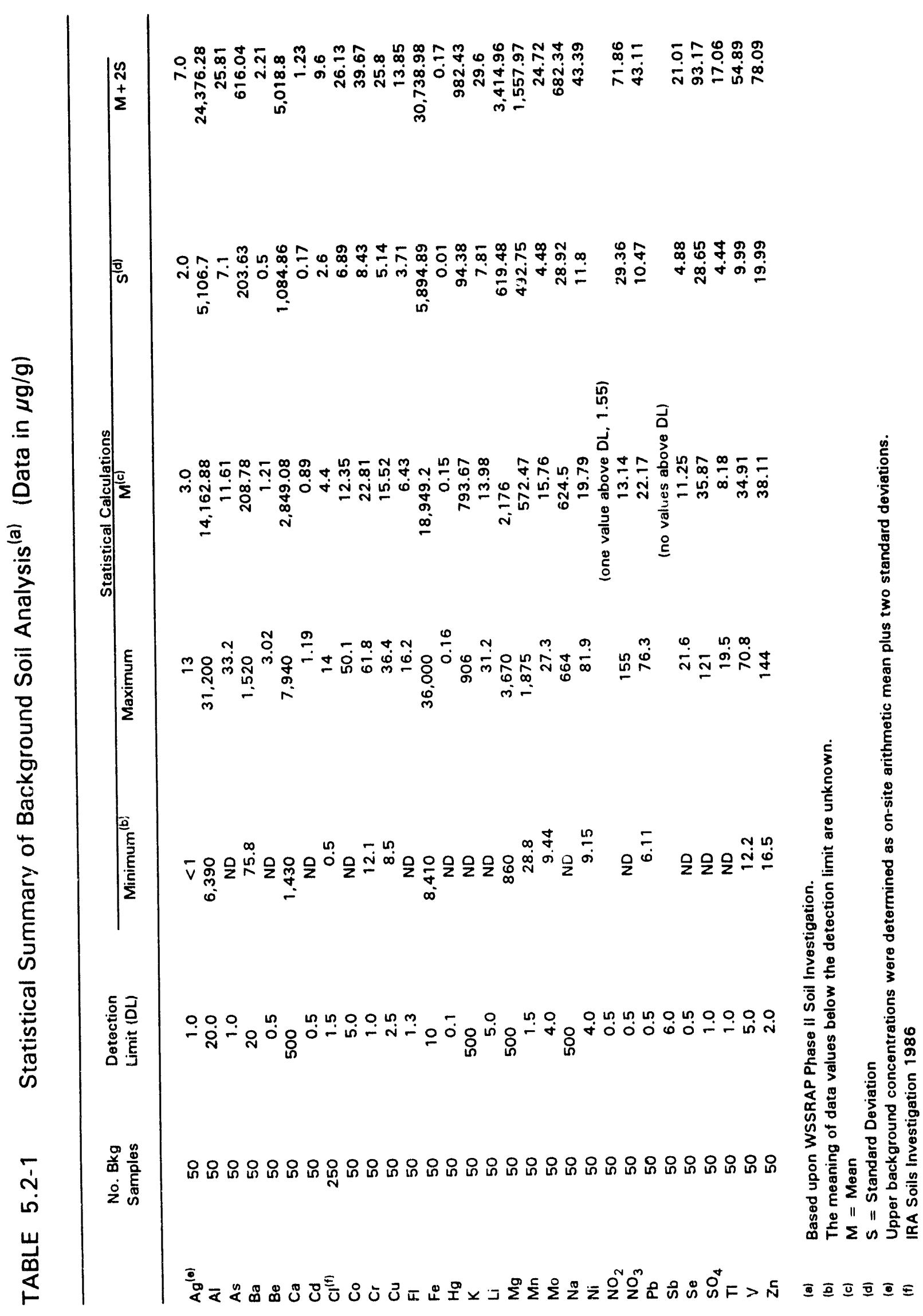




\section{TABLE 5.2-2 Soil Contaminant Concentrations Above Background}

\begin{tabular}{|c|c|c|c|c|}
\hline $\begin{array}{l}\text { Location }^{(a)} \\
\text { Number }\end{array}$ & Depth & Conc. & Units & Parameter \\
\hline 1 & $\begin{array}{l}0.0 .7 .0 \\
0.0 .7 .0\end{array}$ & $\begin{array}{l}200 \\
52.0\end{array}$ & $\begin{array}{l}\mu g / k g \\
\mu g / k g\end{array}$ & $\begin{array}{l}\text { Acetone } \\
\text { Methylene Chloride }\end{array}$ \\
\hline 2 & $\begin{array}{l}0.0,7.0 \\
0.0,7.0\end{array}$ & $\begin{array}{l}155 \\
39.0\end{array}$ & $\begin{array}{l}\mu \mathrm{g} / \mathrm{kg} \\
\mu \mathrm{g} / \mathrm{kg}\end{array}$ & $\begin{array}{l}\text { Acetone } \\
\text { Methylene Chloride }\end{array}$ \\
\hline 3 & $\begin{array}{l}0.0,7.0 \\
0.0,7.0 \\
0.0,7.0 \\
0.0,7.0 \\
0.0,7.0\end{array}$ & $\begin{array}{c}51.0 \\
0.13 \\
7.00 \\
3040 \\
99.1\end{array}$ & $\begin{array}{c}\mu g / k g \\
\mu g / g \\
\mu g / k g \\
\mu g / g \\
\mu g / g\end{array}$ & $\begin{array}{l}\text { Acetone } \\
\text { Mercury } \\
\text { Methylene Chloride } \\
\text { Potassium } \\
\text { Sulfate }\end{array}$ \\
\hline 4 & $\begin{array}{l}0.0,7.0 \\
0.0,7.0\end{array}$ & $\begin{array}{l}80.0 \\
39.0\end{array}$ & $\begin{array}{l}\mu g / k g \\
\mu g / k g\end{array}$ & $\begin{array}{l}\text { Acetone } \\
\text { Methylene Chloride }\end{array}$ \\
\hline 5 & $\begin{array}{l}0.0,7.0 \\
0.0,7.0 \\
0.0,7.0 \\
0.0,7.0 \\
8.0,15.0 \\
8.0,15.0\end{array}$ & $\begin{array}{r}47.0 \\
66.7 \\
2130 \\
13.0 \\
81.0 \\
18.0\end{array}$ & $\begin{array}{c}\mu g / k g \\
\mu g / g \\
\mu g / g \\
\mu g / k g \\
\mu g / k g \\
\mu g / k g\end{array}$ & $\begin{array}{l}\text { Acetone } \\
\text { Cobalt } \\
\text { Manganese } \\
\text { Methylene Chloride } \\
\text { Acetone } \\
\text { Methylene Chloride }\end{array}$ \\
\hline 6 & $\begin{array}{c}0.0,2.0 \\
6.0,8.0 \\
6.0,8.0 \\
6.0,8.0 \\
8.0,10.0 \\
10.0,12.0\end{array}$ & $\begin{array}{c}6180 \\
2.38 \\
25900 \\
4040 \\
1.88 \\
1.68\end{array}$ & $\begin{array}{l}\mu g / g \\
\mu g / g \\
\mu g / g \\
\mu g / g \\
\mu g / g \\
\mu g / g\end{array}$ & $\begin{array}{l}\text { Magnesium } \\
\text { 1,3,5-Trinitrobenzene } \\
\text { Aluminum } \\
\text { Magnesium } \\
\text { 1,3,5-Trinitrobenzene } \\
\text { 1,3,5-Trinitrobenzene }\end{array}$ \\
\hline 7 & $\begin{array}{l}0.0,2.0 \\
8.0,10.0\end{array}$ & $\begin{array}{r}3590 \\
12.3\end{array}$ & $\begin{array}{l}\mu g / g \\
\mu g / g\end{array}$ & $\begin{array}{l}\text { Magnesium } \\
\text { Chloride }\end{array}$ \\
\hline 8 & $\begin{array}{c}0.0,2.0 \\
2.0,4.0 \\
4.0,6.0 \\
6.0,8.0 \\
6.0,8.0 \\
8.0,10.0 \\
10.0,12.0 \\
12.0,14.0 \\
12.0,14.0 \\
12.0,14.0\end{array}$ & $\begin{array}{r}4350 \\
3700 \\
4080 \\
44600 \\
349 \\
162 \\
200 \\
143 \\
3820 \\
2290\end{array}$ & $\begin{array}{l}\mu g / g \\
\mu g / g \\
\mu g / g \\
\mu g / g \\
\mu g / g \\
\mu g / g \\
\mu g / g \\
\mu g / g \\
\mu g / g \\
\mu g / g\end{array}$ & $\begin{array}{l}\text { Magnesium } \\
\text { Magnesium } \\
\text { Magnesium } \\
\text { Iron } \\
\text { Sulfate } \\
\text { Sulfate } \\
\text { Sulfate } \\
\text { Lead } \\
\text { Magnesium } \\
\text { Manganese }\end{array}$ \\
\hline 9 & $\begin{array}{l}0.0,2.0 \\
0.0,2.0 \\
0.0,2.0 \\
2.0,4.0 \\
4.0,6.0 \\
4.0,6.0 \\
4.0,6.0 \\
6.0,8.0 \\
8.0,10.0 \\
8.0,10.0\end{array}$ & $\begin{array}{c}26.4 \\
5360 \\
1030 \\
29.6 \\
36.6 \\
300 \\
24.2 \\
30.1 \\
10.2 \\
1590\end{array}$ & $\begin{array}{l}\mu g / g \\
\mu g / g \\
\mu g / g \\
\mu g / g \\
\mu g / g \\
\mu g / g \\
\mu g / g \\
\mu g / g \\
\mu g / g \\
\mu g / g\end{array}$ & $\begin{array}{l}\text { Arsenic } \\
\text { Calcium } \\
\text { Potassium } \\
\text { Arsenic } \\
\text { Arsenic } \\
\text { Sulfate } \\
\text { Thallium } \\
\text { Arsenic } \\
\text { Chloride } \\
\text { Potassium }\end{array}$ \\
\hline
\end{tabular}


TABLE 5.2-2 Soil Contaminant Concentrations Above Background (Continued)

\begin{tabular}{|c|c|c|c|c|}
\hline $\begin{array}{l}\text { Location } \\
\text { Number }\end{array}$ & Depth & Conc. & Units & Parameter \\
\hline 10 & $\begin{array}{l}0.0,2.0 \\
0.0,2.0\end{array}$ & $\begin{array}{r}29300 \\
5970\end{array}$ & $\begin{array}{l}\mu g / g \\
\mu g / \theta\end{array}$ & $\begin{array}{l}\text { Aluminum } \\
\text { Magnesium }\end{array}$ \\
\hline 11 & $\begin{array}{l}0.0,2.0 \\
2.0,4.0\end{array}$ & $\begin{array}{l}4440 \\
3490\end{array}$ & $\begin{array}{l}\mu g / g \\
\mu g / g\end{array}$ & $\begin{array}{l}\text { Magnesium } \\
\text { Magnesium }\end{array}$ \\
\hline 12 & $\begin{array}{c}2.0,4.0 \\
6.0,8.0 \\
8.0,10.0 \\
10.0,12.0 \\
12.0,14.0\end{array}$ & $\begin{array}{r}3480 \\
156 \\
3620 \\
3940 \\
3810\end{array}$ & $\begin{array}{l}\mu g / g \\
\mu g / g \\
\mu g / g \\
\mu g / g \\
\mu g / g\end{array}$ & $\begin{array}{l}\text { Magnesium } \\
\text { Sulfate } \\
\text { Plagnesium } \\
\text { Magnesium } \\
\text { Magnesium }\end{array}$ \\
\hline 13 & $\begin{array}{l}0.0,7.0 \\
0.0,7.0 \\
0.0 .7 .0 \\
0.0 .7 .0 \\
0.0,7.0\end{array}$ & $\begin{array}{c}15000 \\
670 \\
2700 \\
8.00 \\
235\end{array}$ & $\begin{array}{c}\mu g / k g \\
\mu g / k g \\
\mu g / k g \\
\mu g / k g \\
\mu g / g\end{array}$ & $\begin{array}{l}\text { Bis (2-Ethylhexyl) Phthalate } \\
\text { Butylbenzylphthalate } \\
\text { Di-N-Butyl Phthalate } \\
\text { Methylene Chloride } \\
\text { Sulfate }\end{array}$ \\
\hline 14 & $\begin{array}{l}0.0,7.0 \\
0.0,7.0 \\
8.0,15.0 \\
8.0,15.0 \\
8.0,15.0 \\
8.0,15.0 \\
8.0,15.0 \\
8.0,15.0\end{array}$ & $\begin{array}{c}49.0 \\
25.0 \\
142 \\
4.73 \\
39.1 \\
58600 \\
2360 \\
50.0\end{array}$ & $\begin{array}{l}\mu g / k g \\
\mu g / k g \\
\mu g / k g \\
\mu g / g \\
\mu g / g \\
\mu g / g \\
\mu g / g \\
\mu g / k g\end{array}$ & $\begin{array}{l}\text { Acetone } \\
\text { Methylene Chloride } \\
\text { Acetone } \\
\text { Beryllium } \\
\text { Cobalt } \\
\text { Iron } \\
\text { Manganese } \\
\text { Methylene Chloride }\end{array}$ \\
\hline 15 & $\begin{array}{l}0.0,7.0 \\
0.0,7.0\end{array}$ & $\begin{array}{r}27.1 \\
3180\end{array}$ & $\begin{array}{l}\mu g / g \\
\mu g / g\end{array}$ & $\begin{array}{l}\text { Cobalt } \\
\text { Manganese }\end{array}$ \\
\hline 16 & $\begin{array}{l}0.0,7.0 \\
0.0,7.0 \\
0.0,7.0 \\
0.0,7.0 \\
0.0,7.0 \\
0.0,7.0 \\
0.0,7.0 \\
8.0,15.0 \\
8.0,15.0 \\
8.0,15.0 \\
8.0,15.0 \\
8.0,15.0\end{array}$ & $\begin{array}{c}45.0 \\
1100 \\
6750 \\
3720 \\
12.0 \\
1350 \\
128 \\
55.0 \\
1300 \\
9.00 \\
25.6 \\
25.0\end{array}$ & $\begin{array}{c}\mu g / k g \\
\mu g / k g \\
\mu g / g \\
\mu g / g \\
\mu g / k g \\
\mu g / g \\
\mu g / k g \\
\mu g / k g \\
\mu g / k g \\
\mu g / k g \\
\mu g / g \\
\mu g / k g\end{array}$ & $\begin{array}{l}\text { Acetone } \\
\text { Bis (2-Ethylhexyl) Phthalate } \\
\text { Calcium } \\
\text { Megnesium } \\
\text { Methylene Chloride } \\
\text { Potassium } \\
\text { Toluene } \\
\text { Acetone } \\
\text { Bis (2-Ethylhexyl) Phthalate } \\
\text { Methylene Chloride } \\
\text { Molybdenum } \\
\text { Toluene }\end{array}$ \\
\hline 17 & $\begin{array}{l}0.0,2.0 \\
0.0,2.0 \\
0.0,2.0 \\
0.0,2.0 \\
2.0,4.0 \\
2.0,4.0 \\
2.0,4.0 \\
2.0,4.0\end{array}$ & $\begin{array}{c}35.6 \\
5775 \\
3742 \\
1130 \\
34.1 \\
3520 \\
1420 \\
24.3\end{array}$ & $\begin{array}{l}\mu g / g \\
\mu g / g \\
\mu g / g \\
\mu g / g \\
\mu g / g \\
\mu g / g \\
\mu g / g \\
\mu g / g\end{array}$ & $\begin{array}{l}\text { Arsenic } \\
\text { Calcium } \\
\text { Magnesium } \\
\text { Potassium } \\
\text { Arsenic } \\
\text { Magnesium } \\
\text { Potassium } \\
\text { Seleni.um }\end{array}$ \\
\hline
\end{tabular}


TABLE 5.2-2 Soil Contaminant Concentrations Above Background (Continued)

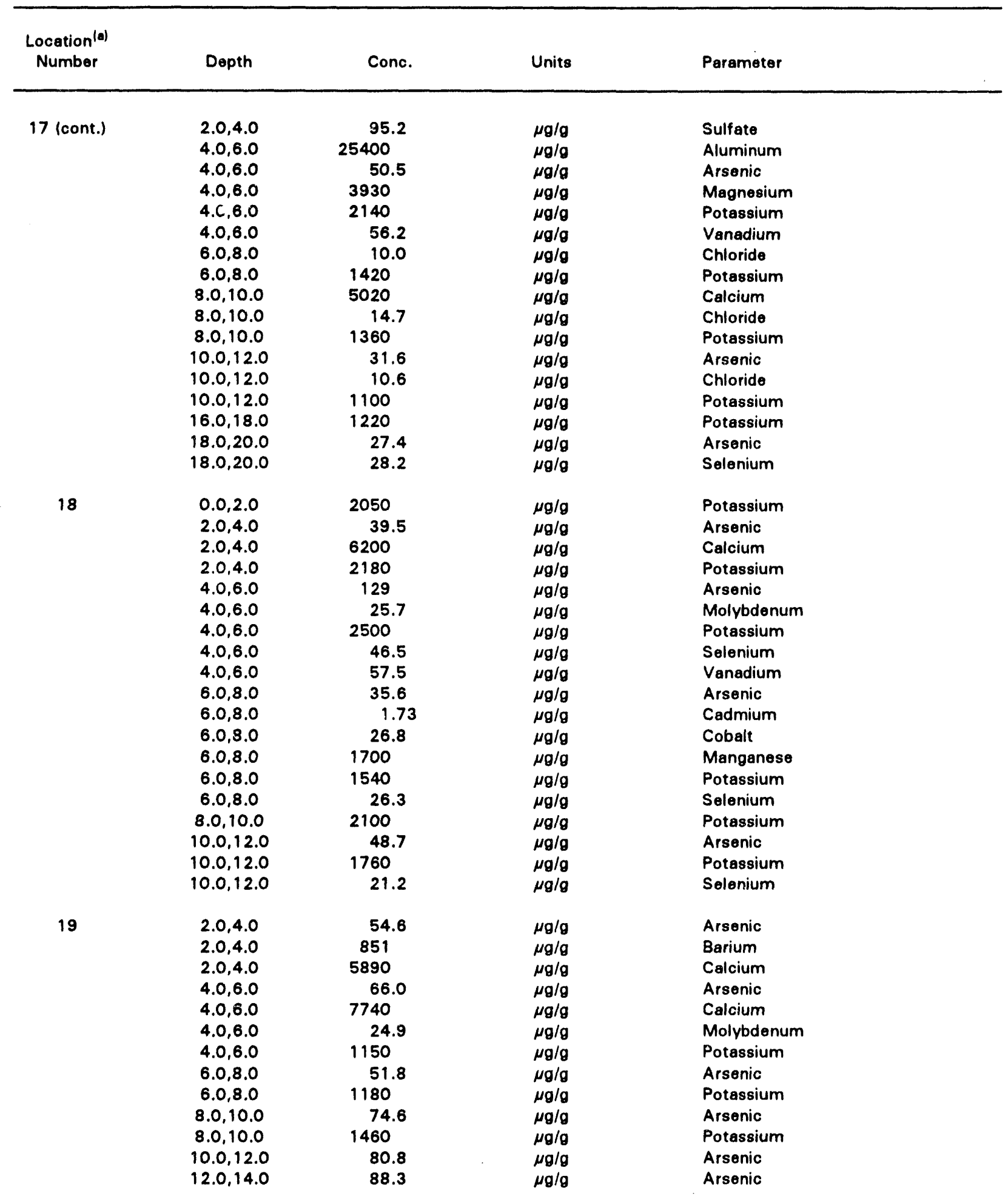


TABLE 5.2-2 Soil Contaminant Concentrations Above Background (Continued)

\begin{tabular}{|c|c|c|c|c|}
\hline $\begin{array}{l}\text { Location }^{(a)} \\
\text { Number }\end{array}$ & Depth & Conc. & Units & Parameter \\
\hline 19 (cont.) & $\begin{array}{l}12.0,14.0 \\
12.0,14.0 \\
12.0,14.0 \\
12.0,14.0 \\
12.0,14.0 \\
14.0,16.0 \\
14.0,16.0 \\
16.0,18.0 \\
18.0,20.0 \\
18.0,20.0 \\
18.0,20.0 \\
18.0,20.0 \\
18.0,20.0 \\
18.0,20.0 \\
20.0,22.0 \\
20.0,22.0\end{array}$ & $\begin{array}{c}38.1 \\
37100 \\
60.1 \\
6210 \\
79.8 \\
25800 \\
59.3 \\
53.8 \\
99.9 \\
39.8 \\
30.4 \\
47700 \\
87.4 \\
121 \\
59.17 \\
12300\end{array}$ & $\begin{array}{l}\mu g / g \\
\mu g / g \\
\mu g / g \\
\mu g / g \\
\mu g / g \\
\mu g / g \\
\mu g / g \\
\mu g / g \\
\mu g / g \\
\mu g / g \\
\mu g / g \\
\mu g / g \\
\mu g / g \\
\mu g / g \\
\mu g / g \\
\mu g / g\end{array}$ & $\begin{array}{l}\text { Cobalt } \\
\text { Iron } \\
\text { Lead } \\
\text { Manganese } \\
\text { Vanadium } \\
\text { Aluminum } \\
\text { Arsenic } \\
\text { Lithium } \\
\text { Arsenic } \\
\text { Chromium } \\
\text { Cobalt } \\
\text { Iron } \\
\text { Lead } \\
\text { Vanadium } \\
\text { Arsenic } \\
\text { Potassium }\end{array}$ \\
\hline 20 & $\begin{array}{l}0.0,7.0 \\
0.0,7.0\end{array}$ & $1250^{6.00}$ & $\begin{array}{c}\mu g / k g \\
\mu g / g\end{array}$ & $\begin{array}{l}\text { Methylene Chloride } \\
\text { Potassium }\end{array}$ \\
\hline 21 & $\begin{array}{l}0.0,2.0 \\
0.0,2.0 \\
0.0,2.0 \\
0.0,2.0 \\
2.5,3.0 \\
2.5,3.0 \\
2.5,3.0 \\
2.5,3.0 \\
4.5,5.0 \\
4.5,5.0\end{array}$ & $\begin{array}{c}15.0 \\
1300 \\
18.0 \\
184 \\
21.0 \\
10.0 \\
900 \\
13.0 \\
1600 \\
9.00\end{array}$ & $\begin{array}{l}\mu \mathrm{g} / \mathrm{kg} \\
\mu \mathrm{g} / \mathrm{kg} \\
\mu \mathrm{g} / \mathrm{kg} \\
\mu \mathrm{g} / \mathrm{g} \\
\mu \mathrm{g} / \mathrm{kg} \\
\mu \mathrm{g} / \mathrm{kg} \\
\mu \mathrm{g} / \mathrm{kg} \\
\mu \mathrm{g} / \mathrm{kg} \\
\mu \mathrm{g} / \mathrm{kg} \\
\mu \mathrm{g} / \mathrm{kg}\end{array}$ & $\begin{array}{l}\text { Acetone } \\
\text { Bis (2-Ethylhexyl) Phthalate } \\
\text { Methylene Chloride } \\
\text { Sulfate } \\
\text { Acetone } \\
\text { Beta-BHC } \\
\text { Bis (2-Ethylhexyl) Phthalate } \\
\text { Methylene Chloride } \\
\text { Bis (2-Ethylhexyl) Phthalate } \\
\text { Methylene Chloride }\end{array}$ \\
\hline 22 & $\begin{array}{l}0.0,7.0 \\
0.0,7.0 \\
0.0,7.0 \\
0.0,7.0 \\
0.0,7.0\end{array}$ & $\begin{array}{c}530 \\
11900 \\
3700 \\
8.00 \\
1350\end{array}$ & $\begin{array}{c}\mu \mathrm{g} / \mathrm{kg} \\
\mu \mathrm{g} / \mathrm{g} \\
\mu \mathrm{g} / \mathrm{g} \\
\mu \mathrm{g} / \mathrm{kg} \\
\mu \mathrm{g} / \mathrm{g}\end{array}$ & $\begin{array}{l}\text { Bis (2-Ethylhexyl) Phthalate } \\
\text { Calcium } \\
\text { Magnesium } \\
\text { Methylene Chloride } \\
\text { Potassium }\end{array}$ \\
\hline 23 & $\begin{array}{l}0.0,0.5 \\
0.0,0.5 \\
0.0,0.5 \\
2.0,2.5 \\
2.0,2.5 \\
4.5,5.0\end{array}$ & $\begin{array}{c}870 \\
0.15 \\
19.0 \\
3300 \\
1170 \\
560\end{array}$ & $\begin{array}{c}\mu g / k g \\
\mu g / g \\
\mu g / k g \\
\mu g / k g \\
\mu g / g \\
\mu g / k g\end{array}$ & $\begin{array}{l}\text { Bis (2-Ethylhexyl) Phthalate } \\
\text { Mercury } \\
\text { Methylene Chloride } \\
\text { Bis (2-Ethylhexyl) Phthalate } \\
\text { Potassium } \\
\text { Bis (2-Ethylhexyl) Phthalate }\end{array}$ \\
\hline 24 & $\begin{array}{l}0.0,1.5 \\
0.0,1.5\end{array}$ & $\begin{array}{l}560 \\
0.23\end{array}$ & $\begin{array}{c}\mu \mathrm{g} / \mathrm{kg} \\
\mu \mathrm{g} / \mathrm{g}\end{array}$ & $\begin{array}{l}\text { Aroclor-1254 } \\
\text { Mercury }\end{array}$ \\
\hline 25 & $\begin{array}{l}0.0,7.0 \\
0.0,7.0 \\
0.0,7.0 \\
0.0,7.0\end{array}$ & $\begin{array}{c}590 \\
7860 \\
12.9 \\
1060\end{array}$ & $\begin{array}{l}\mu g / k g \\
\mu g / g \\
\mu g / g \\
\mu g / g\end{array}$ & $\begin{array}{l}\text { Bis (2-Ethylhexyl) Phthalate } \\
\text { Calcium } \\
\text { Chloride } \\
\text { Potassium }\end{array}$ \\
\hline
\end{tabular}


TABLE 5.2-2 Soil Contaminant Concentrations Above Background (Continued)

\begin{tabular}{|c|c|c|c|c|}
\hline $\begin{array}{l}\text { Location }{ }^{(a)} \\
\text { Number }\end{array}$ & Depth & Conc. & Units & Parameter \\
\hline 25 (cont.) & $\begin{array}{l}0.0,7.0 \\
8.0,15.0 \\
8.0,15.0 \\
8.0,15.0\end{array}$ & $\begin{array}{c}11.0 \\
15.0 \\
2000 \\
6.00\end{array}$ & $\begin{array}{l}\mu g / k g \\
\mu g / k g \\
\mu g / k g \\
\mu g / k g\end{array}$ & $\begin{array}{l}\text { Toluene } \\
\text { Acetone } \\
\text { Bis (2-Ethylhexyl) Phthalate } \\
\text { Methylene Chloride }\end{array}$ \\
\hline 26 & $\begin{array}{l}2.0,4.0 \\
2.0,4.0 \\
2.0,4.0 \\
2.0,4.0 \\
4.0,6.0 \\
4.0,6.0 \\
6.0,8.0 \\
8.0,10.0\end{array}$ & $\begin{array}{r}440 \\
7940 \\
3670 \\
1150 \\
2600 \\
1940 \\
1640 \\
1740\end{array}$ & $\begin{array}{c}\mu g / k g \\
\mu g / g \\
\mu g / g \\
\mu g / g \\
\mu g / k g \\
\mu g / g \\
\mu g / g \\
\mu g / g\end{array}$ & $\begin{array}{l}\text { Bis (2-Ethylhexyl) Phthalate } \\
\text { Calcium } \\
\text { Magnesium } \\
\text { Potassium } \\
\text { Bis (2-Ethylhexyl) Phthalate } \\
\text { Potassium } \\
\text { Potassium } \\
\text { Potassium }\end{array}$ \\
\hline 27 & $\begin{array}{l}0.0,7.0 \\
0.0,7.0 \\
0.0,7.0 \\
0.0,7.0\end{array}$ & $\begin{array}{c}24.0 \\
1100 \\
16300 \\
18.0\end{array}$ & $\begin{array}{c}\mu \mathrm{g} / \mathrm{kg} \\
\mu \mathrm{g} / \mathrm{kg} \\
\mu \mathrm{g} / \mathrm{g} \\
\mu \mathrm{g} / \mathrm{kg}\end{array}$ & $\begin{array}{l}\text { Acetone } \\
\text { Bis (2-Ethylhexyl) Phthalate } \\
\text { Calcium } \\
\text { Methylene Chloride }\end{array}$ \\
\hline 28 & $\begin{array}{l}0.0,0.5 \\
0.0,0.5 \\
0.0,0.5 \\
0.0,0.5 \\
0.0,0.5 \\
0.0,0.5 \\
2.0,2.5 \\
2.0,2.5 \\
2.0,2.5 \\
2.0,2.5\end{array}$ & $\begin{array}{c}17.0 \\
2400 \\
16000 \\
5270 \\
39.0 \\
1050 \\
16.0 \\
267 \\
1700 \\
15.0\end{array}$ & $\begin{array}{c}\mu g / k g \\
\mu g / k g \\
\mu g / g \\
\mu g / g \\
\mu g / k g \\
\mu g / g \\
\mu g / k g \\
\mu g / k g \\
\mu g / k g \\
\mu g / k g\end{array}$ & $\begin{array}{l}\text { Acetone } \\
\text { Bis (2-Ethylhexyl) Phthalate } \\
\text { Calcium } \\
\text { Magnesium } \\
\text { Methylene Chloride } \\
\text { Potassium } \\
\text { Acetone } \\
\text { Beta-BHC } \\
\text { Bis (2-Ethylhexyl) Phthalate } \\
\text { Methylene Chloride }\end{array}$ \\
\hline 29 & $\begin{array}{l}3.0,7.0 \\
3.0,7.0 \\
3.0,7.0 \\
3.0,7.0 \\
8.0,15.0 \\
8.0,15.0 \\
8.0,15.0 \\
8.0,15.0\end{array}$ & $\begin{array}{c}1400 \\
3530 \\
67.0 \\
1520 \\
1700 \\
13.0 \\
45.0 \\
1300\end{array}$ & $\begin{array}{c}\mu g / k g \\
\mu g / g \\
\mu g / k g \\
\mu g / g \\
\mu g / k g \\
\mu g / g \\
\mu g / k g \\
\mu g / g\end{array}$ & $\begin{array}{l}\text { Bis (2-Ethylhexyl) Phthalate } \\
\text { Magnesium } \\
\text { Methylene Chloride } \\
\text { Potassium } \\
\text { Bis (2-Ethylhexyl) Phthalate } \\
\text { Chloride } \\
\text { Methylene Chloride } \\
\text { Potassium }\end{array}$ \\
\hline 30 & $\begin{array}{l}0.0,0.5 \\
0.0,0.5 \\
0.0,0.5 \\
0.0,0.5 \\
0.0,0.5 \\
0.0,0.5 \\
0.0,0.5 \\
0.0,0.5 \\
0.0,0.5 \\
0.0,0.5 \\
0.0,0.5 \\
0.0,0.5\end{array}$ & $\begin{array}{r}1900 \\
3400 \\
8200 \\
5100 \\
4600 \\
2100 \\
3900 \\
400 \\
6490 \\
8000 \\
820 \\
11000\end{array}$ & $\begin{array}{l}\mu g / k g \\
\mu g / k g \\
\mu g / k g \\
\mu g / k g \\
\mu g / k g \\
\mu g / k g \\
\mu g / k g \\
\mu g / k g \\
\mu g / g \\
\mu g / k g \\
\mu g / k g \\
\mu g / k g\end{array}$ & $\begin{array}{l}\text { Acenaphthene } \\
\text { Anthrecene } \\
\text { Benzo(A)Anthracene } \\
\text { Benzo(A)Pyrene } \\
\text { Benzo(B)Fluoranihene } \\
\text { Benzo(G,H,I)Perylene } \\
\text { Benzo(K)Fluoranthene } \\
\text { Bis (2-Ethylhexyl; Phthalate } \\
\text { Calcium } \\
\text { Chrysene } \\
\text { Dibenzofuran } \\
\text { Fluoranthene }\end{array}$ \\
\hline
\end{tabular}


TABLE 5.2-2 Soil Contaminant Concentrations Above Background (Continued)

\begin{tabular}{|c|c|c|c|c|}
\hline $\begin{array}{l}\text { Location } \\
\text { Number }\end{array}$ & Depth & Conc. & Units & Parameter \\
\hline \multirow[t]{13}{*}{30 (cont.) } & $0.0,0.5$ & 1600 & $\mu \mathrm{g} / \mathbf{k g}$ & Fluorene \\
\hline & $0.0,0.5$ & 3200 & $\mu g / k g$ & Indeno(1,2,3-CD)Pyrene \\
\hline & $0.0,0.5$ & 40.0 & $\mu g / k g$ & Methylene Chloride \\
\hline & $0.0,0.5$ & 1015 & $\mu \mathrm{g} / \mathrm{g}$ & Potassium \\
\hline & $0.0,0.5$ & 19400 & $\mu \mathrm{g} / \mathrm{kg}$ & Pyrene \\
\hline & $2.0,2.5$ & 530 & $\mu \mathrm{g} / \mathrm{kg}$ & Bis (2-Ethylhexyl) Phthalate \\
\hline & $2.0,2.5$ & 48.0 & $\mu \mathrm{g} / \mathrm{kg}$ & Methvlene Chloride \\
\hline & $4.5,5.0$ & 490 & $\mu g / k g$ & Bis (2-Ethylhexyl) Phthalate \\
\hline & $4.5,5.0$ & 5280 & $\mu g / \theta$ & Calcium \\
\hline & $4.5,5.0$ & 2210 & $\mu g / g$ & Manganese \\
\hline & $4.5,5.0$ & 21.0 & $\mu \mathrm{g} / \mathrm{kg}$ & Methylene Chloride \\
\hline & $4.5,5.0$ & 28.2 & $\mu g / \theta$ & Molybdenum \\
\hline & $4.5,5.0$ & 1060 & $\mu g / g$ & Potassium \\
\hline \multirow[t]{18}{*}{31} & $0.0,0.5$ & 23.0 & $\mu \mathrm{g} / \mathrm{kg}$ & Acetone \\
\hline & $0.0,0.5$ & 1300 & $\mu \mathrm{g} / \mathrm{kg}$ & Bis (2-Ethylhexyl) Phthalate \\
\hline & $0.0,0.5$ & 1.39 & $\mu g / g$ & Cadmium \\
\hline & $0.0,0.5$ & 10300 & $\mu g / g$ & Calcium \\
\hline & $0.0,0.5$ & 1.05 & $\mu g / g$ & Morcury \\
\hline & $0.0,0.5$ & 86.0 & $\mu g / k g$ & Methylene Chloride \\
\hline & $0.0,0.5$ & 997 & $\mu g / \theta$ & Potassium \\
\hline & $2.0,2.5$ & 700 & $\mu \mathrm{g} / \mathrm{kg}$ & Bis (2-Ethylhexyl) Phthalate \\
\hline & $2.0,2.5$ & 0.14 & $\mu g / g$ & Mercury \\
\hline & $2.0,2.5$ & 1060 & $\mu g / g$ & Potassium \\
\hline & $4.5,5.0$ & 2400 & $\mu \mathrm{g} / \mathrm{kg}$ & Bis (2-Ethylhexyl) Phthalate \\
\hline & $4.5,5.0$ & 1.69 & $\mu g / g$ & Cadmium \\
\hline & $4.5,5.0$ & 8930 & $\mu g / g$ & Calcium \\
\hline & $4.5,5.0$ & 30.3 & $\mu g / g$ & Copper \\
\hline & $4.5,5.0$ & 37200 & $\mu_{g} / g$ & Iron \\
\hline & $4.5,5.0$ & 4310 & $\mu g / g$ & Magnesium \\
\hline & $4.5,5.0$ & 59.0 & $\mu \mathrm{g} / \mathrm{kg}$ & Methylene Chloride \\
\hline & $4.5,5.0$ & 1200 & $\mu g / g$ & Potassium \\
\hline \multirow[t]{17}{*}{32} & $0.0,1.0$ & 62.1 & $\mu g / g$ & Arsenic \\
\hline & 0.0 .1 .0 & 7470 & $\mu \mathrm{g} / \mathrm{g}$ & Calcium \\
\hline & $0.0,1.0$ & 32.2 & $\mu g / g$ & Molybdenum \\
\hline & $0.0,1.0$ & 1140 & $\mu g / g$ & Potassium \\
\hline & $0.0,1.0$ & 119 & $\mu \mathrm{g} / \mathrm{g}$ & Sulfate \\
\hline & $0.0,1.0$ & 70.6 & $\mu g / g$ & Vanadium \\
\hline & $2.0,2.5$ & 19500 & $\mu g / g$ & Calcium \\
\hline & $2.0,2.5$ & 42.9 & $\mu g / g$ & Chloride \\
\hline & $2.0,2.5$ & 46.3 & $\mu g / g$ & Chromium \\
\hline & $2.0,2.5$ & 290 & $\mu g / g$ & Sulfate \\
\hline & $4.5,5.0$ & 5.09 & $\mu g / g$ & Beryllium \\
\hline & $4.5,5.0$ & 14600 & $\mu \mathrm{g} / \mathrm{g}$ & Calcium \\
\hline & $4.5,5.0$ & 14.5 & $\mu \mathrm{g} / \mathrm{g}$ & Chloride \\
\hline & $4.5,5.0$ & 14700 & $\mu \mathrm{g} / \mathrm{g}$ & Magnesium \\
\hline & $4.5,5.0$ & 2280 & $\mu g / g$ & Manganese \\
\hline & $4.5,5.0$ & 61.1 & $\mu g / g$ & Nickol \\
\hline & $4.5,5.0$ & 1400 & $\mu g / g$ & Potassium \\
\hline
\end{tabular}


TABLE 5.2-2 Soil Contaminant Concentrations Above Background (Continued)

\begin{tabular}{|c|c|c|c|c|}
\hline $\begin{array}{l}\text { Location(a) } \\
\text { Number }\end{array}$ & Depth & Conc. & Units & Paramoter \\
\hline \multirow[t]{6}{*}{33} & $0.0,1.0$ & 324 & $\mu g / \mathbf{k g}$ & Aroclor-1248 \\
\hline & $0.0,1.0$ & 625 & $\mu \mathrm{g} / \mathrm{kg}$ & Aroclor-1254 \\
\hline & $0.0,1.0$ & 354 & $\mu \mathrm{g} / \mathrm{kg}$ & Pyrone \\
\hline & $2.0,2.5$ & 51300 & $\mu g / g$ & Calcium \\
\hline & $2.0,2.5$ & 4190 & $\mu g / g$ & Magnesium \\
\hline & $4.5,5.0$ & 10.0 & $\mu g / k g$ & Methylene Chloride \\
\hline \multirow[t]{11}{*}{34} & $0.0,0.5$ & 20.0 & $\mu g / k g$ & Acetone \\
\hline & $0.0,0.5$ & 1200 & $\mu g / k g$ & Bis (2-Ethylhexy) Phthalato \\
\hline & $0.0,0.5$ & 630 & $\mu \mathrm{g} / \mathbf{k g}$ & Di-N-Butyl Phthalato \\
\hline & $0.0,0.5$ & 28.0 & $\mu g / k g$ & Methylene Chloride \\
\hline & $2.0,4.0$ & 15.0 & $\mu g / k g$ & Acotone \\
\hline & $2.0,4.0$ & 3000 & $\mu \mathrm{g} / \mathbf{k g}$ & Bis (2-Ethylhexyl) Phthalate \\
\hline & $2.0,4.0$ & 93.0 & $\mu \mathrm{g} / \mathrm{kg}$ & Methvlene Chloride \\
\hline & $2.0,4.0$ & 111 & $\mu g / g$ & Sulfate \\
\hline & $4.5,5.0$ & 18.0 & $\mu \mathrm{g} / \mathrm{kg}$ & Acotone \\
\hline & $4.5,5.0$ & 790 & $\mu \mathrm{g} / \mathrm{kg}$ & Bis (2-Ethylhexyl) Phthalate \\
\hline & $4.5,5.0$ & 55.0 & $\mu \mathrm{g} / \mathbf{k g}$ & Methylene Chloride \\
\hline \multirow[t]{4}{*}{35} & $0.0,2.0$ & 930 & $\mu g / k_{g}$ & Aroclor-1254 \\
\hline & $4.0,6.0$ & 9.00 & $\mu \mathrm{g} / \mathrm{kg}$ & Methylene Chloride \\
\hline & $6.0,8.0$ & 8.00 & $\mu g / k g$ & Methylene Chloride \\
\hline & $8.0,10.0$ & 7.00 & $\mu g / k g$ & Methylene Chloride \\
\hline \multirow[t]{12}{*}{36} & $0.0,0.5$ & 690 & $\mu \mathbf{g} / \mathbf{k g}$ & Aroclor-1254 \\
\hline & $0.0,0.5$ & 890 & $\mu \mathrm{g} / \mathrm{kg}$ & Bis (2-Ethylhexyl) Phthalate \\
\hline & $0.0,0.5$ & 23900 & $\mu g / g$ & Calcium \\
\hline & $0.0,0.5$ & 4030 & $\mu g / g$ & Magnesium \\
\hline & $0.0,0.5$ & 1210 & $\mu g / g$ & Potassium \\
\hline & $2.0,2.5$ & 31.0 & $\mu g / k g$ & Endosulfan I \\
\hline & $2.0,2.5$ & 3480 & $\mu \mathrm{g} / \mathrm{g}$ & Magnesium \\
\hline & 2.0 .2 .5 & 27.0 & $\mu g / g$ & Molybdenum \\
\hline & $2.0,2.5$ & 1080 & $\mu g / g$ & Potassium \\
\hline & $4.5,5.0$ & 560 & $\mu g / k_{g}$ & Bis (2-Ethylhexyl) Phthaiate \\
\hline & $4.5,5.0$ & 62800 & $\mu g / g$ & Calcium \\
\hline & $4.5,5.0$ & 17800 & $\mu g / g$ & Magnesium \\
\hline \multirow[t]{5}{*}{37} & $0.0,1.0$ & 1100 & $\mu \mathrm{g} / \mathbf{k g}$ & Bis (2-Ethylhexyl) Phthalate \\
\hline & $0.0,1.0$ & 157 & $\mu \mathrm{g} / \mathrm{kg}$ & Toluene \\
\hline & $2.0,2.5$ & 14000 & $\mu \mathrm{g} / \mathrm{kg}$ & Bis (2-Ethylhexyl) Phthalate \\
\hline & $2.0,2.5$ & 14.0 & $\mu \mathrm{g} / \mathrm{kg}$ & Methylene Chloride \\
\hline & $3.0,5.0$ & 1100 & $\mu \mathrm{g} / \mathrm{kg}$ & Bis (2-Ethylhexyl) Phthalate \\
\hline 38 & $0.0,5.0$ & 7400 & $\mu g / k_{g}$ & Aroclor- 1260 \\
\hline \multirow[t]{2}{*}{39} & $0.0,7.0$ & 31600 & $\mu g / g$ & Calcium \\
\hline & $8.0,15.0$ & 18.3 & $\mu g / \theta$ & Fluoride \\
\hline \multirow[t]{3}{*}{40} & $0.0,7.0$ & 17.0 & $\mu \mathrm{g} / \mathrm{kg}$ & Acetone \\
\hline & $0.0,7.0$ & 25600 & $\mu \mathrm{g} / \mathrm{g}$ & Aluminum \\
\hline & $0.0,7.0$ & 820 & $\mu \mathrm{g} / \mathrm{kg}$ & Bis (2-Ethylhexyl) Phthalate \\
\hline
\end{tabular}




\section{TABLE 5.2-2 Soil Contaminant Concentrations Above Background (Continued)}

\begin{tabular}{|c|c|c|c|c|}
\hline $\begin{array}{l}\text { Location } \\
\text { Number }\end{array}$ & Depth & Conc. & Units & Parameter \\
\hline 40 (cont.) & $\begin{array}{l}0.0,7.0 \\
0.0,7.0 \\
0.0,7.0 \\
0.0,7.0 \\
0.0,7.0 \\
0.0,7.0 \\
0.0,7.0 \\
8.0,15.0 \\
8.0,15.0 \\
16.0,17.0\end{array}$ & $\begin{array}{c}16100 \\
4990 \\
19.0 \\
27.7 \\
1060 \\
121 \\
122 \\
3900 \\
16.0 \\
32.7\end{array}$ & $\begin{array}{c}\mu g / g \\
\mu g / g \\
\mu g / k g \\
\mu g / g \\
\mu g / g \\
\mu g / g \\
\mu g / g \\
\mu g / k g \\
\mu g / k g \\
\mu g / g\end{array}$ & $\begin{array}{l}\text { Calcium } \\
\text { Magnesium } \\
\text { Methylene Chloride } \\
\text { Molybdenum } \\
\text { Potassium } \\
\text { Sulfate } \\
\text { Zinc } \\
\text { Bis (2-Ethylhexyl) Phthalate } \\
\text { Methylene Chloride } \\
\text { Chloride }\end{array}$ \\
\hline 41 & $\begin{array}{l}0.0,0.7 \\
0.0,0.7 \\
0.0,0.7 \\
0.0,0.7 \\
0.0,0.7 \\
0.0,0.7 \\
0.0,0.7 \\
8.0,15.0 \\
8.0,15.0 \\
8.0,15.0 \\
8.0,15.0 \\
8.0,15.0\end{array}$ & $\begin{array}{c}43.0 \\
9300 \\
11900 \\
40.1 \\
4240 \\
8.00 \\
1710 \\
52.0 \\
25060 \\
1500 \\
24.0 \\
30.3\end{array}$ & $\begin{array}{c}\mu g / k g \\
\mu g / k g \\
\mu g / g \\
\mu g / g \\
\mu g / g \\
\mu g / k g \\
\mu g / g \\
\mu g / k g \\
\mu g / g \\
\mu g / k g \\
\mu g / k g \\
\mu g / g\end{array}$ & $\begin{array}{l}\text { Acetone } \\
\text { Bis (2-Ethylhexyl) Phthalate } \\
\text { Calcium } \\
\text { Chromium } \\
\text { Magnesium } \\
\text { Methylene Chloride } \\
\text { Potessium } \\
\text { Acetone } \\
\text { Aluminum } \\
\text { Bis (2-Ethylhexyl) Phthalate } \\
\text { Methylene Chloride } \\
\text { Molybdenum }\end{array}$ \\
\hline 42 & $\begin{array}{l}0.0,7.0 \\
0.0,7.0 \\
0.0,7.0 \\
8.0,15.0 \\
8.0,15.0\end{array}$ & $\begin{array}{c}930 \\
46.0 \\
133 \\
9900 \\
19.0\end{array}$ & $\begin{array}{c}\mu \mathrm{g} / \mathrm{kg} \\
\mu \mathrm{g} / \mathrm{kg} \\
\mu \mathrm{g} / \mathrm{g} \\
\mu \mathrm{g} / \mathrm{kg} \\
\mu \mathrm{g} / \mathrm{kg}\end{array}$ & $\begin{array}{l}\text { Bis (2-Ethylhexyl) Phthalate } \\
\text { Methylene Chloride } \\
\text { Sulfate } \\
\text { Bis (2-Ethylhexyl) Phthalate } \\
\text { Methylene Chloride }\end{array}$ \\
\hline 43 & $\begin{array}{l}0.0,7.0 \\
0.0,7.0 \\
0.0,7.0\end{array}$ & $\begin{array}{r}460 \\
15900 \\
1830\end{array}$ & $\begin{array}{c}\mu \mathrm{g} / \mathrm{kg} \\
\mu \mathrm{g} / \mathrm{g} \\
\mu \mathrm{g} / \mathrm{g}\end{array}$ & $\begin{array}{l}\text { Bis (2-Ethylhexyl) Phthalate } \\
\text { Calcium } \\
\text { Sulfate }\end{array}$ \\
\hline 44 & $\begin{array}{l}0.0,7.0 \\
0.0,7.0 \\
0.0,7.0 \\
0.0,7.0\end{array}$ & $\begin{array}{l}19.0 \\
360 \\
9150 \\
15.0\end{array}$ & $\begin{array}{c}\mu g / k g \\
\mu g / k g \\
\mu g / g \\
\mu g / k g\end{array}$ & $\begin{array}{l}\text { Acetone } \\
\text { Bis (2-Ethylhexyl) Phthalate } \\
\text { Calcium } \\
\text { Methylene Chloride }\end{array}$ \\
\hline 45 & $\begin{array}{l}0.0,0.5 \\
0.0,0.5 \\
0.0,0.5 \\
0.0,0.5 \\
4.5,5.0 \\
4.5,5.0 \\
4.5,5.0 \\
4.5,5.0 \\
4.5,5.0\end{array}$ & $\begin{array}{c}3000 \\
14900 \\
6890 \\
1160 \\
100 \\
6780 \\
3830 \\
6.00 \\
1660\end{array}$ & $\begin{array}{c}\mu g / k g \\
\mu g / g \\
\mu g / g \\
\mu g / g \\
\mu g / k g \\
\mu g / g \\
\mu g / g \\
\mu g / k g \\
\mu g / g\end{array}$ & $\begin{array}{l}\text { Aroclor-1260 } \\
\text { Calcium } \\
\text { Magnesium } \\
\text { Potassium } \\
\text { Acetone } \\
\text { Calcium } \\
\text { Magnesium } \\
\text { Methylene Chloride } \\
\text { Potassium }\end{array}$ \\
\hline 46 & $2.0,4.0$ & 3.45 & $\mu g / g$ & 2,4-Dinitrotoluene \\
\hline
\end{tabular}


TABLE 5.2-2 Soil Contaminant Concentrations Above Background (Continued)

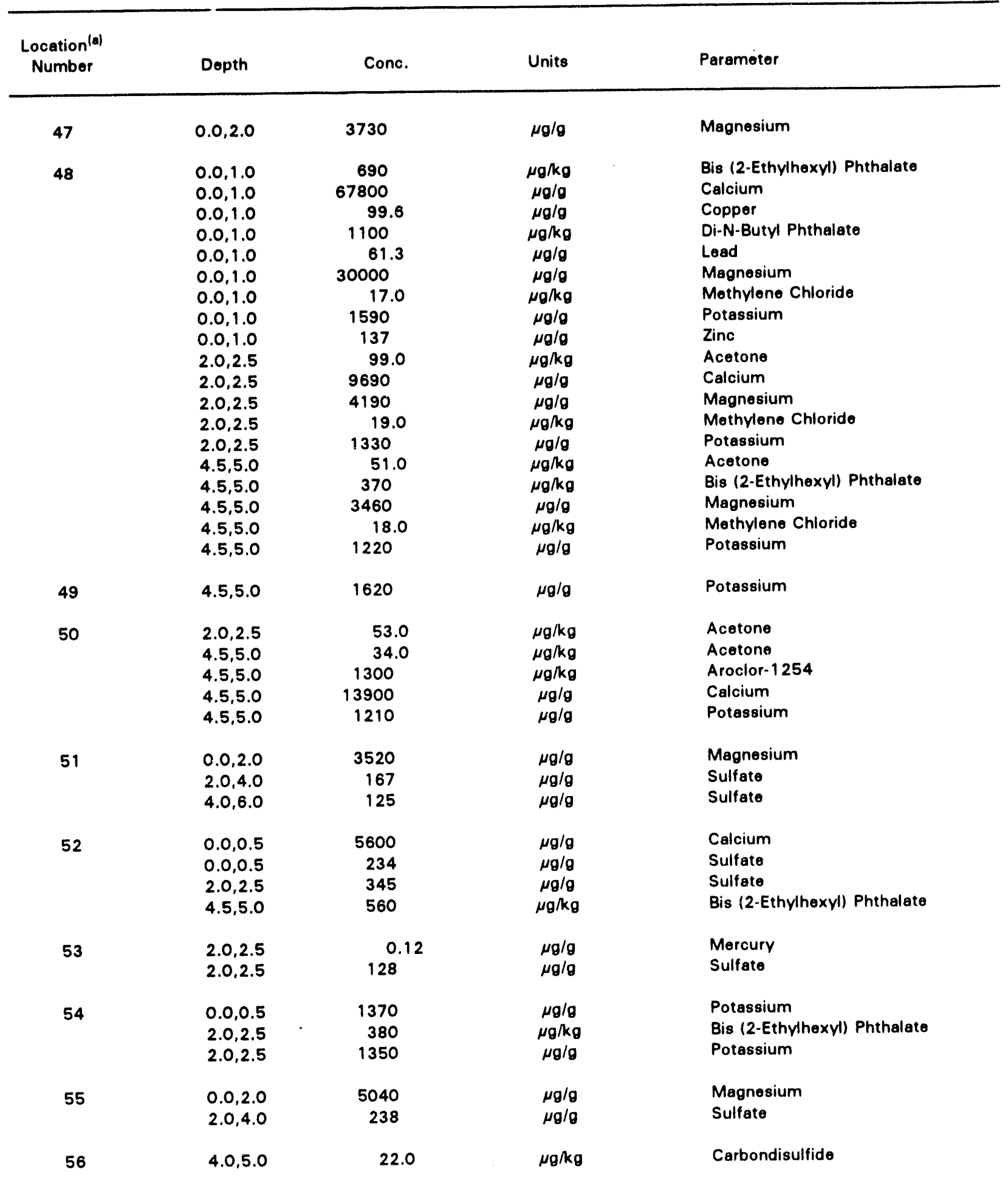


TABLE 5.2-2 Soil Contaminant Concentrations Above Background (Continued)

\begin{tabular}{|c|c|c|c|c|}
\hline $\begin{array}{l}\text { Location }^{(8)} \\
\text { Number }\end{array}$ & Depth & Conc. & Units & Parameter \\
\hline 57 & $\begin{array}{l}0.0 .1 .0 \\
4.5,5.0\end{array}$ & $\begin{array}{r}1300 \\
390\end{array}$ & $\begin{array}{l}\mu \mathrm{g} / \mathbf{k g} \\
\mu \mathrm{g} / \mathrm{kg}\end{array}$ & $\begin{array}{l}\text { Aroclor-1260 } \\
\text { Aroclor-1260 }\end{array}$ \\
\hline 58 & $\begin{array}{l}0.0,7.0 \\
0.0,7.0 \\
0.0,7.0 \\
0.0,7.0\end{array}$ & $\begin{array}{c}30.0 \\
2220 \\
6.00 \\
1320\end{array}$ & $\begin{array}{c}\mu \mathrm{g} / \mathrm{kg} \\
\mu \mathrm{g} / \mathrm{g} \\
\mu \mathrm{g} / \mathrm{kg} \\
\mu \mathrm{g} / \mathrm{g}\end{array}$ & $\begin{array}{l}\text { Acetone } \\
\text { Manganese } \\
\text { Methylene Chloride } \\
\text { Potassium }\end{array}$ \\
\hline 59 & $\begin{array}{l}5.0,7.0 \\
5.0,7.0 \\
5.0,7.0 \\
5.0,7.0 \\
5.0,7.0 \\
5.0,7.0\end{array}$ & $\begin{array}{c}30.0 \\
5500 \\
59.2 \\
0.98 \\
10.0 \\
146\end{array}$ & $\begin{array}{c}\mu g / k g \\
\mu g / g \\
\mu g / g \\
\mu g / g \\
\mu g / k g \\
\mu g / g\end{array}$ & $\begin{array}{l}\text { Acetone } \\
\text { Calcium } \\
\text { Chromium } \\
\text { Mercury } \\
\text { Methylene Chloride } \\
\text { Sulfate }\end{array}$ \\
\hline 60 & $\begin{array}{l}2.0,2.5 \\
2.0,2.5 \\
2.0,2.5 \\
2.0,2.5 \\
2.0,2.5 \\
4.5,5.0 \\
4.5,5.0 \\
4.5,5.0\end{array}$ & $\begin{array}{c}720 \\
24100 \\
3760 \\
9.00 \\
1430 \\
1700 \\
11.8 \\
10.0\end{array}$ & $\begin{array}{c}\mu \mathrm{g} / \mathrm{kg} \\
\mu \mathrm{g} / \mathrm{g} \\
\mu \mathrm{g} / \mathrm{g} \\
\mu \mathrm{g} / \mathrm{kg} \\
\mu \mathrm{g} / \mathrm{g} \\
\mu \mathrm{g} / \mathrm{kg} \\
\mu \mathrm{g} / \mathrm{g} \\
\mu \mathrm{g} / \mathrm{kg}\end{array}$ & $\begin{array}{l}\text { Bis (2-Ethylhexyl) Phthalate } \\
\text { Calcium } \\
\text { Magnesium } \\
\text { Methylene Chloride } \\
\text { Potassium } \\
\text { Bis (2-Ethylhexyl) Phthalate } \\
\text { Chloride } \\
\text { Methylene Chloride }\end{array}$ \\
\hline 61 & $\begin{array}{l}1.0,1.5 \\
1.0,1.5 \\
1.0,1.5 \\
1.0,1.5 \\
1.0,1.5 \\
1.0,1.5 \\
1.0,1.5 \\
1.0,1.5 \\
1.0,1.5 \\
1.0,1.5 \\
1.0,1.5 \\
1.0,1.5 \\
1.0,1.5 \\
1.0,1.5 \\
1.0,1.5 \\
1.0,1.5 \\
2.0,2.5 \\
2.0,2.5 \\
2.0,2.5 \\
2.0,2.5 \\
2.0,2.5 \\
4.5,5.0 \\
4.5,5.0 \\
4.5,5.0 \\
4.5,5.0 \\
4.5,5.0 \\
4.5,5.0\end{array}$ & $\begin{array}{c}60.0 \\
550 \\
5.64 \\
77200 \\
256 \\
21.9 \\
46990 \\
192 \\
10200 \\
0.19 \\
21.0 \\
25.4 \\
1700 \\
333 \\
63.0 \\
1000 \\
56.0 \\
110.1 \\
2920 \\
232 \\
250 \\
66.0 \\
16.8 \\
15.0 \\
3940 \\
110 \\
29.0\end{array}$ & $\begin{array}{c}\mu g / k g \\
\mu g / k g \\
\mu g / g \\
\mu g / g \\
\mu g / g \\
\mu g / g \\
\mu g / g \\
\mu g / g \\
\mu g / g \\
\mu g / g \\
\mu g / k g \\
\mu g / g \\
\mu g / g \\
\mu g / g \\
\mu g / k g \\
\mu g / g \\
\mu g / k g \\
\mu g / g \\
\mu g / g \\
\mu g / g \\
\mu g / k g \\
\mu g / k g \\
\mu g / g \\
\mu g / k g \\
\mu g / g \\
\mu g / g \\
\mu g / k g\end{array}$ & $\begin{array}{l}\text { Acetone } \\
\text { Bis (2-Ethylhexyl) Phthalate } \\
\text { Cadmium } \\
\text { Calcium } \\
\text { Copper } \\
\text { Fluoride } \\
\text { Iron } \\
\text { Lead } \\
\text { Magnesium } \\
\text { Mercury } \\
\text { Methylene Chloride } \\
\text { Molybdenum } \\
\text { Potassium } \\
\text { Sulfate } \\
\text { Trichloroethene } \\
\text { Zinc } \\
\text { Acetone } \\
\text { Molybdenum } \\
\text { Potassium } \\
\text { Sulfate } \\
\text { Trichloroethene } \\
\text { Acetone } \\
\text { Fluoride } \\
\text { Methylene Chloride } \\
\text { Potassium } \\
\text { Sulfate } \\
\text { Trichloroethene }\end{array}$ \\
\hline
\end{tabular}


TABLE 5.2-2 Soil Contaminant Concentrations Above Background (Continued)

\begin{tabular}{|c|c|c|c|c|}
\hline $\begin{array}{l}\text { Location } \\
\text { Number }\end{array}$ & Depth & Conc. & Units & Parameter \\
\hline 62 & $\begin{array}{l}0.0,7.0 \\
0.0,7.0 \\
0.0,7.0\end{array}$ & $\begin{array}{r}27 \\
24700 \\
27.7\end{array}$ & $\begin{array}{l}\mu g / \mathbf{k g} \\
\mu g / g \\
\mu g / g\end{array}$ & $\begin{array}{l}\text { 1,2-Dichloroothene (TOTAL) } \\
\text { Aluminum } \\
\text { Copper }\end{array}$ \\
\hline 63 & $\begin{array}{l}0.0,0.5 \\
0.0,0.5 \\
2.0,2.5\end{array}$ & $\begin{array}{l}810 \\
6.00 \\
360\end{array}$ & $\begin{array}{l}\mu \mathrm{g} / \mathrm{kg} \\
\mu \mathrm{g} / \mathrm{kg} \\
\mu \mathrm{g} / \mathrm{kg}\end{array}$ & $\begin{array}{l}\text { Bis (2-Ethylhexyl) Phthalate } \\
\text { Methylene Chloride } \\
\text { Bis (2-Ethylhexyl) Phthalate }\end{array}$ \\
\hline 64 & $\begin{array}{l}0.0,0.5 \\
0.0,0.5 \\
2.0,2.5 \\
2.0,2.5 \\
4.5,5.0 \\
4.5,5.0\end{array}$ & $\begin{array}{c}11100 \\
3760 \\
630 \\
0.13 \\
1100 \\
19.3\end{array}$ & $\begin{array}{c}\mu \mathrm{g} / \mathrm{g} \\
\mu \mathrm{g} / \mathrm{g} \\
\mu \mathrm{g} / \mathrm{kg} \\
\mu \mathrm{g} / \mathrm{g} \\
\mu \mathrm{g} / \mathrm{kg} \\
\mu \mathrm{g} / \mathrm{g}\end{array}$ & $\begin{array}{l}\text { Calcium } \\
\text { Magnesium } \\
\text { Bis (2-Ethylhexyl) Phthalate } \\
\text { Mercury } \\
\text { Bis (2-Ethylhexyl) Phthalate } \\
\text { Fluoride }\end{array}$ \\
\hline 65 & $\begin{array}{c}0.0,7.0 \\
8.0,15.0\end{array}$ & $\begin{array}{l}118 \\
153\end{array}$ & $\begin{array}{l}\mu g / g \\
\mu g / g\end{array}$ & $\begin{array}{l}\text { Sulfate } \\
\text { Sulfate }\end{array}$ \\
\hline 66 & $\begin{array}{l}0.0,7.0 \\
0.0,7.0 \\
8.0,15.0\end{array}$ & $\begin{array}{l}2.19 \\
233 \\
20.1\end{array}$ & $\begin{array}{l}\mu g / g \\
\mu g / g \\
\mu g / g\end{array}$ & $\begin{array}{l}\text { Cadmium } \\
\text { Sulfate } \\
\text { Fluoride }\end{array}$ \\
\hline 67 & $\begin{array}{l}0.0,7.0 \\
0.0,7.0 \\
8.0,15.0\end{array}$ & $\begin{array}{l}6.00 \\
5.65 \\
20.3\end{array}$ & $\begin{array}{l}\mu g / k g \\
\mu g / g \\
\mu g / g\end{array}$ & $\begin{array}{l}\text { Methylene Chloride } \\
\text { Nitrite } \\
\text { Fluoride }\end{array}$ \\
\hline 68 & $\begin{array}{l}8.0,15.0 \\
8.0,15.0 \\
0.0,7.0 \\
0.0,7.0\end{array}$ & $\begin{array}{c}5300 \\
14.0 \\
14.2 \\
144\end{array}$ & $\begin{array}{l}\mu g / g \\
\mu g / k g \\
\mu g /= \\
\mu g / g\end{array}$ & $\begin{array}{l}\text { Calcium } \\
\text { Methylene Chloride } \\
\text { Chloride } \\
\text { Sulfate }\end{array}$ \\
\hline 69 & $\begin{array}{l}2.0,2.5 \\
2.0,2.5 \\
2.0,2.5 \\
4.0,5.0 \\
4.0,5.0 \\
4.0,5.0\end{array}$ & $\begin{array}{c}1140 \\
5290 \\
995 \\
26100 \\
25.9 \\
1480\end{array}$ & $\begin{array}{l}\mu g / g \\
\mu g / g \\
\mu g / g \\
\mu g / g \\
\mu g / g \\
\mu g / g\end{array}$ & $\begin{array}{l}\text { Barium } \\
\text { Calcium } \\
\text { Potassium } \\
\text { Aluminum } \\
\text { Molybdenum } \\
\text { Potassium }\end{array}$ \\
\hline 70 & $\begin{array}{l}0.0,0.5 \\
0.0,0.5 \\
0.0,0.5 \\
0.0,0.5 \\
4.5,5.0 \\
4.5,5.0\end{array}$ & $\begin{array}{r}2800 \\
9660 \\
4700 \\
1470 \\
5490 \\
280\end{array}$ & $\begin{array}{l}\mu \mathrm{g} / \mathrm{kg} \\
\mu \mathrm{g} / \mathrm{g} \\
\mu \mathrm{g} / \mathrm{g} \\
\mu \mathrm{g} / \mathrm{g} \\
\mu \mathrm{g} / \mathrm{g} \\
\mu \mathrm{g} / \mathrm{kg}\end{array}$ & $\begin{array}{l}\text { Aroclor-1260 } \\
\text { Calcium } \\
\text { Magnesium } \\
\text { Potassium } \\
\text { Calcium } \\
\text { Methylene Chloride }\end{array}$ \\
\hline 71 & $\begin{array}{l}0.0,0.5 \\
0.0,0.5 \\
0.0,0.5 \\
0.0,0.5 \\
0.0,0.5 \\
2.0,2.5\end{array}$ & $\begin{array}{c}19600 \\
5340 \\
0.11 \\
23.2 \\
1380 \\
183\end{array}$ & $\begin{array}{c}\mu g / g \\
\mu g / g \\
\mu g / g \\
\mu g / k g \\
\mu g / g \\
\mu g / g\end{array}$ & $\begin{array}{l}\text { Calcium } \\
\text { Magnesium } \\
\text { Mercury } \\
\text { Methylene Chloride } \\
\text { Potassium } \\
\text { Nitrate }\end{array}$ \\
\hline
\end{tabular}


TABLE 5.2-2 Soil Contaminant Concentrations Above Background (Continued)

\begin{tabular}{|c|c|c|c|c|}
\hline $\begin{array}{c}\text { Location }{ }^{(a)} \\
\text { Number }\end{array}$ & Depth & Conc. & Units & Parameter \\
\hline 71 (cont.) & $\begin{array}{l}2.0,2.5 \\
4.5,5.0 \\
4.5,5.0 \\
4.5,5.0 \\
4.5,5.0 \\
4.5,5.0 \\
4.5,5.0 \\
4.5,5.0\end{array}$ & $\begin{array}{c}139 \\
26200 \\
5190 \\
2460 \\
28.5 \\
555 \\
1260 \\
67.1\end{array}$ & $\begin{array}{l}\mu g / g \\
\mu g / g \\
\mu g / g \\
\mu g / g \\
\mu g / g \\
\mu g / g \\
\mu g / g \\
\mu g / g\end{array}$ & $\begin{array}{l}\text { Sulfate } \\
\text { Aluminum } \\
\text { Barium } \\
\text { Manganese } \\
\text { Molybdenum } \\
\text { Nitrate } \\
\text { Potassium } \\
\text { Vanadium }\end{array}$ \\
\hline 72 & $\begin{array}{l}0.0,1.0 \\
0.0,1.0 \\
2.0,2.5 \\
4.0,5.0 \\
4.0,5.0\end{array}$ & $\begin{array}{c}9360 \\
3590 \\
2.13 \\
5320 \\
0.11\end{array}$ & $\begin{array}{l}\mu g / g \\
\mu g / g \\
\mu g / g \\
\mu g / g \\
\mu g / g\end{array}$ & $\begin{array}{l}\text { Calcium } \\
\text { Magnesium } \\
\text { Cadmium } \\
\text { Calcium } \\
\text { Mercury }\end{array}$ \\
\hline 73 & $\begin{array}{l}0.0 .0 .5 \\
0.0,0.5 \\
0.0,0.5 \\
2.0,2.5 \\
4.5,5.0\end{array}$ & $\begin{array}{l}7240 \\
26.8 \\
5060 \\
4500 \\
4840\end{array}$ & $\begin{array}{l}\mu g / g \\
\mu g / g \\
\mu g / g \\
\mu g / g \\
\mu g / g\end{array}$ & $\begin{array}{l}\text { Calcium } \\
\text { Copper } \\
\text { Potassium } \\
\text { Potassium } \\
\text { Potassium }\end{array}$ \\
\hline 74 & $\begin{array}{l}0.0,1.0 \\
0.0,1.0 \\
0.0,1.0 \\
0.0,1.0 \\
0.0,1.0\end{array}$ & $\begin{array}{c}1100 \\
22100 \\
4640 \\
6.00 \\
2210\end{array}$ & $\begin{array}{c}\mu \mathrm{g} / \mathrm{kg} \\
\mu \mathrm{g} / \mathrm{g} \\
\mu \mathrm{g} / \mathrm{g} \\
\mu \mathrm{g} / \mathrm{kg} \\
\mu \mathrm{g} / \mathrm{g}\end{array}$ & $\begin{array}{l}\text { Bis (2-Ethylhexyl) Phthalate } \\
\text { Calcium } \\
\text { Magnesium } \\
\text { Methylene Chloride } \\
\text { Potassium }\end{array}$ \\
\hline 75 & $\begin{array}{l}0.0,0.5 \\
0.0,0.5 \\
0.0,0.5 \\
0.0,0.5 \\
0.0,0.5\end{array}$ & $\begin{array}{c}3500 \\
11500 \\
4060 \\
1220 \\
79.0\end{array}$ & $\begin{array}{c}\mu g / k g \\
\mu g / g \\
\mu g / g \\
\mu g / g \\
\mu g / k g\end{array}$ & $\begin{array}{l}\text { Aroclor-1260 } \\
\text { Calcium } \\
\text { Magnesium } \\
\text { Potassium } \\
\text { Toluene }\end{array}$ \\
\hline 76 & $\begin{array}{l}0.0,0.5 \\
0.0,0.5 \\
2.0,3.5 \\
2.0,3.5 \\
2.0,3.5 \\
2.0,3.5 \\
2.0,3.5 \\
4.5,5.0 \\
4.5,5.0 \\
4.5,5.0 \\
4.5,5.0 \\
4.5,5.0\end{array}$ & $\begin{array}{c}6970 \\
16.0 \\
29600 \\
25.1 \\
15.0 \\
26.6 \\
1180 \\
34700 \\
27.0 \\
8.00 \\
31.0 \\
1230\end{array}$ & $\begin{array}{c}\mu g / g \\
\mu g / k g \\
\mu g / g \\
\mu g / g \\
\mu g / k g \\
\mu g / g \\
\mu g / g \\
\mu g / g \\
\mu g / g \\
\mu g / k g \\
\mu g / g \\
\mu g / g\end{array}$ & $\begin{array}{l}\text { Calcium } \\
\text { Methylane Chloride } \\
\text { Aluminum } \\
\text { Chloride } \\
\text { Methylene Chloride } \\
\text { Molybdenum } \\
\text { Potassium } \\
\text { Aluminum } \\
\text { Chloride } \\
\text { Methylene Chloride } \\
\text { Molybdenum } \\
\text { Potassium }\end{array}$ \\
\hline 77 & $\begin{array}{l}1.0,1.5 \\
1.0,1.5 \\
1.0,1.5 \\
1.0,1.5 \\
3.0,3.5\end{array}$ & $\begin{array}{c}26600 \\
8170 \\
26.7 \\
1020 \\
1050\end{array}$ & $\begin{array}{l}\mu \mathrm{g} / \mathrm{g} \\
\mu \mathrm{g} / \mathrm{g} \\
\mu \mathrm{g} / \mathrm{g} \\
\mu \mathrm{g} / \mathrm{g} \\
\mu \mathrm{g} / \mathrm{g}\end{array}$ & $\begin{array}{l}\text { Aluminum } \\
\text { Calcium } \\
\text { Molybdenum } \\
\text { Potassium } \\
\text { Potassium }\end{array}$ \\
\hline
\end{tabular}


TABLE 5.2-2 Soil Contaminant Concentrations Above Background (Continued)

\begin{tabular}{|c|c|c|c|c|}
\hline $\begin{array}{l}\text { Location }^{(a)} \\
\text { Number }\end{array}$ & Depth & Conc. & Units & Parameter \\
\hline 77 (cont.) & $\begin{array}{l}5.5,6.0 \\
5.5,6.0 \\
5.5,6.0\end{array}$ & $\begin{array}{c}31200 \\
29.5 \\
1520\end{array}$ & $\begin{array}{l}\mu g / g \\
\mu g / g \\
\mu g / g\end{array}$ & $\begin{array}{l}\text { Aluminum } \\
\text { Molybdenum } \\
\text { Potassium }\end{array}$ \\
\hline 78 & $\begin{array}{l}1.0,1.5 \\
1.0,1.5 \\
3.0,4.0 \\
3.0,4.0 \\
3.0,4.0 \\
5.5,6.0 \\
5.5,6.0 \\
5.5,6.0\end{array}$ & $\begin{array}{c}15500 \\
44.6 \\
28600 \\
39.9 \\
27.1 \\
24400 \\
970 \\
1020\end{array}$ & $\begin{array}{l}\mu g / g \\
\mu g / g \\
\mu g / g \\
\mu g / g \\
\mu g / g \\
\mu g / g \\
\mu g / k g \\
\mu g / g\end{array}$ & $\begin{array}{l}\text { Calcium } \\
\text { Fluoride } \\
\text { Aluminum } \\
\text { Chromium } \\
\text { Molybdenum } \\
\text { Aluminum } \\
\text { Bis (2-Ethylhexyl) Phthalate } \\
\text { Potassium }\end{array}$ \\
\hline 79 & $\begin{array}{l}0.0,0.5 \\
0.0,0.5 \\
0.0,0.5 \\
0.0,0.5 \\
2.0,2.5 \\
2.1,2.5 \\
2.0,2.5 \\
2.0,2.5 \\
4.5,5.0 \\
4.5,5.0\end{array}$ & $\begin{array}{c}27.0 \\
2350 \\
129 \\
23.0 \\
26200 \\
27.0 \\
4410 \\
30.4 \\
9240 \\
4750\end{array}$ & $\begin{array}{c}\mu g / \mathbf{k g} \\
\mu g / g \\
\mu g / g \\
\mu g / k g \\
\mu g / g \\
\mu g / g \\
\mu g / g \\
\mu g / g \\
\mu g / g \\
\mu g / g\end{array}$ & $\begin{array}{l}\text { Acetone } \\
\text { Manganese } \\
\text { Sulfate } \\
\text { Toluene } \\
\text { Aluminum } \\
\text { Copper } \\
\text { Maynesium } \\
\text { Molybdenum } \\
\text { Calcium } \\
\text { Magnesium }\end{array}$ \\
\hline 80 & $\begin{array}{l}0.0,0.5 \\
0.0,0.5 \\
0.0,0.5 \\
2.0,2.5 \\
2.0,2.5 \\
2.0,2.5 \\
2.0,2.5 \\
2.0,2.5 \\
4.5,5.0 \\
4.5,5.0\end{array}$ & $\begin{array}{c}10300 \\
146 \\
251 \\
56200 \\
18700 \\
1470 \\
103 \\
78.5 \\
1160 \\
154\end{array}$ & $\begin{array}{l}\mu g / g \\
\mu g / g \\
\mu g / g \\
\mu g / g \\
\mu g / g \\
\mu g / g \\
\mu g / g \\
\mu g / g \\
\mu g / g \\
\mu g / g\end{array}$ & $\begin{array}{l}\text { Calcium } \\
\text { Sulfate } \\
\text { Zinc } \\
\text { Calcium } \\
\text { Magnesium } \\
\text { Potassium } \\
\text { Sulfate } \\
\text { Zinc } \\
\text { Potassium } \\
\text { Sulfate }\end{array}$ \\
\hline 81 & $\begin{array}{l}0.0,2.0 \\
2.0,4.0 \\
2.0,4.0 \\
4.0,6.0 \\
4.0,6.0\end{array}$ & $\begin{array}{l}6.00 \\
180 \\
11.0 \\
32.0 \\
20.0\end{array}$ & $\begin{array}{l}\mu g / \mathrm{kg} \\
\mu g / \mathrm{kg} \\
\mu g / \mathrm{kg} \\
\mu g / \mathrm{kg} \\
\mu g / \mathrm{kg}\end{array}$ & $\begin{array}{l}\text { Methylene Chloride } \\
\text { Aroctor-1254 } \\
\text { Methylene Chloride } \\
\text { Acetone } \\
\text { Methylene Chloride }\end{array}$ \\
\hline 82 & $\begin{array}{l}0.0,2.0 \\
0.0,2.0\end{array}$ & $\begin{array}{r}2030 \\
726\end{array}$ & $\begin{array}{l}\mu g / g \\
\mu g / g\end{array}$ & $\begin{array}{l}\text { Manganese } \\
\text { Sulfate }\end{array}$ \\
\hline 83 & $\begin{array}{l}0.0,0.5 \\
0.0,0.5 \\
0.0,0.5 \\
0.0,0.5 \\
0.0,0.5 \\
0.0,0.5 \\
2.0,2.5\end{array}$ & $\begin{array}{c}25300 \\
340 \\
25900 \\
6680 \\
19.0 \\
1360 \\
12.0\end{array}$ & $\begin{array}{c}\mu g / g \\
\mu g / k g \\
\mu g / g \\
\mu g / g \\
\mu g / k g \\
\mu g / g \\
\mu g / k g\end{array}$ & $\begin{array}{l}\text { Aluminum } \\
\text { Aroclor-1254 } \\
\text { Calcium } \\
\text { Magnesium } \\
\text { Methylene Chloride } \\
\text { Potessium } \\
\text { Methylene Chloride }\end{array}$ \\
\hline
\end{tabular}


TABLE 5.2-2 Soil Contaminant Concentrations Above Background (Continued)

\begin{tabular}{|c|c|c|c|c|}
\hline $\begin{array}{c}\text { Location }^{(a)} \\
\text { Number }\end{array}$ & Depth & Conc. & Units & Parameter \\
\hline 83 (cont.) & $\begin{array}{l}2.0,2.5 \\
4.5,5.0 \\
4.5,5.0\end{array}$ & $\begin{array}{l}248 \\
460 \\
8.00\end{array}$ & $\begin{array}{l}\mu g / g \\
\mu g / k g \\
\mu g / k g\end{array}$ & $\begin{array}{l}\text { Sulfate } \\
\text { Bis (2-Ethylhexyl) Phthalate } \\
\text { Methylene Chloride }\end{array}$ \\
\hline 84 & $\begin{array}{l}2.0,2.5 \\
2.0,2.5 \\
2.0,2.5 \\
3.5,5.0 \\
3.5,5.0\end{array}$ & $\begin{array}{r}54.4 \\
20.0 \\
162 \\
5070 \\
22.0\end{array}$ & $\begin{array}{c}\mu g / g \\
\mu g / k g \\
\mu g / g \\
\mu g / g \\
\mu g / k g\end{array}$ & $\begin{array}{l}\text { Chromium } \\
\text { Methylene Chloride } \\
\text { Sulfate } \\
\text { Calcium } \\
\text { Methylene Chloride }\end{array}$ \\
\hline 85 & $\begin{array}{l}2.0,2.5 \\
2.0,2.5 \\
4.5,5.0 \\
4.5,5.0 \\
4.5,5.0 \\
4.5,5.0\end{array}$ & $\begin{array}{c}16.0 \\
345 \\
32.0 \\
530 \\
15.3 \\
8.00\end{array}$ & $\begin{array}{c}\mu g / k g \\
\mu g / g \\
\mu g / k g \\
\mu g / k g \\
\mu g / g \\
\mu g / k g\end{array}$ & $\begin{array}{l}\text { Methylene Chloride } \\
\text { Sulfate } \\
\text { Acetone } \\
\text { Bis (2-Ethylhexyl) Phthalate } \\
\text { Chloride } \\
\text { Methylene Chloride }\end{array}$ \\
\hline 86 & $\begin{array}{l}1.0,2.0 \\
1.0,2.0 \\
3.0,4.5 \\
3.0,4.5 \\
3.0,4.5 \\
3.0,4.5\end{array}$ & $\begin{array}{c}20800 \\
2.11 \\
52400 \\
7200 \\
8.00 \\
1040\end{array}$ & $\begin{array}{c}\mu g / g \\
\mu g / g \\
\mu g / g \\
\mu g / g \\
\mu g / k g \\
\mu g / g\end{array}$ & $\begin{array}{l}\text { Calcium } \\
\text { Mercury } \\
\text { Calcium } \\
\text { Magnesium } \\
\text { Methylene Chloride } \\
\text { Potassium }\end{array}$ \\
\hline 87 & $\begin{array}{c}8.0,15.0 \\
8.0,15.0 \\
8.0,15.0 \\
16.0,17.0\end{array}$ & $\begin{array}{c}5200 \\
20.2 \\
1390 \\
19.2\end{array}$ & $\begin{array}{l}\mu g / g \\
\mu g / g \\
\mu g / g \\
\mu g / g\end{array}$ & $\begin{array}{l}\text { Calcium } \\
\text { Fluoride } \\
\text { Potassium } \\
\text { Fluoride }\end{array}$ \\
\hline 88 & $4.5,5.0$ & 42.6 & $\mu g / g$ & Chloride \\
\hline 89 & $\begin{array}{l}8.0,15.0 \\
8.0,15.0\end{array}$ & $\begin{array}{l}10.9 \\
427\end{array}$ & $\begin{array}{l}\mu_{g} / g \\
\mu_{g} / g\end{array}$ & $\begin{array}{l}\text { Chloride } \\
\text { Nitrate }\end{array}$ \\
\hline 90 & $\begin{array}{l}0.0,7.0 \\
0.0,7.0 \\
0.0,7.0 \\
0.0,7.0 \\
0.0,7.0 \\
0.0,7.0 \\
8.0,15.0\end{array}$ & $\begin{array}{c}26500 \\
5840 \\
0.18 \\
8.00 \\
29.0 \\
1770 \\
17.4\end{array}$ & $\begin{array}{c}\mu g / g \\
\mu g / g \\
\mu g / g \\
\mu g / k g \\
\mu g / g \\
\mu g / g \\
\mu g / g\end{array}$ & $\begin{array}{l}\text { Calcium } \\
\text { Magnesium } \\
\text { Mercury } \\
\text { Methylene Chloride } \\
\text { Molybdenum } \\
\text { Potassium } \\
\text { Fluoride }\end{array}$ \\
\hline 91 & $\begin{array}{l}0.0,7.0 \\
0.0,7.0 \\
0.0,7.0\end{array}$ & $\begin{array}{c}35.0 \\
0.12 \\
1310\end{array}$ & $\begin{array}{c}\mu g / k g \\
\mu g / g \\
\mu g / g\end{array}$ & $\begin{array}{l}\text { Carbondisulfide } \\
\text { Mercury } \\
\text { Potassium }\end{array}$ \\
\hline 92 & $\begin{array}{l}0.0,0.5 \\
2.0,3.0 \\
2.0,3.0 \\
2.0,3.0 \\
2.0,3.0\end{array}$ & $\begin{array}{c}8.00 \\
9820 \\
55.1 \\
10.0 \\
278\end{array}$ & $\begin{array}{c}\mu g / k g \\
\mu g / g \\
\mu g / g \\
\mu g / k g \\
\mu g / g\end{array}$ & $\begin{array}{l}\text { Methylene Chloride } \\
\text { Calcium } \\
\text { Chromium } \\
\text { Methylene Chloride } \\
\text { Sulfate }\end{array}$ \\
\hline
\end{tabular}


TABLE 5.2-2 Soil Contaminant Concentrations Above Background (Continued)

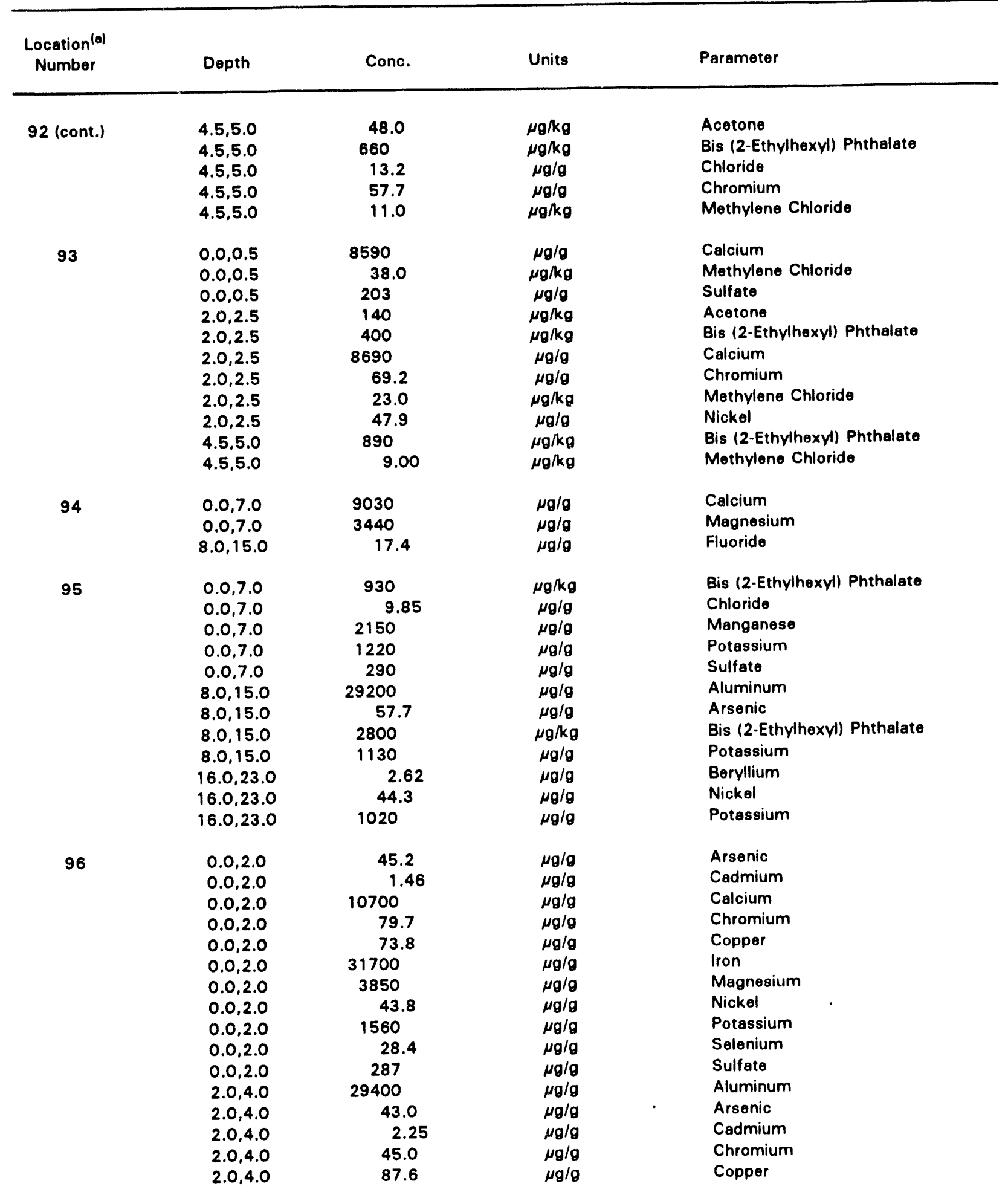


TABLE 5.2-2 Soil Contaminant Concentrations Above Background (Continued)

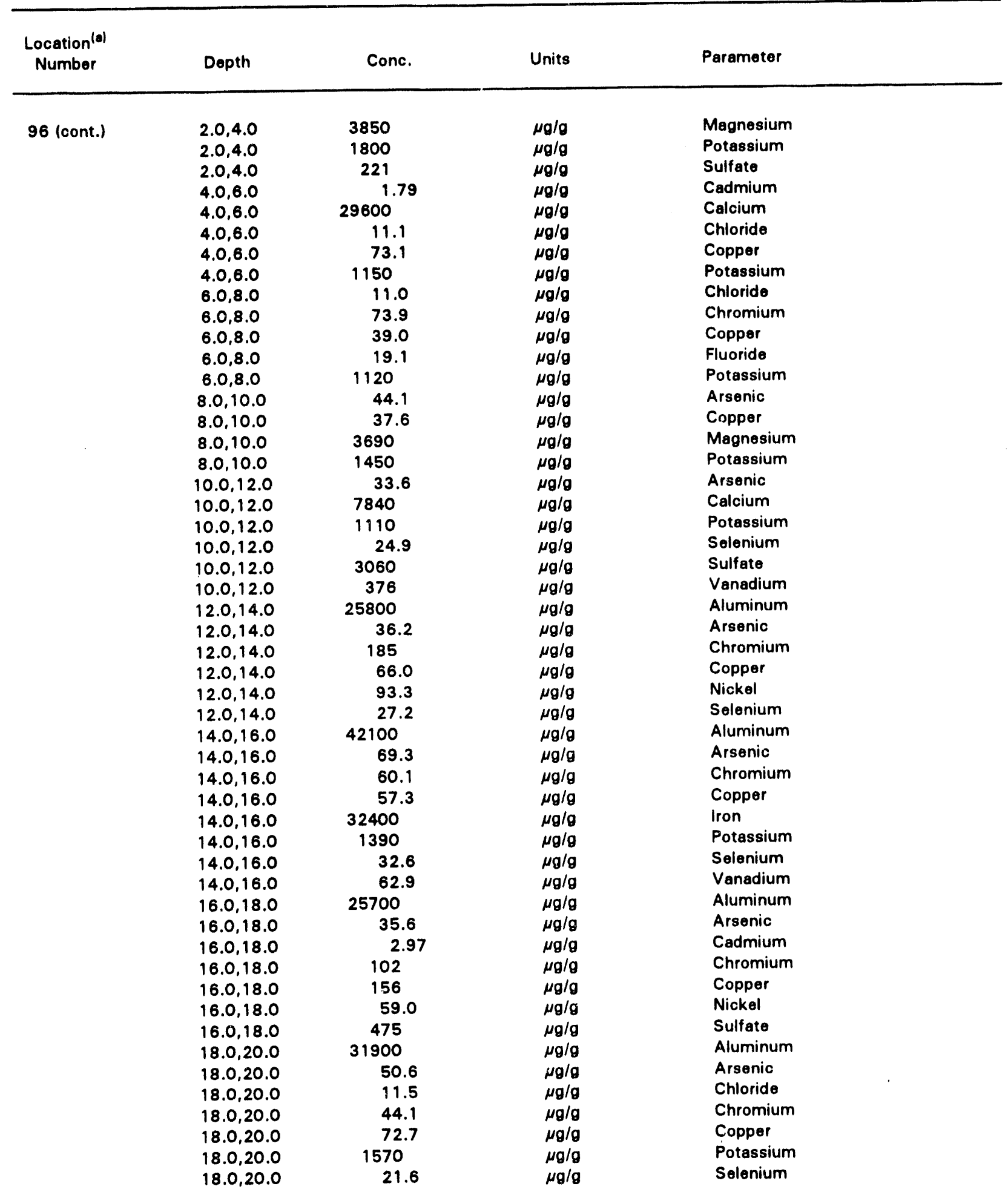


TABLE 5.2-2 Soil Contaminant Concentrations Above Background (Continued)

\begin{tabular}{|c|c|c|c|c|}
\hline $\begin{array}{l}\text { Location(a) } \\
\text { Numbor }\end{array}$ & Dopth & Conc. & Units & Parameter \\
\hline \multirow[t]{11}{*}{96 (cont.) } & $18.0,20.0$ & 124 & $\mu g / g$ & Sulfate \\
\hline & $18.0,20.0$ & 58.9 & $\mu g / g$ & Vanadium \\
\hline & $20.0,22.0$ & 30900 & $\mu g / g$ & Aluminum \\
\hline & $20.0,22.0$ & 68.4 & $\mu g / g$ & Arsenic \\
\hline & $20.0,22.0$ & 2.00 & $\mu g / g$ & Cadmium \\
\hline & $20.0,22.0$ & 41.7 & $\mu g / g$ & Chromium \\
\hline & $20.0,22.0$ & 70.6 & $\mu g / g$ & Copper \\
\hline & $20.0,22.0$ & 1460 & $\mu g / g$ & Potassium \\
\hline & $20.0,22.0$ & 25.7 & $\mu g / g$ & Solonium \\
\hline & $20.0,22.0$ & 164 & $\mu g / g$ & Sulfate \\
\hline & $20.0,22.0$ & 56.7 & $\mu g / g$ & Vanadium \\
\hline \multirow[t]{6}{*}{97} & 0.0 .7 .0 & 8.00 & $\mu g / k g$ & Methylene Chloride \\
\hline & $8.0,15.0$ & 25600 & $\mu g / g$ & Calcium \\
\hline & 8.0 .15 .0 & 3900 & $\mu g / g$ & Magnesium \\
\hline & 8.0 .15 .0 & 0.13 & $\mu \mathrm{g} / \mathrm{g}$ & Mercury \\
\hline & $8.0,15.0$ & 14.0 & $\mu g / k g$ & Methylene Chloride \\
\hline & 8.0 .15 .0 & 1720 & $\mu g / g$ & Potassium \\
\hline \multirow[t]{9}{*}{98} & 0.0 .7 .0 & 39.0 & $\mu g / k g$ & Acetone \\
\hline & 0.0 .7 .0 & 14.4 & $\mu g / g$ & Fluoride \\
\hline & $0.0,7.0$ & 8.00 & $\mu \mathrm{g} / \mathrm{kg}$ & Methylene Chloride \\
\hline & $0.0,7.0$ & 142 & $\mu g / g$ & Sulfato \\
\hline & $8.0,15.0$ & 61.0 & $\mu g / k g$ & Acetone \\
\hline & $8.0,15.0$ & 29.9 & $\mu \mathrm{g} / \mathrm{g}$ & Fluoride \\
\hline & $8.0,15.0$ & 0.13 & $\mu g / g$ & Mercury \\
\hline & 8.0 .15 .0 & 9.00 & $\mu \mathrm{g} / \mathrm{kg}$ & Methylene Chloride \\
\hline & $16.0,19.0$ & 23.2 & $\mu g / g$ & Fluoride \\
\hline \multirow[t]{20}{*}{99} & 2.0 .2 .5 & 1300 & $\mu g / k g$ & Aroclor-1254 \\
\hline & 2.0 .2 .5 & 9590 & $\mu g / g$ & Calcium \\
\hline & $2.0,2.5$ & 4650 & $\mu g / g$ & Magnesium \\
\hline & 2.0 .2 .5 & 6.00 & $\mu \mathrm{g} / \mathrm{kg}$ & Methylene Chloride \\
\hline & $2.0,2.5$ & 1370 & $\mu g / g$ & Potassium \\
\hline & 2.0 .2 .5 & 4800 & $\mu g / k g$ & Trichloroethene \\
\hline & $4.0,5.5$ & 1600 & $\mu g / k g$ & 1,2-Dichloroethene (TOTAL) \\
\hline & $4.0,5.5$ & 120 & $\mu g / k g$ & Acetone \\
\hline & 4.0 .5 .5 & 24800 & $\mu g / g$ & Aluminum \\
\hline & $4.0,5.5$ & 1.52 & $\mu g / g$ & Cadmium \\
\hline & $4.0,5.5$ & 9980 & $\mu g / g$ & Calcium \\
\hline & $4.0,5.5$ & 63.4 & $\mu g / g$ & Chromium \\
\hline & $4.0,5.5$ & 4830 & $\mu g / g$ & Magnesium \\
\hline & $4.0,5.5$ & 7.00 & $\mu \mathrm{g} / \mathrm{kg}$ & Methylene Chloride \\
\hline & 4.0 .5 .5 & 1560 & $\mu g / g$ & Potassium \\
\hline & $4.0,5.5$ & 320 & $\mu g / k g$ & Trichloroethene \\
\hline & $6.5,7.0$ & 6200 & $\mu g / k g$ & 1,2-Dichloroethene (TOTAL) \\
\hline & $6.5,7.0$ & 54.0 & $\mu g / k g$ & Acetone \\
\hline & $6.5,7.0$ & 30.0 & $\mu g / k g$ & Trichloroethene \\
\hline & $6.5,7.0$ & 56.3 & $\mu g / g$ & Vanadium \\
\hline
\end{tabular}


TABLE 5.2-2 Soil Contaminant Concentrations Above Background (Continued)

\begin{tabular}{|c|c|c|c|c|}
\hline $\begin{array}{l}\text { Location } \\
\text { Number }\end{array}$ & Depth & Conc. & Units & Parameter \\
\hline \multirow[t]{11}{*}{100} & $1.5,3.0$ & 180 & $\mu g / k g$ & 1,2-Dichloroethene (TOTAL) \\
\hline & $1.5,3.0$ & 5830 & $\mu g / g$ & Calcium \\
\hline & $1.5,3.0$ & 2.65 & $\mu g / g$ & Nitrite \\
\hline & $1.5,3.0$ & 148 & $\mu g / g$ & Sulfate \\
\hline & 4.5.5.0 & 120 & $\mu g / k g$ & Acetone \\
\hline & 4.5.5.0 & 5160 & $\mu g / g$ & Calcium \\
\hline & 4.5.5.0 & 1900 & $\mu \mathrm{g} / \mathrm{g}$ & Manganese \\
\hline & 4.5.5.0 & 36.0 & $\mu g / k g$ & Methylene Chloride \\
\hline & 4.5.5.0 & 1120 & $\mu g / g$ & Potassium \\
\hline & $6.0,6.5$ & 29.0 & $\mu \mathrm{g} / \mathbf{k g}$ & Acetone \\
\hline & $6.0,6.5$ & 26.9 & $\mu g / g$ & Copper \\
\hline \multirow[t]{2}{*}{101} & 0.0 .7 .0 & 1010 & $\mu \mathrm{g} / \mathrm{kg}$ & Bis (2-Ethylhexyl) Phthalate \\
\hline & $0.0,7.0$ & 1340 & $\mu g / g$ & Potassium \\
\hline \multirow[t]{23}{*}{102} & $0.0,7.0$ & 81.1 & $\mu \mathrm{g} / \mathrm{g}$ & Arsenic \\
\hline & $0.0,7.0$ & 26700 & $\mu g / g$ & Calcium \\
\hline & $0.0,7.0$ & 3430 & $\mu g / g$ & Magnesium \\
\hline & $0.0,7.0$ & 0.18 & $\mu g / g$ & Mercury \\
\hline & $0.0,7.0$ & 7.00 & $\mu \mathrm{g} / \mathrm{kg}$ & Methylene Chloride \\
\hline & $0.0,7.0$ & 28.1 & $\mu g / g$ & Molybdenum \\
\hline & $0.0,7.0$ & 687 & $\mu g / g$ & Nitrate \\
\hline & 0.0 .7 .0 & 1080 & $\mu g / g$ & Potassium \\
\hline & $0.0,7.0$ & 183 & $\mu g / g$ & Sulfato \\
\hline & $8.0,15.0$ & 70.1 & $\mu g / g$ & Arsenic \\
\hline & $8.0,15.0$ & 3490 & $\mu g / k g$ & Bis (2-Ethylhexyl) Phthalate \\
\hline & $8.0,15.0$ & 5100 & $\mu g / g$ & Calcium \\
\hline & $8.0,15.0$ & 11.3 & $\mu g / g$ & Chloride \\
\hline & $8.0,15.0$ & 9.00 & $\mu \mathrm{g} / \mathrm{kg}$ & Methylene Chloride \\
\hline & $8.0,15.0$ & 25.6 & $\mu \mathrm{g} / \mathrm{g}$ & Molybdenum \\
\hline & $8.0,15.0$ & 3830 & $\mu \mathrm{g} / \mathrm{g}$ & Nitrate \\
\hline & 8.0 .15 .0 & 3.27 & $\mu g / g$ & Nitrite \\
\hline & $8.0,15.0$ & 932 & $\mu g / g$ & Sodium \\
\hline & $8.0,15.0$ & 274 & $\mu g / g$ & Sulfate \\
\hline & 16.0 .17 .0 & 9.76 & $\mu g / g$ & Chloride \\
\hline & $16.0,17.0$ & 3380 & $\mu g / g$ & Nitrate \\
\hline & $16.0,17.0$ & 2.11 & $\mu \mathrm{g} / \mathrm{g}$ & Nitrite \\
\hline & 16.0 .17 .0 & 221 & $\mu g / g$ & Sulfate \\
\hline \multirow[t]{12}{*}{103} & $0.0,7.0$ & 699 & $\mu \mathrm{g} / \mathrm{kg}$ & Bis (2-Ethylhexyl) Phthalate \\
\hline & $0.0,7.0$ & 5720 & $\mu g / g$ & Calcium \\
\hline & $0.0,7.0$ & 29.6 & $\mu g / g$ & Copper \\
\hline & $0.0,7.0$ & 3460 & $\mu g / g$ & Magnesium \\
\hline & $0.0,7.0$ & 29.6 & $\mu \mathrm{g} / \mathrm{g}$ & Molybdenum \\
\hline & $0.0,7.0$ & 1100 & $\mu g / g$ & Potassium \\
\hline & $0.0,7.0$ & 142 & $\mu g / g$ & Sulfate \\
\hline & 8.0 .15 .0 & 69.5 & $\mu \mathrm{g} / \mathrm{g}$ & Arsenic \\
\hline & $8.0,15.0$ & 190 & $\mu g / g$ & Nitrate \\
\hline & 8.0 .15 .0 & 149 & $\mu g / g$ & Sulfate \\
\hline & $16.0,17.0$ & 11.1 & $\mu g / g$ & Chloride \\
\hline & $16.0,17.0$ & 452 & $\mu g / g$ & Nitrate \\
\hline
\end{tabular}


TABLE 5.2-2 Soil Contaminant Concentrations Above Background (Continued)

\begin{tabular}{|c|c|c|c|c|}
\hline $\begin{array}{l}\text { Location }{ }^{(a)} \\
\text { Number }\end{array}$ & Depth & Conc. & Units & Parameter \\
\hline \multirow[t]{51}{*}{104} & $0.0,1.0$ & 5390 & $\mu g / g$ & Calcium \\
\hline & 0.0 .1 .0 & 42. 8 & $\mu g / g$ & Chromium \\
\hline & $0.0,1.0$ & 1070 & $\mu g / g$ & Potassium \\
\hline & $0.0,2.0$ & 39.5 & $\mu \mathrm{g} / \boldsymbol{g}$ & Arsenic \\
\hline & $0.0,2.0$ & 2.61 & $\mu g / g$ & Cadmium \\
\hline & $0.0,2.0$ & 63.1 & $\mu g / g$ & Copper \\
\hline & 0.0 .2 .0 & 3590 & $\mu \mathrm{g} / \mathrm{g}$ & Magnesium \\
\hline & $0.0,2.0$ & 1640 & $\mu g / g$ & Potassium \\
\hline & 0.0 .2 .0 & 346 & $\mu \mathrm{g} / \mathrm{g}$ & Sulfate \\
\hline & $2.0,3.0$ & 24800 & $\mu g / g$ & Aluminum \\
\hline & $2.0,3.0$ & 1500 & $\mu g / k g$ & Bis (2-Ethylhexyl) Phthalate \\
\hline & $2.0,3.0$ & 24.8 & $\mu g / g$ & Molybdenum \\
\hline & 2.0 .3 .0 & 1290 & $\mu c / g$ & Potassium \\
\hline & 2.0 .4 .0 & 28900 & $\mu g / g$ & Aluminum \\
\hline & 2.0 .4 .0 & 46.2 & $\mu g / g$ & Arsenic \\
\hline & $2.0,4.0$ & 1.37 & $\mu g / g$ & Cadmium \\
\hline & $2.0,4.0$ & 39.7 & $\mu \mathrm{g} / \mathrm{g}$ & Chromium \\
\hline & $2.0,4.0$ & 36.3 & $\mu g / \theta$ & Copper \\
\hline & $2.0,4.0$ & 1060 & $\mu g / g$ & Potassium \\
\hline & $2.0,4.0$ & 232 & $\mu g / g$ & Sulfate \\
\hline & $4.0,5.0$ & 820 & $\mu \mathrm{g} / \mathrm{kg}$ & \\
\hline & 4.0 .5 .0 & 29.1 & $\mu g / g$ & $\begin{array}{l}\text { Molybdenum } \\
\text { Mols }\end{array}$ \\
\hline & $4.0,5.0$ & 1120 & $\mu g / g$ & $\begin{array}{l}\text { Molybdenum } \\
\text { Potassium }\end{array}$ \\
\hline & $4.0,6.0$ & 31800 & $\mu g / g$ & $\begin{array}{l}\text { Potassium } \\
\text { Aluminum }\end{array}$ \\
\hline & $4.0,6.0$ & 50.1 & $\mu g / g$ & $\begin{array}{l}\text { Aluminum } \\
\text { Arsenic }\end{array}$ \\
\hline & $4.0,6.0$ & 51.6 & $\mu g / g$ & $\begin{array}{l}\text { Arsenic } \\
\text { Chromium }\end{array}$ \\
\hline & $4.0,6.0$ & 65.1 & $\mu g / g$ & $\begin{array}{l}\text { Chromium } \\
\text { Copper }\end{array}$ \\
\hline & $4.0,6.0$ & 4180 & $\mu g / g$ & $\begin{array}{l}\text { Copper } \\
\text { Magnesium }\end{array}$ \\
\hline & $4.0,6.0$ & 2000 & $\mu g / g$ & $\begin{array}{l}\text { Magnesium } \\
\text { Potassium }\end{array}$ \\
\hline & $4.0,6.0$ & 21.4 & $\mu g / g$ & $\begin{array}{l}\text { Potassium } \\
\text { Selenium }\end{array}$ \\
\hline & $4.0,6.0$ & 62.7 & $\mu_{g} / g$ & $\begin{array}{l}\text { Selenium } \\
\text { Vanadium }\end{array}$ \\
\hline & $6.0,8.0$ & 27900 & $\mu g / g$ & $\begin{array}{l}\text { Vanadium } \\
\text { Aluminum }\end{array}$ \\
\hline & $6.0,8.0$ & 52.7 & $\mu g / g$ & \\
\hline & $6.0,8.0$ & 2.09 & $\mu g / g$ & $\begin{array}{l}\text { Arsenic } \\
\text { Cadmium }\end{array}$ \\
\hline & $6.0,8.0$ & 101 & $\mu g / g$ & $\begin{array}{l}\text { Cadmium } \\
\text { Chromium }\end{array}$ \\
\hline & $6.0,8.0$ & 74.0 & & \\
\hline & $6.0,8.0$ & 53.2 & $\mu g / g$ & Magnesium \\
\hline & & 2060 & $\mu g / g$ & Nickel \\
\hline & $6.0,8.0$ & 22.6 & $\mu g / g$ & Potassium \\
\hline & $6.0,8.0$ & $\begin{array}{l}22.6 \\
22.9\end{array}$ & $\mu g / g$ & Selenium \\
\hline & $6.0,8.0$ & $\begin{array}{l}22.9 \\
577\end{array}$ & $\mu g / g$ & Thallium \\
\hline & $8.0,10.0$ & 57.7 & $\mu g / g$ & Vanadium \\
\hline & 8.0 .10 .0 & 36.3 & $\mu g / g$ & Arsenic \\
\hline & $8.0,10.0$ & 2.36 & $\mu g / g$ & Cadmium \\
\hline & & 6020 & $\mu g / \theta$ & Calcium \\
\hline & $8.0,10.0$ & 95.6 & $\mu g / g$ & Chromium \\
\hline & $8.0,10.0$ & 87.9 & $\mu g / g$ & Copper \\
\hline & $8.0,10.0$ & 3590 & $\mu \mathrm{g} / \mathrm{g}$ & Niagnesium \\
\hline & 8.0,10.0 & 59.5 & $\mu g / g$ & Nickel \\
\hline & $8.0,10.0$ & 1470 & $\mu g / g$ & Potassium \\
\hline & $10.0,12.0$ & 21500 & $\mu g / \theta$ & Calcium \\
\hline
\end{tabular}


TABLE 5.2-2 Soil Contaminant Concentrations Above Background (Continued)

\begin{tabular}{|c|c|c|c|c|}
\hline $\begin{array}{l}\text { Location }^{(a)} \\
\text { Number }\end{array}$ & Depth & Conc. & Units & Parameter \\
\hline 104 (cont.) & $\begin{array}{l}10.0,12.0 \\
10.0,12.0 \\
10.0,12.0 \\
12.0,14.0 \\
12.0,14.0 \\
12.0,14.0 \\
12.0,14.0 \\
12.0,14.0 \\
12.0,14.0 \\
12.0,14.0 \\
12.0,14.0 \\
12.0,14.0 \\
14.0,16.0 \\
14.0,16.0 \\
14.0,16.0 \\
14.0,16.0 \\
14.0,16.0 \\
14.0,16.0 \\
14.0,16.0 \\
16.0,18.0 \\
16.0,18.0 \\
16.0,18.0 \\
16.0,18.0 \\
16.0,18.0 \\
16.0,18.0 \\
16.0,18.0 \\
18.0,20.0 \\
18.0,20.0 \\
18.0,20.0 \\
18.0,20.0 \\
18.0,20.0 \\
20.0,22.0 \\
20.0,22.0 \\
20.0,22.0\end{array}$ & $\begin{array}{c}49.8 \\
4470 \\
1600 \\
26.5 \\
1.92 \\
12600 \\
59.5 \\
36400 \\
3580 \\
4760 \\
1520 \\
29.0 \\
28600 \\
48.8 \\
3.02 \\
45.6 \\
82.2 \\
3960 \\
1530 \\
24500 \\
37.1 \\
2.94 \\
65.7 \\
3850 \\
1600 \\
21.2 \\
28.6 \\
3.11 \\
2.08 \\
53.4 \\
1300 \\
30.0 \\
34.1 \\
1050\end{array}$ & $\begin{array}{l}\mu g / g \\
\mu g / g \\
\mu g / g \\
\mu g / g \\
\mu g / g \\
\mu g / g \\
\mu g / g \\
\mu g / g \\
\mu g / g \\
\mu g / g \\
\mu g / g \\
\mu g / g \\
\mu g / g \\
\mu g / g \\
\mu g / g \\
\mu g / g \\
\mu g / g \\
\mu g / g \\
\mu g / g \\
\mu g / g \\
\mu g / g \\
\mu g / g \\
\mu g / g \\
\mu g / g \\
\mu g / g \\
\mu g / g \\
\mu g / g \\
\mu g / g \\
\mu g / g \\
\mu g / g \\
\mu g / g \\
\mu g / g \\
\mu g / g \\
\mu g / g\end{array}$ & $\begin{array}{l}\text { Copper } \\
\text { Magnesium } \\
\text { Potessium } \\
\text { Arsenic } \\
\text { Cadmium } \\
\text { Calcium } \\
\text { Copper } \\
\text { Iron } \\
\text { Magnesium } \\
\text { Menganese } \\
\text { Potassium } \\
\text { Selenium } \\
\text { Aluminum } \\
\text { Arsenic } \\
\text { Cadmium } \\
\text { Chromium } \\
\text { Copper } \\
\text { Magnesium } \\
\text { Potassium } \\
\text { Aluminum } \\
\text { Arsenic } \\
\text { Cadmium } \\
\text { Copper } \\
\text { Megnesium } \\
\text { Potassium } \\
\text { Selenium } \\
\text { Arsenic } \\
\text { Beryllium } \\
\text { Cadmium } \\
\text { Copper } \\
\text { Potassium } \\
\text { Arsenic } \\
\text { Copper } \\
\text { Potassium }\end{array}$ \\
\hline 105 & $\begin{array}{c}0.0,2.0 \\
0.0,2.0 \\
2.0,4.0 \\
6.0,8.0 \\
8.0,10.0 \\
8.0,10.0 \\
10.0,12.0 \\
10.0,12.0 \\
10.0,12.0 \\
12.0,14.0 \\
12.0,14.0 \\
14.0,16.0 \\
14.0,16.0 \\
14.0,16.0 \\
14.0,16.0 \\
16.0,18.0\end{array}$ & $\begin{array}{c}1090 \\
327 \\
202 \\
1080 \\
3470 \\
1540 \\
48.9 \\
22.4 \\
65.9 \\
26.4 \\
1030 \\
29.1 \\
2.33 \\
60.4 \\
1050 \\
24.1\end{array}$ & $\begin{array}{l}\mu g / g \\
\mu g / g \\
\mu g / g \\
\mu g / g \\
\mu g / g \\
\mu g / g \\
\mu g / g \\
\mu g / g \\
\mu g / g \\
\mu g / g \\
\mu g / g \\
\mu g / g \\
\mu g / g \\
\mu g / g \\
\mu g / g \\
\mu g / g\end{array}$ & $\begin{array}{l}\text { Potassium } \\
\text { Sulfate } \\
\text { Sulfate } \\
\text { Potassium } \\
\text { Magnesium } \\
\text { Potassium } \\
\text { Arsenic } \\
\text { Selenium } \\
\text { Vanadium } \\
\text { Arsenic } \\
\text { Potassium } \\
\text { Arsenic } \\
\text { Beryllium } \\
\text { Cobalt } \\
\text { Potassium } \\
\text { Thallium }\end{array}$ \\
\hline
\end{tabular}


TABLE 5.2-2 Soil Contaminant Concentrations Above Background (Continued)

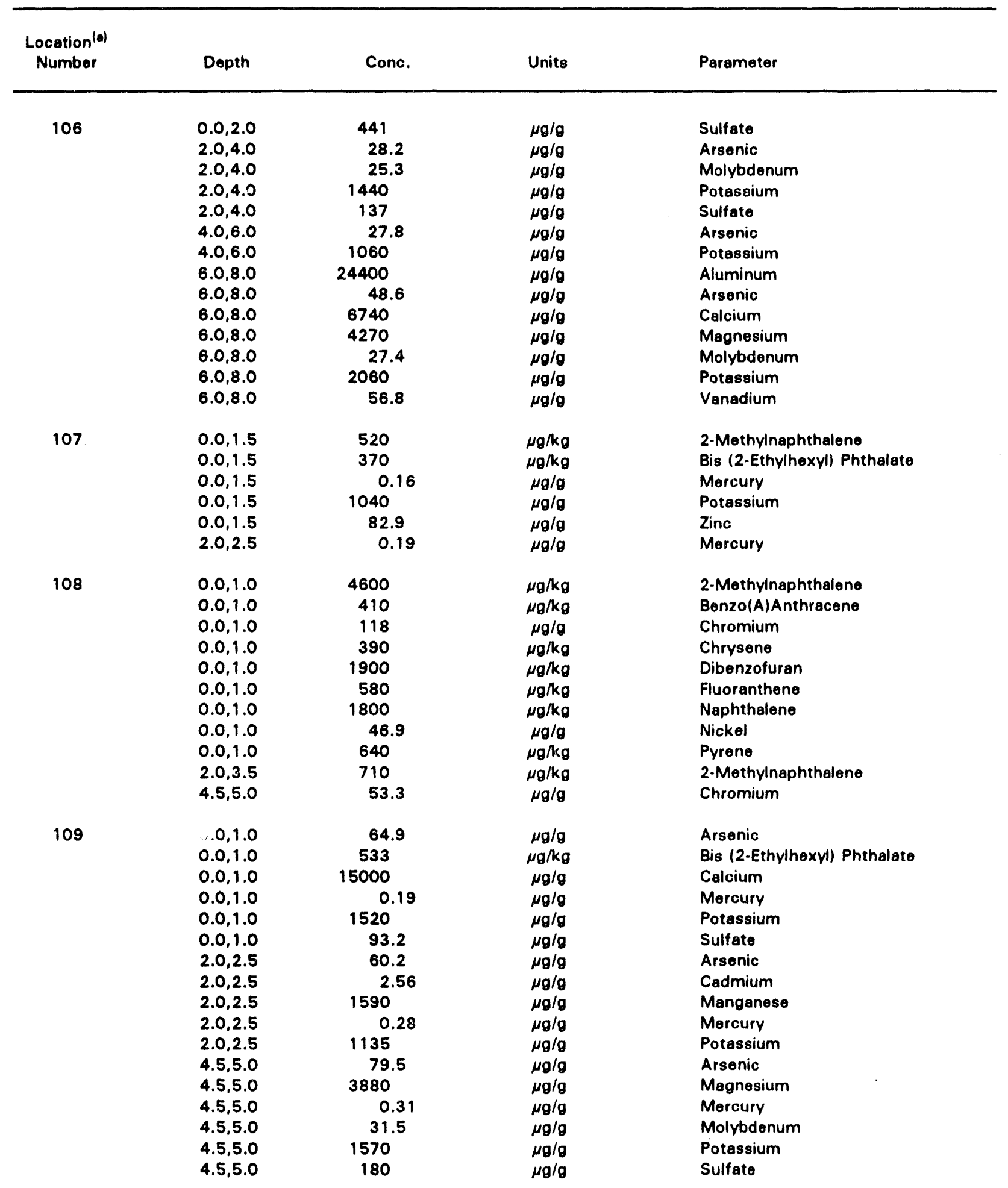


TABLE 5.2-2 Soil Contaminant Concentrations Above Background (Continued)

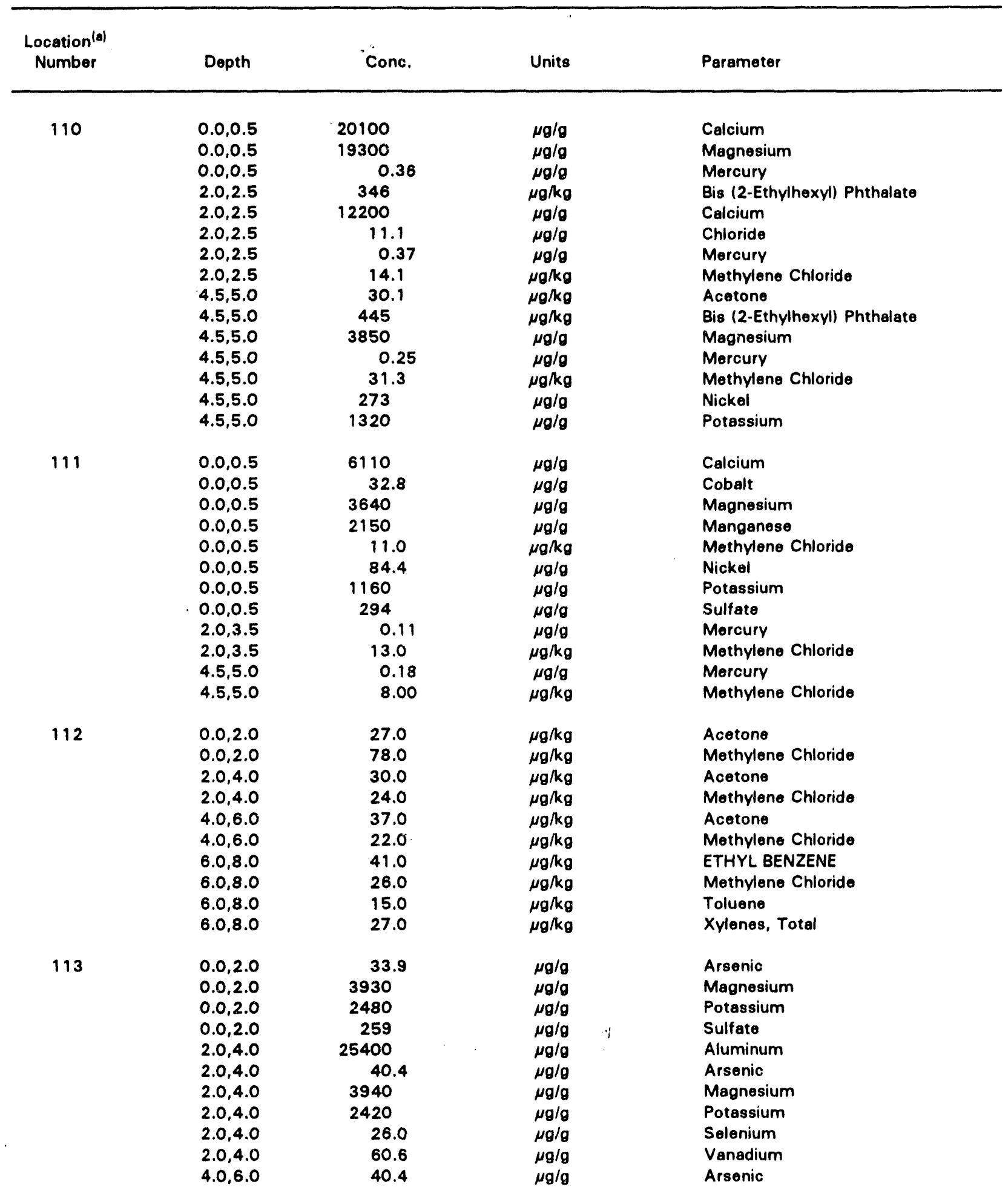


TABLE 5.2-2 Soil Contaminant Concentrations Above Background (Continued)

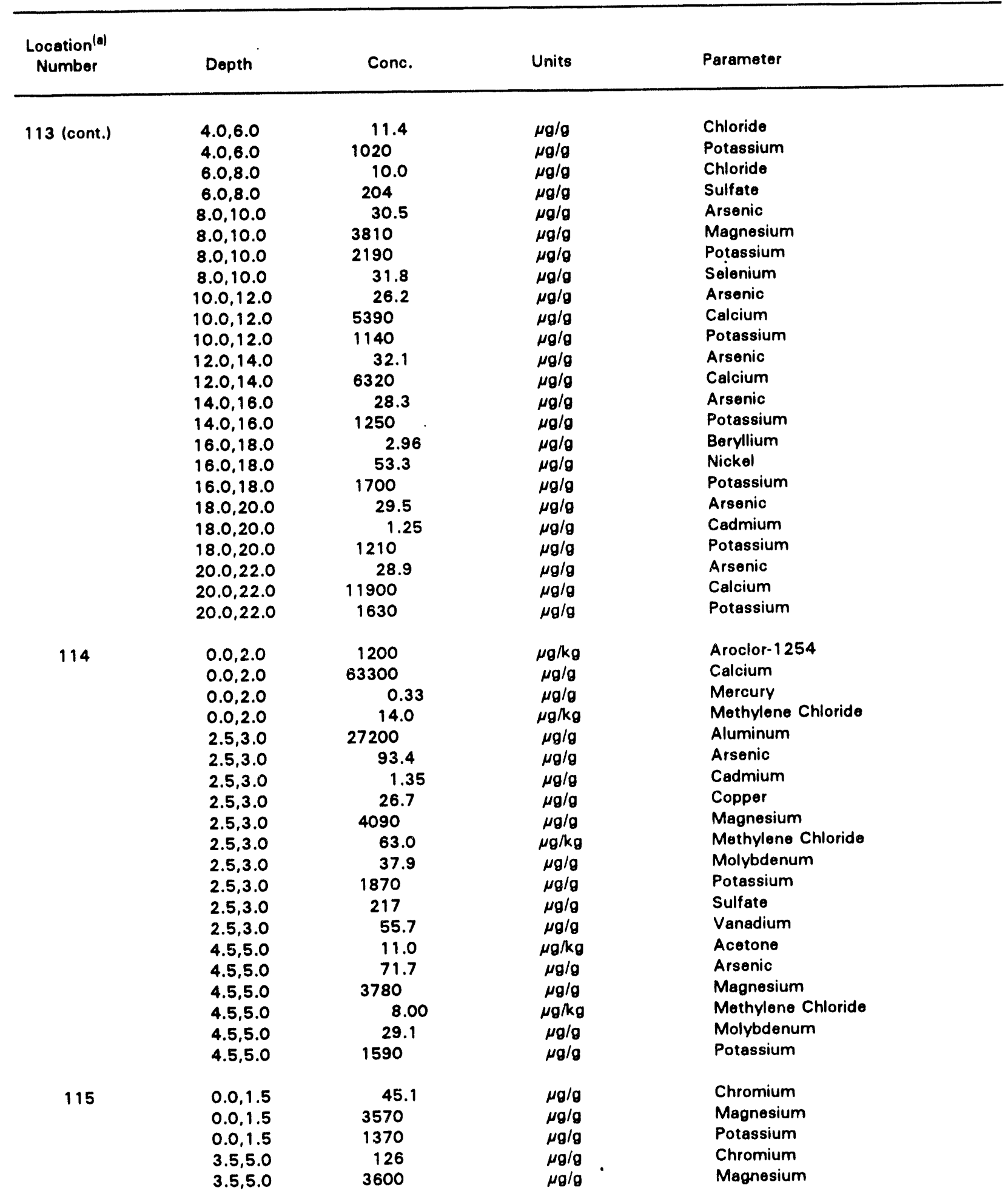


TABLE 5.2-2 Soil Contaminant Concentrations Above Background (Continued)

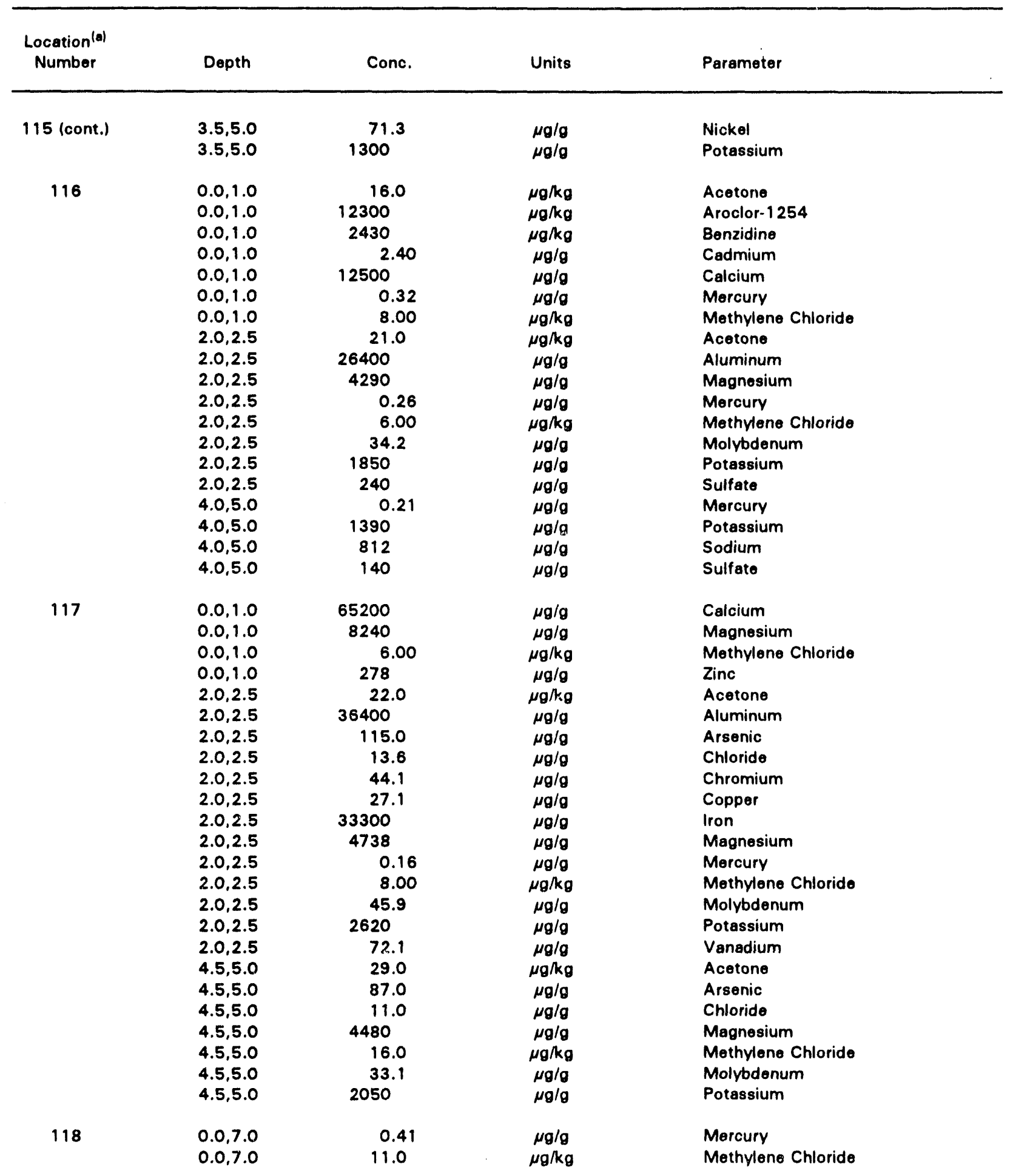


TABLE 5.2-2 Soil Contaminant Concentrations Above Background (Continued)

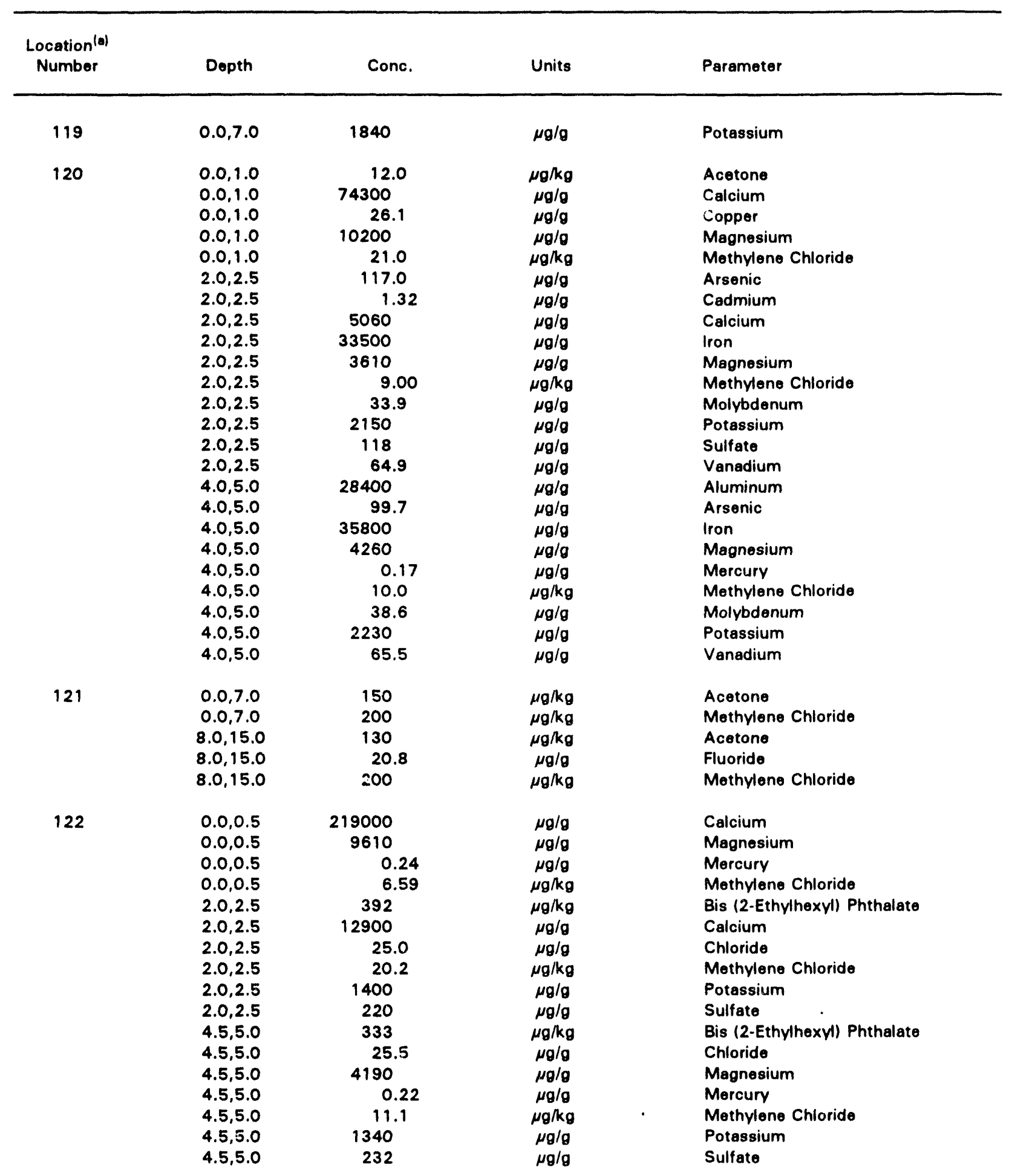


TABLE 5.2-2 Soil Contaminant Concentrations Above Background (Continued)

\begin{tabular}{|c|c|c|c|c|}
\hline $\begin{array}{l}\text { Location } \\
\text { Numbir) }\end{array}$ & Depth & Conc. & Units & Parameter \\
\hline 123 & $\begin{array}{l}0.0,7.0 \\
8.0,15.0\end{array}$ & $\begin{array}{l}110 \\
16.8\end{array}$ & $\begin{array}{c}\mu g / k g \\
\mu g / g\end{array}$ & $\begin{array}{l}\text { Methylene Chloride } \\
\text { Fluoride }\end{array}$ \\
\hline 124 & $\begin{array}{l}0.0,7.0 \\
0.0,7.0 \\
0.0,7.0 \\
0.0,7.0\end{array}$ & $\begin{array}{c}26600 \\
19.0 \\
25.7 \\
1590\end{array}$ & $\begin{array}{c}\mu \mathrm{g} / \mathrm{g} \\
\mu \mathrm{g} / \mathbf{k g} \\
\mu \mathrm{g} / \mathrm{g} \\
\mu \mathrm{g} / \mathrm{g}\end{array}$ & $\begin{array}{l}\text { Aluminum } \\
\text { Methylene Chloride } \\
\text { Molybdenum } \\
\text { Potassium }\end{array}$ \\
\hline 125 & $\begin{array}{c}0.0,2.0 \\
2.0,4.0 \\
2.0,4.0 \\
4.0,6.0 \\
8.0,10.0 \\
8.0,10.0\end{array}$ & $\begin{array}{r}1440 \\
4040 \\
1700 \\
1160 \\
27300 \\
25.3\end{array}$ & $\begin{array}{l}\mu g / g \\
\mu g / g \\
\mu g / g \\
\mu g / g \\
\mu g / g \\
\mu g / g\end{array}$ & $\begin{array}{l}\text { Potassium } \\
\text { Magnesium } \\
\text { Potassium } \\
\text { Potassium } \\
\text { Aluminum } \\
\text { Molybdenum }\end{array}$ \\
\hline 126 & $\begin{array}{c}0.0,2.0 \\
2.0,4.0 \\
4.0,6.0 \\
8.0,10.0 \\
8.0,10.0 \\
8.0,10.0 \\
10.0,12.0 \\
10.0,12.0 \\
10.0,12.0 \\
10.0,12.0\end{array}$ & $\begin{array}{c}1190 \\
93.5 \\
31.1 \\
27600 \\
26.9 \\
1030 \\
33600 \\
37.1 \\
29.4 \\
1410\end{array}$ & $\begin{array}{l}\mu g / g \\
\mu g / g \\
\mu g / g \\
\mu g / g \\
\mu g / g \\
\mu g / g \\
\mu g / g \\
\mu g / g \\
\mu g / g \\
\mu g / g\end{array}$ & $\begin{array}{l}\text { Potassium } \\
\text { Sulfate } \\
\text { Cobalt } \\
\text { Aluminum } \\
\text { Arsenic } \\
\text { Potassium } \\
\text { Aluminum } \\
\text { Arsenic } \\
\text { Molybdenum } \\
\text { Potassium }\end{array}$ \\
\hline 127 & $\begin{array}{l}0.0,2.0 \\
4.0,6.0\end{array}$ & $\begin{array}{r}1070 \\
43.9\end{array}$ & $\begin{array}{l}\mu g / g \\
\mu g / g\end{array}$ & $\begin{array}{l}\text { Potassium } \\
\text { Lead }\end{array}$ \\
\hline 128 & $\begin{array}{l}0.0,2.0 \\
2.0,4.0 \\
2.0,4.0 \\
2.0,4.0 \\
4.0,6.0 \\
4.0,6.0 \\
4.0,6.0 \\
4.0,6.0 \\
4.0,6.0 \\
6.0,8.0 \\
6.0,8.0 \\
6.0,8.0\end{array}$ & $\begin{array}{c}19600 \\
26.6 \\
17900 \\
100 \\
35000 \\
29.4 \\
33.1 \\
1250 \\
98.3 \\
26100 \\
32.7 \\
25.4\end{array}$ & $\begin{array}{l}\mu g / g \\
\mu g / g \\
\mu g / g \\
\mu g / g \\
\mu g / g \\
\mu g / g \\
\mu g / g \\
\mu g / g \\
\mu g / g \\
\mu g / g \\
\mu g / g \\
\mu g / g\end{array}$ & $\begin{array}{l}\text { Calcium } \\
\text { Arsenic } \\
\text { Calcium } \\
\text { Sulfate } \\
\text { Aluminum } \\
\text { Arsenic } \\
\text { Molybdenum } \\
\text { Potassium } \\
\text { Sulfate } \\
\text { Aluminum } \\
\text { Arsenic } \\
\text { Molybdenum }\end{array}$ \\
\hline 129 & $\begin{array}{l}0.0,2.0 \\
2.0,4.0 \\
2.0,4.0 \\
2.0,4.0 \\
2.0,4.0 \\
2.0,4.0 \\
4.0,6.0 \\
4.0,6.0 \\
4.0,6.0\end{array}$ & $\begin{array}{c}1280 \\
31.6 \\
35.4 \\
1670 \\
128 \\
56.0 \\
6890 \\
1270 \\
178\end{array}$ & $\begin{array}{l}\mu \mathrm{g} / \mathrm{g} \\
\mu \mathrm{g} / \mathrm{g} \\
\mu \mathrm{g} / \mathrm{g} \\
\mu \mathrm{g} / \mathrm{g} \\
\mu \mathrm{g} / \mathrm{g} \\
\mu \mathrm{g} / \mathrm{g} \\
\mu \mathrm{g} / \mathrm{g} \\
\mu \mathrm{g} / \mathrm{g} \\
\mu \mathrm{g} / \mathrm{g}\end{array}$ & $\begin{array}{l}\text { Potassium } \\
\text { Arsenic } \\
\text { Cobalt } \\
\text { Potassium } \\
\text { Sul.ate } \\
\text { Vanadium } \\
\text { Calcium } \\
\text { Potassium } \\
\text { Sulfate }\end{array}$ \\
\hline
\end{tabular}


TABLE 5.2-2 Soil Contaminant Concentrations Above Background (Continued)

\begin{tabular}{|c|c|c|c|c|}
\hline $\begin{array}{l}\text { Location(日) } \\
\text { Number }\end{array}$ & Depth & Conc. & Units & Parameter \\
\hline 129 (cont.) & $\begin{array}{l}6.0,8.0 \\
6.0,8.0 \\
8.0,10.0 \\
8.0,10.0\end{array}$ & $\begin{array}{r}25300 \\
1020 \\
25900 \\
985\end{array}$ & $\begin{array}{l}\mu g / \theta \\
\mu g / g \\
\mu g / g \\
\mu g / g\end{array}$ & $\begin{array}{l}\text { Aluminum } \\
\text { Potassium } \\
\text { Aluminum } \\
\text { Potassium }\end{array}$ \\
\hline 130 & $\begin{array}{l}0.0,7.0 \\
0.0,7.0 \\
0.0,7.0 \\
0.0,7.0 \\
0.0,7.0 \\
0.0,7.0\end{array}$ & $\begin{array}{c}320 \\
15400 \\
14.1 \\
4770 \\
0.20 \\
51.0\end{array}$ & $\begin{array}{c}\mu g / k g \\
\mu g / g \\
\mu g / g \\
\mu g / g \\
\mu g / g \\
\mu g / k g\end{array}$ & $\begin{array}{l}\text { Acetone } \\
\text { Calcium } \\
\text { Chloride } \\
\text { Magnesium } \\
\text { Mercury } \\
\text { Methylene Chloride }\end{array}$ \\
\hline 131 & $\begin{array}{l}0.0,2.0 \\
2.0,4.0 \\
2.0,4.0 \\
6.0,8.0 \\
6.0,8.0 \\
8.0,10.0\end{array}$ & $\begin{array}{c}9140 \\
1130 \\
129 \\
25800 \\
26.9 \\
25200\end{array}$ & 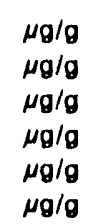 & $\begin{array}{l}\text { Calcium } \\
\text { Potassium } \\
\text { Sulfate } \\
\text { Aluminum } \\
\text { Arsonic } \\
\text { Aluminum }\end{array}$ \\
\hline 132 & $\begin{array}{l}0.0,1.0 \\
0.0,1.0 \\
4.5,5.0\end{array}$ & $\begin{array}{c}866 \\
7.00 \\
153\end{array}$ & $\begin{array}{c}\mu \mathrm{g} / \mathrm{kg} \\
\mu \mathrm{g} / \mathrm{kg} \\
\mu \mathrm{g} / \mathrm{g}\end{array}$ & $\begin{array}{l}\text { 2-Methylnaphthalene } \\
\text { Methylene Chloride } \\
\text { Sulfate }\end{array}$ \\
\hline 133 & $\begin{array}{l}0.0,2.0 \\
0.0,2.0 \\
0.0,2.0 \\
0.0,2.0 \\
0.0,2.0 \\
0.0,2.0 \\
0.0,2.0 \\
0.0,2.0 \\
2.0,4.0 \\
2.0,4.0 \\
4.0,6.0 \\
4.0,6.0 \\
6.0,8.0 \\
8.0,10.0\end{array}$ & $\begin{array}{c}34900 \\
41.500 \\
27.400 \\
3810 \\
27.000 \\
996.000 \\
30.0 \\
58.700 \\
305.000 \\
159 \\
26500 \\
14.7 \\
30200 \\
26.8\end{array}$ & $\begin{array}{l}\mu \mathrm{g} / \mathrm{g} \\
\mu \mathrm{g} / \mathrm{g} \\
\mu \mathrm{g} / \mathrm{g} \\
\mu \mathrm{g} / \mathrm{g} \\
\mu \mathrm{g} / \mathrm{g} \\
\mu \mathrm{g} / \mathrm{g} \\
\mu \mathrm{g} / \mathrm{g} \\
\mu \mathrm{g} / \mathrm{g} \\
\mu \mathrm{g} / \mathrm{g} \\
\mu \mathrm{g} / \mathrm{g} \\
\mu \mathrm{g} / \mathrm{g} \\
\mu \mathrm{g} / \mathrm{g} \\
\mu \mathrm{g} / \mathrm{g} \\
\mu \mathrm{g} / \mathrm{g}\end{array}$ & $\begin{array}{l}\text { Aluminum } \\
\text { Chromium } \\
\text { Cobalt } \\
\text { Magnesium } \\
\text { Molybdenum } \\
\text { Potassium } \\
\text { Seienium } \\
\text { Vanadium } \\
\text { Chromium } \\
\text { Nickel } \\
\text { Aluminum } \\
\text { Fluoride } \\
\text { Aluminum } \\
\text { Selenium }\end{array}$ \\
\hline 134 & $\begin{array}{l}0.0,7.0 \\
0.0,7.0 \\
0.0,7.0 \\
0.0,7.0 \\
0.0,7.0\end{array}$ & $\begin{array}{l}62.5 \\
375 \\
10.8 \\
0.17 \\
47.5\end{array}$ & $\begin{array}{l}\mu g / k g \\
\mu g / k g \\
\mu g / g \\
\mu g / g \\
\mu g / k g\end{array}$ & $\begin{array}{l}\text { Acetone } \\
\text { Bis (2-Ethylhexyl) Phthalate } \\
\text { Chloride } \\
\text { Mercury } \\
\text { Methylene Chloride }\end{array}$ \\
\hline 135 & $\begin{array}{l}0.0,7.0 \\
0.0,7.0 \\
0.0,7.0 \\
0.0,7.0 \\
0.0,7.0\end{array}$ & $\begin{array}{c}25300 \\
4280 \\
120 \\
28.1 \\
59.1\end{array}$ & $\begin{array}{c}\mu g / g \\
\mu g / g \\
\mu g / k g \\
\mu g / g \\
\mu g / g\end{array}$ & $\begin{array}{l}\text { Aluminum } \\
\text { Magnesium } \\
\text { Methylene Chloride } \\
\text { Molybdenum } \\
\text { Vanadium }\end{array}$ \\
\hline
\end{tabular}


TABLE 5.2-2 Soil Contaminant Concentrations Above Background (Continued)

\begin{tabular}{|c|c|c|c|c|}
\hline $\begin{array}{l}\text { Location }^{(a)} \\
\text { Number }\end{array}$ & Depth & Conc. & Units & Parameter \\
\hline 136 & $\begin{array}{l}0.0,7.0 \\
0.0,7.0 \\
0.0,7.0 \\
8.0,15.0\end{array}$ & $\begin{array}{c}1560 \\
14.0 \\
111 \\
6.00\end{array}$ & $\begin{array}{c}\mu g / g \\
\mu g / k g \\
\mu g / g \\
\mu g / k g\end{array}$ & $\begin{array}{l}\text { Manganese } \\
\text { Methylene Chloride } \\
\text { Sulfate } \\
\text { Methylene Chloride }\end{array}$ \\
\hline 137 & $\begin{array}{l}0.0,0.5 \\
0.0,0.5 \\
0.0,0.5 \\
0.0,0.5 \\
0.0,0.5 \\
0.0,0.5 \\
0.0,0.5 \\
0.0,0.5 \\
0.0,0.5 \\
2.0,2.5 \\
2.0,2.5 \\
2.0,2.5 \\
2.0,2.5 \\
2.0,2.5 \\
2.0,2.5 \\
2.0,2.5 \\
2.0,2.5 \\
2.0,3.0 \\
4.5,5.0 \\
4.5,5.0\end{array}$ & $\begin{array}{c}33.0 \\
28500 \\
1880000 \\
14100 \\
4050 \\
1840 \\
9.00 \\
2520 \\
67.6 \\
51.0 \\
27500 \\
620 \\
3650 \\
12500 \\
15.0 \\
2160 \\
60.5 \\
3650 \\
50.0 \\
150\end{array}$ & $\begin{array}{c}\mu g / k g \\
\mu g / g \\
\mu g / k g \\
\mu g / g \\
\mu g / g \\
\mu g / g \\
\mu g / k g \\
\mu g / g \\
\mu g / g \\
\mu g / k g \\
\mu g / g \\
\mu g / k g \\
\mu g / g \\
\mu g / g \\
\mu g / k g \\
\mu g / g \\
\mu g / g \\
\mu g / g \\
\mu g / k g \\
\mu g / k g\end{array}$ & $\begin{array}{l}\text { Acetone } \\
\text { Aluminum } \\
\text { Bis (2-Ethylhexyl) Phthalate } \\
\text { Calcium } \\
\text { Magnesium } \\
\text { Manganese } \\
\text { Methylene Chloride } \\
\text { Potessium } \\
\text { Vanadium } \\
\text { Acetone } \\
\text { Aluminum } \\
\text { Bis (2-Ethylhexyl) Phthalate } \\
\text { Magnesium } \\
\text { Manganese } \\
\text { Methylene Chloride } \\
\text { Potassium } \\
\text { Vanadium } \\
\text { Magnesium } \\
\text { Acetone } \\
\text { Heptachlor }\end{array}$ \\
\hline 138 & $\begin{array}{l}0.0,7.0 \\
0.0,7.0 \\
0.0,7.0 \\
0.0,7.0 \\
8.0,15.0 \\
8.0,15.0 \\
8.0,15.0 \\
8.0,15.0 \\
8.0,15.0 \\
8.0,15.0\end{array}$ & $\begin{array}{c}48.0 \\
11.4 \\
1750 \\
140 \\
67.0 \\
29100 \\
0.12 \\
190 \\
1160 \\
14.4\end{array}$ & $\begin{array}{c}\mu g / k g \\
\mu g / g \\
\mu g / g \\
\mu g / k g \\
\mu g / k g \\
\mu g / g \\
\mu g / g \\
\mu g / k g \\
\mu g / g \\
\mu g / g\end{array}$ & $\begin{array}{l}\text { Acetone } \\
\text { Chloride } \\
\text { Manganese } \\
\text { Methylene Chloride } \\
\text { Acetone } \\
\text { Aluminum } \\
\text { Mercury } \\
\text { Methylene Chloride } \\
\text { Potassium } \\
\text { Total Organic Halogens }\end{array}$ \\
\hline 139 & $\begin{array}{l}0.0,7.0 \\
0.0,7.0 \\
0.0,7.0 \\
0.0,7.0 \\
8.0,15.0 \\
8.0,15.0\end{array}$ & $\begin{array}{c}14.0 \\
2910 \\
782 \\
102 \\
10.0 \\
1390\end{array}$ & $\begin{array}{c}\mu g / k g \\
\mu g / g \\
\mu g / g \\
\mu g / g \\
\mu g / k g \\
\mu g / g\end{array}$ & $\begin{array}{l}\text { Methylene Chloride } \\
\text { Nitrate } \\
\text { Sodium } \\
\text { Sulfate } \\
\text { Methylene Chloride } \\
\text { Nitrate }\end{array}$ \\
\hline 140 & $\begin{array}{c}0.0,7.0 \\
0.0,7.0 \\
0.0,7.0 \\
8.0,15.0 \\
8.0,15.0 \\
8.0,15.0 \\
16.0,23.0\end{array}$ & $\begin{array}{l}22.0 \\
42.5 \\
18.0 \\
39.0 \\
44.0 \\
10.0 \\
22.0\end{array}$ & $\begin{array}{c}\mu g / k g \\
\mu g / g \\
\mu g / k g \\
\mu g / k g \\
\mu g / g \\
\mu g / k g \\
\mu g / k g\end{array}$ & $\begin{array}{l}\text { Acetone } \\
\text { Chromium } \\
\text { Methylene Chloride } \\
\text { Acetone } \\
\text { Arsenic } \\
\text { Methylene Chloride } \\
\text { Acetone }\end{array}$ \\
\hline
\end{tabular}


TABLE 5.2-2 Soil Contaminant Concentrations Above Background (Continued)

\begin{tabular}{|c|c|c|c|c|}
\hline $\begin{array}{l}\text { Locetion }^{(a)} \\
\text { Number }\end{array}$ & Depth & Conc. & Units & Paramoter \\
\hline \multirow[t]{11}{*}{140 (cont.) } & $16.0,23.0$ & 73.0 & $\mu g / g$ & Arsenic \\
\hline & $16.0,23.0$ & 689 & $\mu g / g$ & Barium \\
\hline & $16.0,23.0$ & 4.88 & $\mu g / g$ & Bervllium \\
\hline & $16.0,23.0$ & 10600 & $\mu g / g$ & Calcium \\
\hline & $16.0,23.0$ & 71.2 & $\mu g / g$ & Cobait \\
\hline & $16.0,23.0$ & 3260 & $\mu \mathrm{g} / \mathrm{g}$ & Manganese \\
\hline & $16.0,23.0$ & 7.00 & $\mu \mathrm{g} / \mathrm{kg}$ & Mothylene Chloride \\
\hline & $16.0,23.0$ & 34.1 & $\mu g / g$ & Molybdenum \\
\hline & $16.0,23.0$ & 116 & $\mu g / \sigma$ & Nickol \\
\hline & $16.0,23.0$ & 166 & $\mu g / g$ & Nitrate \\
\hline & $16.0,23.0$ & 95.2 & $\mu g / g$ & Zinc \\
\hline \multirow[t]{12}{*}{141} & $0.0,7.0$ & 110 & $\mu g / k g$ & Acetone \\
\hline & $0.0,7.0$ & 27300 & $\mu \mathrm{g} / \mathrm{g}$ & Aluminum \\
\hline & $0.0,7.0$ & 14.0 & $\mu g / g$ & Fluoride \\
\hline & $0.0,7.0$ & 180 & $\mu \mathrm{g} / \mathrm{kg}$ & Methylene Chloride \\
\hline & $0.0,7.0$ & 26.5 & $\mu g / g$ & Molybdenum \\
\hline & $0.0,7.0$ & 57.6 & $\mu g / g$ & Vanadium \\
\hline & $8.0,15.0$ & 33800 & $\mu g / g$ & Aluminum \\
\hline & $8.0,15.0$ & 2.51 & $\mu g / g$ & Bervllium \\
\hline & $8.0,15.0$ & 21.2 & $\mu g / g$ & Fluoride \\
\hline & 8.0 .15 .0 & 31000 & $\mu g / g$ & Iron \\
\hline & $8.0,15.0$ & 130 & $\mu \mathrm{g} / \mathrm{kg}$ & Methylene Chloride \\
\hline & $8.0,15.0$ & 33.7 & $\mu g / g$ & Molybdenum \\
\hline \multirow[t]{3}{*}{142} & 0.0 .7 .0 & 33.0 & $\mu g / \mathbf{k g}$ & Acetone \\
\hline & $0.0,7.0$ & 28.0 & $\mu g / k g$ & Chloroform \\
\hline & 0.0 .7 .0 & 47.0 & $\mu g / k g$ & Methylene Chloride \\
\hline \multirow[t]{4}{*}{143} & $0.0,7.0$ & 26.0 & $\mu \mathrm{g} / \mathrm{kg}$ & Acetone \\
\hline & $0.0,7.0$ & 42.0 & $\mu g / k g$ & Chloroform \\
\hline & $0.0,7.0$ & 110 & $\mu g / k g$ & Methylene Chloride \\
\hline & 0.0 .7 .0 & 107 & $\mu g / g$ & Sulfate \\
\hline \multirow[t]{5}{*}{144} & $0.0,7.0$ & 600 & $\mu g / k g$ & Acetone \\
\hline & $0.0,7.0$ & 37.0 & $\mu g / g$ & Arsenic \\
\hline & $0.0,7.0$ & 260 & $\mu \mathrm{g} / \mathrm{kg}$ & Methylene Chloride \\
\hline & $0.0,7.0$ & 158 & $\mu g / g$ & Sulfate \\
\hline & 0.0 .7 .0 & 138 & $\mu \mathrm{g} / \mathrm{kg}$ & Toluene \\
\hline \multirow[t]{5}{*}{145} & $0.0,7.0$ & 12.9 & $\mu g / g$ & Chloride \\
\hline & $0.0,7.0$ & 48.7 & $\mu g / g$ & Cobalt \\
\hline & 0.0 .7 .0 & 2730 & $\mu g / g$ & Manganese \\
\hline & 0.0 .7 .0 & 0.18 & $\mu g / \theta$ & Mercury \\
\hline & 0.0 .7 .0 & 186 & $\mu g / g$ & Sulfate \\
\hline \multirow[t]{5}{*}{146} & $0.0,2.0$ & 28900 & $\mu g / g$ & Aluminum \\
\hline & $0.0,2.0$ & 3930 & $\mu g / g$ & Magnesium \\
\hline & $0.0,2.0$ & 1690 & $\mu \mathrm{g} / \mathrm{g}$ & Manganese \\
\hline & $2.0,4.0$ & 26900 & $\mu g / g$ & Aluminum \\
\hline & $6.0,8.0$ & 31800 & $\mu g / g$ & Aluminum \\
\hline
\end{tabular}


TABLE 5.2-2 Soil Contaminant Concentrations Above Background (Continued)

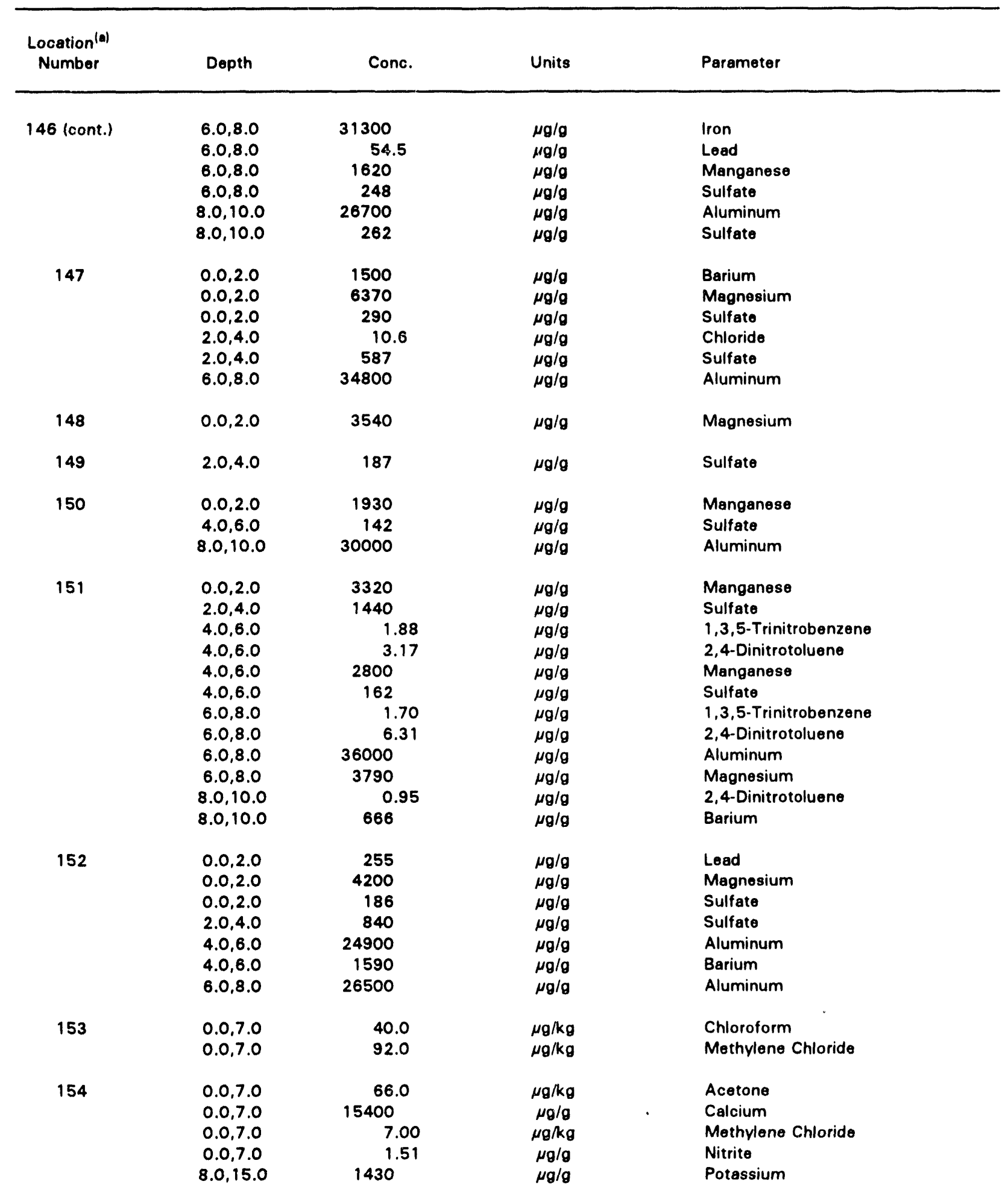


TABLE 5.2-2 Soil Contaminant Concentrations Above Background (Continued)

\begin{tabular}{|c|c|c|c|c|}
\hline $\begin{array}{l}\text { Location } \\
\text { Number }\end{array}$ & Depth & Conc. & Units & Parameter \\
\hline 154 (cont.) & $\begin{array}{l}16.0,22.0 \\
16.0,22.0\end{array}$ & $\begin{array}{l}71.0 \\
13.9\end{array}$ & $\begin{array}{c}\mu g / k g \\
\mu g / g\end{array}$ & $\begin{array}{l}\text { Acetone } \\
\text { Fluoride }\end{array}$ \\
\hline 155 & $\begin{array}{l}0.0,1.0 \\
0.0,1.0 \\
0.0,1.0\end{array}$ & $\begin{array}{l}60.0 \\
3800 \\
1420\end{array}$ & $\begin{array}{l}\mu g / g \\
\mu g / g \\
\mu g / g\end{array}$ & $\begin{array}{l}\text { Lead } \\
\text { Magnesium } \\
\text { Sulfate }\end{array}$ \\
\hline 156 & $\begin{array}{l}0.0,7.0 \\
0.0,7.0 \\
8.0,15.0\end{array}$ & $\begin{array}{l}14.0 \\
14.0 \\
2.60\end{array}$ & $\begin{array}{c}\mu \mathrm{g} / \mathrm{kg} \\
\mu \mathrm{g} / \mathrm{kg} \\
\mu \mathrm{g} / \mathrm{g}\end{array}$ & $\begin{array}{l}\text { Acetone } \\
\text { Methylene Chloride } \\
\text { Nitrite }\end{array}$ \\
\hline 157 & $\begin{array}{l}0.0 .7 .0 \\
0.0 .7 .0\end{array}$ & $\begin{array}{l}40.1 \\
3600\end{array}$ & $\begin{array}{l}\mu g / g \\
\mu g / g\end{array}$ & $\begin{array}{l}\text { Copper } \\
\text { Potassium }\end{array}$ \\
\hline 158 & $0.0,1.0$ & 1900 & $\mu g / g$ & Lead \\
\hline 159 & $\begin{array}{l}0.0,7.0 \\
0.0,7.0 \\
0.0,7.0 \\
8.0,15.0 \\
8.0,15.0 \\
8.0,15.0\end{array}$ & $\begin{array}{c}375 \\
0.13 \\
247 \\
73.0 \\
1.31 \\
23.0\end{array}$ & $\begin{array}{c}\mu \mathrm{g} / \mathrm{kg} \\
\mu \mathrm{g} / \mathrm{g} \\
\mu \mathrm{g} / \mathrm{g} \\
\mu \mathrm{g} / \mathrm{kg} \\
\mu \mathrm{g} / \mathrm{g} \\
\mu \mathrm{g} / \mathrm{kg}\end{array}$ & $\begin{array}{l}\text { Acetone } \\
\text { Mercury } \\
\text { Sulfate } \\
\text { Acetone } \\
\text { Cadmium } \\
\text { Methylene Chloride }\end{array}$ \\
\hline 160 & $\begin{array}{l}0.0,2.0 \\
2.0,4.0 \\
2.0,4.0 \\
4.0,6.0\end{array}$ & $\begin{array}{c}2180 \\
1.21 \\
647 \\
2.02\end{array}$ & $\begin{array}{l}\mu g / g \\
\mu g / g \\
\mu g / g \\
\mu g / g\end{array}$ & $\begin{array}{l}\text { Manganese } \\
\text { 1,3,5-Trinitrobenzene } \\
2,4,6 \text {-Trinitrotoluene } \\
2,4,6 \text {-Trinitrotoluene }\end{array}$ \\
\hline 161 & $\begin{array}{l}0.0,2.0 \\
2.0,4.0 \\
4.0,6.0 \\
4.0,6.0 \\
4.0,6.0 \\
6.0,8.0\end{array}$ & $\begin{array}{r}52.7 \\
28.2 \\
35.6 \\
35800 \\
42.6 \\
28.9\end{array}$ & $\begin{array}{l}\mu g / g \\
\mu g / g \\
\mu g / g \\
\mu g / g \\
\mu g / g \\
\mu g / g\end{array}$ & $\begin{array}{l}\text { Chromium } \\
\text { Arsenic } \\
\text { Arsenic } \\
\text { Calcium } \\
\text { Chromium } \\
\text { Arsenic }\end{array}$ \\
\hline 162 & $\begin{array}{l}0.0,7.0 \\
0.0,7.0 \\
0.0,7.0 \\
8.0,15.0 \\
8.0,15.0 \\
8.0,15.0\end{array}$ & $\begin{array}{c}21.0 \\
5470 \\
0.20 \\
61.0 \\
5200 \\
0.12\end{array}$ & $\begin{array}{c}\mu g / k g \\
\mu g / g \\
\mu g / g \\
\mu g / g \\
\mu g / g \\
\mu g / g\end{array}$ & $\begin{array}{l}\text { Acetone } \\
\text { Calcium } \\
\text { Mercury } \\
\text { Arsenic } \\
\text { Calcium } \\
\text { Mercury }\end{array}$ \\
\hline 163 & $\begin{array}{l}0.0,1.0 \\
0.0,1.0\end{array}$ & $\begin{array}{l}52.1 \\
2590\end{array}$ & $\begin{array}{l}\mu g / g \\
\mu g / g\end{array}$ & $\begin{array}{l}\text { Lead } \\
\text { Sulfate }\end{array}$ \\
\hline 164 & $\begin{array}{c}0.0,2.0 \\
8.0,10.0\end{array}$ & $\begin{array}{r}6280 \\
34000\end{array}$ & $\begin{array}{l}\mu g / g \\
\mu g / g\end{array}$ & $\begin{array}{l}\text { Magnesium } \\
\text { Iron }\end{array}$ \\
\hline 165 & $\begin{array}{l}0.0,2.0 \\
0.0,2.0 \\
0.0,2.0\end{array}$ & $\begin{array}{r}24600 \\
16.1 \\
18.2\end{array}$ & $\begin{array}{l}\mu g / g \\
\mu g / g \\
\mu g / g\end{array}$ & $\begin{array}{l}\text { Aluminum } \\
\text { Chloride } \\
\text { Fluoride }\end{array}$ \\
\hline
\end{tabular}




\section{TABLE 5.2-2 Soil Contaminant Concentrations Above Background (Continued)}

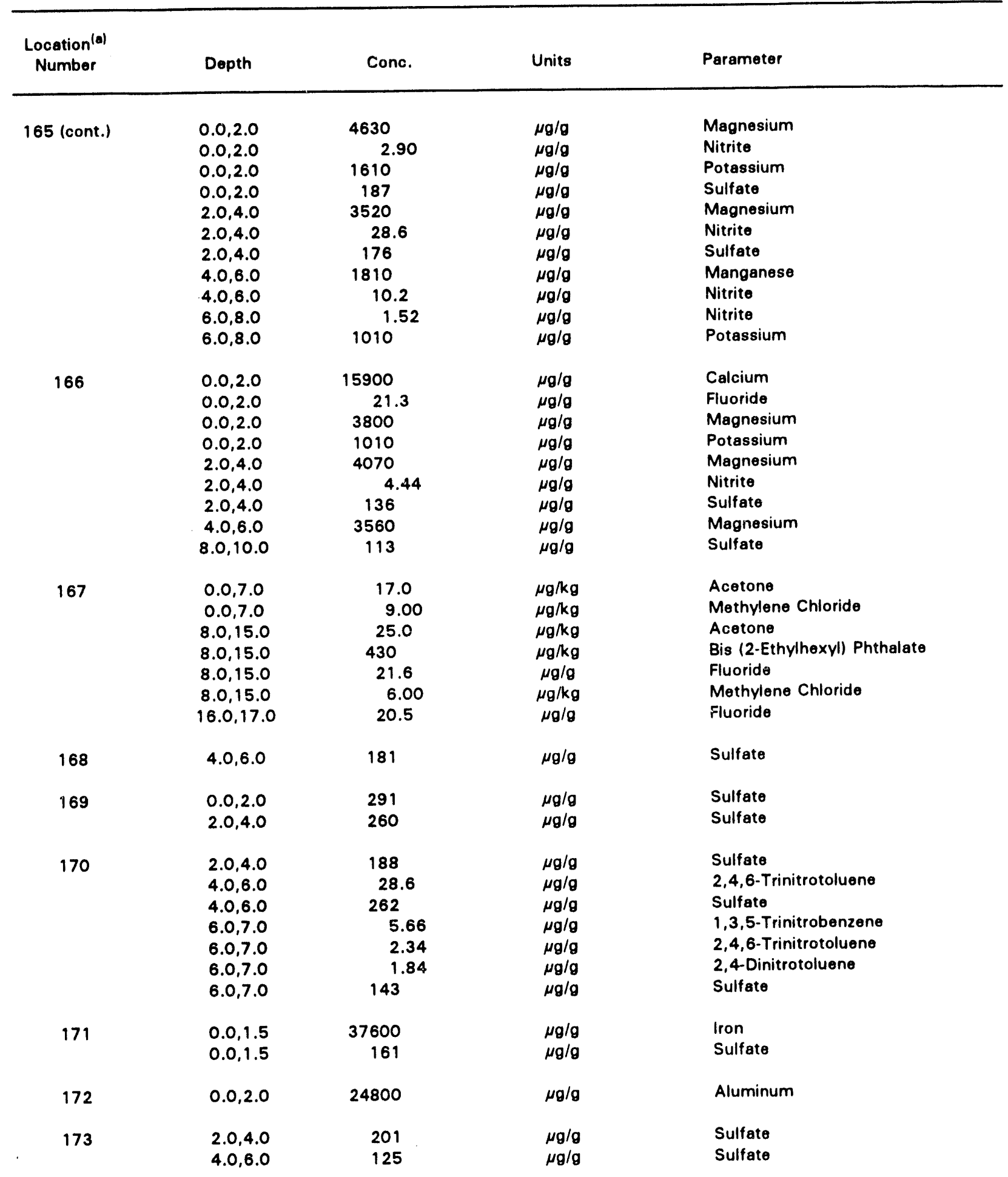


TABLE 5.2-2 Soil Contaminant Concentrations Above Background (Continued)

\begin{tabular}{|c|c|c|c|c|}
\hline $\begin{array}{l}\text { Location(a) } \\
\text { Number }\end{array}$ & Depth & Conc. & Units & Parumeter \\
\hline 174 & $\begin{array}{l}0.0,7.0 \\
0.0 .7 .0 \\
0.0,7.0 \\
0.0 .7 .0 \\
0.0,7.0 \\
8.0,15.0 \\
8.0,15.0 \\
8.0,15.0 \\
8.0,15.0\end{array}$ & $\begin{array}{c}35000 \\
560 \\
0.15 \\
11.0 \\
409 \\
25.0 \\
35057 \\
3687 \\
0.44\end{array}$ & $\begin{array}{c}\mu g / g \\
\mu g / k g \\
\mu g / g \\
\mu g / k g \\
\mu g / g \\
\mu g / k g \\
\mu g / g \\
\mu g / g \\
\mu g / g\end{array}$ & $\begin{array}{l}\text { Aluminum } \\
\text { Bis (2-Ethylhexyl) Phthalate } \\
\text { Mercury } \\
\text { Methylene Chloride } \\
\text { Sulfate } \\
\text { Acetone } \\
\text { Aluminum } \\
\text { Magnesium } \\
\text { Mercury }\end{array}$ \\
\hline 175 & $\begin{array}{l}0.0,7.0 \\
0.0,7.0 \\
0.0,7.0\end{array}$ & $\begin{array}{r}43.8 \\
92.1 \\
1100\end{array}$ & $\begin{array}{c}\mu g / \mathbf{k g} \\
\mu g / \mathbf{k g} \\
\mu g / g\end{array}$ & $\begin{array}{l}\text { Acetone } \\
\text { Methylene Chloride } \\
\text { Potassium }\end{array}$ \\
\hline 176 & $\begin{array}{l}0.0 .7 .0 \\
8.0,15.0 \\
8.0,15.0 \\
8.0,15.0 \\
8.0,13.0 \\
8.0,15.0 \\
8.0,15.0 \\
8.0,15.0\end{array}$ & $\begin{array}{c}8.00 \\
30.0 \\
25900 \\
1.44 \\
81.59 \\
16.0 \\
27.4 \\
142\end{array}$ & $\begin{array}{c}\mu g / k g \\
\mu g / k g \\
\mu g / g \\
\mu g / g \\
\mu g / g \\
\mu g / k g \\
\mu g / g \\
\mu g / g\end{array}$ & $\begin{array}{l}\text { Methylene Chloride } \\
\text { Acetone } \\
\text { Aluminum } \\
\text { Cadmium } \\
\text { Lead } \\
\text { Methylene Chloride } \\
\text { Molybdenum } \\
\text { Nitrate }\end{array}$ \\
\hline 177 & $\begin{array}{l}0.0,7.0 \\
0.0 .7 .0 \\
0.0 .7 .0\end{array}$ & $\begin{array}{l}27.0 \\
28.0 \\
259\end{array}$ & $\begin{array}{c}\mu g / \mathbf{k g} \\
\mu g / \mathbf{k g} \\
\mu \mathrm{g} / \mathrm{g}\end{array}$ & $\begin{array}{l}\text { Acetone } \\
\text { Methylene Chloride } \\
\text { Sulfate }\end{array}$ \\
\hline 178 & $\begin{array}{l}0.0,7.0 \\
0.0 .7 .0 \\
0.0 .7 .0\end{array}$ & $\begin{array}{c}21.0 \\
9.00 \\
162\end{array}$ & $\begin{array}{c}\mu g / k g \\
\mu g / k g \\
\mu g / g\end{array}$ & $\begin{array}{l}\text { Acetone } \\
\text { Methylene Chloride } \\
\text { Sulfate }\end{array}$ \\
\hline 179 & $\begin{array}{l}0.0,7.0 \\
0.0,7.0 \\
0.0,7.0 \\
8.0 .15 .0 \\
8.0,15.0\end{array}$ & $\begin{array}{l}13.0 \\
39.5 \\
13.0 \\
26.0 \\
30.0\end{array}$ & $\begin{array}{c}\mu g / k g \\
\mu g / g \\
\mu g / k g \\
\mu g / k g \\
\mu g / g\end{array}$ & $\begin{array}{l}\text { Acetone } \\
\text { Arsenic } \\
\text { A lethylene Chloride } \\
\text { Acetone } \\
\text { Arsenic }\end{array}$ \\
\hline 180 & $\begin{array}{l}0.0 .7 .0 \\
8.0 .15 .0 \\
8.0 .15 .0\end{array}$ & $\begin{array}{l}15.0 \\
10.2 \\
11.3\end{array}$ & $\begin{array}{l}\mu g / k g \\
\mu g / k g \\
\mu g / k g\end{array}$ & $\begin{array}{l}\text { Acetone } \\
\text { Acetone } \\
\text { Methylene Chloride }\end{array}$ \\
\hline 181 & $0.0,1.0$ & 5910 & $\mu g / g$ & Magnesium \\
\hline 182 & $\begin{array}{l}4.0,6.0 \\
4.0,6.0\end{array}$ & $103^{1.73}$ & $\begin{array}{l}\mu g / g \\
\mu g / g\end{array}$ & $\begin{array}{l}\text { 2,6-Dinitrotoluene } \\
\text { Sulfate }\end{array}$ \\
\hline 183 & $\begin{array}{l}2.0,4.0 \\
4.0,6.0\end{array}$ & $\begin{array}{l}210 \\
115\end{array}$ & $\begin{array}{l}\mu g / g \\
\mu g / g\end{array}$ & $\begin{array}{l}\text { Sulfate } \\
\text { Sulfate }\end{array}$ \\
\hline
\end{tabular}


TABLE 5.2-2 Soil Contaminant Concentrations Above Background (Continued)

\begin{tabular}{|c|c|c|c|c|}
\hline $\begin{array}{l}\text { Location }^{\text {(a) }} \\
\text { Number }\end{array}$ & Depth & Conc. & Units & Parameter \\
\hline 184 & $\begin{array}{l}0.0,7.0 \\
0.0,7.0 \\
0.0,7.0\end{array}$ & $\begin{array}{l}22.0 \\
54.5 \\
2320\end{array}$ & $\begin{array}{c}\mu g / k g \\
\mu g / g \\
\mu g / g\end{array}$ & $\begin{array}{l}\text { Acetone } \\
\text { Arsenic } \\
\text { Potassium }\end{array}$ \\
\hline 185 & $\begin{array}{l}0.0,7.0 \\
0.0,7.0 \\
0.0,7.0 \\
8.0,15.0 \\
8.0,15.0 \\
8.0,15.0 \\
8.0,15.0 \\
8.0,15.0\end{array}$ & $\begin{array}{c}61.0 \\
35.0 \\
1110 \\
82.0 \\
25100 \\
530 \\
240 \\
1160\end{array}$ & $\begin{array}{c}\mu g / k g \\
\mu g / k g \\
\mu g / g \\
\mu g / k g \\
\mu g / g \\
\mu g / k g \\
\mu g / k g \\
\mu g / g\end{array}$ & $\begin{array}{l}\text { Acetone } \\
\text { Methylene Chloride } \\
\text { Potassium } \\
\text { Acetone } \\
\text { Aluminum } \\
\text { Bis (2-Ethylhexyl) Phthalate } \\
\text { Methylene Chloride } \\
\text { Potassium }\end{array}$ \\
\hline 186 & $\begin{array}{l}0.0,7.0 \\
0.0 .7 .0\end{array}$ & $\begin{array}{l}8.00 \\
1460\end{array}$ & $\begin{array}{c}\mu \mathrm{g} / \mathrm{kg} \\
\mu \mathrm{g} / \mathrm{g}\end{array}$ & $\begin{array}{l}\text { Methylene Chloride } \\
\text { Potassium }\end{array}$ \\
\hline 187 & $\begin{array}{l}0.0,1.0 \\
0.0,1.0\end{array}$ & $\begin{array}{r}747 \\
1.88\end{array}$ & $\begin{array}{l}\mu g / g \\
\mu g / g\end{array}$ & $\begin{array}{l}\text { Lead } \\
\text { Nitrite }\end{array}$ \\
\hline 188 & $\begin{array}{l}0.0,7.0 \\
0.0,7.0\end{array}$ & $\begin{array}{l}1.29 \\
41.0\end{array}$ & 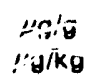 & $\begin{array}{l}\text { Cadmium } \\
\text { Methylene Chloride }\end{array}$ \\
\hline 189 & $\begin{array}{l}0.0,7.0 \\
0.0,7.0 \\
0.0,7.0 \\
0.0,7.0\end{array}$ & $\begin{array}{c}31.0 \\
26.6 \\
8.00 \\
99.0\end{array}$ & $\begin{array}{c}\mu g / k_{g} \\
\mu g / g \\
\mu g / k g \\
\mu g / k g\end{array}$ & $\begin{array}{l}\text { Acetone } \\
\text { Arsenic } \\
\text { Methylene Chloride } \\
\text { Toluene }\end{array}$ \\
\hline 190 & $\begin{array}{l}0.0,2 \\
0.0,2.0 \\
0.0,2.0 \\
0.0,2.0 \\
0.0,2.0 \\
0.0,2.0 \\
0.0,2.0 \\
0.0,2.0 \\
0.0,2.0 \\
0.0,2.0 \\
2.0,3.0 \\
2.0,3.0 \\
2.0,3.0 \\
2.0,3.0 \\
2.0,3.0 \\
2.0,3.0 \\
2.0,3.0 \\
2.0,3.0 \\
2.0,3.0 \\
2.0,3.0 \\
2.0,3.0 \\
2.0,3.0\end{array}$ & $\begin{array}{c}7.69 \\
15500 \\
120 \\
85.9 \\
14.6 \\
111 \\
3850 \\
37.0 \\
177 \\
250 \\
1.69 \\
28700 \\
83.3 \\
239 \\
83800 \\
337 \\
3890 \\
35.1 \\
92.7 \\
47.1 \\
356 \\
949\end{array}$ & $\begin{array}{l}\mu g / g \\
\mu g / g \\
\mu g / g \\
\mu g / g \\
\mu g / g \\
\mu g / g \\
\mu g / g \\
\mu g / g \\
\mu g / g \\
\mu g / g \\
\mu g / g \\
\mu g / g \\
\mu g / g \\
\mu g / g \\
\mu g / g \\
\mu g / g \\
\mu g / g \\
\mu g / g \\
\mu g / g \\
\mu g / g \\
\mu g / g \\
\mu g / g\end{array}$ & $\begin{array}{l}\text { Silver } \\
\text { Calcium } \\
\text { Chromium } \\
\text { Copper } \\
\text { Fluoride } \\
\text { Lead } \\
\text { Magnesium } \\
\text { Molybdenum } \\
\text { Nickel } \\
\text { Zinc } \\
\text { Cadmium } \\
\text { Calcium } \\
\text { Chromium } \\
\text { Copper } \\
\text { Iron } \\
\text { Lead } \\
\text { Magnesium } \\
\text { Molybdenum } \\
\text { Nickel } \\
\text { Selenium } \\
\text { Sulfate } \\
\text { Zinc }\end{array}$ \\
\hline
\end{tabular}


TABLE 5.2-2 Soil Contaminant Concentrations Above Background (Continued)

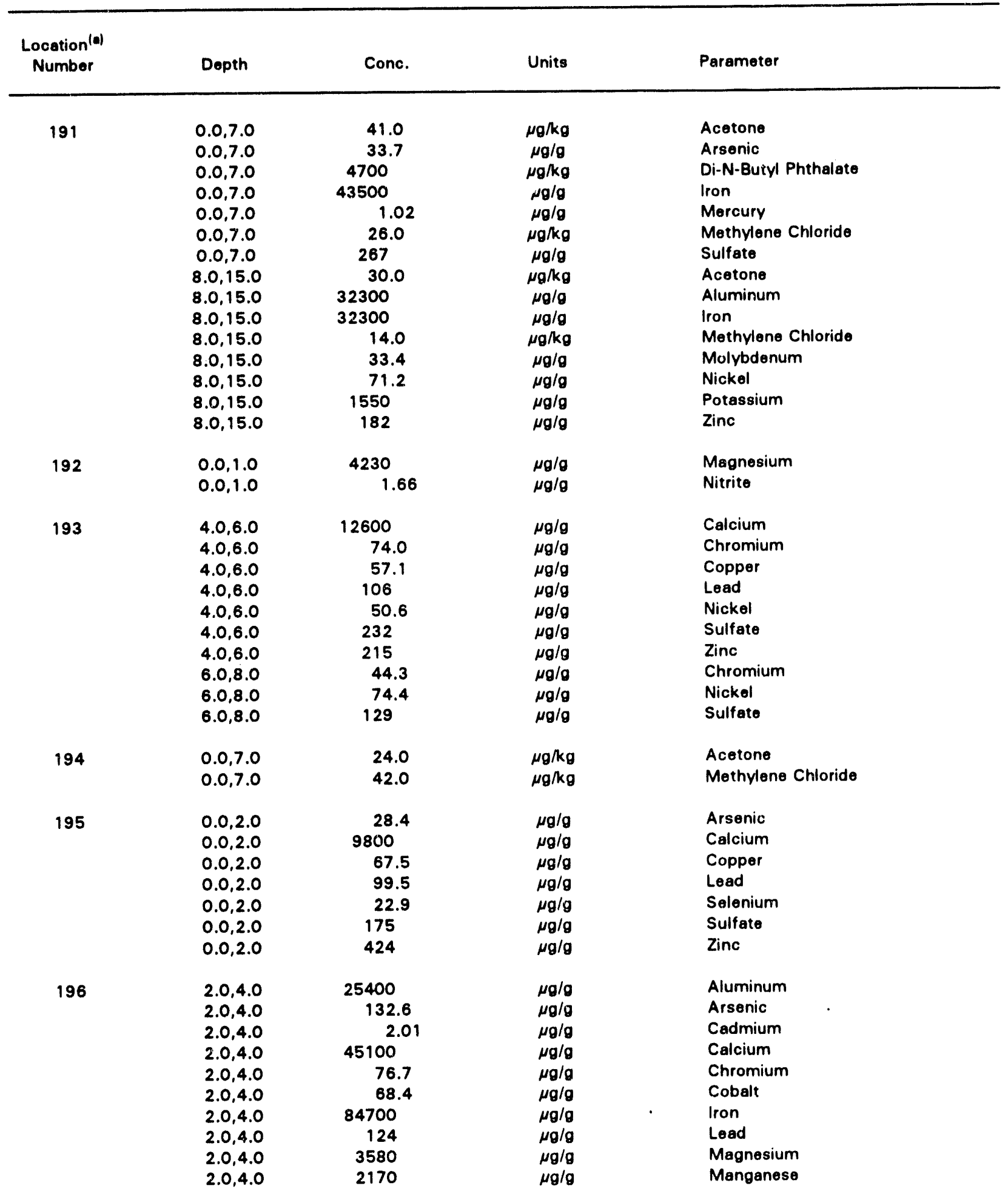


TABLE 5.2-2 Soil Contaminant Concentrations Above Background (Continued)

\begin{tabular}{|c|c|c|c|c|}
\hline $\begin{array}{l}\text { Locetion( }{ }^{(\bullet)} \\
\text { Number }\end{array}$ & Depth & Conc. & Units & Parameter \\
\hline 196 (cont.) & $\begin{array}{l}2.0,4.0 \\
2.0,4.0 \\
2.0,4.0 \\
2.0,4.0 \\
2.0,4.0 \\
2.0,4.0 \\
4.0,6.0 \\
4.0,6.0\end{array}$ & $\begin{array}{c}116 \\
118 \\
1098 \\
341 \\
80.2 \\
1050 \\
42.5 \\
327\end{array}$ & $\begin{array}{l}\mu g / g \\
\mu g / g \\
\mu g / g \\
\mu g / g \\
\mu g / g \\
\mu g / g \\
\mu g / g \\
\mu g / g\end{array}$ & $\begin{array}{l}\text { Molybdenum } \\
\text { Nickel } \\
\text { Sodium } \\
\text { Sulfate } \\
\text { Thallium } \\
\text { Zinc } \\
\text { Arsenic } \\
\text { Sulfate }\end{array}$ \\
\hline 197 & $\begin{array}{l}2.0,4.0 \\
2.0,4.0 \\
2.0,4.0 \\
6.0,8.0\end{array}$ & $\begin{array}{c}25.9 \\
51.0 \\
145 \\
40.4\end{array}$ & $\begin{array}{l}\mu g / g \\
\mu g / g \\
\mu g / g \\
\mu g / g\end{array}$ & $\begin{array}{l}\text { Arsenic } \\
\text { Chromium } \\
\text { Sulfate } \\
\text { Cobalt }\end{array}$ \\
\hline 198 & $\begin{array}{l}0.0,7.0 \\
0.0,7.0 \\
8.0,15.0 \\
8.0,15.0 \\
8.0,15.0\end{array}$ & $\begin{array}{c}43.0 \\
108 \\
17.0 \\
3.02 \\
11.0\end{array}$ & $\begin{array}{c}\mu g / k g \\
\mu g / g \\
\mu g / k g \\
\mu g / g \\
\mu g / k g\end{array}$ & $\begin{array}{l}\text { Methylene Chloride } \\
\text { Sulfate } \\
\text { Acetone } \\
\text { Cadmium } \\
\text { Methylene Chloride }\end{array}$ \\
\hline 199 & $\begin{array}{l}0.0,1.5 \\
0.0,1.5 \\
0.0,1.5 \\
0.0,1.5 \\
2.0,2.5 \\
4.5,5.0 \\
4.5,5.0\end{array}$ & $\begin{array}{c}470 \\
18.0 \\
6.00 \\
25.0 \\
27.0 \\
10.0 \\
26.0\end{array}$ & $\begin{array}{c}\mu g / k g \\
\mu g / k g \\
\mu g / k g \\
\mu g / g \\
\mu g / g \\
\mu g / k g \\
\mu g / g\end{array}$ & $\begin{array}{l}\text { Aroclor-1254 } \\
\text { Beta-BHC } \\
\text { Methylene Chloride } \\
\text { Molybdenum } \\
\text { Molybdenum } \\
\text { Methylene Chloride } \\
\text { Molybdenum }\end{array}$ \\
\hline 200 & $\begin{array}{l}0.0,7.0 \\
0.0,7.0 \\
8.0,14.0 \\
8.0,14.0\end{array}$ & $\begin{array}{c}2400 \\
7.00 \\
58.7 \\
33.9\end{array}$ & $\begin{array}{c}\mu g / g \\
\mu g / \mathbf{g g} \\
\mu \mathrm{g} / \mathrm{g} \\
\mu \mathrm{g} / \mathrm{g}\end{array}$ & $\begin{array}{l}\text { Manganese } \\
\text { Methylene Chloride } \\
\text { Chromium } \\
\text { Molybdenum }\end{array}$ \\
\hline 201 & $8.0,15.0$ & 1.28 & $\mu g / g$ & Cadmium \\
\hline 202 & $\begin{array}{c}0.0,7.0 \\
0.0,7.0 \\
8.0,15.0 \\
8.0,15.0 \\
16.0,17.0\end{array}$ & $\begin{array}{l}31.0 \\
0.16 \\
14.4 \\
11.0 \\
14.4\end{array}$ & $\begin{array}{c}\mu g / k g \\
\mu g / g \\
\mu g / g \\
\mu g / k g \\
\mu g / g\end{array}$ & $\begin{array}{l}\text { Acetone } \\
\text { Mercury } \\
\text { Fluoride } \\
\text { Methylene Chloride } \\
\text { Fluoride }\end{array}$ \\
\hline 203 & $\begin{array}{c}4.0,6.0 \\
6.0,8.0 \\
8.0,10.0 \\
10.0,12.0 \\
18.0,20.0 \\
18.0,20.0\end{array}$ & $\begin{array}{c}219 \\
332 \\
282 \\
99.5 \\
100 \\
2500\end{array}$ & $\begin{array}{l}\mu g / g \\
\mu g / g \\
\mu g / g \\
\mu g / g \\
\mu g / g \\
\mu g / g\end{array}$ & $\begin{array}{l}\text { Sulfate } \\
\text { Sulfate } \\
\text { Sulfate } \\
\text { Sulfate } \\
\text { Lead } \\
\text { Manganese }\end{array}$ \\
\hline 204 & $\begin{array}{c}8.0,10.0 \\
8.0,10.0 \\
10.0,12.0\end{array}$ & $\begin{array}{l}72.9 \\
127 \\
248\end{array}$ & $\begin{array}{l}\mu g / g \\
\mu g / g \\
\mu g / g\end{array}$ & $\begin{array}{l}\text { Lead } \\
\text { Sulfate } \\
\text { Sulfate }\end{array}$ \\
\hline
\end{tabular}


TABLE 5.2-2 Soil Contaminant Concentrations Above Background (Continued)

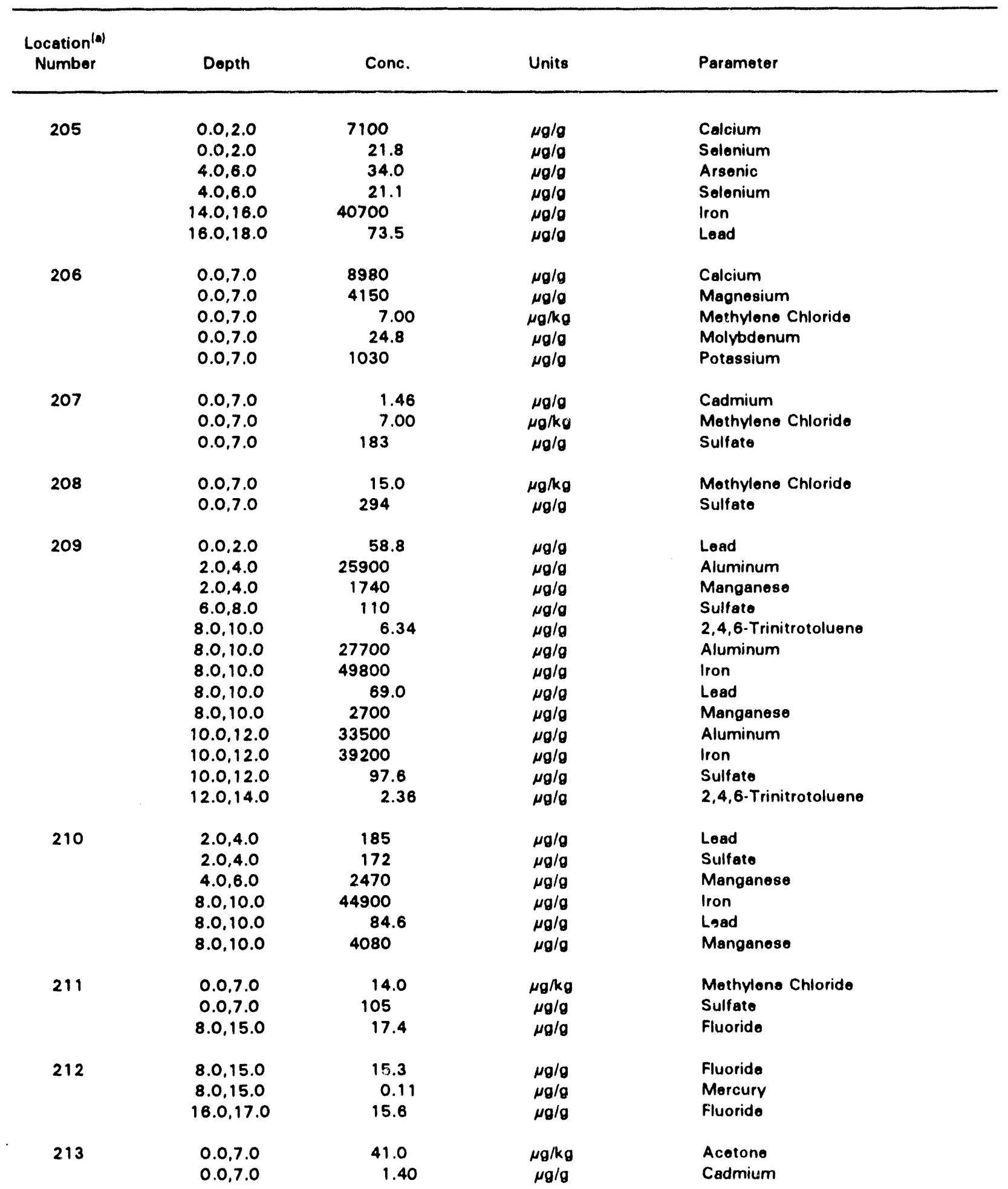


TABLE 5.2-2 Soil Contaminant Concentrations Above Background (Continued)

\begin{tabular}{|c|c|c|c|c|}
\hline $\begin{array}{l}\text { Location }^{(a)} \\
\text { Number }\end{array}$ & Depth & Conc. & Units & Parameter \\
\hline 213 (cont.) & $\begin{array}{c}0.0,7.0 \\
0.0,7.0 \\
0.0,7.0 \\
0.0,7.0 \\
12.0,15.0 \\
12.0,15.0 \\
12.0,15.0 \\
12.0,15.0 \\
12.0,15.0\end{array}$ & $\begin{array}{c}40400 \\
4020 \\
7.00 \\
226 \\
48.0 \\
1.50 \\
51.6 \\
2390 \\
6.00\end{array}$ & $\begin{array}{c}\mu \mathrm{g} / \mathrm{g} \\
\mu \mathrm{g} / \mathrm{g} \\
\mu \mathrm{g} / \mathbf{k g} \\
\mu \mathrm{g} / \mathrm{g} \\
\mu \mathrm{g} / \mathbf{k g} \\
\mu \mathrm{g} / \mathrm{g} \\
\mu \mathrm{g} / \mathrm{g} \\
\mu \mathrm{g} / \mathrm{g} \\
\mu \mathrm{g} / \mathrm{kg}\end{array}$ & $\begin{array}{l}\text { Calcium } \\
\text { Magnesium } \\
\text { Methylene Chloride } \\
\text { Sulfate } \\
\text { Acetone } \\
\text { Cadmium } \\
\text { Leạd } \\
\text { Manganese } \\
\text { Methylene Chloride }\end{array}$ \\
\hline 214 & $\begin{array}{l}4.0,6.0 \\
6.0,8.0\end{array}$ & $\begin{array}{r}85600 \\
1630\end{array}$ & $\begin{array}{l}\mu g / g \\
\mu g / g\end{array}$ & $\begin{array}{l}\text { Aluminum } \\
\text { Manganese }\end{array}$ \\
\hline 215 & $\begin{array}{l}0.0,7.0 \\
0.0,7.0 \\
0.0,7.0\end{array}$ & $\begin{array}{l}27.0 \\
20.0 \\
72.0\end{array}$ & $\begin{array}{l}\mu \mathrm{g} / \mathrm{kg} \\
\mu \mathrm{g} / \mathrm{kg} \\
\mu \mathrm{g} / \mathrm{kg}\end{array}$ & $\begin{array}{l}\text { Acetone } \\
\text { Chloroform } \\
\text { Methylene Chloride }\end{array}$ \\
\hline 216 & $\begin{array}{l}0.0 .7 .0 \\
0.0 .7 .0\end{array}$ & $\begin{array}{l}29.3 \\
118\end{array}$ & $\begin{array}{l}\mu g / g \\
\mu g / g\end{array}$ & $\begin{array}{l}\text { Molybdenum } \\
\text { Sulfate }\end{array}$ \\
\hline 217 & $0.0,1.0$ & 125 & $\mu g / g$ & Lead \\
\hline 218 & $2.0,4.0$ & 98.5 & $\mu g / g$ & Sulfate \\
\hline 219 & $6.0,8.0$ & 175 & $\mu g / g$ & Sulfate \\
\hline $\begin{array}{l}220 \\
221\end{array}$ & $\begin{array}{l}0.0,1.0 \\
0.0,2.0 \\
0.0,2.0 \\
4.0,6.0\end{array}$ & $\begin{array}{c}57.8 \\
214 \\
1630 \\
54.6\end{array}$ & $\begin{array}{l}\mu g / g \\
\mu g / g \\
\mu g / g \\
\mu g / g\end{array}$ & $\begin{array}{l}\text { Lead } \\
\text { Lead } \\
\text { Manganese } \\
\text { Lead }\end{array}$ \\
\hline 222 & $\begin{array}{l}2.0,4.0 \\
4.0,6.0 \\
6.0,8.0 \\
6.0,8.0\end{array}$ & $\begin{array}{r}104 \\
255 \\
38500 \\
123\end{array}$ & $\begin{array}{l}\mu g / g \\
\mu g / g \\
\mu g / g \\
\mu g / g\end{array}$ & $\begin{array}{l}\text { Sulfate } \\
\text { Sulfate } \\
\text { Iron } \\
\text { Sulfate }\end{array}$ \\
\hline 223 & $\begin{array}{l}8.0,10.0 \\
8.0,10.0 \\
8.0,10.0 \\
8.0,10.0 \\
8.0,10.0 \\
8.0,10.0 \\
8.0,10.0 \\
8.0,10.0 \\
8.0,10.0\end{array}$ & $\begin{array}{c}12.0 \\
34.7 \\
1.83 \\
16.3 \\
0.18 \\
6.00 \\
152 \\
14.2 \\
107\end{array}$ & $\begin{array}{c}\mu g / k g \\
\mu g / g \\
\mu g / g \\
\mu g / g \\
\mu g / g \\
\mu g / k g \\
\mu g / g \\
\mu g / g \\
\mu g / g\end{array}$ & $\begin{array}{l}\text { Acetone } \\
\text { Arsenic } \\
\text { Cadmium } \\
\text { Fluoride } \\
\text { Mercury } \\
\text { Methylene Chloride } \\
\text { Nitrate } \\
\text { Nitrite } \\
\text { Zinc }\end{array}$ \\
\hline 224 & $\begin{array}{c}0.0,7.0 \\
8.0,15.0 \\
8.0,15.0 \\
8.0,15.0 \\
8.0,15.0\end{array}$ & $\begin{array}{c}33.0 \\
35.0 \\
35000 \\
91.8 \\
2.47\end{array}$ & $\begin{array}{c}\mu g / k g \\
\mu g / k g \\
\mu g / g \\
\mu g / g \\
\mu g / g\end{array}$ & $\begin{array}{l}\text { Acetone } \\
\text { Acetone } \\
\text { Aluminum } \\
\text { Arsenic } \\
\text { Bervllium }\end{array}$ \\
\hline
\end{tabular}


TABLE 5.2-2 Soil Contaminant Concentrations Above Background (Continued)

\begin{tabular}{|c|c|c|c|c|}
\hline $\begin{array}{l}\text { Location } \\
\text { Number }\end{array}$ & Depth & Conc. & Units & Parameter \\
\hline \multirow[t]{14}{*}{224 (cont.) } & $8.0,15.0$ & 3.19 & $\mu \boldsymbol{g} / \boldsymbol{g}$ & Cadmium \\
\hline & $8.0,15.0$ & 7800 & $\mu \mathrm{g} / \mathrm{g}$ & Calcium \\
\hline & $8.0,15.0$ & 44.5 & $\mu g / g$ & Cobalt \\
\hline & $8.0,15.0$ & 32.9 & $\mu g / g$ & Copper \\
\hline & $8.0,15.0$ & 35500 & $\mu g / g$ & Iron \\
\hline & $8.0,15.0$ & 5120 & $\mu g / g$ & Magnesium \\
\hline & 8.0 .15 .0 & 2260 & $\mu g / g$ & Manganese \\
\hline & $8.0,15.0$ & 42.0 & $\mu g / k g$ & Methylene Chloride \\
\hline & $8.0,15.0$ & 43.1 & $\mu g / g$ & Molybdenum \\
\hline & $8.0,15.0$ & 119 & $\mu g / g$ & Nickel \\
\hline & $8.0,15.0$ & 1370 & $\mu \mathrm{g} / \mathrm{g}$ & Potassium \\
\hline & $8.0,15.0$ & 816 & $\mu \mathrm{g} / \mathrm{g}$ & Sodium \\
\hline & $8.0,15.0$ & 56.5 & $\mu g / g$ & Vanadium \\
\hline & $8.0,15.0$ & 180 & $\mu g / g$ & Zinc \\
\hline \multirow[t]{10}{*}{225} & 0,2 & 13600 & $\mu g / g$ & Calcium \\
\hline & 2,4 & 30000 & $\mu g / g$ & Aluminum \\
\hline & 2,4 & 21.1 & $\mu \mathrm{g} / \mathrm{g}$ & Fluoride \\
\hline & 2,4 & 3490 & $\mu \mathrm{g} / \mathrm{g}$ & Magnesium \\
\hline & 2,4 & 33.5 & $\mu g / g$ & Molybdenum \\
\hline & 4,6 & 17.6 & $\mu g / g$ & Fluoride \\
\hline & 4,6 & 0.15 & $\mu g / g$ & Mercury \\
\hline & 6,8 & 9.61 & $\mu g / g$ & Chloride \\
\hline & 6,8 & 19.6 & $\mu g / g$ & Fluoride \\
\hline & 6,8 & 0.19 & $\mu g / g$ & Mercury \\
\hline \multirow[t]{5}{*}{226} & 2,4 & 12.7 & $\mu \mathrm{g} / \mathrm{g}$ & Chloride \\
\hline & 2,4 & 163 & $\mu g / g$ & Sulfate \\
\hline & 4,6 & 19.3 & $\mu \mathrm{g} / \boldsymbol{g}$ & Chloride \\
\hline & 6,8 & 13.0 & $\mu g / g$ & Chloride \\
\hline & 6,8 & 14.8 & $\mu g / g$ & Fluoride \\
\hline \multirow[t]{12}{*}{227} & 0,2 & 1.50 & $\mu g / g$ & Cadmium \\
\hline & 0,2 & 3610 & $\mu g / g$ & Magnesium \\
\hline & 2,4 & 0.15 & $\mu g / g$ & Mercury \\
\hline & 4,6 & 25500 & $\mu g / g$ & Aluminum \\
\hline & 4,6 & 1.30 & $\mu g / g$ & Cadmium \\
\hline & 4,6 & 27.5 & $\mu \mathrm{g} / \mathrm{g}$ & Cobalt \\
\hline & 4,6 & 1770 & $\mu \mathrm{g} / \mathrm{g}$ & Manganese \\
\hline & 4,6 & 0.14 & $\mu \mathrm{g} / \mathrm{g}$ & Mercury \\
\hline & 4,6 & 35.5 & $\mu g / g$ & Molybdenum \\
\hline & 4,6 & 65.1 & $\mu g / g$ & Vanadium \\
\hline & 6,8 & 87.4 & $\mu g / g$ & Arsenic \\
\hline & 6,8 & 0.13 & $\mu \mathrm{g} / \mathrm{g}$ & Mercury \\
\hline \multirow[t]{6}{*}{228} & 2,4 & 127 & $\mu g / g$ & Sulfate \\
\hline & 4,6 & 15.6 & $\mu g / g$ & Fluoride \\
\hline & 4,6 & 0.16 & $\mu \mathrm{g} / \mathrm{g}$ & Mercury \\
\hline & 4,6 & 136 & $\mu g / g$ & Sulfate \\
\hline & 6,8 & 9.87 & $\mu \mathrm{g} / \mathrm{g}$ & Chloride \\
\hline & 6,8 & 0.20 & $\mu g / g$ & Mercury \\
\hline
\end{tabular}


TABLE 5.2-2 Soil Contaminant Concentrations Above Background (Continued)

\begin{tabular}{|c|c|c|c|c|}
\hline $\begin{array}{l}\text { Location } \\
\text { Number }\end{array}$ & Depth & Conc. & Units & Parameter \\
\hline 228 (cont.) & $\begin{array}{c}8,10 \\
10,12\end{array}$ & $\begin{array}{l}0.18 \\
0.21\end{array}$ & $\begin{array}{l}\mu g / g \\
\mu g / g\end{array}$ & $\begin{array}{l}\text { Mercury } \\
\text { Mercury }\end{array}$ \\
\hline 229 & $\begin{array}{l}0,2 \\
0,2 \\
2,4 \\
2,4 \\
2,4 \\
2,4 \\
2,4 \\
4,6 \\
4,6 \\
4,6 \\
4,6 \\
6,8 \\
6,8 \\
6,8 \\
6,8 \\
6,8\end{array}$ & $\begin{array}{c}550 \\
17.0 \\
1300 \\
25.7 \\
10.0 \\
707 \\
327 \\
900 \\
48.7 \\
430 \\
18.0 \\
1200 \\
12.0 \\
540 \\
16.2 \\
12.0\end{array}$ & $\begin{array}{c}\mu \mathrm{g} / \mathrm{kg} \\
\mu \mathrm{g} / \mathrm{kg} \\
\mu \mathrm{g} / \mathrm{kg} \\
\mu \mathrm{g} / \mathrm{g} \\
\mu \mathrm{g} / \mathrm{kg} \\
\mu \mathrm{g} / \mathrm{g} \\
\mu \mathrm{g} / \mathrm{g} \\
\mu \mathrm{g} / \mathrm{kg} \\
\mu \mathrm{g} / \mathrm{g} \\
\mu \mathrm{g} / \mathrm{kg} \\
\mu \mathrm{g} / \mathrm{kg} \\
\mu \mathrm{g} / \mathrm{kg} \\
\mu \mathrm{g} / \mathrm{kg} \\
\mu \mathrm{g} / \mathrm{kg} \\
\mu \mathrm{g} / \mathrm{g} \\
\mu \mathrm{g} / \mathrm{kg}\end{array}$ & $\begin{array}{l}\text { Bis (2-Ethylhexyl) Phthalate } \\
\text { Methylene Chloride } \\
\text { Bis (2-Ethylhexyl) Phthalate } \\
\text { Chloride } \\
\text { Methylene Chloride } \\
\text { Sodium } \\
\text { Sulfate } \\
\text { Bis (2-Ethylhexyl) Phthalate } \\
\text { Chloride } \\
\text { Di-N-Butyl Phthalate } \\
\text { Methylene Chloride } \\
\text { Bis (2-Ethylhexyl) Phthalate } \\
\text { Chloroethene } \\
\text { Di-N-Butyl Phthalate } \\
\text { Fluoride } \\
\text { Methylene Chloride }\end{array}$ \\
\hline 230 & $\begin{array}{c}6,8 \\
8,10\end{array}$ & $\begin{array}{c}21.4 \\
0.21\end{array}$ & $\begin{array}{l}\mu g / g \\
\mu g / g\end{array}$ & $\begin{array}{l}\text { Fluoride } \\
\text { Mercury }\end{array}$ \\
\hline 231 & $\begin{array}{c}0,2 \\
2,4 \\
2,4 \\
2,4 \\
4,6 \\
4,6 \\
4,6 \\
6,8 \\
6,8 \\
8,10 \\
8,10 \\
10,12 \\
10,12 \\
10,12 \\
12,14 \\
12,14 \\
14,16\end{array}$ & $\begin{array}{c}0.19 \\
36.5 \\
0.20 \\
140 \\
64.0 \\
0.14 \\
106 \\
750 \\
0.17 \\
600 \\
60.0 \\
430 \\
45.3 \\
0.15 \\
550 \\
0.11 \\
0.14\end{array}$ & $\begin{array}{c}\mu g / g \\
\mu g / g \\
\mu g / g \\
\mu g / g \\
\mu g / g \\
\mu g / g \\
\mu g / g \\
\mu g / k g \\
\mu g / g \\
\mu g / k g \\
\mu g / g \\
\mu g / k g \\
\mu g / g \\
\mu g / g \\
\mu g / k g \\
\mu g / g \\
\mu g / g\end{array}$ & $\begin{array}{l}\text { Mercury } \\
\text { Copper } \\
\text { Mercury } \\
\text { Sulfate } \\
\text { Chromium } \\
\text { Mercury } \\
\text { Sulfate } \\
\text { Bis (2-Ethylhexyl) Phthalate } \\
\text { Mercury } \\
\text { Bis (2-Ethylhexyl) Phthalate } \\
\text { Venadium } \\
\text { Bis (2-Ethylhexyl) Phthalate } \\
\text { Chloride } \\
\text { Mercury } \\
\text { Bis (2-Ethylhexyl) Phthalate } \\
\text { Mercury } \\
\text { Mercury }\end{array}$ \\
\hline 232 & $\begin{array}{l}0,2 \\
2,4 \\
2,4 \\
2,4 \\
2,4 \\
2,4 \\
2,4 \\
4,6 \\
4,6\end{array}$ & $\begin{array}{c}2.22 \\
3.30 \\
3.37 \\
3.44 \\
2.52 \\
69.5 \\
3.77 \\
2.24 \\
834\end{array}$ & $\begin{array}{l}\mu \mathrm{g} / \mathrm{g} \\
\mu \mathrm{g} / \mathrm{g} \\
\mu \mathrm{g} / \mathrm{g} \\
\mu \mathrm{g} / \mathrm{g} \\
\mu \mathrm{g} / \mathrm{g} \\
\mu \mathrm{g} / \mathrm{g} \\
\mu \mathrm{g} / \mathrm{g} \\
\mu \mathrm{g} / \mathrm{g} \\
\mu \mathrm{g} / \mathrm{g}\end{array}$ & $\begin{array}{l}\text { Cadmium } \\
\text { 1,3,5-Trinitrobenzene } \\
\text { 1,3-Dinitrobenzene } \\
\text { 2,4,6-Trinitrotoluene } \\
\text { Cadmium } \\
\text { Copper } \\
\text { Nitrobenzene } \\
\text { Cadmium } \\
\text { Nitrate }\end{array}$ \\
\hline
\end{tabular}


TABLE 5.2-2 Soil Contaminant Concentrations Above Background (Continued)

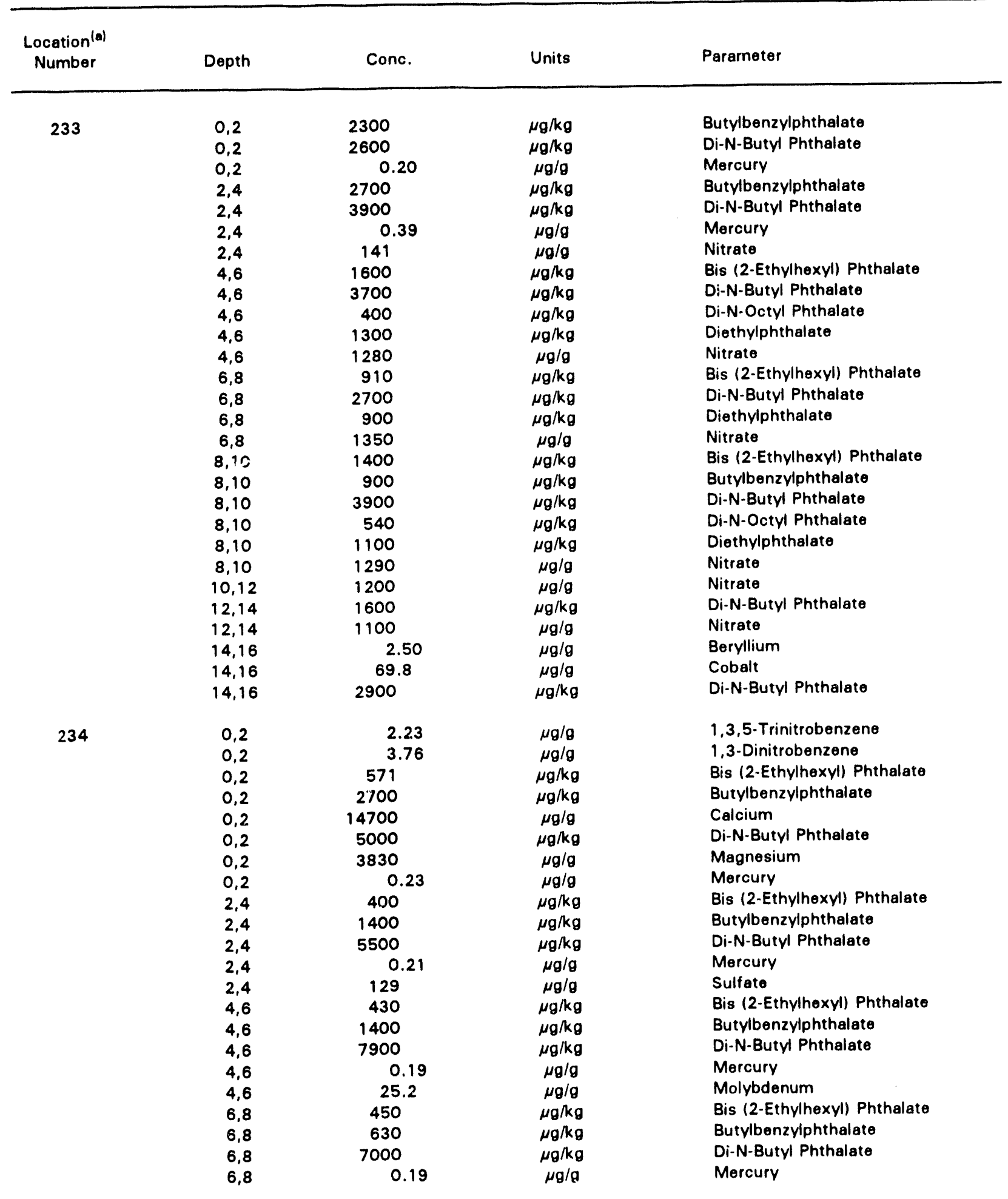


TABLE 5.2-2 Soil Contaminant Concentrations Above Background (Continued)

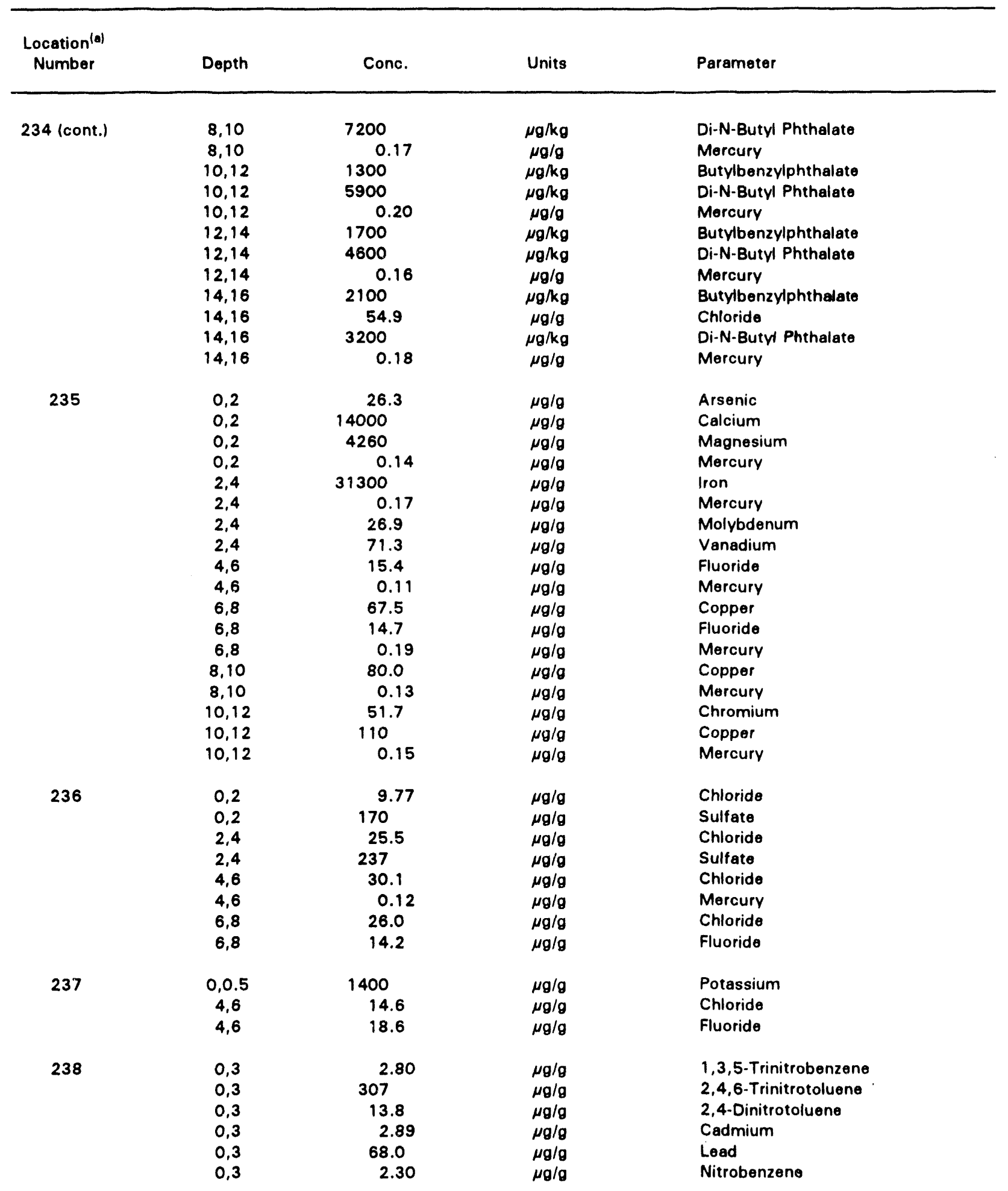


TABLE 5.2-2 Soil Contaminant Concentrations Above Background (Continued)

\begin{tabular}{|c|c|c|c|c|}
\hline $\begin{array}{l}\text { Location }^{(a)} \\
\text { Number }\end{array}$ & Depth & Conc. & Units & Parameter \\
\hline \multirow[t]{20}{*}{238 (cont.) } & 0,3 & 980 & $\mu g / g$ & Sodium \\
\hline & 0,3 & 210 & $\mu g / g$ & Sulfate \\
\hline & 3,5 & 1.90 & $\mu g / g$ & 1,3,5-Trinitrobenzene \\
\hline & 3,5 & 3.43 & $\mu g / g$ & Cadmium \\
\hline & 3,5 & 43.5 & $\mu g / g$ & Lead \\
\hline & 3,5 & 72.4 & $\mu g / g$ & Nitrato \\
\hline & 3,5 & 1060 & $\mu g / g$ & Potassium \\
\hline & 3,5 & 1000 & $\mu g / g$ & Sodium \\
\hline & 3,5 & 282 & $\mu g / g$ & Sulfate \\
\hline & 5,8 & 1.20 & $\mu g / g$ & 1,3,5-Trinitrobenzene \\
\hline & 5,8 & 2.34 & $\mu g / \theta$ & Cadmium \\
\hline & 5,8 & 1.00 & $\mu \mathrm{g} / \mathrm{g}$ & Mercury \\
\hline & 5,8 & 1700 & $\mu g / g$ & Potassium \\
\hline & 5,8 & 707 & $\mu g / g$ & Sodium \\
\hline & 5,8 & 190 & $\mu g / g$ & Sulfato \\
\hline & 8,13 & 3.48 & $\mu g / g$ & Cadmium \\
\hline & 8,13 & 16.8 & $\mu g / g$ & Chloride \\
\hline & 8,13 & 65.3 & $\mu \mathrm{g} / \mathrm{g}$ & Lead \\
\hline & 8,13 & 10.2 & $\mu \mathrm{g} / \mathrm{g}$ & Silver \\
\hline & 8,13 & 276 & $\mu \mathrm{g} / \mathrm{g}$ & Sulfato \\
\hline \multirow[t]{11}{*}{239} & 1,2 & 2.20 & $\mu g / g$ & 1,3,5-Trinitrobenzene \\
\hline & 1,2 & 17.2 & $\mu g / g$ & $2,4,6$-Trinitrotoluene \\
\hline & 1.2 & 3.00 & $\mu g / \theta$ & 2,4-Dinitrotoluene \\
\hline & 1,2 & 3.16 & $\mu \mathrm{g} / \mathrm{g}$ & Cadmium \\
\hline & 1,2 & 968 & $\mu g / g$ & Sodium \\
\hline & 2,3 & 3.70 & $\mu g / g$ & 1,3,5-Trinitrobenzene \\
\hline & 2,3 & 13.7 & $\mu g / g$ & $2,4,6$-Trinitrotoluene \\
\hline & 2,3 & 2.20 & $\mu g / \theta$ & 2.4-Dinitrotoluene \\
\hline & 2,3 & 3.00 & $\mu g / g$ & Cadmium \\
\hline & 2,3 & 19.0 & $\mu g / g$ & Fluoride \\
\hline & 2,3 & 1400 & $\mu g / g$ & Sodium \\
\hline \multirow[t]{8}{*}{240} & 0,2 & 28.2 & $\mu g / g$ & Arsenic \\
\hline & 0.2 & 0.69 & $\mu g / g$ & Mercury \\
\hline & 0.2 & 136 & $\mu g / g$ & Sulfate \\
\hline & 2,4 & 0.12 & $\mu_{g} / g$ & Mercury \\
\hline & 2,4 & 1540 & $\mu g / g$ & Sulfate \\
\hline & 4,6 & 22.5 & $\mu g / g$ & Chloride \\
\hline & 4,6 & 0.13 & $\mu \mathrm{g} / \mathrm{g}$ & Mercury \\
\hline & 6,8 & 0.17 & $\mu g / g$ & Mercury \\
\hline \multirow[t]{9}{*}{241} & $1.5,2.5$ & 1.40 & $\mu g / g$ & 2,4-Dinitrotoluene \\
\hline & $1.5,2.5$ & 2.59 & $\mu \mathrm{g} / \mathrm{g}$ & Cadmium \\
\hline & $1.5,2.5$ & 984 & $\mu g / g$ & Sodium \\
\hline & $1.5,2.5$ & 139 & $\mu g / g$ & Sulfate \\
\hline & $2.5,3$ & 2.30 & $\mu g / g$ & 2,4-Dinitrotoluene \\
\hline & $2.5,3$ & 3.93 & $\mu \mathrm{g} / \mathrm{g}$ & Cadmium \\
\hline & $2.5,3$ & 1230 & $\mu \mathrm{g} / \mathrm{g}$ & Potassium \\
\hline & $2.5,3$ & 1050 & $\mu g / g$ & Sodium \\
\hline & $2.5,3$ & 114 & $\mu g / g$ & Sulfate \\
\hline
\end{tabular}


TABLE 5.2-2 Soil Contaminant Concentrations Above Background (Continued)

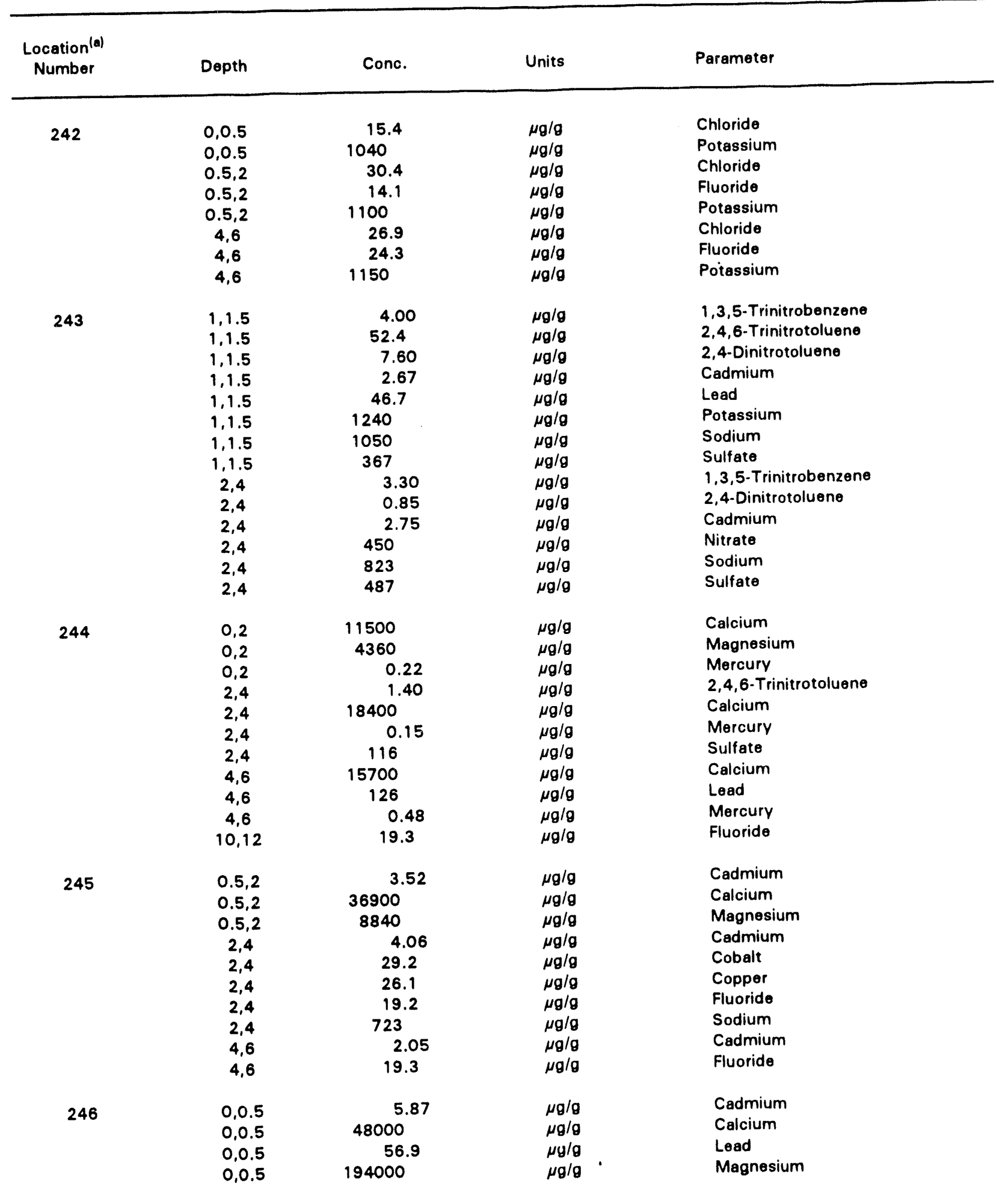


TABLE 5.2-2 Soil Contaminant Concentrations Above Background (Continued)

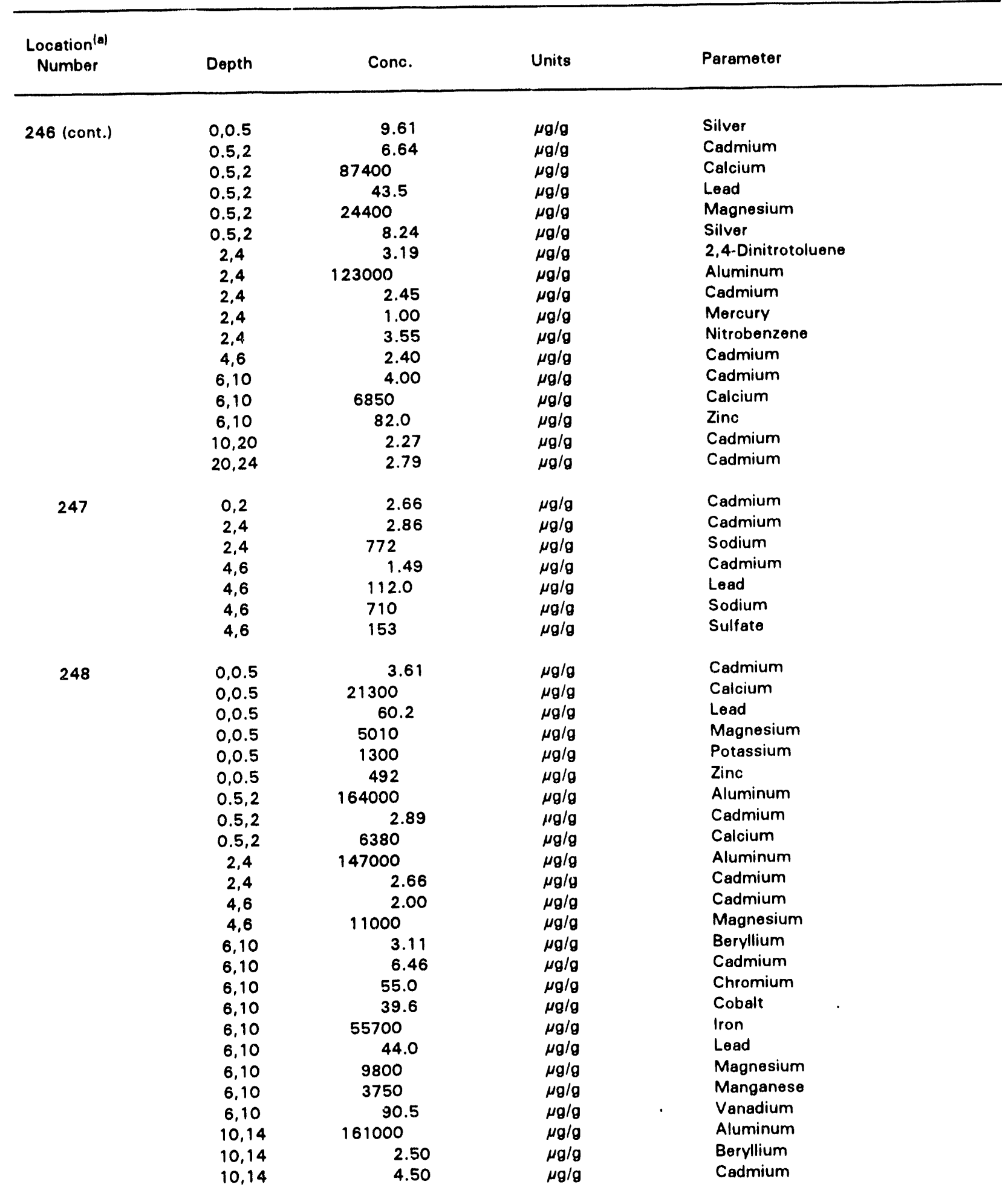


TABI.E 5.2-2 Soil Contaminant Concentrations Above Background (Continued)

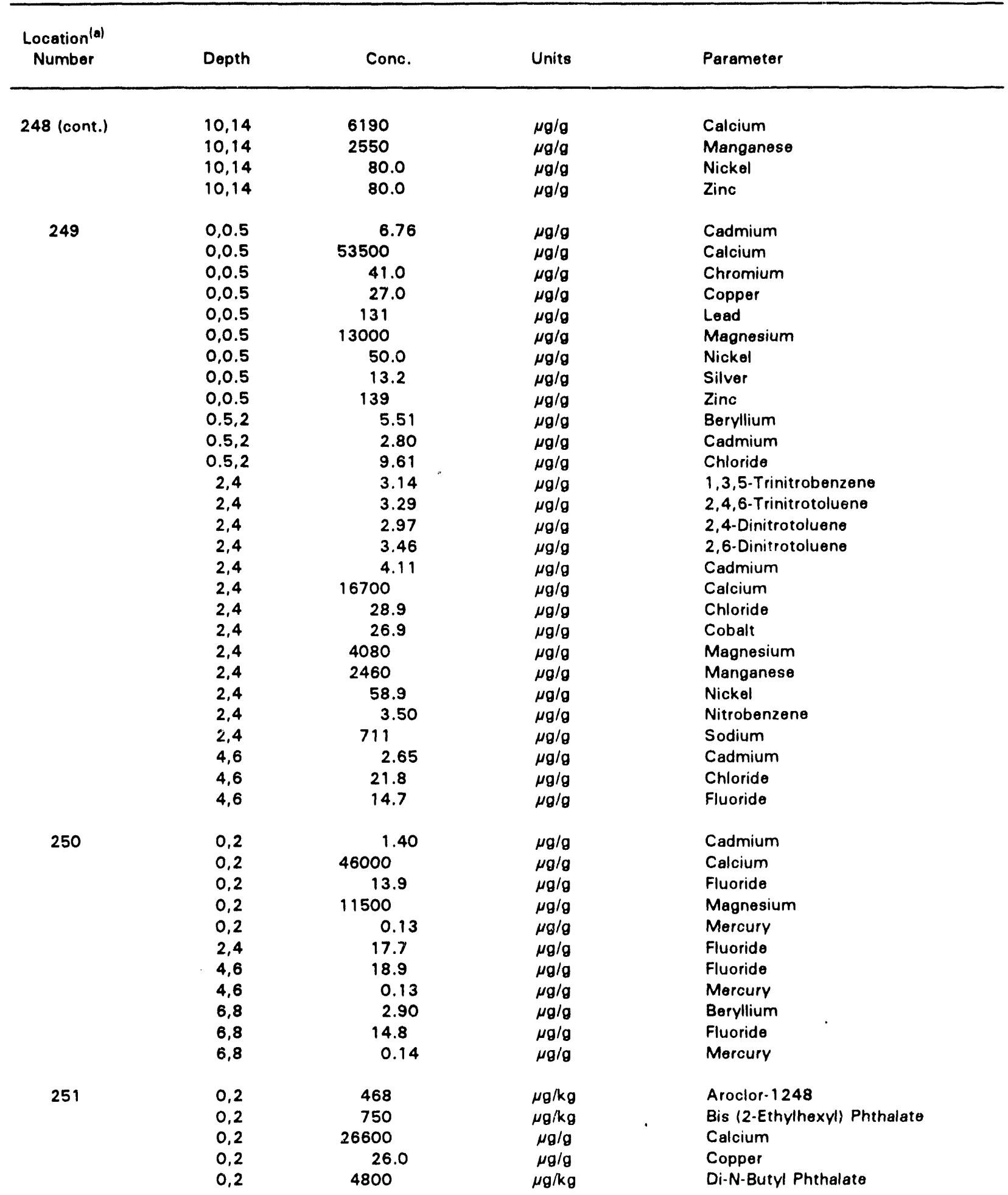


TABLE 5.2-2 Soil Contaminant Concentrations Above Background (Continued)

\begin{tabular}{|c|c|c|c|c|}
\hline $\begin{array}{l}\text { Location }^{(\theta)} \\
\text { Number }\end{array}$ & Depth & Conc. & Units & Parameter \\
\hline 251 (cont.) & $\begin{array}{l}0,2 \\
0,2 \\
0,2 \\
2,4 \\
2,4 \\
2,4 \\
4,6 \\
4,6 \\
4,6 \\
4,6 \\
6,8 \\
6,8 \\
8,10 \\
8,10 \\
8,10 \\
10,12\end{array}$ & $\begin{array}{c}440 \\
0.31 \\
104.2 \\
570 \\
7900 \\
3120 \\
600 \\
880 \\
1200 \\
2020 \\
2200 \\
17000 \\
27.5 \\
3480 \\
66.2 \\
2 \% 00\end{array}$ & $\begin{array}{c}\mu g / k g \\
\mu g / g \\
\mu g / g \\
\mu g / k g \\
\mu g / k g \\
\mu g / g \\
\mu g / k g \\
\mu g / k g \\
\mu g / k g \\
\mu g / g \\
\mu g / k g \\
\mu g / k g \\
\mu g / g \\
\mu g / g \\
\mu g / g \\
\mu g / k g\end{array}$ & $\begin{array}{l}\text { Diethylphthalate } \\
\text { Mercury } \\
\text { Sulfate } \\
\text { Bis (2-Ethylhexyl) Phthalate } \\
\text { Di-N-Butyl Phthalate } \\
\text { Menganese } \\
\text { Bis (2-Ethylhexyl) Phthalate } \\
\text { Butylbenzylphthalate } \\
\text { Di-N-Butyl Phthalate } \\
\text { Manganese } \\
\text { Bis (2-Ethylhexyl) Phthalate } \\
\text { Di-N-Butyl Phthalate } \\
\text { Cobalt } \\
\text { Manganese } \\
\text { Vanadium } \\
\text { Di-N-Butyl Phthalate }\end{array}$ \\
\hline 252 & $\begin{array}{l}0,2 \\
2,4 \\
2,4 \\
4,6 \\
6,8 \\
6,8\end{array}$ & $\begin{array}{c}27.1 \\
35.1 \\
113 \\
0.29 \\
26.0 \\
0.30\end{array}$ & $\begin{array}{l}\mu \mathrm{g} / \mathrm{g} \\
\mu \mathrm{g} / \mathrm{g} \\
\mu \mathrm{g} / \mathrm{g} \\
\mu \mathrm{g} / \mathrm{g} \\
\mu \mathrm{g} / \mathrm{g} \\
\mu \mathrm{g} / \mathrm{g}\end{array}$ & $\begin{array}{l}\text { Molybdenum } \\
\text { Copper } \\
\text { Sulfate } \\
\text { Mercury } \\
\text { Copper } \\
\text { Mercury }\end{array}$ \\
\hline 253 & 0,2 & 6510 & $\mu g / g$ & Calcium \\
\hline 254 & $\begin{array}{l}0,2 \\
0,2 \\
2,4 \\
2,4 \\
2,4 \\
4,6 \\
6,8 \\
6,8\end{array}$ & $\begin{array}{c}6420 \\
0.12 \\
18500 \\
5110 \\
514 \\
263 \\
5500 \\
112\end{array}$ & $\begin{array}{l}\mu g / g \\
\mu g / g \\
\mu g / g \\
\mu g / g \\
\mu g / g \\
\mu g / g \\
\mu g / g \\
\mu g / g\end{array}$ & $\begin{array}{l}\text { Calcium } \\
\text { Mercury } \\
\text { Calcium } \\
\text { Magnesium } \\
\text { Sulfate } \\
\text { Sulfate } \\
\text { Calcium } \\
\text { Sulfate }\end{array}$ \\
\hline 255 & $\begin{array}{c}0,0.5 \\
0.5,2 \\
2,4 \\
4,6 \\
4,6 \\
4,6\end{array}$ & $\begin{array}{l}2.09 \\
2.83 \\
2.24 \\
2.42 \\
17.0 \\
14.0\end{array}$ & $\begin{array}{l}\mu g / g \\
\mu g / g \\
\mu g / g \\
\mu g / g \\
\mu g / g \\
\mu g / g\end{array}$ & $\begin{array}{l}\text { Cadmium } \\
\text { Cadmium } \\
\text { Cadmium } \\
\text { Cadmium } \\
\text { Chloride } \\
\text { Fluoride }\end{array}$ \\
\hline 256 & $\begin{array}{l}1,2 \\
1,2 \\
1,2 \\
1,2 \\
2,4 \\
2,4 \\
4,6 \\
4,6 \\
4,6\end{array}$ & $\begin{array}{c}5.03 \\
37300 \\
13800 \\
79.7 \\
1.95 \\
509 \\
2.23 \\
7030 \\
329\end{array}$ & $\begin{array}{l}\mu g / g \\
\mu g / g \\
\mu g / g \\
\mu g / g \\
\mu g / g \\
\mu g / g \\
\mu g / g \\
\mu g / g \\
\mu g / g\end{array}$ & $\begin{array}{l}\text { Cadmium } \\
\text { Calcium } \\
\text { Magnesium } \\
\text { Zinc } \\
\text { Cadmium } \\
\text { Sulfate } \\
\text { Cadmium } \\
\text { Calcium } \\
\text { Sulfate }\end{array}$ \\
\hline
\end{tabular}


TABLE 5.2-2 Soil Contaminant Concentrations Above Background (Continued)

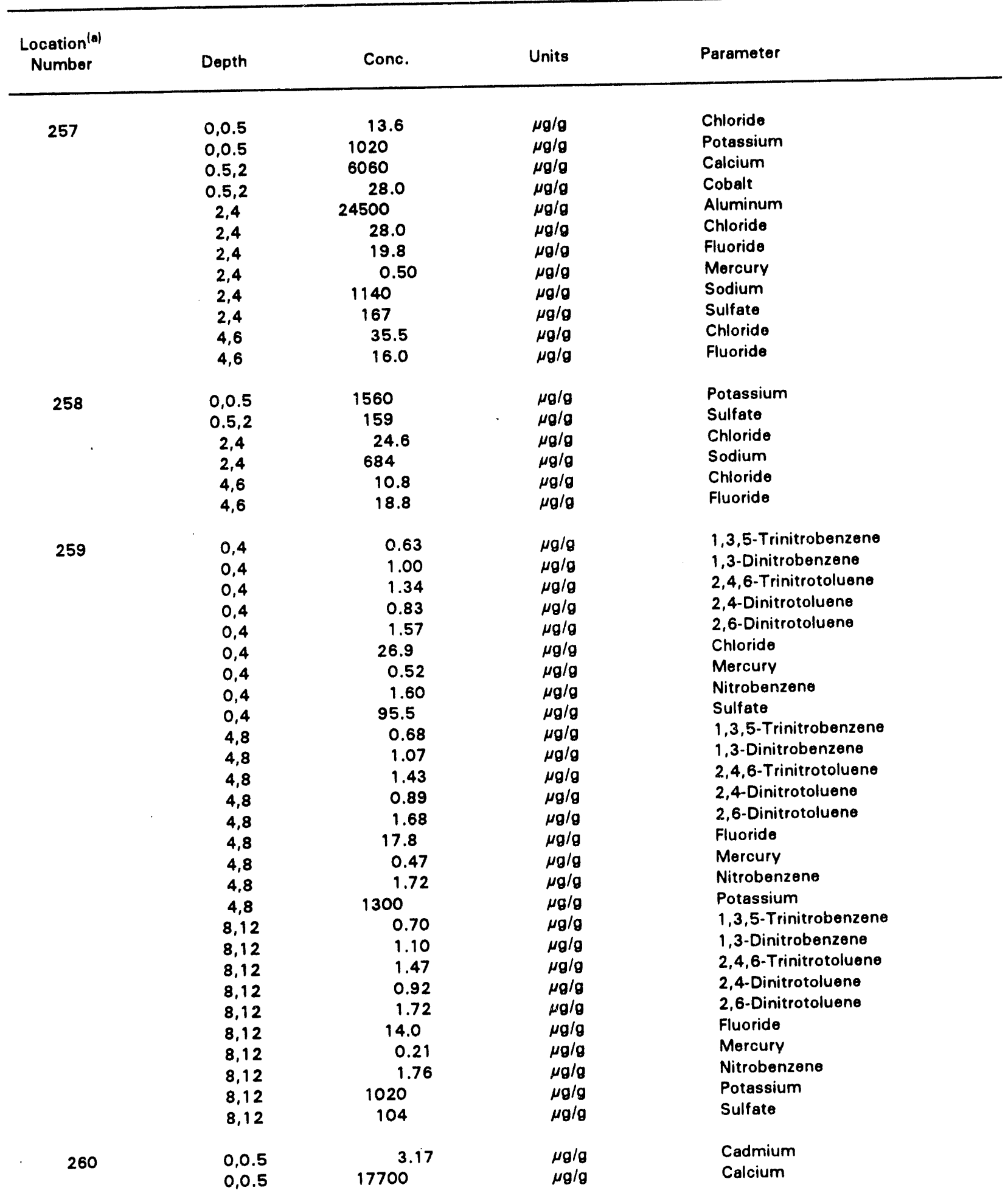


TABLE 5.2-2 Soil Contaminant Concentrations Above Background (Continued)

\begin{tabular}{|c|c|c|c|c|}
\hline $\begin{array}{l}\text { Location }^{(a)} \\
\text { Number }\end{array}$ & Depth & Conc. & Units & Parameter \\
\hline 260 (cont.) & $\begin{array}{c}0,0.5 \\
0,0.5 \\
0.5,2 \\
0.5,2 \\
0.5,2 \\
0.5,2 \\
0.5,2 \\
0.5,2 \\
2,4 \\
2,4 \\
2,4 \\
2,4 \\
2,4 \\
4,6 \\
4,6\end{array}$ & $\begin{array}{c}56.4 \\
1680 \\
2.49 \\
16700 \\
14.7 \\
46.9 \\
901 \\
199 \\
3.63 \\
28.7 \\
68.1 \\
1670 \\
7.63 \\
0.65 \\
15.8\end{array}$ & $\begin{array}{l}\mu g / g \\
\mu g / g \\
\mu g / g \\
\mu g / g \\
\mu g / g \\
\mu g / g \\
\mu g / g \\
\mu g / g \\
\mu g / g \\
\mu g / g \\
\mu g / g \\
\mu g / g \\
\mu g / g \\
\mu g / g \\
\mu g / g\end{array}$ & $\begin{array}{l}\text { Lead } \\
\text { Potassium } \\
\text { Cadmium } \\
\text { Calcium } \\
\text { Fluoride } \\
\text { Lead } \\
\text { Sodium } \\
\text { Sulfate } \\
\text { Cadmium } \\
\text { Cobalt } \\
\text { Lead } \\
\text { Manganese } \\
\text { Silver } \\
\text { 1,3,5-Trinitrobenzene } \\
\text { Chloride }\end{array}$ \\
\hline 261 & $\begin{array}{c}0,0.5 \\
0,0.5 \\
0,0.5 \\
0.5,2 \\
0.5,2 \\
0.5,2 \\
0.5,2 \\
0.5,2 \\
2,4 \\
2,4 \\
4,6 \\
4,6 \\
4,6 \\
6,10 \\
6,10 \\
10,20 \\
10,20 \\
10,20 \\
10,20 \\
10,20 \\
10,20\end{array}$ & $\begin{array}{c}2.46 \\
9200 \\
339 \\
3.95 \\
30500 \\
53.6 \\
3520 \\
10.9 \\
2.75 \\
110 \\
3.92 \\
106 \\
71.1 \\
2.10 \\
14.0 \\
2.80 \\
4.11 \\
5350 \\
31.0 \\
54.0 \\
62.0\end{array}$ & $\begin{array}{l}\mu g / g \\
\mu g / g \\
\mu g / g \\
\mu g / g \\
\mu g / g \\
\mu g / g \\
\mu g / g \\
\mu g / g \\
\mu g / g \\
\mu g / g \\
\mu g / g \\
\mu g / g \\
\mu g / g \\
\mu g / g \\
\mu g / g \\
\mu g / g \\
\mu g / g \\
\mu g / g \\
\mu g / g \\
\mu g / g \\
\mu g / g\end{array}$ & $\begin{array}{l}\text { Cadmium } \\
\text { Calcium } \\
\text { Zinc } \\
\text { Cadmium } \\
\text { Calcium } \\
\text { Lead } \\
\text { Magnesium } \\
\text { silyer } \\
\text { Cadmium } \\
\text { Sulfate } \\
\text { Cadmium } \\
\text { Cobalt } \\
\text { Lead } \\
\text { Cadmium } \\
\text { Fluoride } \\
\text { Beryllium } \\
\text { Cadmium } \\
\text { Calcium } \\
\text { Cobalt } \\
\text { Lead } \\
\text { Nickel }\end{array}$ \\
\hline 262 & $\begin{array}{c}0.5,2 \\
8,9 \\
8,9 \\
8,9 \\
8,9\end{array}$ & $\begin{array}{c}9.0 \\
3.20 \\
44.7 \\
4550 \\
710\end{array}$ & $\begin{array}{l}\mu g / g \\
\mu g / g \\
\mu g / g \\
\mu g / g \\
\mu g / g\end{array}$ & $\begin{array}{l}\text { Silver } \\
\text { Cadmium } \\
\text { Lead } \\
\text { Manganese } \\
\text { Sodium }\end{array}$ \\
\hline 263 & $\begin{array}{c}0,0.5 \\
0,0.5 \\
0,0.5 \\
0,0.5 \\
0,0.5 \\
0.5,2 \\
2,4\end{array}$ & $\begin{array}{c}180000 \\
27.0 \\
5700 \\
1400 \\
84.0 \\
185000 \\
3.92\end{array}$ & $\begin{array}{l}\mu g / g \\
\mu g / g \\
\mu g / g \\
\mu g / g \\
\mu g / g \\
\mu g / g \\
\mu g / g\end{array}$ & $\begin{array}{l}\text { Calcium } \\
\text { Copper } \\
\text { Magnesium } \\
\text { Sodium } \\
\text { Zinc } \\
\text { Calcium } \\
\text { Cadmium }\end{array}$ \\
\hline
\end{tabular}


TABLE 5.2-2 Soil Contaminant Concentrations Above Background (Continued)

\begin{tabular}{|c|c|c|c|c|}
\hline $\begin{array}{l}\text { Location }{ }^{(a)} \\
\text { Number }\end{array}$ & Depth & Conc. & Units & Parameter \\
\hline 263 (cont.) & $\begin{array}{l}2,4 \\
2,4 \\
4,6 \\
4,6 \\
4,6 \\
4,6\end{array}$ & $\begin{array}{c}14.1 \\
15.2 \\
3.60 \\
11.0 \\
4540 \\
718\end{array}$ & $\begin{array}{l}\mu g / g \\
\mu g / g \\
\mu g / g \\
\mu g / g \\
\mu g / g \\
\mu g / g\end{array}$ & $\begin{array}{l}\text { Chloride } \\
\text { Fluoride } \\
\text { Cadmium } \\
\text { Chloride } \\
\text { Manganese } \\
\text { Sodium }\end{array}$ \\
\hline 264 & $\begin{array}{c}0,0.5 \\
0,0.5 \\
0,0.5 \\
0.5,2 \\
0.5,2 \\
2,4 \\
4,6 \\
4,6 \\
4,6 \\
4,6\end{array}$ & $\begin{array}{c}5.23 \\
3.45 \\
1570 \\
3.42 \\
1110 \\
2.39 \\
2.50 \\
9.77 \\
115 \\
94.2\end{array}$ & $\begin{array}{l}\mu g / g \\
\mu g / g \\
\mu g / g \\
\mu g / g \\
\mu g / g \\
\mu g / g \\
\mu g / g \\
\mu g / g \\
\mu g / g \\
\mu g / g\end{array}$ & $\begin{array}{l}\text { Beryllium } \\
\text { Cadmium } \\
\text { Manganese } \\
\text { Cadmium } \\
\text { Potassium } \\
\text { Cadmium } \\
\text { Cadmium } \\
\text { Chloride } \\
\text { Lead } \\
\text { Sulfate }\end{array}$ \\
\hline 265 & $\begin{array}{l}0,2 \\
0,2 \\
0,2 \\
4,6 \\
4,6 \\
8,10 \\
8,10 \\
8,10 \\
8,10 \\
8,10 \\
8,10\end{array}$ & $\begin{array}{c}270 \\
1.50 \\
0.11 \\
36.0 \\
27.0 \\
3.00 \\
5260 \\
750 \\
32100 \\
25.3 \\
67.2\end{array}$ & $\begin{array}{c}\mu \mathrm{g} / \mathrm{kg} \\
\mu \mathrm{g} / \mathrm{g} \\
\mu \mathrm{g} / \mathrm{g} \\
\mu \mathrm{g} / \mathrm{g} \\
\mu \mathrm{g} / \mathrm{g} \\
\mu \mathrm{g} / \mathrm{g} \\
\mu \mathrm{g} / \mathrm{g} \\
\mu \mathrm{g} / \mathrm{kg} \\
\mu \mathrm{g} / \mathrm{g} \\
\mu \mathrm{g} / \mathrm{g} \\
\mu \mathrm{g} / \mathrm{g}\end{array}$ & $\begin{array}{l}\text { Aroclor-1254 } \\
\text { Cadmium } \\
\text { Mercury } \\
\text { Cobalt } \\
\text { Molybdenum } \\
\text { Beryllium } \\
\text { Calcium } \\
\text { Diethyiphthalate } \\
\text { Iron } \\
\text { Molybdenum } \\
\text { Nickel }\end{array}$ \\
\hline 266 & $\begin{array}{l}0,2 \\
2,4 \\
4,6 \\
4,6 \\
4,6 \\
6,8 \\
6,8 \\
6,8 \\
6,8 \\
6,8 \\
8,10\end{array}$ & $\begin{array}{c}0.15 \\
0.12 \\
25500 \\
5400 \\
32.0 \\
5270 \\
30800 \\
28.4 \\
96.4 \\
84.7 \\
2.78\end{array}$ & $\begin{array}{l}\mu g / g \\
\mu g / g \\
\mu g / g \\
\mu g / g \\
\mu g / g \\
\mu g / g \\
\mu g / g \\
\mu g / g \\
\mu g / g \\
\mu g / g \\
\mu g / g\end{array}$ & $\begin{array}{l}\text { Mercury } \\
\text { Mercury } \\
\text { Aluminum } \\
\text { Calcium } \\
\text { Molybdenum } \\
\text { Calcium } \\
\text { Iron } \\
\text { Molybdenum } \\
\text { Nitrate } \\
\text { Zinc } \\
\text { Beryllium }\end{array}$ \\
\hline 267 & $\begin{array}{l}0,2 \\
0,2 \\
0,2 \\
0,2 \\
2,4 \\
2,4 \\
2,4 \\
4,6 \\
4,6\end{array}$ & $\begin{array}{c}240 \\
670 \\
31.9 \\
1670 \\
70.0 \\
380 \\
1060 \\
1660 \\
370\end{array}$ & $\begin{array}{c}\mu g / k g \\
\mu g / k g \\
\mu g / g \\
\mu g / g \\
\mu g / k g \\
\mu g / k g \\
\mu g / g \\
\mu g / k g \\
\mu g / k g\end{array}$ & $\begin{array}{l}\text { Aldrin } \\
\text { Bis (2-Ethylhexyl) Phthalate } \\
\text { Molybdenum } \\
\text { Potassium } \\
\text { Aldrin } \\
\text { Bis (2-Ethylhexyl) Phthalate } \\
\text { Potassium } \\
\text { Aldrin } \\
\text { Bis (2-Ethylhexyl) Phthalate }\end{array}$ \\
\hline
\end{tabular}




\section{TABLE 5.2-2 Soil Contaminant Concentrations Above Background (Continued)}

\begin{tabular}{|c|c|c|c|c|}
\hline $\begin{array}{l}\text { Location(a) } \\
\text { Number }\end{array}$ & Depth & Conc. & Units & Parameter \\
\hline 267 (cont.) & $\begin{array}{c}6,8 \\
6,8 \\
8,10 \\
10,12 \\
10,12\end{array}$ & $\begin{array}{l}610 \\
26.0 \\
740 \\
20.0 \\
660\end{array}$ & $\begin{array}{l}\mu g / k g \\
\mu g / g \\
\mu g / k g \\
\mu g / k g \\
\mu g / k g\end{array}$ & $\begin{array}{l}\text { Bis (2-Ethylhexyl) Phthalate } \\
\text { Molybdenum } \\
\text { Bis (2-Ethylhexyl) Phthalate } \\
\text { Aldrin } \\
\text { Bis (2-Ethylhexyl) Phthalate }\end{array}$ \\
\hline 268 & $\begin{array}{l}6,8 \\
6,8\end{array}$ & $\begin{array}{c}0.13 \\
55.8\end{array}$ & $\begin{array}{l}\mu g / g \\
\mu g / g\end{array}$ & $\begin{array}{l}\text { Mercury } \\
\text { Vanadium }\end{array}$ \\
\hline 269 & $\begin{array}{l}0,2 \\
0,2 \\
2,4 \\
2,4 \\
2,4 \\
6,8 \\
6,8 \\
6,8\end{array}$ & $\begin{array}{c}26.8 \\
1060 \\
3730 \\
1280 \\
148 \\
1.30 \\
27.1 \\
25.2\end{array}$ & $\begin{array}{l}\mu g / g \\
\mu g / g \\
\mu g / g \\
\mu g / g \\
\mu g / g \\
\mu g / g \\
\mu g / g \\
\mu g / g\end{array}$ & $\begin{array}{l}\text { Molybdenum } \\
\text { Potassium } \\
\text { Magnesium } \\
\text { Potassium } \\
\text { Sulfate } \\
\text { Cadmium } \\
\text { Cobalt } \\
\text { Molybdenum }\end{array}$ \\
\hline 270 & $\begin{array}{l}0,2 \\
0,2 \\
2,4\end{array}$ & $\begin{array}{l}0.11 \\
123 \\
150\end{array}$ & $\begin{array}{l}\mu g / g \\
\mu g / g \\
\mu g / g\end{array}$ & $\begin{array}{l}\text { Mercury } \\
\text { Sulfate } \\
\text { Sulfate }\end{array}$ \\
\hline 271 & $\begin{array}{c}0.5,2 \\
0.5,2 \\
2,4 \\
2,4 \\
4,6 \\
4,6 \\
4,6 \\
6,10 \\
6,10 \\
10,14 \\
10,14 \\
10,14 \\
10,14 \\
10,14 \\
14.3,16 \\
14.3,16 \\
14.3,16\end{array}$ & $\begin{array}{c}2.43 \\
0.27 \\
2.34 \\
0.27 \\
3.02 \\
10500 \\
787 \\
3.80 \\
842 \\
2.96 \\
2.94 \\
1.60 \\
1.35 \\
92.7 \\
3.58 \\
29.5 \\
1.31\end{array}$ & $\begin{array}{l}\mu \mathrm{g} / \mathrm{g} \\
\mu \mathrm{g} / \mathrm{g} \\
\mu \mathrm{g} / \mathrm{g} \\
\mu \mathrm{g} / \mathrm{g} \\
\mu \mathrm{g} / \mathrm{g} \\
\mu \mathrm{g} / \mathrm{g} \\
\mu \mathrm{g} / \mathrm{g} \\
\mu \mathrm{g} / \mathrm{g} \\
\mu \mathrm{g} / \mathrm{g} \\
\mu \mathrm{g} / \mathrm{g} \\
\mu \mathrm{g} / \mathrm{g} \\
\mu g / g \\
\mu \mathrm{g} / \mathrm{g} \\
\mu \mathrm{g} / \mathrm{g} \\
\mu \mathrm{g} / \mathrm{g} \\
\mu \mathrm{g} / \mathrm{g} \\
\mu \mathrm{g} / \mathrm{g}\end{array}$ & $\begin{array}{l}\text { Cadmium } \\
\text { Mercury } \\
\text { Cadmium } \\
\text { Mercury } \\
\text { Cadmium } \\
\text { Calcium } \\
\text { Sodium } \\
\text { Cadmium } \\
\text { Sodium } \\
\text { 1,3,5-Trinitrobenzene } \\
\text { 2,4-Dinitrotoluene } \\
\text { Cadmium } \\
\text { Mercury } \\
\text { Nitrate } \\
\text { Cadmium } \\
\text { Cobalt } \\
\text { Mercury }\end{array}$ \\
\hline 272 & $\begin{array}{c}0,0.5 \\
0,0.5 \\
0.5,2 \\
0.5,2 \\
0.5,2 \\
0.5,2 \\
2,4 \\
2,4 \\
4,6 \\
4,6\end{array}$ & $\begin{array}{c}2.22 \\
34500 \\
3.51 \\
29000 \\
3920 \\
100.0 \\
2.35 \\
35000 \\
2.40 \\
34900\end{array}$ & $\begin{array}{l}\mu g / g \\
\mu g / g \\
\mu g / g \\
\mu g / g \\
\mu g / g \\
\mu g / g \\
\mu g / g \\
\mu g / g \\
\mu g / g \\
\mu g / g\end{array}$ & $\begin{array}{l}\text { Cadmium } \\
\text { Calcium } \\
\text { Cadmium } \\
\text { Calcium } \\
\text { Magnesium } \\
\text { Zinc } \\
\text { Cadmium } \\
\text { Calcium } \\
\text { Cadmium } \\
\text { Calcium }\end{array}$ \\
\hline
\end{tabular}


TABLE 5.2-2 Soil Contaminant Concentrations Above Background (Continued)

\begin{tabular}{|c|c|c|c|c|}
\hline $\begin{array}{l}\text { Location }^{(a)} \\
\text { Number }\end{array}$ & Depth & Conc. & Units & Parameter \\
\hline 272 (cont.) & $\begin{array}{l}4,6 \\
4,6\end{array}$ & $\begin{array}{r}1090 \\
141\end{array}$ & $\begin{array}{l}\mu \boldsymbol{g} / \boldsymbol{g} \\
\mu \boldsymbol{g} / \boldsymbol{g}\end{array}$ & $\begin{array}{l}\text { Potassium } \\
\text { Sulfate }\end{array}$ \\
\hline 273 & $\begin{array}{l}2,4 \\
6,8\end{array}$ & $\begin{array}{l}1.04 \\
97.7\end{array}$ & $\begin{array}{l}\mu g / g \\
\mu g / g\end{array}$ & $\begin{array}{l}\text { 2,4-Dinitrotoluene } \\
\text { Sulfate }\end{array}$ \\
\hline 274 & $\begin{array}{l}0,2 \\
0,2 \\
0,2 \\
0,2 \\
0,2 \\
2,4 \\
2,4 \\
2,4 \\
2,4 \\
8,10\end{array}$ & $\begin{array}{c}11900 \\
4140 \\
33.1 \\
1390 \\
97.8 \\
15800 \\
4860 \\
24.9 \\
1440 \\
29.3\end{array}$ & $\begin{array}{l}\mu g / g \\
\mu g / g \\
\mu g / g \\
\mu g / g \\
\mu g / g \\
\mu g / g \\
\mu g / g \\
\mu g / g \\
\mu g / g \\
\mu g / g\end{array}$ & $\begin{array}{l}\text { Calcium } \\
\text { Magnesium } \\
\text { Molvbdenum } \\
\text { Potassium } \\
\text { Sulfate } \\
\text { Calcium } \\
\text { Magnesium } \\
\text { Molybdenum } \\
\text { Potassium } \\
\text { Molybdonum }\end{array}$ \\
\hline 275 & $\begin{array}{c}0,0.5 \\
0,0.5 \\
0.5,2 \\
0.5,2 \\
0.5,2 \\
0.5,2 \\
2,4 \\
2,4 \\
2,4 \\
2,4 \\
2,4 \\
2,4 \\
2,4 \\
2,4 \\
2,4 \\
2,4 \\
4,6 \\
4,6 \\
4,6 \\
4,6 \\
4,6\end{array}$ & $\begin{array}{c}2.10 \\
182 \\
3.43 \\
12800 \\
30.0 \\
44.6 \\
10.5 \\
9300 \\
42.0 \\
35.0 \\
34.0 \\
82200 \\
43034 \\
1400 \\
341 \\
337 \\
3.74 \\
20600 \\
3900 \\
193 \\
83.0\end{array}$ & $\begin{array}{l}\mu g / g \\
\mu g / g \\
\mu g / g \\
\mu g / g \\
\mu g / g \\
\mu g / g \\
\mu g / g \\
\mu g / g \\
\mu g / g \\
\mu g / g \\
\mu g / g \\
\mu g / g \\
\mu g / g \\
\mu g / g \\
\mu g / g \\
\mu g / g \\
\mu g / g \\
\mu g / g \\
\mu g / g \\
\mu g / g \\
\mu g / g\end{array}$ & $\begin{array}{l}\text { Cadmium } \\
\text { Zinc } \\
\text { Cadmium } \\
\text { Calcium } \\
\text { Copper } \\
\text { Lead } \\
\text { Cadmium } \\
\text { Calcium } \\
\text { Chromium } \\
\text { Cobalt } \\
\text { Copper } \\
\text { Iron } \\
\text { Lead } \\
\text { Potassium } \\
\text { Sulfate } \\
\text { Zinc } \\
\text { Cadmium } \\
\text { Calcium } \\
\text { Magnesium } \\
\text { Sulfate } \\
\text { Zinc }\end{array}$ \\
\hline 276 & $\begin{array}{c}0,0.5 \\
0,0.5 \\
0,0.5 \\
0.5,2 \\
0.5,2 \\
0.5,2 \\
0.5,2 \\
2.4 \\
4,6 \\
4,6 \\
4,6\end{array}$ & $\begin{array}{c}2.31 \\
37400 \\
1070 \\
3.41 \\
30800 \\
1600 \\
59.0 \\
2.40 \\
29.0 \\
2550 \\
365\end{array}$ & $\begin{array}{l}\mu g / g \\
\mu g / g \\
\mu g / g \\
\mu g / g \\
\mu g / g \\
\mu g / g \\
\mu g / g \\
\mu g / g \\
\mu g / g \\
\mu g / g \\
\mu g / g\end{array}$ & $\begin{array}{l}\text { Cadmium } \\
\text { Calcium } \\
\text { Potassium } \\
\text { Cadmium } \\
\text { Iron } \\
\text { Potassium } \\
\text { Vanadium } \\
\text { Cadmium } \\
\text { Cobalt } \\
\text { Manganese } \\
\text { Sulfate }\end{array}$ \\
\hline
\end{tabular}


TABLE 5.2-2 Soil Contaminant Concentrations Above Background (Continued)

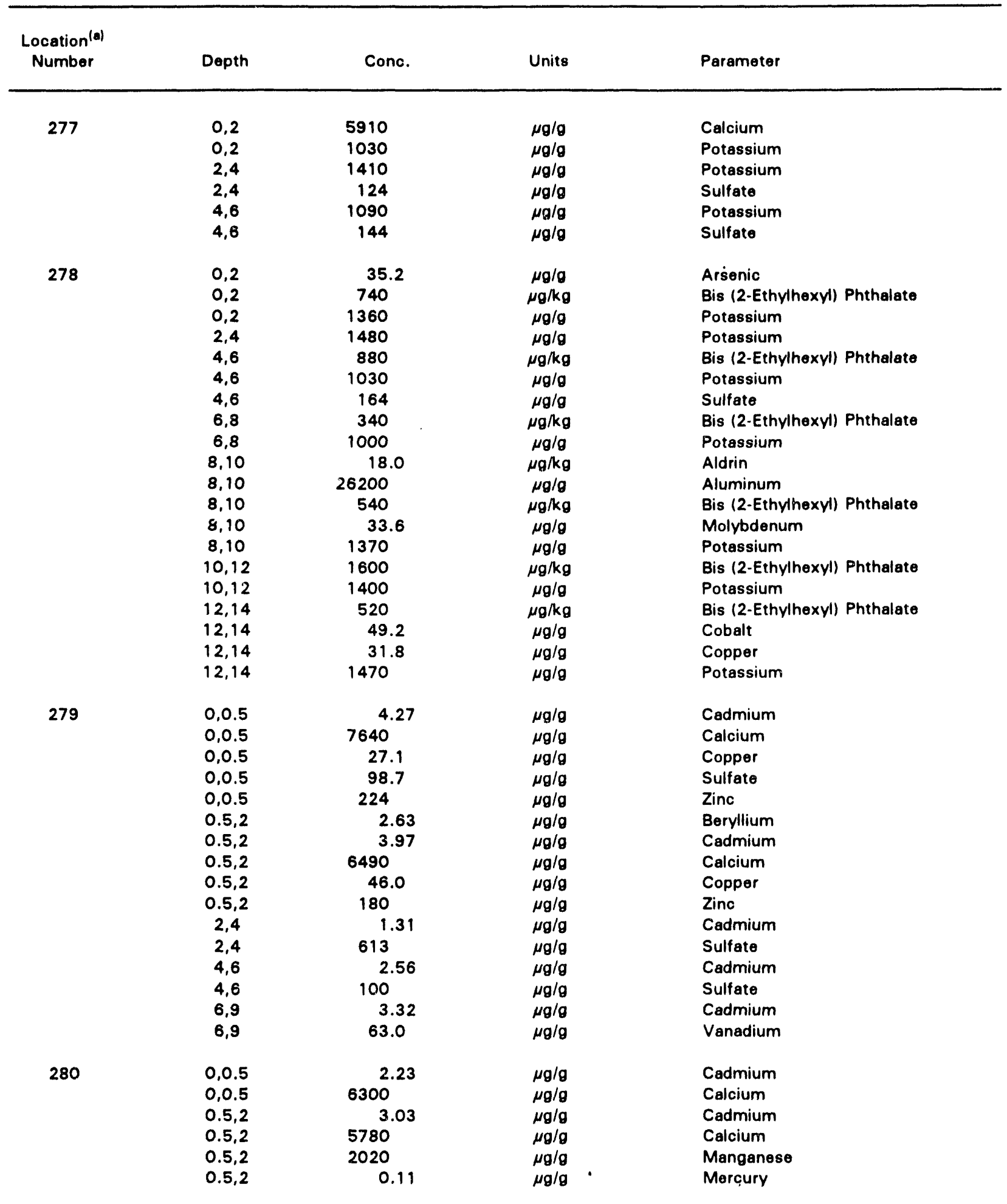


TABLE 5.2-2 Soil Contaminant Concentrations Above Background (Continued)

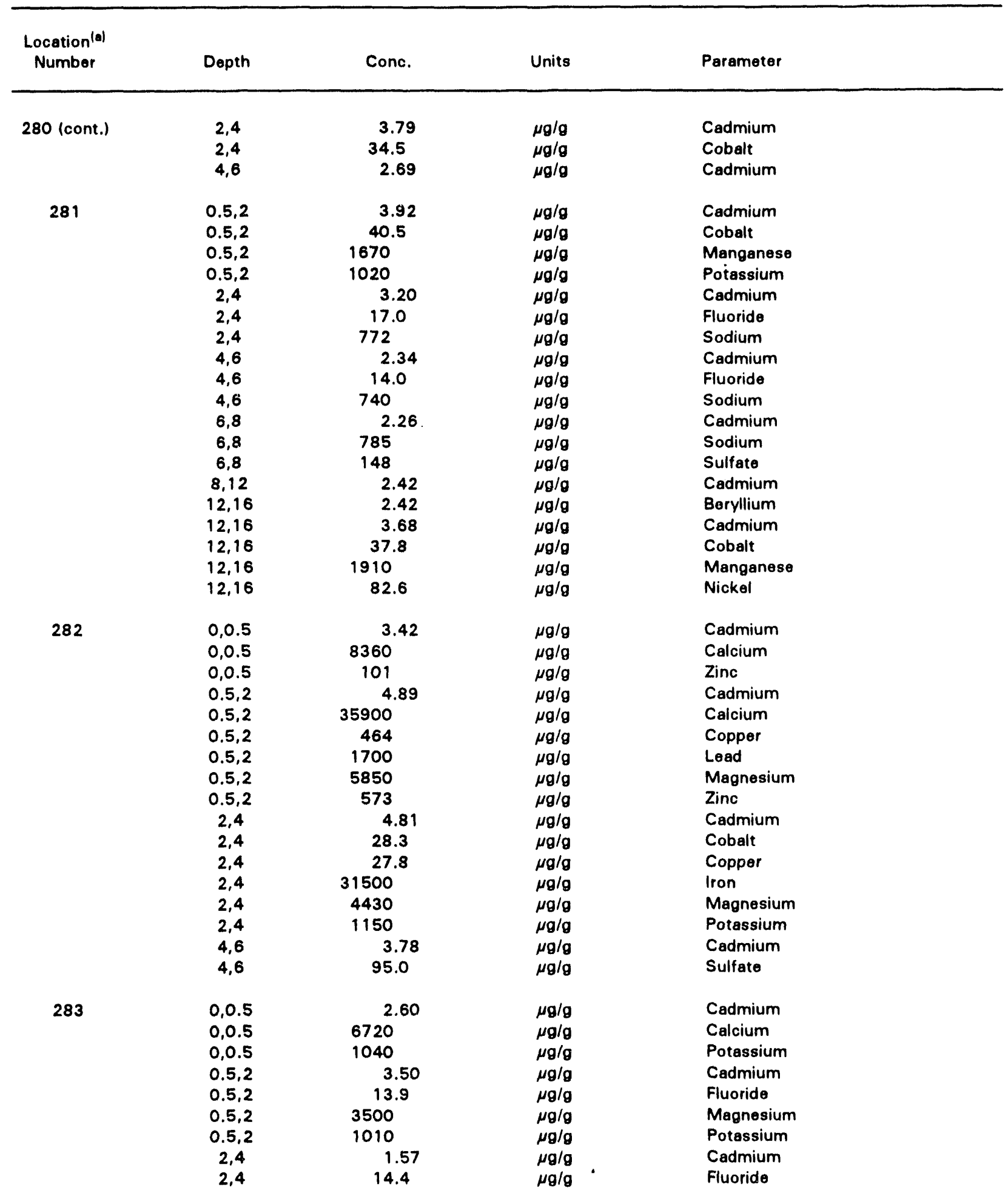


TABLE 5.2-2 Soil Contaminant Concentrations Above Background (Continued)

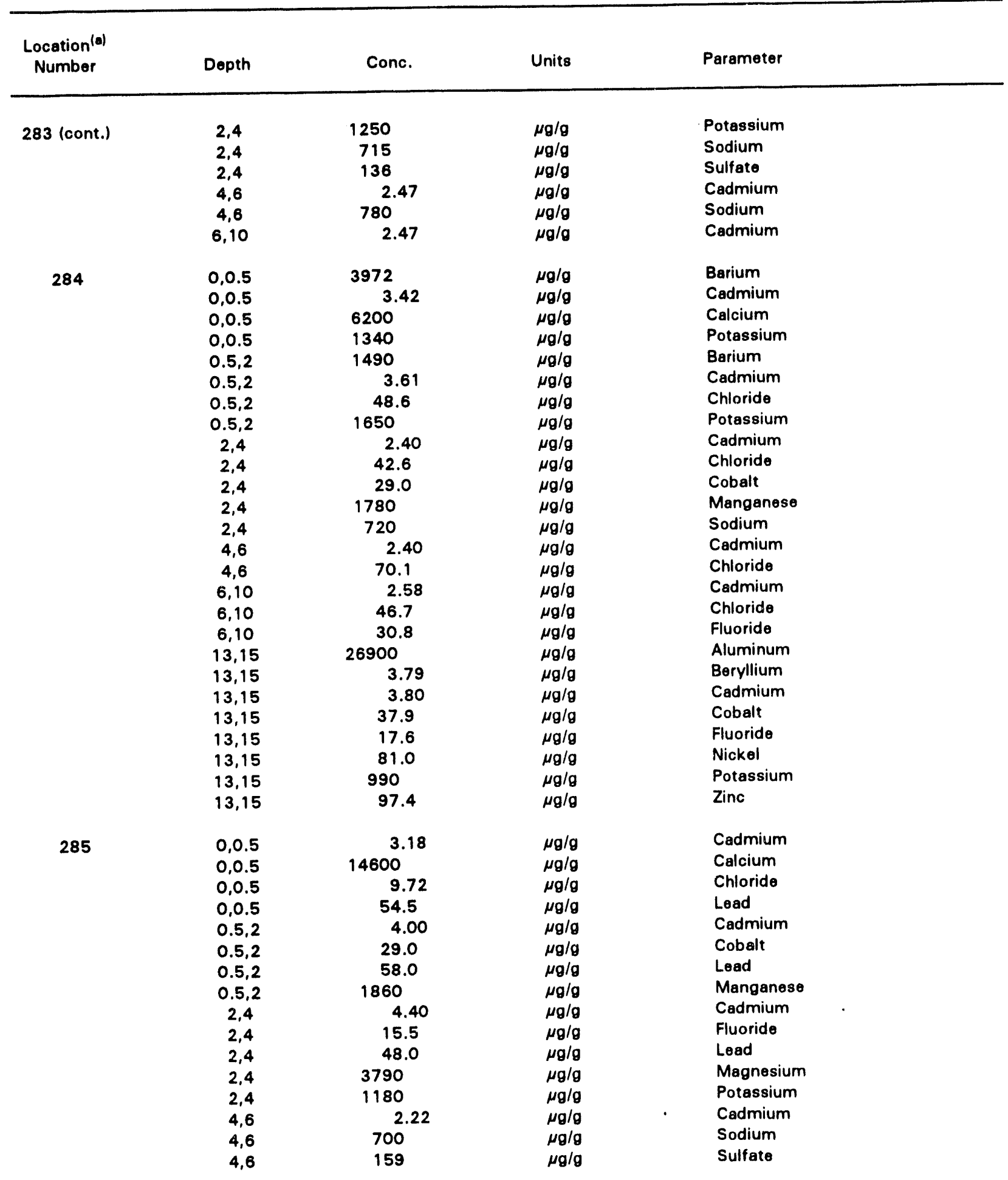


TABLE 5.2-2 Soil Contaminant Concentrations Above Background (Continued)

\begin{tabular}{|c|c|c|c|c|}
\hline $\begin{array}{l}\text { Location }^{(a)} \\
\text { Number }\end{array}$ & Depth & Conc. & Units & Parameter \\
\hline \multirow[t]{12}{*}{286} & 5,6 & 3.55 & $\mu g / g$ & Cadmium \\
\hline & 5,6 & 36200 & $\mu g / g$ & Calcium \\
\hline & 5,6 & 8580 & $\mu \mathrm{g} / \mathrm{g}$ & Magnesium \\
\hline & 5,6 & 101 & $\mu g / g$ & Sulfate \\
\hline & 6,8 & 2.04 & $\mu g / g$ & Cadmium \\
\hline & 8,10 & 2.71 & $\mu g / g$ & Cadmium \\
\hline & 10,12 & 2.93 & $\mu g / g$ & Cadmium \\
\hline & 10,12 & 86.3 & $\mu g / g$ & Nitrate \\
\hline & 10,12 & 683 & $\mu g / g$ & Sodium \\
\hline & 12,16 & 3.13 & $\mu g / g$ & Cadmium \\
\hline & 12,16 & 43.6 & $\mu g / g$ & Nickol \\
\hline & 12,16 & 145 & $\mu g / g$ & Nitrate \\
\hline \multirow[t]{17}{*}{287} & $0,0.5$ & 2.40 & $\mu g / g$ & Cadmium \\
\hline & $0,0.5$ & 6100 & $\mu g / g$ & Calcium \\
\hline & $0,0.5$ & 48.0 & $\mu g / g$ & Copper \\
\hline & $0,0.5$ & 1100 & $\mu \mathrm{g} / \mathrm{g}$ & Lead \\
\hline & $0,0.5$ & 71.0 & $\mu \mathrm{g} / \mathrm{g}$ & Lithium \\
\hline & $0,0.5$ & 1110 & $\mu g / g$ & Potassium \\
\hline & $0.5,2$ & 32.3 & $\mu g / g$ & $2,4,6$-Trinitrçoluene \\
\hline & $0.5,2$ & 2.29 & $\mu \mathrm{g} / \mathrm{g}$ & Cadmium \\
\hline & $0.5,2$ & 7000 & $\mu \mathrm{g} / \mathrm{g}$ & Calcium \\
\hline & $0.5,2$ & 585 & $\mu g / g$ & Lead \\
\hline & 2,4 & 3.42 & $\mu g / g$ & Cadmium \\
\hline & 2,4 & 13300 & $\mu g / g$ & Calcium \\
\hline & 2,4 & 285 & $\mu g / g$ & Lead \\
\hline & 2,4 & 990 & $\mu g / g$ & Potassium \\
\hline & 4,6 & 2.32 & $\mu g / g$ & Cadmium \\
\hline & 4,6 & 10500 & $\mu g / g$ & Calcium \\
\hline & 4,6 & 127 & $\mu g / g$ & Lead \\
\hline \multirow[t]{9}{*}{288} & $0,0.5$ & 2.20 & $\mu \mathrm{g} / \mathrm{g}$ & Cadmium \\
\hline & $0,0.5$ & 1760 & $\mu \mathrm{g} / \mathrm{g}$ & Manganese \\
\hline & $0.5,2$ & 2.57 & $\mu \mathrm{g} / \mathrm{g}$ & Cadmium \\
\hline & $0.5,2$ & 5840 & $\mu \mathrm{g} / \mathrm{g}$ & Calcium \\
\hline & $0.5,2$ & 26.3 & $\mu \mathrm{g} / \mathrm{g}$ & Cobalt \\
\hline & $0.5,2$ & 2920 & $\mu g / g$ & Manganese \\
\hline & 2,4 & 2.77 & $\mu g / g$ & Cadmium \\
\hline & 2,4 & 26.8 & $\mu g / g$ & Cobalt \\
\hline & 4,6 & 1.98 & $\mu g / g$ & Cadmium \\
\hline
\end{tabular}

(a) Location number corresponds to number presented on Figures 5.2-1 through 5.2-31. 


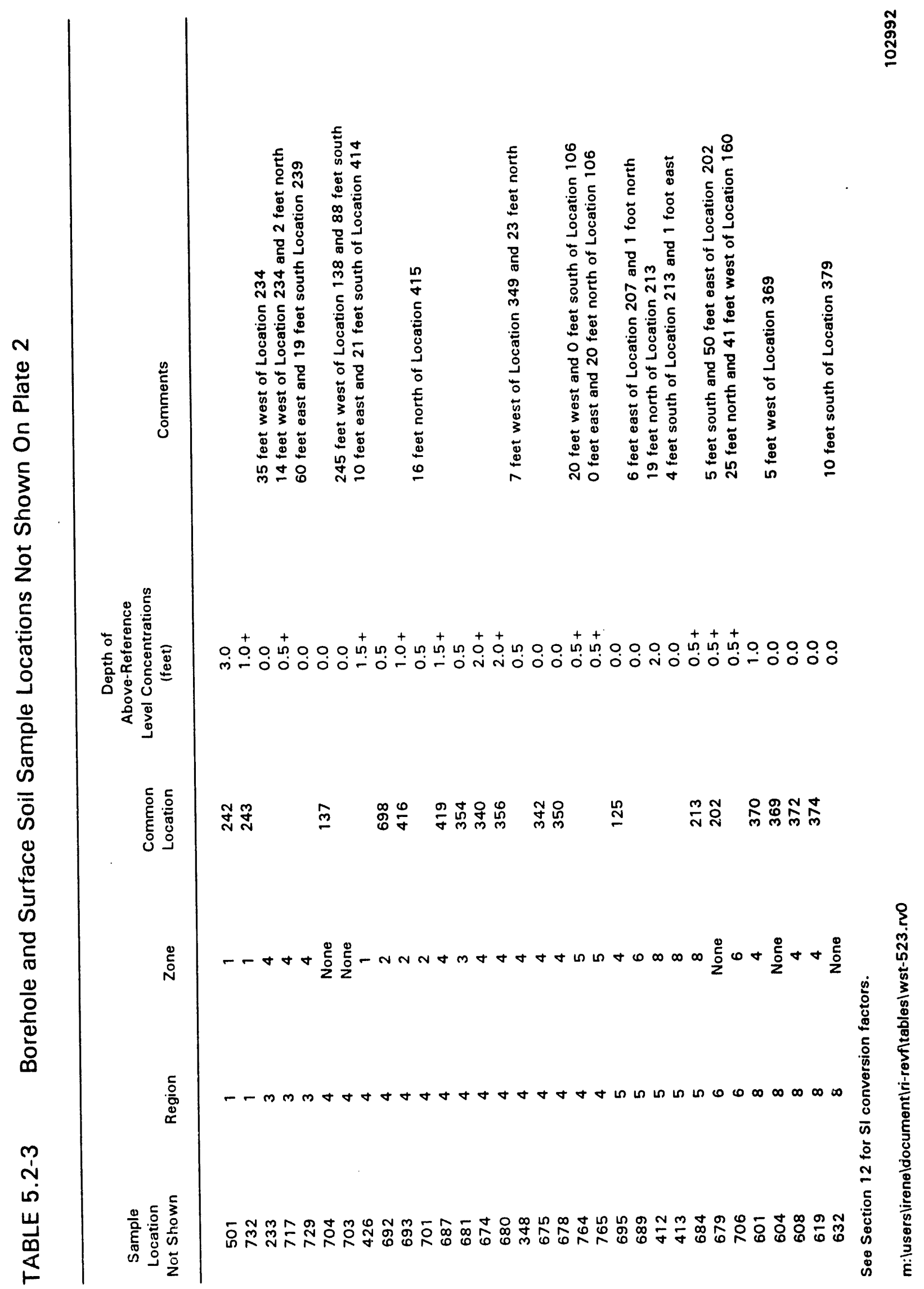




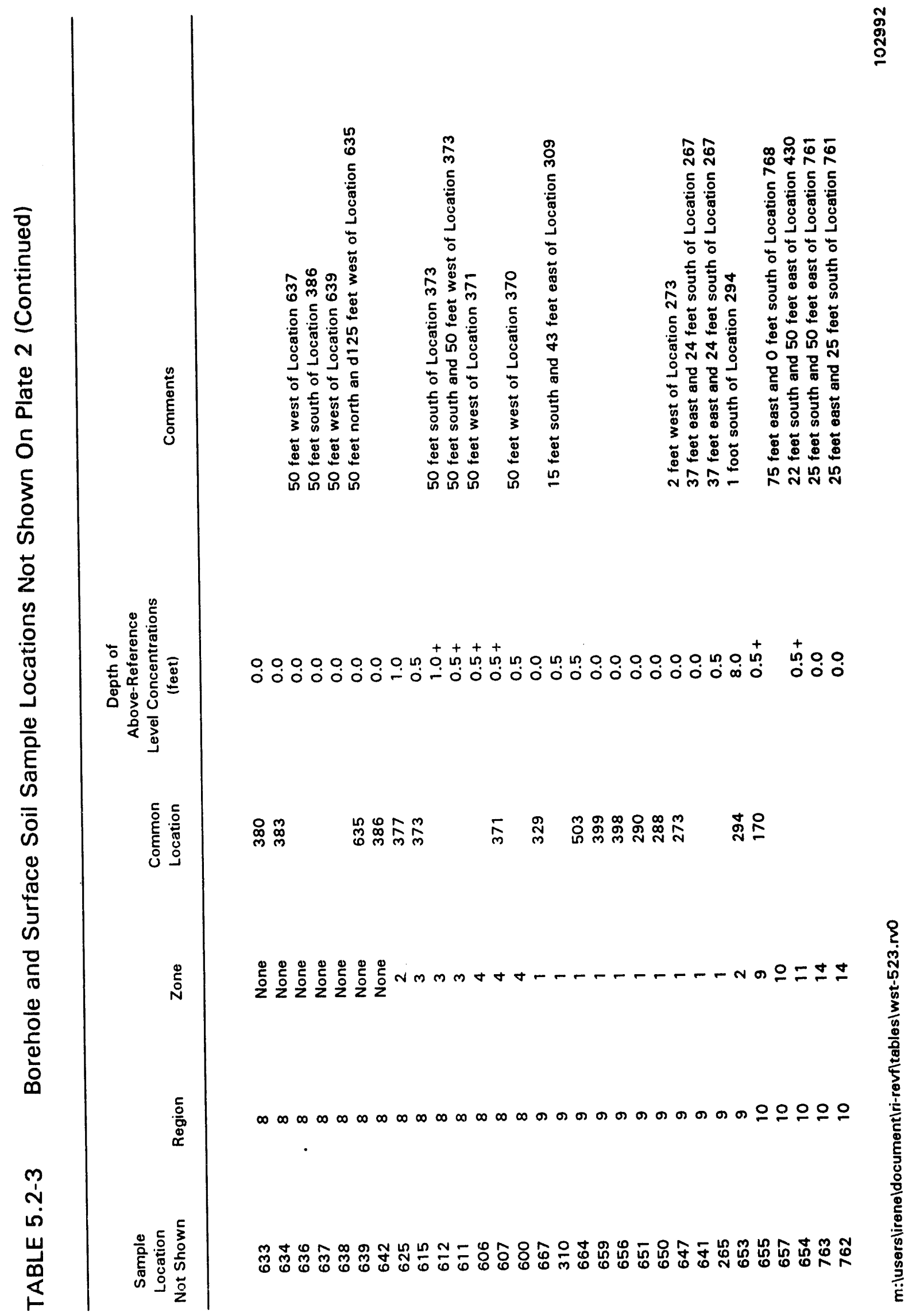




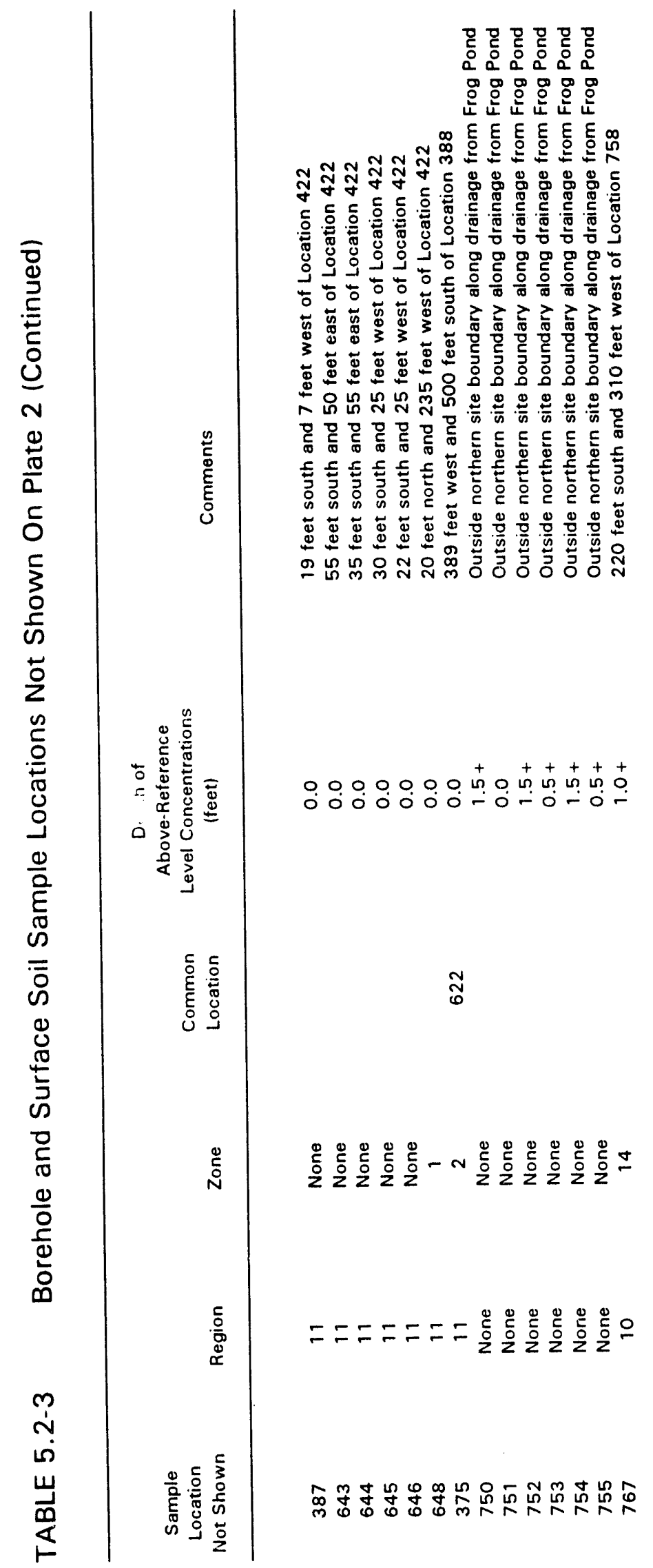


TABLE 5.2-4 Summary of Estimated Areas, Depths, and Volumes in Zones

\begin{tabular}{|c|c|c|c|c|}
\hline Region & Zone & Area $\left(\mathrm{ft}^{2}\right)$ & Uepth (ft) & Volume (cy) \\
\hline \multirow[t]{2}{*}{1} & 1 & 65,491 & 3.0 & 7,277 \\
\hline & 2 & 17,015 & 0.5 & 315 \\
\hline \multirow[t]{4}{*}{3} & 1 & 25,757 & 1.0 & 954 \\
\hline & 2 & 5,000 & 1.0 & 185 \\
\hline & 3 & 9,348 & 0.5 & 173 \\
\hline & 4 & 36,885 & 4.0 & 5,464 \\
\hline \multirow[t]{6}{*}{4} & 1 & 7,525 & 5.0 & 1,394 \\
\hline & 2 & 305,791 & 0.5 & 5,663 \\
\hline & 3 & 55,408 & 0.5 & 1,026 \\
\hline & 4 & 182,290 & 2.5 & 16,879 \\
\hline & 5 & 7,621 & 0.5 & 141 \\
\hline & 6 & 47,820 & 0.5 & 886 \\
\hline \multirow[t]{11}{*}{5} & 1 & 16,156 & 1.0 & 898 \\
\hline & 2 & 117,033 & 0.5 & 2,167 \\
\hline & 3 & 6,597 & 4.0 & 977 \\
\hline & 4 & 26,486 & 0.5 & 490 \\
\hline & 5 & 51,908 & 1.5 & 2,884 \\
\hline & 6 & 27,683 & 0.5 & 513 \\
\hline & 7 & 9,606 & 0.5 & 178 \\
\hline & 8 & 23,349 & 2.0 & 1,730 \\
\hline & 9 & 183 & 11.0 & 75 \\
\hline & 10 & 938 & 0.5 & 17 \\
\hline & 11 & 2,505 & 1.0 & 92 \\
\hline \multirow[t]{6}{*}{6} & 1 & 20,667 & 0.5 & 383 \\
\hline & 2 & 65,947 & 1.0 & 2,442 \\
\hline & 3 & 8,955 & 2.0 & 663 \\
\hline & 4 & 40,379 & 0.5 & 748 \\
\hline & 5 & 24,466 & 0.5 & 453 \\
\hline & 6 & 5,851 & 0.5 & 108 \\
\hline \multirow[t]{4}{*}{8} & 1 & 16,380 & 0.5 & 303 \\
\hline & 2 & 16,475 & 2.0 & 1,220 \\
\hline & 3 & 29,000 & 1.0 & 1,075 \\
\hline & 4 & 31,847 & 0.5 & 590 \\
\hline \multirow[t]{2}{*}{9} & 1 & 277.350 & 0.5 & 5,136 \\
\hline & 2 & 18,539 & 8.0 & 5,493 \\
\hline \multirow[t]{11}{*}{10} & 1 & 11,175 & 1.0 & 414 \\
\hline & 2 & 14,552 & 0.5 & 270 \\
\hline & 3 & 8,636 & 1.0 & 320 \\
\hline & 4 & 2,533 & 0.5 & 47 \\
\hline & 5 & 12,819 & 1.5 & 712 \\
\hline & 6 & 16,233 & 4.0 & 2,405 \\
\hline & 7 & 5,688 & 0.5 & 105 \\
\hline & 8 & 10,042 & 1.0 & 372 \\
\hline & 9 & 16,384 & 2.0 & 1,214 \\
\hline & 10 & 23,050 & 1.0 & 854 \\
\hline & 11 & 56,156 & 1.0 & 2,080 \\
\hline \multirow[t]{2}{*}{10} & 12 & 4,904 & 1.0 & 182 \\
\hline & 13 & 3,099 & 0.5 & 115 \\
\hline milusers & mentir & rvo & & \\
\hline
\end{tabular}


TABLE 5.2-4 Summary of Estimated Areas, Depths, and Volumes in Zones (Continued)

\begin{tabular}{ccccc}
\hline Region & Zone & Area $\left(\mathrm{ft}^{2}\right)$ & Depth (tt) & Volume (cy) \\
\hline & 14 & 11,162 & 0.5 & 207 \\
11 & 1 & 91,321 & 0.5 & 1,691 \\
& 2 & 46,938 & 0.5 & 869 \\
TOTAL & & $1,938,943$ & & 80,849
\end{tabular}

See Section 12 for SI conversion factors.

Notes: 1) Regions 2 and 7 do not contain zones

2) Zone areas, depths and boundaries are based on reference-level concentrations created for presentation purposes only. Some of these parameters will most likely change if site-specific guidelines to be be determined later differ from reference-level concentrations. 


\section{TABLE 5.2-5 Summary of Contamination on Vicinity Property DA 1}

\begin{tabular}{|c|c|c|c|}
\hline \multirow[b]{2}{*}{ Location } & \multicolumn{2}{|c|}{ Volume } & \multirow{2}{*}{$\begin{array}{c}\text { Average U-238 } \\
\text { Concentration } \\
(p \mathrm{Ci} / \mathrm{g})\end{array}$} \\
\hline & $\mathrm{m}^{3}$ & $\left(y d^{3}\right)$ & \\
\hline Buried Mound & 317 & $(415)$ & 676 \\
\hline \multicolumn{4}{|l|}{ Northern and W/estern } \\
\hline Mound Perimeter & 337 & $(440)$ & 75 \\
\hline Exterior one & 138 & $(180)$ & 22 \\
\hline Along Railroad Tracks & 85 & $(111)$ & 38 \\
\hline \multirow[t]{2}{*}{ Drainage Ditch } & 15 & (19) & NE \\
\hline & 892 & $(1165)$ & $281^{(a)}$ \\
\hline
\end{tabular}

\footnotetext{
(a) Volume - weighted average

NE - Not estimated
}

See Section 12 for SI conversion factors.

Source: MKF and JEG 1987j 


\section{TABLE 5.2-6 Summary of Contamination on Vicinity Property DA 2}

\begin{tabular}{|c|c|c|}
\hline Location & $m^{\text {Volume }}\left(y d^{3}\right)$ & $\begin{array}{c}\text { Average U-238 } \\
\text { Concentration } \\
\text { (pCi/g) }\end{array}$ \\
\hline Zone 1 & 31 & 20 \\
\hline Zone 2 & 26 & 18 \\
\hline Zone 3 & 50 & 96 \\
\hline Zone 4 & 71 & 26 \\
\hline Zone $5^{(a)}$ & 6 & 76 \\
\hline & - & - \\
\hline TOTAL & (184) & 45 (b) \\
\hline
\end{tabular}

(a) Average Ra-226 concentration of $18 \mathrm{pCi} / \mathrm{g}$

(b) Volume - weighted average

See Section 12 for SI conversion factors.

Source: $\quad$ MKF and JEG $1987 f$ 
TABLE 5.2-7 Summary of Vicinity Property Estimated Areas, Depths, Volumes, and Average Primary Contaminant Concentrations

\begin{tabular}{|c|c|c|c|c|c|}
\hline $\begin{array}{c}\text { Vicinity } \\
\text { Property } \\
\text { Identification }\end{array}$ & $\begin{array}{c}\text { Area } \\
m^{2}\left(\mathrm{ft}^{2}\right)\end{array}$ & $\begin{array}{c}\text { Average depth } \\
\mathrm{cm}(\mathrm{ft})\end{array}$ & $\begin{array}{l}\text { Volume } \\
\mathrm{m}^{3}\left(y d^{3}\right)\end{array}$ & $\begin{array}{c}\text { Primary } \\
\text { Contaminant }\end{array}$ & $\begin{array}{c}\text { Average } \\
\text { Concentration } \\
\text { pCi/g }\end{array}$ \\
\hline DA 1 & $1,808(19,458)$ & $49(1.6)$ & $892(1,165)$ & $U .238$ & 281 \\
\hline DA 2 & $699(7,533)$ & $20(0.7)$ & $141(182)$ & U-238 & 45 \\
\hline DA 3 & $77(825)$ & $46(1.5)$ & $35(46)$ & $U-238$ & 62 \\
\hline$D A 4^{(a)}$ & -- & $\cdots$ & -- & $\cdots$ & $-\cdot$ \\
\hline DA 5 & $743(8,000)$ & 91 (3) & $680(889)$ & $R a-226$ & $<30$ \\
\hline DA 6 & $613(6,600)$ & $91(3)$ & $560(733)$ & (b) & (b) \\
\hline$D A 7^{|c|}$ & $3.3(35)$ & $30(1)$ & $1(1.5)$ & $\mathrm{Ra}-226$ & 38 \\
\hline $\operatorname{DOC} 1^{|c|}$ & $167(1,800)$ & $46(1.5)^{(d)}$ & $76(100)^{(d)}$ & (b) & (b) \\
\hline $\operatorname{Doc} 2^{(0)}$ & (b) & (b) & (b) & Ra. 226 & 82 \\
\hline DOC 3 & $10.4(114)$ & $72(2.4)$ & $7.7(10)$ & U-238 & 208 \\
\hline DOC 4 & $628(6,766)$ & $30(1)$ & $193(251)$ & $\mathrm{Ra} \cdot 226$ & 113 \\
\hline DOC 5 & $5^{(d)}(54)$ & $15^{\text {(d) }}(0.5)$ & $1^{(d)}(1)$ & Th-230 & 59 \\
\hline DOC 6 & $1(9)$ & $76(2.5)$ & $1(1)$ & (b) & (b) \\
\hline $\operatorname{DOC} 7^{(\mathrm{a})}$ & $\cdots$ & $\ldots$ & $\ldots$ & $\ldots$ & $\ldots$ \\
\hline $\operatorname{DOC} 8^{(c)}$ & $2.4(27)$ & $63(2)$ & $1.5(2)$ & Ra-226 & 63 \\
\hline $\begin{array}{l}\text { DOC } g^{(c)} \\
\text { (Femme } \\
\text { Osage Slough) }\end{array}$ & $\cdots$ & $\cdots$ & $\cdots$ & -- & -- \\
\hline DOC 10 & $0.5(5)$ & $30(1)$ & $0.2(0.2)$ & Th-230 & 36 \\
\hline $\begin{array}{l}\text { Southeast } \\
\text { Drainage }\end{array}$ & $11,150(120,000)$ & $61(2)$ & $6,800(8,900)$ & (b) & (b) \\
\hline $\operatorname{Total}^{(\theta)}$ & $15,735(169,364)$ & $\cdots$ & $9,311(12,180)$ & & \\
\hline
\end{tabular}

(a) Included in southeast drainage date

(b) Not estimated

(c) Remediated

(d) Estimated

(e) Totals do not include previously remediated areas 


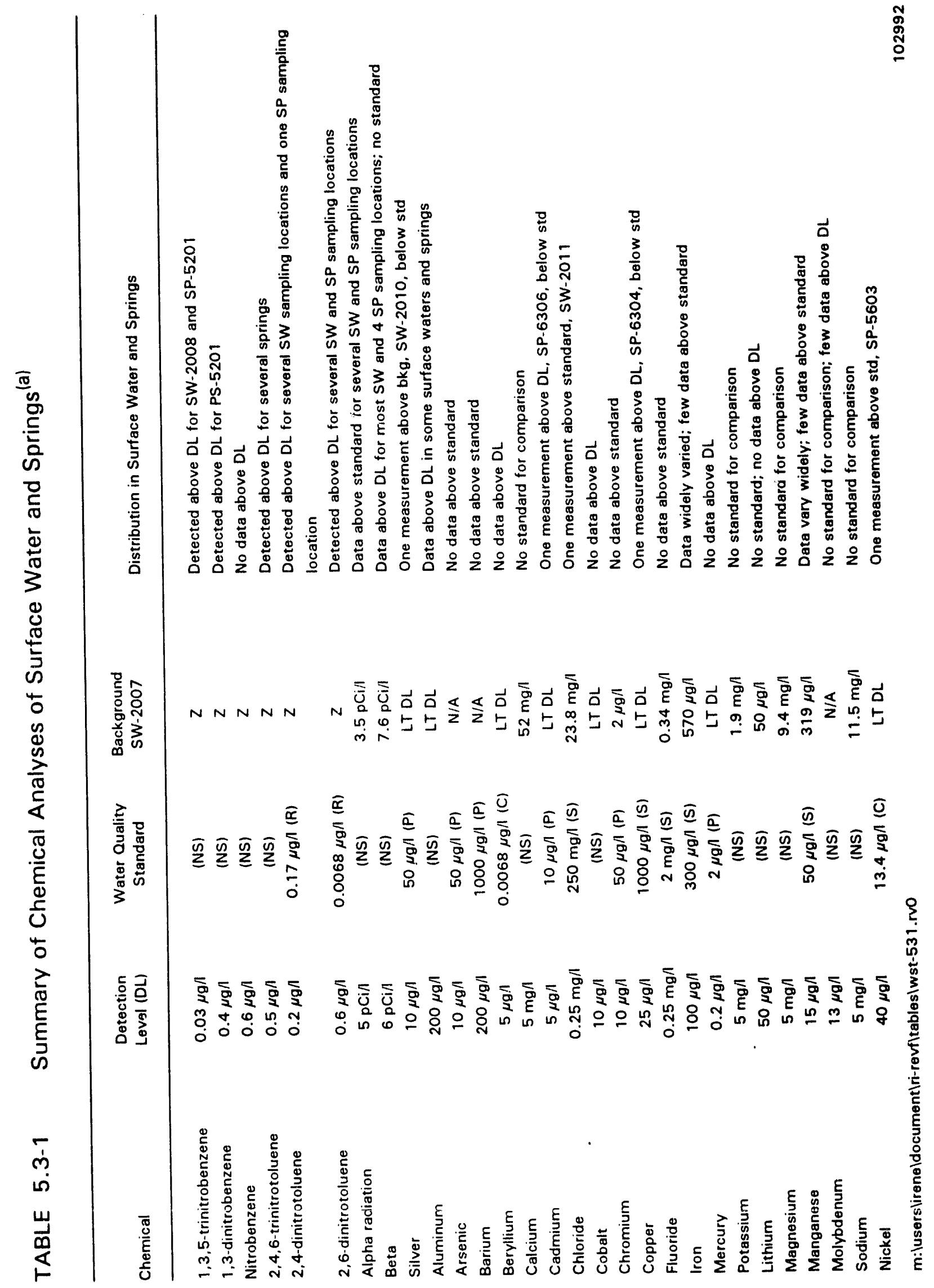



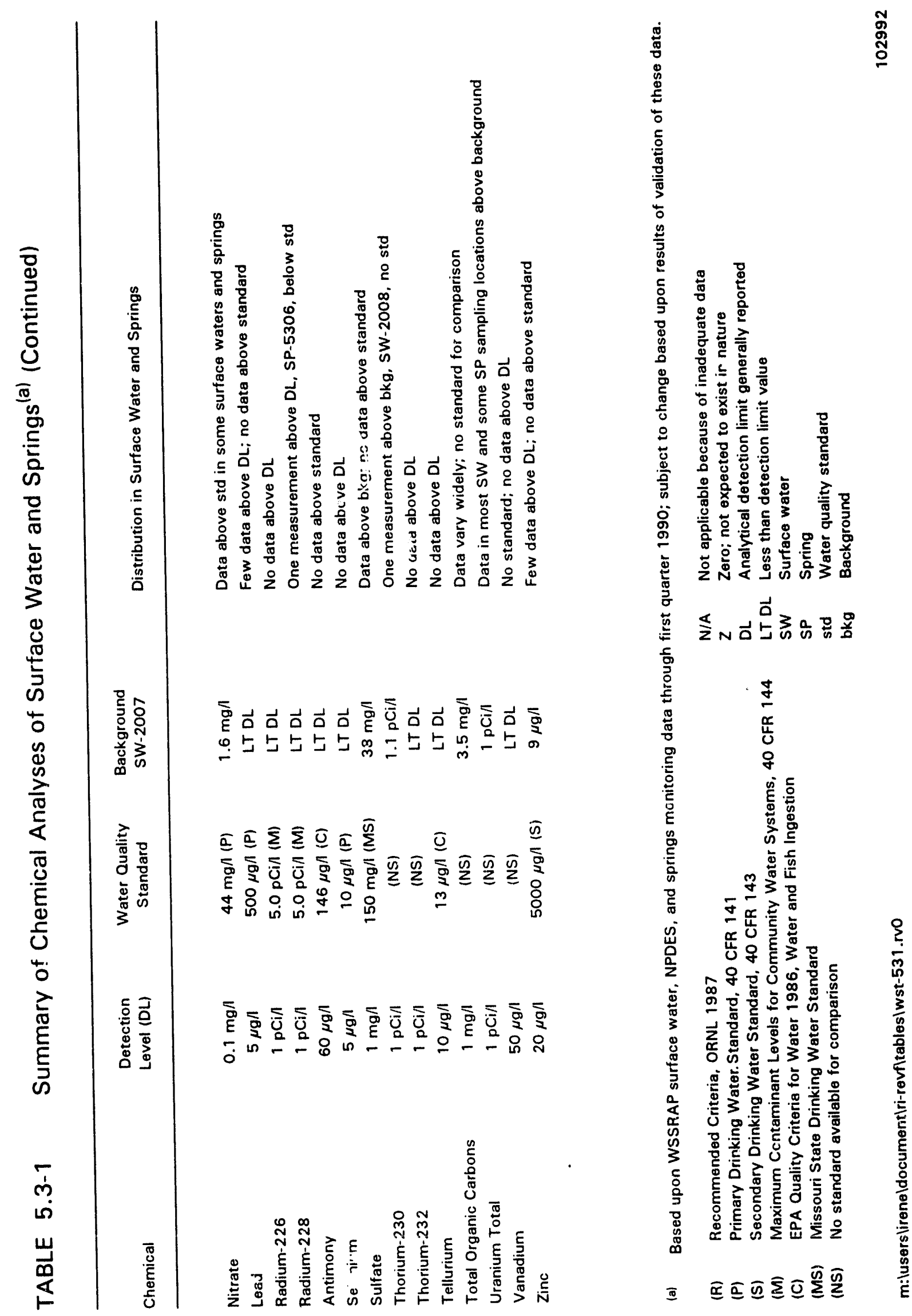
TABLE 5.3-2 Analyses of Uranium $(\mathrm{pCi} / \mathrm{l})$ as Presented in Figures 5.3-2 and 5.3-3 ${ }^{(a)}$

\begin{tabular}{|c|c|c|c|c|c|}
\hline \multirow{2}{*}{$\begin{array}{l}\text { SAMPLE } \\
\text { LOCATION }\end{array}$} & \multirow{2}{*}{$\begin{array}{c}\text { DETECT/ } \\
\text { ANALYSES }^{(b)}\end{array}$} & \multicolumn{3}{|c|}{ VALUE PRESENTED ON MAPS $(a)$} & \multirow{2}{*}{$\begin{array}{l}\text { STANDARD } \\
\text { DEVIATION }\end{array}$} \\
\hline & & MINIMUM & MAXIMUM & MEAN & \\
\hline NP-0001 & $15 / 17$ & $N D^{(c)}$ & 1200.00 & 505.60 & 307.97 \\
\hline NP-0002 & $59 / 64$ & ND & 1500.00 & 147.04 & 197.04 \\
\hline NP-0003 & $25 / 25$ & 16.00 & 3500.00 & 1.081 .53 & 1034.89 \\
\hline NP-0004 & $11 / 14$ & ND & 9.80 & 5.33 & 2.34 \\
\hline NP-0005 & $21 / 23$ & ND & 1200.00 & 436.43 & 264.62 \\
\hline SP.5101 & $0 / 1$ & ND & ND & NA (d) & NA \\
\hline SP-5201 & $0 / 5$ & ND & ND & NA & NA \\
\hline SP-5202 & $1 / 1$ & 1.10 & 1.10 & NA & NA \\
\hline SP.5203 & $3 / 6$ & ND & 11.00 & 4.98 & 5.21 \\
\hline SP-5204 & $0 / 1$ & ND & ND & NA & NA \\
\hline SP.5205 & $1 / 1$ & 1.90 & 1.90 & NA & NA \\
\hline SP-5206 & $1 / 1$ & 2.90 & 2.90 & NA & NA \\
\hline SP-5207 & $1 / 1$ & 3.30 & 3.30 & NA & NA \\
\hline SP-5301 & $11 / 11$ & 80.00 & 578.00 & 309.91 & 142.73 \\
\hline SP. 5302 & $8 / 8$ & 96.00 & 515.00 & 311.88 & 144.26 \\
\hline SP. 5303 & $9 / 9$ & 95.00 & 312.00 & 223.89 & 65.87 \\
\hline SP-5304 & $8 / 8$ & 39.40 & 244.00 & 125.05 & 59.54 \\
\hline SP-5306 & $0 / 1$ & ND & ND & NA & NA \\
\hline SP-5401 & $0 / 1$ & ND & ND & NA & NA \\
\hline SP-5402 & $0 / 1$ & ND & ND & NA & NA \\
\hline SP-5403 & $0 / 1$ & ND & ND & NA & NA \\
\hline SP-5405 & $0 / 1$ & ND & ND & NA & NA \\
\hline SP-5501 & $0 / 2$ & ND & ND & NA & NA \\
\hline SP-5502 & $0 / 1$ & ND & ND & NA & NA \\
\hline SP-5503 & $2 / 3$ & ND & 6.80 & 4.25 & 3.61 \\
\hline SP-5601 & $0 / 2$ & ND & ND & NA & NA \\
\hline SP-5602 & $0 / 1$ & ND & ND & NA & NA \\
\hline SP-5603 & $0 / 2$ & ND & ND & NA & NA \\
\hline SP-5604 & $0 / 1$ & ND & ND & NA & NA \\
\hline SP-6301 & $19 / 19$ & 13.60 & 160.00 & 66.37 & 40.19 \\
\hline SP-6302 & $6 / 6$ & 10.00 & 90.00 & 39.13 & 30.84 \\
\hline SP- 6303 & $6 / 8$ & ND & 5.80 & 3.30 & 1.85 \\
\hline SP- 6304 & $1 / 1$ & 1.40 & 1.40 & NA & NA \\
\hline SP-6306 & $5 / 8$ & ND & 12.90 & 6.44 & 4.87 \\
\hline SP-6307 & $0 / 1$ & ND & ND & NA & NA \\
\hline SP-6308 & $0 /:$ & ND & ND & NA & NA \\
\hline SP-6501 & $0 / 2$ & ND & ND & NA & NA \\
\hline SP-6601 & $1 / 2$ & ND & 1.10 & NA & NA \\
\hline SW-2001 & $13 / 14$ & ND & 4.30 & 2.46 & 1.25 \\
\hline SW-2002 & $8 / 8$ & 6.40 & 240.00 & 104.70 & 91.69 \\
\hline SW-2003 & $13 / 13$ & 8.00 & 69.00 & 18.28 & 15.76 \\
\hline SW-2004 & $14 / 14$ & 8.90 & 39.00 & 20.93 & 8.25 \\
\hline SW-2005 & $14 / 14$ & 4.10 & 53.72 & 16.51 & 12.29 \\
\hline SW-2006 & $5 / 14$ & ND & 4.40 & 2.07 & 1.50 \\
\hline SW-2007 & $2 / 14$ & ND & 1.80 & 1.45 & 0.49 \\
\hline SW-2010 & $5 / 5$ & 5.40 & 3200.00 & 1354.68 & 1491.09 \\
\hline SW-2011 & $4 / 4$ & 28.56 & 319.00 & 216.39 & 129.31 \\
\hline SW-2012 & $1 / 1$ & 11.20 & 11.20 & NA & NA \\
\hline
\end{tabular}


TABLE 5.3-2 Analyses of Uranium $(\mathrm{pCi} / \mathrm{l})$ as Presented in Figures 5.3-2 and 5.3-3 (Continued)

\begin{tabular}{|c|c|c|c|c|c|}
\hline \multirow{2}{*}{$\begin{array}{l}\text { SAMPLE } \\
\text { LOCATION }\end{array}$} & \multirow{2}{*}{$\begin{array}{c}\text { DETECT/ } \\
\text { ANALYSES }\end{array}$} & \multicolumn{3}{|c|}{ VALUE PRESENTED ON MAPS ${ }^{(a)}$} & \multirow{2}{*}{$\begin{array}{l}\text { STANDARD } \\
\text { DEVIATION }\end{array}$} \\
\hline & & MINIMUM & MAXIMUM & MEAN & \\
\hline SW-3001 & $3 / 3$ & 198.00 & 405.00 & 275.80 & 112.67 \\
\hline SW-3002 & $3 / 3$ & 570.00 & 2312.00 & 1350.67 & 884.94 \\
\hline SW-3003 & $3 / 3$ & 57.00 & 2580.00 & 947.00 & 1416.13 \\
\hline SW-3004 & $3 / 3$ & 202.00 & 2700.00 & 1420.67 & 1250.10 \\
\hline
\end{tabular}

\footnotetext{
(a) The data summarized in this table and presented on maps reflect data from the fourth quarter of 1988 to the first quarter of 1990.

(b) Does not include QA data, a dplicates and matrix spikes.

(c) Not detected

(d) Not applicable
} 
TABLE 5.3-3 Analyses of Nitrate $(\mathrm{mg} / \mathrm{l})$ as Presented in Figures 5.3-5 and 5.3-6

\begin{tabular}{|c|c|c|c|c|c|}
\hline \multirow{2}{*}{$\begin{array}{l}\text { SAMPLE } \\
\text { LOCATION }\end{array}$} & \multirow{2}{*}{$\begin{array}{c}\text { DETECT// } \\
\text { ANALYSES } \\
\text { AN) }\end{array}$} & \multicolumn{3}{|c|}{ VALUE PRESENTED ON MAPS $|b|$} & \multirow{2}{*}{$\begin{array}{l}\text { STANDARD } \\
\text { DEVIATION }\end{array}$} \\
\hline & & MINIMUM & MAXIMUM & MEAN & \\
\hline NP-0001 & $13 / 19$ & $N D^{(c)}$ & 7.90 & 4.29 & 1.75 \\
\hline NP.0002 & $25 / 34$ & ND & 698.00 & 49.15 & 165.50 \\
\hline NP.0003 & $22 / 30$ & ND & 9010.00 & 728.00 & 2212.99 \\
\hline NP.0004 & $13 / 17$ & ND & 373.00 & 30.20 & 103.01 \\
\hline NP.0005 & $25 / 28$ & ND & 2060.00 & 2.06 .41 & 553.26 \\
\hline SP.5101 & $1 / 1$ & 1.50 & 1.50 & iNA (d) & NA \\
\hline SP.5201 & $4 / 4$ & 0.15 & 2.20 & 0.89 & 0.92 \\
\hline SP. 5202 & $1 / 1$ & 4.00 & 4.00 & NA & NA \\
\hline SP.5203 & $4 / 4$ & 0.50 & 53.80 & 14.10 & 26.47 \\
\hline SP. 5204 & $1 / 1$ & 1.30 & 1.30 & NA & NA \\
\hline SP.5205 & $0 / 1$ & ND & ND & NA & NA \\
\hline SP.5206 & $1 / 1$ & 2.50 & 2.50 & NA & NA \\
\hline SP. 5207 & $1 / 1$ & 2.50 & 2.50 & NA & NA \\
\hline SP.5301 & $6 / 6$ & 1.50 & 35.40 & 12.42 & 12.89 \\
\hline SP. 5302 & $4 / 4$ & 2.00 & 31.30 & 14.84 & 13.78 \\
\hline SP. 5303 & $4 / 4$ & 3.00 & 17.60 & 9.30 & 6.07 \\
\hline SP. 5304 & $5 / 5$ & 1.85 & 9.10 & 5.17 & 3.07 \\
\hline SP.5306 & $1 / 1$ & 0.70 & 0.70 & NA & NA \\
\hline SP-5401 & $0 / 1$ & ND & ND & NA & NA \\
\hline SP. 5402 & $1 / 1$ & 0.20 & 0.20 & NA & NA \\
\hline SP. 5403 & $1 / 1$ & 1.70 & 1.70 & NA & NA \\
\hline SP. 5405 & $1 / 1$ & 0.70 & 0.70 & NA & NA \\
\hline SP.5501 & $2 / 2$ & 2.50 & 2.60 & 2.55 & 0.07 \\
\hline SP.5502 & $1 / 1$ & 0.30 & 0.30 & NA & NA \\
\hline SP.5503 & $2 / 2$ & 2.30 & 2.70 & 2.50 & 0.28 \\
\hline SP.5601 & $2 / 2$ & 0.90 & 0.90 & 0.90 & 0.00 \\
\hline SP. 5602 & $1 / 1$ & 1.80 & 1.80 & NA & NA \\
\hline SP-5603 & $2 / 2$ & 0.40 & 0.84 & 0.62 & 0.31 \\
\hline SP. 5604 & $1 / 1$ & 5.40 & 5.40 & NA & NA \\
\hline SP-6301 & $15 / 16$ & ND & 10200.00 & 739.81 & 2617.87 \\
\hline SP- 6302 & $3 / 3$ & 15.00 & 15.60 & 15.27 & 0.31 \\
\hline SP- 6303 & $6 / 6$ & 7.40 & 66.60 & 36.77 & 25.16 \\
\hline SP. 6304 & $1 / 1$ & 6.00 & 6.00 & NA & NA \\
\hline SP-6306 & $6 / 7$ & ND & 14.50 & 4.22 & 5.22 \\
\hline SP- 6307 & $1 / 1$ & 2.60 & 2.60 & NA & NA \\
\hline SP. 6308 & $0 / 1$ & ND & ND & NA & NA \\
\hline SP-6501 & $2 / 2$ & 3.00 & 3.70 & 3.35 & 0.49 \\
\hline SP-6601 & $2 / 2$ & 3.80 & 5.50 & 4.65 & 1.20 \\
\hline SW-2001 & $8 / 9$ & ND & 6.90 & 3.30 & 2.25 \\
\hline SW-2002 & $4 / 5$ & ND & 4.00 & 1.62 & 8.59 \\
\hline SW-2003 & $5 / 10$ & ND & 3.00 & 1.39 & 0.97 \\
\hline SW-2004 & $10 / 10$ & 0.70 & 8.90 & 3.87 & 2.67 \\
\hline SW-2005 & $7 / 9$ & ND & 1.50 & 0.64 & 0.45 \\
\hline SW-2006 & $3 / 10$ & ND & 0.90 & 0.42 & 0.42 \\
\hline SW-2007 & $7 / 9$ & ND & 5.00 & 2.80 & 1.58 \\
\hline SW-2010 & $3 / 5$ & ND & 48.60 & 18.80 & 25.81 \\
\hline SW-2011 & $2 / 4$ & ND & 0.79 & 0.70 & 0.13 \\
\hline SW-2012 & $0 / 1$ & ND & ND & NA & NA \\
\hline
\end{tabular}


TABLE 5.3-3 Analyses of Nitrate $(\mathrm{mg} / \mathrm{l})$ as Presented in Figures 5.3-5 and 5.3-6 (Continued)

\begin{tabular}{|c|c|c|c|c|c|}
\hline \multirow{2}{*}{$\begin{array}{l}\text { SAMPLE } \\
\text { LOCATION }\end{array}$} & \multirow{2}{*}{$\begin{array}{c}\text { DETECT/ } \\
\text { ANALYSES }^{(\mathrm{a})}\end{array}$} & \multicolumn{3}{|c|}{ VALUE PRESENTED ON MAPS } & \multirow{2}{*}{$\begin{array}{l}\text { STANDARD } \\
\text { DEVIATION }\end{array}$} \\
\hline & & MINIMUM & MAXIMUM & MEAN & \\
\hline SW-3001 & $3 / 3$ & 248.00 & 1668.00 & 1052.00 & 728.43 \\
\hline SW-3002 & $0 / 3$ & ND & ND & NA & NA \\
\hline SW-3003 & $3 / 3$ & 19.60 & 3700.00 & 1851.53 & 1840.26 \\
\hline SW-3004 & $3 / 3$ & 0.25 & 7240.00 & 2413.51 & 4179.87 \\
\hline
\end{tabular}

(a) Does not include QA data, duplicates, and matrix spikes.

(b) The data summarized in this table and presented on maps reflect data from the fourth quarter of 1988 to the first quarter of 1990 .

(c) Not detected.

(d) Not applicable. 
TABLE 5.3-4 Analyses of 2,4,6-Trinitrotoluene $(\mu \mathrm{g} / \mathrm{l})$ as Presented in Figures 5.3-8 and 5.3-9

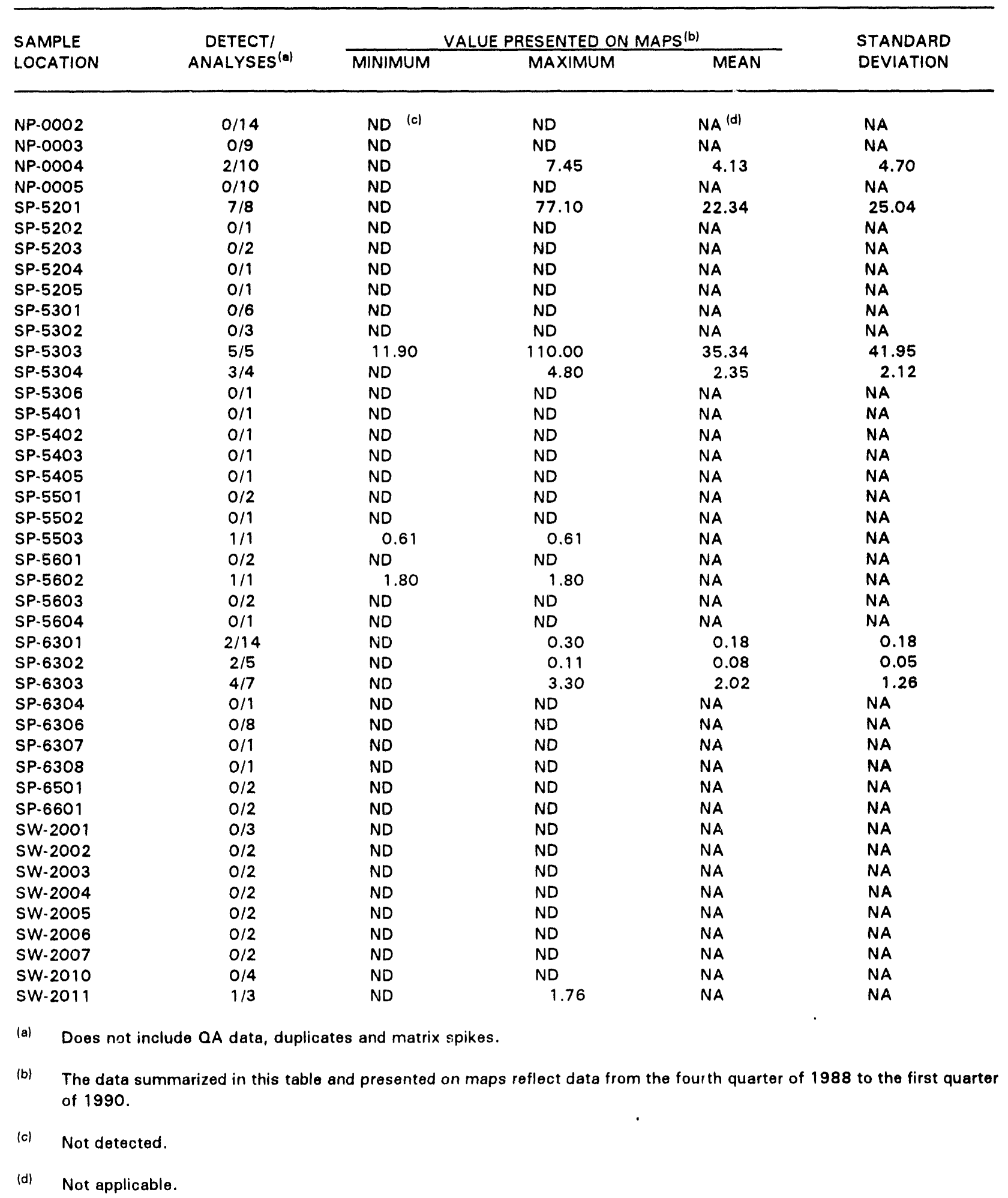


TABLE 5.3-5 Analyses of 1,3,5-Trinitrobenzene $(\mu \mathrm{g} / 1)$ as Presented in Figures 5.3-8 and 5.3-9

\begin{tabular}{|c|c|c|c|c|c|c|}
\hline \multirow{2}{*}{\multicolumn{2}{|c|}{$\begin{array}{l}\text { SAMPLE } \\
\text { LOCATION }\end{array}$}} & \multirow{2}{*}{$\begin{array}{c}\text { DETECT/ } \\
\text { ANALYSES }^{(0)}\end{array}$} & \multicolumn{3}{|c|}{ VALUE PRESENTED ON MAPS ${ }^{(b)}$} & \multirow{2}{*}{$\begin{array}{l}\text { STANDARD } \\
\text { DEVIATION }\end{array}$} \\
\hline & & & MINIMUM & MAXIMUM & $\overline{M E A N}$ & \\
\hline \multicolumn{2}{|c|}{ NP-0001 } & $1 / 11$ & ND & 0.84 & NA & NA \\
\hline \multirow{2}{*}{\multicolumn{2}{|c|}{$\begin{array}{l}\text { NP.0002 } \\
\text { NP-0003 }\end{array}$}} & $2 / 14$ & ND & 1.42 & 0.81 & 0.86 \\
\hline & & $0 / 9$ & ND & ND & NA & NA \\
\hline \multicolumn{2}{|c|}{ NP-0004 } & $2 / 10$ & ND & 0.65 & 0.35 & 0.43 \\
\hline \multicolumn{2}{|c|}{ NP-0005 } & $0 / 10$ & ND & ND & NA & NA \\
\hline \multicolumn{2}{|c|}{ SP-5201 } & $7 / 8$ & ND & 3.26 & 1.40 & 1.04 \\
\hline \multicolumn{2}{|c|}{ SP-5202 } & $0 / 1$ & ND & ND & NA & NA \\
\hline \multicolumn{2}{|c|}{ SP. 5203} & $0 / 2$ & ND & ND & NA & NA \\
\hline \multicolumn{2}{|c|}{ SP.5204 } & $0 / 1$ & ND & ND & NA & NA \\
\hline \multicolumn{2}{|c|}{ SP. 5205} & $0 / 1$ & ND & ND & NA & NA \\
\hline \multicolumn{2}{|c|}{ SP-5301 } & $0 / 6$ & ND & ND & NA & NA \\
\hline \multicolumn{2}{|c|}{ SP. 5302} & $0 / 4$ & ND & ND & NA & P.A \\
\hline \multicolumn{2}{|c|}{ SP-5303 } & $2 / 6$ & ND & 0.47 & 0.35 & 0.18 \\
\hline \multicolumn{2}{|c|}{ SP-5304 } & $1 / 4$ & ND & 0.42 & NA & NA \\
\hline \multicolumn{2}{|c|}{ SP-5306 } & $0 / 1$ & ND & ND & NA & NA \\
\hline \multicolumn{2}{|c|}{ SP-5401 } & $0 / 1$ & ND & ND & NA & NA \\
\hline \multicolumn{2}{|c|}{ SP-5402 } & $0 / 1$ & $\cdot I D$ & ND & NA & NA \\
\hline \multicolumn{2}{|c|}{ SP-5403 } & $0 / 1$ & ND & ND & NA & NA \\
\hline$S P-$ & 105 & $0 / 1$ & ND & ND & NA & NA \\
\hline$S P-$ & 501 & $0 / 2$ & ND & ND & NA & NA \\
\hline & 502 & $0 / 1$ & ND & ND & NA & NA \\
\hline & 503 & $0 / 1$ & ND & ND & NA & NA \\
\hline & 501 & $0 / 2$ & ND & ND & NA & NA \\
\hline & 302 & $0 / 1$ & ND & ND & NA & NA \\
\hline & 303 & $0 / 2$ & ND & ND & NA & NA \\
\hline$S P-$ & 504 & $0 / 1$ & ND & ND & NA & NA \\
\hline SP- & 301 & $1 / 14$ & ND & 0.02 & NA & NA \\
\hline$S P-$ & 302 & $0 / 5$ & ND & ND & NA & NA \\
\hline SP- & 303 & $2 / 7$ & ND & 0.23 & 0.17 & 0.08 \\
\hline & 304 & $0 / 1$ & ND & ND & NA & NA \\
\hline SP. & 306 & $0 / 8$ & ND & ND & NA & NA \\
\hline SP. & 307 & $0 / 1$ & ND & ND & NA & NA \\
\hline SP. & 308 & $0 / 1$ & ND & ND & NA & NA \\
\hline SP- & 501 & $0 / 2$ & ND & ND & NA & NA \\
\hline & 601 & $0 / 2$ & ND & ND & NA & NA \\
\hline & 001 & $0 / 3$ & ND & ND & NA & NA \\
\hline SW & 002 & $0 / 2$ & ND & ND & NA & NA \\
\hline sw & 003 & $0 / 2$ & ND & ND & NA & NA \\
\hline sw & .004 & $0 / 2$ & ND & ND & NA & NA \\
\hline SW & .005 & $0 / 2$ & ND & ND & NA & NA \\
\hline SW & .006 & $0 / 2$ & ND & ND & NA & NA \\
\hline SW & 007 & $0 / 2$ & ND & ND & NA & NA \\
\hline SW & 010 & $0 / 4$ & ND & ND & NA & NA \\
\hline sw. & 011 & $1 / 3$ & ND & 4.97 & NA & NA \\
\hline (a) & Does $n$ & O QA data, dupl & tes, and matr & & & \\
\hline (b) & $\begin{array}{l}\text { The dat } \\
\text { of } 1990\end{array}$ & arized in this tab & and presentec & os reflect data & e fourth quar & 1988 to the first quarter \\
\hline (c) & Not det & & & & & \\
\hline (d) & Not ap & & & & & \\
\hline
\end{tabular}


TABLE 5.3-6 Analyses of 2,4-Dinitrotoluene $(\mu \mathrm{g} / 1)$ as Presented in Figures 5.3-8 and 5.3-9

\begin{tabular}{|c|c|c|c|c|c|c|}
\hline \multirow{2}{*}{\multicolumn{2}{|c|}{$\begin{array}{l}\text { SAMPLE } \\
\text { LOCATION }\end{array}$}} & \multirow{2}{*}{$\begin{array}{c}\text { DETECT/ } \\
\text { ANALYSES }\end{array}$} & \multicolumn{3}{|c|}{ VALUE PRESENTED ON MAPS ${ }^{(b)}$} & \multirow{2}{*}{$\begin{array}{l}\text { STANDARD } \\
\text { DEVIATION }\end{array}$} \\
\hline & & & MINIMUM & MAXIMUM & $\overline{M E A N}$ & \\
\hline \multicolumn{2}{|c|}{ NP-0002 } & $0 / 14$ & ND $(c)$ & ND & NA $(d)$ & NA \\
\hline \multicolumn{2}{|c|}{ NP-0003 } & $0 / 9$ & ND & ND & NA & NA \\
\hline \multicolumn{2}{|c|}{ NP-0004 } & $0 / 10$ & ND & ND & NA & NA \\
\hline \multicolumn{2}{|c|}{ NP.0005 } & $0 / 10$ & ND & ND & NA & NA \\
\hline \multicolumn{2}{|c|}{ SP-5201 } & $1 / 8$ & ND & 1.73 & NA & NA \\
\hline \multicolumn{2}{|c|}{ SP-5202 } & $0 / 1$ & ND & ND & NA & NA \\
\hline \multicolumn{2}{|c|}{ SP-5203 } & $0 / 2$ & ND & ND & NA & NA \\
\hline \multicolumn{2}{|c|}{ SP-5204 } & $0 / 1$ & ND & ND & NA & NA \\
\hline \multicolumn{2}{|c|}{ SP-5205 } & $0 / 1$ & ND & ND & NA & NA \\
\hline \multicolumn{2}{|c|}{ SP-5301 } & $0 / 6$ & ND & ND & NA & NA \\
\hline \multicolumn{2}{|c|}{ SP-5302 } & $1 / 4$ & ND & 0.89 & NA & NA \\
\hline \multicolumn{2}{|c|}{ SP-5303 } & $2 / 6$ & ND & 11.00 & 6.22 & 6.77 \\
\hline \multicolumn{2}{|c|}{ SP-5304 } & $0 / 4$ & ND & ND & NA & NA \\
\hline \multicolumn{2}{|c|}{ SP-5306 } & $0 / 1$ & ND & ND & NA & NA \\
\hline \multicolumn{2}{|c|}{ SP-5401 } & $0 / 1$ & ND & ND & NA & NA \\
\hline \multicolumn{2}{|c|}{ SP-5402 } & $0 / 1$ & ND & ND & NA & NA \\
\hline \multicolumn{2}{|c|}{ SP-5403 } & $0 / 1$ & ND & ND & NA & NA \\
\hline SP- & 405. & $0 / 1$ & ND & ND & NA & NA \\
\hline SP- & 501 & $0 / 2$ & ND & ND & NA & NA \\
\hline SP. & 502 & $0 / 1$ & ND & ND & NA & NA \\
\hline SP- & 503 & $0 / 1$ & ND & ND & NA & NA \\
\hline SP- & 601 & $0 / 2$ & ND & ND & NA & NA \\
\hline SP- & 192 & $1 / 1$ & 0.50 & 0.50 & NA & NA \\
\hline SP- & 603 & $0 / 2$ & ND & ND & NA & NA \\
\hline SP.5 & 604 & $0 / 1$ & ND & ND & NA & NA \\
\hline SP- & 301 & $5 / 15$ & ND & 1.44 & 0.71 & 0.44 \\
\hline SP. & 302 & $2 / 5$ & ND & 1.42 & 1.01 & 0.58 \\
\hline SP-C & 303 & $0 / 7$ & ND & ND & NA & NA \\
\hline$S P-C$ & 304 & $0 / 1$ & ND & ND & NA & NA \\
\hline SP-C & 306 & $0 / 8$ & ND & ND & NA & NA \\
\hline SP-C & 307 & $0 / 1$ & ND & ND & NA & NA \\
\hline SP-C & 308 & $0 / 1$ & ND & ND & NA & NA \\
\hline SP-C & 501 & $0 / 2$ & ND & ND & NA & NA \\
\hline$S P-$ & 601 & $0 / 2$ & ND & ND & NA & NA \\
\hline sw. & 2001 & $0 / 3$ & ND & ND & NA & NA \\
\hline sw & 2002 & $0 / 2$ & ND & ND & NA & NA \\
\hline sW & 2003 & $1 / 2$ & ND & 0.58 & NA & NA \\
\hline sW & 2004 & $0 / 2$ & ND & ND & NA & NA \\
\hline sw & 2005 & $0 / 2$ & ND & ND & NA & NA \\
\hline SW & 2006 & $1 / 2$ & ND & 0.06 & NA & NA \\
\hline sw & 2007 & $0 / 2$ & ND & ND & NA & NA \\
\hline sw & 2010 & $0 / 4$ & ND & ND & NA & NA \\
\hline sw & 2011 & $0 / 3$ & ND & ND & NA & NA \\
\hline lai & Does & lo QA data, dup & tes, and mat & & & \\
\hline (b) & $\begin{array}{l}\text { The d } \\
\text { of } 19 s\end{array}$ & larized in this tat & and presente & os reflect dat & e fourth quar & 1988 to the first quarter \\
\hline (c) & Not de & & & & & \\
\hline (d) & Not a & & & & & \\
\hline
\end{tabular}


TABLE 5.3-7 Analyses of 2,6-Dinitrotoluene $(\mu \mathrm{g} / \mathrm{l})$ as Presented in Figures 5.3-8 and 5.3-9

\begin{tabular}{|c|c|c|c|c|c|c|}
\hline \multirow{2}{*}{\multicolumn{2}{|c|}{$\begin{array}{l}\text { SAMPLE } \\
\text { LOCATION }\end{array}$}} & \multirow{2}{*}{$\begin{array}{c}\text { DETECT/ } \\
\text { ANALYSES } \\
\end{array}$} & \multicolumn{3}{|c|}{ VALUE PRESENTED ON MAPS ${ }^{(b)}$} & \multirow{2}{*}{$\begin{array}{l}\text { STANDARD } \\
\text { DEVIATION }\end{array}$} \\
\hline & & & MINIMUM & MAXIMUM & MEAN & \\
\hline \multicolumn{2}{|c|}{ NP-0002 } & $0 / 14$ & ND $|c|$ & ND & $N A^{(d)}$ & NA \\
\hline \multicolumn{2}{|c|}{ NP.0003 } & $0 / 9$ & ND & ND & NA & NA \\
\hline \multicolumn{2}{|c|}{ NP.0004 } & $0 / 10$ & ND & ND & NA & NA \\
\hline \multicolumn{2}{|c|}{ NP-0005 } & $0 / 10$ & ND & ND & NA & NA \\
\hline \multicolumn{2}{|c|}{ SP.5201 } & $4 / 8$ & ND & 1.26 & 0.81 & $-\quad 0.45$ \\
\hline \multicolumn{2}{|c|}{ SP-5202 } & $0 / 1$ & ND & ND & NA & NA \\
\hline \multicolumn{2}{|c|}{ SP.5203 } & $0 / 2$ & ND & ND & NA & NA \\
\hline \multicolumn{2}{|c|}{ SP-5204 } & $0 / 1$ & ND & ND & NA & NA \\
\hline \multicolumn{2}{|c|}{ SP-5205 } & $0 / 1$ & ND & ND & NA & NA \\
\hline \multicolumn{2}{|c|}{ SP-5301 } & $0 / 6$ & ND & ND & NA & NA \\
\hline \multicolumn{2}{|c|}{ SP-5302 } & $0 / 4$ & ND & ND & NA & NA \\
\hline \multicolumn{2}{|c|}{ SP.5303 } & $2 / 6$ & ND & 11.00 & 5.81 & 7.35 \\
\hline \multicolumn{2}{|c|}{ SP. 5304} & $1 / 4$ & ND & 0.40 & NA & NA \\
\hline \multicolumn{2}{|c|}{ SP-5306 } & $0 / 1$ & ND & ND & NA & NA \\
\hline \multicolumn{2}{|c|}{ SP-5401 } & $0 / 1$ & ND & ND & NA & NA \\
\hline \multicolumn{2}{|c|}{ SP-5402 } & $0 / 1$ & ND & ND & NA & NA \\
\hline \multicolumn{2}{|c|}{ SP. 5403} & $0 / 1$ & ND & ND & NA & NA \\
\hline$S P-$ & 405 & $0 / 1$ & ND & ND & NA & NA \\
\hline$S P-$ & 501 & $0 / 2$ & ND & ND & NA & NA \\
\hline$S P-S$ & 502 & $0 / 1$ & ND & ND & NA & NAA \\
\hline SP-S & 503 & $0 / 1$ & ND & ND & NA & NA \\
\hline$S P$ : & 601 & $0 / 2$ & ND & ND & NA & NA \\
\hline$S P-:$ & 602 & $1 / 1$ & 3.50 & 3.50 & NA & NA \\
\hline SP. 5 & 603 & $0 / 2$ & ND & ND & iA & NA \\
\hline SP. & 604 & $0 / 1$ & ND & ND & NA & NA \\
\hline$S P-G$ & 301 & $6 / 15$ & ND & 17.60 & 3.62 & 6.86 \\
\hline$S P-G$ & 302 & $1 / 5$ & ND & 0.53 & NA & NA \\
\hline$S P-G$ & 303 & $3 / 7$ & ND & 0.59 & 0.38 & 0.19 \\
\hline SP-C & 304 & $0 / 1$ & ND & ND & NA & NA \\
\hline SP-E & 306 & $0 / 8$ & ND & ND & NA & NA \\
\hline SP-C & 307 & $0 / 1$ & ND & ND & NA & NA \\
\hline SP-C & 308 & $0 / 1$ & ND & ND & NA & NA \\
\hline$S P-C$ & 501 & $0 / 2$ & ND & ND & NA & NA \\
\hline SP-C & 601 & $0 / 2$ & ND & ND & NA & NA \\
\hline sw. & .001 & $0 / 3$ & ND & ND & NA & NA \\
\hline sw & 1002 & $0 / 2$ & ND & ND & NA & NA \\
\hline sw. & .003 & $0 / 2$ & ND & ND & NA & NA \\
\hline sw. & 004 & $0 / 2$ & ND & ND & NA & NA \\
\hline sw. & 005 & $0 / 2$ & ND & ND & NA & NA \\
\hline sW & .006 & $0 / 2$ & ND & ND & NA & NA \\
\hline sw. & 007 & $0 / 2$ & ND & ND & NA & NA \\
\hline sw. & 010 & $0 / 4$ & ND & ND & NA & NA \\
\hline SW- & 011 & $1 / 3$ & ND & 0.39 & NA & NA \\
\hline (a) & Does $n$ & o QA data, dupl & es, and matr & & & \\
\hline (b) & $\begin{array}{l}\text { The da } \\
\text { of } 199\end{array}$ & arized in this tab & nd presented & Ss reflect data & e fourth quar & 1988 to the first quarter \\
\hline |c) & Not de & & & & & \\
\hline (d) & Not ap & & & ' & $\cdot$ & \\
\hline
\end{tabular}



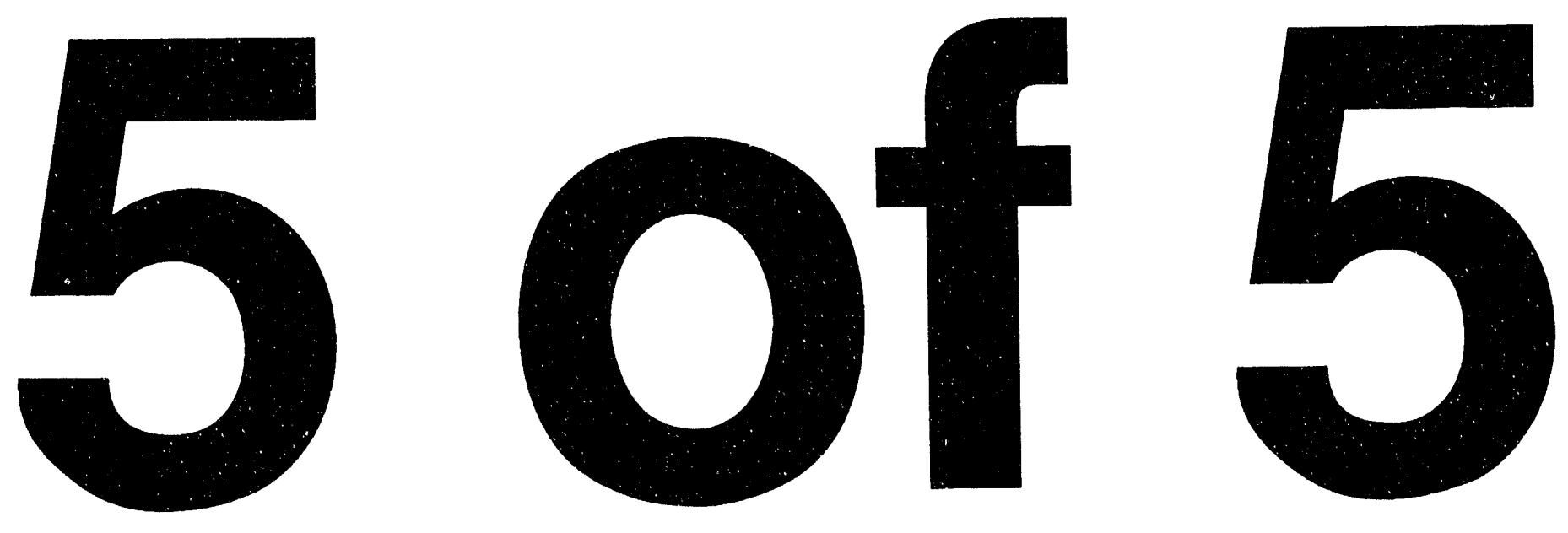
TABLE 5.3-8 Analyses of 1,3-Dinitrobenzene $(\mu \mathrm{g} / 1)$ as Presented in Figures 5.38 and $5.3-9$

\begin{tabular}{|c|c|c|c|c|c|}
\hline \multirow{2}{*}{$\begin{array}{l}\text { SAMPLE } \\
\text { LOCATION }\end{array}$} & \multirow{2}{*}{$\begin{array}{c}\text { DETECT// } \\
\text { ANALYSES }\end{array}$} & \multicolumn{3}{|c|}{ VALUE PRESENTED ON MAPS ${ }^{|6|}$} & \multirow{2}{*}{$\begin{array}{l}\text { STANDARD } \\
\text { DEVIATION }\end{array}$} \\
\hline & & MINIMUM & MAXIMUM & MEAN & \\
\hline NP-0001 & $0 / 11$ & ND $|c|$ & ND & $N A^{(d)}$ & NA \\
\hline NP-0002 & $0 / 14$ & ND & ND & NA & NA \\
\hline NP.0003 & $0 / 9$ & ND & ND & NA & NA \\
\hline NP-0004 & $0 / 10$ & ND & ND & NA & NA \\
\hline NP.0005 & $0 / 10$ & ND & ND & NA & NA \\
\hline SP-5201 & $3 / 8$ & ND & 1.21 & 0.85 & 0.32 \\
\hline SP.5202 & $0 / 1$ & ND & ND & NA & NA \\
\hline SP.5203 & $0 / 2$ & ND & ND & NA & NA \\
\hline SP.5204 & $0 / 1$ & ND & ND & NA & NA \\
\hline SP-5205 & $0 / 1$ & ND & ND & NA & NA \\
\hline SP-5301 & $0 / 6$ & ND & ND & NA & NA \\
\hline SP. 5302 & $0 / 4$ & ND & ND & NA & NA \\
\hline SP-5303 & $1 / 6$ & ND & 0.81 & NA & NA \\
\hline SP.5304 & $0 / 4$ & ND & ND & NA & NA \\
\hline SP.5306 & $0 / 1$ & ND & ND & NA & NA \\
\hline SP.5401 & $0 / 1$ & ND & ND & NA & NA \\
\hline SP-5402 & $0 / 1$ & ND & ND & NA & NA \\
\hline SP.5403 & $0 / 1$ & ND & ND & NA & NA \\
\hline SP-5405 & $0 / 1$ & ND & ND & NA & NA \\
\hline SP.5501 & $0 / 2$ & ND & ND & NA & NA \\
\hline SP. 5502 & $0 / 1$ & ND & ND & NA & NA \\
\hline SP-5503 & $0 / 1$ & ND & ND & NA & NA \\
\hline SP-5601 & $0 / 2$ & ND & ND & NA & NA \\
\hline SP-5602 & $0 / 1$ & ND & ND & NA & NA \\
\hline SP.5603 & $0 / 2$ & ND & ND & NA & NA \\
\hline SP-5604 & $0 / 1$ & ND & ND & NA & NA \\
\hline SP.6301 & $1 / 14$ & ND & 0.18 & NA & NA \\
\hline SP.6302 & $0 / 5$ & ND & ND & NA & NA \\
\hline SP-6303 & $1 / 7$ & ND & 0.45 & NA & NA \\
\hline SP- 6304 & $0 / 1$ & ND & ND & NA & NA \\
\hline SP.6306 & $0 / 8$ & ND & ND & NA & NA \\
\hline SP.6307 & $0 / 1$ & ND & ND & NA & NA \\
\hline SP-6308 & $0 / 1$ & ND & ND & NA & NA \\
\hline SP.6501 & $0 / 2$ & ND & ND & NA & NA \\
\hline SP.6601 & $0 / 2$ & ND & ND & NA & NA \\
\hline SW-2001 & $0 / 3$ & ND & ND & NA & NA \\
\hline SW-2002 & $0 / 2$ & ND & ND & NA & NA \\
\hline SW-2003 & $0 / 2$ & ND & NO & NA & NA \\
\hline SW-2004 & $0 / 2$ & ND & ND & NA & NA \\
\hline SW-2005 & $0 / 2$ & ND & ND & NA & NA \\
\hline SW-2006 & $1 / 2$ & ND & 0.27 & NA & NA \\
\hline SW-2007 & $0 / 2$ & ND & ND & NA & NA \\
\hline sW-2010 & $0 / 4$ & ND & ND & NA & NA \\
\hline SW-2011 & $0 / 3$ & ND & ND & NA & NA \\
\hline
\end{tabular}

\footnotetext{
(a) Does not include QA data, duplicates, and matrix spikes.

(b) The data summarized in this table and presented on maps reflect data from the fourth quarter of 1988 to the first quarter of 1990 .

(c) Not detected.

(d) Not applicable.
}

m:lusers lireneldocument $\backslash r i-r e v f \backslash t a b l e s \backslash w s t-538 . r v o$ 
TABLE 5, 3-9 Analyses of Nitrobenzene $(\mu \mathrm{g} / 1)$ as Presented in Figures 5.3-8 and 5.3-9

\begin{tabular}{|c|c|c|c|c|c|}
\hline \multirow{2}{*}{$\begin{array}{l}\text { SAMPLE } \\
\text { LOCATION }\end{array}$} & \multirow{2}{*}{$\begin{array}{c}\text { DETECT/ } \\
\text { ANALYSES }\end{array}$} & \multicolumn{3}{|c|}{ VALUE PRESENTED ON MAPS ${ }^{(0)}$} & \multirow{2}{*}{$\begin{array}{l}\text { STANDARD } \\
\text { DEVIATION }\end{array}$} \\
\hline & & MINIMUM & MAXIMUM & MEAN & \\
\hline NP.0001 & $0 / 11$ & ND $|c|$ & ND & NA (d) & NA \\
\hline NP.0002 & $0 / 14$ & ND & ND & NA & NA \\
\hline NP-0003 & $0 / 9$ & ND & ND & NA & NA \\
\hline NP-0004 & $0 / 10$ & ND & ND & NA & NA \\
\hline NP.0005 & $0 / 10$ & ND & ND & NA & NA \\
\hline SP.5201 & $1 / 8$ & ND & 1.39 & NA & NA \\
\hline SP. 5202 & $0 / 1$ & ND & ND & NA & NA \\
\hline SP. 5203 & $0 / 2$ & ND & ND & NA & NA \\
\hline SP.5204 & $0 / 1$ & ND & ND & NA & NA \\
\hline SP-5205 & $0 / 1$ & ND & ND & NA & NA \\
\hline SP.5301 & $0 / 6$ & ND & ND & NA & NA \\
\hline SP. 5302 & $0 / 5$ & ND & ND & NA & NA \\
\hline SP. 5303 & $1 / 6$ & ND & 0.87 & NA & NA \\
\hline SP. 5304 & $0 / 4$ & ND & ND & NA & NA \\
\hline SP.5306 & $0 / 1$ & ND & ND & NA & NA \\
\hline SP.5401 & $0 / 1$ & ND & ND & NA & NA \\
\hline SP. 5402 & $0 / 1$ & ND & ND & NA & NA \\
\hline SP. 5403 & $0 / 1$ & ND & ND & NA & NA \\
\hline SP. 5405 & $0 / 1$ & ND & ND & NA & NA \\
\hline SP.5501 & $0 / 2$ & ND & ND & NA & NA \\
\hline SP. 5502 & $0 / 1$ & No & ND & NA & NA \\
\hline SP.5503 & $0 / 1$ & ND & ND & NA & NA \\
\hline SP.5601 & $0 / 2$ & ND & ND & NA & NA \\
\hline SP. 5602 & $0 / 1$ & ND & ND & NA & NA \\
\hline SP. 5603 & $0 / 2$ & ND & ND & NA & NA \\
\hline SP. 5604 & $0 / 1$ & ND & ND & NA & NA \\
\hline SP. 6301 & $0 / 14$ & ND & ND & NA & NA \\
\hline S1 - 6302 & $0 / 4$ & ND & ND & NA & NA \\
\hline SP. 6303 & $1 / 7$ & ND & 0.39 & NA & NA \\
\hline SP. 6304 & $0 / 1$ & ND & ND & NA & NA \\
\hline SP. 6306 & $0 / 8$ & ND & ND & NA & NA \\
\hline SP. 6307 & $0 / 1$ & ND & ND & NA & NA \\
\hline SP. 6308 & $0 / 1$ & ND & ND & NA & NA \\
\hline SP.6501 & $0 / 2$ & ND & ND & NA & NA \\
\hline SP.6601 & $0 / 2$ & ND & ND & NA & NA \\
\hline sW-2001 & $0 / 3$ & ND & ND & NA & NA \\
\hline SW-2002 & $0 / 2$ & ND & ND & NA & NA \\
\hline SW-2003 & $0 / 2$ & ND & ND & NA & NA \\
\hline SW-2004 & $0 / 2$ & ND & ND & NA & NA \\
\hline SW-2005 & $0 / 2$ & ND & ND & NA & NA \\
\hline SW-2006 & $0 / 2$ & ND & ND & NA & NA \\
\hline SW-2007 & $0 / 2$ & ND & ND & NA & NA \\
\hline SW-2010 & $0 / 4$ & ND & ND & NA & NA \\
\hline SW-2011 & $0 / 3$ & ND & ND & NA & NA \\
\hline
\end{tabular}

\footnotetext{
(a) Does not include QA data, duplicates, and matrix spikes.

(b) The data summarized in this table and presented on maps reflect data from the fourth quarter of 1988 to the first quarter . of 1990 .

(c) Not detected.

(d) Not applicable.
} 


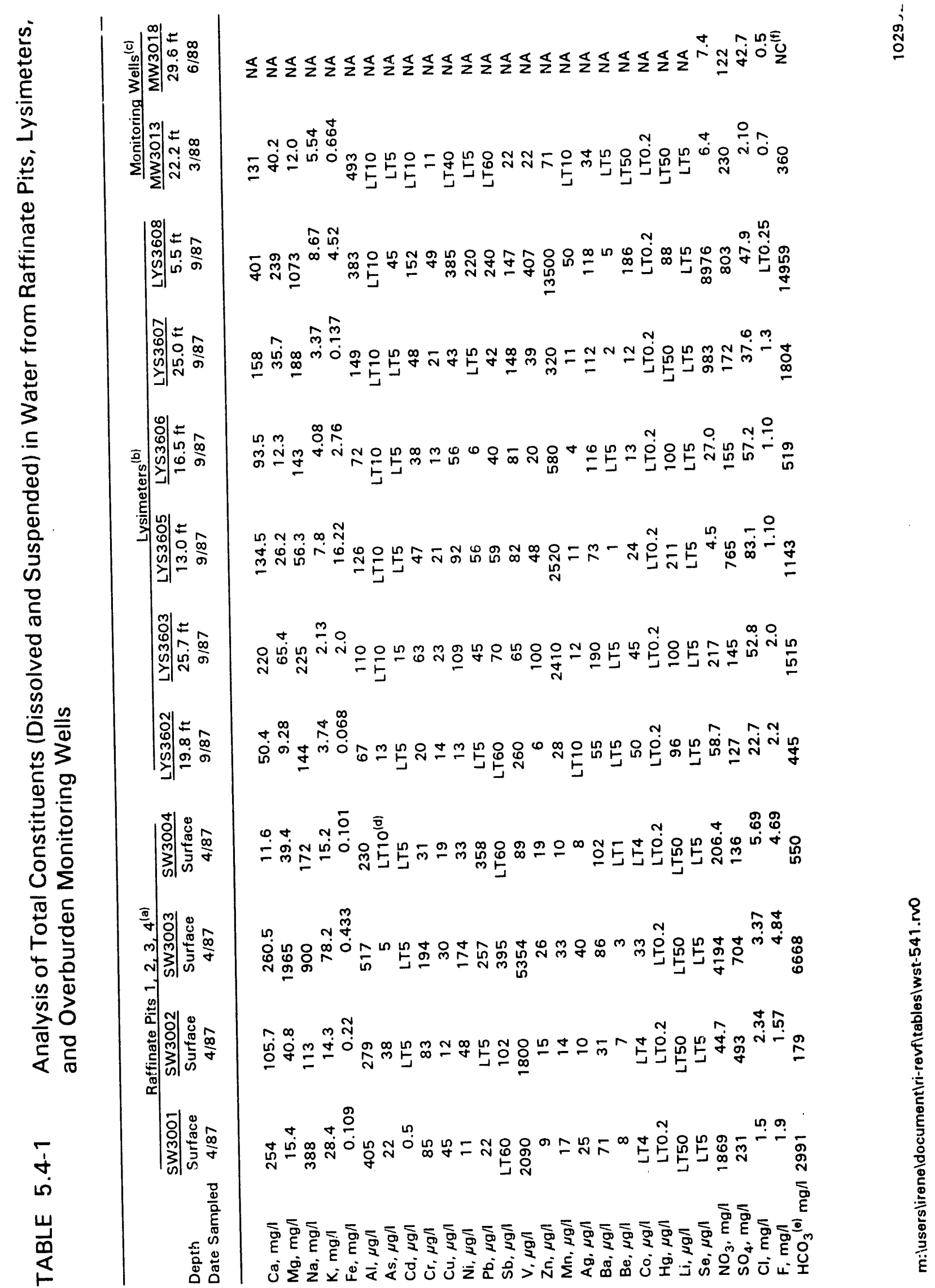




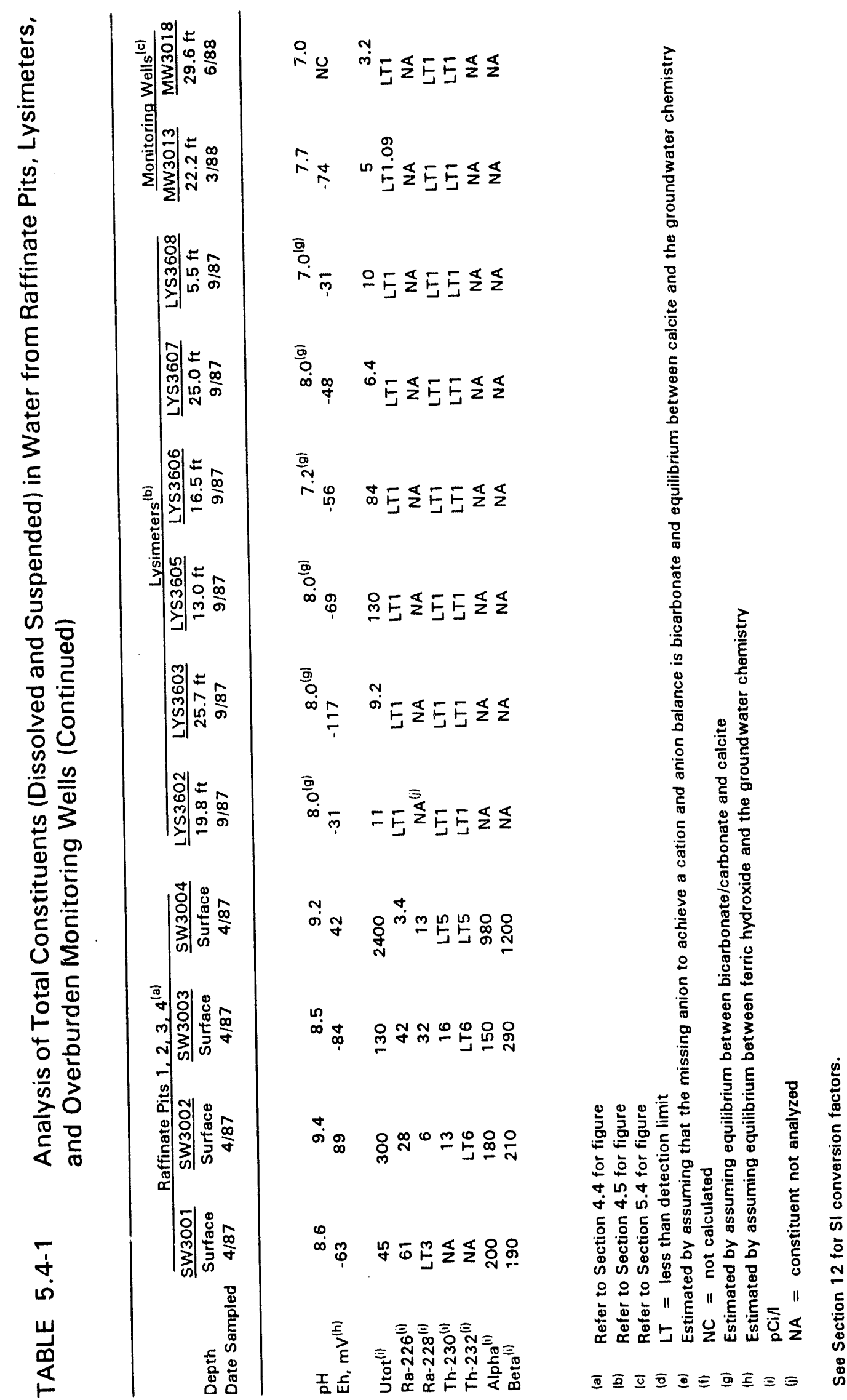

ô
พิ
ㅇ 
TABLE 5.4-2 Summary of Cinemical Analyses of Groundivater ${ }^{(a)}$

\begin{tabular}{|c|c|c|c|c|}
\hline Chemical & $\begin{array}{l}\text { Detection } \\
\text { Level (DL) }\end{array}$ & $\begin{array}{l}\text { Water Quality } \\
\text { Standard }\end{array}$ & $\begin{array}{l}\text { Background } \\
\text { App. D }\end{array}$ & Distribution in Groundwater \\
\hline $1,3,5-$ TNB & $0.03 \mu \mathrm{g} / 1$ & $N S^{(b)}$ & $Z^{(c)}$ & Detected in groundwater on and off site \\
\hline 1,3-TNB & $0.4 \mu \mathrm{g} / \mathrm{I}$ & NS & $\mathbf{z}$ & Detected in groundwater on and off site \\
\hline NB & $0.6 \mu \mathrm{g} / 1$ & NS & z & Detected in groundwater on and off site \\
\hline $2,4,6 \cdot$ TNT & $0.5 \mu \mathrm{g} / 1$ & NS & $\mathbf{Z}$ & Dotected in groundwater on and off site \\
\hline 2,4-DNT & $0.2 \mu \mathrm{g} / 1$ & $0.17 \mu \mathrm{g} / /^{(d)}$ & z & Detected in groundwater on and off site \\
\hline 2,6-DNT & $0.6 \mu \mathrm{g} / 1$ & $0.0068 \mu \mathrm{g} / \mathrm{l}^{(\mathrm{d})}$ & z & Detected in groundwater on and off site \\
\hline Alpha & $5 \mathrm{pCill}$ & $15 \mathrm{pCi} / /^{(\theta)}$ & $N / A^{(f)}$ & No data above standard \\
\hline Beta & $8 \mathrm{pCi} / \mathrm{l}$ & NS & N/A & Seven measurements above $D L^{(g)}$; no standard \\
\hline $\mathrm{Ag}$ & $10 \mu \mathrm{g} / 1$ & $50 \mu \mathrm{g} / /^{(h)}$ & N/A & No data above standard \\
\hline Al & $200 \mu \mathrm{g} / 1$ & NS & $150 \mu \mathrm{g} / 1$ & Data above bkg (i) near raff. pits and SE $1 / 4$ site \\
\hline As & $10 \mu \mathrm{g} / 1$ & $50 \mu g / /^{(h)}$ & N/A & One value above DL, MW 4022, below standard \\
\hline $\mathrm{Ba}$ & $200 \mu \mathrm{g} / 1$ & $1000 \mu \mathrm{g} / /^{(\mathrm{h})}$ & $\mathrm{mN} / \mathrm{A}$ & Two measurements above standard, MW 3009 \\
\hline $\mathrm{Be}$ & $5 \mu \mathrm{g} / 1$ & $0.0068 \mu \mathrm{g} / /^{(1)}$ & $N / A$ & No data above $\mathrm{DL}$ \\
\hline $\mathrm{Ca}$ & $5 \mu \mathrm{g} / 1$ & NS & N/A & No standard for comparison \\
\hline $\mathrm{Cd}$ & $5 \mu \mathrm{g} / 1$ & $10 \mu \mathrm{g} / 1^{(\mathrm{h})}$ & $N / A$ & $\begin{array}{l}\text { Three measurements above DL; MW 2003, } 3007 . \\
3008\end{array}$ \\
\hline $\mathrm{Cl}$ & $0.25 \mathrm{mg} / \mathrm{l}$ & $250 \mathrm{mg} / /^{(k)}$ & N/A & Mean of measurements in MW 2006 below standard \\
\hline Co & $50 \mu_{\mathrm{g}} / 1$ & NS & $N / A$ & No data above DL \\
\hline $\mathrm{Cr}$ & $10 \mu \mathrm{g} / 1$ & $50 \mu g /^{(h)}$ & $55 \mu \mathrm{g} / 1$ & Data from near raff. pits \& Frog Pond above bkg \\
\hline $\mathrm{Cu}$ & $25 \mu \mathrm{g} / \mathrm{l}$ & $1000 \mu \mathrm{g} / /^{(k)}$ & $20 ; \mathrm{g} / \mathrm{l}$ & Few data above DL; no data above standard \\
\hline CYN & $0.01 \mathrm{mg} / \mathrm{l}$ & $200 \mu \mathrm{g} / /^{(h)}$ & $N / A$ & No data above DL \\
\hline$F$ & $0.25 \mathrm{mg} / \mathrm{l}$ & $2 \mathrm{mg} / /^{(k)}$ & N/A & Few data from near raff. pits above standard \\
\hline $\mathrm{Fe}$ & $100 \mu \mathrm{g} / \mathrm{l}$ & $300 \mu_{g} /^{(k)}$ & $N / A$ & Data widely varied; few data above standard \\
\hline $\mathrm{Hg}$ & $0.2 \mu \mathrm{g} / 1$ & $2 \mu g / /^{(h)}$ & $N / A$ & No data above standard; fow data above $D L$ \\
\hline K & $5 \mathrm{mg} / \mathrm{l}$ & NS & N/A & No standard for comparison \\
\hline $\mathrm{Li}$ & $50 \mu \mathrm{g} / 1$ & NS & N/A & No standard; few data near raff. pits above DL \\
\hline $\mathrm{Mg}$ & $5 \mathrm{mg} / \mathrm{l}$ & NS & N/A & $\begin{array}{l}\text { No standard for comparison } \\
\text {. }\end{array}$ \\
\hline Mn & $15 \mu \mathrm{g} / 1$ & $50 \mu \mathrm{g} / /^{(k)}$ & N/A & Data vary widely; féw data above standard \\
\hline Mo & $13 \mu \mathrm{g} / 1$ & NS & $N / A$ & No standard; higher values near raff. pits \\
\hline
\end{tabular}


TABLE 5.4-2 Summary of Chemical Analyses of Groundwater (Continued)

\begin{tabular}{|c|c|c|c|c|}
\hline Chemical & $\begin{array}{l}\text { Detection } \\
\text { Level (DL) }\end{array}$ & $\begin{array}{c}\text { Water Quality } \\
\text { Standard }\end{array}$ & $\begin{array}{c}\text { Background } \\
\text { App. D }\end{array}$ & Distribution in Groundwater \\
\hline $\mathrm{Na}$ & $5 \mathrm{mg} / \mathrm{l}$ & NS & N/A & No standard for comparison \\
\hline $\mathrm{Ni}$ & $40 \mu \mathrm{g} / 1$ & $13.4 \mu \mathrm{g} /(\mathrm{i})$ & $65 \mu \mathrm{g} / 1$ & Data above standard on and off site \\
\hline NO3 & $0.1 \mathrm{mg} / \mathrm{l}$ & $44 \mathrm{mg} / /^{(h)}$ & $9 \mathrm{mg} / \mathrm{l}$ & Data above standard on and off site \\
\hline $\mathrm{Pb}$ & $5 \mu \mathrm{g} / \mathrm{l}$ & $500 \mu \mathrm{g} / /^{(h)}$ & N/A & Few data above $O L ;$ no data above standard \\
\hline Phenol & $0.01 \mathrm{mg} / \mathrm{l}$ & $3.5 \mathrm{mg} / /^{(i)}$ & N/A & No data above $D L]$ \\
\hline $\mathrm{Ra}-226$ & $1 \mathrm{pCi} / \mathrm{l}$ & $5.0 \mathrm{pCi} / /^{(\theta)}$ & N/A & Two measurements above DL\} \\
\hline $\mathrm{Sb}$ & $60 \mu \mathrm{g} / 1$ & $146 \mu g / l^{(i)}$ & $N / A$ & Two data values raff. pits above standard \\
\hline Se & $5 \mu \mathrm{g} / 1$ & $10 \mu g / /^{(h)}$ & $N / A$ & One value above standard, MW 4022 \\
\hline SO4 & $1 \mathrm{mg} / \mathrm{l}$ & $150 \mathrm{mg} / \mathrm{l}^{\prime \prime \prime}$ & $55 \mathrm{mg} / \mathrm{l}$ & Many data above standard \\
\hline Th-230 & $1 \mathrm{pCi} / \mathrm{I}$ & NS & N/A & Few data above DL; no standard for comparison \\
\hline Th-232 & $1 \mathrm{pCi} / \mathrm{I}$ & NS & N/A & Few data above DL; no standard for comparison \\
\hline $\mathrm{TI}$ & $10 \mu \mathrm{g} / 1$ & $13 \mu \mathrm{g} / \mathrm{l}^{(\mathrm{j})}$ & N/A & One value above standard, MW 4022 \\
\hline TOC & $1 \mathrm{mg} / \mathrm{l}$ & NS & N/A & Data vary widely; no standard for comparison \\
\hline UTot & $2 \mathrm{pCi} / \mathrm{l}$ & NS & $5.5 \mathrm{pCi} / 1$ & Data near raff. pits and in WSCP above bkg \\
\hline V & $50 \mu \mathrm{g} / 1$ & NS & N/A & Few data near raffinate pits above DL \\
\hline $\mathrm{Zn}$ & $20 \mu \mathrm{g} / 1$ & $5000 \mu \mathrm{g} / /^{(k)}$ & $45 \mu \mathrm{g} / 1$ & Few data above bkg; no data above standard \\
\hline
\end{tabular}

\footnotetext{
(a) Based upon WSSRAP Groundwater Monitoring Data through First Quarter 1990; subject to change based upon results of validation of these data.

NS = No standard available for comparison

$Z=$ Zero; not expected to exist in nature

Recommended Criteria, ORNL 1987

Maximum Contaminant Levels for Community Water Systems, 40 CFR 144

N/A = Not applicable because of inadequate data

$D L=$ Analytical detection limit generally reported

Primary Drinking Water Standard, 40 CFR 141

bkg = Background

EPA Quality Criteria for Water 1986; Water and Fish Ingestion

Secondary Drinking Water Standard, 40 CFR 143

Missouri State Drinking Water Standard
} 
TABLE 5.4-3 Analyses of Uranium ( $\mathrm{pCi} / \mathrm{l}$ ) as Presented in Figures 5.4-1 and 5.4-2

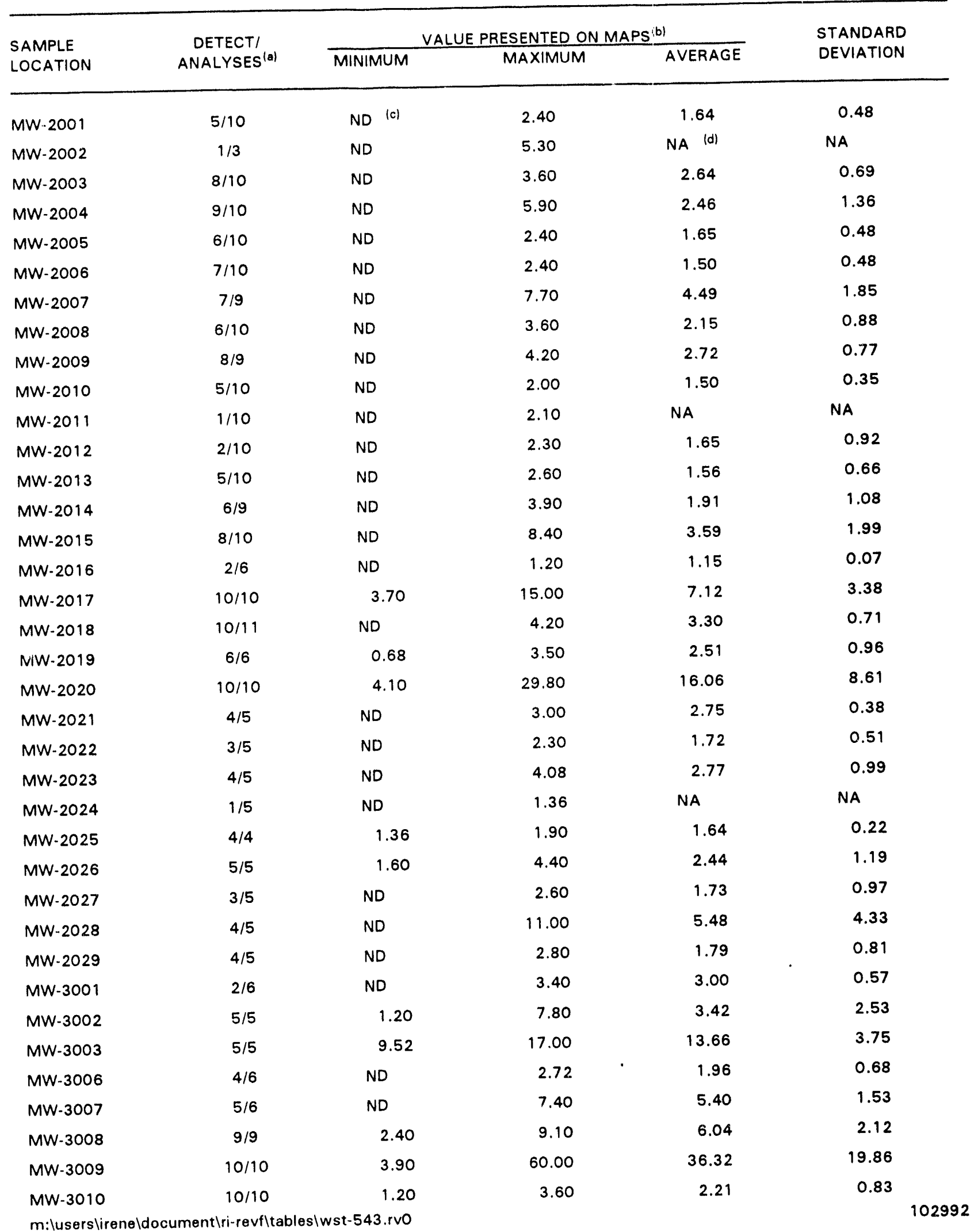


TABLE 5.4-3 Analyses of Uranium ( $\mathrm{pCi} / \mathrm{l})$ as Presented in Figures 5.4-1 and 5.4-2 (Continued)

\begin{tabular}{|c|c|c|c|c|c|}
\hline \multirow{2}{*}{$\begin{array}{l}\text { SAMPLE } \\
\text { LOCATION }\end{array}$} & \multirow{2}{*}{$\begin{array}{c}\text { DETECT/ } \\
\text { ANALYSES }^{(a)}\end{array}$} & \multicolumn{3}{|c|}{ VALUE PRESENTED ON MAPS ${ }^{(b)}$} & \multirow{2}{*}{$\begin{array}{l}\text { STANDARD } \\
\text { DEVIATION }\end{array}$} \\
\hline & & MINIMUM & MAXIMUM & AVERAGE & \\
\hline MW-3013 & $9 / 9$ & 2.00 & 33.00 & 7.12 & 9.86 \\
\hline MW-3018 & $5 / 5$ & 3.20 & 5.30 & 4.64 & 0.86 \\
\hline$M W-3019$ & $4 / 4$ & 5.50 & 9.52 & 7.56 & 1.65 \\
\hline MW-4001 & $5 / 7$ & ND & 1.50 & 1.18 & 0.19 \\
\hline$M W \cdot 4002$ & $3 / 7$ & ND & 1.50 & 1.01 & 0.49 \\
\hline$M W-4003$ & $1 / 5$ & ND & 1.70 & NA & NA \\
\hline MW-4004 & $4 / 4$ & 1.90 & 4.30 & 2.93 & 1.17 \\
\hline MW-4005 & $5 / 5$ & 1.00 & 3.00 & 1.97 & 0.72 \\
\hline$M W-4006$ & $0 / 5$ & ND & ND & NA & NA \\
\hline$M W-4007$ & $5 / 5$ & 0.68 & 3.40 & 1.62 & 1.04 \\
\hline$M W-4008$ & $4 / 5$ & ND & 4.08 & 3.12 & 0.97 \\
\hline MW-4009 & $4 / 4$ & 1.40 & 3.40 & 2.15 & 0.87 \\
\hline$M W-4010$ & $4 / 4$ & 2.50 & 5.40 & 4.02 & 1.31 \\
\hline$M W-4011$ & $4 / 5$ & ND & 4.40 & 3.15 & 0.85 \\
\hline$M W-4012$ & $4 / 4$ & 1.30 & 3.50 & 2.41 & 0.95 \\
\hline$M W-4013$ & $3 / 5$ & ND & 1.80 & 1.60 & 0.20 \\
\hline$M W-4014$ & $0 / 5$ & ND & ND & NA & NA \\
\hline$M W-4015$ & $3 / 5$ & ND & 1.60 & 1.35 & 0.25 \\
\hline$M W-4016$ & $5 / 5$ & 4.60 & 5.44 & 4.99 & 0.38 \\
\hline$M W-4017$ & $5 / 5$ & 1.40 & 3.40 & 2.56 & 0.82 \\
\hline$M W-4018$ & $4 / 5$ & ND & 2.30 & 1.84 & 0.43 \\
\hline$M W-4019$ & $9 / 9$ & 1.50 & 5.20 & 2.81 & 1.34 \\
\hline$M W-4020$ & $5 / 5$ & 13.00 & 28.00 & 21.26 & 7.05 \\
\hline$M W \cdot 4021$ & $5 / 5$ & 2.90 & 18.00 & 10.22 & 5.37 \\
\hline$M W-4022$ & $4 / 5$ & ND & 16.70 & 6.98 & 6.59 \\
\hline$M W-4023$ & $4 / 5$ & ND & 3.20 & 2.48 & 0.51 \\
\hline (a) Does & de QA data, dur & es and matrir & & & \\
\hline The c & narized in this te & nd presented & reflect deta $t$ & he first quar & 90. \\
\hline Not o & & & & & \\
\hline (d) Not a & & & & & \\
\hline
\end{tabular}


TABLE 5.4-4 Analyses of Nitrate $(\mathrm{mg} / \mathrm{l})$ as Presented in Figures 5.4-3 and 5.4-4

\begin{tabular}{|c|c|c|c|c|c|}
\hline \multirow{2}{*}{$\begin{array}{l}\text { SAMPLE } \\
\text { LOCATION }\end{array}$} & \multirow{2}{*}{$\begin{array}{c}\text { DETECT/ } \\
\text { ANALYSES }^{(8)}\end{array}$} & \multicolumn{3}{|c|}{ VALUE PRESENTED ON MAPS ${ }^{(b)}$} & \multirow{2}{*}{$\begin{array}{l}\text { STANDARD } \\
\text { DEVIATION }\end{array}$} \\
\hline & & MINIMUM & MAXIMUM & AVERAGE & \\
\hline MW-2001 & $11 / 11$ & 6.50 & 50.20 & 29.81 & 12.46 \\
\hline$M W-2002$ & $3 / 3$ & 1010.00 & 3580.00 & 2024.33 & 1367.85 \\
\hline MW-2003 & $11 / 11$ & 1380.00 & 3940.00 & 2780.55 & 707.43 \\
\hline MW-2004 & $11 / 11$ & 0.80 & 5.30 & 3.36 & 1.27 \\
\hline MW-2005 & $9 / 10$ & ND $|c|$ & 529.00 & 162.26 & 145.24 \\
\hline MW-2006 & $11 / 11$ & 0.64 & 36.30 & 23.70 & 12.25 \\
\hline MW-2007 & $1 / 9$ & ND & 0.30 & NA (d) & NA \\
\hline MW-2008 & $9 / 9$ & 3.60 & 32.10 & 18.18 & 8.06 \\
\hline MW-2009 & $8 / 8$ & 5.30 & 8.80 & 7.52 & 1.31 \\
\hline$M W-2010$ & $11 / 11$ & 1.00 & 5.70 & 3.81 & 1.44 \\
\hline MW-2011 & $10 / 10$ & 0.80 & 26.50 & 18.84 & 9.12 \\
\hline$M W-2012$ & $10 / 10$ & 0.50 & 4.00 & 1.73 & 1.24 \\
\hline$M W-2013$ & $10 / 10$ & 0.90 & 4.40 & 2.94 & 1.05 \\
\hline$M W-2014$ & $9 / 9$ & 2.20 & 10.40 & 8.37 & 2.43 \\
\hline$M W-2015$ & $9 / 9$ & 0.75 & 1.80 & 1.33 & 0.33 \\
\hline$M W-2016$ & $2 / 6$ & ND & 2490.00 & 1666.00 & 1165.31 \\
\hline MW.2017 & $10 / 10$ & 0.90 & 7.20 & 3.47 & 2.35 \\
\hline$M W-2018$ & $10 / 10$ & 1.51 & 4.10 & 2.29 & 0.72 \\
\hline MW-2019 & $3 / 7$ & ND & 1.00 & 0.87 & 0.12 \\
\hline$M W-2020$ & $10 / 10$ & 0.50 & 5.20 & 2.40 & 1.75 \\
\hline$M W-2021$ & $0 / 5$ & ND & ND & NA & NA \\
\hline$M W-2022$ & $0 / 5$ & ND & ND & NA & NA \\
\hline$M W-2023$ & $4 / 6$ & ND & 1.30 & 0.53 & 0.53 \\
\hline$M W-2024$ & $0 / 5$ & ND & ND & NA & NA \\
\hline$M W-2025$ & $0 / 4$ & ND & ND & NA & NA \\
\hline$M W-2026$ & $1 / 5$ & ND & 0.50 & $\mathrm{NA}$ & NA \\
\hline$M W-2027$ & $4 / 5$ & ND & 0.53 & 0.31 & 0.16 \\
\hline$M W-2028$ & $1 / 5$ & ND & 6.10 & NA & NA \\
\hline$M W-2029$ & $1 / 5$ & ND & 0.11 & NA & NA \\
\hline$M W-3001$ & $6 / 6$ & 129.00 & 2150.00 & 908.83 & 713.45 \\
\hline$M W-3002$ & $2 / 5$ & ND & 0.20 & 0.19 & 0.01 \\
\hline$M W-3003$ & $3 / 4$ & ND & 2170.00 & 2035.00 & 143.09 \\
\hline MW-3006 & $2 / 5$ & ND & 4.00 & 2.65 & 1.91 \\
\hline$M W-3007$ & $5 / 5$ & 2650.00 & 5560.00 & 4254.00 & 1044.81 \\
\hline MW-3008 & $9 / 9$ & 597.00 & 6010.00 & 4611.78 & 1636.69 \\
\hline$M W-3009$ & $9 / 9$ & 16.70 & 10846.00 & 1637.56 & 3523.07 \\
\hline MW-3010 & $8 / 8$ & 4.10 & 13.90 & 7.23 & 4.19 \\
\hline
\end{tabular}

m:luserslireneldocument|ri-revfltablesiwst-544.rvo 
TABLE 5.4-4 Analyses of Nitrate $(\mathrm{mg} / \mathrm{l})$ as Presented in Figures 5.4-3 and 5.4-4 (Continued)

\begin{tabular}{|c|c|c|c|c|c|}
\hline \multirow{2}{*}{$\begin{array}{l}\text { SAMPLE } \\
\text { LOCATION }\end{array}$} & \multirow{2}{*}{$\begin{array}{c}\text { DETECT/ } \\
\text { ANALYSES }\end{array}$} & \multicolumn{3}{|c|}{ VALUE PRESENTED ON MAPS ${ }^{(b)}$} & \multirow{2}{*}{$\begin{array}{l}\text { STANDARD } \\
\text { DEVIATION }\end{array}$} \\
\hline & & MINIMUM & MAXIMUM & AVERAGE & \\
\hline$M W-3013$ & $7 / 7$ & 6.40 & 15.50 & 11.03 & 3.70 \\
\hline$M W-3018$ & $4 / 4$ & 7.40 & 1150.00 & 451.53 & 545.01 \\
\hline MW-3019 & $3 / 4$ & ND & 0.59 & 0.38 & 0.20 \\
\hline$M W-4001$ & $6 / 6$ & 56.00 & 182.00 & 139.33 & 43.20 \\
\hline$M W-4002$ & $7 / 7$ & 7.40 & 26.70 & 13.68 & 6.85 \\
\hline$M W-4003$ & $5 / 5$ & 2.99 & 2100.00 & 422.52 & 937.74 \\
\hline MW-4004 & $6 / 6$ & 0.50 & 2.30 & 1.49 & 0.71 \\
\hline$M W-4005$ & $6 / 6$ & 0.98 & 8.70 & 5.03 & 3.09 \\
\hline MW-4006 & $5 / 5$ & $18.80^{\circ}$ & 30.30 & 22.50 & 4.54 \\
\hline$M W-4007$ & $0 / 5$ & ND & ND & NA & NA \\
\hline$M W-4008$ & $1 / 5$ & ND & 3.20 & NA & NA \\
\hline$M W-4009$ & $3 / 4$ & ND & 0.60 & 0.44 & 0.14 \\
\hline MW-4010 & $5 / 6$ & ND & 1.30 & 0.60 & 0.52 \\
\hline$M W-4011$ & $4 / 5$ & ND & 133.00 & 87.53 & 47.37 \\
\hline$M W-4012$ & $3 / 5$ & ND & 0.55 & 0.34 & 0.21 \\
\hline$M W-4013$ & $4 / 5$ & ND & 657.00 & 499.00 & 124.35 \\
\hline MW-4014 & $4 / 4$ & 7.90 & 14.30 & 11.18 & 3.02 \\
\hline$M W-4015$ & $5 / 5$ & 1.30 & 22.40 & 9.54 & 8.25 \\
\hline$M W-4016$ & $0 / 5$ & ND & ND & NA & NA \\
\hline$M W-4017$ & $5 / 5$ & 0.47 & 3.40 & 2.15 & 1.08 \\
\hline$M W-4018$ & $5 / 5$ & 1.30 & 9.70 & 7.24 & 3.38 \\
\hline MW.4019 & $9 / 9$ & 0.34 & 177.00 & 20.26 & 58.78 \\
\hline$M W \cdot 4020$ & $3 / 5$ & ND & 5.50 & 2.46 & 2.74 \\
\hline MW-4021 & $1 / 6$ & ND & 1.40 & NA & NA \\
\hline$M W-4022$ & $5 / 6$ & ND & 3.00 & 1.39 & 1.29 \\
\hline$M W-4023$ & $6 / 6$ & 7.33 & 28.90 & 19.84 & 8.85 \\
\hline (a) Does & de QA data, du & es and matri & & & \\
\hline The c & narized in this & nd presente & s reflect data $t$ & the first quar & 190. \\
\hline Not c & & & & & \\
\hline (d) Not : & & & & & \\
\hline
\end{tabular}


TABLE 5.4-5 Mnalyses of Sulfate $(\mathrm{mg} / \mathrm{l})$ as Presented in Figures 5.4-5 and 5.4-6

\begin{tabular}{|c|c|c|c|c|c|}
\hline \multirow{2}{*}{$\begin{array}{l}\text { SAMPLE } \\
\text { LOCATION }\end{array}$} & \multirow{2}{*}{$\begin{array}{c}\text { DETECT/ } \\
\text { ANALYSES }\end{array}$} & \multicolumn{3}{|c|}{ VALUE PRESENTED ON MAPS ${ }^{(b)}$} & \multirow{2}{*}{$\begin{array}{l}\text { STANDARD } \\
\text { DEVIATION }\end{array}$} \\
\hline & & MINIMUM & MAXIMUM & AVERAGE & \\
\hline$M W-2001$ & $11 / 11$ & 4.40 & 22.50 & 7.97 & 5.63 \\
\hline MW-2002 & $3 / 3$ & 95.00 & 198.00 & 160.67 & 57.05 \\
\hline MW-2003 & $10 / 10$ & 70.00 & 298.00 & 200.57 & 84.50 \\
\hline MW-2004 & $12 / 12$ & 1.90 & 6.70 & 3.07 & 1.63 \\
\hline MW-2005 & $10 / 10$ & 2.30 & 7.00 & 3.41 & 1.42 \\
\hline MW-2006 & $9 / 10$ & ND (c) & 52.50 & 40.86 & 9.52 \\
\hline MW-2007 & $9 / 9$ & 14.10 & 17.90 & 15.70 & 1.25 \\
\hline MW-2008 & $9 / 9$ & 9.10 & 795.00 & 131.47 & 252.93 \\
\hline MW-2009 & $9 / 9$ & 38.20 & 193.00 & 100.77 & 39.86 \\
\hline$M W \cdot 2010$ & $11 / 11$ & 27.50 & 56.80 & 39.65 & 9.32 \\
\hline MW-2011 & $10 / 10$ & 8.60 & 51.20 & 18.52 & 14.42 \\
\hline$M W-2012$ & $9 / 9$ & 51.00 & 111.00 & 69.36 & 19.17 \\
\hline$M W-2013$ & $11 / 11$ & 11.90 & 52.00 & 28.04 & 10.39 \\
\hline MW-2014 & $8 / 8$ & 27.10 & 40.00 & 33.69 & 3.71 \\
\hline MW-2015 & $9 / 9$ & 84.70 & 158.00 & 108.02 & 21.52 \\
\hline MW-2016 & $8 / 8$ & 30.30 & 112.00 & 43.58 & 28.63 \\
\hline MW-2017 & $10 / 10$ & 462.00 & 1830.00 & 775.30 & 408.30 \\
\hline MW-2018 & $11 / 11$ & 9.30 & 18.80 & 10.63 & 2.73 \\
\hline$M W-2019$ & $7 / 7$ & 7.80 & 22.60 & 16.37 & 6.09 \\
\hline MW-2020 & $10 / 10$ & 144.00 & 336.00 & 236.40 & 76.68 \\
\hline$M W-2021$ & $5 / 5$ & 12.00 & 25.10 & 15.36 & 5.48 \\
\hline$M W-2022$ & $5 / 5$ & 12.50 & 17.80 & 14.74 & 2.19 \\
\hline$M W-2023$ & $6 / 6$ & 22.10 & 55.40 & 31.30 & 13.04 \\
\hline MW-2024 & $5 / 5$ & 23.20 & 38.60 & 32.98 & 5.89 \\
\hline MW-2025 & $4 / 4$ & 16.10 & 18.70 & 16.90 & 1.23 \\
\hline MW-2026 & $5 / 5$ & 11.90 & 13.70 & 13.18 & 0.77 \\
\hline MW-2027 & $4 / 4$ & 10.50 & 14.90 & 12.68 & 1.81 \\
\hline$M W-2028$ & $5 / 5$ & 95.70 & 117.00 & 109.14 & 8.17 \\
\hline MW-2029 & $5 / 5$ & 16.60 & 27.10 & 23.22 & 4.12 \\
\hline$M W-3001$ & $6 / 6$ & 19.80 & 53.90 & 27.65 & 13.22 \\
\hline$M W-3002$ & $4 / 5$ & ND & 19.50 & 19.30 & 0.22 \\
\hline MW-3003 & $5 / 5$ & 153.00 & 232.00 & 187.80 & 29.15 \\
\hline MW-3006 & $6 / 6$ & 29.40 & 80.40 & 57.35 & 20.81 \\
\hline MW-3007 & $5 / 5$ & 24.40 & 866.00 & 284.70 & 341.97 \\
\hline MW-3008 & $9 / 9$ & 11.40 & 100.00 & 49.21 & 28.42 \\
\hline MW-3009 & $8 / 8$ & 33.20 & 71.90 & 49.40 & 14.46 \\
\hline
\end{tabular}


TABLE $5.4-5$

Analyses of Sulfate $(\mathrm{mg} / \mathrm{l})$ as Presented in Figures 5.4-5 and 5.4-6 (Continued)

\begin{tabular}{|c|c|c|c|c|c|}
\hline \multirow{2}{*}{$\begin{array}{l}\text { SAMPLE } \\
\text { LOCATION }\end{array}$} & \multirow{2}{*}{$\begin{array}{c}\text { DETECT/ } \\
\text { ANALYSES }^{(\theta)}\end{array}$} & \multicolumn{3}{|c|}{ VALUE PRESENTED ON MAPS ${ }^{(b)}$} & \multirow{2}{*}{$\begin{array}{l}\text { STAANDARD } \\
\text { DEVIATION }\end{array}$} \\
\hline & & MINIMUM & MAXIMUM & AVERAGE & \\
\hline MW-3010 & $9 / 9$ & 6.30 & 23.80 & 11.43 & 6.73 \\
\hline$M W-3013$ & $8 / 8$ & 230.00 & 915.00 & 672.38 & 237.38 \\
\hline$M W-3018$ & $3 / 3$ & 122.00 & 187.00 & 160.33 & 34.03 \\
\hline MW-3019 & $4 / 4$ & 5.20 & 10.30 & 7.45 & 2.16 \\
\hline MW-4001 & $7 / 7$ & 50.10 & 159.00 & 77.19 & 37.24 \\
\hline MW-4002 & $7 / 7$ & 15.00 & 28.70 & 21.13 & 5.31 \\
\hline$M W-4003$ & $5 / 5$ & 27.60 & 36.00 & 30.80 & 3.35 \\
\hline MW-4004 & $6 / 6$ & 25.10 & 35.20 & 29.25 & 3.40 \\
\hline MW-4005 & $6 / 6$ & 16.10 & 22.00 & 18.98 & 2.26 \\
\hline MW-4006 & $6 / 6$ & 31.30 & 129.00 & 49.63 & 38.91 \\
\hline$M W-4007$ & $5 / 5$ & 3.00 & 14.80 & 10.92 & 4.80 \\
\hline$M V-O O B$ & $5 / 5$ & 12.20 & 16.90 & 14.86 & 1.78 \\
\hline MW 4 & $5 / 5$ & 12.90 & 39.00 & 23.10 & 10.90 \\
\hline$M W-4 L, O$ & $6 / 6$ & 24.10 & 42.70 & 29.37 & 7.00 \\
\hline MW-4011 & $5 / 5$ & 45.80 & 52.40 & 49.32 & 2.76 \\
\hline$M W-4012$ & $5 / 5$ & 39.90 & 57.50 & 48.36 & 6.47 \\
\hline$M W-4013$ & $5 / 5$ & 43.20 & 67.70 & 55.62 & 9.41 \\
\hline$M W-4014$ & $5 / 5$ & 21.50 & 36.10 & 27.58 & 5.48 \\
\hline$M W-4015$ & $4 / 5$ & ND & 18.60 & 10.23 & 6.30 \\
\hline$M W \cdot 4016$ & $5 / 5$ & 11.60 & 52.00 & 27.40 & 15.72 \\
\hline MW-4017 & $5 / 5$ & 5.10 & 10.20 & 8.06 & 2.14 \\
\hline$M W-4018$ & $5 / 5$ & 8.20 & 18.90 & 12.86 & 3.92 \\
\hline$M W-4019$ & $9 / 9$ & 7.00 & 11.50 & 9.15 & 1.41 \\
\hline$M W-4020$ & $5 / 5$ & 121.00 & 181.00 & 142.70 & 23.67 \\
\hline$M W-4021$ & $6 / 6$ & 51.70 & 343.00 & 261.62 & 106.00 \\
\hline$M W-4022$ & $6 / 6$ & 32.30 & 70.90 & 52.55 & 15.34 \\
\hline$M W-4023$ & $6 / 6$ & 23.50 & 285.00 & 112.82 & 88.96 \\
\hline
\end{tabular}

\footnotetext{
(a) Does not include QA data, duplicates and matrix spikes.

(b) The date summarized in this table and presented on maps reflect data through the first quarter of 1990 .

(c) Not detected.
} 
TABLE 5.4-6 Analyses of Chromium $(\mu \mathrm{g} / \mathrm{l})$ as Presented in Figure 5.4-7

\begin{tabular}{|c|c|c|c|c|c|}
\hline \multirow{2}{*}{$\begin{array}{l}\text { SAMPLE } \\
\text { LOCATION }\end{array}$} & \multirow{2}{*}{$\begin{array}{c}\text { DETECT/ } \\
\text { ANALYSES }\end{array}$} & \multicolumn{3}{|c|}{ VALUE PRESENTED ON MAPS ${ }^{(b)}$} & \multirow{2}{*}{$\begin{array}{l}\text { STANDARD } \\
\text { DEVIATION }\end{array}$} \\
\hline & & MINIMUM & MAXIMUM & AVERAGE & \\
\hline MW-2001 & $1 / 3$ & ND (c) & 34.00 & NA (d) & NA \\
\hline$M W-2002$ & $1 / 2$ & ND & 61.00 & NA & NA \\
\hline$M W-2003$ & $2 / 3$ & ND & 91.00 & 58.00 & 46.67 \\
\hline$M W-2004$ & $2 / 3$ & ND & 131.00 & 86.50 & 62.93 \\
\hline$M W-2005$ & $1 / 2$ & ND & 75.00 & NA & NA \\
\hline$M W-2006$ & $2 / 3$ & ND & 36.00 & 26.50 & 13.44 \\
\hline MW-2007 & $1 / 3$ & ND & 22.00 & NA & NA \\
\hline MW-2008 & $1 / 3$ & ND & 40.00 & NA & NA \\
\hline$M W \cdot 2009$ & $1 / 3$ & ND & 15.00 & NA & NA \\
\hline$M W-2010$ & $2 / 3$ & ND & 72.00 & 50.00 & 31.11 \\
\hline MW-2011 & $1 / 3$ & ND & 15.00 & NA & NA \\
\hline$M W-2012$ & $1 / 2$ & ND & 39.00 & NA & NA \\
\hline MW.2013 & $1 / 3$ & ND & 22.00 & NA & NA \\
\hline$M W-2014$ & $1 / 3$ & ND & 40.00 & NA & NA \\
\hline MW-2015 & $1 / 3$ & ND & 46.00 & NA & NA \\
\hline$M W-2016$ & $1 / 1$ & 31.00 & 31.00 & NA & NA \\
\hline$M W-2017$ & $1 / 3$ & ND & 37.00 & NA & NA \\
\hline$M W-2018$ & $1 / 3$ & ND & 35.00 & NA & NA \\
\hline$M W-2019$ & $0 / 1$ & ND & ND & NA & NA \\
\hline$M W-2020$ & $2 / 3$ & ND & 121.00 & 84.00 & 52.33 \\
\hline$M W-2021$ & $0 /:$ & ND & ND & NA & NA \\
\hline$M W-2022$ & $0 / 1$ & ND & ND & NA & NA \\
\hline$M W-2023$ & $1 / 1$ & 10.50 & 10.50 & NA & NA \\
\hline$M W-2024$ & $0 / 1$ & ND & ND & NA & NA \\
\hline$M W-2026$ & $0 / 1$ & ND & ND & NA & NA \\
\hline$M W-2027$ & $0 / 1$ & ND & ND & NA & NA \\
\hline$M W-2028$ & $0 / 1$ & ND & ND & NA & NA \\
\hline$M W-2029$ & $0 / 1$ & ND & ND & NA & NA \\
\hline$M W-3001$ & $0 / 1$ & ND & ND & NA & NA \\
\hline$M W-3002$ & $0 / 1$ & ND & ND & NA & NA \\
\hline$M W-3003$ & $0 / 1$ & ND & ND & NA & NA \\
\hline MW-3006 & $1 / 1$ & 37.20 & 37.20 & NA & NA \\
\hline MW-3007 & $2 / 2$ & 35.00 & 133.00 & 84.00 & 69.30 \\
\hline MW-3008 & $2 / 3$ & ND & 137.00 & 94.50 & 60.10 \\
\hline$M W-3009$ & $1 / 3$ & ND & 45.00 & NA & NA \\
\hline$M W-3010$ & $1 / 3$ & ND & 25.00 & NA & NA \\
\hline
\end{tabular}


TABLE 5.4-6 Analyses of Chromium $(\mu \mathrm{g} / \mathrm{l})$ as Presented in Figure 5.4-7 (Continued)

\begin{tabular}{|c|c|c|c|c|c|}
\hline \multirow{2}{*}{$\begin{array}{l}\text { SAMPLE } \\
\text { LOCATION }\end{array}$} & \multirow{2}{*}{$\begin{array}{c}\text { DETECT/ } \\
\text { ANALYSES } \\
\text { (a) }\end{array}$} & \multicolumn{3}{|c|}{ VALUE PRESENTED ON MAPS ${ }^{(b)}$} & \multirow{2}{*}{$\begin{array}{l}\text { STANDARD } \\
\text { DEVIATION }\end{array}$} \\
\hline & & MINIMUM & MAXIMUM & AVERAGE & \\
\hline$M W-3013$ & $1 / 3$ & ND & 79.00 & NA & NA \\
\hline MW-3019 & $0 / 1$ & ND & ND & NA & NA \\
\hline$M W \cdot 4001$ & $1 / 3$ & ND & 40.00 & NA & NA \\
\hline$M W-4002$ & $1 / 3$ & ND & 29.00 & NA & NA \\
\hline$M W \cdot 4003$ & $1 / 1$ & 34.00 & 34.00 & NA & NA \\
\hline$M W-4004$ & $0 / 1$ & ND & ND & NA & NA \\
\hline MW-4005 & $0 / 1$ & ND & ND & NA & NA \\
\hline MW-4006 & $1 / 2$ & ND & 26.00 & NA & NA \\
\hline$M W-4007$ & $0 / 1$ & ND & ND & NA & NA \\
\hline$M W-4008$ & $0 / 1$ & ND & ND & NA & NA \\
\hline$M W-4009$ & $1 / 1$ & 10.20 & 10.20 & NA & NA \\
\hline$M W-4010$ & $0 / 1$ & ND & ND & NA & NA \\
\hline$M W-4011$ & $0 / 1$ & ND & ND & NA & NA \\
\hline$M W-4013$ & $0 / 1$ & ND & ND & NA & NA \\
\hline$M W-4014$ & $0 / 1$ & ND & ND & NA & NA \\
\hline$M W-4015$ & $0 / 1$ & ND & ND & NA & NA \\
\hline$M W-4016$ & $0 / 1$ & ND & ND & NA & NA \\
\hline MW-4017 & $0 / 1$ & ND & ND & NA & NA \\
\hline$M W \cdot 4018$ & $0 / 1$ & ND & ND & NA & NA \\
\hline$M W-4019$ & $2 / 3$ & ND & 27.00 & 22.35 & 6.58 \\
\hline$M W-4020$ & $1 / 1$ & 24.10 & 24.10 & NA & NA \\
\hline$M W-4021$ & $1 / 1$ & 14.40 & 14.40 & NA & NA \\
\hline$M W-4022$ & $1 / 1$ & 16.80 & 16.80 & NA & NA \\
\hline$M W-4023$ & $1 / 1$ & 29.40 & 29.40 & NA & NA \\
\hline
\end{tabular}

\footnotetext{
(a) Does not include QA data, duplicates and matrix spikes.

(b) The data summarized in this table and presented on maps reflect data through the first quarter of 1990 .

(c) Not detected.

(d) Not applicable.
} 
TABLE 5.4-7 Analyses of Nickel $(\mu \mathrm{g} / \mathrm{l})$ as Presented in Figure 5.4-8

\begin{tabular}{|c|c|c|c|c|c|}
\hline \multirow{2}{*}{$\begin{array}{l}\text { SAMPLE } \\
\text { LOCATION }\end{array}$} & \multirow{2}{*}{$\begin{array}{c}\text { DETECT/ } \\
\text { ANALYSES(a) }\end{array}$} & \multicolumn{3}{|c|}{ VALUE PRESENTED ON MAPS ${ }^{(b)}$} & \multirow{2}{*}{$\begin{array}{l}\text { STANDARD } \\
\text { DEVIATION }\end{array}$} \\
\hline & & MINIMUM & MAXIMUM & AVERAGE & \\
\hline$M W-2001$ & $0 / 3$ & ND $|c|$ & ND & NA (d) & NA \\
\hline MW-2002 & $0 / 2$ & ND & ND & NA & NA \\
\hline MW-2003 & $0 / 2$ & ND & ND & NA & NA \\
\hline MW-2004 & $1 / 2$ & ND & 57.80 & NA & NA \\
\hline MW-2005 & $1 / 2$ & ND & 52.00 & NA & NA \\
\hline MW-2006 & $2 / 3$ & ND & 45.00 & 44.00 & 1.41 \\
\hline MW-2007 & $1 / 3$ & ND & 54.00 & NA & NA \\
\hline$M W-2008$ & $1 / 3$ & ND & 88.00 & NA & NA \\
\hline MW-2009 & $0 / 2$ & ND & ND & NA & NA \\
\hline$M W-2010$ & $3 / 3$ & 43.00 & 172.00 & 109.00 & 64.55 \\
\hline MW-2011 & $0 / 2$ & ND & ND & NA & NA \\
\hline MW-2012 & $0 / 1$ & ND & ND & NA & NA \\
\hline$M W-2013$ & $0 / 2$ & ND & ND & NA & NA \\
\hline MW-2014 & $0 / 3$ & ND & ND & NA & NA \\
\hline$M W-2015$ & $1 / 3$ & ND & 50.00 & NA & NA \\
\hline MW-2016 & $0 / 1$ & ND & ND & NA & NA \\
\hline MW-2017 & $1 / 3$ & ND & 60.00 & NA & NA \\
\hline$M W-2018$ & $0 / 3$ & ND & ND & NA & NA \\
\hline$M W \cdot 2019$ & $0 / 1$ & ND & ND & NA & NA \\
\hline$M W-2020$ & $1 / 3$ & ND & 40.40 & NA & NA \\
\hline MW-2021 & $0 / 1$ & ND & ND & NA & NA \\
\hline$M W-2022$ & $0 / 1$ & ND & ND & NA & NA \\
\hline$M W-2023$ & $0 / 1$ & ND & ND & NA & NA \\
\hline$M W-2024$ & $0 / 1$ & ND & ND & NA & NA \\
\hline$M W-2026$ & $0 / 1$ & ND & ND & NA & NA \\
\hline$M W-2027$ & $0 / 1$ & ND & ND & NA & NA \\
\hline MW-2028 & $0 / 1$ & ND & ND & NA & NA \\
\hline$M W-2029$ & $0 / 1$ & ND & ND & NA & NA \\
\hline$M W-3001$ & $0 / 1$ & ND & ND & NA & NA \\
\hline$M W-3002$ & $0 / 1$ & ND & ND & NA & NA \\
\hline$M W-3003$ & $1 / 1$ & 45.90 & 45.90 & NA & NA \\
\hline MW-3006 & $0 / 1$ & ND & ND & NA & NA \\
\hline MW-3007 & $1 / 2$ & ND & 100.00 & NA & NA \\
\hline$M W-3008$ & $1 / 3$ & ND & 107.00 & NA & NA \\
\hline MW-3009 & $1 / 3$ & ND & 48.00 & NA & NA \\
\hline MW-3010 & $0 / 3$ & ND & ND & NA & NA \\
\hline
\end{tabular}


TABLE 5.4-7 Analyses of Nickel $(\mu \mathrm{g} / \mathrm{l})$ as Presented in Figure 5.4-8 (Continued)

\begin{tabular}{|c|c|c|c|c|c|}
\hline \multirow{2}{*}{$\begin{array}{l}\text { SAMPLE } \\
\text { LOCATION }\end{array}$} & \multirow{2}{*}{$\begin{array}{c}\text { DETECT/ } \\
\text { ANALYSES }\end{array}$} & \multicolumn{3}{|c|}{ VALUE PRESENTED ON MAPS $(b)$} & \multirow{2}{*}{$\begin{array}{l}\text { STANDARD } \\
\text { DEVIATION }\end{array}$} \\
\hline & & MINIMUM & MAXIMUM & AVERAGE & \\
\hline MW-3013 & $1 / 3$ & ND & 60.00 & NA & NA \\
\hline MW-3019 & $0 / 1$ & ND & ND & NA & NA \\
\hline$M W-4001$ & $0 / 3$ & ND & ND & NA & NA \\
\hline MW-4002 & $0 / 3$ & ND & ND & NA & NA \\
\hline$M W-4003$ & $0 / 1$ & ND & ND & NA & NA \\
\hline MW-4004 & $0 / 1$ & ND & ND & NA & NA \\
\hline MW-4005 & $0 / 1$ & ND & ND & NA & NA \\
\hline MW-4006 & $0 / 2$ & ND & ND & NA & NA \\
\hline MW-4007 & $0 / 1$ & ND & ND & NA & NA \\
\hline MW-4008 & $0 / 1$ & ND & ND & NA & NA \\
\hline MW-4009 & $0 / 1$ & ND & ND & NA & NA \\
\hline MW-4010 & $0 / 1$ & ND & ND & NA & NA \\
\hline$M W-4011$ & $0 / 1$ & ND & ND & NA & NA \\
\hline$M W \cdot 4013$ & $0 / 1$ & ND & ND & NA & NA \\
\hline$M W-4014$ & $1 / 1$ & 82.70 & 82.70 & NA & NA \\
\hline$M W-4015$ & $0 / 1$ & ND & ND & NA & NA \\
\hline$M W-4016$ & $0 / 1$ & ND & ND & NA & NA \\
\hline$M W-4017$ & $0 / 1$ & ND & ND & NA & NA \\
\hline$M W-4018$ & $0 / 1$ & ND & ND & NA & NA \\
\hline$M W-4019$ & $0 / 3$ & ND & ND & NA & NA \\
\hline$M W-4020$ & $0 / 1$ & ND & ND & NA & NA \\
\hline MW-4021 & $0 / 1$ & ND & ND & NA & NA \\
\hline$M W-4022$ & $0 / 1$ & ND & ND & NA & NA \\
\hline$M W-4023$ & $1 / 1$ & 44.70 & 44.70 & NA & NA \\
\hline
\end{tabular}

\footnotetext{
(a) Does not include QA data, duplicates and matrix spikes.

(b) The data summarized in this table and presented on maps reflect data through the first quarter of 1990 .

(c) Not detucted.

(d) Not applicable.
} 
TABLE 5.4-8 Analyses of Aluminum $(\mu \mathrm{g} / \mathrm{g})$ as Presented in Figure 5.4-9

\begin{tabular}{|c|c|c|c|c|c|}
\hline \multirow{2}{*}{$\begin{array}{l}\text { SAMPLE } \\
\text { LOCATION }\end{array}$} & \multirow{2}{*}{$\begin{array}{c}\text { DETECTI/ } \\
\text { ANALYSES(a) }\end{array}$} & \multicolumn{3}{|c|}{ VALUE PRESENTED ON MAPS ${ }^{(b)}$} & \multirow{2}{*}{$\begin{array}{l}\text { STANDARD } \\
\text { DEVIATION }\end{array}$} \\
\hline & & MINIMUM & MAXIMUM & AVERAGE & \\
\hline MW-2001 & $0 / 2$ & ND (c) & ND & NA (d) & NA \\
\hline MW-2002 & $1 / 2$ & ND & 244.00 & NA & NA \\
\hline MW-2003 & $2 / 3$ & ND & 517.00 & 396.50 & 170.41 \\
\hline MW-2004 & $0 / 2$ & ND & ND & NA & NA \\
\hline MW-2005 & $0 / 3$ & ND & ND & NA & NA \\
\hline$M W-2006$ & $0 / 2$ & ND & ND & NA & NA \\
\hline MW-2007 & $0 / 2$ & ND & ND & NA & NA \\
\hline$M W-2008$ & $0 / 2$ & ND & ND & NA & NA \\
\hline MW-2009 & $0 / 2$ & ND & ND & NA & NA \\
\hline MW-2010 & $0 / 2$ & ND & ND & NA & NA \\
\hline MW-2011 & $0 / 2$ & ND & ND & NA & NA \\
\hline$M W-2012$ & $0 / 2$ & ND & ND & NA & NA \\
\hline$M W-2013$ & $0 / 3$ & ND & ND & NA & NA \\
\hline$M W-2014$ & $0 / 2$ & ND & ND & NA & NA \\
\hline$M W \cdot 2015$ & $0 / 3$ & ND & ND & NA & NA \\
\hline$M W-2017$ & $0 / 3$ & ND & ND & NA & NA \\
\hline$M W-2018$ & $0 / 3$ & ND & ND & NA & NA \\
\hline$M W-2019$ & $0 / 1$ & ND & ND & NA & NA \\
\hline$M W-2020$ & $0 / 3$ & ND & ND & NA & NA \\
\hline MW-2021 & $0 / 1$ & ND & ND & NA & NA \\
\hline MW-2022 & $0 / 1$ & ND & ND & NA & NA \\
\hline$M W-2023$ & $0 / 1$ & ND & ND & NA & NA \\
\hline MW-2024 & $0 / 1$ & ND & ND & NA & NA \\
\hline$M W-2026$ & $0 / 1$ & ND & ND & NA & NA \\
\hline$M W-2027$ & $0 / 1$ & ND & ND & NA & NA \\
\hline$M W-2028$ & $0 / 1$ & ND & ND & NA & NA \\
\hline MW-2029 & $0 / 1$ & ND & ND & NA & NA \\
\hline$M W-3001$ & $0 / 1$ & ND & ND & NA & NA \\
\hline$M W-3002$ & $0 / 1$ & ND & ND & NA & NA \\
\hline$M W-3003$ & $0 / 1$ & ND & ND & NA & NA \\
\hline MW-3006 & $0 / 1$ & ND & ND & NA & NA \\
\hline MW-3007 & $1 / 2$ & ND & 344.00 & NA & NA \\
\hline MW-3008 & $2 / 3$ & ND & 382.00 & 305.50 & 108.19 \\
\hline$M W-3009$ & $0 / 3$ & ND & ND & NA & NA \\
\hline MW-3010 & $0 / 2$ & ND & ND & NA & NA \\
\hline$M W-3013$ & $3 / 3$ & 234.00 & 493.00 & 342.33 & 134.59 \\
\hline
\end{tabular}


TABLE 5.4-8 Analyses of Aluminum $(\mu \mathrm{g} / 1)$ as Presented in Figure 5.4-9 (Continued)

\begin{tabular}{|c|c|c|c|c|c|}
\hline \multirow{2}{*}{$\begin{array}{l}\text { SAMPLE } \\
\text { LOCATION }\end{array}$} & \multirow{2}{*}{$\begin{array}{c}\text { DETECT/ } \\
\text { ANALYSES } \\
\text { (a) }\end{array}$} & \multicolumn{3}{|c|}{ VALUE PRESENTED ON MAPS ${ }^{(b)}$} & \multirow{2}{*}{$\begin{array}{l}\text { STANDARD } \\
\text { DEVIATION }\end{array}$} \\
\hline & & MINIMUM & MAXIMUM & AVERAGE & \\
\hline$M W-3019$ & $0 / 1$ & ND & ND & NA & NA \\
\hline MW-4001 & $0 / 2$ & ND & ND & NA & NA \\
\hline$M W-4002$ & $1 / 2$ & ND & 290.00 & NA & NA \\
\hline$M W-4004$ & $0 / 1$ & ND & ND & NA & NA \\
\hline MW-4005 & $1 / 1$ & 665.00 & 665.00 & NA & NA \\
\hline$M W-4006$ & $1 / 1$ & 358.00 & 358.00 & NA & NA \\
\hline$M W-4007$ & $0 / 1$ & ND & ND & NA & NA \\
\hline MW-4008 & $0 / 1$ & ND & ND & NA & NA \\
\hline$M W-4009$ & $0 / 1$ & ND & ND & NA & NA \\
\hline$M W-4010$ & $0 / 1$ & ND & ND & NA & NA \\
\hline$M W-4011$ & $0 / 1$ & ND & ND & NA & NA \\
\hline$M W-4013$ & $0 / 1$ & ND & ND & NA & NA \\
\hline$M W-4014$ & $0 / 1$ & ND & ND & NA & NA \\
\hline$M W-4015$ & $0 / 1$ & ND & ND & NA & NA \\
\hline$M W-4016$ & $0 / 1$ & ND & ND & NA & NA \\
\hline$M W-4017$ & $0 / 1$ & ND & ND & NA & NA \\
\hline$M W-4018$ & $0 / 1$ & ND & ND & NA & NA \\
\hline$M W-4019$ & $1 / 2$ & ND & 1350.00 & NA & NA \\
\hline$M W-4020$ & $0 / 1$ & ND & ND & NA & NA \\
\hline$M W-4021$ & $0 / 1$ & ND & ND & NA & NA \\
\hline$M W-4022$ & $0 / 1$ & ND & ND & NA & NA \\
\hline$M W-4023$ & $0 / 1$ & ND & ND & NA & NA \\
\hline
\end{tabular}

\footnotetext{
(a) Does not include QA data, duplicates and matrix spikes.

(b) The data summarized in this table and presented on maps reflect data through the first quarter of 1990 .

(c) Not detected.

(d) Not applicable.
} 
TABLE 5.4-9 Analyses of 2,4,6-Trinitrotoluene $(\mu \mathrm{g} / \mathrm{l})$ as Presented in Figures 5.4-10 and 5.4-11

\begin{tabular}{|c|c|c|c|c|c|}
\hline \multirow{2}{*}{$\begin{array}{l}\text { SAMPLE } \\
\text { LOCATION }\end{array}$} & \multirow{2}{*}{$\begin{array}{c}\text { DETECTI' } \\
\text { ANALYSES }\end{array}$} & \multicolumn{3}{|c|}{ VALUE PRESENTED ON MAPS ${ }^{(b)}$} & \multirow{2}{*}{$\begin{array}{l}\text { STANDARD } \\
\text { DEVIATION }\end{array}$} \\
\hline & & MINIMUM & MAXIMUM & AVERAGE & \\
\hline MW-2001 & $0 / 10$ & ND (c) & ND & NA (d) & NA \\
\hline MW-2002 & $1 / 3$ & ND & 0.60 & NA & NA \\
\hline MW-2003 & $0 / 10$ & ND & ND & NA & NA \\
\hline MW-2004 & $0 / 10$ & ND & ND & NA & NA \\
\hline MW-2005 & $0 / 10$ & ND & ND & NA & NA \\
\hline MW-2006 & $2 / 10$ & ND & 0.78 & 0.71 & 0.11 \\
\hline MW-2007 & $0 / 9$ & ND & ND & NA & NA \\
\hline MW-2008 & $1 / 10$ & ND & 0.62 & NA & NA \\
\hline MW-2009 & $1 / 9$ & ND & 7.10 & NA & NA \\
\hline$M W-2010$ & $3 / 10$ & ND & 1.70 & 0.96 & 0.64 \\
\hline MW-2011 & $8 / 70$ & ND & 4.20 & 2.83 & 0.77 \\
\hline$M W-2012$ & $9 / 10$ & ND & 1.80 & 1.06 & 0.39 \\
\hline MW-2013 & $10 / 10$ & 2.28 & 64.90 & 22.98 & 22.91 \\
\hline$M W-2014$ & $3 / 9$ & ND & 2.33 & 1.91 & 0.38 \\
\hline$M W-2015$ & $0 / 10$ & ND & ND & NA & NA \\
\hline MW-2016 & $0 / 6$ & ND & ND & NA & NA \\
\hline$M W \cdot 2017$ & $0 / 10$ & ND & ND & NA & NA \\
\hline$M W-2018$ & $0 / 10$ & IND & ND & NA & NA \\
\hline$M W-2019$ & $0 / 6$ & ND & ND & NA & NA \\
\hline MW-2020 & $0 / 10$ & ND & ND & NA & NA \\
\hline MW-2021 & $0 / 4$ & ND & ND & NA & NA \\
\hline$M W-2022$ & $0 / 5$ & ND & ND & NA & NA \\
\hline$M W-2023$ & $0 / 6$ & ND & ND & NA & NA \\
\hline MW-2024 & $0 / 5$ & ND & ND & NA & NA \\
\hline MW.2025 & $0 / 4$ & NC & ND & NA & NA \\
\hline$M W-2026$ & $0 / 5$ & NO & ND & NA & NA \\
\hline$M W-2027$ & $0 / 5$ & ND & ND & NA & NA \\
\hline MW-2028 & $0 / 5$ & ND & ND & NA & NA \\
\hline$M W-2029$ & $0 / 5$ & ND & ND & NA & NA \\
\hline$M W-3001$ & $1 / 6$ & ND & 18.00 & NA & NA \\
\hline$M W-3002$ & $0 / 5$ & ND & ND & NA & NA \\
\hline$M W-3003$ & $0 / 5$ & ND & ND & NA & NA \\
\hline MW-3006 & $1 / 6$ & ND & 1.50 & NA & NA \\
\hline MW-3007 & $0 / 6$ & ND & ND . & NA & NA \\
\hline$M W \cdot 3008$ & $0 / 9$ & ND & ND & NA & NA \\
\hline MW-3009 & $0 / 10$ & ND & ND & NA & NA \\
\hline$M W-3010$ & $0 / 9$ & ND & ND & NA & NA \\
\hline
\end{tabular}


TABLE 5.4-9 Analyses of 2,4,6-Trinitrotoluene $(\mu \mathrm{g} / 1)$ as Presented in Figures 5.4-10 and 5.4-11 (Continued)

\begin{tabular}{|c|c|c|c|c|c|}
\hline \multirow{2}{*}{$\begin{array}{l}\text { SAMPLE } \\
\text { LOCATION }\end{array}$} & \multirow{2}{*}{$\begin{array}{c}\text { DETECT/ } \\
\text { ANALYSES } \\
\text { (a) }\end{array}$} & \multicolumn{3}{|c|}{ VALUE PRESENTED ON MAPS ${ }^{(b)}$} & \multirow{2}{*}{$\begin{array}{l}\text { STANDARD } \\
\text { DEVIATION }\end{array}$} \\
\hline & & MINIMUM & MAXIMUM & AVERAGE & \\
\hline MW-3013 & $0 / 9$ & ND & ND & NA & NA \\
\hline MW-3018 & $3 / 5$ & ND & 27.50 & 13.50 & 12.12 \\
\hline$M W-3019$ & $0 / 4$ & ND & ND & NA & NA \\
\hline$M W-4001$ & $5 / 6$ & ND & 1.75 & 1.50 & 0.22 \\
\hline$M W-4002$ & $4 / 7$ & ND & 2.70 & 1.65 & 0.99 \\
\hline$M W-4003$ & $0 / 5$ & ND & ND & NA & NA \\
\hline$M W-4004$ & $0 / 6$ & ND & ND & NA & NA \\
\hline$M W-4005$ & $0 / 6$ & ND & ND & NA & NA \\
\hline$M W-4006$ & $1 / 6$ & ND & 1.20 & NA & NA \\
\hline MW-4007 & $0 / 5$ & ND & ND & NA & NA \\
\hline$M W-4008$ & $0 / 5$ & ND & ND & NA & NA \\
\hline MW-4009 & $1 / 5$ & ND & 7.77 & NA & NA \\
\hline$M W-4010$ & $0 / 5$ & ND & ND & NA & NA \\
\hline$M W-4011$ & $0 / 5$ & ND & ND & NA & NA \\
\hline$M W-4012$ & $0 / 5$ & ND & ND & NA & NA \\
\hline$M W \cdot 4013$ & $0 / 5$ & ND & ND & NA & NA \\
\hline$M W-4014$ & $0 / 5$ & ND & ND & NA & NA \\
\hline$M W-4015$ & $0 / 5$ & ND & ND & NA & NA \\
\hline$M W-4016$ & $0 / 5$ & ND & ND & NA & NA \\
\hline$M W-4017$ & $0 / 5$ & ND & ND & NA & NA \\
\hline$M W-4018$ & $0 / 5$ & ND & ND & NA & NA \\
\hline MW.4019 & $0 / 9$ & ND & ND & NA & NA \\
\hline$M W \cdot 4020$ & $0 / 5$ & ND & ND & NA & NA \\
\hline$M W-4021$ & $0 / 7$ & ND & ND & NA & NA \\
\hline$M W-4022$ & $0 / 5$ & ND & ND & NA & NA \\
\hline$M W-4023$ & $0 / 6$ & ND & ND & NA & NA \\
\hline
\end{tabular}

\footnotetext{
(a) Does not include QA data, duplicates and matrix spikes.

(b) The data summarized in this table and presented on maps reflect data through the first quarter of 1990 .

(c) Not detected.

(d) Not applicable.
} 
TABLE 5.4-10 Analyses of 1,3,5-Trinitrobenzene $(\mu \mathrm{g} / \mathrm{ll})$ as Presented in Figures 5.4-12 and 5.4-13

\begin{tabular}{|c|c|c|c|c|c|}
\hline \multirow{2}{*}{$\begin{array}{l}\text { SAMPLE } \\
\text { LOCATION }\end{array}$} & \multirow{2}{*}{$\begin{array}{c}\text { DETECT/ } \\
\text { ANALYSES }\end{array}$} & \multicolumn{3}{|c|}{ VALUE PRESENTED ON MAPS $(b)$} & \multirow{2}{*}{$\begin{array}{l}\text { STANDARD } \\
\text { DEVIATION }\end{array}$} \\
\hline & & MINIMUM & MAXIMUM & AVERAGE & \\
\hline MW-2001 & $2 / 10$ & $N D^{(c)}$ & 0.05 & 0.05 & 0.00 \\
\hline$M W \cdot 2002$ & $1 / 3$ & ND & 0.02 & NA ${ }^{(d)}$ & NA \\
\hline MW-2003 & $1 / 10$ & ND & 0.06 & NA & NA \\
\hline$M W-2004$ & $1 / 10$ & ND & 0.14 & NA & NA \\
\hline MW-2005 & $2 / 10$ & ND & 0.18 & 0.14 & 0.06 \\
\hline MW-2006 & $10 / 10$ & 2.60 & 16.00 & 7.69 & 4.63 \\
\hline MW-2007 & $1 / 9$ & ND & 0.13 & NA & NA \\
\hline MW-2008 & $6 / 11$ & ND & 1.51 & 0.79 & 0.51 \\
\hline$M W-2009$ & $2 / 9$ & ND & 0.78 & 0.48 & 0.42 \\
\hline MW-2010 & $3 / 10$ & ND & 0.40 & 0.26 & 0.18 \\
\hline MW-2011 & $4 / 10$ & ND & 0.55 & 0.40 & 0.20 \\
\hline MW-2012 & $7 / 9$ & ND & 4.56 & 1.47 & 1.41 \\
\hline$M W \cdot 2013$ & $10 / 10$ & 2.30 & 35.10 & 12.58 & 11.40 \\
\hline MW-2014 & $8 / 9$ & ND & 4.76 & 1.56 & 1.64 \\
\hline MW-2015 & $1 / 10$ & ND & 0.21 & NA & NA \\
\hline MW-2016 & $0 / 6$ & ND & ND & NA & NA \\
\hline$M W \cdot 2017$ & $2 / 10$ & ND & 0.13 & 0.12 & 0.01 \\
\hline MW-2018 & $2 / 10$ & ND & 0.21 & 0.14 & 0.11 \\
\hline$M W-2019$ & $3 / 6$ & ND & 0.15 & 0.09 & 0.06 \\
\hline$M W-2020$ & $0 / 10$ & ND & ND & NA & NA \\
\hline MW-2021 & $0 / 4$ & ND & ND & NA & NA \\
\hline$M W \cdot 2022$ & $2 / 5$ & ND & 0.21 & 0.12 & 0.13 \\
\hline$M W \cdot 2023$ & $1 / 6$ & ND & 0.32 & NA & NA \\
\hline$M W-2024$ & $2 / 5$ & ND & 0.07 & 0.04 & 0.03 \\
\hline$M W \cdot 2025$ & $0 / 4$ & ND & ND & NA & NA \\
\hline$M W-2026$ & $1 / 5$ & ND & 0.04 & NA & NA \\
\hline MW-2027 & $0 / 5$ & ND & ND & NA & NA \\
\hline$M W-2028$ & $1 / 5$ & ND & 0.03 & NA & NA \\
\hline$M W-2029$ & $0 / 5$ & ND & ND & NA & NA \\
\hline$M W-3001$ & $4 / 5$ & ND & 0.17 & 0.12 & 0.07 \\
\hline$M W-3002$ & $2 / 5$ & ND & 0.53 & 0.29 & 0.34 \\
\hline MW-3003 & $0 / 5$ & ND & ND & NA & NA \\
\hline MW-3006 & $3 / 6$ & ND & 0.63 & 0.29 & 0.30 \\
\hline$M W-3007$ & $1 / 6$ & ND & 0.10 & NA & NA \\
\hline$M W \cdot 3008$ & $1 / 9$ & ND & 0.06 & NA & NA \\
\hline
\end{tabular}


TABLE 5.4-10 Analyses of 1,3,5-Trinitrobenzene $(\mu \mathrm{g} / \mathrm{l})$ as Presented in Figures 5.4-12 and 5.4-13 (Continued)

\begin{tabular}{|c|c|c|c|c|c|}
\hline \multirow{2}{*}{$\begin{array}{l}\text { SAMPLE } \\
\text { LOCATION }\end{array}$} & \multirow{2}{*}{$\begin{array}{c}\text { DETECT/ } \\
\text { ANALYSES }^{(0)}\end{array}$} & \multicolumn{3}{|c|}{ VALUE PRESENTED ON MAPS ${ }^{(b)}$} & \multirow{2}{*}{$\begin{array}{l}\text { STANDARD } \\
\text { DEVIATION }\end{array}$} \\
\hline & & MINIMUM & MAXIMUM & AVERAGE & \\
\hline MW-3009 & $3 / 10$ & ND & 0.22 & 0.11 & 0.10 \\
\hline$M W-3010$ & $0 / 9$ & ND & ND & NA & NA \\
\hline$M W-3013$ & $0 / 9$ & ND & ND & NA & NA \\
\hline$M W-3018$ & $1 / 5$ & ND & 3.60 & NA & NA \\
\hline MW-3019 & $1 / 4$ & ND & 0.04 & NA & NA \\
\hline MW-4001 & $7 / 7$ & 10.70 & 60.50 & 31.47 & 22.23 \\
\hline$M W-4002$ & $2 / 6$ & ND & 0.30 & 0.16 & 0.20 \\
\hline MW-4003 & $1 / 5$ & ND & 0.05 & NA & NA \\
\hline$M W-4004$ & $0 / 6$ & ND & ND & NA & NA \\
\hline MW-4005 & $2 / 6$ & ND & 0.18 & 0.10 & 0.11 \\
\hline$M W-4006$ & $6 / 6$ & 1.30 & 17.30 & 6.77 & 6.76 \\
\hline MW-4007 & $2 / 5$ & ND & 0.17 & 0.10 & 0.11 \\
\hline$M W-4008$ & $0 / 5$ & ND & ND & NA & NA \\
\hline$M W-4009$ & $2 / 5$ & ND & 16.50 & 8.39 & 11.48 \\
\hline$M W-4010$ & $1 / 6$ & ND & 0.05 & NA & NA \\
\hline$M W-4011$ & $0 / 5$ & ND & ND & NA & NA \\
\hline MW-4012 & $0 / 5$ & ND & ND & NA & NA \\
\hline$M W-4013$ & $4 / 5$ & ND & 89.00 & 51.58 & 25.43 \\
\hline MW-4014 & $2 / 5$ & ND & 0.38 & 0.33 & 0.08 \\
\hline$M W-4015$ & $3 / 5$ & ND & 0.13 & 0.12 & 0.02 \\
\hline$M W-4016$ & $0 / 5$ & ND & ND & NA & NA \\
\hline$M W-4017$ & $1 / 5$ & ND & 0.05 & NA & NA \\
\hline$M W-4018$ & $1 / 5$ & ND & 0.13 & NA & NA \\
\hline$M W-4019$ & $2 / 9$ & ND & 0.08 & 0.08 & 0.00 \\
\hline$M W-4020$ & $1 / 5$ & ND & 0.11 & NA & NA \\
\hline$M W-4021$ & $3 / 8$ & ND & 0.84 & 0.34 & 0.43 \\
\hline$M W-4022$ & $1 / 5$ & ND & 0.06 & NA & NA \\
\hline$M W \cdot 4023$ & $2 / 6$ & ND & 0.29 & 0.19 & 0.15 \\
\hline Does & ude QA data, dur & tes and matrir & & & \\
\hline The d & marized in this t & and presentec & s reflect data & the first quar & 1990. \\
\hline Not d & & & & & \\
\hline Not a & & & & & \\
\hline
\end{tabular}


TABLE 5.4-11 Analyses of 2,4-Dinitrotoluene $(\mu \mathrm{g} / 1)$ as Presented in Figures 5.4-14 and 5.4-15

\begin{tabular}{|c|c|c|c|c|c|}
\hline \multirow{2}{*}{$\begin{array}{l}\text { SAMPLE } \\
\text { LOCATION }\end{array}$} & \multirow{2}{*}{$\begin{array}{c}\text { CETECTI' } \\
\text { ANALYSES }\end{array}$} & \multicolumn{3}{|c|}{ VALUE PRESENTED ON MAPS ${ }^{|b|}$} & \multirow{2}{*}{$\begin{array}{l}\text { STANDARD } \\
\text { DEVIATION }\end{array}$} \\
\hline & & MINIMUM & MAXIMUM & AVERAGE & \\
\hline$M W-2001$ & $1 / 10$ & ND (c) & 2.10 & NA (d) & NA \\
\hline MW-2002 & $0 / 3$ & ND & ND & NA & NA \\
\hline MW-2003 & $8 / 10$ & ND & 0.60 & 0.45 & 0.15 \\
\hline$M W-2004$ & $0 / 10$ & ND & ND & NA & NA \\
\hline MW-2005 & $2 / 10$ & ND & 0.40 & 0.24 & 0.23 \\
\hline MW-2006 & $2 / 10$ & ND & 3.70 & 2.14 & 2.21 \\
\hline MW-2007 & $1 / 9$ & ND & 0.30 & NA & NA \\
\hline MW-2008 & $2 / 10$ & ND & 0.24 & 0.19 & 0.08 \\
\hline MW-2009 & $3 / 9$ & ND & 134.00 & 44.90 & 77.16 \\
\hline MW-2010 & $1 / 10$ & ND & 0.30 & NA & NA \\
\hline$M W-2011$ & $2 / 10$ & ND & 3.53 & 2.87 & 0.94 \\
\hline$M W-2012$ & $5 / 10$ & ND & 7.60 & 4.18 & 3.34 \\
\hline$M W-2013$ & 9/9 & 43.10 & 388.00 & 163.40 & 119.67 \\
\hline$M W-2014$ & $2 / 9$ & ND & 1.10 & 0.74 & 0.52 \\
\hline$M W-2015$ & $0 / 10$ & ND & ND & NA & NA \\
\hline$M W-2016$ & $0 / 6$ & ND & ND & NA & NA \\
\hline MW-2017 & $1 / 10$ & ND & 0.40 & NA & NA \\
\hline$M W-2018$ & $0 / 10$ & ND & ND & NA & NA \\
\hline$M W-2019$ & $0 / 5$ & ND & ND & NA & NA \\
\hline$M W-2020$ & $0 / 10$ & ND & ND & NA & NA \\
\hline$M W-2021$ & $0 / 4$ & ND & ND & NA & NA \\
\hline$M W-2022$ & $0 / 5$ & ND & ND & NA & NA \\
\hline$M W-2023$ & $0 / 6$ & ND & ND & NA & NA \\
\hline$M W-2024$ & $0 / 5$ & ND & ND & NA & NA \\
\hline$M W-2025$ & $0 / 4$ & ND & ND & NA & NA \\
\hline$M W-2026$ & $0 / 5$ & ND & ND & NA & NA \\
\hline$M W-2027$ & $0 / 5$ & ND & ND & NA & NA \\
\hline MW-2028 & $0 / 5$ & ND & ND & NA & NA \\
\hline$M W-2029$ & $1 / 5$ & ND & 0.59 & NA & NA \\
\hline$M W-3001$ & $4 / 6$ & ND & 2.20 & 0.95 & 0.86 \\
\hline$M W-3002$ & $0 / 5$ & ND & ND & NA & NA \\
\hline MW-3003 & $0 / 5$ & ND & ND & NA & NA \\
\hline MW-3006 & $1 / 6$ & ND & 0.58 & NA & NA \\
\hline MW-3007 & $4 / 6$ & ND & 1.80 & 1.41 & 0.38 \\
\hline$M W-3008$ & $3 / 9$ & ND & 0.63 & 0.36 & 0.29 \\
\hline MW-3009 & $3 / 10$ & ND & 0.60 & 0.39 & 0.28 \\
\hline$M W-3010$ & $0 / 9$ & ND & ND & NA & NA \\
\hline
\end{tabular}


TABLE 5.4-11 Analyses of 2,4-Dinitrotoluene $(\mu \mathrm{g} / 1)$ as Presented in Figures 5.4-14 and 5.4-15 (Continued)

\begin{tabular}{|c|c|c|c|c|c|}
\hline \multirow{2}{*}{$\begin{array}{l}\text { SAMPLE } \\
\text { LOCATION }\end{array}$} & \multirow{2}{*}{$\begin{array}{c}\text { DETECT/ } \\
\text { ANALYSES }^{(a)}\end{array}$} & \multicolumn{3}{|c|}{ VALUE PRESENTED ON MAPS ${ }^{(b)}$} & \multirow{2}{*}{$\begin{array}{l}\text { STANDARD } \\
\text { DEVIATION }\end{array}$} \\
\hline & & MINIMUM & MAXIMUM & AVERAGE & \\
\hline$M W-3013$ & $1 / 9$ & ND & 0.30 & NA & NA \\
\hline$M W-3018$ & $3 / 5$ & ND & 83.90 & 70.87 & 22.57 \\
\hline MW-3019 & $0 / 4$ & ND & ND & NA & NA \\
\hline$M W-4001$ & $7 / 7$ & 0.85 & 3.27 & 2.20 & 0.90 \\
\hline$M W-4002$ & $3 / 7$ & ND & 0.70 & 0.39 & 0.27 \\
\hline$M W-4003$ & $1 / 5$ & ND & 0.30 & NA & NA \\
\hline MW-4004 & $0 / 6$ & ND & ND & NA & NA \\
\hline$M W-4005$ & $0 / 6$ & ND & ND & NA & NA \\
\hline$M W-4006$ & $3 / 6$ & ND & 0.70 & 0.49 & 0.19 \\
\hline$M W-4007$ & 0,5 & ND & ND & NA & NA \\
\hline$M W-4008$ & $0 / 5$ & ND & ND & NA & NA \\
\hline$M W-4009$ & $0 / 5$ & ND & ND & NA & NA \\
\hline$M W-4010$ & $0 / 5$ & ND & ND & NA & NA \\
\hline$M W-4011$ & $0 / 5$ & ND & ND & NA & NA \\
\hline$M W-4012$ & $0 / 5$ & ND & ND & NA & NA \\
\hline$M W \cdot 4013$ & $0 / 5$ & ND & ND & NA & NA \\
\hline$M W-4014$ & $0 / 5$ & ND & ND & NA & NA \\
\hline$M W-4015$ & $0 / 5$ & ND & ND & NA & NA \\
\hline$M W-4016$ & $0 / 5$ & ND & ND & NA & NA \\
\hline MW-4017 & $0 / 5$ & ND & ND & NA & NA \\
\hline$M W-4018$ & $0 / 5$ & ND & ND & NA & NA \\
\hline$M W-4019$ & $1 / 9$ & ND & 0.58 & NA & NA \\
\hline$M W-4020$ & $0 / 5$ & ND & ND & NA & NA \\
\hline MW-4021 & $0 / 7$ & ND & ND & NA & NA \\
\hline$M W-4022$ & $0 / 5$ & ND & ND & NA & NA \\
\hline$M W-4023$ & $1 / 6$ & ND & 0.06 & NA & NA \\
\hline
\end{tabular}

\footnotetext{
(a) Does not include QA data, duplicates and matrix spikes.

(b) The data summarized in this table and presented on maps reflect data through the first quarter of 1990.

(c) Not detected.

(d) Not applicable.
} 
TABLE 5.4-12 Analyses of 2,6-Dinitrotoluene $(\mu \mathrm{g} / 1)$ as Presented in Figures 5.4-16 and 5.4-17

\begin{tabular}{|c|c|c|c|c|c|}
\hline \multirow{2}{*}{$\begin{array}{l}\text { SAMPLE } \\
\text { LOCATION }\end{array}$} & \multirow{2}{*}{$\begin{array}{c}\text { DETECT/ } \\
\text { ANALYSES }^{(a)}\end{array}$} & \multicolumn{3}{|c|}{ VALUE PRESENTED ON MAPS ${ }^{|b|}$} & \multirow{2}{*}{$\begin{array}{l}\text { STANDARD } \\
\text { DEVIATION }\end{array}$} \\
\hline & & MINIMUM & MAXIMUM & AVERAGE & \\
\hline MW-2001 & $2 / 10$ & ND (c) & 23.70 & 13.05 & 15.06 \\
\hline MW-2002 & $1 / 3$ & ND & 0.46 & NA (d) & NA \\
\hline MW-2003 & $10 / 10$ & 0.61 & 25.00 & 3.21 & 7.66 \\
\hline MW-2004 & $1 / 10$ & ND & 20.60 & NA & NA \\
\hline MW-2005 & $1 / 10$ & ND & 0.90 & NA & NA \\
\hline MW-2006 & $9 / 10$ & ND & 50.10 & 10.91 & 15.52 \\
\hline MW-2007 & $0 / 9$ & ND & ND & NA & NA \\
\hline MW-2008 & $4 / 10$ & ND & 1.21 & 1.02 & 0.14 \\
\hline MW-2009 & $4 / 8$ & ND & 0.90 & 0.61 & 0.30 \\
\hline MW-2010 & $4 / 10$ & ND & 12.90 & 3.87 & 6.02 \\
\hline MW-2011 & $9 / 10$ & ND & 73.30 & 19.66 & 24.67 \\
\hline$M W-2012$ & $2 / 10$ & ND & 7.52 & 4.88 & 3.74 \\
\hline$M W-2013$ & $10 / 10$ & 7.83 & 290.00 & 101.46 & 95.60 \\
\hline$M W-2014$ & $5 / 9$ & ND & 1.66 & 1.16 & 0.39 \\
\hline$M W-2015$ & $1 / 9$ & ND & 0.62 & NA & NA \\
\hline$M W-2016$ & $1 / 6$ & ND & 1.00 & NA & NA \\
\hline MW-2017 & $0 / 10$ & ND & ND & NA & NA \\
\hline$M W-2018$ & $1 / 10$ & ND & 16.40 & NA & NA \\
\hline MW-2019 & $0 / 6$ & ND & ND & NA & NA \\
\hline$M W \cdot 2020$ & $0 / 10$ & ND & ND & NA & NA \\
\hline MW-2021 & $0 / 4$ & ND & ND & NA & NA \\
\hline MW-2022 & $0 / 5$ & ND & ND & NA & NA \\
\hline$M W-2023$ & $0 / 6$ & ND & ND & NA & NA \\
\hline$M W-2024$ & $0 / 5$ & ND & ND & NA & NA \\
\hline MW-2025 & $0 / 4$ & ND & ND & NA & NA \\
\hline$M W-2026$ & $0 / 5$ & ND & ND & NA & NA \\
\hline$M W-2027$ & $0 / 5$ & ND & ND & NA & NA \\
\hline$M W-2028$ & $0 / 5$ & ND & ND & NA & NA \\
\hline MW-2029 & $0 / 5$ & ND & ND & NA & NA \\
\hline MW-3001 & $1 / 6$ & ND & 2.90 & NA & NA \\
\hline MW-3002 & $0 / 5$ & ND & ND & NA & NA \\
\hline MW-3003 & $0 / 5$ & ND & ND & NA & NA \\
\hline MW-3006 & $1 / 6$ & ND & 2.90 & NA & NA \\
\hline MW-3007 & $5 / 6$ & ND & 3.30 & 2.32 & 0.68 \\
\hline$M W-3008$ & $0 / 9$ & ND & ND & NA & NA \\
\hline MW-3009 & $0 / 10$ & ND & ND & NA & NA \\
\hline MW-3010 & $0 / 9$ & ND & ND & NA & NA \\
\hline
\end{tabular}


TABLE 5.4-12 Analyses of 2,6-Dinitrotoluene $(\mu \mathrm{g} / \mathrm{l})$ as Presented in Figures 5.4-16 and 5.4-17 (Continued)

\begin{tabular}{|c|c|c|c|c|c|}
\hline \multirow{2}{*}{$\begin{array}{l}\text { SAMPLE } \\
\text { LOCATION }\end{array}$} & \multirow{2}{*}{$\begin{array}{c}\text { DETECTI } \\
\text { ANALYSES(e) }\end{array}$} & \multicolumn{3}{|c|}{ VALUE PRESENTED ON MAPS ${ }^{(b)}$} & \multirow{2}{*}{$\begin{array}{l}\text { STANDAR } \\
\text { DEVIATIO }\end{array}$} \\
\hline & & MINIMUM & MAXIMUM & AVERAGE & \\
\hline$M W-3013$ & $1 / 9$ & ND & 0.62 & NA & NA \\
\hline$M W \cdot 3018$ & $4 / 5$ & ND & 1150.00 & 751.75 & 296.29 \\
\hline MW-3019 & $0 / 4$ & ND & ND & NA & NA \\
\hline MW-4001 & $6 / 7$ & ND & 4.20 & 3.35 & 0.80 \\
\hline$M W-40 C 2$ & $2 / 7$ & ND & 1.30 & 1.13 & 0.25 \\
\hline MW-4003 & $1 / 5$ & ND & 0.80 & NA & NA \\
\hline MW-4004 & $1 / 6$ & ND & 0.94 & NA & NA \\
\hline MW.4005 & $0 / 6$ & ND & ND & NA & NA \\
\hline$M W-4006$ & $5 / 6$ & ND & 7.22 & 4.14 & 1.82 \\
\hline MW.4007 & $0 / 5$ & ND & ND & NA & NA \\
\hline MW-4008 & $0 / 5$ & ND & ND & NA & NA \\
\hline MW-4009 & $1 / 5$ & ND & 1.70 & NA & NA \\
\hline$M W-4010$ & $0 / 5$ & ND & ND & NA & NA \\
\hline$M W \cdot 4011$ & $0 / 5$ & ND & ND & NA & NA \\
\hline$M W-4012$ & $0 / 5$ & ND & ND & NA & NA \\
\hline$M W-4013$ & $3 / 5$ & ND & 1.21 & 1.00 & 0.28 \\
\hline$M W-4014$ & $0 / 5$ & ND & ND & NA & NA \\
\hline$M W \cdot 4015$ & $4 / 5$ & ND & 6.01 & 2.52 & 2.38 \\
\hline$M W \cdot 4016$ & $0 / 5$ & ND & ND & NA & NA \\
\hline MW-4017 & $0 / 5$ & ND & ND & NA & NA \\
\hline$M W \cdot 4018$ & $0 / 5$ & ND & ND & NA & NA \\
\hline$M W-4019$ & $0 / 9$ & ND & ND & NA & NA \\
\hline$M W \cdot 4020$ & $0 / 5$ & ND & ND & NA & NA \\
\hline MW-4021 & $0 / 7$ & ND & ND & NA & NA \\
\hline MW-4022 & $0 / 5$ & ND & ND & NA & NA \\
\hline$M W-4023$ & $1 / 6$ & ND & 0.02 & NA & NA \\
\hline
\end{tabular}

\footnotetext{
(a) Does not include QA data, duplicates and matrix spikes.

(b) The data summarized in this table and presented on maps reflect data through the first quarter of 1990.

(c) Not detected

(d) Not applicable
} 
TABLE 5.4-13 Analyses of 1,3-Dinitrobenzene $(\mu \mathrm{g} / 1)$ as Presented in Figures 5.4-18 and 5.4-19

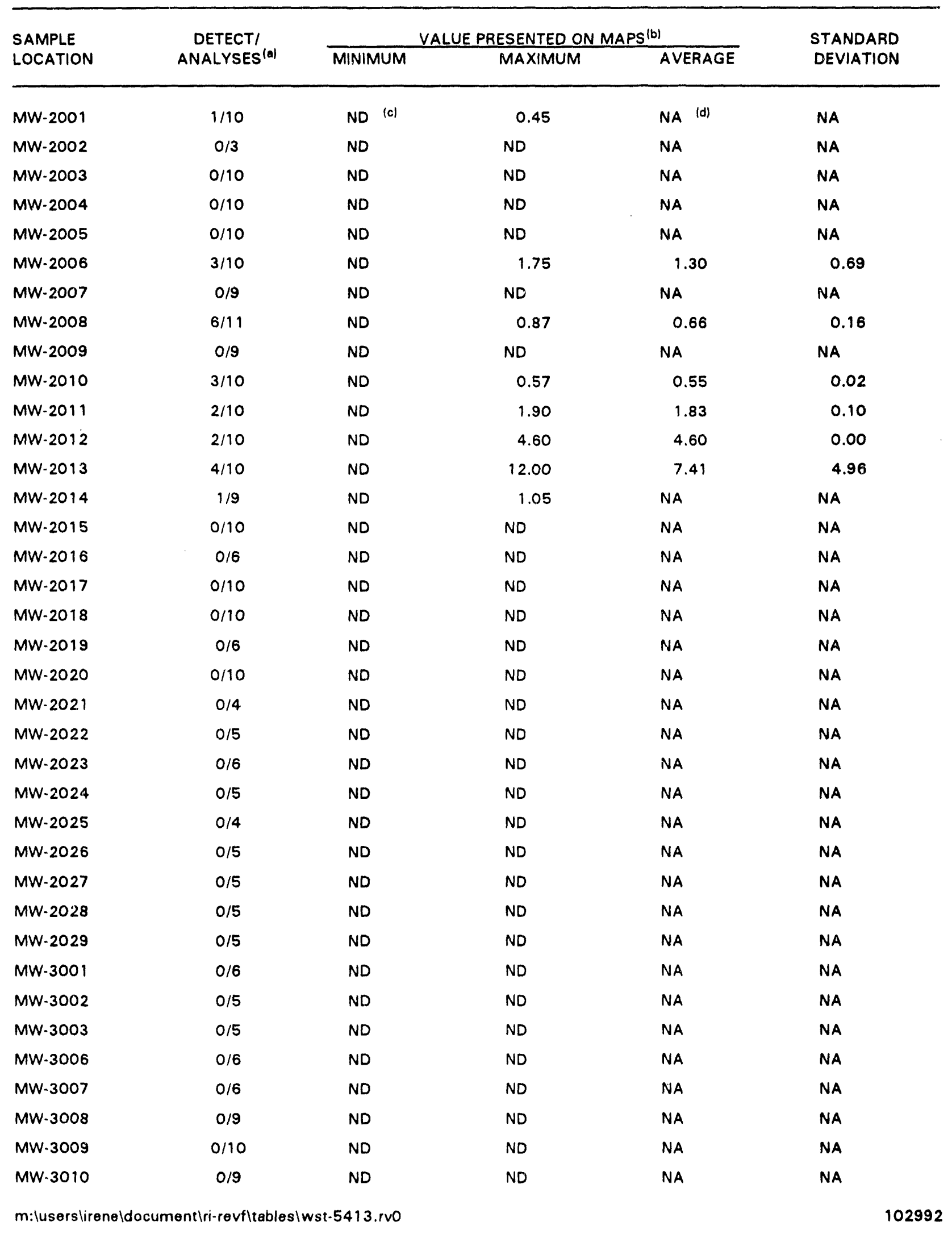


TABLE 5.4-13 Analyses of 1,3-Dinitrobenzene $(\mu \mathrm{g} / 1)$ as Presented in Figures 5.4-18 and 5.4-19 (Continued)

\begin{tabular}{|c|c|c|c|c|c|}
\hline \multirow{2}{*}{$\begin{array}{l}\text { SAMPLE } \\
\text { LOCATION }\end{array}$} & \multirow{2}{*}{$\begin{array}{c}\text { DETECT/ } \\
\text { ANALYSES }^{(8)}\end{array}$} & \multicolumn{3}{|c|}{ VALUE PRESENTED ON MAPS ${ }^{(b)}$} & \multirow{2}{*}{$\begin{array}{l}\text { STANDARD } \\
\text { DEVIATION }\end{array}$} \\
\hline & & MINIMUM & MAXIMUM & AVERAGE & \\
\hline$M W-3013$ & $0 / 9$ & ND & ND & NA & NA \\
\hline MW-3018 & $1 / 5$ & ND & 1.50 & NA & NA \\
\hline MW-3019 & $0 / 4$ & ND & ND & NA & NA \\
\hline$M W-4001$ & $5 / 7$ & ND & 5.14 & 3.13 & 1.29 \\
\hline MW-4002 & $0 / 7$ & ND & ND & NA & NA \\
\hline$M W-4003$ & $1 / 5$ & ND & 0.14 & NA & NA \\
\hline MW-4004 & $0 / 6$ & ND & ND & NA & NA \\
\hline MW-4005 & $0 / 6$ & ND & ND & NA & NA \\
\hline MW-4006 & $4 / 6$ & ND & 3.20 & 2.24 & 0.81 \\
\hline$M W-4007$ & $0 / 5$ & ND & ND & NA & NA \\
\hline$M W-4008$ & $0 / 5$ & ND & ND & NA & NA \\
\hline MW-4009 & $0 / 5$ & ND & ND & NA & NA \\
\hline$M W-4010$ & $0 / 6$ & ND & ND & NA & NA \\
\hline$M W-4011$ & $0 / 5$ & ND & ND & NA & NA \\
\hline$M W-4012$ & $0 / 5$ & NO & ND & NA & NA \\
\hline$M W-4013$ & $2 / 5$ & ND & 0.80 & 0.76 & 0.06 \\
\hline$M W-4014$ & $0 / 5$ & ND & ND & NA & NA \\
\hline$M W-4015$ & $2 / 5$ & ND & 0.66 & 0.46 & 0.29 \\
\hline$M W-4016$ & $0 / 5$ & ND & ND & NA & NA \\
\hline$M W-4017$ & $0 / 5$ & ND & ND & NA & NA \\
\hline$M W-4018$ & $0 / 5$ & ND & ND & NA & NA \\
\hline$M W-4019$ & $0 / 9$ & ND & ND & NA & NA \\
\hline$M W-4020$ & $0 / 5$ & ND & ND & NA & NA \\
\hline$M W-4021$ & $0 / 7$ & ND & ND & NA & NA \\
\hline$M W-4022$ & $0 / 5$ & ND & ND & NA & NA \\
\hline$M W-4023$ & $0 / 6$ & ND & ND & NA & NA \\
\hline
\end{tabular}

\footnotetext{
(a) Does not include QA data, duplicates and matrix spikes.

(b) The data summarized in this table and presented on maps reflect data through the first quarter of 1990.

(c) Not detected.

(d) Not applicable.
} 
TABLE 5.4-14 Analyses of Nitrobenzene $(\mu \mathrm{g} / 1)$ as Presented in Figures 5.4-20 and 5.4-21

\begin{tabular}{|c|c|c|c|c|c|}
\hline \multirow{2}{*}{$\begin{array}{l}\text { SAMPLE } \\
\text { LOCATION }\end{array}$} & \multirow{2}{*}{$\begin{array}{c}\text { DETECTI' } \\
\text { ANALYSES }^{(a)}\end{array}$} & \multicolumn{3}{|c|}{ VALUE PRESENTED ON MAPS(b) } & \multirow{2}{*}{$\begin{array}{l}\text { STANDARD } \\
\text { DEVIATION }\end{array}$} \\
\hline & & MINIMUM & MAXIMUM & AVERAGE & \\
\hline MW-2001 & $1 / 10$ & ND $\quad(c)$ & 1.00 & $N A$ (d) & NA \\
\hline MW-2002 & $0 / 3$ & ND & ND & NA & NA \\
\hline$M W-2003$ & $0 / 10$ & ND & ND & NA & NA \\
\hline MW.2004 & $0 / 10$ & ND & ND & NA & NA \\
\hline MW-2005 & $0 / 10$ & ND & ND & NA & NA \\
\hline MW-2006 & $3 / 10$ & ND & 8.30 & 5.20 & 3.37 \\
\hline MW-2007 & $0 / 9$ & ND & ND & NA & NA \\
\hline$M W-2008$ & $1 / 10$ & ND & 1.95 & NA & NA \\
\hline MW.2009 & $1 / 9$ & ND & 22.20 & NA & NA \\
\hline MW-2010 & $0 / 10$ & ND & ND & NA & NA \\
\hline MW-2011 & $0 / 10$ & ND & ND & NA & NA \\
\hline$M W-2012$ & $1 / 10$ & ND & 1.22 & NA & NA \\
\hline$M W-2013$ & $3 / 10$ & ND & 5.04 & 3.95 & 1.86 \\
\hline MW-2014 & 1/9 & ND & 4.00 & NA & NA \\
\hline$M W-2015$ & $0 / 10$ & ND & ND & NA & NA \\
\hline MW-2016 & $0 / 6$ & ND & ND & NA & NA \\
\hline MW-2017 & $0 / 10$ & ND & ND & NA & NA \\
\hline$M W-2018$ & $0 / 10$ & ND & ND & NA & NA \\
\hline MW-2019 & $0 / 6$ & ND & ND & NA & NA \\
\hline MW.2020 & $1 / 10$ & ND & 1.70 & NA & NA \\
\hline$M W-2021$ & $0 / 4$ & ND & ND & NA & NA \\
\hline$M W-2022$ & $2 / 5$ & ND & 6.00 & 3.70 & 3.26 \\
\hline$M W-2023$ & $0 / 6$ & ND & ND & NA & NA \\
\hline MW-2024 & $0 / 5$ & ND & ND & NA & NA \\
\hline$M W-2025$ & $0 / 4$ & ND & ND & NA & NA \\
\hline$M W-2026$ & $0 / 5$ & ND & ND & NA & NA \\
\hline MW-2027 & $1 / 5$ & ND & 1.46 & NA & NA \\
\hline$M W-2028$ & $2 / 5$ & ND & 1.22 & 1.16 & 0.09 \\
\hline MW- 2029 & $0 / 5$ & ND & ND & NA & NA \\
\hline$M W-3001$ & $1 / 6$ & NO & 4.60 & NA & NA \\
\hline$M W-3002$ & $1 / 5$ & ND & 2.17 & NA & NA \\
\hline MW-3003 & $0 / 5$ & ND & ND & NA & NA \\
\hline MW-3006 & $2 / 6$ & ND & 5.28 & 3.64 & 2.32 \\
\hline MW-3007 & $0 / 6$ & ND & ND & NA & NA \\
\hline MW-3008 & $0 / 9$ & ND & ND & NA & NA \\
\hline
\end{tabular}


TABLE 5.4-14 Analyses of Nitrobenzene $(\mu \mathrm{g} / 1)$ as Presented in Figures 5.4-20 and 5.4-21 (Continued)

\begin{tabular}{|c|c|c|c|c|c|}
\hline \multirow{2}{*}{$\begin{array}{l}\text { SAMPLE } \\
\text { LOCATION }\end{array}$} & \multirow{2}{*}{$\begin{array}{c}\text { DETECTI } \\
\text { ANALYSES }\end{array}$} & \multicolumn{3}{|c|}{ VALUE PRESENTED ON MAPS $(b)$} & \multirow{2}{*}{$\begin{array}{l}\text { STANDARD } \\
\text { DEVIATION }\end{array}$} \\
\hline & & MINIMUM & MAXIMUM & AVERAGE & \\
\hline MW-3009 & $0 / 10$ & ND & ND & NA & NA \\
\hline MW-3010 & $0 / 9$ & ND & ND & NA & NA \\
\hline MW-3013 & $0 / 9$ & ND & ND & NA & NA \\
\hline MW-3018 & $3 / 5$ & ND & 31.30 & 30.30 & 0.87 \\
\hline MW.3019 & $1 / 4$ & ND & 0.86 & NA & NA \\
\hline MW-4001 & $4 / 8$ & ND & 7.64 & 5.97 & 1.44 \\
\hline MW-4002 & $0 / 7$ & ND & ND & NA & NA \\
\hline$M W-4003$ & $0 / 5$ & ND & ND & NA & NA \\
\hline$M W-4004$ & $0 / 6$ & ND & ND & NA & NA \\
\hline MW-4005 & $0 / 6$ & ND & ND & NA & NA \\
\hline$M W-4006$ & $4 / 6$ & ND & 3.71 & 3.12 & 0.52 \\
\hline MW-4007 & $0 / 5$ & ND & ND & NA & NA \\
\hline$M W-4008$ & $0 / 5$ & ND & ND & NA & NA \\
\hline MW-4009 & $0 / 5$ & ND & ND & NA & NA \\
\hline$M W-4010$ & $0 / 6$ & ND & ND & NA & NA \\
\hline MW-4011 & $0 / 5$ & ND & ND & NA & NA \\
\hline$M W-4012$ & $0 / 5$ & ND & ND & NA & NA \\
\hline$M W-4013$ & $0 / 5$ & ND & ND & NA & NA \\
\hline MW-4014 & $0 / 5$ & NO & ND & NA & NA \\
\hline$M W-4015$ & $1 / 5$ & ND & 0.41 & NA & NA \\
\hline$M W-4016$ & $0 / 5$ & ND & ND & NA & NA \\
\hline MW-4017 & $0 / 5$ & ND & ND & NA & NA \\
\hline$M W-4018$ & $0 / 5$ & ND & ND & NA & NA \\
\hline$M W-4019$ & $0 / 9$ & ND & ND & NA & NA \\
\hline$M W \cdot 4020$ & $0 / 5$ & ND & ND & NA & NA \\
\hline MW-4021 & $2 / 7$ & ND & 1.71 & 1.71 & 0.00 \\
\hline MW-4022 & $0 / 5$ & ND & ND & NA & NA \\
\hline$M W \cdot 4023$ & $0 / 6$ & ND & ND & NA & NA \\
\hline (a) Does r & include $Q A$ data, dupi & $s$ and matrix & & & \\
\hline The $d \varepsilon$ & summarized in this tat & id presented & eflect data th & e fourth qua & 1988. \\
\hline (c) Not de & ted. & & & & \\
\hline (d) Not ap & able. & & & & \\
\hline
\end{tabular}


TABLE 5.5-1 Vegetation Surveyed at the Weldon Spring Chemical Plant Area

\author{
Alfalfa \\ American elm \\ American plum \\ American sycamore \\ Beauty berry \\ Bellwort \\ Black locust \\ Box elder \\ Broad-leaved arrowhead \\ Broad-leaved cattail \\ Canada rush \\ Common cattail \\ Common dandelion \\ Common milkweed \\ Common mullein \\ Common sunflower \\ Common thistle \\ Cut-leaved water horehound \\ Daisy fleabane \\ Dodder \\ Eastern cottonwood \\ Eastern red cedar \\ Flowering dogwood \\ Goldenrod \\ Green ash \\ Ground cherry \\ Hackberry \\ Hedge bindweed \\ Hog peanut \\ Honey locust \\ Horse nettle \\ Horseweed \\ Ironweed \\ Mountain bluegrass \\ Nodding foxtail \\ Panic grass \\ Paper mulberry \\ Partridge pea \\ Pawpaw \\ Persimmon \\ Plantain \\ Poison ivy \\ Pokeweed \\ Post oak
}

Queen Anne's lace

Ragweed

Renunculus

Red clover

Red-tip grass

Redbud

Rosa sp.

Roughleaf dogwood

Sassafras

Scurfy pea

Sedge

Shagbark hickory

Shingle oak

Short-leaved pine

Skullcap

Slender nettle

Smooth hedge nettle

Smooth sumac

Spanish needles

Spotted touch-me-not

Stinging nettle

Sugar maple

Swamp smartweed

Sweetgum

Tall eriogonum (bluestem)

Tartarian honeysuckle

Thoroughwort

Tickseed sunflower

Trailing bush clover

Trumpet creeper

Virginia creeper

White avens

White sweet clover

Wild grape

Willow

Yarrow

Yellow rocket

Yellow sweet clover 


\section{TABLE 5.5-2 Bird and Waterfowl Species Observed at the Weldon Spring Chemical Plant Area}

\author{
American coot \\ American crow \\ American goldfinch \\ American kestrel \\ American robin \\ American wigeon \\ Barn swallow \\ Belted kingfisher \\ Black-capped chickadee \\ Blue jay \\ Blue-winged teal \\ Bobwhite quail \\ Bufflehead \\ Canada goose \\ Canvasback \\ Cedar waxwing \\ Common nighthawk \\ Common snipe \\ Dark-eyed junco \\ Downy woodpecker \\ Eastern bluebird \\ Eastern kingbird \\ Eastern meadowlark \\ European starling \\ Field sparrow \\ Great blue heron \\ Great crested flycatcher \\ Great-horned owl \\ Green-backed heron \\ Hairy woodpecker \\ Hooded merganser
}

\author{
House sparrow \\ Killdeer \\ Lesser scoup \\ Mallard \\ Meadowlark \\ Mockingbird \\ Mourning dove \\ Nighthawk \\ Northern cardinal \\ Northern flicker \\ Northern oriole \\ Northern shoveler \\ Nothern pintail \\ Pied-billed grebe \\ Pileated woodpecker \\ Red-headed woodpecker \\ Red-tailed hawk \\ Red-winged blackbird \\ Ring-necked duck \\ Rock dove \\ Ruby-throated hummingbird \\ Ruddy duck \\ Spotted sandpiper \\ Tufted titmouse \\ White-crowned sparrow \\ White-throated sparrow \\ Wild turkey \\ Wood duck \\ Yellow-billed cuckoo \\ Yellow-rumped warbler
}




\section{Table 5.5-3 List of Birds Observed During the Haul Road Ecological Survey June 24-28, 1991}

\begin{tabular}{|c|c|c|c|c|}
\hline Common Namo/(Scientific Name) & $\begin{array}{l}\text { Total } \\
\text { Seen }\end{array}$ & $\begin{array}{c}\text { Relative } \\
\text { Abundance }\end{array}$ & $\begin{array}{c}\text { Breeding } \\
\text { Status }\end{array}$ & $\begin{array}{c}\text { Habitat } \\
\text { Type }\end{array}$ \\
\hline Turkey vulture (Cathartes aura) & 1 & $\mathrm{co}$ & 0 & $\mathbf{F}$ \\
\hline Red-tailed hawk (Buteo jamaicensis) & 1 & $\mathbf{c a}$ & 0 & $w$ \\
\hline American kestrel (Falco sparverius) & 2 & $u$ & 0 & $w$ \\
\hline American woodcock (Scolopax minor) & 1 & ca & 0 & w \\
\hline Northern bob white (Colinus virginianus) & 15 & c & $R$ & 0 \\
\hline Rock dove (Columba livia) & 2 & u & $\mathbf{R}$ & $\mathbf{F}$ \\
\hline Mourning dove (Zenaida macroura) & 7 & c & $\mathbf{R}$ & o.w \\
\hline Yellow-billed cuckoo (Coccyzus americanus) & 9 & c & c & $w$ \\
\hline Eastern screech owl (Otus asio) & 2 & $u$ & $\mathbf{R}$ & w \\
\hline Barred owl (Strix varia) & 4 & c & $\mathbf{R}$ & $w$ \\
\hline Whip-poor-will (Caprimulgus vociferus) & 1 & $\mathrm{ca}$ & $\mathbf{R}$ & $0 . W, F$ \\
\hline Chimney swift (Chaetura pelagica) & 14 & c & $\mathbf{R}$ & $0, F$ \\
\hline Ruby-throated hummingbird (Archilochus colubris) & 1 & $\mathrm{ca}$ & 0 & 0 \\
\hline Belted kingfisher (Ceryle alcyon) & 1 & ca & 0 & $W, F$ \\
\hline Red-headed woodpecker (Melanerpes erythrocephalus) & 3 & u & $\mathbf{R}$ & $w$ \\
\hline Red-bellied woodpecker (Melanerpes carolinus) & 1 & $\mathrm{ca}$ & R & w \\
\hline Northern flicker (Colaptes auratus) & 12 & c & $R$ & w \\
\hline Downy woodpecker (Picoides pubescens) & 3 & $u$ & $\mathbf{R}$ & w \\
\hline Halry woodpecker (Picoides villosus) & 3 & $u$ & $\mathbf{R}$ & w \\
\hline Pileated woodpecker (Dryocopus pileatus) & 3 & $\mathrm{u}$ & $\mathbf{R}$ & W \\
\hline Eastern wood-pewee (Contopus virens) & 3 & $u$ & $\mathbf{R}$ & $0, W$ \\
\hline Eastern phoebe (Sayornis phoebe) & 1 & ca & $\mathbf{R}$ & $w$ \\
\hline Great crested flycatcher (Myiarchus crinitus) & 3 & u & $\mathbf{R}$ & $0, W$ \\
\hline Eastern kingbird (Tyrannus tyrannus) & 3 & $u$ & $\mathbf{R}$ & w \\
\hline Northern rough-winged swallow (Stelgidopteryx serripennis) & 5 & c & $\mathbf{R}$ & 0 \\
\hline Blue jay (Cyanocitta cristata) & 29 & $\mathrm{c}$ & $\mathbf{R}$ & $0, w$ \\
\hline American crow (Corvus brachyrhynchos) & 9 & c & $\mathbf{R}$ & $0, W$ \\
\hline Black-capped chickadee (Parus atricapillus) & 1 & $\mathbf{c o}$ & $\mathbf{R}$ & w \\
\hline Carolina chickadee (Parus carolinensis) & 5 & c & $\mathbf{R}$ & w \\
\hline Tufted titmouse (Parus bicolor) & 21 & c & $R$ & w \\
\hline
\end{tabular}


Table 5.5-3 List of Birds Observed During the Haul Road Ecological Survey June 24-28, 1991 (Continued)

\begin{tabular}{|c|c|c|c|c|}
\hline Common Name/(Scientific Name) & $\begin{array}{l}\text { Total } \\
\text { Seen }\end{array}$ & $\begin{array}{c}\text { Relative } \\
\text { Abundance }\end{array}$ & $\begin{array}{l}\text { Breoding } \\
\text { Status }\end{array}$ & $\begin{array}{c}\text { Habitat } \\
\text { Type }\end{array}$ \\
\hline White-breasted nuthatch (Sitta carolinensis) & 6 & c & $\mathbf{R}$ & W \\
\hline House wren (Troglodytes aedon) & 3 & $u$ & c & $w$ \\
\hline Blue-gray gnatcatcher (Polioptila caerulea) & 1 & ca & $\mathbf{R}$ & W \\
\hline Eastern bluebird (Sialia sialis) & 7 & c & $\mathbf{R}$ & 0 \\
\hline Wood thrush (Hylocichla mustelina) & 16 & c & $\mathbf{R}$ & $w$ \\
\hline American robin (Turdus migratorius) & 13 & c & C & $0, w$ \\
\hline Gray catbird (Dumetella carolinensis) & 4 & c & $\mathbf{R}$ & w \\
\hline Northern mockingbird (Mimus polyglottos) & 2 & u & $\mathbf{R}$ & 0 \\
\hline European starling (Sturnus vulgaris) & 5 & c & $\mathbf{R}$ & $\mathbf{F}$ \\
\hline White-eyed vireo (Vireo griseus) & 1 & $\mathrm{ca}$ & $\mathbf{R}$ & $w$ \\
\hline Red-eyed vireo (Vireo olivaceus) & 3 & u & $R$ & $w$ \\
\hline Blue-winged warbler (Vermivora pinus) & 6 & c & $\mathbf{R}$ & w \\
\hline Yellow warbler (Dendroica petechia) & 8 & c & $\mathbf{R}$ & W \\
\hline Yellow-throated warbler (Dendroica dominica) & 1 & $\mathrm{ca}$ & $\mathbf{R}$ & W \\
\hline American redstart (Setophaga ruticilla) & 1 & $\mathrm{ca}$ & $R$ & $w$ \\
\hline Ovenbird (Seiurus aurocapillus) & 4 & $c$ & $\mathbf{R}$ & w \\
\hline Kentucky warbler (Oporornis formosus) & 3 & $u$ & $\mathbf{R}$ & $w$ \\
\hline Common vellowthroat (Geoth/ypis trichas) & 4 & c & $\mathbf{R}$ & $w$ \\
\hline Yellow-breasted chat (/cteria virens) & 6 & $c$ & $\mathbf{R}$ & $0, W$ \\
\hline Summer tanager (Piranga rubra) & 15 & c & $\mathbf{R}$ & $0, W$ \\
\hline Scarlet tanager (Piranga olivacea) & 6 & $\mathrm{c}$ & $\mathbf{R}$ & $0, w$ \\
\hline Northern cardinal (Cardinalis cardinalis) & 16 & c & C & O.W \\
\hline Rose-breasted grosbeak (Pheucticus ludovicianus) & 3 & u & $\mathbf{R}$ & $0, W$ \\
\hline Blue grosbeak (Guiraca caerulea) & 2 & $u$ & $\mathbf{R}$ & w \\
\hline Indigo bunting (Passerina cyanea) & 6 & c & $\mathbf{R}$ & $0, W$ \\
\hline Rufous-sided to whee (Pipilo erythrophthalmus) & 8 & c & $\mathbf{R}$ & $0, w$ \\
\hline Chipping sparrow (Spizella passerina) & 1 & $\mathrm{ca}$ & 0 & 0 \\
\hline Field sparrow (Spizella pusilla) & 7 & c & R & 0 \\
\hline
\end{tabular}




\section{Table 5.5-3 List of Birds Observed During the Haul Road Ecological Survey June 24-28, 1991 (Continued)}

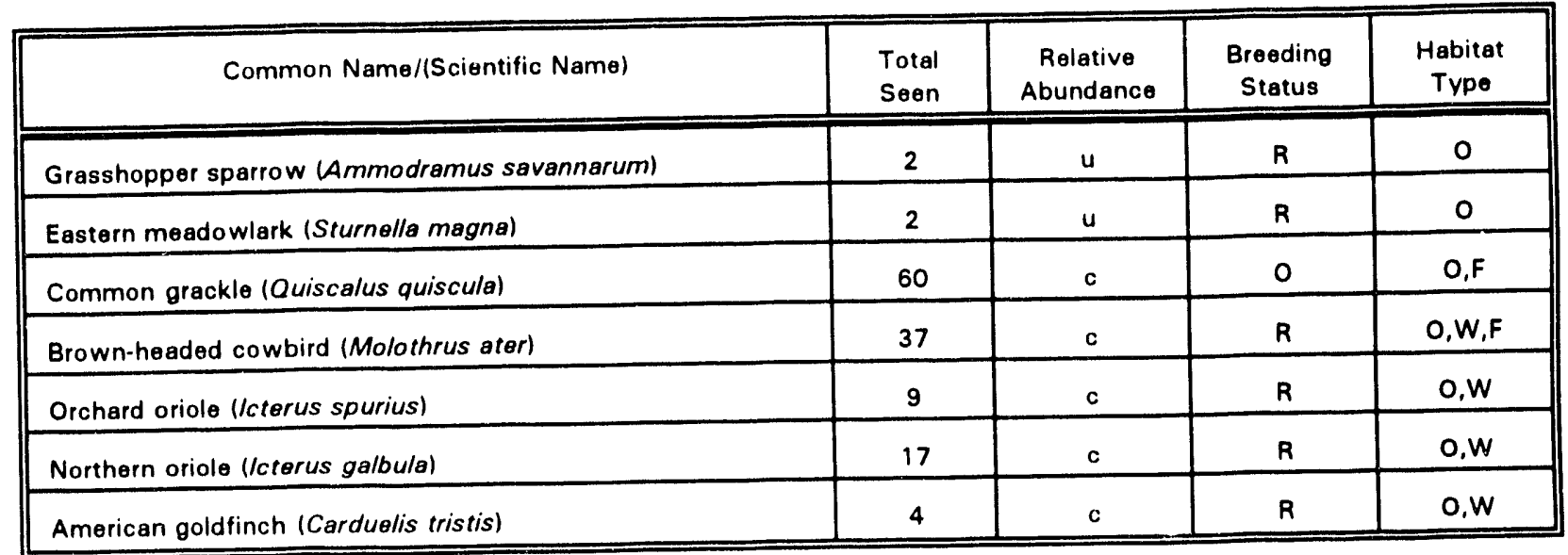

NOTES:

Abundance Status. This scale is relative only to this survey.

c $=$ common species seen or heard more than 4 times.

$u=$ uncommon species seen or heard 2 to 3 times.

ca $=$ casual species was seen or heard only once.

Breeding Status

$\mathrm{C}=$ Confirmed Breeder. Presence of an active nest, newly fledged or flightless young, adult carrving nest matter or carrying food for young.

$\mathrm{R}=$ Probable Breeder. Present during the breeding within normal range during breeding season, males

singling or defending a territory.
- Possible Breeder. Present during the breeding season within normal range but no direct evidence of breeding activity was observed.

Habitat Trpe

$W=$ Mixed deciduous woodland.

$\mathrm{O}=$ Old succassional field.

$F=$ Bird seer llying overhead, not in any one habitat. 
TABLE 5.5-4 List of Mammals Found in the Weldon Spring Wildlife Area

\begin{tabular}{ll}
\hline Common Name & Genus and Species \\
\hline Opossum & Didelphis virginiana \\
Eastern Mole & Scalopus aquaticus \\
Big Brown Bet & Eptesicus fuscus \\
Eastern Cottontail Rabbit & Sylvilagus floridanus \\
Eastern Chipmunk & Tamies striatus \\
Woodchuck & Marmota monax \\
Eestern Gray Squirrel & Sciurus carolinensis \\
Fox Squirrel & Sciurus niger \\
Southern Flying Squirrel & Glaucomys volans \\
Beaver & Castor canadensis \\
Western Harvest Mouse & Reithrodontomys megalotis \\
Deer Mouse & Peromyscus maniculetus \\
White-footed Mouse & Peromyscus leucopus \\
Prairie Vole & Microtus ochrogaster \\
Muskrat & Ondatra zibethicus \\
House Mouse & Mus musculus \\
Coyote & Canis latrans \\
Red Fox & Vulpes vulpes \\
Gray Fox & Urocyon cinereoargenteus \\
Raccoon & Proycon lotor \\
Striped Skunk & Mephitis mephitis \\
White-tailed Deer & Ococoileus virginianus \\
&
\end{tabular}

Source: Herron 1991b 
TABLE 5.5-5

List of Reptiles and Amphibians Found in the Weldon Spring Wildlife Area

Ringed Salamander

Spotted Salamander

Smallmouth Salamander

Eastern Tiger Salamender

Central Newt

Slimy Salamander

Eastern American Toad

Fowler's Toad

Blanchard's Cricket Frog

Northern Spring Peeper

Eastern Gray Troefrog

Western Chorus Frog

Plains Leopard Frog

Bullfrog

Green Frog

Pickerel Frog

Southern Leopard Frog

Wood Frog

Common Snapping Turtle

Western Painted Turtle

Three-toed Box Turile

Ornate Box Turtle

Red-eared Slider

Midland Smooth Softshell

Northern Fence Lizard

Five-lined Skink

Broadhead Skink

Ground Skink

Six-lined Racerunner

Western Slender Glass Lizard

Western Worm Snake

Eastern Yellowbelly Racer

Prairie Ringneck Snake

Black Rat Snake

Eastern Hognose Snake

Prairie Kingsnake

Speckled Kingsnake

Diamondback Water Snako

Northern Water Snake

Rough Green Snake

Midland Brown Snake

Northern Redbelly Snake

Western Ribbon Snake

Eastern Garter Snake

Western Earth Snake

Osage Copperhead

Timber Rattlesnake
Ambystoma annulatum

Ambystome maculatum

Ambystoma texanum

Ambystoma tigrinum tigrinum

Notophthalmus viridescens louisianensis

Plethodon glutinosus glutinosus

Bufo americanus americanus

Bufo woodhousei fowleri

Acris crepitans blenchardi

Hyla crucifor crucifer

Hyla versicolor

Pseudacris triseriata triseriata

Rana blairi

Rana catesbeiana

Rana clamitans melanota

Rana palustric

Rana sphenocephala

Rana sylvatica

Chelydra serpentina serpentina

Chrysemys picta bellii

Terrapene carolina triunguis

Terrapene ornata ornata

Trachemys scripta elegans

Trionyx muticus muticus

Sceloporus undulatus hyacin thinus

Eumeces fasciatus

Eumeces laticeps

Scincella lateralis

Cnemidophorus sexlineatus sexlineatus

Ophisaurus attenuatus attenuatus

Carphophis amoenus vermis

Coluber constrictor flaviventris

Diadophis punctatus arnyi

Elaphe obsolete obsoleta

Heterodon platyrhinos

Lampropeltis calligaster calligaster

Lampropeltis getulus holbrooki

Nerodia rhombifer rhombifer

Nerodia sipedon sipedon

Opheodrys aestivus

Storeria dekayi wrightorum

Storeria occipitomaculata occipitomaculata

Thamnophis proximus proximus

Thamnophis sirtalis sirtalis

Virginia valeriae elegans

Agkistrodon contortrix phaeogaster

Crotalus horridus

Source: Herron 1991b 
TABLE 5.5-6 Threatened, Endangered, or Special Concern Species Reported from St. Charles County, Missouri, and Potentially Occurring on or near the Weldon Spring Site

\begin{tabular}{|c|c|c|}
\hline \multicolumn{3}{|c|}{ STATUS } \\
\hline SPECIES & FEDERAL (n) & STATE $^{(b)}$ \\
\hline \multicolumn{3}{|l|}{ Plante } \\
\hline Starwort (variety) & $T$ & Endangered \\
\hline Rose turtlehead & $3 c$ & Endangered \\
\hline Arrow arum & $\cdot-$ & Rare \\
\hline Star duckweed & .. & Rare \\
\hline Bugseed (variety) & $\because$ & Watch List \\
\hline Adder's tongue fern (variety) & -- & Watch List \\
\hline Salt meadow grass (variety) & -. & Undetermined \\
\hline \multicolumn{3}{|l|}{ Fish } \\
\hline Pallid sturgeon & $E$ & Endangered \\
\hline Pugnose minnow & $\cdot \cdot$ & Watch List \\
\hline Sturgeon chub & $\mathrm{C} 2$ & Rare \\
\hline Sicklefin chub & $\mathrm{C}_{2}$ & Rare \\
\hline Alligator gar & .. & Rare \\
\hline Brown bullhead & -. & Raro \\
\hline Alabama shad & -. & Rare \\
\hline Starhead topminnow & $\cdots$ & Watch List \\
\hline Western sand darter & $\cdot \cdot$ & Watch List \\
\hline Paddlefish & $3 C$ & Watch List \\
\hline \multicolumn{3}{|l|}{ Reptiles and Amphibians } \\
\hline Western fox snake & .. & Endangered \\
\hline Eastern massauga rattlesnake & $\mathrm{C}_{2}$ & Endangered \\
\hline Western smooth green snake & $\cdot \cdot$ & Endangered \\
\hline Wood frog & $\cdot-$ & Rare \\
\hline Northern crawfish frog & -. & Watch List \\
\hline Blanding's turtle & -. & Endangered \\
\hline Alligator snapping turtle & $\mathrm{C} 2$ & Rare \\
\hline \multicolumn{3}{|l|}{ Mammals } \\
\hline Long-tailed weasel & .. . & Rare \\
\hline
\end{tabular}


TABLE 5.5-6 Threatened, Endangered, or Special Concern Species Reported from St. Charles County, Missouri, and Potentially Occurring on or near the Weldon Spring Site (Continued)

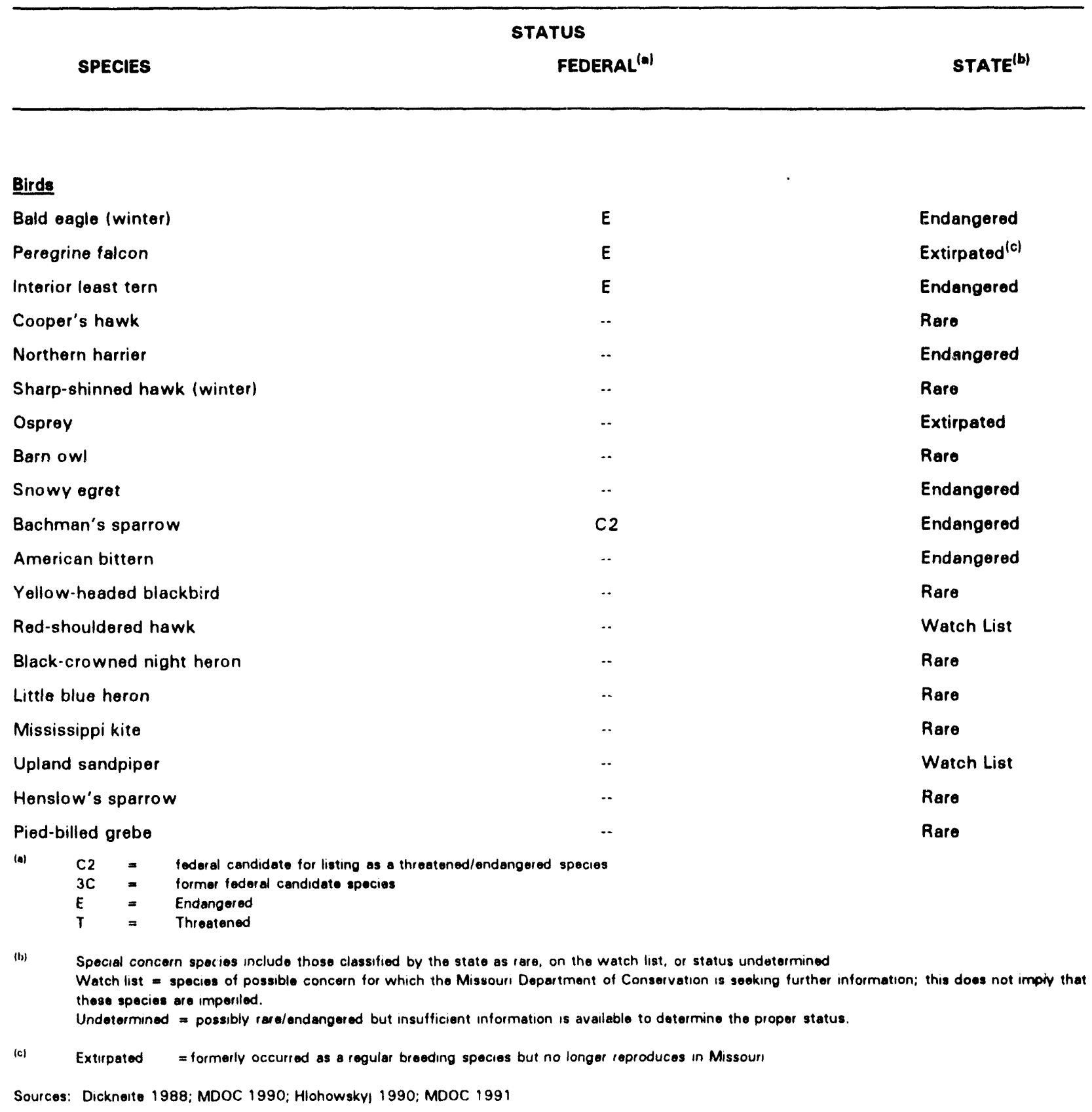




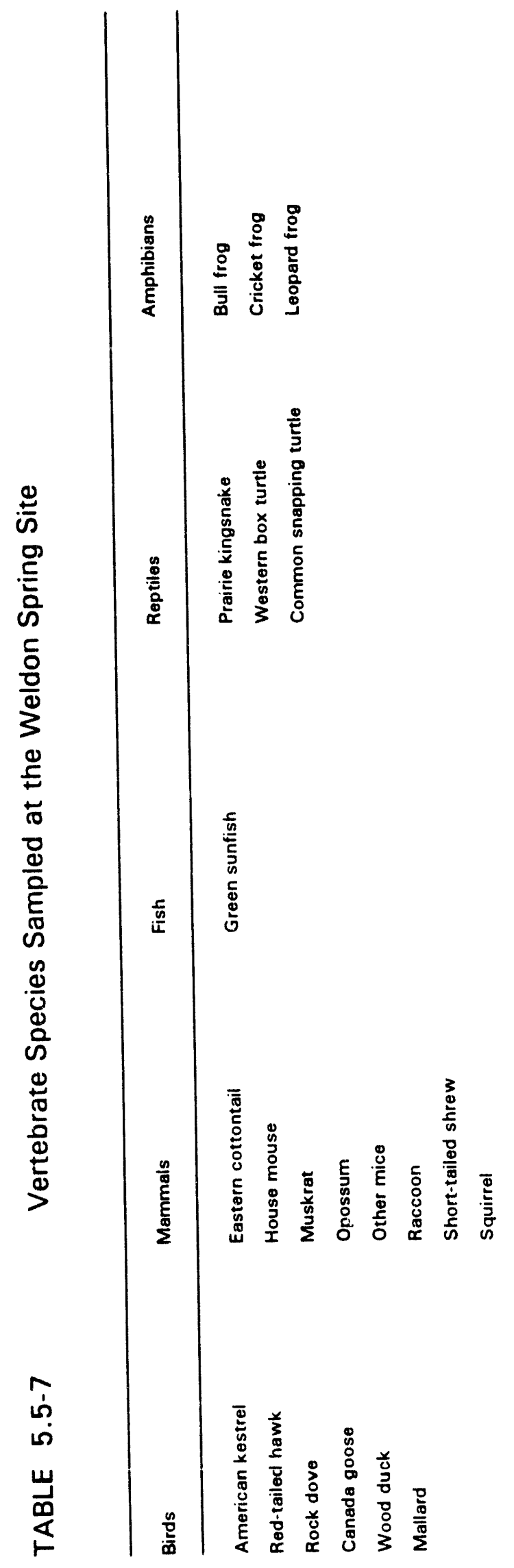

옹

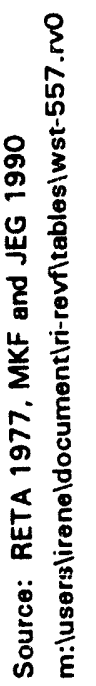




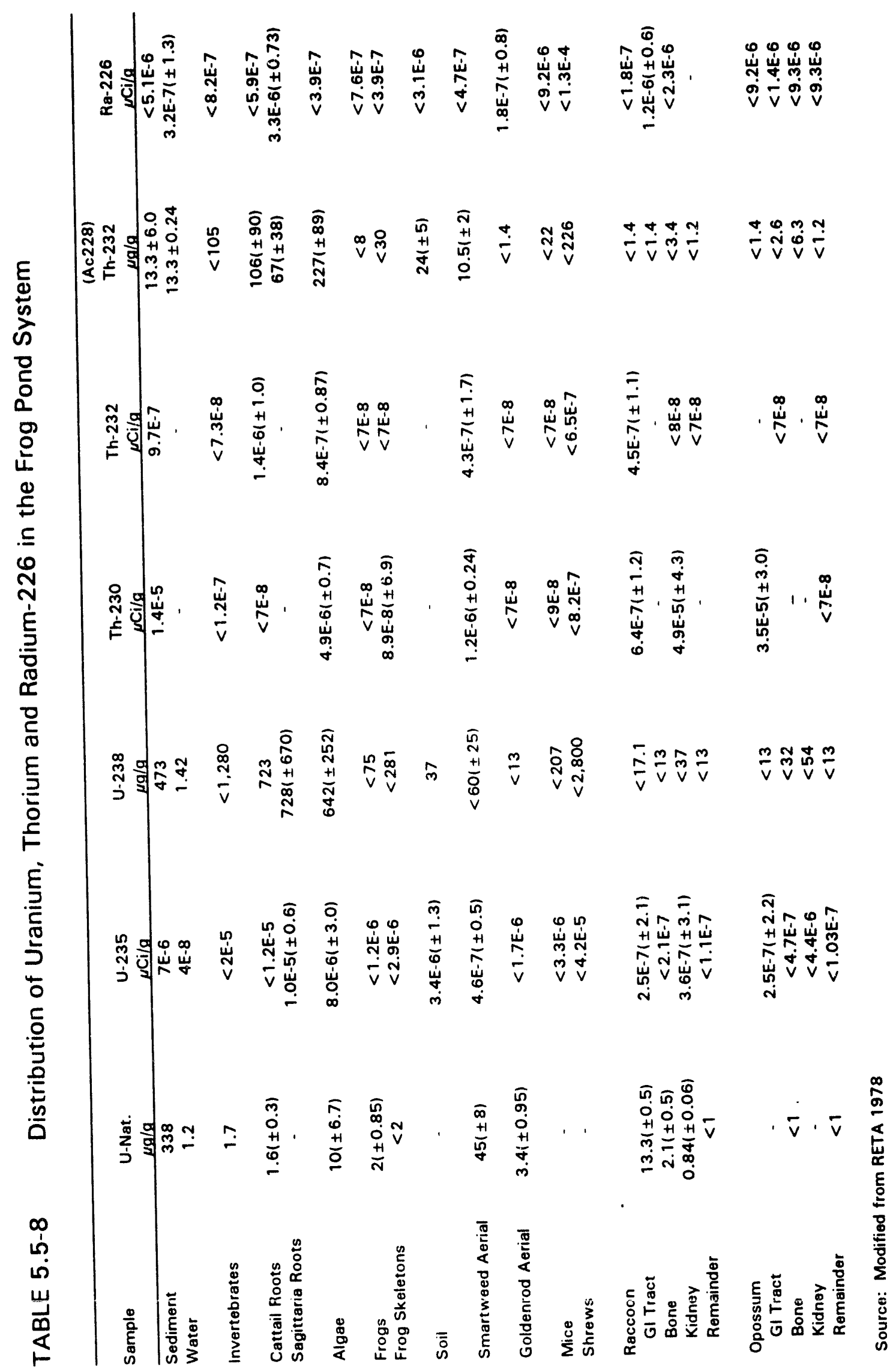

옹 


\section{TABLE 5.5-9 Natural Uranium Concentrations in Fish and Water Bodies of the Busch Wildlife Area}

\begin{tabular}{|c|c|c|c|}
\hline & $\begin{array}{c}\text { nat- }- \text { in water }_{(\mathrm{a})} \\
(\mathrm{pC \textrm {Ci }} /)^{(1)}\end{array}$ & $\begin{array}{l}\text { Fish } \\
\text { Type }\end{array}$ & $\begin{array}{c}\text { nat-U in fish } \\
\quad(p C i / g)\end{array}$ \\
\hline Lake 34 & $18-33$ & $\begin{array}{l}\text { Bluegill" } \\
\text { Tiger Musky } \\
\text { Channel Catfish } \\
\text { Grass Carp }\end{array}$ & $\begin{array}{l}0.06 \\
0.008 \\
0.005 \\
0.005\end{array}$ \\
\hline Lake 25 & $8-21$ & $\begin{array}{l}\text { Largemouth Bass } \\
\text { Channel Catfish }\end{array}$ & $\begin{array}{l}0.005 \\
0.002\end{array}$ \\
\hline Lake 36 & $9-53$ & $\begin{array}{l}\text { Largemouth Bass } \\
\text { Channel Catfish }\end{array}$ & $\begin{array}{l}0.005 \\
0.002\end{array}$ \\
\hline Femme Osage Slough & $8-47$ & $\begin{array}{l}\text { Bluegill" } \\
\text { Carp } \\
\text { Buffalo }\end{array}$ & $\begin{array}{l}0.02 \\
0.012 \\
0.009\end{array}$ \\
\hline $\begin{array}{l}\text { Background (Little } \\
\text { Dixie Lake) }\end{array}$ & -. & $\begin{array}{l}\text { Bluegill " } \\
\text { Largemouth Bass } \\
\text { Channel Catfish }\end{array}$ & $\begin{array}{l}0.02 \\
0.005 \\
0.009\end{array}$ \\
\hline
\end{tabular}

- Bluegill were not filleted; they were decapitated, eviscerated and scaled

$\begin{array}{ll}\text { Source: } & \text { (a) DOE 1987c } \\ & \text { (b) deRoos, C. 1984 }\end{array}$ 
TABLE 5.5-10 Radiological Concentrations in Fish Biota

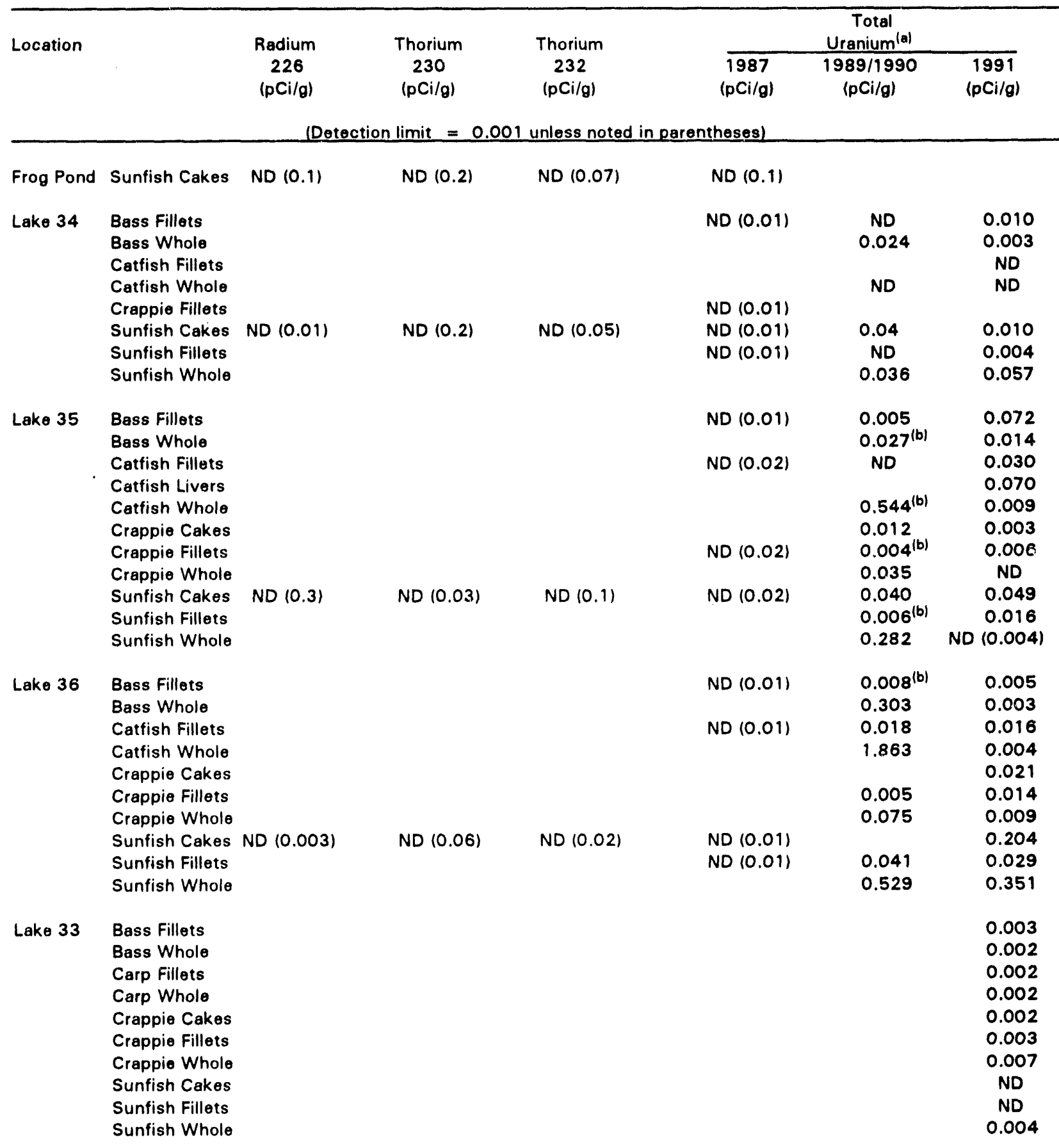


TABLE 5.5-10

Radiological Concentrations in Fish Biota (Continued)

\begin{tabular}{|c|c|c|c|c|c|c|}
\hline Location & Radium & Thorium & Thorium & & $\begin{array}{c}\text { Total } \\
\text { Uranium }^{(0)}\end{array}$ & \\
\hline & $\begin{array}{c}226 \\
(\mathrm{pCi} / g)\end{array}$ & $\begin{array}{c}230 \\
(\mathrm{pCi} / \mathrm{g})\end{array}$ & $\begin{array}{c}232 \\
(\mathrm{pCi} / \mathrm{g})\end{array}$ & $\begin{array}{l}1987 \\
(\mathrm{pCi} / \mathrm{g})\end{array}$ & $\begin{array}{c}1989 / 1990 \\
(\mathrm{pCi} / \mathrm{g})\end{array}$ & $\begin{array}{l}1991 \\
(\mathrm{pCi} / \mathrm{g})\end{array}$ \\
\hline
\end{tabular}

Lake 37 Bass Fillets

Bass Whole

Catfish Fillets

Catfish Whole

Sunfish Cakes

Sunfish Fillets

Sunfish Whole
ND (0.02)

ND (0.2)

ND (0.2)

ND (0.3)

ND (0.01)

$\begin{array}{cc}\text { ND } & \text { ND } \\ \text { ND } & 0.007 \\ 0.003 & \\ 0.013 & 0.003\end{array}$

$0.013 \quad 0.003$

ND $\quad 0.003$

(a) $1989=$ Uranium-234 concentration included in total uranium

(b) 2 samples; all other data based on 1 sample.

m:luserslireneldocument|ri-revf|tablesiwst-5510.rvo 


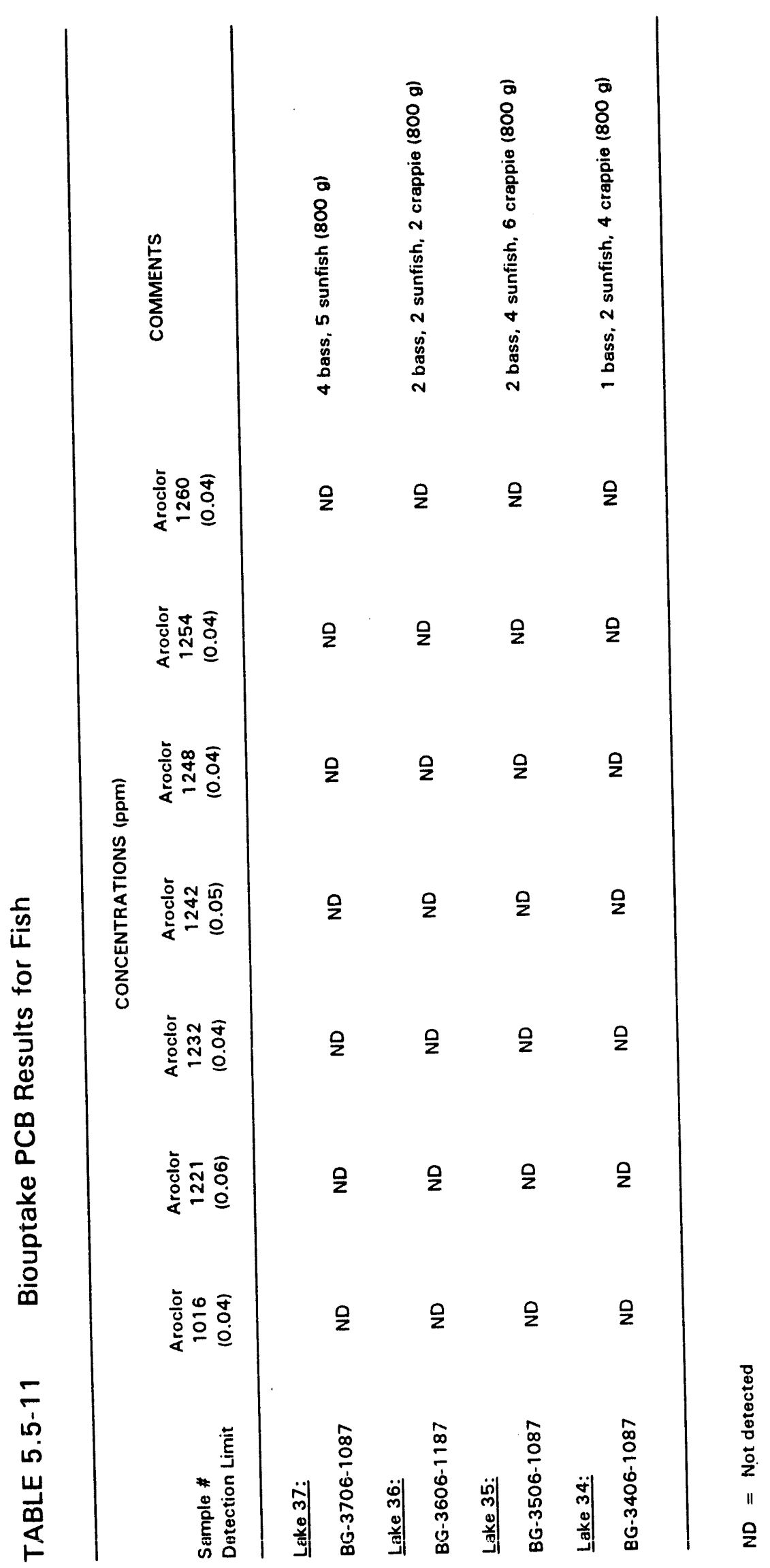

พ
ำ
음

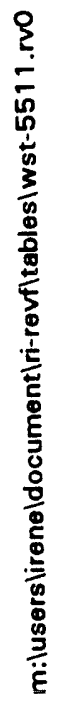




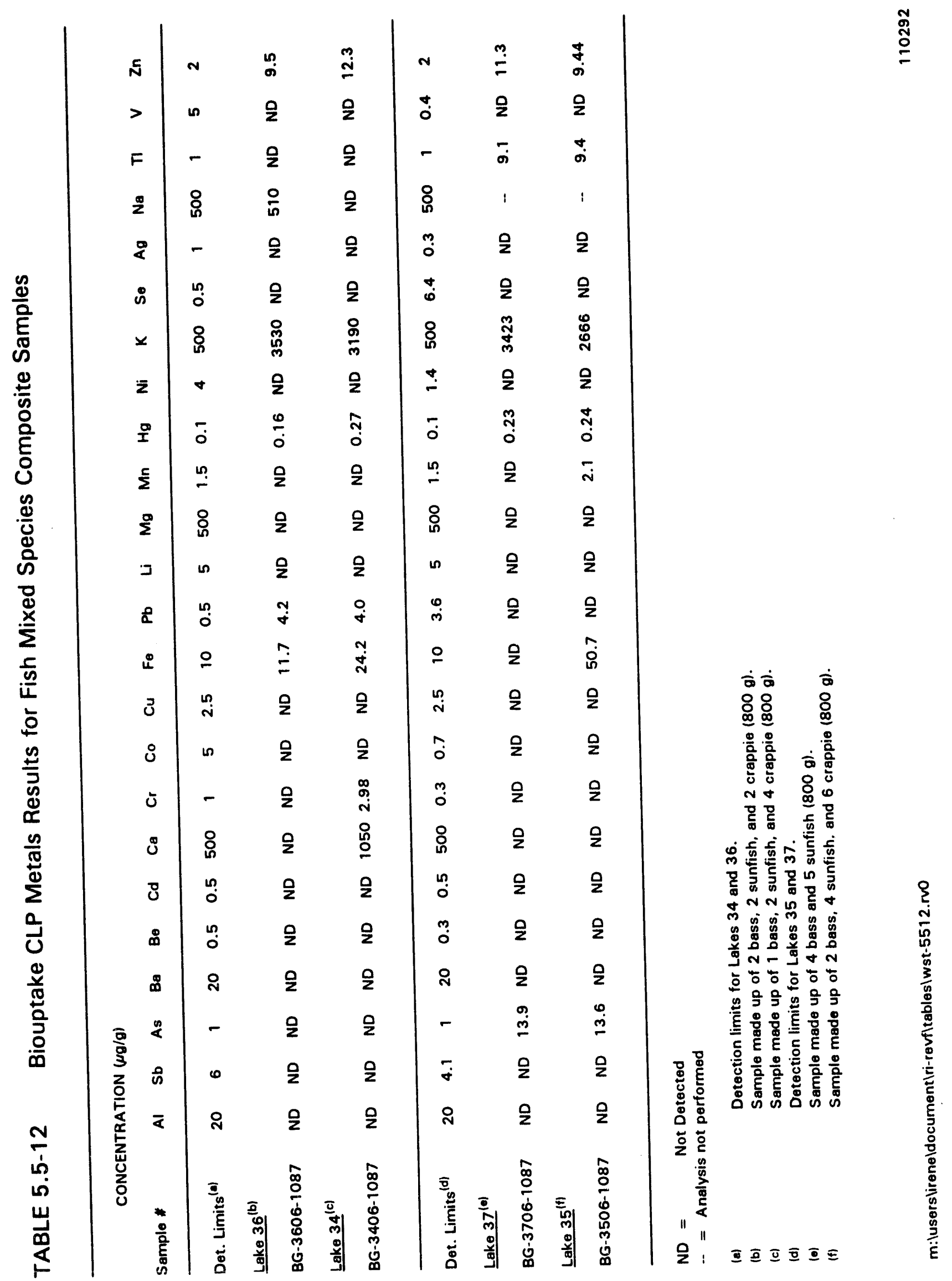


TABLE 5.5-13 Comparison of Bioaccumulation Factors (B.F.) for Uranium

\begin{tabular}{|c|c|c|c|c|}
\hline Area & Species & $\begin{array}{c}\text { Gilbert } \\
\text { B.F. }\end{array}$ & $\begin{array}{l}\text { NCRP } \\
\text { B.F. }\end{array}$ & $\begin{array}{c}\text { WSSRAP } \\
\text { B.F. }\end{array}$ \\
\hline Frog Pond & Sunfish Cakes & 2.0 & $0.7-38$ & 0.23 \\
\hline \multirow[t]{8}{*}{ Lake 34} & Bass Fillets & 2.0 & $0.5-0.7$ & 0.59 \\
\hline & Bass Whole & 2.0 & $0.5-0.7$ & 0.15 \\
\hline & Catfish Fillets & 2.0 & $0.7-38$ & 0.008 \\
\hline & Catfish Whole & 2.0 & $0.7 \cdot 38$ & 0.008 \\
\hline & Crappie Fillets & 2.0 & $0.5-0.7$ & 0.076 \\
\hline & Sunfish Cakes & 2.0 & $0.7-38$ & 0.167 \\
\hline & Sunfish Fillets & 2.0 & $0.7-38$ & 0.40 \\
\hline & Sunfish Whole & 2.0 & $0.7 \cdot 38$ & 0.348 \\
\hline \multirow[t]{11}{*}{ Lake 35} & Bass Fillets & 2.0 & $0.5-0.7$ & 1.132 \\
\hline & Bass Whole & 2.0 & $0.5 \cdot 0.7$ & 0.728 \\
\hline & Catfish Fillets & 2.0 & $0.7-38$ & 0.556 \\
\hline & Catfish Livers & 2.0 & $0.7 \cdot 38$ & 1.931 \\
\hline & Catfish Whole & 2.0 & $0.7-38$ & 10.93 \\
\hline & Crappie Cakes & 2.0 & $0.5 \cdot 0.7$ & 0.201 \\
\hline & Crappie Fillets & 2.0 & $0.5-0.7$ & 0.231 \\
\hline & Crappie Whole & 2.0 & $0.5 \cdot 0.7$ & 0.648 \\
\hline & Sunfish Cakes & 2.0 & $0.7 \cdot 38$ & 1.089 \\
\hline & Sunfish Fillets & 2.0 & $0.7 \cdot 38$ & 0.375 \\
\hline & Sunfish Whole & 2.0 & $0.7 \cdot 38$ & 5.259 \\
\hline \multirow[t]{10}{*}{ Lake 36} & Bass Fillets & 2.0 & $0.5-0.7$ & 0.034 \\
\hline & Bass Whole & 2.0 & $0.5-0.7$ & 0.970 \\
\hline & Catfish Fillets & 2.0 & $0.7 \cdot 38$ & 0.073 \\
\hline & Catfish Whole & 2.0 & $0.7 \cdot 38$ & 5.925 \\
\hline & Crappie Cakes & 2.0 & $0.5-0.7$ & 0.98 \\
\hline & Crappie Fillets & 2.0 & $0.5-0.7$ & 0.046 \\
\hline & Crappie Whole & 2.0 & $0.5-0.7$ & 0.198 \\
\hline & Sunfish Cakes & 2.0 & $0.7 \cdot 38$ & 0.663 \\
\hline & Sunfish Fillets & 2.0 & $0.7 \cdot 38$ & 0.177 \\
\hline & Sunfish Whole & 2.0 & $0.7-38$ & 4.190 \\
\hline
\end{tabular}

- B.F. are average concentrations and non-detects at lower limit of detection.

Adapted from Gilbert, et al. 1985 and NCRP 1985 


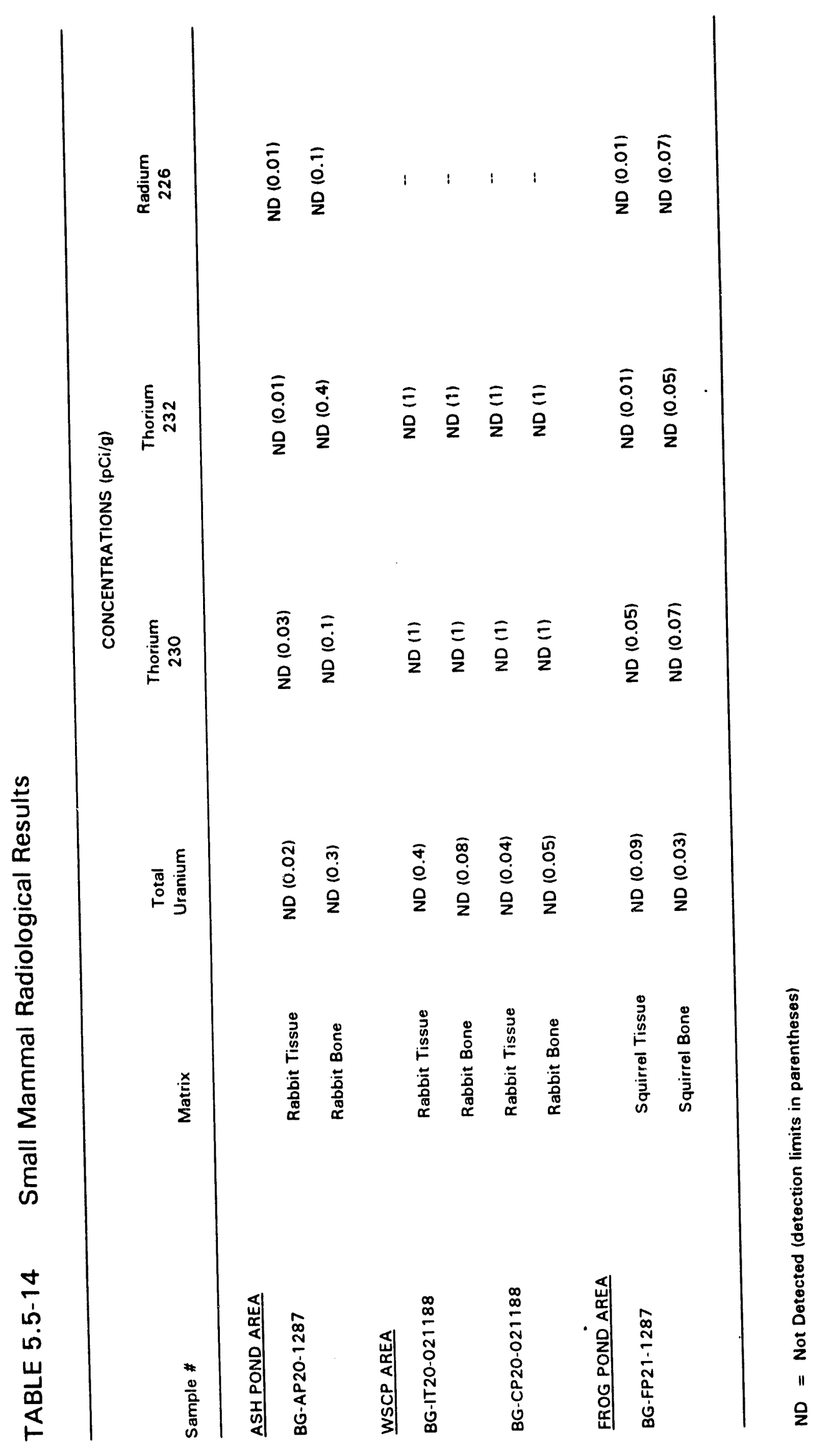




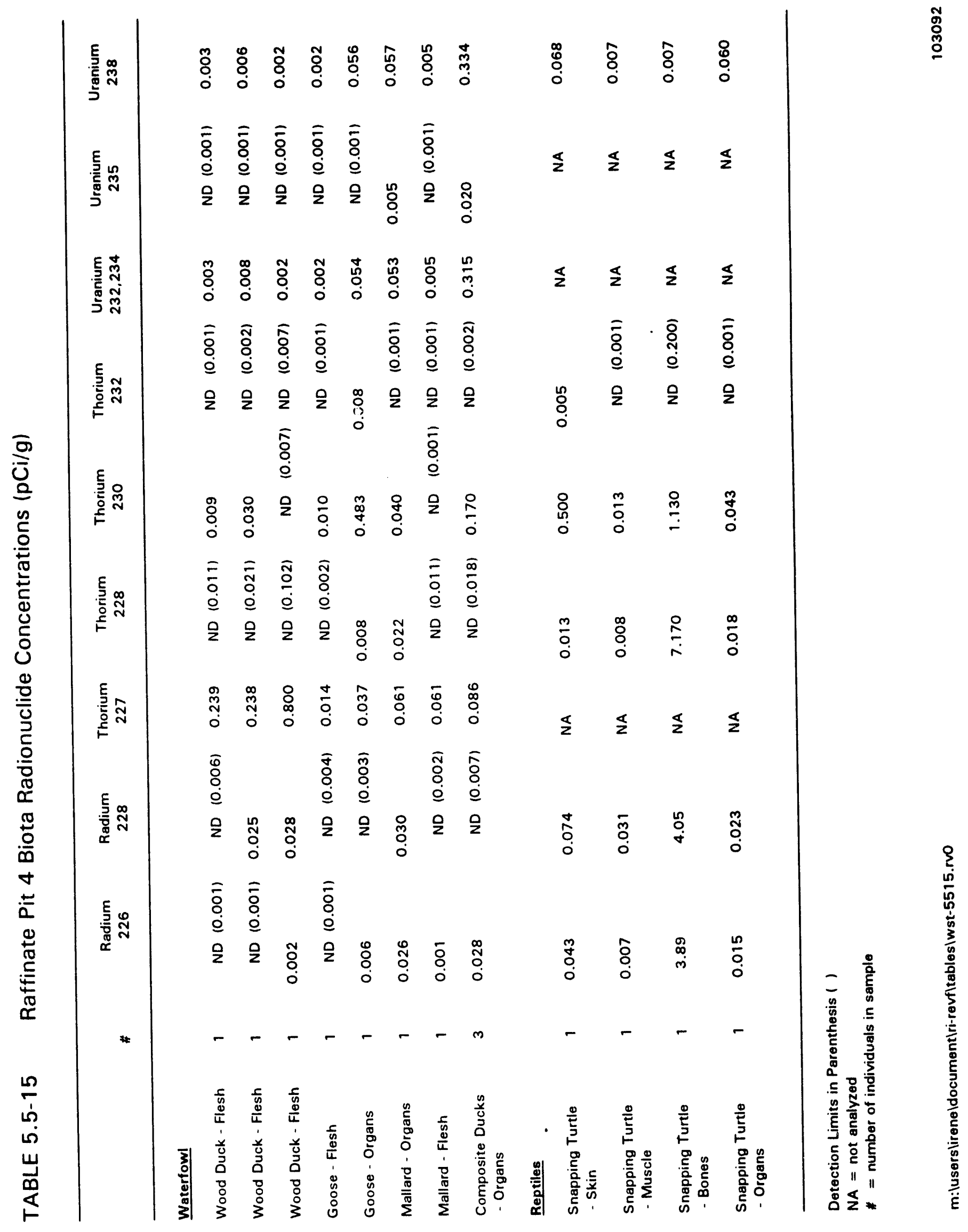




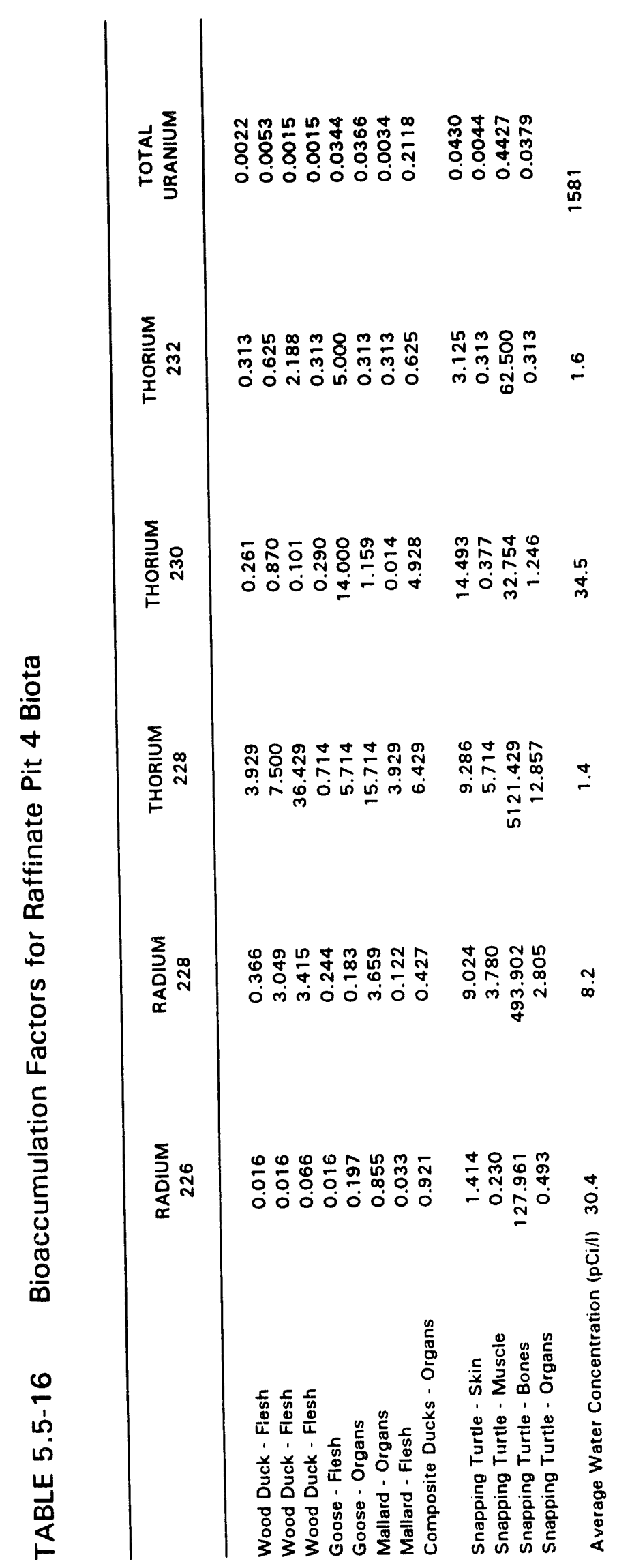


TABLE 5.6-1 Radon Measurements at the Weldon Spring Site in $1989^{(a)}$

\begin{tabular}{|c|c|c|c|c|c|c|c|}
\hline \multirow[b]{2}{*}{$\begin{array}{c}\text { Location } \\
\text { I.D. }\end{array}$} & \multirow{2}{*}{$\frac{1 \text { st Quarter }}{\text { pCin }}$} & \multirow{2}{*}{$\frac{\text { 2nd Querter }}{\mathrm{pCin}}$} & \multirow{2}{*}{ 3rd Quarter } & \multirow{2}{*}{ 4th Quarter } & \multicolumn{2}{|c|}{ A.nnual Average(b) } & \multirow{2}{*}{$\begin{array}{c}\text { Percent } \\
\text { of } \\
\text { Guidelinelc }\end{array}$} \\
\hline & & & & & $\mathrm{pCi} / \mathrm{l}$ & $\begin{array}{l}\text { Std. } \\
\text { Dov. }\end{array}$ & \\
\hline \multicolumn{8}{|c|}{ QUARRY } \\
\hline RD- 1001 & 1.0 & 1.5 & 2.0 & 1.5 & 1.5 & 0.2 & 32 \\
\hline RD- 1002 & 1.8 & 1.3 & 1.3 & 2.1 & 1.6 & 0.2 & 35 \\
\hline RD-1003 & 4.7 & 0.8 & 1.3 & 1.6 & 2.1 & 0.4 & 52 \\
\hline RD- 1004 & 1.1 & 0.5 & 0.7 & 1.2 & 0.8 & 0.1 & 8 \\
\hline RD-1005 & 1.8 & 0.7 & 0.8 & 1.3 & 1.1 & 0.2 & 18 \\
\hline RD-1006 & 0.4 & 0.4 & 0.7 & 1.1 & 0.6 & 0.1 & - \\
\hline
\end{tabular}

\begin{tabular}{|c|c|c|c|c|c|c|c|}
\hline \multicolumn{8}{|c|}{ CHEMICAL PLANT } \\
\hline RD-2001 & 0.7 & 0.4 & 0.4 & 0.7 & 0.5 & 0.1 & - \\
\hline RD-2002 & 0.5 & 0.4 & 0.6 & 0.6 & 0.5 & 0.1 & - \\
\hline RD-2003 & 0.6 & 0.4 & 1.1 & 0.6 & 0.6 & 0.1 & - \\
\hline RD. 2004 & 0.3 & 0.4 & 0.8 & 0.6 & 0.5 & 0.1 & - \\
\hline RD-2005 & 0.3 & 0.3 & 0.5 & 0.5 & 0.4 & 0.1 & - \\
\hline RD-2006 & 0.4 & 0.5 & 0.7 & 0.7 & 0.6 & 0.1 & - \\
\hline
\end{tabular}

RAFFINATE PITS

\begin{tabular}{llllllll}
\hline RD-3001 & 0.6 & 0.3 & 0.5 & 0.6 & 0.5 & 0.1 & - \\
RD-3002 & 0.3 & 0.3 & 0.5 & 0.6 & 0.4 & 0.1 & -1 \\
RD-3003 & 0.3 & 0.4 & 0.7 & 0.6 & 0.5 & 0.1 & - \\
RD-3004 & 0.3 & 0.4 & 0.7 & 0.4 & 0.4 & & 0.1 \\
\hline
\end{tabular}

OFF SITE

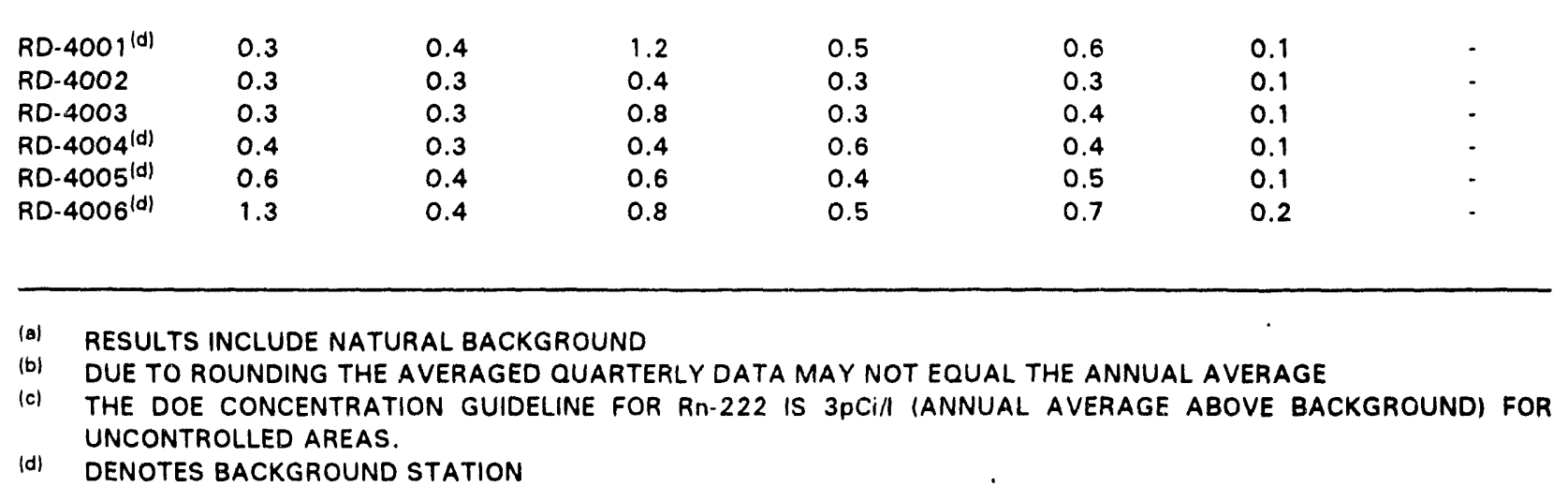

See Figure 5.6-1 
TABLE 5.6-2 Gamma Radiation Exposure Rate Monitoring Results ${ }^{(a)}$ (mR/year)

\begin{tabular}{|c|c|c|c|c|c|c|}
\hline $\begin{array}{l}\text { LOCATION } \\
\text { I.D. }\end{array}$ & $\begin{array}{c}\text { FIRST } \\
\text { QUARTER }\end{array}$ & $\begin{array}{l}\text { SECOND } \\
\text { QUARTER }\end{array}$ & $\begin{array}{l}\text { THIRD } \\
\text { QUARTER }\end{array}$ & $\begin{array}{l}\text { FOURTH } \\
\text { QUARTER }\end{array}$ & $\begin{array}{l}\text { ANNUAL } \\
\text { TOTAL (b) }\end{array}$ & $\begin{array}{l}2 \text { ST } \\
\text { DEV }\end{array}$ \\
\hline \multicolumn{7}{|c|}{ QUARRY } \\
\hline TD. 1001 & 27 & $T E^{(c)}$ & 21 & 25 & 98 & 7 \\
\hline TD. 1002 & 27 & $T E^{(c)}$ & 16 & 18 & 80 & 6 \\
\hline TD-1003 & 23 & $T E^{(c)}$ & 17 & 22 & 82 & 8 \\
\hline TD.1004 & 22 & $T E^{(c)}$ & 16 & 18 & 75 & 15 \\
\hline TD-1005 & 23 & $T E^{(c)}$ & 17 & 21 & 80 & 16 \\
\hline TD. 1006 & 20 & $T E^{(c)}$ & 15 & 19 & 71 & 11 \\
\hline
\end{tabular}

CHEMICAL PLANT

\begin{tabular}{lllllll}
\hline & & & & & \\
$T D-2001$ & 18 & $T E^{(c)}$ & 13 & 22 & 71 & 7 \\
$T D-2002$ & 20 & $T E^{(c)}$ & 15 & 17 & 68 & 8 \\
$T D-2003$ & 26 & $T E^{(c)}$ & 13 & 20 & 79 & 20 \\
$T D-2004$ & 20 & $T E^{(c)}$ & 14 & 18 & 69 & 11 \\
$T D-2005$ & 18 & $T E^{(c)}$ & 13 & 16 & 62 & 4 \\
$T D-2006$ & 18 & $T E^{(c)}$ & 15 & 20 & 70 & 11 \\
\hline
\end{tabular}

RAFFINATE PITS

\begin{tabular}{|c|c|c|c|c|c|c|}
\hline TD-3001 & 20 & $T E^{|c|}$ & 15 & 20 & 73 & 12 \\
\hline TD-3002 & 16 & $T E^{(c)}$ & 11 & 15 & 56 & 7 \\
\hline TD-3003 & 18 & $T E^{(c)}$ & 14 & 18 & 67 & 6 \\
\hline TD-3004 & 18 & $T E^{(c)}$ & 15 & 15 & 65 & 10 \\
\hline
\end{tabular}

OFF SITE

\begin{tabular}{|c|c|c|c|c|c|c|}
\hline$T D-4001^{\{d\}}$ & 21 & $T E^{(c)}$ & 15 & 21 & 75 & 12 \\
\hline TD-4002 & 15 & $T E^{(c)}$ & 11 & 13 & 52 & 7 \\
\hline TD-4003 & 18 & $T E^{(c)}$ & 12 & 14 & 59 & 10 \\
\hline TD $-4004^{(d)}$ & . & $T E^{(c)}$ & . & 17 & 70 & 3 \\
\hline TD-4005 (d) & 17 & $T E^{(c)}$ & . & 16 & 66 & 7 \\
\hline TD $-4006^{(d)}$ & 17 & $T E^{(c)}$ & 14 & 16 & 62 & 6 \\
\hline
\end{tabular}

(a)RESULTS INCLUDE NATURAL BACKGROUND

(b) DUE TO ROUNDING THE SUMMED QUARTERLY DATA MAY NOT EQUAL THE ANNUAL TOTAL.

(c) TE = TRANSPORTATION EXPOSURE - NOT REPORTED

IdIDENOTES BACKGROUND STATION

See Figure 5.6-1 
TABLE 5.6-3 Radiological Air Particulate Results 1989

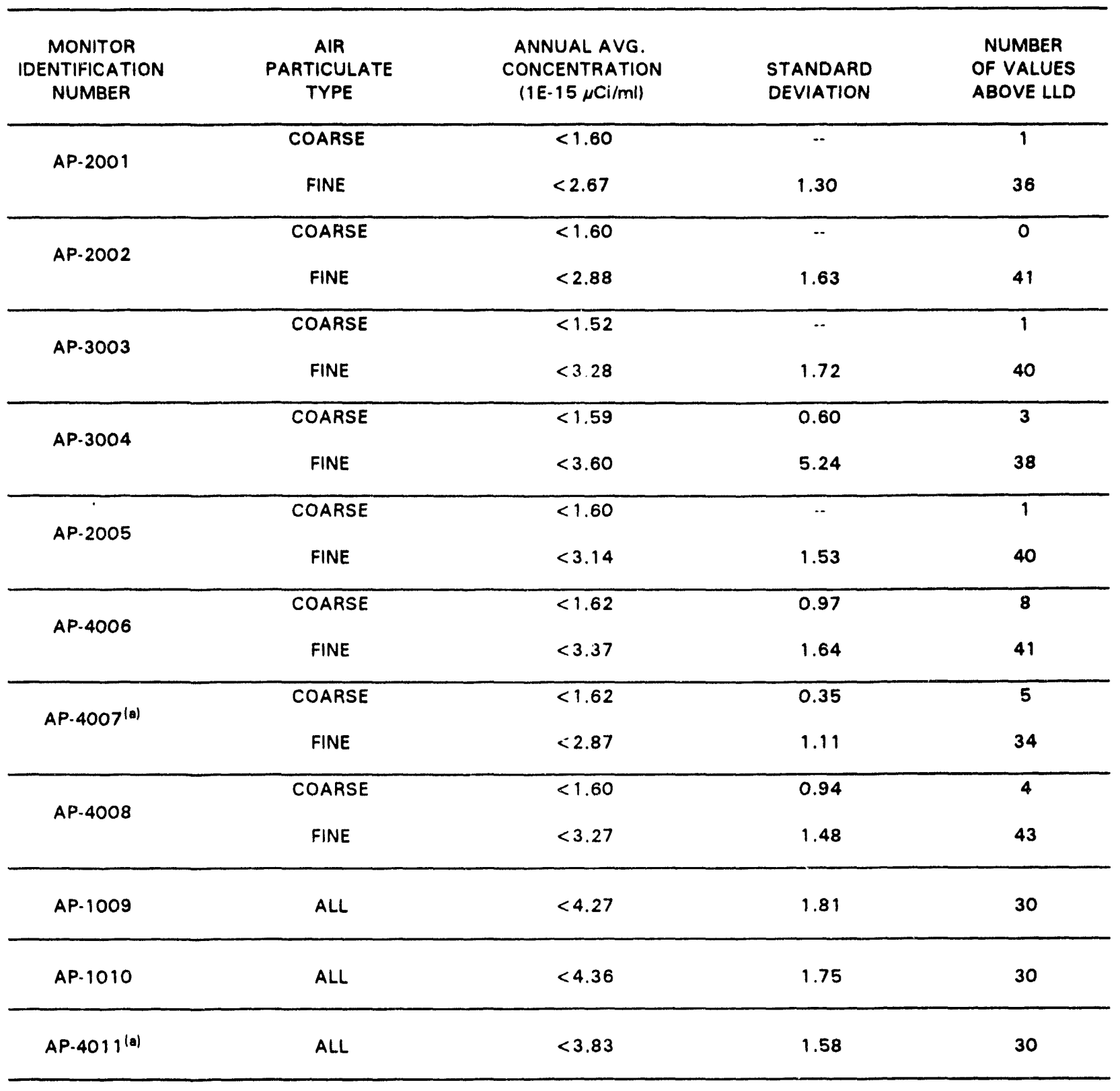

(a) INDICATES BACKGROUND MONITOR STATION 


\section{TABLE 5.6-4 Asbestos Monitoring Results for 1988-1989 (Phase Contrast Microscopy)}

\begin{tabular}{lcccc}
\hline & & 1988 & \\
\hline $\begin{array}{l}\text { Sampling } \\
\text { Location }\end{array}$ & Min & $\begin{array}{c}\text { Concentration }\left(f / \mathrm{cm}^{2}\right)(\mathbf{a})(\mathrm{b}) \\
\text { Max }\end{array}$ & Averago & No. of Samples \\
\hline Site Perimeter & $<0.0005$ & 0.033 & 0.004 & 85 \\
FHHS(c) & $<0.0005$ & 0.013 & 0.0024 & 52
\end{tabular}

1989

\begin{tabular}{|c|c|c|c|c|}
\hline \multirow{2}{*}{$\begin{array}{l}\text { Sampling } \\
\text { Location }\end{array}$} & \multicolumn{3}{|c|}{ Concentration $\left(f / \mathrm{cm}^{2}\right)$} & \multirow[b]{2}{*}{ No. of Samples } \\
\hline & Min & $\operatorname{Max}$ & Average & \\
\hline Site Perimeter & $<0.0005$ & 0.007 & 0.001 & 103 \\
\hline FHHS ${ }^{(c)}$ & $<0.0005$ & 0.017 & 0.001 & 186 \\
\hline
\end{tabular}

Notes:

(a) $f / \mathrm{cm}^{2}=$ fibers per cubic centimeter of air

(b) For those samples with fiber concentrations less than the limit of detection, the limit of detection was used to calculate the overall average fiber concentration.

(c) FHHS $=$ Francis Howell High School 
TABLE 6.2-1 Chemical Properties of Nitroaromatics ${ }^{(a)}$

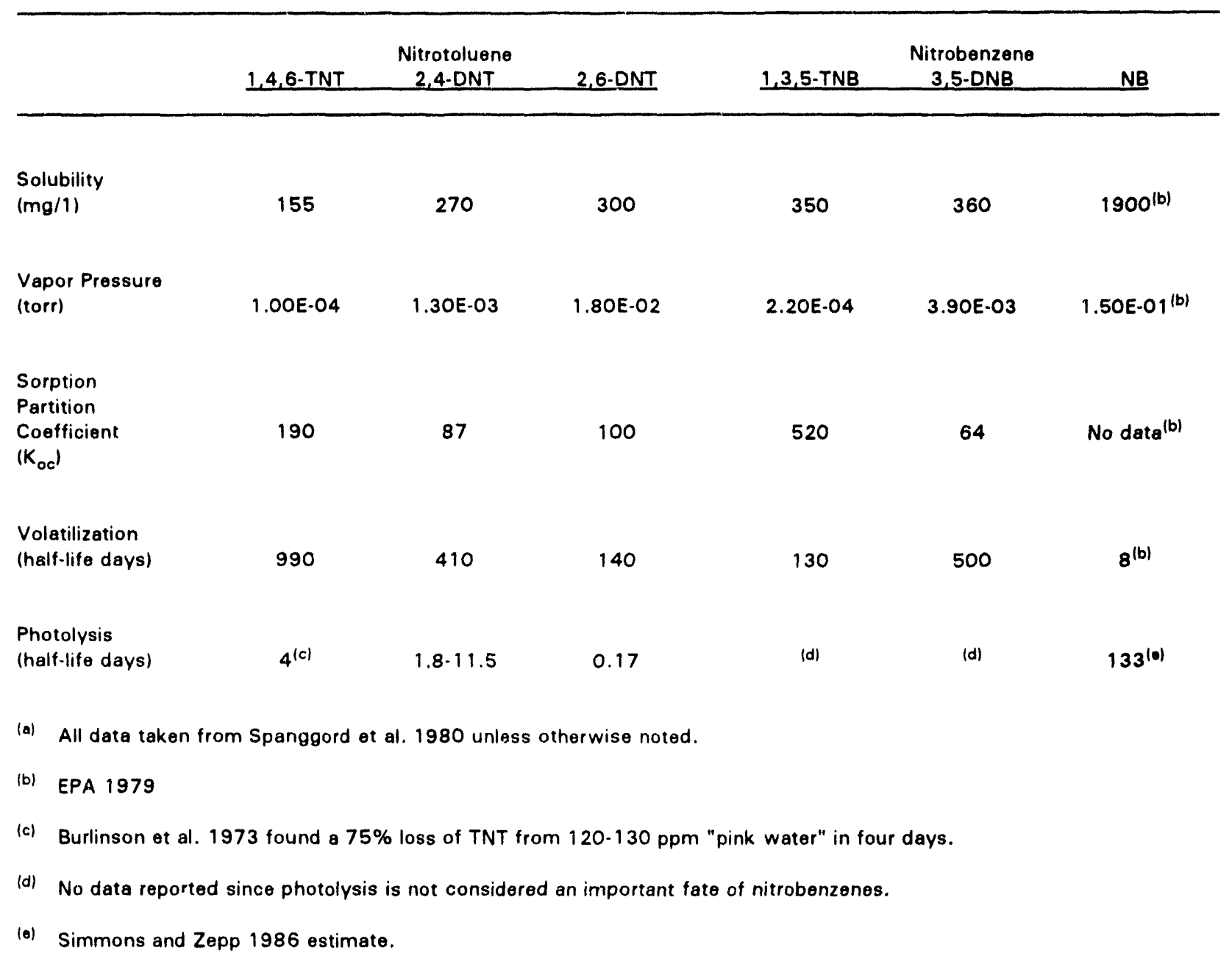



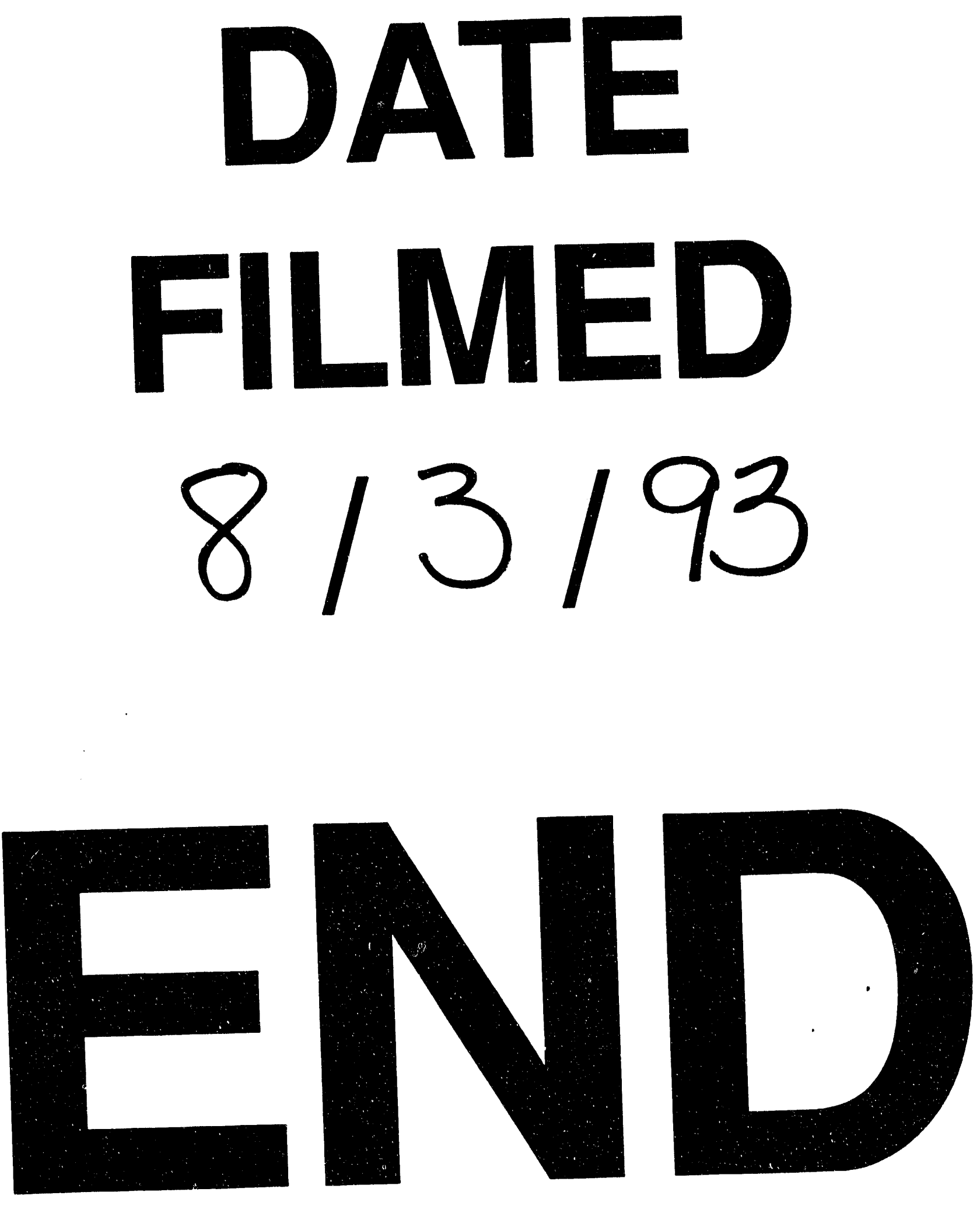
